WSRC-TR-94-0354

Unclassified

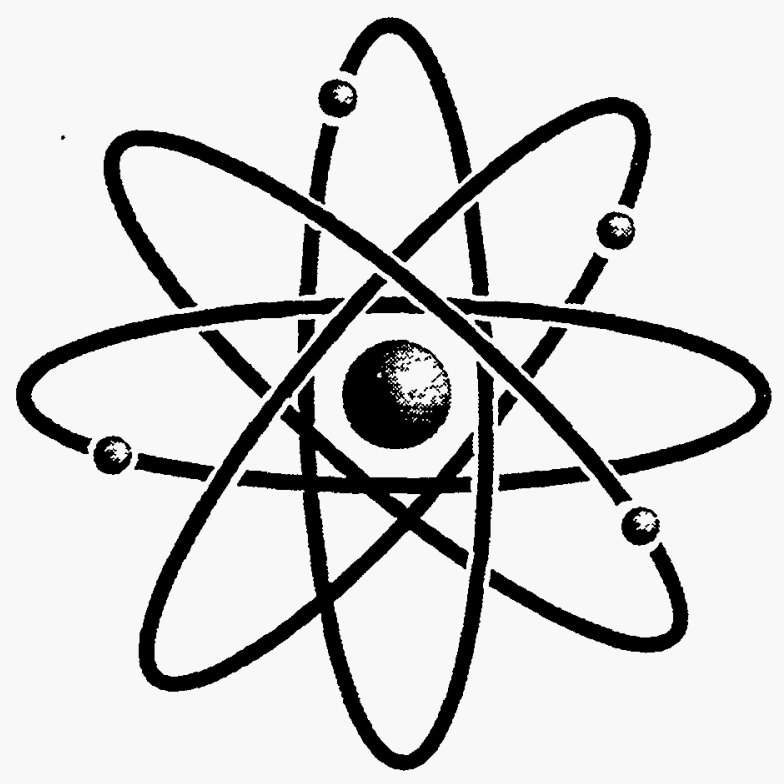

\title{
R-Area Reactor 1993 Annual Groundwater Monitoring Report
}

Publication Date: September 1994

Authorized Derivative Classifier:

Aoseph P. Kanglaiter, Enginuer 10-4-94

UNCLASSIFIED

Does Not Contain Unclassified

Controlled Nuclear Information

Westinghouse Savannah River Company

Savannah River Site

Aiken, SC 29808

MSTER

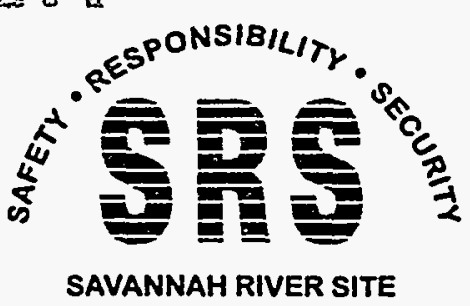

Prepared for the U.S. Department of Energy Under Contract Number DE-AC09-89SR18035 


\section{DISCLAIMER}

This report was prepared as an account of work sponsored by an agency of the United States Government. Neither the United States Government nor any agency thereof, nor any of their employees, makes any warranty, express or implied, or assumes any legal liability or responsibility for the accuracy. completeness, or usefulness of any information, apparatus, product, or process disclosed, or represents that its use would not infringe privately owned rights. Reference herein to any specific commercial product, process, or service by trade name, trademark, manufacturer, or otherwise does not necessarily constitute or imply its endorsement, recommendation, or favoring by the United States Government or any agency thereof. The vicws and opinions of authors expressed herein do not necessarily state or reflect those of the United States Government or any agency thereof.

This report has been reproduced directly from the best available copy.

Available to DOE and DOE contractors from the Office of Scientific and Technical Information. P. O. Box 62. Oak Ridge. TN 37831: prices available from (615) $576-8401$.

Available to the public from the National Technical Information Service, U. S. Department of Commerce, 5285 Port Royal Rd., Springfield, VA 22161 


\section{DISCLAIMER}

Portions of this document may be illegible in electronic image products. Images are produced from the best available original document. 


\section{Table of Contents}

Executive Summary ................................................................................................................. iii

Savannah River Site History ...............................................................................................

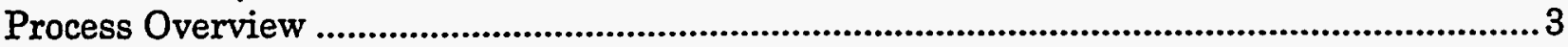

Reactor Area Processes ..........................................................................................................

Assembly ........................................................................................................................

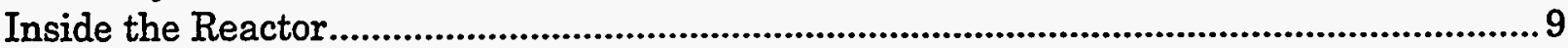

Post-Irradiation Cooling ....................................................................................................... 11

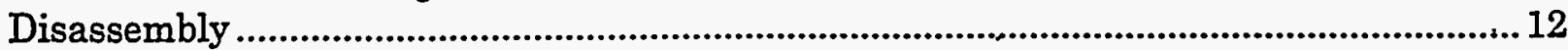

Heavy-Water Purification .................................................................................................. 14

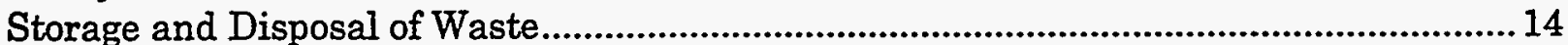

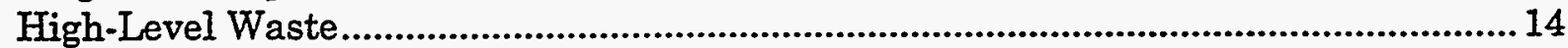

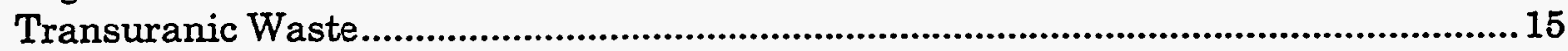

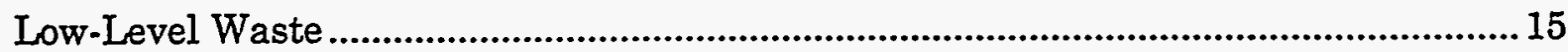

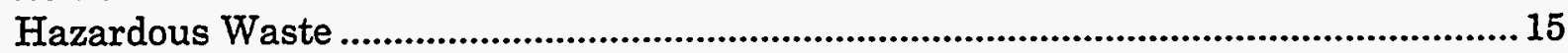

Mixed Waste ...................................................................................................................... 15

Sanitary Waste............................................................................................................. 17

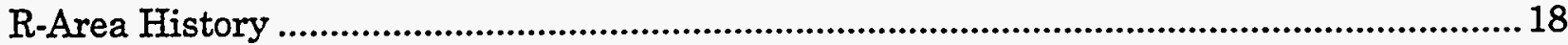

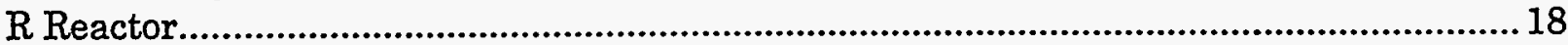

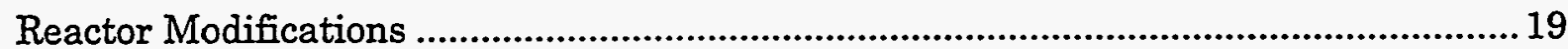

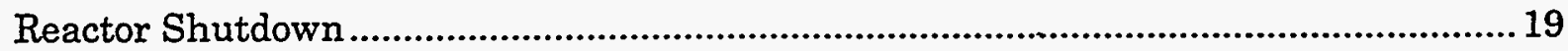

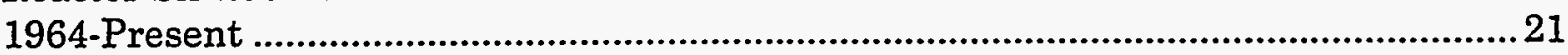

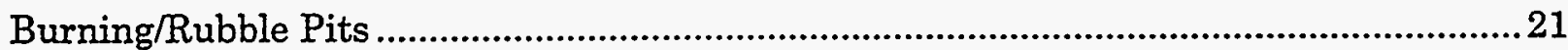

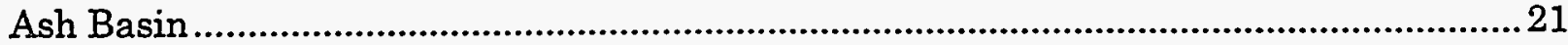

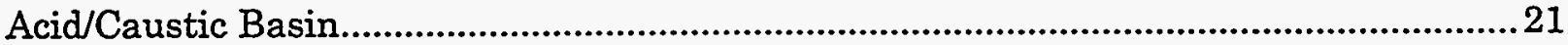

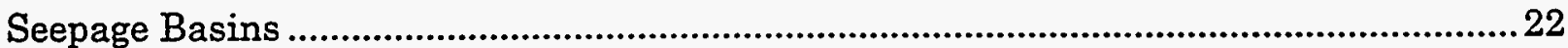

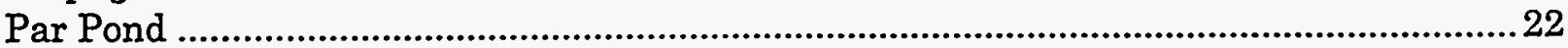

Bingham Pump Outage Pits.............................................................................................. 22

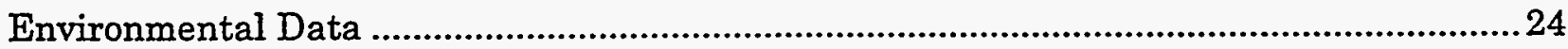

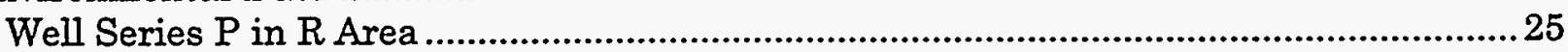

Acid/Caustic Basin (RAC Well Series) ..................................................................................26

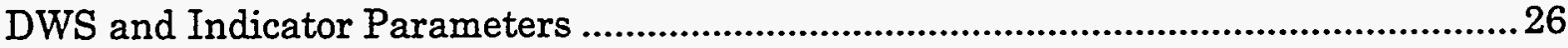

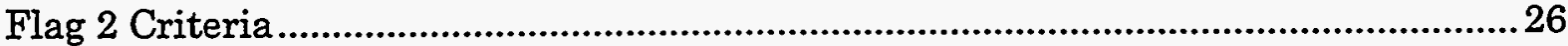

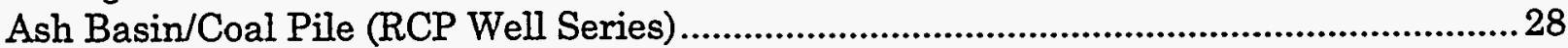

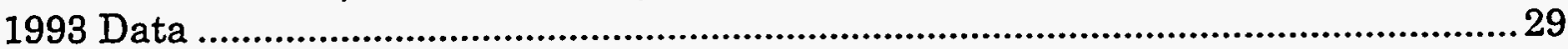

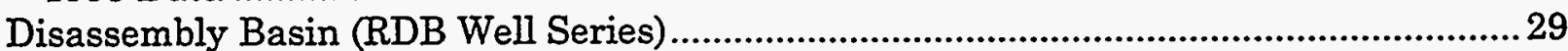

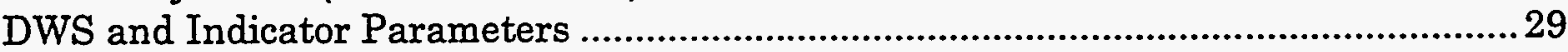

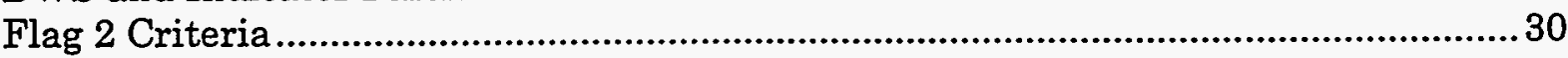

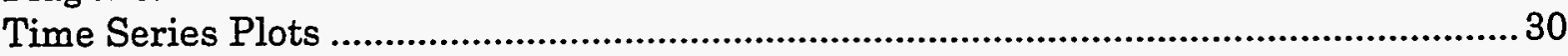

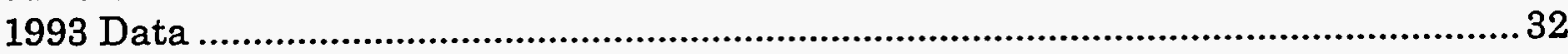




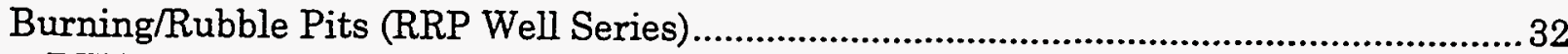

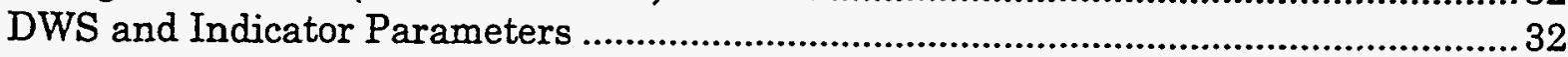

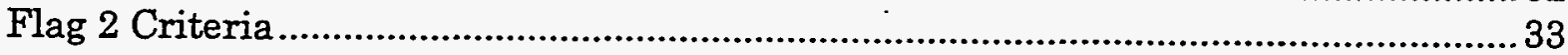

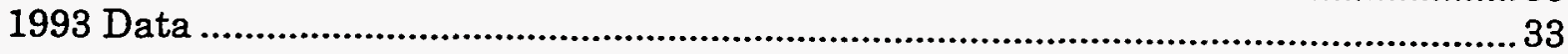

Seepage Basins (RSA, RSB, RSC, RSD, RSE, and RSF Well Series) ............................... 34

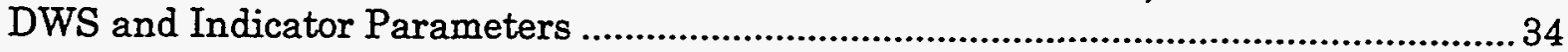

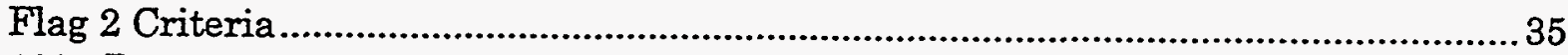

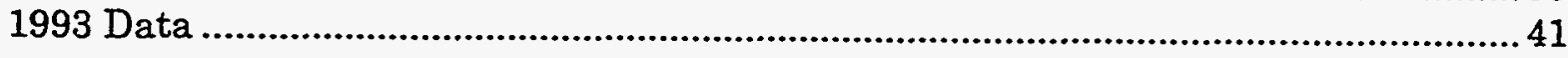

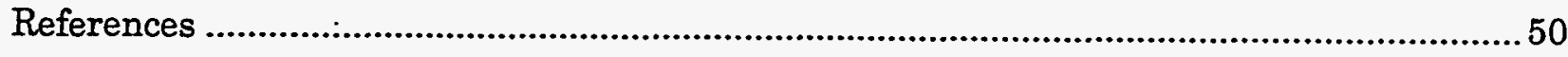

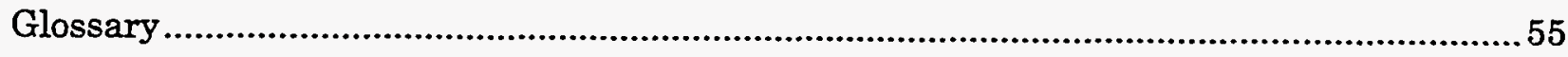

Appendix A: Final Primary Drinking Water Standards ................................................ A-1

Appendix B: Flagging Criteria .................................................................................. B-1

Appendix C: Groundwater Monitoring Results for Individual Wells during 1993 ........... C-1

Appendix D: Time Series Plots ........................................................................ D-1 


\section{Executive Summary}

Groundwater was sampled and analyzed during 1993 from wells monitoring the following locations in R Area: well cluster P20 east of R Area (one well each in the water table and the McBean formation), the R-Area Acid/Caustic Basin (the four water-table wells of the RAC series), the R-Area Ash Basin/Coal.Pile (one well of the RCP series in the Congaree formation and one in the water table), the R-Area Disassembly Basin (the three water-table wells of the RDB series), the RArea Burning/Rubble Pits (the four water-table wells of the RRP series), and the R-Area Seepage Basins (numerous water-table wells in the RSA, RSB, RSC, $\mathrm{RSD}, \mathrm{RSE}$, and RSF series).

Lead was the only constituent detected above its $50 \mu \mathrm{g} / \mathrm{L}$ standard in any but the seepage basin wells; it exceeded that level in one RDB well and in 23 of the seepage basin wells. Cadmium exceeded its drinking water standard (DWS) in 30 of the seepage basin wells, as did mercury in 10. Nitrate-nitrite was above DWS once each in two seepage basin wells.

Tritium was above DWS in six seepage basin wells, as was gross alpha activity in 22. Nonvolatile beta exceeded its screening standard in 29 wells.

Extensive radionuclide analyses were requested during 1993 for the RCP series and most of the seepage basin wells. Strontium-90 in eight wells was the only specific radionuclide other than tritium detected above DWS; it appeared to account for about one-half of the nonvolatile beta activity in those wells. 


\section{R-Area Reactor}

\section{Savannah River Site History}

In September 1949, the Soviet Union completed successful testing of its first atomic weapon. In response to this development, the United States Department of Defense began planning for a more diversified stockpile of nuclear armaments. In January 1950, President Harry Truman directed the United States Atomic Energy Commission (AEC) to increase research on all forms of nuclear defense, particularly the "super" or hydrogen weapon.

On June 12, 1950, the AEC contracted with E.I. du Pont de Nemours and Company to design, construct, and operate the Savannah River Plant for the purpose of producing materials for use in nuclear and thermonuclear weapons. Site surveys began in July 1950. The plant is located in Aiken, Barnwell, and Allendale counties in South Carolina, southeast of Augusta, Georgia (Figure 1).

Construction of the complex began in February 1951, and the heavy water processing plant became the first facility in operation in August 1952. $R$ Reactor was the first production unit to achieve criticality (December 28, 1953) and was followed by $\mathrm{P}, \mathrm{L}, \mathrm{K}$, and $\mathrm{C}$ Reactors, respectively.

In 1975, the duties of the AEC were transferred to the newly established Energy Research and Development Administration and the Nuclear Regulatory Commission. In 1976, these two agencies evolved into the Department of Energy (DOE), which has overseen all functions of the facility since that year. Du Pont operated the complex through March 1989. On April 1, 1989, Westinghouse Savannah River Company (WSRC) became the operating contractor, and the Savannah River Plant was renamed the Savannah River Site (SRS).

During the period of weapons-grade material production, the SRS complex consisted of a heavy water production and rework facility, a fuel and target fabrication plant, five reactors, two chemical separations plants, and a number of waste management facilities. As of August 1994, all SRS production reactors are inactive. $\mathrm{C}, \mathrm{L}, \mathrm{P}$, and $\mathrm{R}$ Reactors are in shutdown status, and $\mathrm{K}$ Reactor remains in cold standby.

Recent functions of the SRS complex have included the recycling of nuclear components and the production of isotopes for medical applications and nonweapons use in the nation's space program. In addition to these tasks, SRS' environmental restoration and waste management activities have been expanded. 
WSRC-TR-94-0354

Unclassified

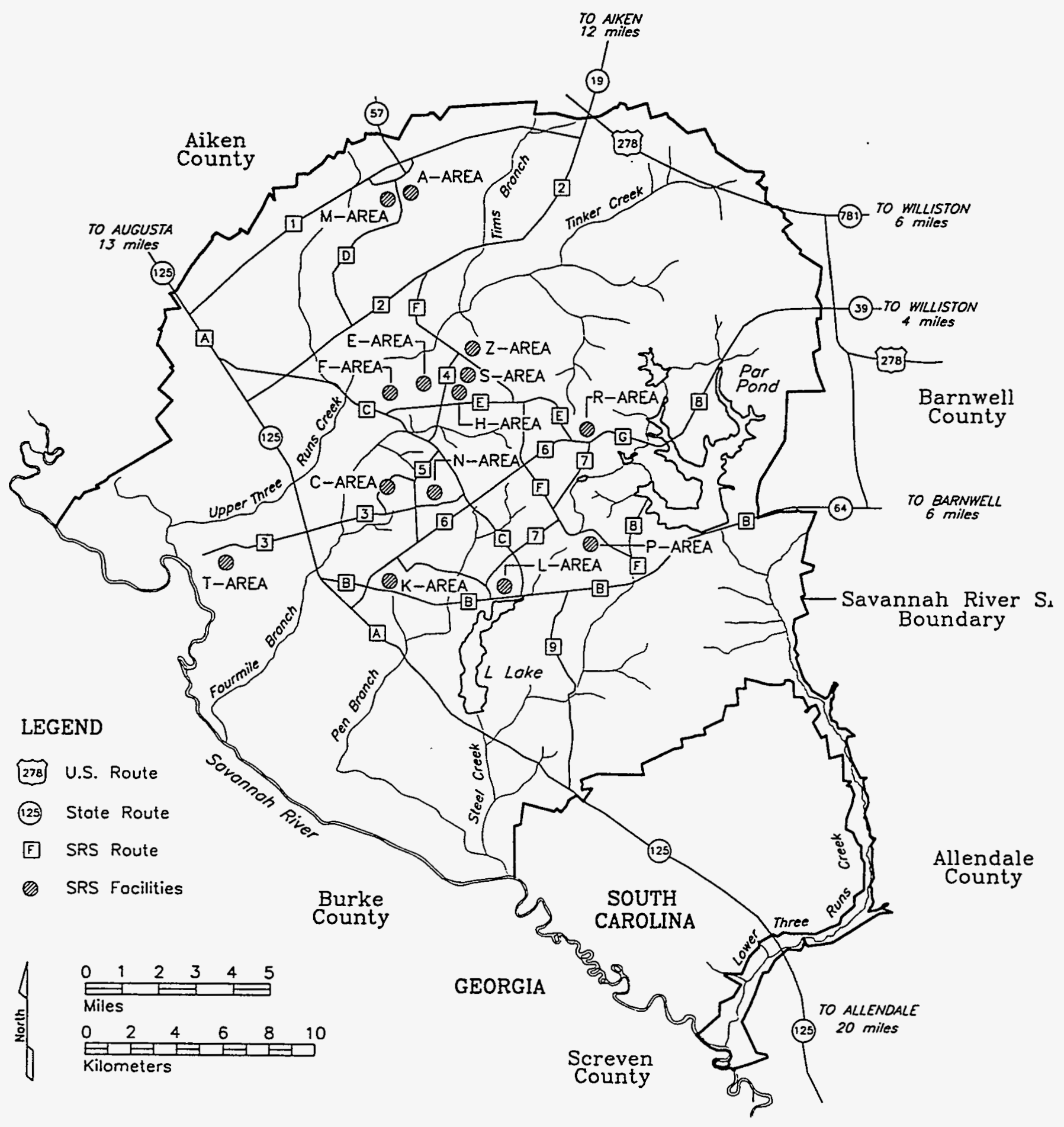

Figure 1. Savannah River Site and Its Environs 


\section{Process Overview}

The primary materials produced at the Savannah River Site during operation were plutonium ( $\mathrm{Pu}-238$ and $\mathrm{Pu}-239)$ and tritium ( $\mathrm{H}-3)$. These products were produced by absorption of neutrons in neptunium (Np-237), uranium (U-238), and lithium (Li-6).

The production process consisted of

- Fabrication of fuel and target materials into elements/assemblies

- Irradiation of these assemblies in the reactors

- Separation of transuranic elements, tritium, and residual uranium from waste byproducts produced by irradiation

- Recovery and purification of heavy water used as a coolant and neutron moderator

- Storage, processing, and disposal of waste

Figure 2 provides an overview of the production process.

During reactor operation, neutrons caused uranium-235 atoms to split into lighter atoms (fission). In addition, each fissioned uranium-235 atom released two to three neutrons. This surge of released neutrons, which split more uranium-235 atoms, resulted in a chain reaction that generated enormcus amounts of energy.

Some neutrons from the chain reaction were captured by uranium-238 atoms. When a uranium atom captured a neutron, the atom became an artificial and unstable isotope known as uranium-239. The uranium-239 emitted a beta particle to form neptunium-239, which in turn emitted another beta particle to form plutonium-239.

Reactors were moderated and heat generated by fissioning was cooled by recirculating heavy water (deuterium oxide or $\mathrm{D}_{2} \mathrm{O}$ ). The combination of high moderating ability and low neutron absorption provided by heavy water permitted flexibility in designing different charges to produce different products. Heat was removed from the heavy water in the reactor coolant system's heat exchangers by a once-through flow of "light" water $\left(\mathrm{H}_{2} \mathrm{O}\right)$ from either the Savannah River or one of two man-made reservoirs, Par Pond or L Lake. Figure 3 illustrates the site-wide flow of cooling water from its surface water sources to the reactor buildings and back to surface water.

At the conclusion of controlled fissioning, fuel and target assemblies were moved from the reactor core to a large pool of water in the reactor building to permit decay of short-lived fission products before the assemblies were delivered to one of the chemical separations plants within the SRS complex. 


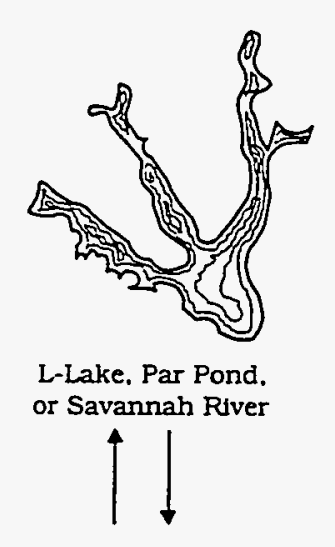

Reactor Area Cooling

Water System (Figure 3)

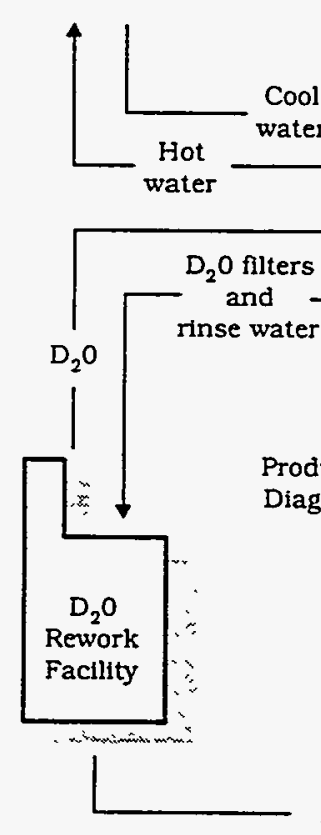

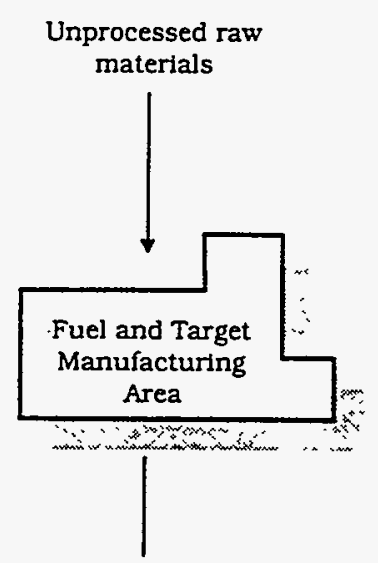

Nonirradiated fuel and targets
Finished products: plutonium-238

plutonium-239 tritium

uranium-233

polonium-210

caltfornium-252

cobalt -60

curium-244

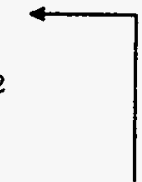

Chemical Separations Area

.

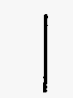

Waste materials generated during separation process

irradiated

fuel and targets

radioactive solid n:aste

Disassembly Basin Diagram (Figure 7)

Diagram (Figure 5)

Low level

radioactive solid waste

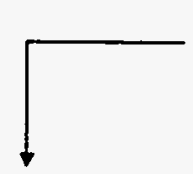

Low level radioactive solid waste

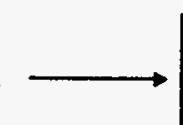

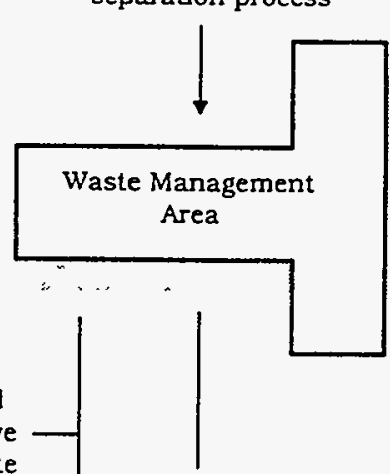

High level radioactive liquid waste

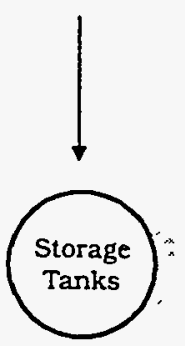

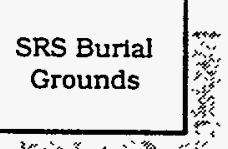

$\sum^{2} \alpha_{2}^{2} x^{2} \times x^{2}$

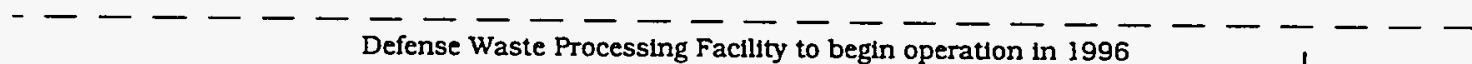

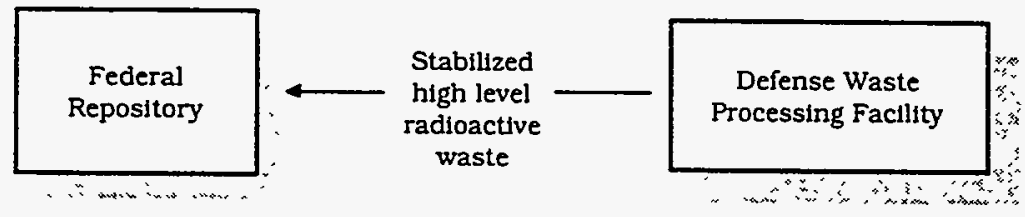

Figure 2. Production Process Diagram 


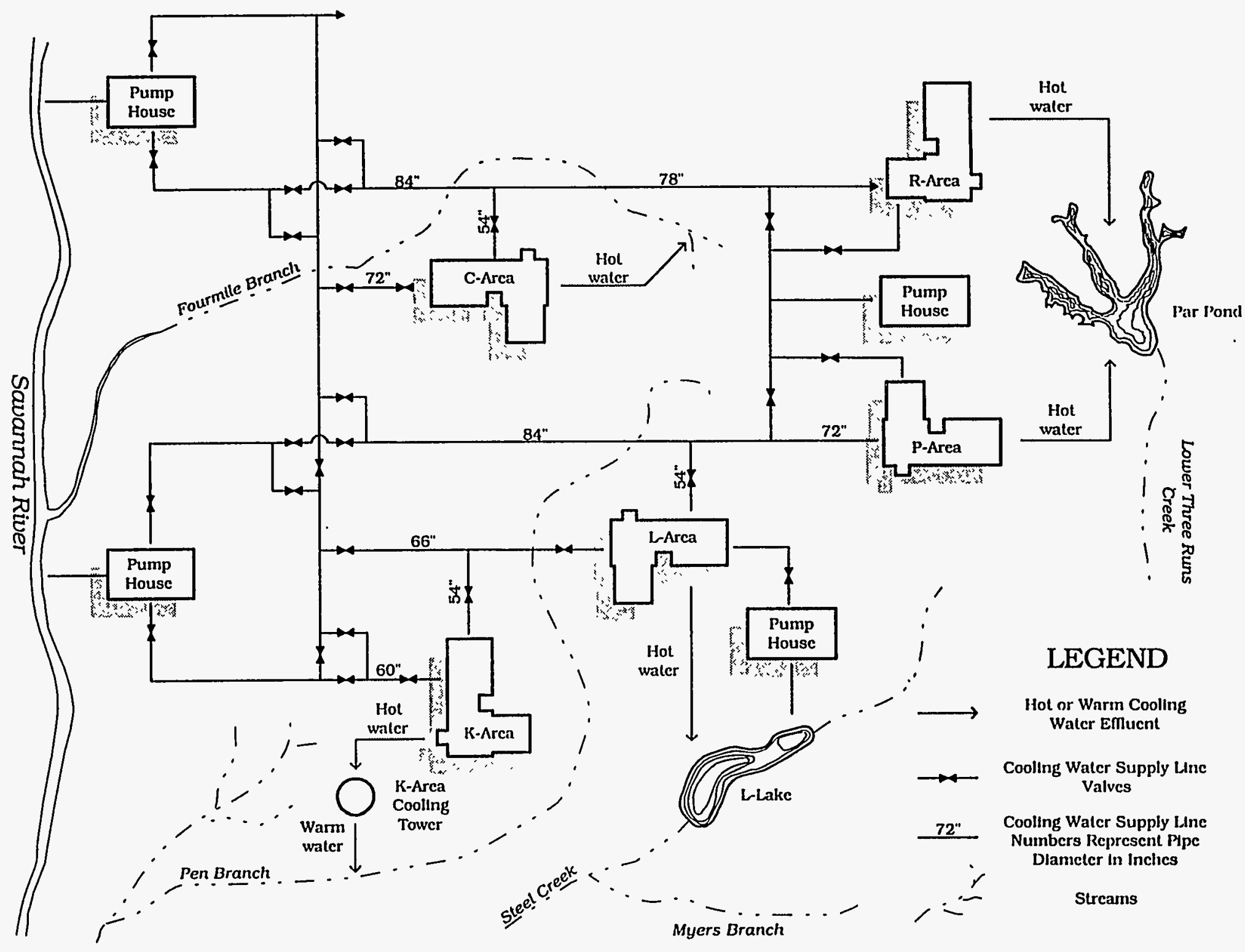

Figure 3. Cooling Water Supply Diagram 
Reactor-produced materials were processed in the Separations Area. Target rods were processed to obtain the desired radioactive products, which were compressed in cylinders (tritium) or converted into solids (plutonium) for shipment to other DOE facilities. Gases were extracted from irradiated reactor elements in an electric furnace in the tritium facilities. Several purification steps produced highpurity tritium.

Aluminum cladding on the uranium target slugs was dissolved in sodium hydroxide solution, followed by dissolution of the target material in nitric acid. Spent uranium fuel also was dissolved in nitric acid. After this dissolution, the subsequent nitric acid solution was treated by a solvent extraction process to yield solutions of plutonium, uranium, or neptunium. This method also produced both high-and low-heat liquid wastes containing fission products. Additional processing transformed plutonium, uranium, and other higher $\mathrm{Z}$ (atomic number) products from solution to solid for shipment, recycling, or processing.

Liquid radioactive wastes produced by the chemical processing were partially concentrated and stored in large underground tanks in the Separations Area, and low-level radioactive solid wastes were disposed of in the centrally located burial grounds. 


\section{Reactor Area Processes}

All five SRS reactors were production reactors used to fabricate nuclear materials for both government and civilian uses. They were designed to produce thermal neutrons that could be used to create a large amount of isotopes.

The two main products were plutonium (produced by the absorption of neutrons in the uranium-238 isotope) and tritium (produced by the absorption of neutrons in the lithium-6 isotope). Other products manufactured at SRS included uranium-233, plutonium-238, curium-244, polonium-210, cobalt-60, and californium-252.

The reactor buildings are large, reinforced concrete structures designed to be blast-resistant and reinforced for seismic activity. Heavy concrete shielding and a confinement system were used within each building to protect personnel from radiation and to minimize atmospheric radioactive releases. A cross section of a reactor building is provided in Figure 4. Each building contained four major process areas:

- The assembly area containing the fuel and target receiving areas, assembly and testing areas, and fuel storage areas

- The production reactor and its supporting operational and safety systems

- The disassembly area consisting of a pool (basin) for the storage and disassembly of irradiated fuel and target elements

- A purification-system area containing heavy-water moderator/coolant $\left(\mathrm{D}_{2} \mathrm{O}\right)$ purification facilities

\section{Assembly}

New fuel for use in the reactor process was received, assembled, inspected, tested, and stored in the reactor building's assembly area. The fuel and target fabrication facility manufactured fuel, target, control, and safety rods for use in the production reactors. Fuel and target materials were organized into the proper operating configurations in the assembly area.

During the production process, fuel assemblies produced neutrons for the target assemblies, causing nuclear reactions that eventually produced the desired materials. Fuel rods consisted of extruded enriched-uranium fuel slugs arrayed in aluminum casing. Target rods usually consisted of uranium or lithium (depending on the product to be produced) inside a lithium-aluminum alloy jacket. Aluminum was used because of its low neutron absorption rate, good heat transfer ability, and ability to be easily processed.

Control rods were used to regulate neutron exchange, and safety rods provided immediate shutdown in case of adverse conditions. Control rods were composed of either cadmium or lithium-aluminum depending on the desired product to be 

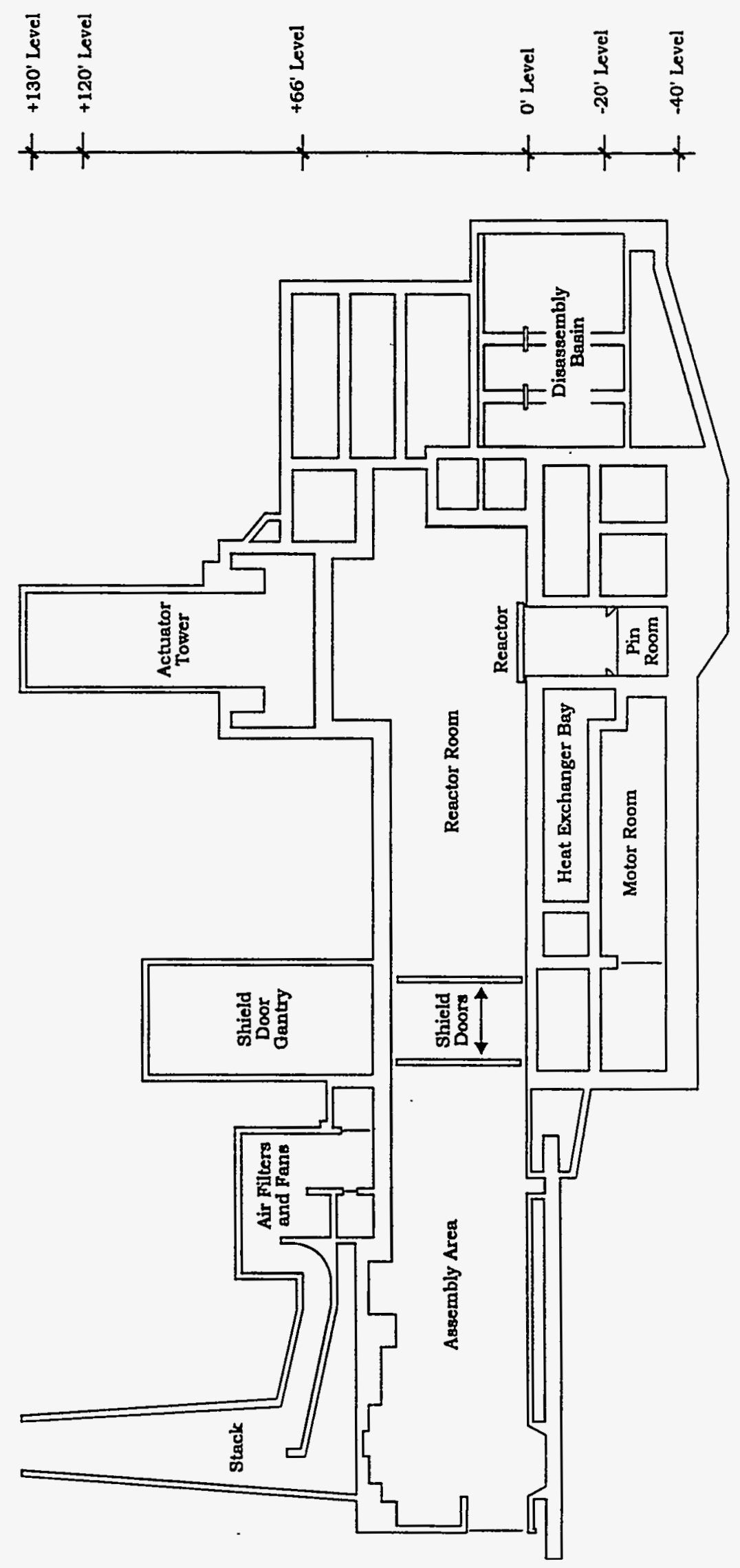

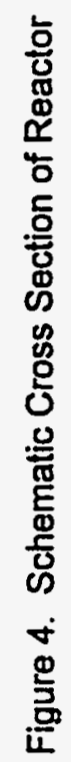


manufactured. Safety rods were fabricated from cadmium and were designed as safeguards against reactor core damage. Cadmium was used in safety and control rods because of its high neutron absorption rate.

Equipment in the assembly area included an inlet conveyor, a charge machine, a discharge machine, and a deposit-and-exit conveyor. The charge and discharge machines were cranes that could perform similar functions, but only the discharge machine could supply heavy- or light-water cooling to an irradiated assembly as it was being removed from the reactor vessel. The charge machine transported assemblies from the assembly area to the reactor.

\section{Inside the Reactor}

The reactor area was located in the central portion of the reactor building and housed the reactor, actuator system, heat exchangers, process pumps and piping, motors, crane and reactor control rooms, crane wash and maintenance areas, and auxiliary equipment and service facilities.

Process monitoring and control of the reactor was accomplished in the central control room. Reactivity instruments, controls, and assembly flow and temperature monitors were grouped together at one end of the control room, and instruments for hydraulic and auxiliary systems were at the opposite end.

The nuclear fission process took place within reactor vessels, $4.5 \mathrm{~m} \mathrm{x} 5 \mathrm{~m}$ cylinders composed of $1 / 2$-in. stainless steel plate and containing a lattice of fuel and target assemblies, control rods, and instrumentation submerged in the primary heavy-water moderator/coolant. Figure 5 illustrates the process.

The reactor vessels were designed so that $\mathrm{D}_{2} \mathrm{O}$ coolant entered through six nozzles at the top of the reactor into a plenum, flowed down coolant channels in the fuel and target assemblies, and discharged into the bulk moderator. Coolant then left through six nozzles at the bottom of the reactor vessel. A gas plenum and top radiation shield were located between the inlet water plenum and the reactor vessel. A radiation shield located under each reactor vessel provided flow and temperature monitoring for each fuel and target position. Figure 6 illustrates a reactor tank.

The assemblies and rods were arranged within a lattice framework to provide the most effective nuclear fission environment. Reactor lattices consisted of approximately 600 fuel and target rods and additional safety rod and control rod clusters that were housed in the reactor tank. Fuel and target rods were arranged in various loading patterns within the reactor to form a charge to initiate the nuclear process. As charging began, the fuel and targets were lifted by the charging machine and inserted into holes in the reactor tank lattice. The flow of $\mathrm{D}_{2} \mathrm{O}$ and $\mathrm{H}_{2} \mathrm{O}$ was raised to operating levels to moderate the reaction and cool the fuel rods. Safety rods were then extracted by the discharging crane in a predetermined order, followed by the control rods. 


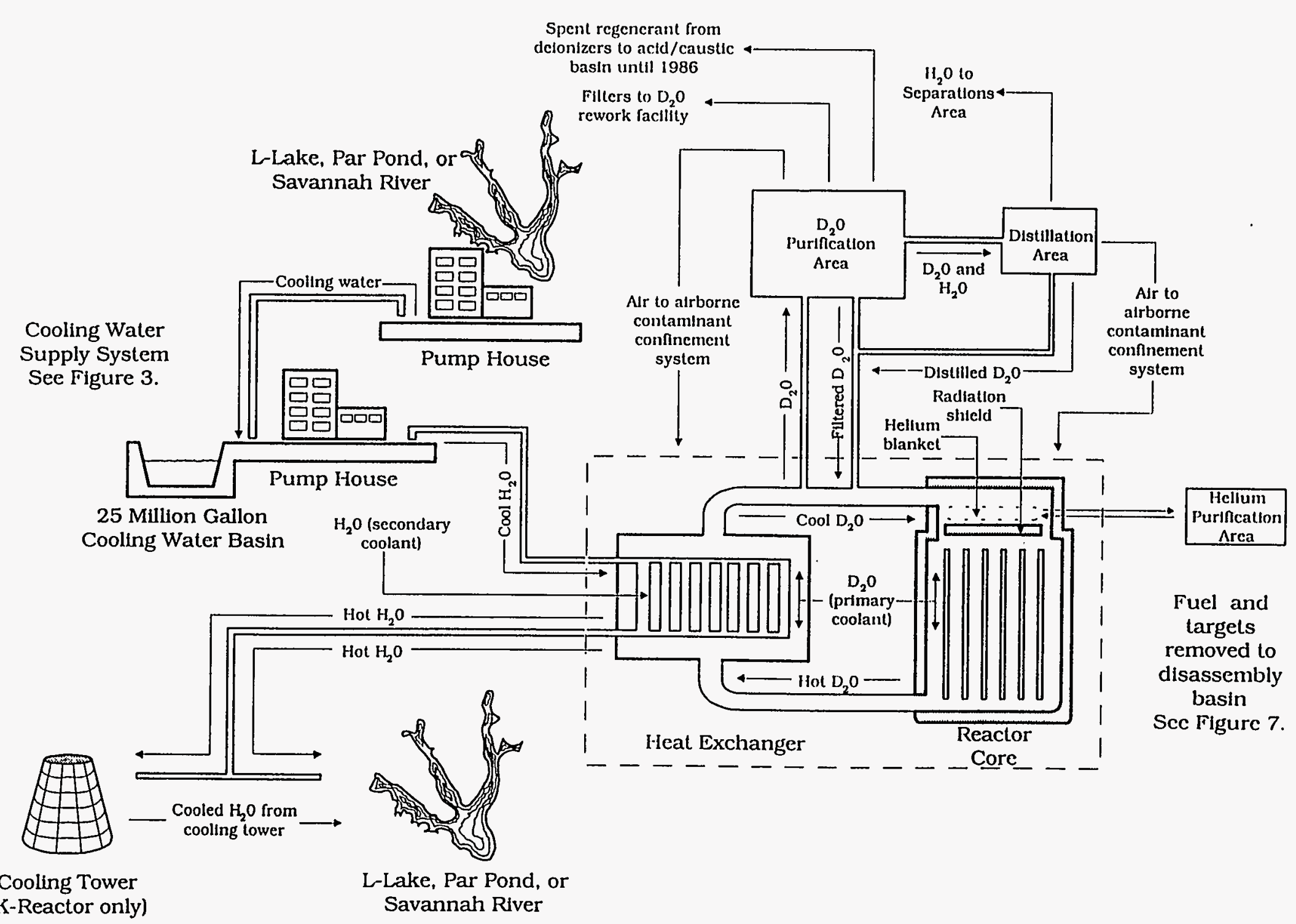

Figure 5. Reactor Cooling System Diagram 


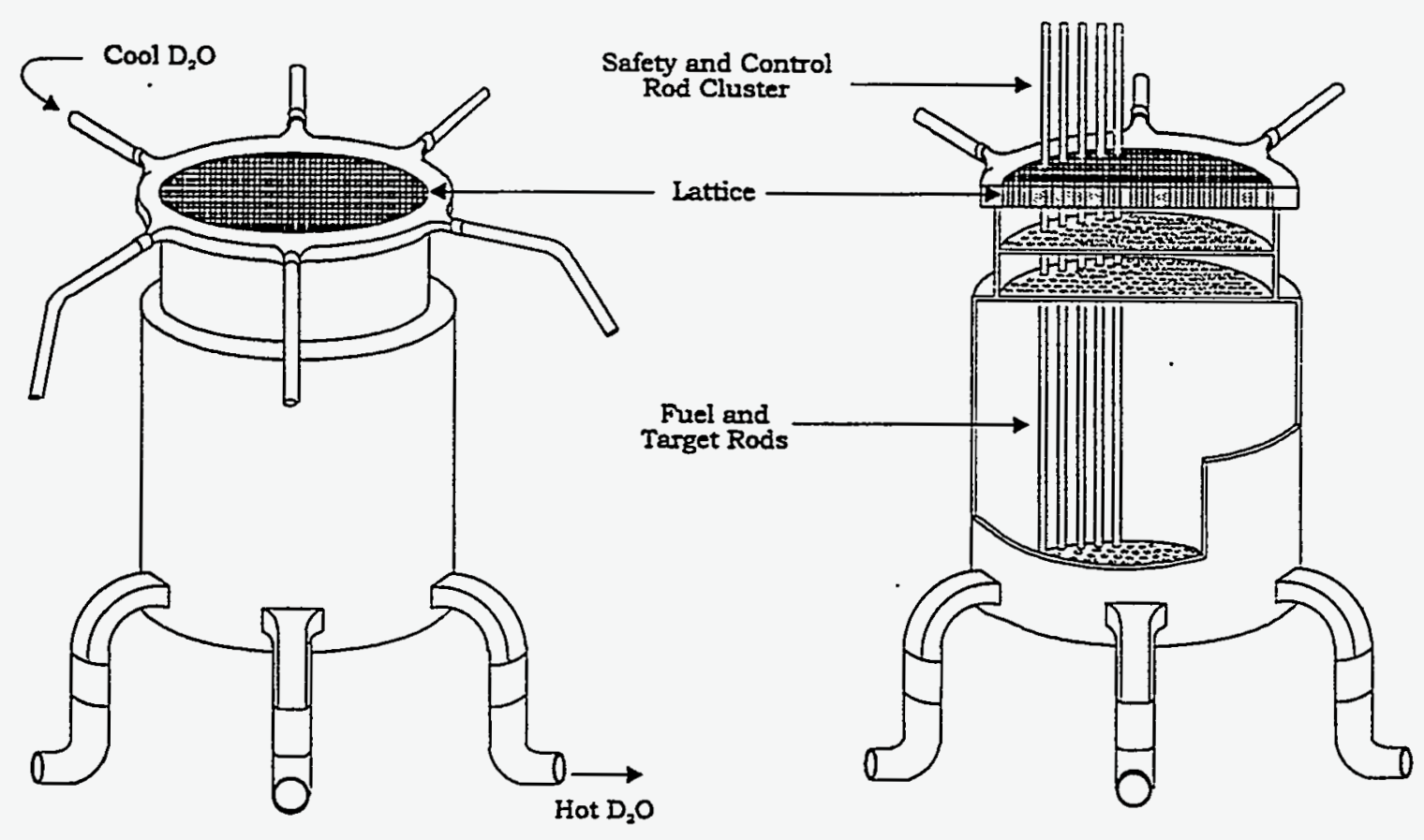

Perspective View

Cutaruay View

Figure 6. Reactor Tank

When the safety and control rod clusters were lifted by the discharging machine, the nuclear reaction began. The number and rate at which the rods were removed were closely specified. After the achievement of criticality, the rate of the reaction was raised by adjusting the control rod pattern.

Neutron-absorbing control rods were inserted into the core to regulate power levels by controlling flux, or flow of neutrons, in the reactor. Each fuel and target position contained control rods that could be moved individually or in groups. Two half-length rods in each position controlled the vertical flux distribution, and full-length rods controlled power and radial flux distribution.

Safety rods provided a primary rapid-shutdown system to prevent core damage to the reactor. They were designed to drop into the reactor core within one second upon indication of adverse conditions. Each reactor had a supply of 66 neutronabsorbing cadmium safety rods.

\section{Post-Irradiation Cooling}

Following irradiation, fuel and target assemblies were allowed to decay to a specified heat-generation rate prior to assembly discharge. After sufficient heatgeneration-rate decay, the discharge machine removed all assemblies from the reactor vessel and rinsed all fuel and target rods with light water. The rinsing 
process allowed heavy water reclamation from the discharged assemblies and minimum carryover of tritiated water to the disassembly basin. The rinse water was collected in a discharge machine water pan and sent to a rinse collection tank. The rinse water was periodically removed from the tank, placed in drums, and transported to the heavy-water rework facility for removal of light water.

Cooling began automatically when an irradiated assembly was completely withidrawn from the reactor. Cooling could also be enacted if an assembly stuck during withdrawal. Both heavy and light cooling water were available on the discharge machine during the discharge operation.

The discharge machine transported irradiated assemblies to the deposit-and-exit conveyor, housed in a water-filled canal, which transported them beneath the reactor-room wall to a water-filled disassembly basin for temporary storage.

\section{Disassembly}

The disassembly basin was used to cool and disassemble all components used in the reaction process. Figure 7 illustrates the flow of cooling water and contaminants associated with activities at the disassembly basin.

The irradiated assemblies were held in the disassembly basin to permit decay of unwanted radionuclides generated by the fission process. Assemblies and components were cooled by natural convection during storage in the disassembly basin. In addition to cooling functions, the water within the basin served to shield personnel from radiation.

After sufficient heat-level decay, the assemblies were disassembled underwater, using submerged power tools. Disassembled components were stored in the basin and allowed to decay until their heat levels were acceptable for movement to the Separations Area.

The disassembled components were then moved from the basin to a transfer bay where they were deposited in shielded shipping casks. These casks were transported to the Separations Area by rail or tractor-trailer.

Discharged assemblies were often covered by a thin liquid film from the reactor vessel. This liquid film contained activated corrosion products, particulate activities, tritium, and other radioisotopes that could escape from irradiated assemblies. Assembly corrosion, cooling, and disassembly of the components added to the contamination of the disassembly basin water.

Due to the transfer of radiation from the irradiated materials to the disassembly basin water, biannual purging of the basin water was necessary to reduce the accumulation of tritium and any other radioactive substances. During disassembly basin purging, basin cooling water was passed through two deionizer beds, monitored, and discharged to a low-level seepage basin. 


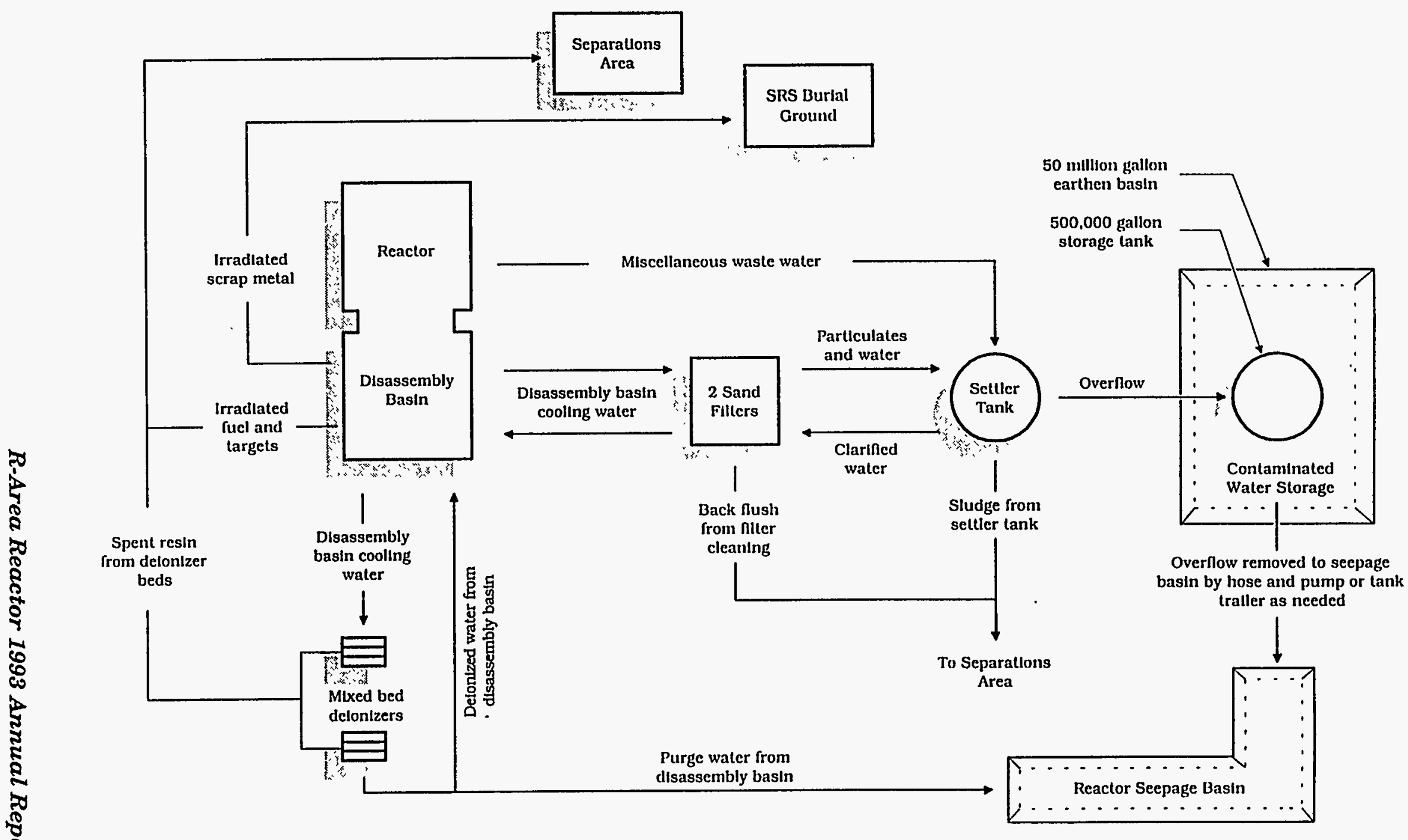

Figure 7. Disassembly Basin Diagram 
Immediately after discharge, the fuel tubes were temporarily stored in a vertical array under water in the disassembly basin. The fuel tubes were subsequently emptied, collected, and stored in buckets.

\section{Heavy-Water Purification}

The function of the reactor building purification system area was to purify and recirculate the heavy water used as reactor coolant throughout the entire irradiation process.

During production, heavy-water moderator/coolant was subjected to several impurities, including the corrosion of aluminum cladding on fuel and target rods, the massing of neutron-absorbing substances that would otherwise be used in nuclear materials production, molecular decomposition of the heavy water into deuterium and oxygen as a result of radiation, neutron activation of component or suspended corrosion products and impurities, and accumulation of light water in the moderator.

During the production phase, heavy-water moderator/coolant was continuously purified through a system of filters in the reactor area. A side stream of heavy water was continuously diverted from the reactor circulating system and sent through a purification system, which circulated the primary coolant/moderator through a prefilter, a deionizer, and an afterfilter located in a shielded purification area. Most of the side stream was returned to the reactor system; the remainder was transported to a distillation area for light water removal.

The deionizer contained mixed-bed ion exchange resin for removal of both cations and anions. The filters retained particles larger than 10 micrometers in diameter. The prefilter removed suspended solids, and the afterfilter retained any resin that might have passed through the deionizer.

\section{Storage and Disposal of Waste}

SRS stores or disposes of six categories of waste:

- High-level waste

- Transuranic waste

- Low-level waste

- Hazardous waste

- Mixed waste

- Sanitary waste

High-Level Waste

The reactor areas did not produce or store high-level waste. 


\section{Transuranic Waste}

Transuranic waste (TRU) is produced in radiological materials areas (RMAs) such as the reactor areas. TRU is defined as radioactive waste containing transuranic elements above specified concentrations. It decays slowly, thus requiring isolation for thousands of years. TRU waste comprises a variety of materials including miscellaneous trash and equipment. It is stored in the Separations Area.

\section{Low-Level Waste}

Low-level waste is any radioactive waste not classified as high-level or transuranic waste. It was generated by reactor operations, isotope production, and research and development projects.

Low-level reactor-produced waste includes contaminated equipment such as resins contaminated with carbon-14, irradiated reactor hardware that does not contain spent fuel, and spent lithium-aluminum targets. Other examples of lowlevel waste include pipe, rags, paper, filters, tools, protective clothing, and suspect materials used within a radiological materials area that cannot be proven to be uncontaminated.

Low-level radioactive solid wastes were disposed of in the centrally located Separations Areas' burial grounds.

\section{Hazardous Waste}

Hazardous waste is defined as any toxic, corrosive, reactive, or ignitable solid material that could damage the environment or pose a risk to human health.

Procedures to screen for radioactivity have been approved at SRS for liquids and solids originating either inside or outside an RMA. If approved for shipment, these wastes may be transported offsite for treatment and disposal. Wastes that fail the screening process must be recategorized as mixed waste.

\section{Mixed Waste}

Mixed waste is a combination of radioactive and hazardous wastes and is produced both inside and outside an RMA. Disposal in the Separations Area burial grounds of mixed wastes from reactor operations, including tritiated pump oil, lead used for shielding, and cadmium from control rods, safety rods, and shielding, was discontinued in March 1986. Mixed waste storage buildings within the boundaries of the burial grounds have been receiving such waste since March 1987. 
WSRC-TR-94-0354

Unclassified

\section{Sanitary Waste}

Sanitary waste is neither radioactive nor hazardous. Solid sanitary waste from throughout SRS is disposed of in the landfill; liquid sanitary waste, including sewage and industrial waste, is treated in SRS sewage treatment plants. 


\section{R-Area History}

$R$ Area (Figure 8) is in the northeast portion of the Savannah River Site. A timeline of major activities in the area is shown in Figure 9.

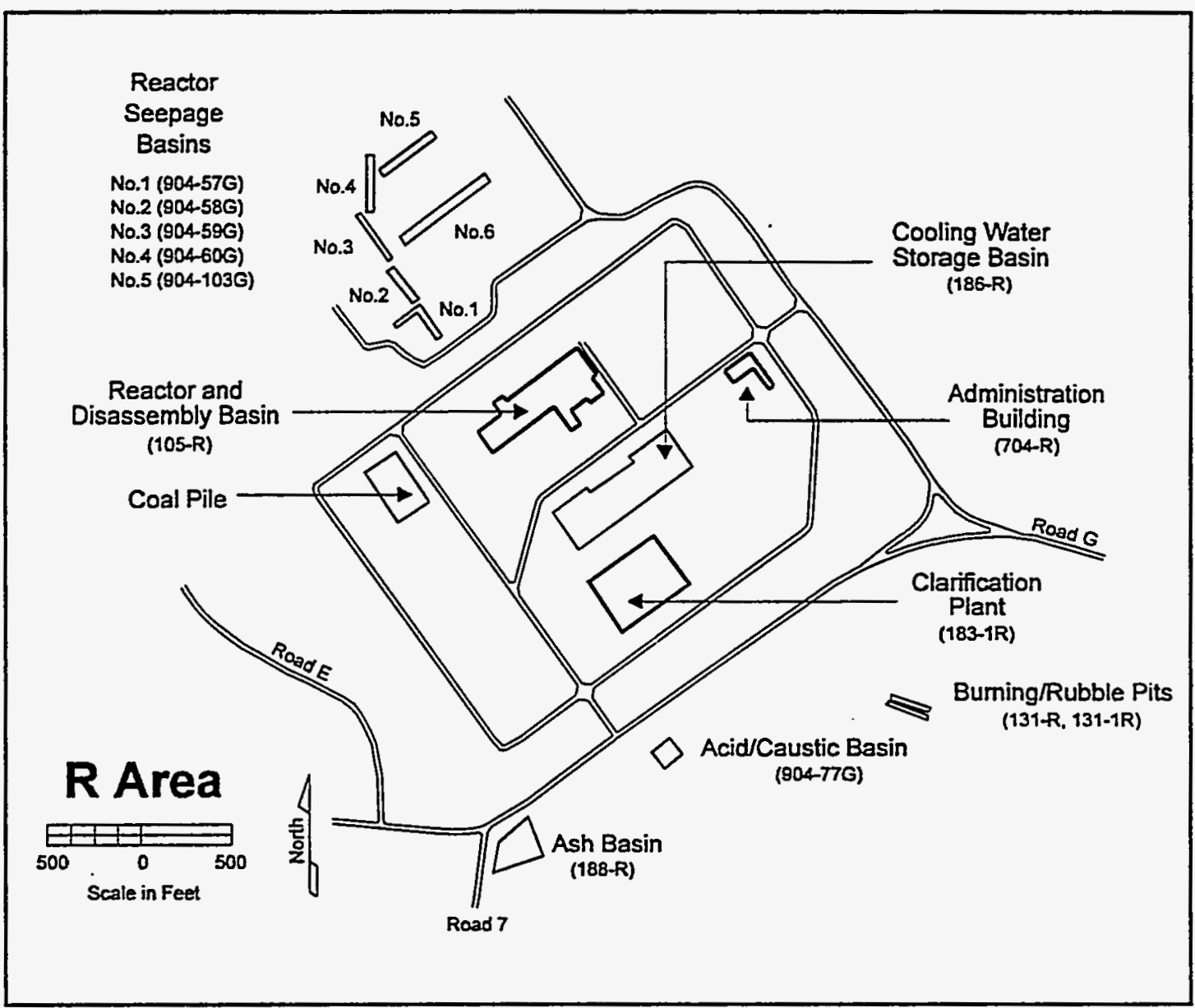

Figure 8. R Area

\section{R Reactor}

$R$ Reactor was the first SRS production unit to achieve criticality (December 28, 1953). It produced plutonium and tritium for national defense until 1964, when it was placed in shutdown status. Equipment for operation and control of the reactor was upgraded several times to meet current standards. 


\section{Reactor Modifications}

\section{Cooling Systems}

Each SRS reactor received major modifications to primary and secondary cooling systems beginning in 1957. Heat exchangers and larger heavy-water pumps (Bingham pumps) were installed at each production facility.

\section{Ventilation Exhaust System}

The ventilation exhaust system was modified in 1960-61 to remove airborne contaminants, particularly iodine-131, by providing moisture separators, particulate filters, and halogen adsorbers (carbon) in process area ventilation exhaust.

\section{Emergency Cooling System}

The emergency cooling system (ECS) received upgrades in 1962-63. A 50-milliongallon earthen cooling basin was constructed for ECS usage.

\section{Remote Detection and Control System (REDAC)}

A remote detection and control system (REDAC) installed in 1962-63 permitted system parameters to be monitored and other functions, such as the addition of light water if necessary, to be handled remotely (from another building) after an area evacuation.

\section{Reactor Shutdown}

$R$ Reactor was placed in shutdown status in 1964 due to poor operating condition. Four major defects were found:

1. An effluent nozzle was leaking $\mathrm{D}_{2} \mathrm{O}$ into the annular cavity in the reactor. Remote television inspection of the nozzle revealed a crack-like line believed to be the source of the leak.

2. The effluent nozzle had been patched at three locations in 1962. At that time, four additional cracked areas were identified but not repaired. A 1964 inspection revealed many discolored, pitted, and crack-like areas in the nozzle material adjacent to each of the three patches. The extent of corrosion was deemed more severe in 1964 than that observed in 1962.

3. Deionized water was leaking from a pipe into the bottom of the reactor vessel. A similar leak at a different location had been repaired in 1960.

4. The top shield had a small leak that was estimated to have existed since $R$ Reactor startup. Light water originally leaked from the shield into the 
1950 through 1965

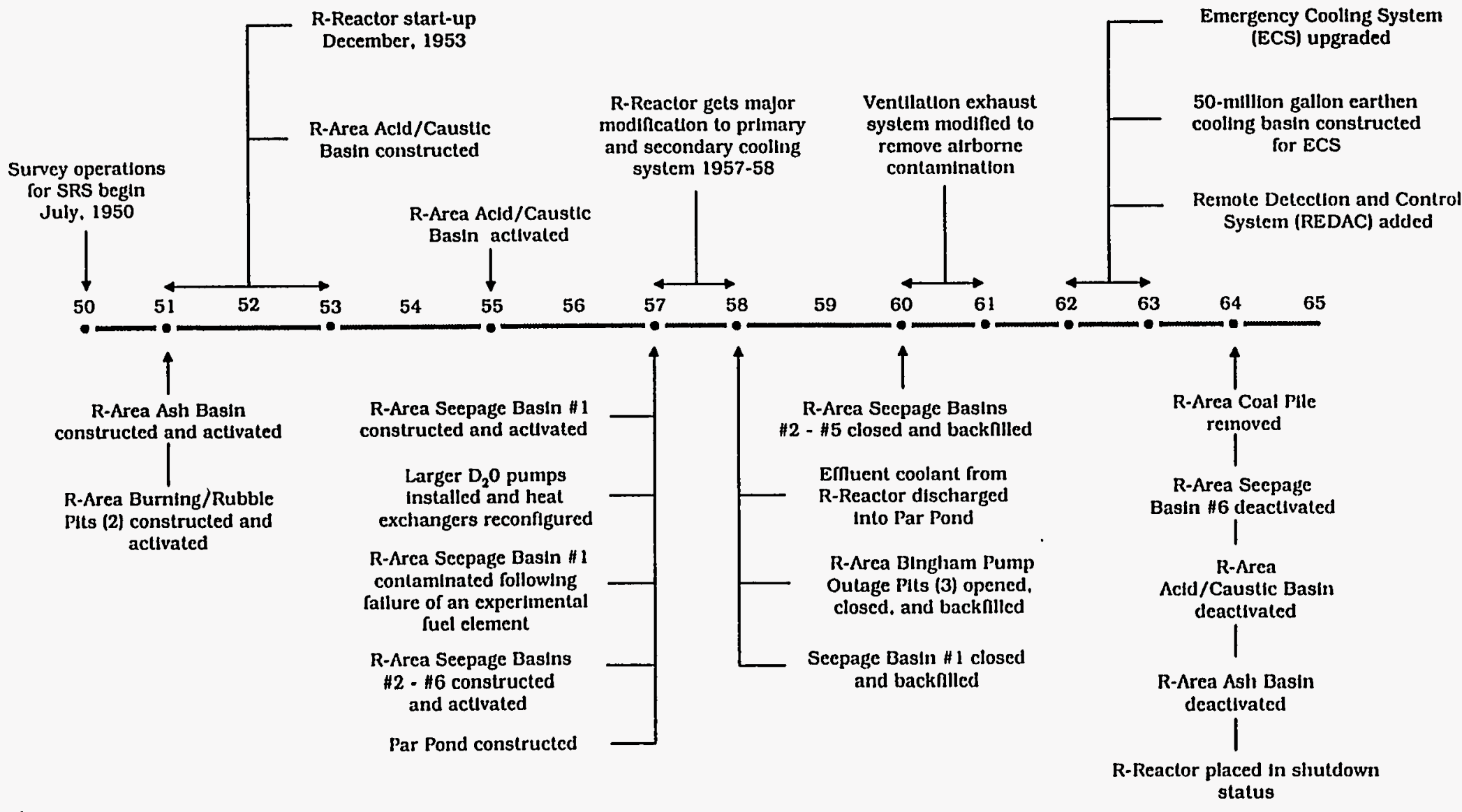

Figure 9. Time Line for $\mathrm{R}$ Area 
reactor moderator. The pressure in the top shield was adjusted to reverse the leakage direction in 1955, and from then until shutdown the leakage was controlled. The point of leakage was thought to be a weld at the outer periphery of the bottom of a permanent tube position.

\section{4-Present}

Since shutdown in 1964, there has been extensive cannibalization of equipment at R Reactor. No habitability systems are operable. There is only limited lighting with no emergency power. The reactor vessel has been emptied, there are no fuel assemblies in the disassembly basin, and the reactor fluid system has been drained. There are 60,000 gallons of moderator stored in drums, and 8,771 drums of depleted uranium oxide are stored in the area.

\section{Burning/Rubble Pits}

An abundance of construction debris necessitated the construction and activation of burning/rubble pits in R Area (Figure 8 and Maps 1 through 10) in 1951. The pits received burnable waste materials such as paper, plastics, wood, rubber, rags, cardboard, oil, degreasers, and drummed solvents. The collected waste materials were incinerated monthly.

Incineration of waste materials in all burning/rubble pits throughout the SRS complex was discontinued in October 1973. A layer of soil was placed over the remainder of the burning pit waste, and the pits began receiving only rubble. Allowable rubble waste included nonsalvageable materials such as bricks, concrete, tile, asphalt, plastics, rubber, wallboard, concrete, empty galvanized steel barrels, and cans.

When pit 131- $R$ reached its capacity, it was closed and covered with soil to grade level. Burning/Rubble Pit 131-1R, which received small quantities of rubble, was not backfilled. All burning/rubble pits in the SRS complex were covered by 1981 .

\section{Ash Basin}

The R-Area Ash Basin (Figure 8 and Maps 1 through 10) was constructed in 1953 to receive fly and bottom ash from the R-Area powerhouse. Each powerhouse was a coal-operated facility that supplied power to the reactor areas. The R-Area Ash Basin was deactivated in 1964.

\section{Acid/Caustic Basin}

The R-Area Acid/Caustic Basin (Figure 8 and Maps 1 through 10) was constructed in 1955 to receive spent regenerant solutions from the water treatment facilities. Wastes discharged to the Acid/Caustic Basin included dilute sulfuric acid and sodium hydroxide used to regenerate ion exchange units, water 
rinses of the ion exchange units both before and after regeneration, steam condensate from the heater in sodium hydroxide storage tanks, and rainfall runoff from storage tank spill containment enclosures. The spent dilute solutions were transported to the acid/caustic basin through acid-resistant sewers.

The basins discharged into local surface streams, and the daily discharges normally equaled the daily influent flows to the basins.

\section{Seepage Basins}

The first R-Area seepage basin (Figure 8 and Maps 1 through 10) was constructed and put into operation in 1957 . The seepage basin was designed to receive lowlevel radioactive purge water from the $R$-Area disassembly basin. The water purge was necessary to keep the tritium concentration in the disassembly basin water below the level that ensured safe working conditions.

In November 1957, an experimental fuel element failed during calorimeter tests, and approximately 200 curies of strontium-90 and 1,000 curies of cesium-137 were released to the basin. To minimize activity released to the environment, four additional R-Area seepage basins were constructed that same year, and the excess water stored in basin 1 was pumped into these units. A sixth and final seepage basin was constructed in 1958.

R-Area Seepage Basin 1 was deactivated and backfilled in January 1958 because of leakage to an abandoned sewer system. In 1960, basins 2 through 5 were deactivated and backfilled. The ground above the five basins was treated with herbicide and covered with asphalt. A kaolinite dike was constructed around basin 1 and the northwest end of basin 3 to contain lateral movement of the radioactive contamination. Basin 6 was last used in 1964 and was backfilled in 1977.

\section{Par Pond}

Par Pond was constructed in 1957 for discharge and recycling of effluent coolant from the $P$ and $R$ Reactors. When it became operational in 1958, Par Pond eliminated the thermal effects of $P$ and $R$ operation on secondary coolant water returned to the Savannah River.

\section{Bingham Pump Outage Pits}

Normally, all radioactive solid waste generated in the reactor areas was sent to the solid waste burial ground, but an exception to this practice was made during the cooling system modifications initiated at all reactor areas in 1957 . Three Bingham pump outage pits were installed at $R$ Area for disposal of debris contaminated with low-level radioactivity. The pits were filled with miscellaneous construction equipment such as pipes, cables, ladders, drums, and boxes of 
miscellaneous hardware. All waste materials with high levels of contamination were sent to the radioactive solid waste burial ground. The Bingham pump outage pits were backfilled with clean soil in 1958. 


\section{Environmental Data}

This report consolidates groundwater monitoring data from 1989 to 1993 to determine the potential contaminants of concern and the potential extent of contamination.

Although there are data for the wells in this area prior to 1989, the data sets are spotty. In addition, the flagging system (see Appendix B) that assures follow-up analysis of constituents that appear above predetermined levels was refined in 1989. Data prior to 1989 can be found in the SRS annual environmental reports.

There are a number of difficulties in characterizing the groundwater data for this area:

- Data for the monitoring wells in $\mathrm{R}$ Area are inconsistent.

- Constituents of concern have not been identified and tracked.

- Constituents have not been regularly sampled in all wells in a series.

- Constituents have not been sampled at regular intervals.

- Except for two wells (P 20C and RCP 1A), all wells in R Area are screened in the water table.

Despite these difficulties, some patterns can be discovered from the data for the past five years. In order to determine constituents of concern, four ways of viewing the groundwater monitoring data are presented:

- Tables of constituents that have appeared above the primary drinking water standards (DWS) or indicator parameters from 1989 to $1993^{1}$

- Tables of constituents that have appeared above Flag 2 criteria from 1989 to 1993

- Time series plots (Appendix D) for potential constituents of concern

- Results for all constituents analyzed for individual wells for all four quarters of 1993

In the tables of constituents that have appeared above the DWS or indicator parameters, there are two sets of standards: the primary drinking water standards applicable during the year under discussion and the indicator parameters (values above those usually found in background wells at SRS).

The DWS for 1993 are presented in Appendix A. The indicator parameters are the following:

- Specific conductance equal to or greater than $100 \mu \mathrm{S} / \mathrm{cm}$

- Alkalinity (as $\mathrm{CaCO}_{3}$ ) at values equal to or greater than $100 \mathrm{mg} / \mathrm{L}$

- Total dissolved solids (TDS) at values equal to or greater than $200 \mathrm{mg} / \mathrm{L}$

1 The absence of a dot for a particular year or quarter in the tables of constituents above standards may mean either that the constituent was not scheduled for analyis during the time period or that it was analyzed for and was below its standard. 
- $\mathrm{pH}$ at values equal to or below 4.0 or equal to or above 8.5

The selection of these values as standards for comparison is somewhat arbitrary; however, these values exceed levels usually found in background wells at SRS. The occurrence of elevated alkalinity (as $\mathrm{CaCO}_{3}$ ), specific conductance, $\mathrm{pH}$, and TDS within a single well may indicate leaching of the grouting material used in well construction rather than degradation of the groundwater.

In the tables of analytes that have appeared above Flag 2 criteria, the standards are those that were applicable during the year under discussion. Flag 2 criteria have changed frequently during the years and can be found in the individual Environmental Protection Department/Environmental Monitoring Section (EPD/EMS) quarterly reports. Flag 2 criteria for 1993 are presented in Appendix B. Despite the problems presented by the changing flagging criteria and the lack of focus on individual wells, the benefits of looking at the Flag 2 criteria summaries are twofold:

- First, once a constituent appears above Flag 2, it is scheduled for regular sampling according to predetermined rules. Therefore, if a constituent appears once in the Flag 2 tables below and then does not reappear, the single appearance in the table may be an anomaly. It is the pattern of appearances above Flag 2 that is the focus of these tables and not the specific analyte levels or wells.

- Second, unlike the DWS tables below, the Flag 2 tables focus on the quarterly schedule of analyses.

Time series plots (Appendix D) provide another way of looking at the data for selected constituents. Trends in the plots are discussed in the sections below.

Finally, Table 1 at the end of this section presents maximum results for constituents exceeding the DWS in individual R-Area wells during 1993.

$R$ Area is located on a groundwater mound with water flowing horizontally away from the southern reactor seepage asins. Flow is toward Mill Creek to the northwest and Par Pond and its tributaries to the southeast and northeast. There is a downward gradient also as detected b the P 20 and RCP cluster wells.

\section{Well Series P in R Area}

The $\mathrm{P}$ well series comprises a large group of baseline hydrogeologic investigation well clusters scattered throughout the site. There are four of these wells (P 20B, 20C, 20D, and 20TD), known as the R-Area Bedrock Exploration Hydrology Wells, in R Area. Two of these wells (P 20C and P 20D) were added to the groundwater monitoring program for sampling and analysis during 1993. P 20C is screened in the McBean formation, and P $20 \mathrm{D}$ is screened in the water table. 
Beginning second quarter 1993, cadmium, gross alpha, lead, nonvolatile beta, $\mathrm{pH}$, specific conductance, and tritium analyses were requested to support the Remedial Investigation (RI) Program for the'R-Reactor Seepage Basins.

During second, third and fourth quarters, the volume of water purged from these wells was estimated because flowmeters were not present. During the third quarter, field notes indicate that well identification signs were missing.

Data from these wells show aluminum and iron above Flag 2 criteria in 1993.

See Table 1 at the end of this section for maximum results for individual wells for 1993.

\section{Acid/Caustic Basin (RAC Well Series)}

Groundwater beneath the R-Area Acid/Caustic Basin (Figure 8 and Maps 1 through 10) has been monitored by the RAC well series since 1983. Horizontal flow in the upper saturated zone beneath the basin is to the south toward Par Pond tributaries. All wells at the basin are water table wells.

\section{DWS and Indicator Parameters}

Analytes Over DWS or Indicator Parameters in Well Series RAC

\begin{tabular}{|c|c|c|c|c|c|}
\hline Analyte & 1989 & 1990 & 1991 & 1992 & 1993 \\
\hline Gross alpha & - & & & - & \\
\hline Lead & $\bullet$ & & & $\bullet$ & \\
\hline Specific conductance & $\because$ & 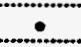 & & 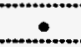 & \\
\hline Total radium & $\bullet$ & $\bullet$ & & & \\
\hline
\end{tabular}

The DWS table shows no consistent constituents of concern at this site.

\section{Flag 2 Criteria}

Analytes Over Flag 2 Criteria in Well Series RAC

\begin{tabular}{|c|c|c|c|c|c|c|c|c|c|c|}
\hline Analyte & Iq89 & $2 \mathrm{q} 89$ & $3 q 89$ & $4 q 89$ & $1 \mathrm{q90}$ & $2 q 90$ & $3 q 90$ & 4q90 & $1 \mathrm{q} 91$ & $2 q 91$ \\
\hline Aluminum & & & & & & & & & & \\
\hline Gross alpha & & $\bullet$ & & $\bullet$ & & & & & & \\
\hline Iron & & $\bullet$ & & & & & & & & \\
\hline Lead & - & $\bullet$ & $\therefore$ & & & & $\cdot$ & & & \\
\hline Manganese & & $\bullet$ & & & & & $\bullet$ & & & \\
\hline Total organic halogens & & $\therefore$ & & & & & & & & \\
\hline Total radium & & $\bullet$ & & - & $\bullet$ & & $\bullet$ & & & \\
\hline
\end{tabular}




\begin{tabular}{|c|c|c|c|c|c|c|c|c|c|c|}
\hline 3q91 & 4q91 & $1 \mathrm{q} 92$ & $2 q 92$ & $3 q 92$ & $4 q 92$ & $1 q 93$ & 2q93 & 3q93 & $4 \mathrm{q} 93$ & Analyte \\
\hline & & & & & & - & & - & & Aluminum \\
\hline & & & & $\bullet$ & & & & & & Gross alpha \\
\hline & $\therefore$ & & $\therefore$. & & - & $\therefore$ & & & & Iron \\
\hline$\therefore$ & $\therefore$ & $\therefore$ & $\therefore$ & & $\therefore$ & .... & & & & Lead \\
\hline & & & & & & $\because$ & & & ........ & Manganese \\
\hline & & & & & & & & & .... & Total organic halogens \\
\hline & & & 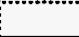 & & & & & “ & 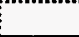 & Total radium \\
\hline
\end{tabular}

Lead appears as a consistent constituent of concern in the Flag 2 table for this site.

\section{Time Series Plots}

Measured levels of selected constituents are plotted over time to illustrate historical trends and significant concentrations in each well series. (See time series plots in Appendix D.) The time series plots indicate the following.

Conductivity remained relatively constant in RAC 2 and RAC 4, but fluctuated in RAC 1 and RAC 3. Measurements ranged from approximately 30 to $170 \mu \mathrm{S} / \mathrm{cm}$ in $\mathrm{RAC} 1$ and from approximately 20 to $120 \mu \mathrm{S} / \mathrm{cm}$ in RAC 3.

Gross alpha activity in RAC 1 exceeded the $15 \mathrm{pCi} / \mathrm{L}$ DWS three times during sampling, reaching a maximum level of approximately $25 \mathrm{pCi} / \mathrm{L}$ during fourth quarter 1984 sampling. RAC 2 levels ranged from approximately 2 to approximately $85 \mathrm{pCi} / \mathrm{L}$, and $\mathrm{RAC} 3$ ranged from approximately 1 to $25 \mathrm{pCi} / \mathrm{L}$. RAC 4 remained consistently below the DWS criterion.

Lead concentrations in all RAC wells fluctuated throughout the reporting period. RAC 1 and RAC 2 were highest late in 1986. RAC 3 and RAC 4 concentrations ranged from approximately detection limit to 56 and $38 \mu \mathrm{g} / \mathrm{L}$, respectively.

All nonvolatile beta activity in RAC wells remained consistently below the DWS of $50 \mathrm{pCi} / \mathrm{L}$, although activity in RAC 2 was observed at approximately $48 \mathrm{pCi} / \mathrm{L}$ during second quarter 1989 sampling.

Field $\mathrm{pH}$ levels in the RAC wells fluctuated between approximately 3.5 and 5.5 $\mathrm{pH}$ units until first quarter 1987. Since that time, $\mathrm{pH}$ levels have been measured between 4.5 and $5.5 \mathrm{pH}$ units.

\section{Data}

RAC wells were scheduled for comprehensive analyses in the first and third quarters of 1993. During the third quarter, these wells were sampled for aluminum, gross alpha, iron, lead, and manganese due to earlier Flag 1 or 2 designation. Field analyses were performed quarterly. During the second quarter, turbidity in RAC 1 exceeded 1,000 NTU. No analytes exceeded DWS during 1993. 


\section{Ash Basin/Coal Pile (RCP Well Series)}

The two RCP wells were installed during 1990, but were not sampled until 1992. Horizontal groundwater flow in the water table appears to be generally westward toward the headwaters of Mill Creek. RCP IA is screened in the Congaree formation; RCP ID is screened in the water table.

Water elevations from the two RCP wells indicate a downward vertical gradient beneath the coal pile boundaries, suggesting a potential for downward migration of groundwater from the upper saturated zone to the Congaree-Fourmile zone.

\section{DWS and Indicator Parameters}

Analytes Over the DWS or Indicator Parameters in Well Series RCP

\begin{tabular}{|c|c|c|c|c|c|}
\hline Analyte & 1989 & 1990 & 1991 & 1992 & 1993 \\
\hline Specific conductance & & & & - & - \\
\hline
\end{tabular}

Groundwater data from 1992-1993 indicate specific conductance above its indicator parameter.

Flag 2 Criteria

Analytes Over Flag 2 Criteria in Well Series RCP

\begin{tabular}{|c|c|c|c|c|c|c|c|c|c|c|}
\hline Analyte & $1 q 89$ & $2 q 89$ & $3 q 89$ & $4 q 89$ & $1 q 90$ & $2 q 90$ & $3 q 90$ & $4 q 90$ & $1 q 91$ & $2 q 91$ \\
\hline Aluminum & & & - & & & & & & & \\
\hline Iron & & & & & & & & & & \\
\hline Manganese & & & & & & & & & & \\
\hline pH & & & & & & & & & & \\
\hline Specific conductance & & & & & & & 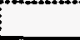 & & & \\
\hline
\end{tabular}

\begin{tabular}{|c|c|c|c|c|c|c|c|c|c|c|}
\hline $3 q 91$ & $4 q 91$ & $1 q 92$ & 2q92 & $3 \mathrm{q} 92$ & $4 \mathrm{q} 92$ & $1 q 93$ & $2 q 93$ & $3 q 93$ & $4 q 93$ & Analyte \\
\hline & & & & & & - & & - & & Aluminum \\
\hline & & & & & $\bullet$ & $\bullet$ & - & 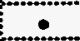 & $\bullet$ & Iron \\
\hline & & & & $\bullet$ & $\bullet$ & $\bullet$ & $\bullet$ & $\bullet$ & & Manganese \\
\hline & & & & & & & & & - & $\mathrm{pH}$ \\
\hline & & & & & & & & & $\because$ & Specific conductance \\
\hline
\end{tabular}

The Flag 2 table shows that iron and manganese have appeared above their flagging criteria several times since late 1992.

\section{Time Series Plots}

The time series plots in Appendix D indicate the following.

Conductivity was measured between approximately 75 and $275 \mu \mathrm{S} / \mathrm{cm}$ in RCP $1 D$ and at approximately $800 \mu \mathrm{S} / \mathrm{cm}$ in RCP 1A during fourth quarter 1993. 
All mercury concentrations in RCP 1D were measured below the $2 \mu \mathrm{g} / \mathrm{L}$ DWS.

Field pH levels in RCP 1D were measured between approximately 5 and $6 \mathrm{pH}$ units, and RCP $1 \mathrm{~A}$ was measured at approximately $11 \mathrm{pH}$ units during fourth quarter 1993.

No tritium activities exceeded the $20 \mathrm{pCi} / \mathrm{mL}$ DWS during the reporting period.

\section{Data}

The RCP wells were scheduled each quarter of 1993 for comprehensive analyses. The analyses were to be used for groundwater assessment in relation to the $R$ Reactor Seepage Basins. During first quarter, manganese was scheduled due to an earlier Flag 2 designation. Sampling for aluminum, iron, lead, and manganese was scheduled for the third quarter because of earlier Flag 1 or 2 designation.

According to field notes, the slow pump rate of RCP $1 \mathrm{~A}$ made sampling during first quarter impossible. RCP ID ran dry after approximately 10 gallons of light brown water were purged, and turbidity for the well varied. During the second quarter, RCP $1 D$ ran dry after 15 gallons of orange-brown water were purged.

RCP $1 \mathrm{~A}$ was not sampled during the third quarter because of mechanical problems, and RCP ID ran dry after 13 gallons of brown water were purged. RCP $1 D$ also ran dry during the fourth quarter after 11 gallons of brown water were purged.

Groundwater data from 1993 indicate specific conductance above its indicator parameter. Iron consistently exceeded its Flag 2 criterion.

\section{Disassembly Basin (RDB Well Series)}

The R-Area Disassembly Basin groundwater has been monitored by the three wells of the RDB well series since 1990. The disassembly basin is near the top of the groundwater mound and the horizontal groundwater flow direction is not clearly defined.

\section{DWS and Indicator Parameters}

\section{Analytes Over the DWS or Indicator Parameters in Well Series RDB}

\begin{tabular}{|c|c|c|c|c|c|}
\hline Analyte & 1989 & 1990 & 1991 & 1992 & 1993 \\
\hline Alkalinity & & - & - & - & - \\
\hline Iead & & & & $\therefore$ & $\therefore$ \\
\hline Specific conductance & & $\ldots$ & $\therefore$ & $\therefore$ & 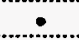 \\
\hline Total dissolved solids & & & & & $\therefore$ \\
\hline Total radium & & $\cdot$ & & & \\
\hline Tritium & & $\bullet$ & $\bullet$ & & \\
\hline
\end{tabular}


The DWS table shows that alkalinity and specific conductance have been consistently above their indicator parameters since 1990.

Flag 2 Criteria

Analytes Over Flag 2 Criteria in Well Series $\mathrm{RDB}$

\begin{tabular}{|c|c|c|c|c|c|c|c|c|c|c|}
\hline Analyte & $1 q 89$ & $2 q 89$ & $3 q 89$ & $4 q 89$ & $1 q 90$ & $2 q 90$ & $3 q 90$ & $4 q 90$ & $1 q^{91}$ & $2 q 91$ \\
\hline Aluminum & & & & & & & & & & . \\
\hline Calcium & & & & & & & & & $\therefore$ & \\
\hline Iron & & & & & $\bullet$ & $\bullet$ & $\therefore$ & $\therefore$ & 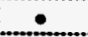 & \\
\hline Lead & & & & & 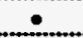 & & & & & \\
\hline Manganese & & & & & 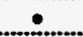 & $\bullet$ & $\therefore$ & $\cdots$ & $\therefore$ & \\
\hline Total radium & & & & & $\because$ & & & & & \\
\hline Tritium & 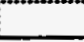 & & 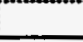 & & $\bullet$ & $\bullet$ & - & $\bullet$ & $\bullet$ & \\
\hline
\end{tabular}

\begin{tabular}{|c|c|c|c|c|c|c|c|c|c|c|}
\hline 3q91 & $4 q 91$ & 1q92 & $2 q 92$ & 3q92 & $4 q 92$ & $1 q 93$ & $2 q 93$ & $3 q 93$ & $4 q 93$ & Analyte \\
\hline & & & & & & $\bullet$ & & $\bullet$ & & Aluminum \\
\hline & & & & & 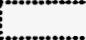 & & & & & Calcium \\
\hline$\therefore$ & & $\because$ & $\because$ & $\because$ & .... & $\therefore$ & $\therefore$. & $\cdots$ & 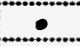 & Iron \\
\hline & & & & & $\bullet$ & & $\bullet$ & $\bullet$ & & Lead \\
\hline$\therefore$ & & $\therefore$ & $\therefore$ & $\therefore$ & $\therefore$ & $\therefore$ & $\therefore$ & $\therefore$ & $\therefore$ & Manganese \\
\hline & & & & & & & 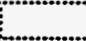 & & & Total radium \\
\hline & & & & & & & & & & Tritiom \\
\hline
\end{tabular}

The Flag 2 table shows that iron, lead, and manganese have frequently been above their Flag 2 criteria.

\section{Time Series Plots}

The time series plots (Appendix D) indicate the following.

Conductivity in RDB wells fluctuated greatly during sampling. Measurements in all wells ranged from approximately $100 \mu \mathrm{S} / \mathrm{cm}$ in $\mathrm{RDB} 3 \mathrm{D}$ during first quarter 1993 to $360 \mu \mathrm{S} / \mathrm{cm}$ in RDB 2D during second quarter 1993.

All gross alpha in $\mathrm{RDB}$ wells remained within the 1 to $3 \mathrm{pCi} / \mathrm{L}$ range with the exception of RDB 3D, which was measured at approximately $4 \mathrm{pCi} / \mathrm{L}$ during third quarter 1993.

No nonvolatile beta activities exceeded the DWS of $50 \mathrm{pCi} / \mathrm{L}$. Nonvolatile beta in $\mathrm{RDB} 2 \mathrm{D}$ was measured between approximately 4 and $11 \mathrm{pCi} / \mathrm{L}$.

Mercury concentrations were observed below the $2 \mu \mathrm{g} / \mathrm{L}$ DWS during the reporting period.

Field pH levels in RDB wells were measured between approximately 6 and $7 \mathrm{pH}$ units during sampling. 


\section{Data}

During each quarter of 1993, comprehensive analyses were requested for the RDB wells, except that herbicides/pesticides, major ions, phenols, total organic halogens, and tritium were scheduled for sampling only in the first quarter. Alpha spectroscopy plutonium, gamma PHA, and strontium-90 were also scheduled for each quarter. During third quarter, aluminum was scheduled for analysis due to earlier Flag 2 designation. Analyses were to be used for groundwater assessment in relation to the R-Reactor Seepage Basins.

All three wells ran dry during each quarter: first quarter, RDB $1 D$ ran dry after 14.5 gallons of light brown water were purged, RDB $2 \mathrm{D}$ after approximately 20 gallons of brown water in which turbidity varied, and $\mathrm{RDB} 3 \mathrm{D}$ after approximately 15 gallons of light brown water.

During second quarter, RDB $1 D$ ran dry after 12.5 gallons of brown water were purged, RDB 2D after 15.5 gallons of brown water, and RDB $3 D$ after 13.5 gallons of brown water. During sampling in the third quarter, RDB $1 D$ ran dry after 13 gallons of brown water were purged, RDB 2D after 12 gallons of yellow brown water, and $\mathrm{RDB} 3 \mathrm{D}$ after 14 gallons of yellow brown water.

During fourth quarter, RDB ID ran dry after 13 gallons of light brown water were purged, RDB 2D after 17 gallons of brown water, and RDB 3D after 12 gallons of brown water.

Quarterly data from 1993 show lead exceeding the drinking standard in well RDB 1D. (See Table 1 at the end of this section.)

\section{Burning/Rubble Pits (RRP Well Series)}

Groundwater below the R-Area Burning/Rubble Pits has been monitored by the four wells of the RRP well series since 1983. Horizontal groundwater flow in the upper saturated zone beneath the pits is to the southeast, toward two small ponds which drain into Par Pond to the east.

\section{DWS and Indicator Parameters}

Analytes Over the DWS or Indicator Parameters in Well Series RRP

\begin{tabular}{|c|c|c|c|c|c|}
\hline Analyte & 1989 & 1990 & 1991 & 1992 & 1993 \\
\hline Chloroethene (Vinyl chloride) & & & $\bullet$ & & \\
\hline Lead & & & $\bullet$ & & \\
\hline Specific conductance & & & & & $\bullet$ \\
\hline
\end{tabular}

The DWS table shows no consistent constituents of concern at this site.

Flag 2 Criteria 
Analytes Over Flag 2 Criteria in Well Series RRP

\begin{tabular}{|c|c|c|c|c|c|c|c|c|c|c|}
\hline Analyte & 1989 & $2 q 89$ & $3 q 89$ & $4 q 89$ & $1 q 90$ & $2 q 90$ & $3 q 90$ & $4 \mathrm{q} 90$ & Iq91 & 2q91 \\
\hline \multicolumn{11}{|c|}{ Chloroethene (Vinyl Chloride) } \\
\hline \multicolumn{11}{|c|}{ 1,1-Dichloroethane } \\
\hline Iron & & $\because$ & & & & & $\therefore$ & & $\therefore$ & \\
\hline Lead & & & & & & & & & $\bullet$ & \\
\hline \multicolumn{11}{|l|}{ Manganese } \\
\hline Silica & & & $"$ & & & 4 & & & $\bullet$ & \\
\hline Total organic halogens & & 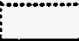 & 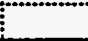 & 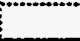 & 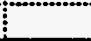 & 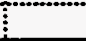 & 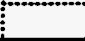 & 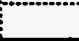 & $\bullet$ & \\
\hline
\end{tabular}

\begin{tabular}{|c|c|c|c|c|c|c|c|c|c|c|}
\hline 3q91 & $4 \mathrm{q} 91$ & $1 q 92$ & 2q92 & $3 q 92$ & $4 \mathrm{q} 92$ & $1 q 93$ & $2 \mathrm{q93}$ & 3q93 & $4 \mathrm{q93}$ & Analyte \\
\hline - & & & & & & & & & & Chloroethene (Vinyl chloride) \\
\hline$\because$ & & & & & & & & & & 1,-Dichloroethan \\
\hline$\bullet$ & & $\bullet$ & & $\therefore$ & & $\therefore$ & & & & Iron \\
\hline & & & & & & & & & & Lead \\
\hline & & & & & & & & & & Manganese \\
\hline & & & & & & & & & & Silica \\
\hline & & & & & & & & & & Total organic halogens \\
\hline
\end{tabular}

The Flag 2 table shows no consistent pattern at this site.

\section{Time Series Plots}

Conductivity in RRP wells remained between approximately 10 and $55 \mu \mathrm{S} / \mathrm{cm}$ during the reporting period until third quarter 1993 sampling, measurements for when RRP 2, RRP 3, and RRP 4 greatly increased.

Lead concentrations in RRP wells varied greatly during sampling. For example, RRP 1 concentrations ranged from approximately $45 \mu \mathrm{g} / \mathrm{L}$ during fourth quarter 1984 to approximately $8 \mu \mathrm{g} / \mathrm{L}$ during third quarter 1992.

No mercury concentrations were detected above the DWS.

Nonvolatile beta activities in RRP wells were consistently detected below the DWS of $50 \mathrm{pCi} / \mathrm{L}$. RRP 4 had the highest level of activity, measuring approximately $11 \mathrm{pCi} / \mathrm{L}$ in first quarter 1985.

Field pH levels in RRP wells were between approximately 3.5 and $5.5 \mathrm{pH}$ units. All $\mathrm{pH}$ levels decreased during first quarter 1986, fourth quarter 1986, and third quarter 1990 sampling.

Tritium activities in all RRP wells greatly exceeded the $20 \mathrm{pCi} / \mathrm{L} \mathrm{DWS}$, ranging from approximately 1,000 to $3,000 \mathrm{pCi} / \mathrm{mL}$.

\section{Data}

The RRP wells were scheduled for comprehensive analyses during first quarter 1993 only. Copper and GCMS VOA analyses were requested in the first quarter for RCRA Facility Investigation/Remedial Investigation (RFI/RI) site 
investigations. During third quarter, iron and lead were scheduled for sampling due to earlier Flag 2 designations.

Specific conductance was found to be above standard in 1993.

\section{Seepage Basins (RSA, RSB, RSC, RSD, RSE, and RSF Well Series)}

R-Area seepage basin groundwater has been monitored by the RSA, RSB, RSC, RSD, RSE, and RSF well series since 1961. However, these well series did not receive routine comprehensive analyses before 1992 or 1993, making it difficult to examine contaminant patterns over time. Currently, there are four wells monitored in the RSA series, three in the RSB series, nine in the RSC series, 13 in the RSD series, 20 in the RSE series, and three in the RSF series.

Water-table gradients vary greatly in the vicinity of the six seepage basins. Basin 2 seems to sit astride a water table divide. In the vicinity of basins $3,4,5$, and 6 there appears to be a slight depression in the groundwater flow gradient. Beneath basins 3, 4, 5, and 6 and the northern portion of basin 2, horizontal flow direction in the upper saturated zone appears to vary between north and northwest, toward Mill Creek.

\section{DWS and Indicator Parameters}

Analytes Over the DWS or Indicator Parameters in the Seepage Basin Well Series

\begin{tabular}{|c|c|c|c|c|c|}
\hline Analyte & 1989 & 1990 & 1991 & 1992 & 1993 \\
\hline Cadmium & & $\therefore$ & $\therefore$ & $\therefore$ & $\therefore$ \\
\hline Gross alpha & $\because$ & $\therefore$ & $\therefore$ & 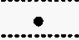 & $\because$ \\
\hline Lead & & & & $\cdots$ & $\because$ \\
\hline Mercury & & & & $\because$ & $\because$. \\
\hline Nitrate-nitrite as nitrogen & & & & & $\because$ \\
\hline Nonvolatile beta & $\therefore$ & $\therefore$ & - & $\therefore$ & $\therefore$ \\
\hline $\mathrm{pH}$ & $\therefore$ & $\therefore$ & - & - & $\therefore$ \\
\hline Potassium-40 & & & & $\therefore$ & \\
\hline Specific conductance & - & - & -.. & $\because$ & $\because$ \\
\hline Strontium-90 & & & & 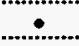 & 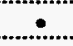 \\
\hline $\begin{array}{l}\text { Total alpha-emitting } \\
\text { radium }\end{array}$ & & & & $\bullet$ & $\cdot$ \\
\hline Total radium & & $\therefore$ & $\therefore$ & & \\
\hline Tritium & $\bullet$ & $\bullet$ & $\bullet$ & $\cdot$ & $\bullet$ \\
\hline
\end{tabular}

During 1989-1993, gross alpha, nonvolatile beta, and tritium were consistently detected above the DWS in R-Area Reactor Seepage Basin wells. Specific conductance and $\mathrm{pH}$ consistently exceeded indicator parameters. Cadmium has been above its DWS since 1990.

Flag 2 Criteria 
Analytes Over Flag 2 Criteria in Well Series RSA

\begin{tabular}{|c|c|c|c|c|c|c|c|c|c|c|}
\hline Analyte & $1 q 89$ & $2 q 89$ & $3 q 89$ & $4 q 89$ & $1 q 90$ & $2 q 90$ & 3q90 & $4 q 90$ & $1 q^{91}$ & $2 q 91$ \\
\hline Aluminum & & & & & & & & & & \\
\hline Cadmium & & & & & & & & $\bullet$ & $\bullet$ & \\
\hline Iron & & & & & & & & & & \\
\hline Lead & & & & & & & & & & \\
\hline Manganese & & & & & & & & & & \\
\hline Mercury & & & & & & & & & & \\
\hline Nonvolatile beta & $\therefore$ & & & & & & & & & \\
\hline Potassium -40 & & & & & & & & & & \\
\hline Strontium-90 & & & & & & & & & & \\
\hline $\begin{array}{l}\text { Total alpha-emitting } \\
\text { radium }\end{array}$ & & & & & & & & & & \\
\hline Total organic carbon & & & & & & & & & & \\
\hline Total radium & & & & & & & & & $\because$ & \\
\hline Tritium & & & & & & - & & & & \\
\hline
\end{tabular}

\begin{tabular}{|c|c|c|c|c|c|c|c|c|c|c|}
\hline 3q91 & $4 q 91$ & 1q92 & $2 q 92$ & $3 q 92$ & $4 q 92$ & $1 \mathrm{q} 93$ & $2 q 93$ & $3 q 93$ & $4 \mathrm{q} 93$ & Analyte \\
\hline & & & & & & $\therefore$ & & - & & Aluminum \\
\hline$\bullet$ & & & $\therefore$ & $\bullet$ & $\therefore$ & & & & & Cadmium \\
\hline & & & $\therefore$ & 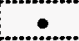 & & $\therefore$ & $\therefore$ & $\therefore$ & & Iron \\
\hline & & & & & & $\because$ & $\because$ & $\therefore$ & & Lead \\
\hline & & & $\therefore$ & & & $\because$ & & & & Manganese \\
\hline & & & $\bullet$ & & $\bullet$ & $\because$ & & & & Mercury \\
\hline & & & & & & & & & & Nonvolatile beta \\
\hline & & & & - & & & & & & Potassium -40 \\
\hline & & & & & & $\bullet$ & & & & Strontium-90 \\
\hline & & & & & & & & & & $\begin{array}{l}\text { Total alpha-emitting } \\
\text { radium }\end{array}$ \\
\hline & & & & $\therefore$ & & & & & & Total organic carbon \\
\hline & & & & & & & & & & Total radium \\
\hline & & & & & & & & - & & Tritium \\
\hline
\end{tabular}

The Flag 2 table for RSA wells does not show consistent contamination over the reporting period.

\section{Analytes Over Flag 2 Criteria in Well Series RSB}

\begin{tabular}{|c|c|c|c|c|c|c|c|c|c|c|}
\hline Analyte & $1 q 89$ & $2 q 89$ & $3 q 89$ & $4 q 89$ & 1q90 & $2 q 90$ & $3 q 90$ & $4 q 90$ & 1q91 & 2q91 \\
\hline Aluminum & & & & & & & & & & \\
\hline Cadmium & & & & & & $\therefore$ & & & & \\
\hline Chromium & & & & & & $\therefore$ & & & & \\
\hline Gross alpha & & & $\therefore$ & & & $\therefore$ & & & & \\
\hline Iron & & & & & & & & & & \\
\hline Lead & & & & & & $\bullet$ & & & & \\
\hline Manganese & & & & & & & & & & \\
\hline Mercury & & & & & & & & & & \\
\hline Nonvolatile beta & & & & & & $\therefore$ & & & & \\
\hline $\begin{array}{l}\text { Total alpha-emitting } \\
\text { radium }\end{array}$ & & & & & & & & & & \\
\hline
\end{tabular}

\begin{tabular}{|c|c|c|c|c|c|c|c|c|c|c|}
\hline $3 q 91$ & 4q91 & 1q92 & 2q92 & 3q92 & $4 q 92$ & Iq93 & $2 q 93$ & $3 q 93$ & $4 \mathrm{q} 93$ & Analyte \\
\hline & & & & & & $\therefore$ & & $\therefore$. & & Alumininum \\
\hline$\bullet$ & & $\bullet$ & • & • & - & $\bullet$ & - & $\bullet$ & $\bullet$ & Cadmium \\
\hline
\end{tabular}




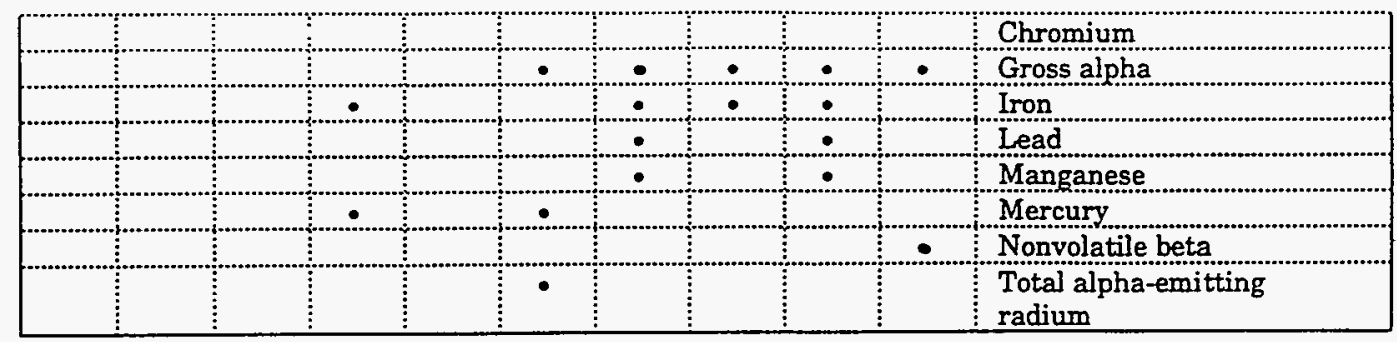

The Flag 2 table for RSB wells shows consistent results above criteria for cadmium in 1992-93, and for gross alpha, iron, and lead in 1993.

\section{Analytes Over Flag 2 Criteria in Well Series RSC}

\begin{tabular}{|c|c|c|c|c|c|c|c|c|c|c|}
\hline Analyte & $1 q 89$ & $2 q 89$ & $3 q 89$ & $4 q 89$ & $1 q 90$ & $2 \mathrm{q} 90$ & $3 q 90$ & $4 q^{90}$ & $1 q 91$ & $2 q 91$ \\
\hline Aluminum. & & & & & & & & & & \\
\hline Cadmium & & & & & & & & & & \\
\hline Gross alpha & & & & & & & & & & \\
\hline Iron & & & & & & & & & & \\
\hline Lead & & & & & & & & & & \\
\hline Manganese & & & & & & & & & & \\
\hline Mercury & & & & & & & & & & \\
\hline Nonvolatile beta & & & & & & & & & $\vdots$ & \\
\hline Specific conductance & & & & & & & & & & \\
\hline Total organic carbon & & & & & & & & & & \\
\hline Total organic halogens & $\vdots$ & & $\vdots$ & ： & $\vdots$ & & $\vdots$ & & 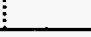 & \\
\hline
\end{tabular}

\begin{tabular}{|c|c|c|c|c|c|c|c|c|c|c|}
\hline $3 q 91$ & $4 q 91$ & $1 q 92$ & $2 q 92$ & 3q92 & $4 q 92$ & $1 q 93$ & $2 q 93$ & $3 q 93$ & $4 q 93$ & Analyte \\
\hline & & & & & & $\cdot$ & & $\bullet$ & & Aluminum \\
\hline & & & & & & $\bullet$ & $\bullet$ & $\bullet$ & $\bullet$ & Cadmium \\
\hline & & & & & & $\because$ & $\bullet$ & 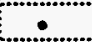 & 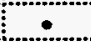 & Gross alpha \\
\hline & & & & & & $\bullet$ & & $\bullet$ & & Iron \\
\hline & & & & & & $\bullet$ & & $\bullet$ & & Lead \\
\hline & & & & & & $\bullet$ & & $\bullet$ & & Manganese \\
\hline & & & & & & $\bullet$ & & $\bullet$ & & Mercury \\
\hline & & & & & & $-\cdots$ & & & $\because$ & Nonvolatile beta \\
\hline & & & & $\bullet$ & & & & & & Specific conductance \\
\hline & & & & & & & & $\therefore$ & & Total organic carbon \\
\hline & & & 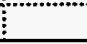 & & 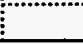 & & & $\bullet$ & & Total organic halogens \\
\hline
\end{tabular}

The Flag 2 table for RSC wells shows gross alpha and cadmium consistently above criteria during 1993. 
Analytes Over Flag 2 Criteria in Well Series RSD

\begin{tabular}{|c|c|c|c|c|c|c|c|c|c|c|}
\hline Analyte & 1989 & $2 q 89$ & $3 q 89$ & $4 q 89$ & $1 q^{90}$ & $2 q 90$ & $3 q 90$ & $4 q 90$ & $1 q 91$ & $2 q 91$ \\
\hline Aluminum & & & & & & & & & & \\
\hline Cadmium & & & & & & $\therefore$ & & & $\therefore$ & \\
\hline Gross alpha & & $\therefore$ & & & & & & & & \\
\hline Iron & & & & & & & & & & \\
\hline Lead & & & & & & & & & & \\
\hline Manganese & & & & & & & & & & \\
\hline Mercury & & & & & & & & & & \\
\hline Nonvolatile beta & $\ldots$ & $\therefore$ & $\therefore$ & $\because$ & $\ldots$ & $\therefore$ & $\therefore$ & $\therefore$ & $\therefore$ & \\
\hline Strontium-90 & & & & & & & & & & \\
\hline Total alpha-emitting radium & & & ל. & & & & & & & \\
\hline Total organic halogens & & & 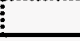 & $\vdots$ & & & 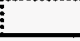 & & & \\
\hline
\end{tabular}

\begin{tabular}{|c|c|c|c|c|c|c|c|c|c|c|}
\hline 3q91 & $4 \mathrm{q} 91$ & 1q92 & $2 q 92$ & 3q92 & $4 q 92$ & 1q93 & $2 q 93$ & 3q93 & $4 q 93$ & Analyte \\
\hline & & & & & & $\bullet$ & & $\therefore$ & & Aluminum \\
\hline (n) & & $\bullet$ & $\therefore$ & $\therefore$ & & 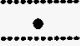 & $\therefore$ & $\therefore$ & -... & Cadmium \\
\hline 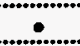 & & & & $\because$ & & 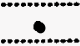 & $\therefore$ & $\therefore$ & - & Gross alpha \\
\hline & & & $\therefore$ & $\bullet$ & - & $\bullet$ & $\bullet$ & 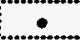 & $\because$ & Iron \\
\hline & & & & & & $\therefore$ & & $\therefore$ & & Lead \\
\hline & & & & & & $\therefore$ & & $\because$ & & Manganese \\
\hline & & & & & $\therefore$ & $\because$ & & & & Mercury \\
\hline$\because$ & & ........... & & $\therefore$ & & $\therefore$ & $\bullet$ & $\because$ & 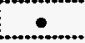 & Nonvolatile beta. \\
\hline & & & & 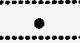 & $\therefore$ & $\because$ & $\therefore$ & $\therefore$ & 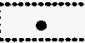 & Strontium-90 \\
\hline & & & & & $\bullet$ & $\bullet$ & & 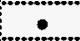 & & Total alpha-emitting radium \\
\hline & & & & & & & & $\bullet$ & & Total organic halogens \\
\hline
\end{tabular}

The Flag 2 table for RSD wells shows contamination with nonvolatile beta nearly every quarter of the reporting period, with strontium-90 during the latter half of 1992 and all of 1993, and with cadmium and iron almost every quarter of $1992-$ 1993.

\section{Analytes Over Flag 2 Criteria in Well Series RSE}

\begin{tabular}{|c|c|c|c|c|c|c|c|c|c|c|}
\hline Analyte & $1 q 89$ & $2 q 89$ & $3 q 89$ & $4 q 89$ & $1 q 90$ & $2 q 90$ & $3 q 90$ & $4 q 90$ & $1 q 91$ & $2 q 91$ \\
\hline Aluminum & & & & & & & & & & \\
\hline Cadmium & & & & & & $\therefore$ & & $\therefore$ & $\therefore$ & \\
\hline Gross alpha & & & & $\therefore$ & & 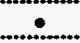 & & & & \\
\hline Iron & & & & & & & & & & \\
\hline Lead & & & & & & & & & & \\
\hline Manganese & & & & & & & & & & \\
\hline Mercury & & & & & & & & & & \\
\hline Nitrate-nitrite as nitro & & & & & & & & & & \\
\hline Nonvolatile beta & $\bullet$ & $\therefore$ & $\therefore$ & $\therefore$ & $\bullet$ & $\therefore$ & $\therefore$ & $\therefore$ & $\therefore$ & \\
\hline Strontium -90 & & & & & & & & & & \\
\hline Total alpha-emitting ra & & & & & & & & & & \\
\hline Total organic carbon & & & & & & & & & & \\
\hline Total organic halogens & & & & & & $\bullet$ & & 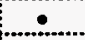 & & \\
\hline Total radium & & & & & & $\therefore$ & & & & \\
\hline Tritium & & & $\bullet$ & & $\bullet$ & 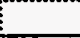 & $\vdots$ & $\bullet$ & $\bullet$ & \\
\hline
\end{tabular}




\begin{tabular}{|c|c|c|c|c|c|c|c|c|c|c|}
\hline $3 q 91$ & $4 \mathrm{q} 91$ & $1 \mathrm{q} 92$ & $2 q 92$ & $3 q 92$ & $4 q 92$ & Iq93 & $2 q 93$ & $3 q 93$ & $4 q 93$ & Analyte \\
\hline & & & & & & $\therefore$ & & $\therefore$ & & Aluminum \\
\hline$\therefore$ & & $\therefore$ & $\therefore$ & $\therefore$ & $\bullet$ & $\therefore$ & $\therefore$ & $\therefore$ & $\therefore$ & Cadmium \\
\hline$\therefore$ & & $\therefore$ & & 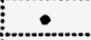 & & $\therefore$ & $\bullet$ & $\therefore$ & $\therefore$ & Gross alpha \\
\hline & & & & $\therefore$ & & $\therefore$ & $\because$ & $\therefore$ & $\therefore$ & Iron \\
\hline & & & & & $\therefore$ & $\therefore$ & $\because$. & $\therefore$ & $\therefore$ & Lead \\
\hline & & & - & - & - & $\therefore$ & $\therefore$ & $\therefore$ & & Manganese \\
\hline & & & & & $\therefore$ & $\therefore$ & & & & Mercury \\
\hline & & & & & & & $\therefore$ & $\therefore$ & & Nitrate-nitrite as nitrogen \\
\hline$\therefore$ & & -.. & $\bullet$ & $\therefore$ & & $\therefore$ & $\therefore$ & $\therefore$ & $\therefore$ & Nonvolatile beta \\
\hline & & & & $\because$ & $\therefore$ & & ?.... & $\therefore$ & $\therefore$ & Strontium-90 \\
\hline & & & & & $\therefore$ & $\therefore$ & $\therefore$ & & & Total alpha-emitting radium \\
\hline & & & - & & & & & $\therefore$ & & Total organic carbon \\
\hline & & & & $\therefore$ & & & & & & Total organic halogens \\
\hline & & & & & & & & & & Total radium \\
\hline$\bullet$ & & $\bullet$ & $\bullet$ & $\cdot$ & $\bullet$ & $\bullet$ & $\bullet$ & $\bullet$ & & Tritium \\
\hline
\end{tabular}

The Flag 2 table for RSE wells shows consistent trends for gross alpha, nonvolatile beta, tritium, strontium-90, cadmium, iron, lead, and manganese.

\section{Analytes Over Flag 2 Criteria in Well Series RSF}

\begin{tabular}{|c|c|c|c|c|c|c|c|c|c|c|}
\hline Analyte & $1 q 89$ & $2 q 89$ & $3 q 89$ & $4 q 89$ & 1q90 & 2q90 & $3 q 90$ & $4 q 90$ & 1q91 & $2 q 91$ \\
\hline Aluminum & & & & & & & & & & \\
\hline Cadmium & & - & & & & & & & & \\
\hline Iron & & & & & & & & & & \\
\hline Mercury & & & & & & & & & & \\
\hline $\mathrm{pH}$ & $\therefore$ & $\therefore$ & $\therefore$ & $\therefore$ & $\therefore$ & $\therefore$ & $\therefore$ & $\therefore$ & & $\therefore$ \\
\hline $\begin{array}{l}\text { Total alpha-emitting } \\
\text { radium }\end{array}$ & & & & & & & & & & \\
\hline Total organic carbon & & & & & & & & & & \\
\hline Tritium & & & & & & & & & & \\
\hline
\end{tabular}

\begin{tabular}{|c|c|c|c|c|c|c|c|c|c|c|}
\hline 3q91 & $4 \mathrm{q} 91$ & $1 \mathrm{q} 92$ & $2 q 92$ & 3q92 & 4992 & $1 q 93$ & $2 q 93$ & 3q93 & $4 q 93$ & Analyte \\
\hline & & & & & & - & & - & & Aluminum \\
\hline & & & & & & & & & & Cadmium \\
\hline & & & & & & $\bullet$ & $\bullet$ & $\bullet$ & & Iron \\
\hline & & & & & & & & $\because$ & & Mercury \\
\hline & & & & & & $\bullet$ & $\bullet$ & & $\bullet$ & $\mathrm{pH}$ \\
\hline & & & & & & & & & & $\begin{array}{l}\text { Total alpha-emitting } \\
\text { radium }\end{array}$ \\
\hline & & & & $\because$ & & & & & & Total organic carbon \\
\hline 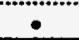 & & $\bullet$ & $\cdot$ & $\bullet$ & $\cdot$ & & $\bullet$ & $\bullet$ & 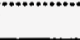 & Tritium \\
\hline
\end{tabular}

The Flag 2 table for RSF wells shows consistent trends for $\mathrm{pH}$ and tritium, with iron and aluminum appearing in 1993.

\section{Time Series Plots}

Cadmium concentrations in RSA wells fluctuated during the reporting period, ranging up to approximately $80 \mu \mathrm{g} / \mathrm{L}$ in RSA 9 during third quarter 1992. In RSB wells, cadmium concentrations in RSB 7 exceeded the DWS several times, reaching approximately $60 \mu \mathrm{g} / \mathrm{L}$ during fourth quarter 1991. RSB 8 and RSB 9 exceeded the DWS criterion during fourth quarter 1993. In the RSD wells, 
cadmium concentration in RSD 1 and RSD 2A exceeded the DWS several three times during sampling, and cadmium concentrations in RSD $2 \mathrm{C}$ increased to approximately $23 \mu \mathrm{g} / \mathrm{L}$ during fourth quarter 1993.

Cadmium concentrations increased in RSE 1A, RSE 1B, RSE 1C, and RSE 2 during fourth quarter 1992. Cadmium levels in RSE 9 rose to approximately 160 $\mu \mathrm{g} / \mathrm{L}$.

Conductivity in the RSA wells remained relatively constant with the exception of first quarter 1988, when RSA 8, RSA 9, and RSA 10 reached high levels. In the RSB wells, RSB 7 conductivity was unusually high during first quarter 1988. Conductivity in RSB 9 fluctuated during the reporting period, ranging from approximately 30 to $90 \mu \mathrm{S} / \mathrm{cm}$.

In the RSC wells, conductivity in RSC 5 measured approximately $2,500 \mu \mathrm{S} / \mathrm{cm}$ during third quarter 1992 sampling, . Conductivity levels in other wells were relatively constant. Conductivity in most RSD wells remained below $100 \mu \mathrm{S} / \mathrm{cm}$ during the reporting period, with the exception of RSD 1, RSD 9, and RSD 11, which were higher during various quarters.

In RSE wells 1 through 8, all conductivity levels were below $100 \mu \mathrm{S} / \mathrm{cm}$ with the exception of one reading for RSE 5 of approximately $250 \mu \mathrm{S} / \mathrm{cm}$ during first quarter 1988. In RSE wells 9 through 25, thre were some high readings during first quarter 1988, 1991, and second quarter 1993.

In the RSF wells, conductivity in RSF 1 fluctuated during 1987 through 1989 sampling, reaching a high of approximately $720 \mu \mathrm{S} / \mathrm{cm}$ during first quarter 1988 . RSF 2 and RSF 3 levels remained relatively constant during the reporting period.

No gross alpha activity in RSA wells exceeded the $15 \mathrm{pCj} / \mathrm{L}$ DWS criterion. In the RSB wells, gross alpha activity in RSB 7 exceeded DWS during third quarter 1989 and second quarter 1990, RSB 8 exceeded DWS during all quarters of 1993, and RSB 9 reached a high of approximately $75 \mathrm{pCi} / \mathrm{L}$ during second quarter 1993.

Gross alpha activities in all RSC wells increased during 1992 and 1993 sampling. RSC 10 reached approximately $114 \mathrm{pCi} / \mathrm{L}$ during first quarter 1993. Gross alpha activity in RSD wells 1 through 5 remained relatively constant with the exception of RSD 2C, which exceeded the $15 \mathrm{pCi} / \mathrm{L}$ DWS approximately five times during sampling, greatly exceeding the DWS during fourth quarter 1991.

Gross alpha activity in RSD wells 6 through 11 remained relatively constant with the exception of RSD 6 and RSD 8, each exceeding the DWS several times during sampling. In RSE wells $1 \mathrm{~A}$ through 6, RSE 4A, RSE 4C, and RSE 6 all exceeded the DWS during third quarter 1991. Gross alpha activity in RSE 6 increased to approximately $280 \mathrm{pCi} / \mathrm{L}$ during first quarter 1993. Gross alpha activity in RSE wells 7 through 25 remained relatively constant during sampling until fourth quarter 1989, when RSE 8 exceeded the DWS. RSE 10, RSE 11, RSE 18, and RSE 19 all exceeded DWS from 1991 to 1994. 
No gross alpha activity in RSF wells exceeded the $15 \mathrm{pCi} / \mathrm{L}$ DWS during sampling. RSF 3 reached approximately $8.5 \mathrm{pCi} / \mathrm{L}$ during fourth quarter 1987.

Lead concentrations in RSA 7, RSA 8, and RSA 9 wells all exceeded the $50 \mu \mathrm{g} / \mathrm{L}$ DWS during 1993 sampling. All RSB wells exceeded DWS during 1993 sampling. RSB 9 reached a high of approximately $500 \mu \mathrm{g} / \mathrm{L}$ during first quarter 1993. All RSC wells, with the exceptions of RSC 2 and RSC 4, exceeded the DWS during 1993 sampling. Lead concentrations in most RSD wells increased during 1993 sampling, with RSD 6 reaching approximately $290 \mu \mathrm{g} / \mathrm{L}$ during first quarter 1993.

Lead concentrations in RSE wells 1A through 6 remained stable throughout sampling with the exception of RSE 6 , which was measured at approximately $1,900 \mu \mathrm{g} / \mathrm{L}$ during first quarter 1993. Lead concentrations in RSE wells 7 through 25 remained relatively constant until 1993, when RSE 18 and RSE 19 exceeded the DWS during second and third quarters.

Mercury levels in RSA 9 reached a high of approximately $8.5 \mu \mathrm{g} / \mathrm{L}$ during fourth quarter 1992 and decreased to approximately $2.5 \mu \mathrm{g} / \mathrm{L}$ during the following sampling quarter.

Mercury concentrations in RSB 8 and RSB 9 were measured below the DWS criterion of $2 \mu \mathrm{g} / \mathrm{L}$ during 1993 sampling. Mercury in RSB 7 exceeded DWS during second and fourth quarters 1992. In the RSC wells, mercury concentrations in RSC 2, RSC 5, and RSC 9 significantly decreased from first to second quarter 1993. All three of these wells were measured above the $2 \mu \mathrm{g} / \mathrm{L}$ DWS during first quarter sampling.

Mercury concentration in RSD 3 exceeded the DWS during fourth quarter 1992. RSD $2 \mathrm{C}$ also exceeded the DWS, measuring approximately $5.5 \mu \mathrm{g} / \mathrm{L}$ during first quarter 1993. In RSE wells.1A through 6, mercury concentrations in RSE IA, RSE 1B, RSE 1C, RSE 2, and RSE 3A noticeably decreased from fourth quarter 1992 to first quarter 1993. RSE $4 A$ and RSE 6 exceeded the DWS during first quarter 1993 sampling, and concentrations in RSE 4A greatly decreased during second quarter 1993. Mercury levels in RSE 13 and RSE 25 were measured above the DWS during first quarter 1993, while generally, mercury in RSE wells 7 through 25 decreased from first to second quarter 1993.

Nonvolatile beta activity in RSA wells remained consistently below the $50 \mathrm{pCi} / \mathrm{L}$ DWS criterion, with the exception of an anomalous reading for RSA 10 of approximately $90 \mathrm{pCi} / \mathrm{L}$ during first quarter 1989. Nonvolatile beta activity in RSB wells remained consistently below the DWS with the exception of an anomalous reading for RSB 7 of approximately $120 \mathrm{pCi} / \mathrm{L}$ during second quarter 1990. Nonvolatile beta activity in RSC 4 decreased sharply during fourth quarter 1983. All other RSC wells remained at relatively consistent levels until 1993, when both RSC 4 and RSC 10 exceeded the DWS.

In RSD wells 1 through 5 , nonvolatile beta activity in RSD $2 \mathrm{C}$ fluctuated throughout the reporting period, ranging from approximately $7,900 \mathrm{pCi} / \mathrm{L}$ during 
third quarter 1979 to approximately detection limit during first quarter 1983. RSD 2B was measured at approximately 4,500 pCi $/ \mathrm{L}$ during third quarter 1987 sampling, exceeding the $50 \mathrm{pCi} / \mathrm{L}$ DWS criterion. Nonvolatile beta activity in RSD wells 6 through 11 fluctuated greatly throughout the reporting period. Activity in RSD 8 reached a high of approximately $2,800 \mathrm{pCi} / \mathrm{L}$ during fourth quarter 1979 sampling. Other wells exceeding the $50 \mathrm{pCi} / \mathrm{L}$ DWS included RSD 6 and RSD 7.

In RSE wells $1 \mathrm{~A}$ through 6 , nonvolatile beta activity in RSE 6 consistently exceeded the $50 \mathrm{pCi} / \mathrm{L}$ DWS, measuring approximately $30,000 \mathrm{pCi} / \mathrm{L}$ during first quarter 1982 sampling. RSE 1B, RSE 4B, and RSE 4C exceeded the DWS during various quarters. Nonvolatile beta activity in RSE wells 7 through 25 remained relatively constant during sampling, with the exception of several fluctuations in RSE 11, and apparently anomalous increases in RSE 13 during first quarter 1988 and RSE 10 during first quarter 1993.

No RSF wells exceeded the DWS for nonvolatile beta during the reporting period.

Field pH levels in RSA wells fluctuated throughout the reporting period. Levels in all wells ranged from approximately 3.5 to $7 \mathrm{pH}$ units. All pH levels decreased during first quarter 1992. RSB pH levels fluctuated between approximately 4.5 and $8 \mathrm{pH}$ units during the reporting period.

RSC pH levels were between approximately 4 and $7 \mathrm{pH}$ units. RSC 9 decreased during third quarter 1991. RSD pH levels were consistently measured between approximately 4 and $7.5 \mathrm{pH}$ units. Most RSD pH levels increased during second quarter 1989, fourth quarter 1990, fourth quarter 1991, and first quarter 1992.

In RSE wells 1 through 7, $\mathrm{pH}$ levels increased during first quarter 1988, first quarter 1989, third quarter 1991, and fourth quarter 1992. In RSE wells 8 through 25, pH levels generally fluctuated between 3.5 and 7. RSE 9 reached a low of approximately 3.5 during first quarter 1988.

RSF pH levels were relatively consistent throughout sampling. RSF 1 was measured between approximately 9 and $12 \mathrm{pH}$ units. RSF 2 was consistently measured between 5 and $6 \mathrm{pH}$ units, and RSF 3 between approximately 6 and 7 .

Tritium activities in many of the R-Area Seepage Basin wells consistently exceeded the $20 \mathrm{pCi} / \mathrm{mL}$ DWS during sampling.

\section{Data}

Quarterly data for 1993 indicate that gross alpha consistently exceeded DWS in wells RSB 8 and RSC 9, and cadmium was above the DWS in well RSC 3 for all four quarters. Wells RSC 7 and RSC 10 showed high amounts of lead during first quarter monitoring. Nonvolatile beta was consistently above the DWS in wells RSD 2A, RSD 2C, RSD 4, RSD 5, RSD 6, RSD 7, RSD 8, RSD 10, RSE 4A, RSE $4 \mathrm{C}$, RSE 11, RSE 13, and RSE 19. See Table 1 at the end of this section. 
For the RSA well series, comprehensive analyses were scheduled during the first quarter of 1993 only. Cadmium, gross alpha, iron, manganese, mercury, nonvolatile beta, total organic halogens, and tritium analyses were scheduled due to earlier Flag 2 designations. Those analytes plus aluminum, lead, radium, and total organic carbon were scheduled during the third quarter due to earlier Flag 2 designations.

During each quarter of 1993 , cadmium, gross alpha, and nonvolatile beta analyses were requested for well RSA 6 to define plumes accurately. Water elevation was also requested for RSA 6 to define groundwater flow. Analyses of RSA 7, 8, 9, and 10 were requested for Comprehensive Environmental Response, Compensation, and Liability Act (CERCLA) investigation.

According to field notes, RSA 6 had not been installed during the first quarter of 1993. In the remaining quarters, several RSA wells ran dry during sampling. During second quarter, RSA 7 ran dry after 32 gallons of water were purged, RSA 8 ran dry after 33 gallons and an elbow cracked on the pump outlet. RSA 9 ran dry after 31 gallons, and RSA 10 ran dry after 17 gallons.

During third quarter, RSA 7 again ran dry after 16 gallons of water were purged, RSA 9 ran dry after 18 gallons, and RSA 10 ran dry after 9 gallons In fourth quarter, RSA 8 ran dry after 2.5 gallons and RSA 9 ran dry after 2.3 gallons were purged.

For the RSB well series, comprehensive analyses were scheduled during the first quarter of 1993 only. Cadmium, gross alpha, iron, manganese, mercury, nonvolatile beta, total organic carbon, total organic halogens, and tritium analyses were scheduled during first quarter due to earlier Flag 2 designation. Those analytes plus aluminum, lead, and radium were scheduled for third quarter due to earlier Flag 2 designations.

During first quarter, turbidity for all RSB wells was measured above 1,000 NTU. During the second quarter, RSB 7 ran dry after 15 gallons of water were purged.

Turbidity was measured above 1,000 NTU for RSB 8 during third quarter. During fourth quarter sampling, RSB 7 ran dry after about one-half gallon was purged, water was muddy in RSB 8, and turbidity for RSB 9 was measured above 1,000 NTU.

For the RSC well series, comprehensive analyses were scheduled for the first quarter of 1993 only.

Cadmium, gross alpha, iron, nitrate as nitrogen, nonvolatile beta, manganese, mercury, total organic carbon, total organic halogens, and tritium analyses were scheduled during first quarter due to earlier Flag 2 designation. Those analytes plus aluminum, lead, and radium were scheduled for third quarter due to earlier Flag 2 designations. 
Cadmium, gross alpha, and nonvolatile beta were requested each quarter to define plumes accurately, and water elevations were requested to define groundwater flow.

According to field notes for first quarter 1993, well RSC 1 had been destroyed and sampling was impossible. During that quarter, the turbidity of RSC 4,5 , and 10 was measured above 1,000 NTU.

Turbidity was also measured above 1,000 NTU for RSC 2, 5, and 9 during third quarter and for $\mathrm{RSC} 2,3,4,5,6,7,8$, and 9 during fourth quarter.

For the RSD well series, comprehensive analyses were scheduled for the first quarter of 1993 only. Cadmium, gross alpha, iron, manganese, mercury, nonvolatile beta, total organic carbon, total organic halogens, and tritium analyses were scheduled for first quarter due to earlier Flag 2 designations. Those analytes plus aluminum, lead, and radium were scheduled for third quarter due to earlier Flag 2 designations.

Cadmium, gross alpha, and nonvolatile beta analyses were requested for all wells except RSD 1 and 3 during each quarter to define plumes accurately. Water elevations for those wells were also requested to define groundwater flow. Analyses of RSD 1 and 3 were requested for CERCLA investigation.

During the first quarter of 1993, RSD 2B ran dry during sampling and turbidity was measured above 1,000 NTU. Turbidity was high in wells RSD 6, 8, 9, and 10 also. During second quarter, RSD 1 ran dry after 17 gallons of water were purged, RSD 2B was bailed dry and sampled after recovery, and RSD 3 ran dry after 26 gallons were purged. RSD 1 and RSD 3 ran dry after 16 gallons were purged during third quarter, and turbidity was measured above 1,000 NTU for wells RSD 8, 9, and 11. During fourth quarter, RSD 1 ran dry after 1.4 gallons were purged, water in RSD 2A was black, and water in RSD 2C, where turbidity was measured above 1,000 NTU, was milky brown. Turbidity was also high in RSD 5, 9, and 10. Water in RSD 9 was muddy and was cloudy in RSD 10 and 11.

For the RSE well series, comprehensive analyses were scheduled for the first quarter of 1993 only. Cadmium, gross alpha, iron, manganese, mercury, nonvolatile beta, total organic carbon, total organic halogens, and tritium analyses were scheduled for first quarter due to earlier Flag 2 designations. Those analytes plus aluminum, lead, and radium were scheduled for third quarter due to earlier Flag 2 designations.

During first quarter, RSE 24 and 25 were scheduled for lead analysis, in addition to the other comprehensive analyses scheduled, due to earlier Flag 2 designation. Aluminum analysis was not scheduled during third quarter.

Cadmium, gross alpha, and nonvolatile beta analyses were requested each quarter for RSE $4 \mathrm{~A}, 4 \mathrm{~B}, 4 \mathrm{C}, 5,6,11,12,13,18$, and 19 to define plumes accurately. The water elevation of those wells was also requested for definition of groundwater flow. 
Comprehensive analyses of $\mathrm{RSE} 1 \mathrm{~A}, 1 \mathrm{~B}, 1 \mathrm{C}, 2,3 \mathrm{~A}, 7,8,9,10,24$, and 25 were requested for CERCLA investigation.

During first quarter, turbidity was measured above 1,000 NTU for RSE 3A and $4 B$, and RSE $4 A, 4 B, 4 C$, and 6 ran dry during sampling. Sampling of RSE 12 was impossible because a sample bailer was stuck in the well. RSE 13 ran dry after 10 gallons of water were purged, RSE 24 ran dry after approximately 40 gallons, and RSE 25 ran dry after approximately 29.5 gallons. RSE 24 and 25 did not have well identification signs, according to field notes.

During second quarter, several RSE wells ran dry during sampling: RSE 1A ran dry after 23 gallons of water were purged, RSE $1 B$ after 18 gallons, RSE 1C after 19 gallons, RSE 2 after 22 gallons, RSE $3 A$ after 18 gallons, RSE 7 after 18 gal, RSE 8 after 29 gallons, RSE 9 after 19 gallons, RSE 10 after 27 gallons, RSE 24 after 35.5 gallons, and RSE 25 after approximately 27.5 gallons of (light brown) water were purged.

Sampling of RSE 12 was again impossible because of a sample bailer stuck in the well. Several wells ran dry during third quarter sampling, including RSE 2 after 27 gallons, RSE $3 A$ after 16 gallons, RSE 24 after 33 gallons, and RSE 25 after 26.5 gallons Turbidity of RSE 19 was measured above 1,000 NTU.

During fourth quarter, RSE 12 was again not sampled because of maintenance or access problems. Wells that ran dry during fourth quarter sampling included RSE $1 \mathrm{~A}$ after 1.4 gallons, RSE $1 \mathrm{~B}$ after .8 gallon, RSE $1 \mathrm{C}$ after 1.2 gallons, RSE 2 after 1.5 gallons, RSE 3A after 1.4 gallons, RSE 9 after .9 gallon, RSE 24 after 32 gallons, and RSE 25 after 26.5 gallons. High turbidity was measured in RSE 18 and 19 during fourth quarter.

RSE 6, as part of the partial implementation of the purge-water containment program, was scheduled for analyses but not sampled during 1993. Only waterlevel measurements were taken for RSE 6 .

For the RSF well series, comprehensive analyses were scheduled for the first quarter of 1993. Cadmium, gross alpha, iron, lead, manganese, mercury, nonvolatile beta, total organic carbon, total organic halogens, and tritium analyses were scheduled for first quarter due to earlier Flag 1 or 2 designations. Those analytes plus aluminum and radium were scheduled for third quarter due to earlier Flag 2 designations. Analyses of most other comprehensives were requested for all quarters for CERCLA investigation.

During first quarter, RSF 1 ran dry after approximately 41 gallons of water were purged and RSF 3 after approximately 37 gallons were purged. According to field notes, well identification signs were missing for the RSF wells.

RSF 1 and RSF 3 ran dry during second quarter sampling, RSF 1 after approximately 40 gallons were purged and RSF 3 after approximately 34 gallons. RSF 3 also ran dry during third quarter after 32 gallons of water were purged. 
During fourth quarter, RSF 1 ran dry after 35 gallons were purged and RSF 3 after 31 gallons. Field notes indicate that no well identification sign was present for RSF 2. 
Table 1. Maximum Results for Constituents Exceeding the DWS in 1993

\begin{tabular}{|c|c|c|c|c|c|c|c|}
\hline Well & Constituent & Unit & $1 \mathrm{Q93}$ & $\underline{2 Q 93}$ & $3 Q 93$ & 4Q93 & DWS \\
\hline RDB 1D & Lead & $\mu g / L$ & - & 129 & 146 & - & 50 \\
\hline RSA 7 & $\begin{array}{l}\text { Lead } \\
\text { Mercury }\end{array}$ & $\begin{array}{l}\mu g / L \\
\mu g / L\end{array}$ & $\begin{array}{l}51 \\
3.9\end{array}$ & - & $\frac{89}{-}$ & - & $\begin{array}{l}50 \\
2\end{array}$ \\
\hline RSA 8 & Lead & $\mu g / L$ & - & 113 & - & - & 50 \\
\hline RSA 9 & $\begin{array}{l}\text { Mercury } \\
\text { Strontium-90 } \\
\text { Tritium }\end{array}$ & $\begin{array}{l}\mu \mathrm{g} / \mathrm{L} \\
\mathrm{pCi} / \mathrm{L} \\
\mathrm{pCi} / \mathrm{mL}\end{array}$ & $\begin{array}{l}2.5 \\
9.1 \mathrm{E}+00 \\
-\end{array}$ & $\overline{-}$ & $\overline{\overline{-}}$ & $\overline{-}$ & $\begin{array}{l}2 \\
8 \mathrm{E}+00 \\
2 \mathrm{E}+01\end{array}$ \\
\hline RSB 7 & Cadmium & $\mu g / L$ & 6.3 & - & - & - & 5 \\
\hline RSB 8 & $\begin{array}{l}\text { Cadmium } \\
\text { Gross alpha } \\
\text { Lead } \\
\text { Nonvolatile beta }\end{array}$ & $\begin{array}{l}\mu \mathrm{g} / \mathrm{L} \\
\mathrm{pCi} / \mathrm{L} \\
\mu \mathrm{g} / \mathrm{L} \\
\mathrm{pCi} / \mathrm{L}\end{array}$ & $\begin{array}{l}\overline{3.1 E}+01 \\
148 \\
-\end{array}$ & $\begin{array}{l}\overline{2.0 \mathrm{E}}+01 \\
-\end{array}$ & $\begin{array}{l}7.1 \\
1.8 \mathrm{E}+01 \\
133 \\
-\end{array}$ & $\begin{array}{l}13 \\
4.8 \mathrm{E}+01 \\
- \\
5.3 \mathrm{E}+01\end{array}$ & $\begin{array}{l}5 \\
1.5 \mathrm{E}+01 \\
50 \\
5 \mathrm{E}+01\end{array}$ \\
\hline RSB 9 & $\begin{array}{l}\text { Cadmium } \\
\text { Gross alpha } \\
\text { Lead }\end{array}$ & $\begin{array}{l}\mu \mathrm{g} / \mathrm{L} \\
\mathrm{pCi} / \mathrm{L} \\
\mu \mathrm{g} / \mathrm{L}\end{array}$ & $\begin{array}{l}6.7 \\
7.8 \mathrm{E}+01 \\
568\end{array}$ & $\begin{array}{l}5.3 \\
- \\
-\end{array}$ & $\overline{-}$ & $\frac{12}{-}$ & $\begin{array}{l}5 \\
1.5 \mathrm{E}+01 \\
50\end{array}$ \\
\hline $\mathrm{RSC} 2$ & $\begin{array}{l}\text { Lead } \\
\text { Mercury } \\
\text { Gross alpha }\end{array}$ & $\begin{array}{l}\mu g / L \\
\mu g / L \\
\mathrm{pCi} / \mathrm{L}\end{array}$ & $\begin{array}{l}104 \\
4.7 \\
8.8 \mathrm{E}+01\end{array}$ & $\frac{\overline{-}}{1.6 \mathrm{E}+01}$ & $\frac{107}{-}$ & $\overline{-}$ & $\begin{array}{l}50 \\
2 \\
1.5 \mathrm{E}+01\end{array}$ \\
\hline RSC 3 & $\begin{array}{l}\text { Cadmium } \\
\text { Lead }\end{array}$ & $\begin{array}{l}\mu g / L \\
\mu g / L\end{array}$ & $\begin{array}{l}15 \\
165\end{array}$ & $\stackrel{6.1}{-}$ & $\begin{array}{l}8.8 \\
-\end{array}$ & $\frac{10}{-}$ & $\begin{array}{l}5 \\
50\end{array}$ \\
\hline $\mathrm{RSC} 4$ & $\begin{array}{l}\text { Cadmium } \\
\text { Gross alpha } \\
\text { Nonvolatile beta }\end{array}$ & $\begin{array}{l}\mu \mathrm{g} / \mathrm{L} \\
\mathrm{pCi} / \mathrm{L} \\
\mathrm{pCi} / \mathrm{L}\end{array}$ & $\begin{array}{l}\overline{2.6 \mathrm{E}}+01 \\
-\end{array}$ & $\begin{array}{l}5.7 \\
- \\
-\end{array}$ & $\begin{array}{l}9.4 \\
-\end{array}$ & $\begin{array}{l}35 \\
3.8 \mathrm{E}+01 \\
5.5 \mathrm{E}+01\end{array}$ & $\begin{array}{l}5 \\
1.5 \mathrm{E}+01 \\
5 \mathrm{E}+01\end{array}$ \\
\hline $\mathrm{RSC} 5$ & $\begin{array}{l}\text { Cadmium } \\
\text { Lead } \\
\text { Mercury } \\
\text { Gross alpha }\end{array}$ & $\begin{array}{l}\mu \mathrm{g} / \mathrm{L} \\
\mu \mathrm{g} / \mathrm{L} \\
\mu \mathrm{g} / \mathrm{L} \\
\mathrm{pCi} / \mathrm{L}\end{array}$ & $\begin{array}{l}8.4 \\
105 \\
11 \\
2.2 \mathrm{E}+01\end{array}$ & $\frac{-}{-}$ & $\begin{array}{l}\overline{96} \\
2.3 \\
-\end{array}$ & $\begin{array}{l}- \\
\overline{-} \\
-\end{array}$ & $\begin{array}{l}5 \\
50 \\
2 \\
1.5 E+01\end{array}$ \\
\hline $\mathrm{RSC} 6$ & Cadmium & $\mu g / L$ & - & 6.2 & 19 & 10 & 5 \\
\hline $\mathrm{RSC} 7$ & $\begin{array}{l}\text { Cadmium } \\
\text { Lead } \\
\text { Gross alpha }\end{array}$ & $\begin{array}{l}\mu \mathrm{g} / \mathrm{L} \\
\mu \mathrm{g} / \mathrm{L} \\
\mathrm{pCi} / \mathrm{L}\end{array}$ & $\begin{array}{l}\overline{365} \\
5.8 \mathrm{E}+01\end{array}$ & $\begin{array}{l}7.7 \\
- \\
-\end{array}$ & $\begin{array}{l}8.8 \\
141 \\
-\end{array}$ & $\begin{array}{l}9.1 \\
- \\
-\end{array}$ & $\begin{array}{l}5 \\
50 \\
1.5 \mathrm{E}+01\end{array}$ \\
\hline
\end{tabular}

- A dash means that a constituent was not analyzed or, if analyzed, did not exceed DWS. 
Table 1. Maximum Results for Constituents Exceeding the DWS in 1993, Continued

\begin{tabular}{|c|c|c|c|c|c|c|c|}
\hline Well & Constituent & Unit & 1098 & $2 Q 93$ & 3093 & 4093 & DWS \\
\hline RSC 8 & $\begin{array}{l}\text { Cadmium } \\
\text { Gross alpha } \\
\text { Lead }\end{array}$ & $\begin{array}{l}\mu \mathrm{g} / \mathrm{L} \\
\mathrm{pCi} / \mathrm{L} \\
\mu \mathrm{g} / \mathrm{L}\end{array}$ & $\begin{array}{l}12 \\
2.8 \mathrm{E}+01 \\
150\end{array}$ & z & $\frac{\bar{z}}{105}$ & $\begin{array}{l}8.5 \\
2.7 \mathrm{E}+01 \\
-\end{array}$ & $\begin{array}{l}5 \\
1.5 \mathrm{E}+01 \\
50\end{array}$ \\
\hline RSC 9 & $\begin{array}{l}\text { Cadmium } \\
\text { Gross alpha } \\
\text { Lead } \\
\text { Mercury }\end{array}$ & $\begin{array}{l}\mu \mathrm{g} / \mathrm{L} \\
\mathrm{pCi} / \mathrm{L} \\
\mu \mathrm{g} / \mathrm{L} \\
\mu \mathrm{g} / \mathrm{L}\end{array}$ & $\begin{array}{l}11 \\
5.0 \mathrm{E}+01 \\
399 \\
5.0\end{array}$ & $\begin{array}{l}8.7 \\
3.4 \mathrm{E}+01 \\
- \\
-\end{array}$ & $\begin{array}{l}5.9 \\
1.5 \mathrm{E}+01 \\
334 \\
-\end{array}$ & $\begin{array}{l}10 \\
7.1 \mathrm{E}+01 \\
- \\
-\end{array}$ & $\begin{array}{l}5 \\
1.5 \mathrm{E}+01 \\
50 \\
2\end{array}$ \\
\hline RSC 10 & $\begin{array}{l}\text { Cadmium } \\
\text { Gross alpha } \\
\text { Lead } \\
\text { Nonvolatile beta }\end{array}$ & $\begin{array}{l}\mu \mathrm{g} / \mathrm{L} \\
\mathrm{pCi} / \mathrm{L} \\
1 \mathrm{~g} / \mathrm{L} \\
\mathrm{pCi} / \mathrm{L}\end{array}$ & $\begin{array}{l}12 \\
1.1 \mathrm{E}+02 \\
930 \\
6.7 \mathrm{E}+01\end{array}$ & $\bar{z}$ & $\overline{-}$ & $\begin{array}{l}5.1 \\
- \\
-\end{array}$ & $\begin{array}{l}5 \\
1.5 \mathrm{E}+01 \\
50 \\
5 \mathrm{E}+01\end{array}$ \\
\hline RSD 1 & $\begin{array}{l}\text { Nonvolatile beta } \\
\text { Strontium-90 }\end{array}$ & $\begin{array}{l}\mathrm{pCi} / \mathrm{L} \\
\mathrm{pCi} / \mathrm{L}\end{array}$ & $\begin{array}{l}5.6 \mathrm{E}+01 \\
3.1 \mathrm{E}+01\end{array}$ & $\overline{2.7 E}+01$ & $\overline{2.1 E}+01$ & $\begin{array}{l}6.1 \mathrm{E}+01 \\
1.8 \mathrm{E}+01\end{array}$ & $\begin{array}{l}5 \mathrm{E}+01 \\
8 \mathrm{E}+00\end{array}$ \\
\hline RSD $2 A$ & $\begin{array}{l}\text { Cadmium } \\
\text { Lead } \\
\text { Nonvolatile beta }\end{array}$ & $\begin{array}{l}\mu \mathrm{g} / \mathrm{L} \\
\mu \mathrm{g} / \mathrm{L} \\
\mathrm{pCi} / \mathrm{L}\end{array}$ & $\begin{array}{l}24 \\
246 \\
2.9 E+02\end{array}$ & $\frac{12}{3.0 E+02}$ & $\frac{6.6}{2.5 E+02}$ & $\frac{20}{3.2 E+02}$ & $\begin{array}{l}5 \\
50 \\
5 E+01\end{array}$ \\
\hline $\mathrm{RSD} 2 \mathrm{~B}$ & $\begin{array}{l}\text { Cadmium } \\
\text { Nonvolatile beta }\end{array}$ & $\begin{array}{l}\mu \mathrm{g} / L \\
\mathrm{pCi} / L\end{array}$ & $\begin{array}{l}5.6 \\
6.3 \mathrm{E}+02\end{array}$ & $\begin{array}{l}8.4 \\
4.1 E+02\end{array}$ & - & - & $\begin{array}{l}5 \\
5 \mathrm{E}+01\end{array}$ \\
\hline $\operatorname{RSD} 2 \mathrm{C}$ & $\begin{array}{l}\text { Cadmium } \\
\text { Gross alpha } \\
\text { Mercury } \\
\text { Nonvolatile beta }\end{array}$ & $\begin{array}{l}\mu \mathrm{g} / \mathrm{L} \\
\mathrm{pCi} / \mathrm{L} \\
\mu \mathrm{g} / \mathrm{L} \\
\mathrm{pCi} / \mathrm{L}\end{array}$ & $\begin{array}{l}- \\
\frac{-}{5.5} \\
5.5 \mathrm{E}+03\end{array}$ & $\begin{array}{l}7.1 \\
4.5 \mathrm{E}+01 \\
\overline{5.2 E}+03\end{array}$ & $\begin{array}{l}\frac{6.1}{-} \\
6.4 \mathrm{E}+03\end{array}$ & $\begin{array}{l}23 \\
7.3 \mathrm{E}+01 \\
\frac{3.4 \mathrm{E}+03}{}\end{array}$ & $\begin{array}{l}5 \\
1.5 \mathrm{E}+01 \\
2 \\
5 \mathrm{E}+01\end{array}$ \\
\hline RSD 3 & Lead & $\mu g / L$ & - & - & 116 & 52 & 50 \\
\hline $\mathrm{RSD}_{4}$ & $\begin{array}{l}\text { Cadmium } \\
\text { Nonvolatile beta }\end{array}$ & $\begin{array}{l}\mu g / L \\
\mathrm{pCi} / L\end{array}$ & $\begin{array}{l}5.5 \\
3.1 E+02\end{array}$ & $\begin{array}{l}6.8 \\
1.3 \mathrm{E}+02\end{array}$ & $\overline{2.4 \mathrm{E}}+02$ & $\begin{array}{l}7.9 \\
3.3 \mathrm{E}+02\end{array}$ & $\begin{array}{l}5 \\
5 \mathrm{E}+01\end{array}$ \\
\hline $\mathrm{RSD} 5$ & $\begin{array}{l}\text { Cadmium } \\
\text { Lead } \\
\text { Gross alpha } \\
\text { Nonvolatile beta }\end{array}$ & $\begin{array}{l}\mu \mathrm{g} / \mathrm{L} \\
\mu \mathrm{g} / \mathrm{L} \\
\mathrm{pCi} / \mathrm{L} \\
\mathrm{pCi} / L\end{array}$ & $\begin{array}{l}-\overline{68} \\
1.6 \mathrm{E}+01 \\
4.6 \mathrm{E}+02\end{array}$ & $\begin{array}{l}5.1 \\
\overline{1.9 \mathrm{E}+02}\end{array}$ & $\frac{17}{-}$ & $\begin{array}{l}- \\
2.3 \mathrm{E}+01 \\
5.1 \mathrm{E}+02\end{array}$ & $\begin{array}{l}5 \\
50 \\
1.5 E+01 \\
5 E+01\end{array}$ \\
\hline RSD 6 & $\begin{array}{l}\text { Cadmium } \\
\text { Gross alpha } \\
\text { Lead } \\
\text { Nonvolatile beta }\end{array}$ & $\begin{array}{l}\mu \mathrm{g} / \mathrm{L} \\
\mathrm{pCi} / \mathrm{L} \\
\mu \mathrm{g} / \mathrm{L} \\
\mathrm{pCi} / \mathrm{L}\end{array}$ & $\begin{array}{l}15 \\
6.0 \mathrm{E}+01 \\
285 \\
3.3 \mathrm{E}+02\end{array}$ & $\begin{array}{l}7.2 \\
- \\
1.4 \mathrm{E}+02\end{array}$ & $\begin{array}{l}- \\
\overline{1.3 E+02}\end{array}$ & $\begin{array}{l}9.6 \\
2.0 \mathrm{E}+01 \\
- \\
2.1 \mathrm{E}+02\end{array}$ & $\begin{array}{l}5 \\
1.5 \mathrm{E}+01 \\
50 \\
5 \mathrm{E}+01\end{array}$ \\
\hline RSD 7 & $\begin{array}{l}\text { Gross alpha } \\
\text { Lead } \\
\text { Nonvolatile beta }\end{array}$ & $\begin{array}{l}\mathrm{pCi} / L \\
\mu \mathrm{g} / \mathrm{L} \\
\mathrm{pCi} / \mathrm{L}\end{array}$ & $\begin{array}{l}- \\
62 \\
1.3 E+02\end{array}$ & $\begin{array}{l}1.8 \mathrm{E}+01 \\
- \\
9.0 \mathrm{E}+01\end{array}$ & $\begin{array}{l}\overline{78} \\
8.8 \mathrm{E}+01\end{array}$ & $\frac{\bar{z}}{1.7 \mathrm{E}+02}$ & $\begin{array}{l}1.5 \mathrm{E}+01 \\
50 \\
5 \mathrm{E}+01\end{array}$ \\
\hline RSD 8 & $\begin{array}{l}\text { Cadmium } \\
\text { Lead }\end{array}$ & $\begin{array}{l}\mu g / L \\
\mu g / L\end{array}$ & $\overline{69}$ & $\overline{-}$ & $\begin{array}{l}5.6 \\
214\end{array}$ & 6.5 & $\begin{array}{l}5 \\
50\end{array}$ \\
\hline
\end{tabular}


Table 1. Maximum Results for Constituents Exceeding the DWS in 1993, Continued

\begin{tabular}{|c|c|c|c|c|c|c|c|}
\hline Well & Constituent & Unit & $1 Q 93$ & $2 Q 93$ & 3093 & $4 Q 93$ & DWS \\
\hline & $\begin{array}{l}\text { Gross alpha } \\
\text { Nonvolatile beta }\end{array}$ & $\begin{array}{l}\mathrm{pCi} / \mathrm{L} \\
\mathrm{pCi} / \mathrm{L}\end{array}$ & $\overline{7.6 \mathrm{E}+02}$ & $\overline{1.1 E}+03$ & $\begin{array}{l}3.4 E+01 \\
8.4 E+02\end{array}$ & $\overline{6.5 E}+02$ & $\begin{array}{l}1.5 E+01 \\
5 E+01\end{array}$ \\
\hline RSD 9 & $\begin{array}{l}\text { Cadmium } \\
\text { Lead } \\
\text { Nonvolatile beta }\end{array}$ & $\begin{array}{l}\mu \mathrm{g} / L \\
\mu \mathrm{g} / \mathrm{L} \\
\mathrm{pCi} / \mathrm{L}\end{array}$ & $\overline{109}$ & $\begin{array}{l}9.6 \\
- \\
-\end{array}$ & $\begin{array}{l}5.0 \\
56 \\
7.7 E+01\end{array}$ & $\begin{array}{l}8.5 \\
-\end{array}$ & $\begin{array}{l}5 \\
50 \\
5 E+01\end{array}$ \\
\hline RSD 10 & $\begin{array}{l}\text { Cadmium } \\
\text { Lead } \\
\text { Nonvolatile beta }\end{array}$ & $\begin{array}{l}\mu \mathrm{g} / \mathrm{L} \\
\mu \mathrm{g} / \mathrm{L} \\
\mathrm{pCi} / \mathrm{L}\end{array}$ & $\frac{\overline{-}}{9.3 \mathrm{E}+01}$ & $\frac{-}{6.5 \mathrm{E}+01}$ & $\begin{array}{l}5.0 \\
67 \\
1.4 E+02\end{array}$ & $\overline{-}$ & $\begin{array}{l}5 \\
50 \\
5 \mathrm{E}+01\end{array}$ \\
\hline RSD 11 & $\begin{array}{l}\text { Cadmium } \\
\text { Lead }\end{array}$ & $\begin{array}{l}\mu g / L \\
\mu g / L\end{array}$ & $\overline{78}$ & $\frac{8.4}{-}$ & $\frac{\dot{ }}{57}$ & - & $\begin{array}{l}5 \\
50\end{array}$ \\
\hline RSE 1A & $\begin{array}{l}\text { Nonvolatile beta } \\
\text { Strontium-90 } \\
\text { Tritium }\end{array}$ & $\begin{array}{l}\mathrm{pCi} / \mathrm{L} \\
\mathrm{pCi} / \mathrm{L} \\
\mathrm{pCi} / \mathrm{mL}\end{array}$ & $\overline{-}$ & $\begin{array}{l}5.3 E+02 \\
2.5 E+02 \\
2.1 E+02\end{array}$ & $\begin{array}{l}3.0 \mathrm{E}+02 \\
1.3 \mathrm{E}+02 \\
-\end{array}$ & $\begin{array}{l}1.4 \mathrm{E}+02 \\
4.9 \mathrm{E}+01 \\
-\end{array}$ & $\begin{array}{l}5 \mathrm{E}+01 \\
8 \mathrm{E}+00 \\
2 \mathrm{E}+01\end{array}$ \\
\hline RSE 1B & $\begin{array}{l}\text { Gross alpha } \\
\text { Nonvolatile beta } \\
\text { Strontium-90 } \\
\text { Tritium }\end{array}$ & $\begin{array}{l}\mathrm{pCi} / \mathrm{L} \\
\mathrm{pCi} / \mathrm{L} \\
\mathrm{pCi} / \mathrm{L} \\
\mathrm{pCi} / \mathrm{mL}\end{array}$ & $\begin{array}{l}2.9 E+01 \\
- \\
-\end{array}$ & $\begin{array}{l}-7.5 \mathrm{E}+01 \\
4.7 \mathrm{E}+01 \\
1.1 \mathrm{E}+04\end{array}$ & $\begin{array}{l}\overline{6.2 E}+01 \\
1.5 \mathrm{E}+01 \\
-\end{array}$ & $\begin{array}{l}- \\
5.8 \mathrm{E}+01 \\
2.8 \mathrm{E}+01 \\
-\end{array}$ & $\begin{array}{l}1.5 \mathrm{E}+01 \\
5 \mathrm{E}+01 \\
8 \mathrm{E}+00 \\
2 \mathrm{E}+01\end{array}$ \\
\hline RSE 1C & $\begin{array}{l}\text { Nitrate-nitrite as } \\
\text { nitrogen } \\
\text { Nonvolatile beta } \\
\text { Strontium-90 } \\
\text { Tritium }\end{array}$ & $\begin{array}{l}\mu \mathrm{g} / \mathrm{L} \\
\mathrm{pCi} / \mathrm{L} \\
\mathrm{pCi} / \mathrm{L} \\
\mathrm{pCi} / \mathrm{mL}\end{array}$ & $\begin{array}{l}- \\
-\end{array}$ & $\begin{array}{l}- \\
2.6 E+02 \\
1.0 E+02 \\
3.2 E+03\end{array}$ & - & $\begin{array}{l}- \\
-\end{array}$ & $\begin{array}{l}5 \mathrm{E}+01 \\
8 \mathrm{E}+00 \\
2 \mathrm{E}+01\end{array}$ \\
\hline RSE 2 & $\begin{array}{l}\text { Nonvolatile beta } \\
\text { Strontium-90 }\end{array}$ & $\begin{array}{l}\mathrm{pCi} / \mathrm{L} \\
\mathrm{pCi} / \mathrm{L}\end{array}$ & - & $\begin{array}{l}9.7 \mathrm{E}+01 \\
4.6 \mathrm{E}+01\end{array}$ & $\overline{2.1 E}+01$ & - & $\begin{array}{l}5 \mathrm{E}+01 \\
8 \mathrm{E}+00\end{array}$ \\
\hline RSE 3A & $\begin{array}{l}\text { Gross alpha } \\
\text { Nonvolatile beta } \\
\text { Strontium-90 }\end{array}$ & $\begin{array}{l}\mathrm{pCi} / L \\
\mathrm{pCi} / L \\
\mathrm{pCi} / L\end{array}$ & $\begin{array}{l}1.8 \mathrm{E}+01 \\
-\end{array}$ & $\overline{-}$ & - & $\begin{array}{l}6.0 \mathrm{E}+01 \\
5.3 \mathrm{E}+01 \\
-\end{array}$ & $\begin{array}{l}1.5 E+01 \\
5 E+01 \\
8 E+00\end{array}$ \\
\hline RSE 4A & $\begin{array}{l}\text { Mercury } \\
\text { Nonvolatile beta }\end{array}$ & $\begin{array}{l}\mu \mathrm{g} / \mathrm{L} \\
\mathrm{pCi} / \mathrm{L}\end{array}$ & $\begin{array}{l}2.8 \\
8.2 E+02\end{array}$ & $\overline{8.1 E}+02$ & $\overline{9.1 E}+01$ & $\overline{1.7 \mathrm{E}+02}$ & $\begin{array}{l}2 \\
5 E+01\end{array}$ \\
\hline RSE 4B & $\begin{array}{l}\text { Cadmium } \\
\text { Gross alpha } \\
\text { Nonvolatile beta }\end{array}$ & $\begin{array}{l}\mu \mathrm{g} / \mathrm{L} \\
\mathrm{pCi} / L \\
\mathrm{pCi} / \mathrm{L}\end{array}$ & $\begin{array}{l}6.1 \\
-\end{array}$ & $\begin{array}{l}8.3 \\
2.2 \mathrm{E}+01 \\
1.2 \mathrm{E}+03\end{array}$ & - & - & $\begin{array}{l}5 \\
1.5 E+01 \\
5 E+01\end{array}$ \\
\hline RSE 4C & $\begin{array}{l}\text { Cadmium } \\
\text { Nonvolatile beta }\end{array}$ & $\begin{array}{l}\mu \mathrm{g} / \mathrm{L} \\
\mathrm{pCi} / \mathrm{L}\end{array}$ & $\begin{array}{l}5.5 \\
3.5 \mathrm{E}+02\end{array}$ & $\overline{8.2 E}+02$ & $\overline{1.6 \mathrm{E}+02}$ & $\begin{array}{l}7.5 \\
1.6 \mathrm{E}+02\end{array}$ & $\begin{array}{l}5 \\
5 E+01\end{array}$ \\
\hline RSE 5 & $\begin{array}{l}\text { Cadmium } \\
\text { Nonvolatile beta }\end{array}$ & $\begin{array}{l}\mu \mathrm{g} / \mathrm{L} \\
\mathrm{pCi} / \mathrm{L}\end{array}$ & $\begin{array}{l}27 \\
8.0 \mathrm{E}+01\end{array}$ & $\begin{array}{l}45 \\
1.3 E+02\end{array}$ & - & - & $\begin{array}{l}5 \\
5 \mathrm{E}+01\end{array}$ \\
\hline
\end{tabular}


Table 1. Maximum Results for Constituents Exceeding the DWS in 1993, Continued

\begin{tabular}{|c|c|c|c|c|c|c|c|}
\hline Well & Constituent & Unit & 1093 & 2093 & 3Q93 & 4093 & DWS \\
\hline RSE 6 & $\begin{array}{l}\text { Cadmium } \\
\text { Lead } \\
\text { Mercury } \\
\text { Gross alpha } \\
\text { Nonvolatile beta }\end{array}$ & $\begin{array}{l}\mu g / L \\
\mu g / L \\
\mu g / L \\
p C i / L \\
\mathrm{pCi} / L\end{array}$ & $\begin{array}{l}12 \\
1,960 \\
2.5 \\
2.8 \mathrm{E}+02 \\
1.6 \mathrm{E}+04\end{array}$ & $\begin{array}{l}6.9 \\
- \\
1.4 E+02 \\
2.9 E+04\end{array}$ & $\begin{array}{l}- \\
- \\
-\end{array}$ & $\begin{array}{l}- \\
- \\
-\end{array}$ & $\begin{array}{l}5 \\
50 \\
2 \\
1.5 E+01 \\
5 \mathrm{E}+01\end{array}$ \\
\hline RSE 9 & Cadmium & $\mu g / L$ & 12 & - & - & 18 & 5 \\
\hline RSE 10 & $\begin{array}{l}\text { Gross alpha } \\
\text { Nitrate-nitrite as } \\
\text { nitrogen } \\
\text { Nonvolatile beta } \\
\text { Strontium-90 } \\
\text { Tritium }\end{array}$ & $\begin{array}{l}\text { pCi/L } \\
\mu g / L \\
\text { pCi } / L \\
\text { pCi } / L \\
\text { pCi/mL }\end{array}$ & $\begin{array}{l}\frac{1.7 \mathrm{E}+01}{-} \\
5.5 \mathrm{E}+01 \\
\frac{-}{4.4 \mathrm{E}+01}\end{array}$ & $\begin{array}{l}1.7 E+04 \\
9.1 E+03 \\
-\end{array}$ & $\begin{array}{l}- \\
6.0 \mathrm{E}+03 \\
1.9 \mathrm{E}+03 \\
2.1 \mathrm{E}+01\end{array}$ & $\overline{-}$ & $\begin{array}{l}5 \mathrm{E}+01 \\
8 \mathrm{E}+00 \\
2 \mathrm{E}+01\end{array}$ \\
\hline RSE 11 & $\begin{array}{l}\text { Cadmium } \\
\text { Gross alpha } \\
\text { Nonvolatile beta }\end{array}$ & $\begin{array}{l}\mu \mathrm{g} / L \\
\mathrm{pCi} / \mathrm{L} \\
\mathrm{pCi} / L\end{array}$ & $\begin{array}{l}5.3 \\
1.8 \mathrm{E}+01 \\
1.0 \mathrm{E}+03\end{array}$ & $\begin{array}{l}\frac{6.6}{8.4 \mathrm{E}}+02 \\
.02\end{array}$ & $\overline{\bar{c}} \overline{7 \mathrm{E}+02}$ & $\overline{\bar{z}} \overline{\mathrm{E}}$ & $\begin{array}{l}5 \\
1.5 \mathrm{E}+01 \\
5 \mathrm{E}+01\end{array}$ \\
\hline RSE 13 & $\begin{array}{l}\text { Cadmium } \\
\text { Mercury } \\
\text { Nonvolatile beta }\end{array}$ & $\begin{array}{l}\mu g / L \\
\mu g / L \\
\mathrm{pCi} / L\end{array}$ & $\begin{array}{l}7.7 \\
2.2 \\
2.2 \mathrm{E}+02\end{array}$ & $\frac{5.3}{3.2 \mathrm{E}+02}$ & $\overline{\overline{-}}$ & $\overline{\overline{s .4 E}+01}$ & $\begin{array}{l}5 \\
2 \\
5 E+01\end{array}$ \\
\hline RSE 18 & $\begin{array}{l}\text { Cadmium } \\
\text { Gross alpha } \\
\text { Lead } \\
\text { Nonvolatile beta }\end{array}$ & $\begin{array}{l}\mu g / L \\
\mathrm{pCi} / \mathrm{L} \\
\mu \mathrm{g} / \mathrm{L} \\
\mathrm{pCi} / \mathrm{L}\end{array}$ & $\begin{array}{l}17 \\
4.2 \mathrm{E}+01 \\
124 \\
-\end{array}$ & $\begin{array}{l}9.4 \\
3.0 \mathrm{E}+01 \\
- \\
-\end{array}$ & $\begin{array}{l}17 \\
1.9 \mathrm{E}+01 \\
51 \\
-\end{array}$ & $\begin{array}{l}48 \\
8.0 \mathrm{E}+01 \\
\frac{-}{5.5 \mathrm{E}+01}\end{array}$ & $\begin{array}{l}5 \\
1.5 \mathrm{E}+01 \\
50 \\
5 \mathrm{E}+01\end{array}$ \\
\hline RSE 19 & $\begin{array}{l}\text { Cadmium } \\
\text { Gross alpha } \\
\text { Lead } \\
\text { Nonvolatile beta }\end{array}$ & $\begin{array}{l}\mu \mathrm{g} / L \\
\mathrm{pCi} / L \\
\mu \mathrm{g} / L \\
\mathrm{pCi} / L\end{array}$ & $\begin{array}{l}13 \\
2.8 \mathrm{E}+01 \\
273 \\
1.3 \mathrm{E}+02\end{array}$ & $\begin{array}{l}\frac{5.7}{-} \\
6.3 E+01\end{array}$ & $\begin{array}{l}8.4 \\
1.8 \mathrm{E}+01 \\
127 \\
1.3 \mathrm{E}+02\end{array}$ & $\begin{array}{l}\overline{4.3 \mathrm{E}}+01 \\
\overline{1.5 \mathrm{E}}+02\end{array}$ & $\begin{array}{l}5 \\
1.5 e+01 \\
50 \\
5 E+01\end{array}$ \\
\hline RSF 1 & Tritium & $\mathrm{pCi} / \mathrm{mL}$ & 一 & $2.3 E+01$ & $3.3 \mathrm{E}+01$ & - & $2 \mathrm{E}+01$ \\
\hline RSF 3 & Mercury & $\mu g / L$ & 一 & 一 & 2.8 & 一 & 2 \\
\hline
\end{tabular}




\section{References}

Arnett, Margaret W., Linda K. Karapatakis, Albert R. Mamatey, James L. Todd, eds. 1992. Savannah River Site Environmental Report for 1991, WSRC-TR-92186, Westinghouse Savannah River Company, Aiken, SC.

Arnett, Margaret W., Linda K. Karapatakis, Albert R. Mamatey, eds. 1993. Savannah River Site Environmental Report for 1992, WSRC-TR-93-075, Westinghouse Savannah River Company, Aiken, SC.

Buckner, M. R. 1988. SRP Reactor Safety Evolution, DPST-83-718 R2, E. I. du Pont de Nemours \& Company, Savannah River Laboratory, Aiken, SC.

Cummins, Carol L., Donna K. Martin, James L. Todd, eds. 1990. Savannah River Site Environmental Report for 1989, WSRC-IM-90-60, Vol. I, Text, Westinghouse Savannah River Company, Aiken, SC.

Cummins, Carol L., Donna K. Martin, James L. Todd, eds. 1991. Savannah River Site Environmental Report for 1990, WSRC-IM-91-28, Westinghouse Savannah River Company, Aiken, SC.

Davis, H. Allen, Donna K. Martin, James L. Todd, eds. 1988. Savannah River Site Environmental Report for 1988, WSRC-RP-89-59-1, Vol. I, Text, Westinghouse Savannah River Company, Aiken, SC.

Dukes, E. K. 1984. The Savannah River Plant Environment, DP-1642, E. I. du Pont de Nemours \& Company, Savannah River Laboratory, Aiken, SC.

Gladden, J. B., W. L. Specht, J. A. Bowers, N. V. Halverson, H. E. Mackey, and E. W. Wilde. 1988. Compliance of the Savannah River Plant L-Reactor Cooling System with Environmental Regulations-Demonstration in Accordance with Section 316(a) of the Clean Water Act-November 1985/December 1987, DP-1766, E. I. du Pont de Nemours \& Company, Savannah River Laboratory, Aiken, SC.

Huber, L. A., W. F. Johnson, and I. W. Marine. 1987. Environmental Information Document: Burning / Rubble Pits, DPST-85-690, E. I. du Pont de Nemours \& Company, Savannah River Laboratory, Aiken, SC.

King, C. M. and J. R. Cook. 1987. Environmental and Waste Management Concern: Neptunium-237 (237Np). Draft, Savannah River Laboratory, E. I. du Pont de Nemours and Company, Aiken, SC

Langley, T. M., and W. L. Marter. 1973. The Savannah River Plant Site, DP1323, E. I. du Pont de Nemours \& Company, Savannah River Plant Laboratory, Aiken, SC. 
Pekalla, R. O., C. E. Jewell, W. G. Holmes, and I. W. Marine. 1986. Environmental Information Document: Bingham Pump Outage Pits, DPST-85-695 (draft), E. I. du Pont de Nemours \& Company, Savannah River Laboratory, Aiken, SC.

Pekalla, R. O., C. E. Jewell, W. G. Holmes, and I. W. Marine. 1987.

Environmental Information Document: Reactor Seepage Basins, DPST-85-707, Savannah River Laboratory, E. I. du Pont de Nemours \& Company, Aiken, SC.

Pekalla, R. O., C. E. Jewell, V. Price, and H. W. Bledsoe. 1987. Environmental Information Document: L-Area Oil and Chemical Basin, DPST-85-700, Savannah River Laboratory, E. I. du Pont de Nemours \& Company, Aiken, SC.

U. S. Department of Energy. 1984. Final Environmental Impact StatementL-Reactor Operation -Savannah River Plant, Aiken, South Carolina, Vol. I, DOE/EIS-0108, U. S. Department of Energy, Washington, D.C.

U. S. Department of Energy. 1990. Final Environmental Impact Statement: Continued Operation of K-, L-, and P-Reactors-Savannah River Site, Aiken, South Carolina, Vol. I, DOE/EIS-0147, U. S. Department of Energy, Washington, D.C.

U. S. Department of Energy. 1994. Department of Energy Programmatic Spent Nuclear Fuel Management and Idaho National Engineering Laboratory Environmental Restoration and Waste Management Programs Draft Environmental Impact Statement, Vol. I, DOE/EIS-0203-D, U. S. Department of Energy, Washington, D.C.

U. S. Department of Energy. 1994. Department of Energy Programmatic Spent Nuclear Fuel Management and Idaho National Engineering Laboratory Environmental Restoration and Waste Management Programs Draft Environmental Impact Statement, Vol. I, Appendix C, Savannah River Site Spent Nuclear Fuel Management Program, DOE/EIS-0203-D, U. S. Department of Energy, Washington, D.C.

U. S. Department of Energy. 1994. Proceedings for the Decommissioning and Deactivation Strategies Seminar for Savannah River Site (SRS) Reactors and Other Facilities, CONF-931267, U. S. Department of Energy, Washington, D.C.

Ward, J. W., and W. F. Johnson. 1986. Environmental Information Document: Acid/Caustic Basins, DPST-85-705 (draft), Savannah River Laboratory, E. I. du Pont de Nemours \& Company, Aiken, SC.

Westinghouse Savannah River Company. 1989a. Savannah River Site's Groundwater Monitoring Program First Quarter, 1989, ESH-EMS-890043, Environmental Monitoring (Health Protection Department) and Exploration Resources, Savannah River Site, Westinghouse Savannah River Company, Aiken, SC. 
Westinghouse Savannah River Company. 1989b. Savannah River Site's Groundwater Monitoring Program Second Quarter, 1989, ESH-EMS-890044, Environmental Monitoring (Health Protection Department) and Exploration Resources, Savannah River Site, Westinghouse Savannah River Company, Aiken, SC.

Westinghouse Savannah River Company. 1989c. Savannah River Site's Groundwater Monitoring Program Third Quarter, 1989, ESH-EMS-890045, Environmental Monitoring (Health Protection Department) and Exploration Resources, Savannah River Site, Westinghouse Savannah River Company, Aiken, $\mathrm{SC}$.

Westinghouse Savannah River Company. 1989d. Savannah River Site's Groundwater Monitoring Program Fourth Quarter, 1989, ESH-EMS-890046, Environmental Monitoring (Health Protection Department) and Exploration Resources, Savannah River Site, Westinghouse Savannah River Company, Aiken, $\mathrm{SC}$.

Westinghouse Savannah River Company. 1990. Savannah River Site's Groundwater Monitoring Program First Quarter, 1990, ESH-EMS-90-0131, Environmental Monitoring (Health Protection Department) and Exploration Resources, Savannah River Site, Westinghouse Savannah River Company, Aiken, $\mathrm{SC}$.

Westinghouse Savannah River Company. 1991a. Savannah River Site's Groundwater Monitoring Program Second Quarter, 1990, ESH-EMS-900132, Environmental Monitoring (Health Protection Department) and Exploration Resources, Savannah River Site, Westinghouse Savannah River Company, Aiken, $\mathrm{SC}$.

Westinghouse Savannah River Company. 1991b. Savannah River Site's Groundwater Monitoring Program Third Quarter, 1990, ESH-EMS-900133, Environmental Monitoring (Health Protection Department) and Exploration Resources, Savannah River Site, Westinghouse Savannah River Company, Aiken, $\mathrm{SC}$.

Westinghouse Savannah River Company. 1991c. Savannah River Site's Groundwater Monitoring Program Fourth Quarter, 1990, ESH-EMS-900134, Environmental Monitoring (Health Protection Department) and Exploration Resources, Savannah River Site, Westinghouse Savannah River Company, Aiken, $\mathrm{SC}$.

Westinghouse Savannah River Company. 1991d. Savannah River Site's Groundwater Monitoring Program First Quarter, 1991, ESH-EMS-910087, Environmental Monitoring (Protection Department) and Exploration Resources, Savannah River Site, Westinghouse Savannah River Company, Aiken, SC. 
Westinghouse Savannah River Company. 1992a. Savannah River Site Environmental Report for 1992-Summary Pamphlet, WSRC-TR-93-076, Westinghouse Savannah River Company, Aiken, SC.

Westinghouse Savannah River Company. 1992b. Savannah River Site's Groundwater Monitoring Program Second Quarter, 1991, ESH-EMS-910088, Environmental Monitoring (Protection Department) and Exploration Resources, Savannah River Site, Westinghouse Savannah River Company, Aiken, SC.

Westinghouse Savannah River Company. 1992c. Savannah River Site's Groundwater Monitoring Program Third Quarter, 1991, ESH-EMS-910089, Environmental Monitoring (Protection Department) and Exploration Resources, Savannah River Site, Westinghouse Savannah River Company, Aiken, SC.

Westinghouse Savannah River Company. 1992d. Savannah River Site's Groundwater Monitoring Program Fourth Quarter, 1991, (U), ESH-EMS-910090, Environmental Protection Department, Environmental Monitoring Section, Westinghouse Savannah River Company, Aiken, SC, June, 1992.

Westinghouse Savannah River Company. 1992e. Savannah River Site's Groundwater Monitoring Program First Quarter, 1992, (U), ESH-EMS-920035, Environmental Protection Department, Environmental Monitoring Section, Westinghouse Savannah River Company, Aiken, SC, October, 1992.

Westinghouse Savannah River Company. 1992f. Savannah River Site's Groundwater Monitoring Program, Second Quarter, 1992, (U), ESH-EMS-920036, Environmental Protection Department, Environmental Monitoring Section, Westinghouse Savannah River Company, Aiken, SC, October, 1992.

Westinghouse Savannah River Company. 1993a. RFI/RI Phase II Plan, L-Area Oil and Chemical Basin, WSRC-RP-90-1028, Rev. 2, Addendum, Westinghouse Savannah River Company, Aiken, SC.

Westinghouse Savannah River Company. 1993b. Savannah River Site's Groundwater Monitoring Program Third Quarter, 1992, (U), ESH-EMS-920037, Environmental Protection Department, Environmental Monitoring Section, Westinghouse Savannah River Company, Aiken, SC, February, 1993.

Westinghouse Savannah River Company. 1993c. Savannah River Site's Groundwater Monitoring Program Fourth Quarter, 1992, (U), ESH-EMS-920038, Environmental Protection Department, Environmental Monitoring Section, Westinghouse Savannah River Company, Aiken, SC, May, 1993.

Westinghouse Savannah River Company. 1993d. Savannah River Site's Groundwater Monitoring Program First Quarter, 1993, (U), ESH-EMS-930096, Environmental Protection Department, Environmental Monitoring Section, Westinghouse Savannah River Company, Aiken, SC, August, 1993. 
Westinghouse Savannah River Company. 1993e. Savannah River Site's Groundwater Monitoring Program Second Quarter, 1993, (U), ESH-EMS-930097, Environmental Protection Department, Environmental Monitoring Section, Westinghouse Savannah River Company, Aiken, SC, October, 1993.

Westinghouse Savannah River Company. 1993f. Working Strategy to Address Groundwater Contamination at the Reactor Areas-Savannah River Site, WSRCRP-93-1261, Westinghouse Savannah River Company, Aiken, SC.

Westinghouse Savannah River Company. 1994a. Savannah River Site's Groundwater Monitoring Program Third Quarter, 1993, (U), ESH-EMS-930098, Environmental Protection Department, Environmental Monitoring Section, Westinghouse Savannah River Company, Aiken, SC, February, 1994.

Westinghouse Savannah River Company. 1994b. Savannah River Site's Groundwater Monitoring Program Fourth Quarter, 1993, ESH-EMS-930099, Environmental Protection Department, Environmental Monitoring Section, Westinghouse Savannah River Company, Aiken, SC, June 1994. 


\section{Glossary}

ANION - ion or group of ions having a negative charge and characteristically moving toward a positive electrode.

BOTTOM ASH - Mineral matter in fossil fuels such as coal or lignite that is converted during combustion to a fused, glassy ash that does not contribute to air pollution.

CATION - ion or group of ions having a positive charge and characteristically moving toward a negative electrode.

CLADDING - protective metal layer covering fuel, target, control, and safety rods.

CONTROL ROD - neutron-absorbing cadmium or lithium-aluminum rod used to regulate the nuclear fission process within the reactor vessel.

CRITICALITY - point at which a nuclear reactor is self-sustaining.

DISCHARGING MACHINE - Remotely or automatically operated device that withdraws fuel and target assemblies from a reactor, cools them, and conveys them to the deposit-and-exit conveyor.

FISSION - nuclear reaction in which an atomic nucleus, especially a heavy nucleus, splits into fragments (usually two fragments of comparable mass) with the release of energy.

FLUX - rate of neutron flow.

FLY ASH - Mineral matter from fossil fuels such as coal or lignite, converted during combustion to a fused, glassy ash small enough to enter the furnace flue, escape through the stack, and enter the atmosphere.

FUEL ROD - protective metal rod containing pellets of fuel for a nuclear reactor.

HEAVY WATER - deuterium oxide $\left(\mathrm{D}_{2} \mathrm{O}\right)$.

HIGH-LEVEL WASTE - The highly radioactive waste material resulting from the reprocessing of spent nuclear fuel. This includes any solid waste derived from the liquid waste produced directly in reprocessing. High-level waste contains a combination of transuranic waste and fission products in concentrations requiring permanent isolation.

LATTICE - spatial arrangement of fissionable and nonfissionable materials in a nuclear reactor. 
LOW-LEVEL WASTE - includes low-activity waste (solid waste radiating $<200$ $\mathrm{mrem} / \mathrm{hr}$ at $5 \mathrm{~cm}$ from an unshielded container), intermediate-level waste (solid waste radiating $>200 \mathrm{mrem} / \mathrm{hr}$ at $5 \mathrm{~cm}$ ), intermediate-level tritium waste (solid waste containing 10 Curies of tritium per container and radiating $<200 \mathrm{mrem} / \mathrm{hr}$ at $5 \mathrm{~cm}$ ), long-lived waste, and soil suspected of being contaminated.

MODERATOR - substance used in a nuclear reactor to slow down fast neutrons and increase the likelihood of fission.

PLENUM - enclosure in which water, air, or other gas is at a pressure greater than that of the outside atmosphere.

POISON - substance that absorbs neutrons without any fission resulting and thereby lowers the reactivity of a nuclear reactor.

PRIMARY COOLANT - deuterium oxide $\left(\mathrm{D}_{2} \mathrm{O}\right)$, or heavy water, used as a cooling source and neutron moderator throughout the production process.

REACTIVITY INSTRUMENTS - instrumentation that monitors the deviation of a nuclear reactor from the critical state.

REWORK - to recycle or reprocess; refers here to heavy water $\left(D_{2} O\right)$.

SAFETY ROD - neutron-absorbing cadmium rod used as a safeguard against reactor core damage.

SCRAM - a sudden shutting down of a nuclear reactor, usually by dropping safety rods, when a predetermined neutron flux or other dangerous condition occurs.

SECONDARY COOLANT - water drawn from the Savannah River, Par Pond, or L Lake and used to receive heat from the primary coolant.

TARGET ROD - lithium-aluminum rod containing materials to be irradiated by the fuel rod.

TRANSURANIC WASTE - waste contaminated with alpha-emitting transuranic radionuclides (having an atomic number greater than 92; e.g., plutonium-239) with half lives greater than 20 years in concentrations greater than $200 \mathrm{nC} / \mathrm{g}$. TRU waste may also contain RCRA-regulated materials. 


\section{Appendix A. Final Primary Drinking} Water Standards

\begin{tabular}{|c|c|c|c|c|}
\hline Analyte & $\underline{\text { Unit }}$ & $\underline{\text { Level }}$ & Status & Source \\
\hline Antimony & $\mu \mathrm{g} / \mathrm{L}$ & 6 & Final & EPA, 1993 \\
\hline Arsenic & $\mu \mathrm{g} / \mathrm{L}$ & 50 & Final & EPA, 1993 \\
\hline Asbestos & fibers $/ \mathrm{L}$ & $7,000,000$ & Final & EPA, 1993 \\
\hline Barium & $\mu \mathrm{g} / \mathrm{L}$ & 2,000 & Final & $\mathrm{EPA}, 1993$ \\
\hline Benzene & $\mu \mathrm{g} / \mathrm{L}$ & 5 & Final & EPA, 1993 \\
\hline Benzo[a]pyrene & $\mu \mathrm{g} / \mathrm{L}$ & 0.2 & Final & EPA, 1993 \\
\hline Beryllium & $\mu \mathrm{g} / \mathrm{L}$ & 4 & Final & EPA, 1993 \\
\hline Bis(2-ethylhexyl) phthalate & $\mu \mathrm{g} / \mathrm{L}$ & 6 & Final & EPA, 1993 \\
\hline Bromodichloromethane & $\mu \mathrm{g} / \mathrm{L}$ & 100 & Final & EPA, 1993 \\
\hline Bromoform & $\mu g / L$ & 100 & Final & EPA, 1993 \\
\hline 2-sec-Butyl-4,6-dinitrophenol & $\mu \mathrm{g} / \mathrm{L}$ & 7 & Final & EPA, 1993 \\
\hline Cadmium & $\mu \mathrm{g} / \mathrm{L}$ & 5 & Final & EPA, 1993 \\
\hline Carbon tetrachloride & $\mu g / L$ & 5 & Final & EPA, 1993 \\
\hline Chlordane & $\mu \mathrm{g} / \mathrm{L}$ & 2 & Final & EPA, 1993 \\
\hline Chlorobenzene & $\mu \mathrm{g} / \mathrm{L}$ & 100 & Final & EPA, 1993 \\
\hline Chloroethene (Vinyl chloride) & $\mu g / L$ & 2 & Final & EPA, 1993 \\
\hline Chloroform & $\mu \mathrm{g} / \mathrm{L}$ & 100 & Final & EPA, 1993 \\
\hline Chromium & $\mu \mathrm{g} / \mathrm{L}$ & 100 & Final & EPA, 1993 \\
\hline Copper & $\mu g / L$ & 1,300 & Final & EPA, 1993 \\
\hline Cyanide & $\mu \mathrm{g} / \mathrm{L}$ & 200 & Final & EPA, 1993 \\
\hline Dibromochloromethane & $\mu \mathrm{g} / \mathrm{L}$ & 100 & Final & EPA, 1993 \\
\hline 1,2-Dibromo-3-chloropropane & $\mu g / L$ & 0.2 & Final & EPA, 1993 \\
\hline 1,2-Dibromoethane & $\mu g / L$ & 0.05 & Final & EPA, 1993 \\
\hline 1,2-Dichlorobenzene & $\mu \mathrm{g} / \mathrm{L}$ & 600 & Final & EPA, 1993 \\
\hline 1,4-Dichlorobenzene & $\mu \mathrm{g} / \mathrm{L}$ & 75 & Final & EPA, 1993 \\
\hline 1,2-Dichloroethane & $\mu \mathrm{g} / \mathrm{L}$ & 5 & Final & EPA, 1993 \\
\hline 1,1-Dichloroethylene & $\mu \mathrm{g} / \mathrm{L}$ & 7 & Final & EPA, 1993 \\
\hline 1,2-Dichloroethylene & $\mu \mathrm{g} / \mathrm{L}$ & 50 & Final & EPA, 1993 \\
\hline cis-1,2-Dichloroethylene & $\mu \mathrm{g} / \mathrm{L}$ & 70 & Final & EPA, 1993 \\
\hline trans-1,2-Dichloroethylene & $\mu \mathrm{g} / \mathrm{L}$ & 100 & Final & EPA, 1993 \\
\hline $\begin{array}{l}\text { Dichloromethane (Methylene } \\
\text { chloride) }\end{array}$ & $\mu \mathrm{g} / \mathrm{L}$ & 5 & Final & EPA, 1993 \\
\hline 2,4-Dichlorophenoxyacetic acid & $\mu \mathrm{g} / \mathrm{L}$ & 70 & Final & EPA, 1993 \\
\hline 1,2-Dichloropropane & $\mu \mathrm{g} / \mathrm{L}$ & 5 & Final & EPA, 1993 \\
\hline Endrin & $\mu \mathrm{g} / \mathrm{L}$ & 2 & Final & EPA, 1993 \\
\hline Ethylbenzene & $\mu \mathrm{g} / \mathrm{L}$ & 700 & Final & EPA, 1993 \\
\hline Fluoride & $\mu g / L$ & 4,000 & Final & EPA, 1993 \\
\hline Gross alphal & $\mathrm{pCi} / \mathrm{L}$ & $1.5 \mathrm{E}+01$ & Final & EPA, 1993 \\
\hline Heptachlor & $\mu \mathrm{g} / \mathrm{L}$ & 0.4 & Final & EPA, 1993 \\
\hline
\end{tabular}

1 The standard given is for gross alpha including radium-226 but excluding radon and uranium. 


\begin{tabular}{|c|c|c|c|c|}
\hline Analvte & Unit & Level & $\underline{\text { Status }}$ & Source \\
\hline Heptachlor epoxide & $\mu \mathrm{g} / \mathrm{L}$ & 0.2 & Final & EPA, 1993 \\
\hline Hexachlorobenzene & $\mu \mathrm{g} / \mathrm{L}$ & 1 & Final & EPA, 1993 \\
\hline Hexachlorocyclopentadiene & $\mu \mathrm{g} / \mathrm{L}$ & 50 & Final & EPA, 1993 \\
\hline Lead & $\mu g / L$ & 50 & Final & SCDHEC, 1981 \\
\hline Lindane & $\mu \mathrm{g} / \mathrm{L}$ & 0.2 & Final & EPA, 1993 \\
\hline Mercury & $\mu \mathrm{g} / \mathrm{L}$ & 2 & Final & EPA, 1993 \\
\hline Methoxychlor & $\mu g / L$ & 40 & Final & EPA, 1993 \\
\hline Nickel & $\mu g / L$ & 100 & Final & EPA, 1993 \\
\hline Nitrate as nitrogen & $\mu g / L$ & 10,000 & Final & EPA, 1993 \\
\hline Nitrate-nitrite as nitrogen & $\mu g / L$ & 10,000 & Final & EPA, 1993 \\
\hline Nitrite as nitrogen & $\mu g / L$ & 1,000 & Final & EPA, 1993 \\
\hline Nonvolatile beta & $\mathrm{pCj} / \mathrm{L}$ & $5 \mathrm{E}+01$ & $\begin{array}{l}\text { Interim } \\
\text { final }\end{array}$ & EPA, 1977 \\
\hline PCB 1016 & $\mu g / L$ & 0.5 & Final & EPA, 1993 \\
\hline PCB 1221 & $\mu \mathrm{g} / \mathrm{L}$ & 0.5 & Final & EPA, 1993 \\
\hline PCB 1232 & $\mu \mathrm{g} / \mathrm{L}$ & 0.5 & Final & EPA, 1993 \\
\hline PCB 1242 & $\mu \mathrm{g} / \mathrm{L}$ & 0.5 & Final & EPA, 1993 \\
\hline РCB 1248 & $\mu \mathrm{g} / \mathrm{L}$ & 0.5 & Final & EPA, 1993 \\
\hline PCB 1254 & $\mu \mathrm{g} / \mathrm{L}$ & 0.5 & Final & EPA, 1993 \\
\hline PCB 1260 & $\mu \mathrm{g} / \mathrm{L}$ & 0.5 & Final & EPA, 1993 \\
\hline PCB 1262 & $\mu g / L$ & 0.5 & Final & EPA, 1993 \\
\hline Pentachlorophenol & $\mu \mathrm{g} / \mathrm{L}$ & 1 & Final & EPA, 1993 \\
\hline Selenium & $\mu g / L$ & 50 & Final & EPA, 1993 \\
\hline Strontium-89/902 & $\mathrm{pCi} / \mathrm{L}$ & $8 \mathrm{E}+00$ & Final & EPA, 1993 \\
\hline Strontium-90 & $\mathrm{pCi} / \mathrm{L}$ & $8 \mathrm{E}+00$ & Final & $\mathrm{EPA}, 1993$ \\
\hline Styrene & $\mu \mathrm{g} / \mathrm{L}$ & 100 & Final & $\mathrm{EPA}, 1993$ \\
\hline 2,3,7,8-TCDD & $\mu g / L$ & 0.00003 & Final & EPA, 1993 \\
\hline Tetrachloroethylene & $\mu \mathrm{g} / \mathrm{L}$ & 5 & Final & EPA, 1993 \\
\hline Thallium & $\mu \mathrm{g} / \mathrm{L}$ & 2 & Final & EPA, 1993 \\
\hline Toluene & $\mu \mathrm{g} / \mathrm{L}$ & 1,000 & Final & EPA, 1993 \\
\hline Toxaphene & $\mu g / L$ & 3 & Final & EPA, 1993 \\
\hline 2,4,5-TP (Silvex) & $\mu \mathrm{g} / \mathrm{L}$ & 50 & Final & EPA, 1993 \\
\hline 1,2,4-Trichlorobenzene & $\mu \mathrm{g} / \mathrm{L}$ & 70 & Final & EPA, 1993 \\
\hline 1,1,1-Trichloroethane & $\mu \mathrm{g} / \mathrm{L}$ & 200 & Final & EPA, 1993 \\
\hline 1,1,2-Trichloroethane & $\mu \mathrm{g} / \mathrm{L}$ & 5 & Final & EPA, 1993 \\
\hline Trichloroethylene & $\mu g / L$ & 5 & Final & EPA, 1993 \\
\hline Tritium & $\mathrm{pCi} / \mathrm{mL}$ & $2 \mathrm{E}+01$ & Final & EPA, 1993 \\
\hline Xylenes & $\mu \mathrm{g} / \mathrm{L}$ & 10,000 & Final & EPA, 1993 \\
\hline
\end{tabular}

\section{References}

EPA (U.S. Environmental Protection Agency), 1977. National Interim Primary Drinking Water Regulations, EPA-570/9-76-003. Washington, DC.

\footnotetext{
2 When the laboratories report results for strontium-89/90, the drinking water standard for strontium-90 is applied.
} 
EPA (U.S. Environmental Protection Agency), 1993. National Primary Drinking Water Regulations, Code of Federal Regulations, Section 40, Part 141, pp. 592-732. Washington, DC.

SCDHEC (South Carolina Department of Health and Environmental Control), 1981. State Primary Drinking Water Regulations, R.61-58.5. Columbia; SC. 
Appendix B. Flagging Criteria

The Savannah River Site Environmental Protection Department/Environmental Monitoring Section (EPD/EMS) flagging criteria are as follows:

- Flag 2 criteria for constituents equal the Safe Drinking Water Act (SDWA) final Primary Drinking Water Standard (PDWS), the SDWA proposed PDWS, or the SDWA Secondary Drinking Water Standard (SDWS). If a constituent does not have a drinking water standard, the Flag 2 criterion equals 10 times the method detection limit (MDL) calculated as the 90th percentile detection limit obtained recently by one of the primary analytical laboratories.

- Flag 1 criteria for constituents equal one-half of the final PDWS, one-half the proposed PDWS, or one-half the SDWS. If a constituent does not have a drinking water standard, the Flag 1 criterion equals 5 times the MDL calculated as the 90 th percentile detection limit obtained recently by one of the primary analytical laboratories.

- Flag 0 criteria are assigned to constituent levels below Flag 1 criteria, constituent levels below the sample detection limits, or constituents having no flagging criteria.

The following parameters are exceptions to the flagging rules:

- EPD/EMS sets flagging criteria for specific conductance and $\mathrm{pH}$. No flags are set for alkalinity, calcium, carbonate, magnesium, potassium, silica, sodium, total dissolved solids, total phosphates (as P), and total phosphorus. Analyses for these parameters are conducted as part of the biennial comprehensive analyses or by special request.

- Aesthetic parameters such as color, corrosivity, Eh, odor, surfactants, and turbidity are not assigned flagging criteria but are analyzed by special request.

- Common laboratory contaminants and cleaners such as dichloromethane (methylene chloride), ketones, phthalates, and toluene are not assigned flagging criteria unless they have primary drinking water standards. These constituents are analyzed by special request.

Analute

Acenaphthene

Acenaphthylene

Acetone

Acetonitrile (Methyl cyanide)

Acetophenone

2-Acetylaminofluorene

\begin{tabular}{|c|c|c|c|}
\hline$\underline{\text { Unit }}$ & Flag 1 & Flag 2 & Source \\
\hline$\mu \mathrm{g} / \mathrm{L}$ & 50 & 100 & EPA Method 8270 \\
\hline$\mu g / L$ & 50 & 100 & EPA Method 8270 \\
\hline$\mu g / L$ & 500 & 1,000 & EPA Method 8240 \\
\hline$\mu g / L$ & 500 & 1,000 & EPA Method 8240 \\
\hline$\mu \mathrm{g} / \mathrm{L}$ & 50 & 100 & EPA Method 8270 \\
\hline$\mu g / L$ & 50 & 100 & EPA Method 8270 \\
\hline
\end{tabular}




\begin{tabular}{|c|c|c|c|c|}
\hline Analite & $\underline{\text { Unit }}$ & $\underline{\text { Flag } 1}$ & Flag 2 & Source \\
\hline Acrolein & $\mu g / L$ & 100 & 200 & EPA Method 8240 \\
\hline Acrylonitrile & $\mu g / L$ & 100 & 200 & EPA Method 8240 \\
\hline Actinium-228 & $\mathrm{pCi} / \mathrm{L}$ & $1.64 \mathrm{E}+03$ & $3.27 E+03$ & Proposed PDWS (EPA, 1991) \\
\hline Alachlor & $\mu \mathrm{g} / \mathrm{L}$ & 1 & 2 & Final PDWS (EPA, 1993a) \\
\hline Aldicarb $b$ & $\mu \mathrm{g} / \mathrm{L}$ & 1.5 & 3 & Final PDWS (EPA, 1993a) \\
\hline Aldicarb sulfoneb & $\mu \mathrm{g} / \mathrm{L}$ & 1 & 2 & Final PDW'S (EPA, 1993a) \\
\hline Aldicarb sulfoxideb & $\mu g / L$ & 2 & 4 & Final PDWS (EPA, 1993a) \\
\hline Aldrin & $\mu g / L$ & 0.25 & 0.5 & EPA Method 8080 \\
\hline Alkalinity (as $\mathrm{CaCO}_{3}$ ) & & No flag & No flag & Set by EPD/EMS \\
\hline Allyl chloride & $\mu g / L$ & 250 & 500 & EPA Method 8240 \\
\hline Aluminum & $\mu \mathrm{g} / \mathrm{L}$ & 25 & 50 & SDWS (EPA, 1993b) \\
\hline Aluminum, dissolved & $\mu \mathrm{g} / \mathrm{L}$ & 25 & 50 & SDWS (EPA, 1993b) \\
\hline Aluminum, total recoverable & $\mu g / L$ & 25 & 50 & SDWS (EPA, 1993b) \\
\hline Americium-241 & $\mathrm{pCi} / /$ & $3.17 E+00$ & $6.34 \mathrm{E}+00$ & Proposed PDWS (EPA, 1991) \\
\hline Americium-243 & $\mathrm{pCi} / \mathrm{L}$ & $3.19 E+00$ & $6.37 \mathrm{E}+00$ & Proposed PDWS (EPA, 1991) \\
\hline 4-Aminobiphenyl & $\mu \mathrm{g} / \mathrm{L}$ & 50 & 100 & EPA Method 8270 \\
\hline Ammonia & $\mu \mathrm{g} / \mathrm{L}$ & 500 & 1,000 & APHA Method 417B \\
\hline Ammonia nitrogen & $\mu \mathrm{g} / \mathrm{L}$ & 500 & 1,000 & EPA Method 350.1 \\
\hline Aniline & $\mu \mathrm{g} / \mathrm{L}$ & 50 & 100 & EPA Method 8270 \\
\hline Anthracene & $\mu \mathrm{g} / \mathrm{L}$ & 50 & 100 & EPA Method 8270 \\
\hline Antimony & $\mu \mathrm{g} / \mathrm{L}$ & 3 & 6 & Final PDWS (EPA, 1993a) \\
\hline Antimony, dissolved & $\mu \mathrm{g} / \mathrm{L}$ & 3 & 6 & Final PDWS (EPA, 1993a) \\
\hline Antimony, total recoverable & $\mu g / L$ & 3 & 6 & Final PDWS (EPA, 1993a) \\
\hline Antimony-125 & $\mathrm{pCi} / \mathrm{L}$ & $1.5 \mathrm{E}+02$ & $3 \mathrm{E}+02$ & Interim Final PDWS (EPA, 1977) \\
\hline Aramite & $\mu g / L$ & 50 & 100 & EPA Method 8270 \\
\hline Arsenic & $\mu \mathrm{g} / \mathrm{L}$ & 25 & 50 & Final PDWS (EPA, 1993a) \\
\hline Arsenic, dissolved & $\mu \mathrm{g} / \mathrm{L}$ & 25 & 50 & Final PDWS (EPA, 1993a) \\
\hline Arsenic, total recoverable & $\mu \mathrm{g} / \mathrm{L}$ & 25 & 50 & Final PDWS (EPA, 1993a) \\
\hline Asbestos & Fibers $/ \mathrm{L}$ & $3,500,000$ & $7,000,000$ & Final PDWS (EPA, 1993a) \\
\hline Atrazine & $\mu g / L$ & 1.5 & 3 & Final PDWS (EPA, 1993a) \\
\hline Azobenzene & $\mu g / L$ & 50 & 100 & EPA Method 625 \\
\hline Barium & $\mu \mathrm{g} / \mathrm{L}$ & 1,000 & 2,000 & Final PDWS (EPA, 1993a) \\
\hline Barium, dissolved & $\mu g / L$ & 1,000 & 2,000 & Final PDWS (EPA, 1993a) \\
\hline Barium, total recoverable & $\mu g / L$ & 1,000 & 2,000 & Final PDWS (EPA, 1993a) \\
\hline Barium-140c & $\mathrm{pCi} / \mathrm{L}$ & $4.5 E+01$ & $9 E+01$ & Interim Final PDWS (EPA, 1977) \\
\hline Benzene & $\mu \mathrm{g} / \mathrm{L}$ & 2.5 & 5 & Final PDWS (EPA, 1993a) \\
\hline alpha-Benzene hexachloride & $\mu g / L$ & 0.25 & 0.5 & EPA Method 8080 \\
\hline beta-Benzene hexachloride & $\mu \mathrm{g} / \mathrm{L}$ & 0.25 & 0.5 & EPA Method 8080 \\
\hline delta-Benzene hexachloride & $\mu g / L$ & 0.25 & 0.5 & EPA Method 8080 \\
\hline Benzidine & $\mu \mathrm{g} / \mathrm{L}$ & 250 & 500 & EPA Method 8270 \\
\hline Benzo[a]anthracene & $\mu g / L$ & 0.05 & 0.1 & Proposed PDWS (EPA, 1990) \\
\hline Benzo[b] fluoranthene & $\mu g / L$ & 0.1 & 0.2 & Proposed PDWS (EPA, 1990) \\
\hline Benzo[k] fluoranthene & $\mu \mathrm{g} / \mathrm{L}$ & 0.1 & 0.2 & Proposed PDWS (EPA, 1990) \\
\hline Benzoic acid & $\mu \mathrm{g} / \mathrm{L}$ & 250 & 500 & EPA Method 8270 \\
\hline Benzo[g,h,i]perylene & $\mu g / L$ & 50 & 100 & EPA Method 8270 \\
\hline Benzo[a]pyrene & $\mu \mathrm{g} / \mathrm{L}$ & 0.1 & 0.2 & Final PDWS (EPA, 1993a) \\
\hline 1,4-Benzoquinone & $\mu \mathrm{g} / \mathrm{L}$ & 50 & 100 & EPA Method 8270 \\
\hline Benzyl alcohol & $\mu \mathrm{g} / \mathrm{L}$ & 50 & 100 & EPA Method 8270 \\
\hline Beryllium & $\mu \mathrm{g} / \mathrm{L}$ & 2 & 4 & Final PDWS (EPA, 1993a) \\
\hline Beryllium, dissolved & $\mu \mathrm{g} / \mathrm{L}$ & 2 & 4 & Final PDWS (EPA, 1993a) \\
\hline Beryllium, total recoverable & $\mu g / L$ & 2 & 4 & Final PDWS (EPA, 1993a) \\
\hline Beryllium-7 & $\mathrm{pCi} / \mathrm{L}$ & $3 E+03$ & $6 \mathrm{E}+03$ & Interim Final PDWS (EPA, 1977) \\
\hline Bis(2-chloroethoxy) methane & $\mu g / L$ & 50 & 100 & EPA Method 8270 \\
\hline Bis(2-chloroethyl) ether & $\mu \mathrm{g} / \mathrm{L}$ & 50 & 100 & EPA Method 8270 \\
\hline Bis(2-chloroisopropyl) ether & $\mu g / L$ & 50 & 100 & EPA Method 8270 \\
\hline Bis(chloromethyl) ether & $\mu \mathrm{g} / \mathrm{L}$ & 50 & 100 & EPA Method 8270 \\
\hline Bis(2-ethylhexyl) phthalate & $\mu g / L$ & 3 & 6 & Final PDWS (EPA, 1993a) \\
\hline Bismuth-214 & $\mathrm{pCi} / \mathrm{L}$ & $9.4 \mathrm{E}+03$ & $1.89 \mathrm{E}+04$ & Proposed PDWS (EPA, 1991) \\
\hline Boron & $\mu g / L$ & 150 & 300 & EPA Method 6010 \\
\hline Boron, dissolved & $\mu \mathrm{g} / \mathrm{L}$ & 150 & 300 & EPA Method 6010 \\
\hline
\end{tabular}


Analvte

Boron, total recoverable

Bromide

Bromodichloromethane

Bromoform

Bromomethane (Methyl bromide)

4-Bromophenyl phenyl ether

2-sec-Butyl-4,6-dinitrophenol

Butylbenzyl phthalate

Cadmium

Cadmium, dissolved

Cadmium, total recoverable

Calcium

Calcium, dissolved

Calcium, total recoverable

Carbon disulfide

Carbofuran

Carbon tetrachloride

Carbon-14

Carbonate

Cerium-141c

Cerium-144

Cesium-134d

Cesium-137

Chlordane

Chloride

4-Chloroaniline

Chlorobenzene

Chlorobenzilate

Chloroethane

Chloroethene (Vinyl chloride)

Chloroethyl vinyl ether

2-Chloroethyl vinyl ether

Chloroform

4-Chloro-m-cresol

Chloromethane (Methyl chloride)

2-Chloronaphthalene

2-Chlorophenol

4-Chlorophenyl phenyl ether

Chloroprene

Chromium

Chromium, dissolved

Chromium, total recoverable

Chromium-51c

Chrysene

Cobalt

Cobalt, dissolved

Cobalt, total recoverable

Cobalt-57

Cobalt-58d

Cobalt. 60

Color

Copper

Copper, dissolved

Copper, total recoverable

Corrosivity

m-Cresol (3-Methylphenol)

o-Cresol (2-Methylphenol)

p-Cresol (4-Methylphenol)

Curium-242

Curium-243

Curium-243/244r

\begin{tabular}{|c|c|c|c|}
\hline Unit & Flag 1 & Flag 2 & Sourcea \\
\hline$\mu g / L$ & 150 & 300 & EPA Method 6010 \\
\hline$\mu \mathrm{g} / \mathrm{L}$ & 5,000 & 10,000 & EPA Method 300.0 \\
\hline$\mu g / L$ & 50 & 100 & Final PDWS (EPA, 1993a) \\
\hline$\mu \mathrm{g} / \mathrm{L}$ & 50 & 100 & Final PDWS (EPA, 1993a) \\
\hline$\mu g / L$ & 5 & 10 & EPA Method 8240 \\
\hline$\mu g / L$ & 50 & 100 & EPA Method 8270 \\
\hline$\mu g / L$ & 3.5 & 7 & Final PDWS (EPA, 1993a) \\
\hline & No flag & No flag & Set by EPD/EMS \\
\hline$\mu g / L$ & 2.5 & 5 & Final PDWS (EPA, 1993a) \\
\hline$\mu g / L$ & 2.5 & $\mathbf{5}$ & Final PDWS (EPA, 1993a) \\
\hline$\mu g / L$ & 2.5 & 5 & Final PDWS (EPA, 1993a) \\
\hline & $\begin{array}{l}\text { No flag } \\
\text { No flag } \\
\text { No flag }\end{array}$ & $\begin{array}{l}\text { No flag } \\
\text { No flag } \\
\text { No flag }\end{array}$ & $\begin{array}{l}\text { Set by EPD/EMS } \\
\text { Set by EPD/EMS } \\
\text { Set by EPD/EMS }\end{array}$ \\
\hline$\mu \mathrm{g} / \mathrm{L}$ & 5 & 10 & EPA Method 8240 \\
\hline$\mu g / L$ & 20 & 40 & Final PDWS (EPA, 1993a) \\
\hline$\mu g / L$ & 2.5 & 5 & Final PDWS (EPA, 1993a) \\
\hline $\mathrm{pCiL}$ & $\begin{array}{l}1 E+03 \\
\text { No flag }\end{array}$ & $\begin{array}{l}2 \mathrm{E}+03 \\
\text { No flag }\end{array}$ & $\begin{array}{l}\text { Interim Final PDWS (EPA, 1977) } \\
\text { Set by EPD/EMS }\end{array}$ \\
\hline $\mathrm{pCi} / \mathrm{L}$ & $1.5 \mathrm{E}+02$ & $3 \mathrm{E}+02$ & Interim Final PDWS (EPA, 1977) \\
\hline $\mathrm{pCi} / \mathrm{L}$ & $1.31 E+02$ & $2.61 E+02$ & Proposed PDWS (EPA, 1991) \\
\hline $\mathrm{pCi} / \mathrm{L}$ & $4.07 \mathrm{E}+01$ & $8.13 E+01$ & Proposed PDWS (EPA, 1991) \\
\hline $\mathrm{pCi} / \mathrm{L}$ & $1 E+02$ & $2 E+02$ & Interim Final PDWS (EPA, 1977) \\
\hline$\mu \mathrm{g} / \mathrm{L}$ & & 2 & Final PDWS (EPA, 1993a) \\
\hline$\mu g / L$ & 125,000 & 250,000 & SDWS (EPA, 1993b) \\
\hline$\mu g / L$ & 50 & 100 & EPA Method 8270 \\
\hline$\mu g / L$ & 50 & 100 & Final PDWS (EPA, 1993a) \\
\hline$\mu \mathrm{g} / \mathrm{L}$ & 50 & 100 & EPA Method 8270 \\
\hline$\mu g / L$ & 5 & 10 & EPA Method 8240 \\
\hline$\mu \mathrm{g} / \mathrm{L}$ & 1 & 2 & Final PDWS (EPA, 1993a) \\
\hline$\mu \mathrm{g} / \mathrm{L}$ & 5 & 10 & EPA Method 8240 \\
\hline$\mu g / L$ & 5 & 10 & EPA Method 8240 \\
\hline$\mu \mathrm{g} / \mathrm{L}$ & 50 & 100 & Final PDWS (EPA, 1993a) \\
\hline$\mu \mathrm{g} / \mathrm{L}$ & 50 & 100 & EPA Method 8270 \\
\hline$\mu \mathrm{g} / \mathrm{L}$ & 5 & 10 & EPA Method 8240 \\
\hline$\mu \mathrm{g} / \mathrm{L}$ & 50 & 100 & EPA Method 8240 \\
\hline$\mu \mathrm{g} / \mathrm{L}$ & 50 & 100 & EPA Method 8270 \\
\hline$\mu g / L$ & 50 & 100 & EPA Method 8270 \\
\hline$\mu \mathrm{g} / \mathrm{L}$ & 1,000 & 2,000 & EPA Method 8240 \\
\hline$\mu \mathrm{g} / \mathrm{L}$ & 50 & 100 & Final PDWS (EPA, 1993a) \\
\hline$\mu \mathrm{g} / \mathrm{L}$ & 50 & 100 & Final PDWS (EPA, 1993a) \\
\hline$\mu \mathrm{g} / \mathrm{L}$ & 50 & 100 & Final PDWS (EPA, 1993a) \\
\hline $\mathrm{pCi} / \mathrm{L}$ & $3 E+03$ & $6 \mathrm{E}+03$ & Interim Final PDWS (EPA, 1977) \\
\hline$\mu g / L$ & 0.1 & 0.2 & Proposed PDWS (EPA, 1990) \\
\hline$\mu \mathrm{g} / \mathrm{L}$ & 20 & 40 & EPA Method 6010 \\
\hline$\mu \mathrm{g} / \mathrm{L}$ & 20 & 40 & EPA Method 6010 \\
\hline$\mu g / L$ & 20 & 40 & EPA Method 6010 \\
\hline $\mathrm{pCi} / \mathrm{L}$ & $5 \mathrm{E}+02$ & $1 \mathrm{E}+03$ & Interim Final PDWS (EPA, 1977) \\
\hline $\mathrm{pCi} / \mathrm{L}$ & $4.5 E+03$ & $9 E+03$ & Interim Final PDWS (EPA, 1977) \\
\hline $\mathrm{pCi} / \mathrm{L}$ & $\begin{array}{l}5 E+01 \\
\text { No flag }\end{array}$ & $\begin{array}{l}\text { 1E+02 } \\
\text { No flag }\end{array}$ & $\begin{array}{l}\text { Interim Final PDWS (EPA, 1977) } \\
\text { Set by EPD/EMS }\end{array}$ \\
\hline$\mu g / L$ & 500 & 1,000 & Final PDWS (SCDHEC, 1981) \\
\hline$\mu g / L$ & 500 & 1,000 & Final PDWS (SCDHEC, 1981) \\
\hline$\mu g / L$ & 500 & 1,000 & Final PDWS (SCDHEC, 1981) \\
\hline$\mu \mathrm{g} / \mathrm{L}$ & $\begin{array}{l}\text { No flag } \\
50\end{array}$ & $\begin{array}{l}\text { No flag } \\
100\end{array}$ & $\begin{array}{l}\text { Set by EPD/EMS } \\
\text { EPA Method } 8270\end{array}$ \\
\hline$\mu \mathrm{g} / \mathrm{L}$ & 50 & 100 & EPA Method 8270 \\
\hline$\mu g / L$ & 50 & 100 & EPA Method 8270 \\
\hline $\mathrm{pCi} / \mathrm{L}$ & $6.65 E+01$ & $1.33 \mathrm{E}+02$ & Proposed PDWS (EPA, 1991) \\
\hline $\mathrm{pCi} / \mathrm{L}$ & $4.15 \mathrm{E}+00$ & $8.3 E+00$ & Proposed PDWS (EPA, 1991) \\
\hline $\mathrm{pCi} / \mathrm{L}$ & $4.15 \mathrm{E}+00$ & $8.3 \mathrm{E}+00$ & Proposed PDWS (EPA, 1991) \\
\hline
\end{tabular}

R-Area Reactor 1993 Annual Report $\bullet$ B-3 


Analyte
Curium-244
Curium-245/246r
Curium-246
Cyanide
Dalapon
p.p'-DDD
p,p'-DDE
p,p'-DDT
Di-n-butyl phthalate
Di-n-octyl phthalate
Diallate
Dibenz[a,h]anthracene
Dibenzofuran
Dibromochloromethane
1,2-Dibromo-3-chloropropane
1,2-Dibromoethane
Dibromomethane
(Methylene bromide)
1,2-Dichlorobenzene
1,3-Dichlorobenzene
1,4-Dichlorobenzene
3,3'-Dichlorobenzidine
trans-1,4-Dichloro-2-butene
Dichlorodifluoromethane
1,1-Dichloroethane
1,2-Dichloroethane
1,1-Dichloroethylene
1,2-Dichloroethylene
cis-1,2-Dichloroethylene
trans-1,2-Dichloroethylene
Dichloromethane
(Methylene chloride)
2,4-Dichlorophenol
2,6-Dichlorophenol
2,4-Dichlorophenoxyacetic acid
1,2-Dichloropropane
cis-1,3-Dichloropropene
trans-1,3-Dichloropropene
Di(2-ethylhexyl) adipate
Dieldrin
Diethyl phthalate
Dimethoate
p-Dimethylaminoazobenzene
p-(Dimethylamino)ethylbenzene
7,12-Dimethylbenz[a]anthracene
3,3'-Dimethylbenzidine
a,a-Dimethylphenethylamine
2,4-Dimethyl phenol
Dimethyl phthalate
1,3-Dinitrobenzene
2,4-Dinitrophenol
2,4-Dinitrotoluene
2,6-Dinitrotoluene
Diquat dibromideb
1,4-Dioxane
Diphenylamine
1,2-Diphenylhydrazine
Dissolved organic carbon
Disulfoton
Eh

\begin{tabular}{|c|c|c|c|}
\hline$\underline{\text { Unit }}$ & Flag 1 & Flag 2 & Source \\
\hline $\mathrm{pCi} / \mathrm{L}$ & $4.92 \mathrm{E}+00$ & $9.84 \mathrm{E}+00$ & Proposed PDWS (EPA, 1991) \\
\hline $\mathrm{pCi} / \mathrm{L}$ & $3.12 \mathrm{E}+00$ & $6.23 E+00$ & Proposed PDWS (EPA, 1991) \\
\hline $\mathrm{pCi} / \mathrm{L}$ & $3.14 \mathrm{E}+00$ & $6.27 \mathrm{E}+00$ & Proposed PDWS (EPA, 1991) \\
\hline$\mu g / L$ & 100 & 200 & Final PDWS (EPA, 1993a) \\
\hline$\mu \mathrm{g} / \mathrm{L}$ & 100 & 200 & Final PDWS (EPA, 1993a) \\
\hline$\mu g / L$ & 0.5 & 1 & EPA Method 8080 \\
\hline$\mu g / L$ & 0.5 & 1 & EPA Method 8080 \\
\hline \multirow[t]{3}{*}{$\mu \mathrm{g} / \mathrm{L}$} & 0.5 & 1 & EPA Method 8080 \\
\hline & No flag & No flag & Set by EPD/EMS \\
\hline & No flag & No flag & Set by EPD/EMS \\
\hline$\mu \mathrm{g} / \mathrm{L}$ & 50 & 100 & EPA Method 8270 \\
\hline$\mu g / L$ & 0.15 & 0.3 & Proposed PDWS (EPA, 1990) \\
\hline$\mu \mathrm{g} / \mathrm{L}$ & 50 & 100 & EPA Method 8270 \\
\hline$\mu \mathrm{g} / \mathrm{L}$ & 50 & 100 & Final PDWS (EPA, 1993a) \\
\hline$\mu \mathrm{g} / \mathrm{L}$ & 0.1 & 0.2 & Final PDWS (EPA, 1993a) \\
\hline$\mu \mathrm{g} / \mathrm{L}$ & 0.025 & 0.05 & Final PDWS (EPA, 1993a) \\
\hline$\mu \mathrm{g} / \mathrm{L}$ & 5 & 10 & EPA Method 8240 \\
\hline$\mu g / L$ & 300 & 600 & Final PDWS (EPA, 1993a) \\
\hline$\mu \mathrm{g} / \mathrm{L}$ & 50 & 100 & EPA Method 8270 \\
\hline$\mu \mathrm{g} / \mathrm{L}$ & 37.5 & 75 & Final PDWS (EPA, 1993a) \\
\hline$\mu g / L$ & 50 & 100 & EPA Method 8270 \\
\hline$\mu \mathrm{g} / \mathrm{L}$ & 150 & 300 & EPA Method 8240 \\
\hline$\mu \mathrm{g} / \mathrm{L}$ & 5 & 10 & EPA Method 8240 \\
\hline$\mu \mathrm{g} / \mathrm{L}$ & 5 & 10 & EPA Method 8240 \\
\hline$\mu \mathrm{g} / \mathrm{L}$ & 2.5 & 5 & Final PDWS (EPA, 1993a) \\
\hline$\mu g / L$ & 3.5 & 7 & Final PDWS (EPA, 1993a) \\
\hline$\mu \mathrm{g} / \mathrm{L}$ & 25 & 50 & Final PDWS (EPA, 1993a) \\
\hline$\mu \mathrm{g} / \mathrm{L}$ & 35 & 70 & Final PDWS (EPA, 1993a) \\
\hline$\mu g / L$ & 50 & 100 & Final PDWS (EPA, 1993a) \\
\hline$\mu g / L$ & 2.5 & 5 & Final PDWS (EPA, 1993a) \\
\hline$\mu g / L$ & 50 & 100 & EPA Method 8270 \\
\hline$\mu g / L$ & 50 & 100 & EPA Method 8270 \\
\hline$\mu g / L$ & 35 & 70 & Final PDWS (EPA, 1993a) \\
\hline$\mu g / L$ & 2.5 & 5 & Final PDWS (EPA, 1993a) \\
\hline$\mu g / L$ & 5 & 10 & EPA Method 8240 \\
\hline$\mu \mathrm{g} / \mathrm{L}$ & 5 & 10 & EPA Method 8240 \\
\hline$\mu g / L$ & 200 & 400 & Final PDWS (EPA, 1993a) \\
\hline \multirow[t]{2}{*}{$\mu \mathrm{g} / \mathrm{L}$} & 2.5 & 5 & EPA Method 8080 \\
\hline & No flag & No flag & Set by EPD/EMS \\
\hline$\mu g / L$ & 50 & 100 & EPA Method 8270 \\
\hline$\mu g / L$ & 50 & 100 & EPA Method 8270 \\
\hline$\mu \mathrm{g} / \mathrm{L}$ & 50 & 100 & EPA Method 8270 \\
\hline$\mu \mathrm{g} / \mathrm{L}$ & 50 & 100 & EPA Method 8270 \\
\hline$\mu \mathrm{g} / \mathrm{L}$ & 50 & 100 & EPA Method 8270 \\
\hline$\mu g / L$ & 50 & 100 & EPA Method 8270 \\
\hline \multirow[t]{2}{*}{$\mu \mathrm{g} / \mathrm{L}$} & 50 & 100 & EPA Method 8270 \\
\hline & No flag & No flag & Set by EPD/EMS \\
\hline$\mu g / L$ & 50 & 100 & EPA Method 8270 \\
\hline$\mu \mathrm{g} / \mathrm{L}$ & 250 & 500 & EPA Method 8270 \\
\hline$\mu \mathrm{g} / \mathrm{L}$ & 50 & 100 & EPA Method 8270 \\
\hline$\mu g / L$ & 50 & 100 & EPA Method 8270 \\
\hline$\mu \mathrm{g} / \mathrm{L}$ & 10 & 20 & Final PDWS (EPA, 1993a) \\
\hline$\mu g / L$ & 50 & 100 & EPA Method 8270 \\
\hline$\mu \mathrm{g} / \mathrm{L}$ & 50 & 100 & EPA Method 8270 \\
\hline$\mu \mathrm{g} / \mathrm{L}$ & 50 & 100 & EPA Method 8270 \\
\hline$\mu \mathrm{g} / \mathrm{L}$ & 5,000 & 10,000 & EPA Method 9060 \\
\hline \multirow[t]{2}{*}{$\mu \mathrm{g} / \mathrm{L}$} & 50 & 100 & EPA Method 8270 \\
\hline & No flag & No flag & Set by EPD/EMS \\
\hline
\end{tabular}


Analvte

Endosulfan I

Endosulfan II

Endosulfan sulfate

Endothall

Endrin

Endrin aldehyde

Endrin ketone

Ethylbenzene

Ethyl methacrylate

Ethyl methanesulfonate

Europium-152

Europium-154

Europium-155

Famphur

Fluoranthene

Fluorene

Fluoride

Glyphosate

Gross alpha

Heptachlor

Heptachlor epoxide

Heptachlorodibenzo-p-dioxin

isomers

1,2,3,4,6,7,8-HPCDD

Heptachlorodibenzo-p-furan isomers

1,2,3,4,6,7,8-HPCDF

Hexachlorobenzene

Hexachlorobutadiene

Hexachlorocyclopentadiene

Hexachlorodibenzo-p-dioxin

isomers

1,2,3,4,7,8-HXCDD

Hexachlorodibenzo-p-furan isomers

1,2,3,4,7,8-HXCDF

Hexachloroethane

Hexachlorophene

Hexachloropropene

2-Hexanone

Indeno[1,2,3-c,d]pyrene

Iodine

Iodine-129

Iodine-131c

Iodomethane (Methyl iodide)

Iron

Iron, dissolved

Iron, total recoverable

Iron-55c

Iron-59c

Isobutyl alcohol

Isodrin

Isophorone

Isosafrole

Kepone

Lanthanum.140c

Lead

Lead, dissolved

Lead, total recoverable

Lead.212

Lindane

\begin{tabular}{|c|c|c|c|}
\hline$\underline{\text { Unit }}$ & Flag 1 & Flag 2 & Source \\
\hline$\mu \mathrm{g} / \mathrm{L}$ & 0.5 & 1 & EPA Method 8080 \\
\hline$\mu \mathrm{g} / \mathrm{L}$ & 0.5 & 1 & EPAMethod 8080 \\
\hline$\mu \mathrm{g} / \mathrm{L}$ & 0.5 & 1 & EPA Method 8080 \\
\hline$\mu g / L$ & 50 & 100 & Final PDWS (EPA, 1993a) \\
\hline$\mu \mathrm{g} / \mathrm{L}$ & 1 & 2 & Final PDWS (EPA, 1993a) \\
\hline$\mu g / L$ & 0.5 & 1 & EPA Method 8080 \\
\hline & No flag & No flag & Set by EPD/EMS \\
\hline$\mu g / L$ & 350 & 700 & Final PDWS (EPA, 1993a) \\
\hline$\mu \mathrm{g} / \mathrm{L}$ & 50 & 100 & EPA Method 8270 \\
\hline$\mu \mathrm{g} / \mathrm{L}$ & 50 & 100 & EPA Method 8270 \\
\hline $\mathrm{pCi} / \mathrm{L}$ & $3 E+01$ & $6 \mathrm{E}+01$ & Interim Final PDWS (EPA, 1977) \\
\hline $\mathrm{pCi} / \mathrm{L}$ & $1 E+02$ & $2 E+02$ & Interim Final PDW'S (EPA, 1977) \\
\hline $\mathrm{pCi} / \mathrm{L}$ & $3 E+02$ & $6 \mathrm{E}+02$ & Interim Final PDWS (EPA, 1977) \\
\hline$\mu \mathrm{g} / \mathrm{L}$ & 50 & 100 & EPA Method 8270 \\
\hline$\mu g / L$ & 50 & 100 & EPA Method 8270 \\
\hline$\mu \mathrm{g} / \mathrm{L}$ & 50 & 100 & EPA Method 8270 \\
\hline$\mu g / L$ & 2,000 & 4,000 & Final PDWS (EPA, 1993a) \\
\hline$\mu \mathrm{g} / \mathrm{L}$ & 350 & 700 & Final PDWS (EPA, 1993a) \\
\hline $\mathrm{pCi} / \mathrm{L}$ & $7.5 E+00$ & $1.5 \mathrm{E}+01$ & Final PDWS (EPA, 1993a) \\
\hline$\mu g / L$ & 0.2 & 0.4 & Final PDWS (EPA, 1993a) \\
\hline$\mu g / L$ & 0.1 & 0.2 & Final PDWS (EPA, 1993a) \\
\hline$\mu g / L$ & 0.00325 & 0.0065 & EPA Method 8280 \\
\hline$\mu g / L$ & 0.00325 & 0.0065 & EPA Method 8280 \\
\hline$\mu \mathrm{g} / \mathrm{L}$ & 0.00225 & 0.0045 & EPA Method 8280 \\
\hline$\mu g / L$ & 0.00225 & 0.0045 & EPA Method 8280 \\
\hline$\mu g / L$ & 0.5 & 1 & Final PDWS (EPA, 1993a) \\
\hline$\mu \mathrm{g} / \mathrm{L}$ & 50 & 100 & EPA Method 8270 \\
\hline$\mu g / L$ & 25 & 50 & Final PDWS (EPA, 1993a) \\
\hline$\mu g / L$ & 0.00225 & 0.0045 & EPA Method 8280 \\
\hline$\mu \mathrm{g} / \mathrm{L}$ & 0.00225 & 0.0045 & EPA Method 8280 \\
\hline$\mu \mathrm{g} / \mathrm{L}$ & 0.002 & 0.004 & EPA Method 8280 \\
\hline$\mu g / L$ & 0.002 & 0.004 & EPA Method 8280 \\
\hline$\mu \mathrm{g} / \mathrm{L}$ & 50 & 100 & EPA Method 8270 \\
\hline$\mu g / L$ & 250 & 500 & EPA Method 8270 \\
\hline$\mu \mathrm{g} / \mathrm{L}$ & 50 & 100 & EPA Method 8270 \\
\hline$\mu g / L$ & 50 & 100 & EPA Method 8240 \\
\hline$\mu \mathrm{g} / \mathrm{L}$ & 50 & 100 & EPA Method 8270 \\
\hline$\mu g / L$ & 250 & 500 & APHA Method 415A \\
\hline $\mathrm{pCi} / 2$ & 5E-01 & $1 E+00$ & Interim Final PDWS (EPA, 1977) \\
\hline $\mathrm{pCi} / \mathrm{L}$ & $1.5 E+00$ & $3 E+00$ & Interim Final PDWS (EPA, 1977) \\
\hline$\mu g / L$ & 75 & 150 & EPA Method 8240 \\
\hline$\mu \mathrm{g} / \mathrm{L}$ & 150 & 300 & SDWS (EPA, 1993b) \\
\hline$\mu \mathrm{g} / \mathrm{L}$ & 150 & 300 & SDWS (EPA, 1993b) \\
\hline$\mu g / L$ & 150 & 300 & SDWS (EPA, 1993b) \\
\hline $\mathrm{pCi} / \mathrm{L}$ & $1 \mathrm{E}+03$ & $2 \mathrm{E}+03$ & Interim Final PDWS (EPA, 1977) \\
\hline $\mathrm{pCi} / \mathrm{L}$ & $1 E+02$ & $2 E+02$ & Interim Final PDWS (EPA, 1977) \\
\hline$\mu g / L$ & 500 & 1,000 & EPA Method 8240 \\
\hline$\mu \mathrm{g} / \mathrm{L}$ & 50 & 100 & EPA Method 8270 \\
\hline$\mu g / L$ & 50 & 100 & EPA Method 8270 \\
\hline$\mu \mathrm{g} / \mathrm{L}$ & 50 & 100 & EPA Method 8270 \\
\hline$\mu g / L$ & 50 & 100 & EPA Method 8270 \\
\hline $\begin{array}{l}\mathrm{pCi} / \mathrm{L} \\
\mu \mathrm{g} / \mathrm{L}\end{array}$ & $\begin{array}{l}3 E+01 \\
25\end{array}$ & $\begin{array}{l}6 \mathrm{E}+01 \\
50\end{array}$ & $\begin{array}{l}\text { Interim Final PDWS (EPA, 1977) } \\
\text { Final PDWS (SCDHEC, 1981) }\end{array}$ \\
\hline$\mu \mathrm{g} / \mathrm{L}$ & 25 & 50 & Final PDWS (SCDHEC, 1981) \\
\hline$\mu g / L$ & 25 & 50 & Final PDWS (SCDHEC, 1981) \\
\hline $\mathrm{pCi} / \mathrm{L}$ & $6.2 E+01$ & $1.23 E+02$ & Proposed PDWS (EPA, 1991) \\
\hline$\mu \mathrm{g} / \mathrm{L}$ & 0.1 & 0.2 & Final PDWS (EPA, 1993a) \\
\hline
\end{tabular}

R-Area Reactor 1993 Annual Report • B-5 
WSRC-TR-94-0351

Unclassified

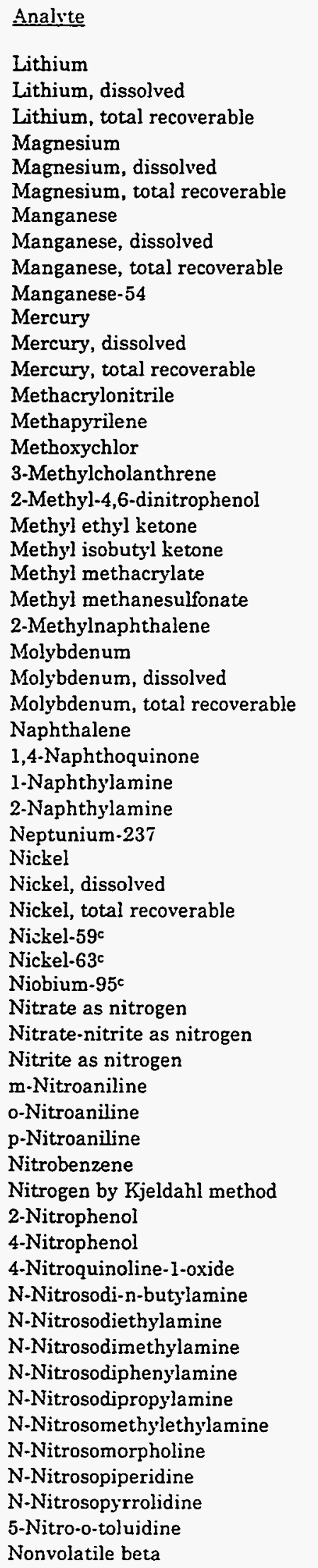

\begin{tabular}{|c|c|c|c|}
\hline Unit & Flag 1 & Flag 2 & Source \\
\hline$\mu \mathrm{g} / \mathrm{L}$ & 25 & 50 & EPA Method 6010 \\
\hline$\mu g / L$ & 25 & 50 & EPA Method 6010 \\
\hline$\mu \mathrm{g} / \mathrm{L}$ & 25 & 50 & EPA Method 6010 \\
\hline & $\begin{array}{l}\text { No flag } \\
\text { No flag } \\
\text { No flag }\end{array}$ & $\begin{array}{l}\text { No flag } \\
\text { No flag } \\
\text { No flag } \\
50\end{array}$ & $\begin{array}{l}\text { Set by EPD/EMS } \\
\text { Set by EPD/EMS } \\
\text { Set by EPD/EMS } \\
\text { SDW' }\end{array}$ \\
\hline $\begin{array}{l}\mu \mathrm{g} / \mathrm{L} \\
\mu \mathrm{g} / \mathrm{L}\end{array}$ & $\begin{array}{l}25 \\
25\end{array}$ & $\begin{array}{l}50 \\
50\end{array}$ & $\begin{array}{l}\text { SDW'S (EPA, 1993b) } \\
\text { SDW'S (EPA, 1993b) }\end{array}$ \\
\hline$\mu \mathrm{g} / \mathrm{L}$ & 25 & 50 & $\begin{array}{l}\text { SDW'S (EPA, 1993b) } \\
\text { SDW'S (EPA, 1993b) }\end{array}$ \\
\hline $\begin{array}{l}\mathrm{pCi} / L \\
\mu \mathrm{g} / \mathrm{L}\end{array}$ & $\begin{array}{l}1.5 \mathrm{E}+02 \\
1\end{array}$ & $\begin{array}{l}3 E \div 02 \\
2\end{array}$ & $\begin{array}{l}\text { Interim Final PDWS (EPA, 1977) } \\
\text { Final PDWS (EPA, 1993a) }\end{array}$ \\
\hline$\mu \mathrm{g} / \mathrm{L}$ & 1 & 2 & Final PDWS (EPA, 1993a) \\
\hline$\mu \mathrm{g} / \mathrm{L}$ & 1 & 2 & Final PDWS (EPA, 1993a) \\
\hline$\mu \mathrm{g} / \mathrm{L}$ & 250 & 500 & EPA Method 8240 \\
\hline$\mu g / L$ & 50 & 100 & EPA Method 8270 \\
\hline$\mu \mathrm{g} / \mathrm{L}$ & 20 & 40 & Final PDWS (EPA, 1993a) \\
\hline$\mu \mathrm{g} / \mathrm{L}$ & 50 & 100 & EPA Method 8270 \\
\hline$\mu \mathrm{g} / \mathrm{L}$ & 250 & 500 & EPA Method 8270 \\
\hline & $\begin{array}{l}\text { No flag } \\
\text { No flag }\end{array}$ & No flag & $\begin{array}{l}\text { Set by EPD/EMS } \\
\text { Set by EPD/EMS }\end{array}$ \\
\hline$\mu g / L$ & 50 & 100 & EPA Method 8270 \\
\hline$\mu \mathrm{g} / \mathrm{L}$ & 50 & 100 & EPA Method 8270 \\
\hline$\mu g / L$ & 50 & 100 & EPA Method 8270 \\
\hline$\mu \mathrm{g} / \mathrm{L}$ & 250 & 500 & EPA Method 6010 \\
\hline$\mu g / L$ & 250 & 500 & EPA Method 6010 \\
\hline$\mu g / L$ & 250 & 500 & EPA Method 6010 \\
\hline$\mu \mathrm{g} / \mathrm{L}$ & 50 & 100 & EPA Method 8270 \\
\hline$\mu g / L$ & 50 & 100 & EPA Method 8270 \\
\hline$\mu g / L$ & 50 & 100 & EPA Method 8270 \\
\hline$\mu \mathrm{g} / \mathrm{L}$ & 50 & 100 & EPA Method 8270 \\
\hline $\begin{array}{l}\mathrm{pCi} / \mathrm{L} \\
\mu \mathrm{g} / \mathrm{L}\end{array}$ & $\begin{array}{l}3.53 E+00 \\
50\end{array}$ & $\begin{array}{l}7.06 \mathrm{E}+00 \\
100\end{array}$ & $\begin{array}{l}\text { Proposed PDWS (EPA, 1991) } \\
\text { Final PDWS (EPA, 1993a) }\end{array}$ \\
\hline$\mu \mathrm{g} / \mathrm{L}$ & 50 & 100 & Final PDWS (EPA, 1993a) \\
\hline$\mu g / L$ & 50 & 100 & Final PDWS (EPA, 1993a) \\
\hline $\mathrm{pCi} / \mathrm{L}$ & $1.5 \mathrm{E}+02$ & $3 E+02$ & Interim Final PDWS (EPA, 1977) \\
\hline $\mathrm{pCi} / \mathrm{L}$ & $2.5 \mathrm{E}+01$ & $5 \mathrm{E}+01$ & Interim Final PDWS (EPA, 1977) \\
\hline $\mathrm{pCi} / \mathrm{L}$ & $1.5 \mathrm{E}+02$ & 3.E+02 & Interim Final PDWS (EPA, 1977) \\
\hline$\mu \mathrm{g} / \mathrm{L}$ & 5,000 & 10,000 & Final PDWS (EPA, 1993a) \\
\hline$\mu \mathrm{g} / \mathrm{L}$ & 5,000 & 10,000 & Final PDWS (EPA, 1993a) \\
\hline$\mu \mathrm{g} / \mathrm{L}$ & 500 & 1,000 & Final PDWS (EPA, 1993a) \\
\hline$\mu \mathrm{g} / \mathrm{L}$ & 50 & 100 & EPA Method 8270 \\
\hline$\mu \mathrm{g} / \mathrm{L}$ & 50 & 100 & EPA Method 8270 \\
\hline$\mu g / L$ & 50 & 100 & EPA Method 8270 \\
\hline$\mu \mathrm{g} / \mathrm{L}$ & 50 & 100 & EPA Method 8270 \\
\hline$\mu g / L$ & 500 & 1,000 & EPA Method 351.2 \\
\hline$\mu \mathrm{g} / \mathrm{L}$ & 50 & 100 & EPA Method 8270 \\
\hline$\mu \mathrm{g} / \mathrm{L}$ & 50 & 100 & EPA Method 8270 \\
\hline$\mu g / L$ & 50 & 100 & EPA Method 8270 \\
\hline$\mu g / L$ & 50 & 100 & EPA Method 8270 \\
\hline$\mu g / L$ & 50 & 100 & EPA Method 8270 \\
\hline$\mu g / L$ & 50 & 100 & EPA Method 8270 \\
\hline$\mu \mathrm{g} / \mathrm{L}$ & 50 & 100 & EPA Method 8270 \\
\hline$\mu \mathrm{g} / \mathrm{L}$ & 50 & 100 & EPA Method 8270 \\
\hline$\mu \mathrm{g} / \mathrm{L}$ & 50 & 100 & EPA Method 8270 \\
\hline$\mu \mathrm{g} / \mathrm{L}$ & 50 & 100 & EPA Method 8270 \\
\hline$\mu \mathrm{g} / \mathrm{L}$ & 50 & 100 & EPA Method 8270 \\
\hline$\mu \mathrm{g} / \mathrm{L}$ & 50 & 100 & EPA Method 8270 \\
\hline$\mu \mathrm{g} / \mathrm{L}$ & 50 & 100 & EPA Method 8270 \\
\hline $\mathrm{pCi} / \mathrm{L}$ & $2.5 \mathrm{E}+01$ & $5 \mathrm{E}+01$ & Interim Final PDWS (EPA, 1977) \\
\hline
\end{tabular}


Analve

Octachlorodibenzo-p-dioxin isomers

Octachlorodibenzo-p-furan

isomers

Odor

Oil \& Grease

Oxamyl

Parathion

Parathion methyl

PCB 1016

PCB 1221

PCB 1232

PCB 1242

PCB 1248 .

PCB 1254

PCB 1260

PCB 1262

Pentachlorobenzene

Pentachlorodibenzo-p-dioxin isomers

1,2,3,7,8-PCDD

Pentachlorodibenzo-p-furan

isomers

1,2,3,7,8-PCDF

Pentachloroethane

Pentachloronitrobenzene

Pentachlorophenol

$\mathrm{pH}$

$\mathrm{pH}$

Phenacetin

Phenanthrene

Phenol

Phenols

p-Phenylenediamine

Phorate

Picloramb

2-Picoline

Plutonium-238

Plutonium-239

Plutonium-239/240

Plutonium-240

Plutonium-241c

Plutonium-242c

Potassium

Potassium, dissolved

Potassium, total recoverable

Potassium-40

Promethium-144

Promethium-146

Promethium-147

Pronamid

Propionitrile

Pyrene

Pyridine

Radium (alpha-emitting)

Radium-226

Radium-228

Radon-222

Ruthenium-103c

Ruthenium-106

Safrole

Selenium

\begin{tabular}{|c|c|c|c|}
\hline Unit & Flag 1 & Flag 2 & Sourcea \\
\hline$\mu g / L$ & 0.005 & 0.01 & EPA Method 8280 \\
\hline$\mu g / L$ & 0.005 & 0.01 & EPA Method 8280 \\
\hline$\mu g / L$ & $\begin{array}{l}\text { No flag } \\
5.000\end{array}$ & $\begin{array}{l}\text { No flag } \\
10,000\end{array}$ & Set by EPDIEMS \\
\hline$\mu \mathrm{g} / \mathrm{L}$ & 100 & 200 & Final PDWS (EPA, 1993a) \\
\hline$\mu g / L$ & 0.25 & 0.5 & EPA Method 8080 \\
\hline$\mu g / L$ & 0.25 & 0.5 & EPA Method 8080 \\
\hline$\mu g / L$ & 0.25 & 0.5 & Final PDWS (EPA, 1993a) \\
\hline$\mu g / L$ & 0.25 & 0.5 & Final PDWS (EPA, 1993a) \\
\hline$\mu \mathrm{g} / \mathrm{L}$ & 0.25 & 0.5 & Final PDWS (EPA, 1993a) \\
\hline$\mu \mathrm{g} / \mathrm{L}$ & 0.25 & 0.5 & Final PDWS (EPA, 1993a) \\
\hline$\mu g / L$ & 0.25 & 0.5 & Final PDWS (EPA, 1993a) \\
\hline$\mu g / L$ & 0.25 & 0.5 & Final PDWS (EPA, 1993a) \\
\hline$\mu g / L$ & 0.25 & 0.5 & Final PDWS (EPA, 1993a) \\
\hline$\mu g / L$ & 0.25 & 0.5 & Final PDWS (EPA, 1993a) \\
\hline$\mu g / L$ & 50 & 100 & EPA Method 8270 \\
\hline$\mu g / L$ & 0.00275 & 0.0055 & EPA Method 8280 \\
\hline$\mu g / L$ & 0.00275 & 0.0055 & EPA Method 8280 \\
\hline$\mu g / L$ & 0.00275 & 0.0055 & EPA Method 8280 \\
\hline$\mu g / L$ & 0.00275 & 0.0055 & EPA Method 8280 \\
\hline$\mu g / L$ & 50 & 100 & EPA Method 8270 \\
\hline$\mu g / 2$ & 50 & 100 & EPA Method 8270 \\
\hline$\mu g / L$ & 0.5 & 1 & Final PDWS (EPA, 1993a) \\
\hline $\mathrm{pH}$ & 8 & 10 & Set by EPD/EMS \\
\hline $\mathrm{pH}$ & 4 & 3 & Set by EPD/EMS \\
\hline$\mu \mathrm{g} / \mathrm{L}$ & 50 & 100 & EPA Method 8270 \\
\hline$\mu g / L$ & 50 & 100 & EPA Method 8270 \\
\hline$\mu \mathrm{g} / \mathrm{L}$ & 50 & 100 & EPA Method 8270 \\
\hline$\mu \mathrm{g} / \mathrm{L}$ & 25 & 50 & EPA Method 420.1 \\
\hline$\mu g / L$ & 50 & 100 & EPA Method 8270 \\
\hline$\mu g / L$ & 0.5 & 1 & EPA Method 8080 \\
\hline$\mu g / L$ & 250 & 500 & Final PDWS (EPA, 1993a) \\
\hline$\mu g / L$ & 50 & 100 & EPA Method 8270 \\
\hline $\mathrm{pCi} / \mathrm{L}$ & $3.51 E+00$ & $7.02 E+00$ & Proposed PDWS (EPA, 1991) \\
\hline $\mathrm{pCi} / \mathrm{L}$ & $3.11 E+01$ & $6.21 E+01$ & Proposed PDWS (EPA, 1991) \\
\hline $\mathrm{pCi} / \mathrm{L}$ & $3.11 E+01$ & $6.21 E+01$ & Proposed PDW' (EPA, 1991) \\
\hline $\mathrm{pCiL}$ & $3.11 E+01$ & $6.22 E+01$ & Proposed PDWS (EPA, 1991) \\
\hline $\mathrm{pCi} / \mathrm{L}$ & $3.13 E+01$ & $6.26 \mathrm{E}+01$ & Proposed PDWS (EPA, 1991) \\
\hline $\mathrm{pCi} / \mathrm{L}$ & $3.27 \mathrm{E}+01$ & $6.54 \mathrm{E}+01$ & Proposed PDWS (EPA, 1991) \\
\hline & No flag & No flag & Set by EPD/EMS \\
\hline & No flag & No flag & Set by EPD/EMS \\
\hline & No flag & No flag & Set by EPD/EMS \\
\hline$p \mathrm{Ci} / L$ & $1.5 \mathrm{E}+02$ & $3 E+02$ & Proposed PDWS (EPA, 1986) \\
\hline $\mathrm{pCi} / \mathrm{L}$ & $5 \mathrm{E}+01$ & $1 E+02$ & EPA Method 901.1 \\
\hline $\mathrm{pCi} / L$ & $5 E+01$ & $1 E+02$ & EPA Method 901.1 \\
\hline $\mathrm{pCi} / \mathrm{L}$ & $5.24 \mathrm{E}+03$ & $5.24 \mathrm{E}+03$ & Proposed PDWS (EPA, 1991) \\
\hline$\mu g / L$ & 50 & 100 & EPA Method 8270 \\
\hline$\mu \mathrm{g} / \mathrm{L}$ & 1,000 & 2,000 & EPA Method 8240 \\
\hline$\mu \mathrm{g} / \mathrm{L}$ & 50 & 100 & EPA Method 8270 \\
\hline$\mu g / L$ & 50 & 100 & EPA Method 8270 \\
\hline $\mathrm{pCi} / \mathrm{L}$ & $1 \mathrm{E}+01$ & $2 \mathrm{E}+01$ & Proposed PDWS (EPA, 1991) \\
\hline $\mathrm{pCi} / 2$ & $1 E+01$ & $2 E+01$ & Proposed PDWS (EPA, 1991) \\
\hline $\mathrm{pCi} / \mathrm{L}$ & $1 E+01$ & $2 \mathrm{E}+01$ & Proposed PDWS (EPA, 1991) \\
\hline $\mathrm{pCi} / \mathrm{L}$ & $1.5 E+02$ & $3 E+02$ & Proposed PDWS (EPA, 1991) \\
\hline $\mathrm{pCi} / L$ & $1 E+02$ & $2 E+02$ & Interim Final PDWS (EPA, 1977) \\
\hline $\mathrm{pCi} / \mathrm{L}$ & $1.5 \mathrm{E}+01$ & $3 E+01$ & Interim Final PDWS (EPA, 1977) \\
\hline$\mu \mathrm{g} / \mathrm{L}$ & 50 & 100 & EPA Method 8270 \\
\hline$\mu \mathrm{g} / \mathrm{L}$ & 25 & 50 & Final PDWS (EPA, 1993a) \\
\hline
\end{tabular}

R-Area Reactor 1993 Annual Report $\bullet$ B-7 
Analyte

Selenium, dissolved

Selenium, total recoverable

Silica

Silica, dissolved

Silica, total recoverable

Silver

Silver, dissolved

Silver, total recoverable

Simazine

Sodium

Sodium, dissolved

Sodium, total recoverable

Sodium-22

Specific conductance

Strontium-89

Strontium-89/90

Strontium-90

Styrene

Sulfate

Sulfide

Sulfotepp

Surfactants

2,3,7,8-TCDD

2,3,7,8-TCDF

Technetium-99

1,2,4,5-Tetrachlorobenzene

Tetrachlorodibenzo-p-dioxin isomers

Tetrachlorodibenzo-p-furan isomers

1,1,1,2-Tetrachloroethane

1,1,2,2-Tetrachloroethane

Tetrachloroethylene

2,3,4,6-Tetrachlorophenol

Thallium

Thallium, dissolved

Thallium, total recoverable

Thionazin

Thorium-228

Thorium-230

Thorium-232

Thorium-234

Tin

Tin, dissolved

Tin, total recoverable

Tin-113c

Toluene

o-Toluidine

Total carbon

Total coliform

Total dissolved solids

Total hydrocarbons

Total inorganic carbon

Total organic carbon

Total organic halogens

Total organic nitrogen

Total petroleum hydrocarbons

Total phosphates (as P)

Total phosphorus

Toxaphene

2,4,5-TP (Silvex)

\begin{tabular}{|c|c|c|c|}
\hline$\underline{\text { Unit }}$ & Flag 1 & Flag 2 & Source \\
\hline$\mu g / L$ & 25 & 50 & Final PDWS (EPA, 1993a) \\
\hline \multirow[t]{2}{*}{$\mu g / L$} & 25 & 50 & Final PDWS (EPA, 1993a) \\
\hline & $\begin{array}{l}\text { No flag } \\
\text { No flag } \\
\text { No flag } \\
50\end{array}$ & $\begin{array}{l}\text { No flag } \\
\text { No flag } \\
\text { No flag } \\
100\end{array}$ & $\begin{array}{l}\text { Set by EPD/EMS } \\
\text { Set by EPD/EMS } \\
\text { Set by EPD/EMS } \\
\text { SDWS (EPA, 1993b) }\end{array}$ \\
\hline$\mu g / L$ & 50 & 100 & SDWS (EPA, 1993b) \\
\hline$\mu g / L$ & 50 & 100 & SDWS (EPA, 1993b) \\
\hline \multirow[t]{2}{*}{$\mu g /$} & 2 & 4 & Final PDWS (EPA, 1993a) \\
\hline & $\begin{array}{l}\text { No flag } \\
\text { No flag } \\
\text { No flag }\end{array}$ & $\begin{array}{l}\text { No flag } \\
\text { No flag } \\
\text { No flag }\end{array}$ & $\begin{array}{l}\text { Set by EPD/EMS } \\
\text { Set by EPD/EMS } \\
\text { Set by EPD/EMS }\end{array}$ \\
\hline $\mathrm{pCi} / \mathrm{L}$ & $2.33 E+02$ & $4.66 \mathrm{E}+02$ & Proposed PDWS (EPA, 1991) \\
\hline$\mu \mathrm{S} / \mathrm{cm}$ & 250 & 500 & Set by EPD/EMS \\
\hline $\mathrm{pCi} / \mathrm{L}$ & $1 E+01$ & $2 E+01$ & Interim Final PDWS (EPA, 1977) \\
\hline $\mathrm{pCi} / \mathrm{L}$ & $4 E+00$ & $8 E+00$ & Final PDWS (EPA, 1993a) \\
\hline $\mathrm{pCi} / \mathrm{L}$ & $4 \mathrm{E}+00$ & $8 \mathrm{E}+00$ & Final PDWS (EPA, 1993a) \\
\hline$\mu g / L$ & 50 & 100 & Final PDWS (EPA, 1993a) \\
\hline$\mu g / L$ & 200,000 & 400,000 & Proposed PDW'S (EPA, 1990) \\
\hline$\mu g / L$ & 5,000 & 10,000 & EPA Method 9030 \\
\hline \multirow[t]{2}{*}{$\mu g / L$} & 50 & 100 & EPA Method 8270 \\
\hline & No flag & No flag & Set by EPD/EMS \\
\hline$\mu g / L$ & 0.000015 & 0.00003 & Final PDWS (EPA, 1993a) \\
\hline$\mu g / L$ & 0.002 & 0.004 & EPA Method 8280 \\
\hline $\mathrm{pCi} / \mathrm{L}$ & $4.5 \mathrm{E}+02$ & $9 \mathrm{E}+02$ & Interim Final PDWS (EPA, 1977) \\
\hline$\mu \mathrm{g} / \mathrm{L}$ & 50 & 100 & EPA Method 8270 \\
\hline$\mu g / L$ & 0.00225 & 0.0045 & EPA Method 8280 \\
\hline$\mu g / L$ & 0.002 & 0.004 & EPA Method 8280 \\
\hline$\mu \mathrm{g} / \mathrm{L}$ & 5 & 10 & EPA Method 8240 \\
\hline$\mu \mathrm{g} / \mathrm{L}$ & 5 & 10 & EPA Method 8240 \\
\hline$\mu g / L$ & 2.5 & 5 & Final PDWS (EPA, 1993a) \\
\hline$\mu \mathrm{g} / \mathrm{L}$ & 50 & 100 & EPA Method 8270 \\
\hline$\mu g / L$ & 1 & 2 & Final PDWS (EPA, 1993a) \\
\hline$\mu g / L$ & 1 & 2 & Final PDWS (EPA, 1993a) \\
\hline$\mu \mathrm{g} / \mathrm{L}$ & 1 & 2 & Final PDWS (EPA, 1993a) \\
\hline$\mu g / L$ & 50 & 100 & EPA Method 8270 \\
\hline $\mathrm{pCi} / \mathrm{L}$ & $6.25 \mathrm{E}+01$ & $1.25 \mathrm{E}+02$ & Proposed PDWS (EPA, 1991) \\
\hline $\mathrm{pCi} / \mathrm{L}$ & $3.96 \mathrm{E}+01$ & $7.92 \mathrm{E}+01$ & Proposed PDWS (EPA, 1991) \\
\hline $\mathrm{pCi} / \mathrm{L}$ & $4.4 \mathrm{E}+01$ & $8.8 \mathrm{E}+01$ & Proposed PDWS (EPA, 1991) \\
\hline $\mathrm{pCi} / \mathrm{L}$ & $2 \mathrm{E}+02$ & $4.01 \mathrm{E}+02$ & Proposed PDWS (EPA, 1991) \\
\hline$\mu \mathrm{g} / \mathrm{L}$ & 10 & 20 & EPA Method 282.2 \\
\hline$\mu \mathrm{g} / \mathrm{L}$ & 10 & 20 & EPA Method 282.2 \\
\hline$\mu \mathrm{g} / \mathrm{L}$ & 10 & 20 & EPA Method 282.2 \\
\hline $\begin{array}{l}\mathrm{pCi} / \mathrm{L} \\
\mu \mathrm{g} / \mathrm{L}\end{array}$ & $\begin{array}{l}1.5 \mathrm{E}+02 \\
500\end{array}$ & $\begin{array}{l}3 E+02 \\
1,000\end{array}$ & $\begin{array}{l}\text { Interim Final PDWS (EPA, 1977) } \\
\text { Final PDWS (EPA, 1993a) }\end{array}$ \\
\hline$\mu \mathrm{g} / \mathrm{L}$ & 50 & 100 & EPA Method 8270 \\
\hline \multirow[t]{3}{*}{$\mu \mathrm{g} / \mathrm{L}$} & 5,000 & 10,000 & EPA Method 9060 \\
\hline & 0 & 0 & Final PDWS (EPA, 1993a) \\
\hline & No flag & No flag & Set by EPD/EMS \\
\hline$\mu g / L$ & 5,000 & 10,000 & EPA Method 418.1 \\
\hline$\mu \mathrm{g} / \mathrm{L}$ & 5,000 & 10,000 & EPA Method 9060 \\
\hline$\mu g / L$ & 5,000 & 10,000 & EPA Method 9060 \\
\hline$\mu \mathrm{g} / \mathrm{L}$ & 25 & 50 & EPA Method 9020 \\
\hline$\mu \mathrm{g} / \mathrm{L}$ & 500 & 1,000 & APHA Method 420 \\
\hline \multirow[t]{3}{*}{$\mu g /$} & 5,000 & 10,000 & EPA Method 418.1 \\
\hline & No flag & No flag & Set by EPD/EMS \\
\hline & No flag & No flag & Set by EPD/EMS \\
\hline & 1.5 & 3 & Final PDWS (EPA, 1993a) \\
\hline$\mu g / L$ & 25 & 50 & Final PDWS (EPA, 1993a) \\
\hline
\end{tabular}


Analyte

Tributyl phosphate

1,2,4-Trichlorobenzene

1,1,1-Trichloroethane

1,1,2-Trichloroethane

Trichloroethylene

Trichlorofluoromethane

2,4,5-Trichlorophenol

2,4,6-Trichlorophenol

2,4,5-Trichlorophenoxyacetic acid

1,2,3-Trichloropropane

$0,0,0$. Triethyl phosphorothioate

1,3,5-Trinitrobenzene

Tritium

Turbiditys

Uranium

Uranium, dissolved

Uranium, total recoverable

Uranium alpha activity

Uranium-233/234e

Uranium-234

Uranium-235

Uranium-238

Vanadium

Vanadium, dissolved

Vanadium, total recoverable

Vinyl acetate

Xylenes

Yttrium-88

Zinc

Zinc, dissolved

Zinc, total recoverable

Zinc-65

Zirconium-95

Zirconium/Niobium-95c

\begin{tabular}{|c|c|c|}
\hline Unit & Flag 1 & Flag 2 \\
\hline$\mu \mathrm{g} / \mathrm{L}$ & 50 & 100 \\
\hline$\mu \mathrm{g} / \mathrm{L}$ & 35 & 70 \\
\hline$\mu \mathrm{g} / \mathrm{L}$ & 100 & 200 \\
\hline$\mu g / L$ & 2.5 & 5 \\
\hline$\mu g / L$ & 2.5 & 5 \\
\hline$\mu \mathrm{g} / \mathrm{L}$ & 5 & 10 \\
\hline$\mu \mathrm{g} / \mathrm{L}$ & 50 & 100 \\
\hline$\mu g / L$ & 50 & 100 \\
\hline$\mu g / L$ & 2.5 & 5 \\
\hline$\mu \mathrm{g} / \mathrm{L}$ & 5 & 10 \\
\hline$\mu g / L$ & 50 & 100 \\
\hline$\mu g / L$ & 50 & 100 \\
\hline $\mathrm{pCi} / \mathrm{mL}$ & $\begin{array}{l}1 E+01 \\
\text { No flag }\end{array}$ & $\begin{array}{l}2 \mathrm{E}+01 \\
\text { No flag }\end{array}$ \\
\hline$\mu g / L$ & 10 & 20 \\
\hline$\mu \mathrm{g} / \mathrm{L}$ & 10 & 20 \\
\hline$\mu g \pi$ & 10 & 20 \\
\hline $\begin{array}{l}\mathrm{p} C \mathrm{C} / \mathrm{L} \\
\mathrm{pCi} / \mathrm{L}\end{array}$ & $\begin{array}{l}1.5 E+01 \\
6.9 E+00\end{array}$ & $\begin{array}{l}3 \mathrm{E}+01 \\
1.38 \mathrm{E}+01\end{array}$ \\
\hline $\mathrm{pCi} / L$ & $6.95 E+00$ & $1.39 \mathrm{E}+01$ \\
\hline $\mathrm{pCi} / \mathrm{L}$ & $7.25 E+00$ & $1.45 \mathrm{E}+01$ \\
\hline $\begin{array}{l}\mathrm{pCi} / L \\
\mu \mathrm{g} / \mathrm{L}\end{array}$ & $\begin{array}{l}7.3 E+00 \\
40\end{array}$ & $\begin{array}{l}1.46 \mathrm{E}+01 \\
80\end{array}$ \\
\hline$\mu \mathrm{g} / \mathrm{L}$ & 40 & 80 \\
\hline$\mu g / L$ & 40 & 80 \\
\hline$\mu \mathrm{g} / \mathrm{L}$ & 5 & 10 \\
\hline$\mu g / L$ & 5,000 & 10,000 \\
\hline $\begin{array}{l}\mathrm{pCi} / \mathrm{L} \\
\mu \mathrm{g} / \mathrm{L}\end{array}$ & $\begin{array}{l}5 E+01 \\
2,500\end{array}$ & $\begin{array}{l}1 E+02 \\
5,000\end{array}$ \\
\hline$\mu g / L$ & 2,500 & 5,000 \\
\hline$\mu \mathrm{g} / \mathrm{L}$ & 2,500 & 5,000 \\
\hline $\mathrm{pCi} / \mathrm{L}$ & $1.5 \mathrm{E}+02$ & $3 E+02$ \\
\hline $\mathrm{pCi} / \mathrm{L}$ & $1 E+02$ & $2 \mathrm{E}+02$ \\
\hline $\mathrm{pCi} / \mathrm{L}$ & $1 E+02$ & $2 E+02$ \\
\hline
\end{tabular}

Source:

EPA Method 8270

Final PDWS (EPA, 1993a)

Final PDWS (EPA, 1993a)

Final PDWS (EPA, 1993a)

Final PDWS (EPA, 1993a)

EPA Method 8240

EPA Method 8270

EPA Method 8270

EPA Method 8150

EPA Method 8240

EPA Method 8270

EPA Method 8270

Final PDWS (EPA, 1993a)

Set by EPD/EMS

Proposed PDWS (EPA, 1991)

Proposed PDWS (EPA, 1991)

Proposed PDWS (EPA, 1991)

Proposed PDWS (EPA, 1991)

Proposed PDWS (EPA, 1991)

Proposed PDWS (EPA, 1991)

Proposed PDWS (EPA, 1991)

Proposed PDWS (EPA, 1991)

EPA Method 6010

EPA Method 6010

EPA Method 6010

EPA Method 8240

Final PDWS (EPA, 1993a)

EPA Method 901.1

SDW'S (EPA, 1993b)

SDWS (EPA, 1993b)

SDWS (EPA, 1993b)

Interim Final PDWS (EPA, 1977)

Interim Final PDWS (EPA, 1977)

Interim Final PDWS (EPA, 1977)

- References for dated sources are at the end of this appendix.

b EMS is currently unable to perform this analysis.

- EMS discontinued monitoring this radionuclide because it is inappropriate for the SRS groundwater monitoring program.

d EPD/EMS set this flagging criterion using the 1991 proposed PDWS because the final PDWS in 1977 may have been in error.

- For double radionuclide analyses where each separate radionuclide has its own standard, the more stringent standard is used.

f The applied standard is for radium-226.

I The primary maximum contaminant level range for turbidity is $1.5 \mathrm{TU}$, which is inappropriate for the SRS groundwater monitoring program.

\section{References}

EPA (U.S. Environmental Protection Agency), 1977. National Interim Primary Drinking Water Regulations, EPA-570/9-76-003. Washington, DC.

EPA (U.S. Environmental Protection Agency), 1986. Water Pollution Control; National Primary Drinking Water Regulations, Radionuclides (Proposed). Federal Register, September 30, 1986, pp. 34835-34862. Washington, DC. 
EPA (U.S. Environmental Protection Agency), 1990. National Primary and Secondary Drinking Water Regulations; Synthetic Organic Chemicals and Inorganic Chemicals (Proposed Rule). Federal Register, July 25, 1990, pp. 30369-30448. Washington, DC.

EPA (U.S. Environmental Protection Agency), 1991. National Primary Drinking Water Regulations; Radionuclides; Proposed Rule. Federal Register, July 18, 1991, pp. 33052-33127. Washington, DC.

EPA (U.S. Environmental Protection Agency), 1993a. National Primary Drinking Water Regulations. Code of Federal Regulations, Section 40, Part 141, pp. 592-732. Washington, DC.

EPA (U.S. Environmental Protection Agency), 1993b. National Secondary Drinking Water Regulations. Code of Federal Regulations, Section 40, Part 143, pp. 774-777. Washington, DC.

SCDHEC (South Carolina Department of Health and Environmental Control), 1981. State Primary Drinking Water Regulations, R.61-58.5. Columbia, SC. 


\section{Appendix C. Groundwater Monitoring Results for Individual Wells during 1993}

WELL P 20C

\begin{tabular}{|c|c|c|}
\hline SRS Coord. & Lat/Longitude & Screen Zone Elevation \\
\hline $\begin{array}{l}\text { N56067.7 } \\
\text { E76814.0 }\end{array}$ & $\begin{array}{l}33.275077 \circ \mathrm{o} \\
81.573452 \circ \mathrm{W}\end{array}$ & $147.8-137.8 \mathrm{ft} \mathrm{msl}$ \\
\hline
\end{tabular}

$\begin{array}{llll}\text { Top of Casing } & \text { Casing } & \text { Pump } & \text { Scroen Zone } \\ 289.1 \mathrm{ft} \mathrm{msl} & \text { 4" PVC } & \text { McBean }\end{array}$

SAMPLE DATE

$06 / 10 / 93 \quad 09 / 09 / 93 \quad 12 / 08 / 93$

FIELD DATA

Analyte
Water olevation
Depth to water
pH
Sp. conductance
Water tomperature
Alkalinity as CaCO
Turbidity
Volume purged
Sampling code

ANALYTICAL DATA
1093

2093
241.1
48.1
6.4
70
20.9
22
1
3.4

1093

$\underline{2093}$
Aluminum, total recoverable

Arsonic, total recoverablo

Barium, total recoverable

Boron, total recovorable

Cadmium, total recoverable

Calcium, total recoverable

Chloride

Chromlum, total recoverable

2,4-Dichlorophenoxyacetic acid

Endrin

Fluoride

Gross alphe

Iron, total recoverable

Lead, total rocoverable

Lindano

Lithium, total recoverable

Magnesium, total rocoverablo

Manganese, total recoverable

Mercury, total recoverable

Mothoxychlor

Nitrate-nitrite as nitrogen

Nonvolatile bote

pH

Phenols

Potassium, total recoverable

Radium, total alpha-emitting

Solenium, totel recovorable

Silica, total recoverable

Silver, total recoverable

Sodium, total recoverable

Specific conductance

Sulfate

Total activity

Total dissolved solids

Total organic carbon

Total organic halogens

Total phosphates (as P)

Toxaphene

2,4,5-TP [Silvex)

Tritium

\section{2}

$$
\begin{aligned}
& 2.9 \\
& 31
\end{aligned}
$$$$
<2.0
$$$$
\begin{aligned}
& <2.0 \\
& 7.240
\end{aligned}
$$

2,350

$<4.0$

$<0.30$

$<0.0060$

$<100$

$3.6 \mathrm{E}+00$

4.030

$<3.0$

$<0.0050$

297

$<0.20$

$<0.50$

$<50$

$7.3 E+00$

6.3

$<5.0$

1.780

$1.5 E+00$

$<2.0$

17.000

$<2.0$

2,150

60

1,110

4.4E + 03

42,000

$<1,000$

$<5.0$

$<50$

$<0.24$

$<0.090$

$<7.0$ E-0 1

3093
238.7
50.4
6.5
73
20.5
25
1
3.6
$x$

3093

$<20$

$<2.0$

$<32$

$<2.0$

6.940

2,110

$<4.0$

$<100$

$<2.0 E+00$

4.120

$<3.0$

10
290

16

0.24

$<50$

3. $1 E+00$

6.3

$<5.0$

1.850

$1.9 E+00$

$<2.0$

16,400

$<2.0$

2.070

54

1.050

$6.4 E+03$

49.000

1.280

$<5.0$

$<50$

$<7.0$ E-01

4093
237.6
51.5
6.3
69
20.0
28
1
3.7

Unit

ft msl

ft

$\mathrm{s} / \mathrm{cm}$

${ }^{\circ} \mathrm{C} / \mathrm{cm}$

mg/L

Well vol.

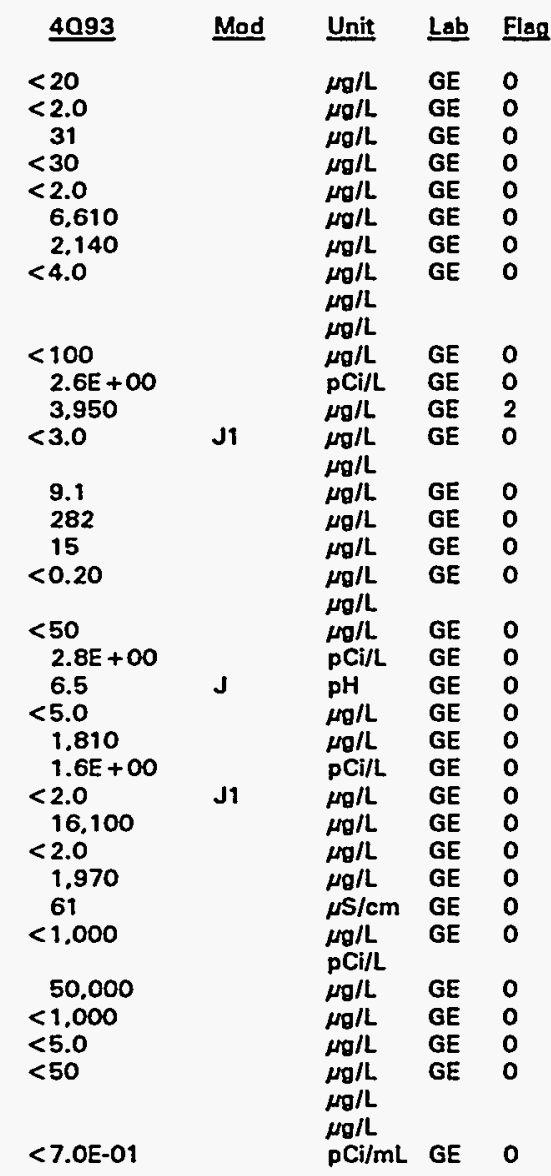

Note: Flagging levels, madifiers, and laboratories are for 4th quarter 1993 data only. See Appendix B for flagging criteria. - = exceeded holding time for 4th quarter 1993.

- = exceeded screening level or final primary drinking water standard for 4th quarter 1993. 
WELL P 2OD

$\begin{array}{llll}\text { SRS Coord. } & \text { Lat/Longitude } & \text { Screen Zone Elevation } \\ \text { N56075.2 } & & \begin{array}{ll}33.275045 \\ \text { oN }\end{array} & 244.1-225.1 \mathrm{ft} \mathrm{msl} \\ \text { E76784.5 } & 81.573545{ }^{\circ} \mathrm{W} & \end{array}$

SAMPLE DATE

FIELD DATA $\underline{1093}$

Water olevation
Dopth to water
pH
Sp. conductance
Water temporature
Alkalinity as $\mathrm{CaCO}$,
Turbidity
Volume purged
Sampling code

\section{ANALYTICAL DATA}

\section{Analyte}

Aluminum, total recoverable Arsenic, total recoverable Barium, total recoverable Boron, total recoverable Cadmium, total recoverable Calcium, total recoverable Chloride

Chromium, total recoverable

2,4-Dichlorophenoxyacetic acid

Endrin

Fluoride

Gross aipha

Iron, total recoverable

Load. total recoverable

Lindane

Lithium, total recoverable

Magnesium, total recoverable

Manganese, total recoverable

Mercury, total recoverable

Methoxychlor

Nitrate-nitrite as nitrogen

Nonvolatile beta

pH

Phenols

Potassium, total recoverable

Radium, total alpha-emitting

Selenium, total recoverable

Silica, total recoverable

Silver, total recoverable

Sodium. total recoverable

Specific conductance

Sulfate

Total activity

Total dissolved solids

Total organic carbon

Total organic halogens

Total phosphates (as P)

Toxaphene

2.4.5-TP (Silvex)

$\begin{array}{llll}\text { Top of Casing } & \text { Casing } & \text { Pump } & \text { Screen Zone } \\ 289.3 \mathrm{ft} \mathrm{msl} & 4^{-} \text {PVC } & \text { Water Table }\end{array}$

$06 / 10 / 93 \quad 09 / 09 / 93 \quad 12 / 08 / 93$

$\begin{array}{llll}\underline{2093} & \underline{3093} & \underline{4093} & \text { Unit } \\ 273.4 & 269.5 & 267.3 & \mathrm{ft} \mathrm{msl} \\ 15.9 & 19.8 & 22.0 & \mathrm{tt} \\ 6.2 & 5.9 & 5.7 & \mathrm{pH} \\ 34 & 35 & 35 & \mu \mathrm{S} / \mathrm{cm} \\ 22.4 & 21.0 & 21.4 & { }^{\circ} \mathrm{C} \\ 7 & 7 & 8 & \mathrm{mg} / \mathrm{L} \\ 2 & 1 & 1 & \mathrm{NTU} \\ 3.0 & 3.6 & 3.9 & \text { Woll vol. }\end{array}$

1093

2093
66
$<2.0$
11
$<2.0$
3.970
1.350
$<4.0$
$<0.30$
$<0.0060$
$<100$
$<2.0 E+00$
15
$<3.0$
$<0.0050$
555
6.4
$<0.20$
$<0.50$
1.200
$<2.0 E+00$
6.1
$<5.0$
$<500$
$1.0 E+00$
$<2.0$
6.250
$<2.0$
1.040
30
$<1.000$
$3.4 E+03$
20.000
$<1.000$
$<5.0$
$<50$
$<0.24$
$<0.090$

3093
68
$<2.0$
6.1
$<30$
$<2.0$
3.690
1.340
$<4.0$

$<100$
$<2.0 E+00$
8.2
$<3.0$
$<5.0$
499
4.1
$<0.20$

1.380
$<2.0 \mathrm{E}+00$
6.1
$<5.0$
$<500$
$<1.0 \mathrm{O}+00$
$<2.0$
6.320
$<2.0$
1.200
32
$<1.000$
$3.1 \mathrm{E}+03$
30.000
1.090
$<5.0$
$<50$

\begin{tabular}{|c|c|c|c|c|}
\hline 4093 & Mad & Unit & $\underline{\text { Lab }}$ & Flo \\
\hline 44 & & $\mu g / L$ & GE & 1 \\
\hline$<2.0$ & & $\mu g / L$ & GE & 0 \\
\hline 7.1 & & $\mu g / L$ & GE & 0 \\
\hline$<30$ & & $\mu g / L$ & GE & 0 \\
\hline$<2.0$ & & $\mu \mathrm{g} / \mathrm{L}$ & GE & 0 \\
\hline 3.570 & & $\mu g / L$ & GE & 0 \\
\hline 1,550 & & $\mu g / L$ & GE & 0 \\
\hline$<4.0$ & & $\begin{array}{c}\mu \mathrm{g} / \mathrm{L} \\
\mu \mathrm{g} / \mathrm{L} \\
\mu \mathrm{g} / \mathrm{L}\end{array}$ & GE & 0 \\
\hline$<100$ & & $\mu \mathrm{g} / \mathrm{L}$ & GE & 0 \\
\hline $\begin{array}{l}<2.0 E+\infty 0 \\
<4.0\end{array}$ & & $\begin{array}{l}\mathrm{pCi} / \mathrm{L} \\
\mu \mathrm{g} / \mathrm{L}\end{array}$ & $\begin{array}{l}G E \\
G E\end{array}$ & $\begin{array}{l}\mathbf{0} \\
0\end{array}$ \\
\hline$<3.0$ & J1 & $\begin{array}{l}\mu g / L \\
\mu g / L\end{array}$ & GE & 0 \\
\hline$<5.0$ & & $\mu_{g} / L$ & GE & 0 \\
\hline $\begin{array}{l}458 \\
3.4\end{array}$ & & $\begin{array}{l}\mu \mathrm{g} / \mathrm{L} \\
\mu \mathrm{g} / \mathrm{L}\end{array}$ & $\begin{array}{l}\text { GE } \\
\text { GE }\end{array}$ & 0 \\
\hline$<0.20$ & & $\begin{array}{l}\mu \mathrm{g} / \mathrm{L} \\
\mu \mathrm{g} / \mathrm{L}\end{array}$ & GE & \\
\hline $\begin{aligned} & 1.200 \\
< & 2.0 E+00\end{aligned}$ & & $\begin{array}{l}\mu \mathrm{g} / \mathrm{L} \\
\mathrm{pC \textrm {C } / \mathrm { L }}\end{array}$ & $\begin{array}{l}\text { GE } \\
\text { GE }\end{array}$ & $\begin{array}{l}0 \\
0\end{array}$ \\
\hline 6.0 & $J$ & $\mathrm{pH}$ & GE & 0 \\
\hline$<5.0$ & & $\mu g / L$ & GE & 0 \\
\hline $\begin{array}{l}<500 \\
<1.0 E+00\end{array}$ & & $\begin{array}{l}\mu \mathrm{g} / \mathrm{L} \\
\mathrm{pCi} / \mathrm{L}\end{array}$ & $\begin{array}{l}\mathrm{GE} \\
\mathrm{GE}\end{array}$ & $\begin{array}{l}0 \\
0\end{array}$ \\
\hline$<2.0$ & J1 & $\mu g / L$ & GE & 0 \\
\hline 6,450 & & $\mu \mathrm{g} / \mathrm{L}$ & GE & 0 \\
\hline$<2.0$ & & $\mu \mathrm{g} / \mathrm{L}$ & GE & 0 \\
\hline 1.250 & & $\mu \mathrm{g} / \mathrm{L}$ & GE & 0 \\
\hline 33 & & $\mu \mathrm{S} / \mathrm{cm}$ & GE & 0 \\
\hline$<1,000$ & & $\begin{array}{l}\mu \mathrm{g} / \mathrm{L} \\
\mathrm{pCi} / \mathrm{L}\end{array}$ & GE & 0 \\
\hline 27.000 & & $\mu_{\mathrm{g}} / \mathrm{L}$ & GE & 0 \\
\hline $\begin{array}{l}<1.000 \\
<5.0\end{array}$ & & $\mu \theta / L$ & GE & $\begin{array}{l}0 \\
0\end{array}$ \\
\hline$<50$ & & $\mu g / L$ & $\begin{array}{l}\text { GE } \\
\text { GE }\end{array}$ & 0 \\
\hline
\end{tabular}

Note: Flagging levels, modifiers, and laboratories are for 4th quarter 1993 data only. See Appendix B for flagging criteria. - = exceeded holding time for 4th quarter 1993.

- =xceeded screening level or final primary drinking water standard for 4th quarter 1993. 
H D Analyto

Tritium $\underline{1093}$

$\underline{2093}$

$2.2 E+00$ $\underline{3093}$

2.1E $+\infty 0$
$4093 \quad$ Mod

$2.2 \mathrm{E}+00$
Unit Lab Flag

$\mathrm{pCi} / \mathrm{mL}$ GE 0

\section{WELL RAC 1}

\begin{tabular}{|c|c|}
\hline SRS Coord. & Lat/Longitude \\
\hline $\begin{array}{l}\text { N55107.3 } \\
\text { E74570.7 }\end{array}$ & $\begin{array}{l}33.269295 \text { oN } \\
81.577499 \text { oW }\end{array}$ \\
\hline
\end{tabular}

SAMPLE DATE

FIELD DATA

\section{Analyze}

Water olevation Dopth to water

$\mathrm{pH}$

Water temperature

Alkalinity as $\mathrm{CaCO}_{3}$

Turbidity

Volume purged

Sampling code
Sp. conductance

\section{Screen Zone Elevstion \\ 277.3-247.3 ft msl}

$03 / 19 / 93$

1093
275.4
8.2
5.1
69
18.3
0
9
2.9

\section{ANALYTICAL DATA}

\section{H D Analyte}

Alkalinity las $\mathrm{CaCO}_{3}$ ) Aluminum, total recoverable Arsenic, total recoverable Barium, total recoverable Cadmium, total recoverable Calcium, total recoverable Chlorido

Chromium, total recoverable 2,4-Dichlorophenoxyacetic acid Endrin

Fluoride

Gross alphe

Iron, total recoverable

Lead, total recoverable

Lindano

Magnesium, total recoverable

Manganese, total recoverable

Mercury, total recoverable

Methoxychlor

Nitrato-nitrite as nitrogen

Nonvolatile bote

pH

Phonols

Potassium, total recoverable

Radium, total alpha-emitting

Solenium, total recoverable

Silica, total recoverable

Silver, total recoverable

Sodium, total recoverable

Specific conductance

Sulfato

Total dissolved solids

Total organic carbon

Total organic halogens
1093

1.9

76

$<2.0$

20

$<2.0$

4,420

2,670

$<4.0$

$<0.30$

$<0.0060$

$<100$

$<2.0 \mathrm{E}+00$

1,640

5.4

$<0.0050$

1.110

15

$<0.20$

$<0.50$

2.540

2. $2 E+\infty 0$

5.1

$<5.0$

584

$<1.0 E+\infty 0$

$<2.0$

7.140

$<2.0$

3.720

58

7,930

41.000

1,630

$<5.0$

$\begin{array}{lrrrr}\text { Top of Casing } & \text { Casing } & \text { Pump } & \text { Screen Zone } \\ 283.6 \mathrm{ft} \mathrm{msl} & \text { 4" PVC } & \text { S } & \text { Water Table } \\ 06 / 10 / 93 & 09 / 14 / 93 & 12 / 16 / 93 & \end{array}$

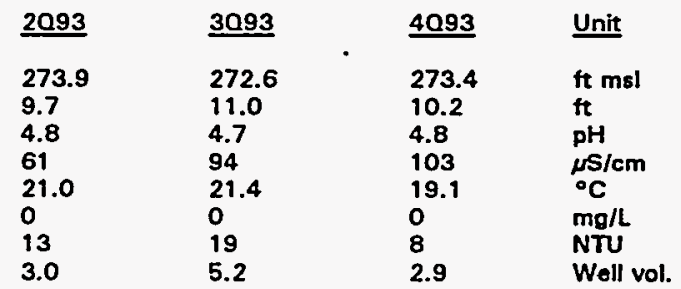

$\underline{2093}$

$\underline{4093}$

Mod Unit Lab Flag

138

$\mathrm{mg} / \mathrm{L}$

$\mu \mathrm{g} / \mathrm{L}$

$\mu \mathrm{g} / \mathrm{L}$

$\mu g / L$

$\mu \mathrm{g} / \mathrm{L}$

$\mu / L$

$\mu g / L$

$\mu \mathrm{g} / \mathrm{L}$

$\mu g / L$

$\mu \mathrm{g} / \mathrm{L}$

$2.0 \mathrm{E}+\infty 0 \quad \begin{aligned} & \mu \mathrm{g} / \mathrm{L} \\ & \mathrm{pCi} / \mathrm{L}\end{aligned}$

151

5.4

25

$\mu g / L$

$\mu \mathrm{g} / \mathrm{L} / \mathrm{L}$

$\mu g / L$

$\mu g / L$

$\mu \mathrm{g} / \mathrm{L}$

$\mu \mathrm{p} / \mathrm{L}$

pH

$\mu g / L$

$\mu \mathrm{g} / \mathrm{L}$

pCi/L

$\mu \mathrm{rg} / \mathrm{L}$

$\mu \mathrm{g} / \mathrm{L}$

$\mu g / L$

$\mu \mathrm{g} / \mathrm{L}$

$\mu \mathrm{s} / \mathrm{cm}$

$\mu \mathrm{g} / \mathrm{L}$

eg/L

$\mu g / L$

$\mu g / L$

Note: Flagging levels, modifiers, and laboratories are for 4th quarter 1993 data only. See Appendix B for flagging criteria.

- = exceeded holding time for 4th quarter 1993.

= exceeded screening level or final primary drinking water standard for 4th quarter 1993. 
H D Analyte

Total phosphates (as $P$ Toxaphene

2,4,5-TP (Silvox)

Tritium

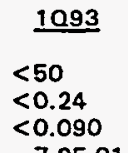

7.9E-01
2093

$\underline{3093}$

$\underline{4093}$

Mod

Unit

$\mu \mathrm{g} / \mathrm{L}$

mo/L

$\mu \mathrm{g} / \mathrm{L}$

$\mathrm{pCi} / \mathrm{mL}$

\section{WELL RAC 2}

SRS Coord.

N55026.3

E74555.5

Lat/Longitude

$33.269091^{\circ} \mathrm{N}$ 81.577382 oW

SAMPLE DATE

FIELD DATA

Analyte

Water elevation

Depth to water

$\mathrm{pH}$

Sp. conductance

Water temperature

Alkalinity as $\mathrm{CaCO}$,

Turbidity

Volume purged

Sampling code

ANALYTICAL DATA

\section{Screen Zone Elevation}

273.4-243.4 ft msl

$03 / 21 / 93$

1093
273.7
6.7
5.3
72
15.9
0
1
2.6

$\underline{2093}$

272.3

8.1

18.1

0

12.5

Top of
280.4
$06 / 10 / 93$

2093
272.3
8.1
4.5
63
18.1
0
2
12.5

$\underline{2093}$

$\underline{3093}$

Cosing

4" PVC

Pump

Screen Zone

Water Table

$\begin{array}{ll}4093 & \text { Unit } \\ 272.6 & \mathrm{ft} \mathrm{msl} \\ 7.8 & \mathrm{ft} \\ 4.8 & \mathrm{pH} \\ 60 & \mu \mathrm{s} / \mathrm{cm} \\ 18.0 & { }^{\circ} \mathrm{C} \\ 0 & \mathrm{mg} / \mathrm{L} \\ 3 & \mathrm{NTU} \\ 11.9 & \text { Well vol. }\end{array}$

$\underline{4093}$
H D Anslyte

Alkalinity (as $\mathrm{CaCO}_{3}$ )

Aluminum, total recoverable

Arsenic, total recoverable

Barium, total recoverable

Cadmium, total recoverable

Calcium, total recoverablo

Chlorido

Chromium total recoverable

2,4-Dichlorophenoxyacetic acid

Endrin

Fluoride

Gross alpha

Iron, total recoverable

Lead, total recoverable

Lindane

Magnesium, total recoverable

Manganese. total recoverable

Mercury, total recoverable

Methoxychlor

Nitrate-nitrite as nitrogen

Nonvolatile bets

$\mathrm{pH}$

Phenols

Potassium, total recoverable

Radium, total alpha-emitting

Solenium, total recovarable

Silica, total recoverable

Silver, total recoverable

Sodium, total recoverable

Specific conductance

Sulfato
1093

2.9

123

$<2.0$

41

$<2.0$

3.500

1.910

$<4.0$

$<0.30$

$<0.0060$

$<100$

2.7E + 00

9.2

11

$<0.0050$

2,940

52

$<0.20$

$<0.50$

3,650

$<2.0 E+00$

5.3

$<5.0$

633

$<1.0 E+\infty 0$

$<2.0$

6.260

$<2.0$

1.720

60

7,840

135

Unit Lab Flag

$\mathrm{mg} / \mathrm{L}$

$\mu g / L$

$\mu \mathrm{g} / \mathrm{L}$

$\mu g / L$

$\mu g / L$

$\mu g / L$

$\mu g / L$

$\mu \mathrm{g} / \mathrm{L}$

$\mu \mathrm{g} / \mathrm{L}$

$\mu g / L$

$\begin{array}{ll}2.2 \mathrm{E}+\infty & \mu \mathrm{\mu} / \mathrm{L} \\ 85 & \mathrm{pCi} / \mathrm{L} \\ \mathrm{\mu g} / \mathrm{L}\end{array}$

$\begin{array}{ll}85 & \mu \mathrm{g} / \mathrm{L} \\ 19 & \mu \mathrm{g} / \mathrm{L} \\ & \end{array}$

$\mu \mathrm{g} / \mathrm{L}$

$\mu g / L$

30

$\mu \mathrm{g} / \mathrm{L}$

$\mu \mathrm{g} / \mathrm{L}$

$\mu \mathrm{g} / \mathrm{L}$

$\mathrm{pCi} / \mathrm{L}$

$\mathrm{pH}$

$\mu \mathrm{g} / \mathrm{L}$

$\mu \mathrm{g} / \mathrm{L}$

pCin

$\mu \mathrm{g} / \mathrm{L}$

$\mu g / L$

$\mu \mathrm{g} / \mathrm{L}$

$\mu \mathrm{g} / \mathrm{L}$

$\mu \mathrm{S} / \mathrm{cm}$

$\mu g / L$

Note: Flagging levels, modifiers, and laboratories are for 4th quarter 1993 data only. See Appendix $B$ for flagging criteria. - = exceeded holding time for 4th quarter 1993.

- =xceeded screening level or final primary drinking water standard for 4th quarter 1993. 


\section{ANALYTICAL OATA}

\section{H D Analvite}

Total dissolved solids Total oroanic carbon

Total organic halogens

Toxephene

2,4,5-TP (Silvox)

Tritium
Total phosphates (as P)

$\quad 1093$
44.000
$<1.000$
5.2
$<50$
$<0.24$
$<0.090$
$<7.0 \mathrm{E}-01$

\section{$\underline{3093}$}

$\underline{2093}$

4093

Mod

Uning/L

$\mu \mathrm{g} / \mathrm{L}$

$\mu \mathrm{gg} / \mathrm{L}$

$\mathrm{\mu g} / \mathrm{L}$

$\mathrm{\mu g} / \mathrm{h}$

${ }_{\mathrm{pCi} / \mathrm{mL}}$

Lab Flag

\section{WELL RAC 3}

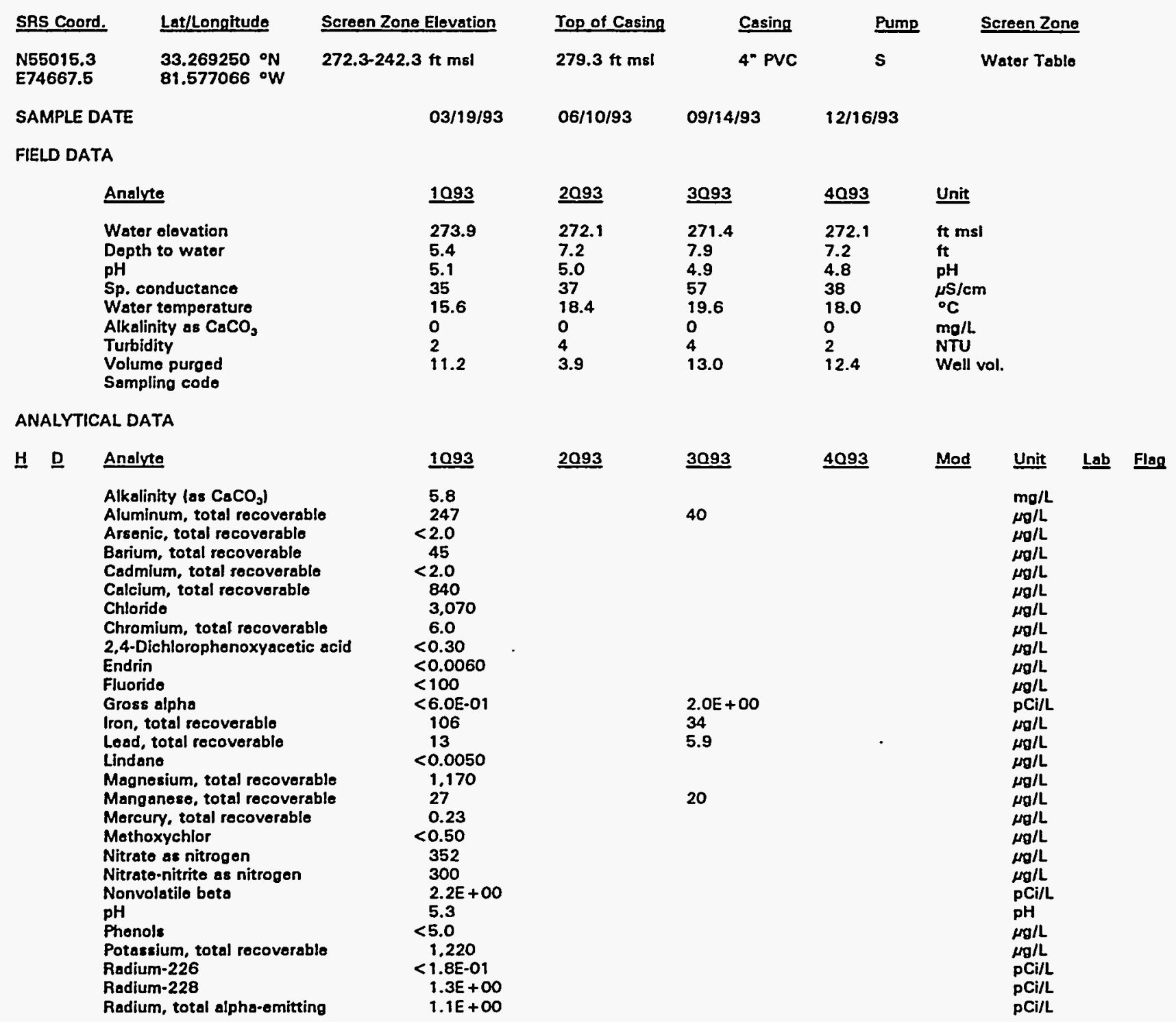

Note: Flagging levels, modifiers, and laboratories are for 4th quarter 1993 data only. See Appendix $B$ for flagging criteria. - = exceeded holding time for 4th quarter 1993.

- = exceeded screening level or final primary drinking water standard for 4th quarter 1993. 


Analyte
Selenium, total recoverable
Silica, total recoverable
Silver, total recovorable
Sodium. total rocoverable
Specific conductance
Sulfate
Total dissolved solids
Total organic carbon
Total organic halogens
Total phosphates (as P)
Toxaphene
2.4,5-TP (Silvox)
Tritium

WELL RAC 4

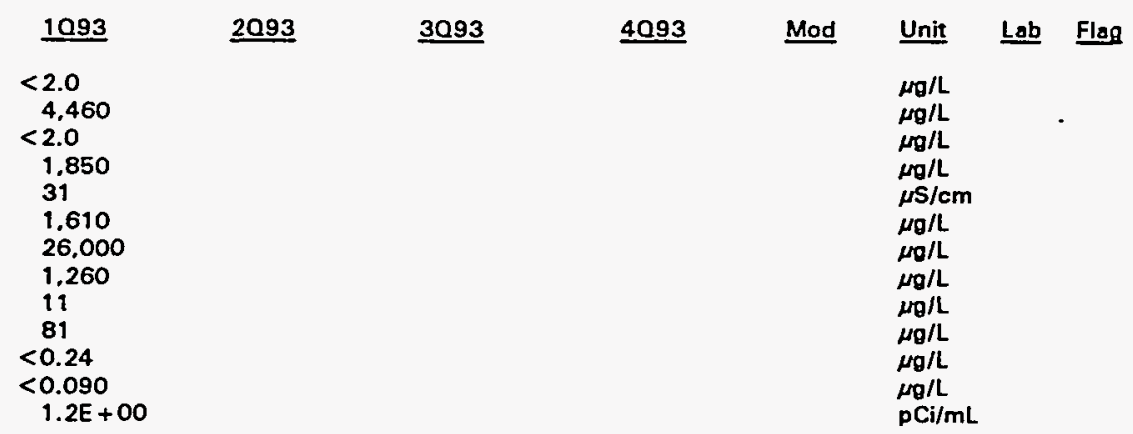

\begin{tabular}{|c|c|c|c|c|c|c|}
\hline SRS Coord. & Lat/Longitude & Screen Zone Elevation & Top of Casing & Casing & Pump & Screen Zone \\
\hline $\begin{array}{l}\text { N54984.0 } \\
\text { E74588.8 }\end{array}$ & $\begin{array}{l}33.269052^{\circ} \mathrm{N} \\
81.577212^{\circ} \mathrm{W}\end{array}$ & $268.2-238.2 \mathrm{ft} \mathrm{msl}$ & 279 t msl & 4" PVC & $\mathbf{s}$ & Water Table \\
\hline SAMPLE DA & & $03 / 21 / 93$ & $06 / 10 / 93$ & $09 / 14 / 93$ & $12 / 16 / 93$ & \\
\hline ELD DATA & & & & & & \\
\hline & & 1093 & 2093 & $\underline{3093}$ & 4093 & $\underline{\text { Unit }}$ \\
\hline $\begin{array}{l}\text { U } \\
\text { D } \\
\text { P } \\
\text { Y } \\
\text { A } \\
\text { V }\end{array}$ & $\begin{array}{l}\text { relevation } \\
\text { to water } \\
\text { onductance } \\
\text { r temperature } \\
\text { nity as } \mathrm{CaCO} \text {, } \\
\text { dity } \\
\text { ne purgod } \\
\text { ling code }\end{array}$ & $\begin{array}{l}273.5 \\
5.6 \\
4.8 \\
50 \\
18.5 \\
0 \\
1 \\
3.0\end{array}$ & $\begin{array}{l}272.0 \\
7.0 \\
4.4 \\
47 \\
19.1 \\
0 \\
1 \\
4.6\end{array}$ & $\begin{array}{l}270.9 \\
8.1 \\
4.6 \\
59 \\
19.2 \\
0 \\
1 \\
3.9\end{array}$ & $\begin{array}{l}271.4 \\
7.6 \\
4.7 \\
54 \\
17.8 \\
0 \\
5 \\
7.4\end{array}$ & $\begin{array}{l}\mathrm{ft} \mathrm{msl} \\
\mathrm{ft} \\
\mathrm{pH} \\
\mu \mathrm{S} / \mathrm{cm} \\
{ }^{\circ} \mathrm{C} \\
\mathrm{mg} / \mathrm{L} \\
\mathrm{NTU} \\
\text { Woll vol. }\end{array}$ \\
\hline
\end{tabular}

ANALYTICAL DATA

H D Analyte

Alkalinity (as $\mathrm{CaCO}_{3}$ )

Aluminum, total recoverable Arsenic, total recoverable Barium, total recoverable

Cadmium, total recoverable Calcium, total recoverable Chloride

Chromium, total recoverable

2,4-Dichlorophenoxyacotic acid

Endrin

Fluorido

Gross alpha

Iron, total recoverable

Lead, total recoverable

Lind ane

Magnesium, total recoverable

Manganese, total recoverable

Morcury, total recoverable

Methoxychlor

Nitrato-nitrito as nitrogen

Nonvolatile beta

pH

$\quad \underline{1093}$
$<1.0$
60
$<2.0$
22
$<2.0$
1.040
2.640
$<4.0$
$<0.30$
$<0.0060$
$<100$
$<2.0 \mathrm{E}+\infty 0$
20
$<3.0$
$<0.0050$
1.090
8.3
0.33
$<0.50$
1.970
$<2.0 \mathrm{E}+\infty 0$
4.9

$\underline{2093}$

$\underline{3093}$

$\underline{4093}$

Mod

Unit Lab Flag $\mathrm{mg} / \mathrm{L}$

$\mu \mathrm{g} / \mathrm{L}$

$\mu g / L$

$\mu \mathrm{g} / \mathrm{L}$

$\mu g / L$

$\mu_{\mathrm{g} / \mathrm{L}}$

$\mu \mathrm{g} / \mathrm{L}$

$\mu g / L$

$\mu \mathrm{g} / \mathrm{L}$

$\mu \mathrm{g} / \mathrm{L}$

$\mu \mathrm{pCi} / \mathrm{L}$

$\mu g / L$

$\mu g / L$

$\mu \mathrm{g} / \mathrm{L}$

$\mu / \mathrm{L}$

$\mu \mathrm{g} / \mathrm{L}$

$\mu g / L$

$\mu g / L$

$\mu \mathrm{g} / \mathrm{L}$

pCi/L

$\mathrm{pH}$

Note: Flagging levels, modifiers, and laboratories are for 4th quarter 1993 data only. See Appendix $B$ for flagging criteria. - = exceeded holding time for 4th quarter 1993.

- =xceeded screening level or final primary drinking water standard for 4th quarter 1993. 


\section{ANALYTICAL DATA}

H D Analyte

Phenols

Potassium, total recoverable

Radium, total alpha-emitting

Selonium, total recoverable

Silica, total recoverable

Silver, total recoverable

Sodium, total recovorable

Spocific conductanco

Sulfato

Total dissolved solids

Total organic carbon

Total organic halogons

Total phosphatos (as P)

Toxaphene

2,4,5-TP (Silvox)

Tritium

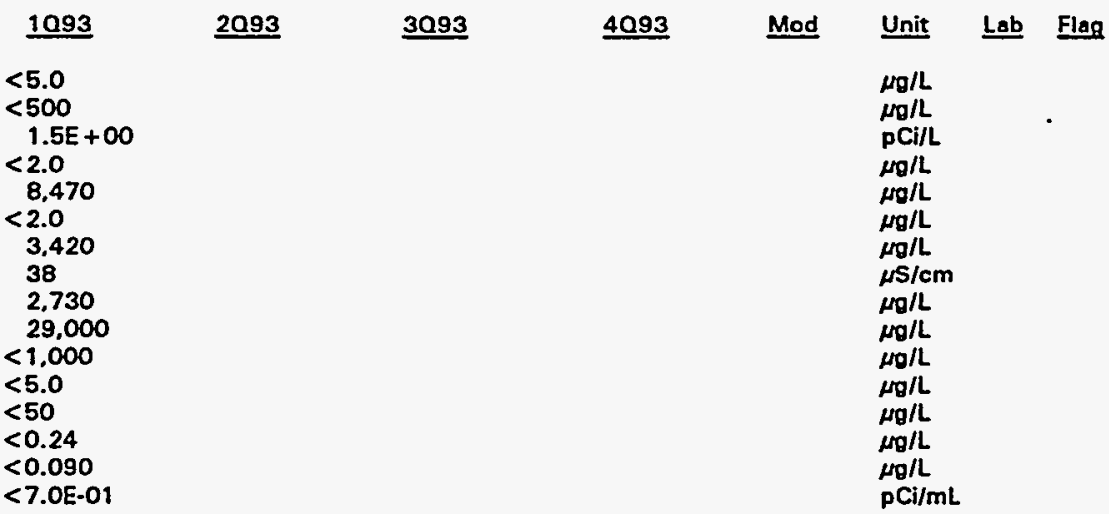

WELL RCP 1A

\begin{tabular}{|c|c|c|c|c|c|c|c|c|c|}
\hline SRS Coord. & Lat/Longitude & Screen Zone Elevation & Top of Casing & Casing & Pump & \multicolumn{4}{|c|}{ Screen Zone } \\
\hline $\begin{array}{l}\text { N56968.1 } \\
\text { E74238.3 }\end{array}$ & $\begin{array}{l}33.272872{ }^{\circ} \mathrm{N} \\
81.581985{ }^{\circ} \mathrm{W}\end{array}$ & $56.8-46.8 \mathrm{ft} \mathrm{msl}$ & $296.9 \mathrm{ft} \mathrm{msl}$ & 4" PVC & $\mathbf{S}$ & \multicolumn{3}{|c|}{ Congaree } & \\
\hline \multicolumn{2}{|c|}{ SAMPLE DATE } & $03 / 19 / 93$ & & $09 / 13 / 93$ & $12 / 27 / 93$ & & & & \\
\hline \multicolumn{10}{|c|}{ FIELD DATA } \\
\hline \multicolumn{2}{|r|}{ Analyte } & 1093 & $\underline{2093}$ & $\underline{3093}$ & 4093 & \multicolumn{4}{|l|}{ Unit } \\
\hline & $\begin{array}{l}\text { Water elovation } \\
\text { Depth to water } \\
\text { pH } \\
\text { Sp. conductance } \\
\text { Water tomperature } \\
\text { Alkalinity as } \mathrm{CaCO}_{3} \\
\text { Turbidity } \\
\text { Volume purged } \\
\text { Sampling code }\end{array}$ & $\begin{array}{l}195.0 \\
101.9\end{array}$ & & $\begin{array}{l}194.0 \\
102.9\end{array}$ & $\begin{array}{l}193.6 \\
103.3 \\
11.6 \\
818 \\
19.7 \\
198 \\
1 \\
2.5\end{array}$ & \multicolumn{3}{|c|}{$\begin{array}{l}\text { ft msl } \\
\text { ft } \\
\text { pH } \\
\text { is/cm } \\
{ }^{\circ} \mathrm{C} \\
\mathrm{mg} / \mathrm{l} \\
\text { NTU } \\
\text { Woll vol. }\end{array}$} & \\
\hline \multicolumn{10}{|c|}{ ANALYTICAL DATA } \\
\hline \multirow[t]{2}{*}{$\underline{H} \quad \underline{D}$} & Analyte & 1093 & $\underline{2093}$ & 3093 & 4093 & Mod & Unit & $\underline{\text { Lab }}$ & Flag \\
\hline & $\begin{array}{l}\text { Actinium-228 } \\
\text { Antimony-125 } \\
\text { Arsenic, total recoverable } \\
\text { Barium, total rocoverable } \\
\text { Cadmium, total recoverable } \\
\text { Calcium, total recoverable } \\
\text { Corium-144 } \\
\text { Cosium-134 } \\
\text { Cosium-137 } \\
\text { Chloride } \\
\text { Chromium, total recoverable } \\
\text { Cobalt-57 } \\
\text { Cobalt-60 } \\
\text { Copper, total rocoverable } \\
\text { Europium-152 } \\
\text { Europium-154 } \\
\text { Europium-155 } \\
\text { Fluoride } \\
\text { Gross alpha }\end{array}$ & & & & $\begin{aligned}<8.4 E+01 \\
<2.5 E+01 \\
<2.0 \\
173 \\
<2.0 \\
\quad 66.200 \\
<5.0 E+01 \\
<1.0 E+01 \\
<1.0 E+01 \\
\quad 1.820 \\
<4.0 \\
<1.0 E+01 \\
<1.2 E+01 \\
<4.0 \\
<5.0 E+01 \\
<2.5 E+01 \\
<2.5 E+01 \\
<266 \\
<2.0 E+00\end{aligned}$ & $\begin{array}{l}\text { Y3 } \\
\text { Y3 } \\
\text { Y3 } \\
\text { Y3 } \\
\text { Y3 }\end{array}$ & $\begin{array}{l}\mathrm{pCi} / \mathrm{L} \\
\mathrm{pCi} / \mathrm{L} \\
\mu \mathrm{g} / \mathrm{L} \\
\mu g / \mathrm{L} \\
\mu \mathrm{g} / \mathrm{L} \\
\mu \mathrm{g} / \mathrm{L} \\
\mathrm{pCi} / \mathrm{L} \\
\mathrm{pCi} / \mathrm{L} \\
\mathrm{pCi} / \mathrm{L} \\
\mu g / \mathrm{L} \\
\mu \mathrm{g} / \mathrm{L} \\
\mathrm{pCi} / \mathrm{L} \\
\mathrm{pCi} / \mathrm{L} \\
\mu g / L \\
\mathrm{pCi} / \mathrm{L} \\
\mathrm{pCi} / \mathrm{L} \\
\mathrm{pCi} / \mathrm{L} \\
\mu g / L \\
\mathrm{pCi} / \mathrm{L}\end{array}$ & $\begin{array}{l}\text { CN } \\
\text { CN } \\
\text { GE } \\
\text { GE } \\
\text { GE } \\
\text { GE } \\
\text { CN } \\
\text { CN } \\
\text { CN } \\
\text { GE } \\
\text { GE } \\
\text { CN } \\
\text { CN } \\
\text { GE } \\
\text { CN } \\
\text { CN } \\
\text { CN } \\
\text { GE } \\
\text { GE }\end{array}$ & $\begin{array}{l}0 \\
0 \\
0 \\
0 \\
0 \\
0 \\
0 \\
0 \\
0 \\
0 \\
0 \\
0 \\
0 \\
0 \\
0 \\
0 \\
0 \\
0 \\
0\end{array}$ \\
\hline
\end{tabular}

Note: Flagging levels, modifiers, and laboratories are for 4 th querter 1993 data only. See Appendix $B$ for flagging criteria.

- =xceeded holding time for 4 th quarter 1993.

- =xceeded screening level or final primary drinking water standard for 4th quarter 1993. 
Well RCP 1A continued

ANALYTICAL DATA

H D Analyte

Iron, total recoverable

Load, total recoverable

Load-212

Magnesium, total recoverable

Manganese, total recoverable

Manganoso-54

Mercury, total recoverable

Nickel, total recoverable

Nitrato-nitrite as nitrogen

Nonvolatile beta

oH

Plutonium-238

Plutonium-239/240

Potassium, total recoverable

Potassium-40

Promothium-144

Promethium-146

Radium, total alpha-emitting

Ruthenium-106

Selenium, total recoverable

Silver, total recoverable

Sodium, total recoverable

Sodium-22

Specific conductance

Strontium-90

Sulfate

Thorium-234

Total activity

Total dissolved solids

Total organic carbon

Total phosphates (as P)

Total suspended solids

Tritium

Yttrium-88

Zinc, total recoverable

Zinc-65

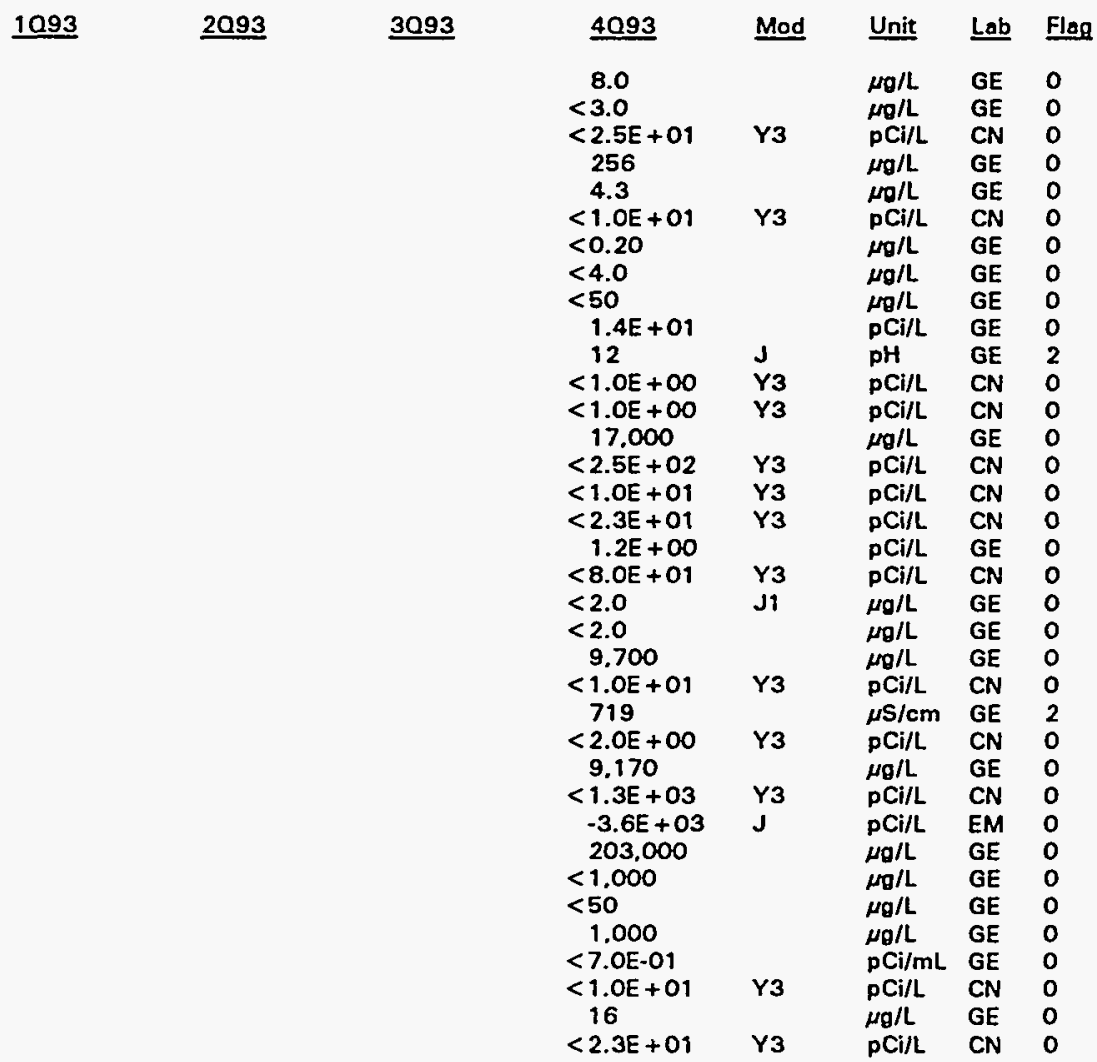

Note: Flagging levels, modifiers, and laboratories are for 4th quarter 1993 data only. See Appendix B for flagging criteria.

- = exceeded holding time for 4th quarter 1993.

- =xceeded screening level or final primary drinking water standard for 4th quarter 1993. 
WELL RCP 1D

\begin{tabular}{|c|c|}
\hline SRS Coord. & Lat/Longitude \\
\hline $\begin{array}{l}\text { N56967.9 } \\
\text { E74223.5 }\end{array}$ & $\begin{array}{l}33.272847 \text { oN } \\
81.582024 \text { ow }\end{array}$ \\
\hline
\end{tabular}

SAMPLE DATE

FIELD DATA

\section{Scroen Zone Elavation \\ 281.3-261.3 ft msl}

$03 / 19 / 93$

\section{Top of Casing}

$296.8 \mathrm{ft} \mathrm{msl}$

$06 / 10 / 93$ $\begin{array}{ll}\text { Casing } & \text { Pump } \\ 4^{*} \text { PVC } & \text { S }\end{array}$

$12 / 27 / 93$
Andyte

Wator olovation

Depth to water

$\mathrm{pH}$

Sp. conductance

Water temperature

Alkalinity as $\mathrm{CaCO}_{3}$

Turbidity

Volume purged

Sampling code

$\begin{array}{lll}1093 & & \underline{2093} \\ 284.8 & & 284.2 \\ 12.0 & & 12.6 \\ 5.6 & & 5.3 \\ 281 & & 210 \\ 18.4 & 23.3 \\ 7 & & 8 \\ 82 & & 228 \\ 0.8 & 1.1 \\ x & x\end{array}$

$\mathbf{3 0 9 3}$
281.4
15.4
5.4
103
23.4
8
113
1.1
$x$

ANALYTICAL DATA

H D

Analyte

Actinium-228

Aluminum, total recoverable

Antimony-125

Arsenic, total recoverable

Barium, total recoverable

Cadmium, total recoverable

Calcium, total recoverable

Corium-144

Cosium-134

Cosium-137

Chlorido

Chromium, total recoverable

Cobalt-57

Cobalt 60

Copper, total recoverable

2,4-Dichlorophenoxyacetic acid

Endrin

Europium-152

Europium-154

Europium-155

Fluorido

Gross alpho

Iron, total recoverable

Lead, total recoverable

Load-212

Lindane

Magnesium, total recoverable

Manganeso, total recoverable

Manganese-54

Morcury, total recoverable

Mothoxychlor

Nickel, total recoverable

Nitrate-nitrite as nitrogen

Nonvolatile bota

pH

Phenols

Plutonium-238

Plutonium-239/240

Potassium, total recovorable

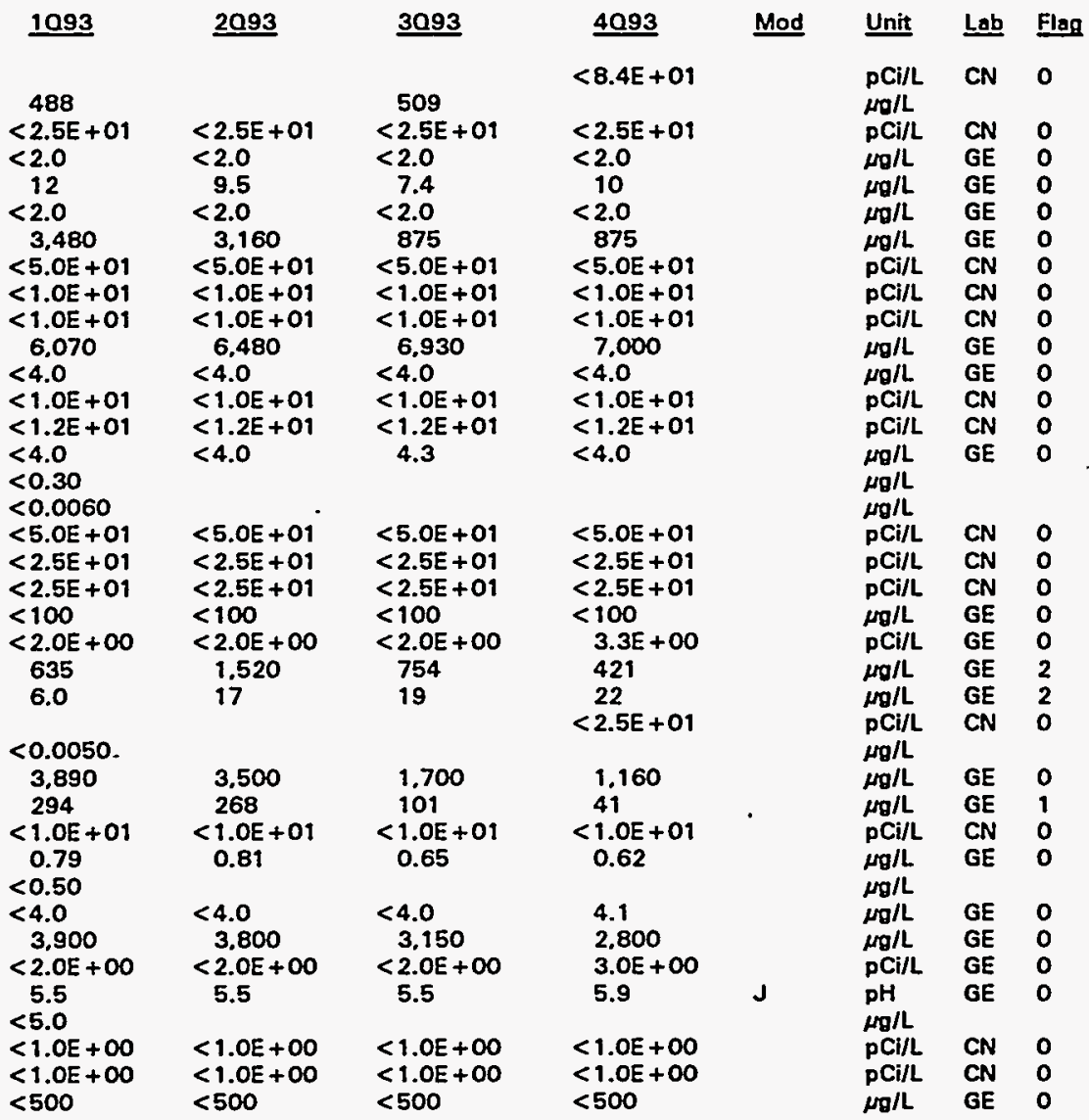

Note: Flagging levels, modifiers, and laboratories are for 4th quarter 1993 data only. See Appendix B for flagging criteria.

- = exceeded holding time for 4th quarter 1993.

- = exceeded screening level or final primary drinking water standard for 4th quarter 1993. 


$\begin{array}{ll}\text { H. } & \text { Analyte } \\ \text { Potassium-40 } \\ \text { Promethium-144 } \\ \text { Promethium-146 } \\ \text { Radium, total alpha-omitting } \\ \text { Ruthenium-106 } \\ \text { Solenium, total recoverable } \\ \text { Silica, total recoverable } \\ \text { Silver, total rocovorable } \\ \text { Sodium, total recoverable } \\ \text { Sodium-22 } \\ \text { Specific conductance } \\ \text { Strontium-90 } \\ \text { Sulfate } \\ \text { Thorium-234 } \\ \text { Total dissolvod solids } \\ \text { Total organic carbon } \\ \text { Total organic halogens } \\ \text { Total phosphates (as P) } \\ \text { Total susponded solids } \\ \text { Toxaphene } \\ \text { 2,4.5-TP (Silvex) } \\ \text { Tritium } \\ \text { Yttrium-88 } \\ \text { Zinc, total recoverable } \\ \text { Zinc-65 }\end{array}$

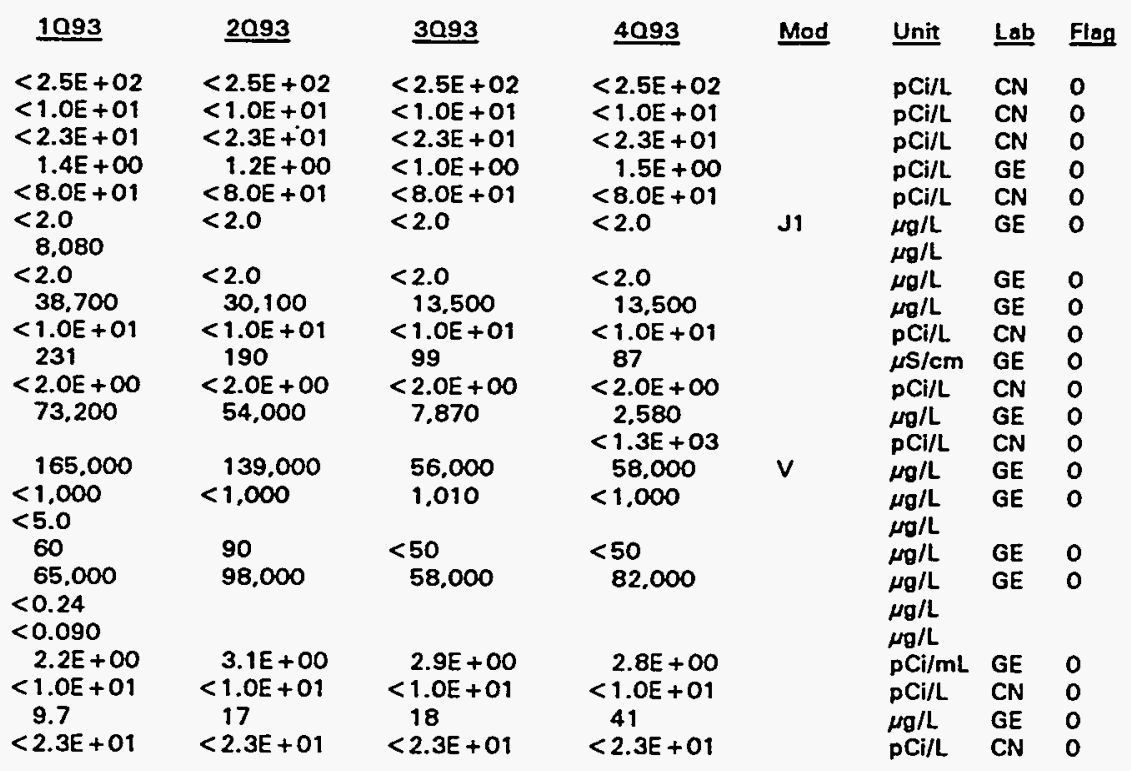

\section{WELL RDB 1D}

\begin{tabular}{|c|c|}
\hline SRS Coord. & Lat/Longitude \\
\hline $\begin{array}{l}N 57097.3 \\
\text { E74844.5 }\end{array}$ & $\begin{array}{l}33.274146 \circ \mathrm{o} \\
81.5806399^{\circ} \mathrm{W}\end{array}$ \\
\hline
\end{tabular}

FIELD DATA

\section{Screen Zone Elevation \\ 285.5-265.5 ft msl}

$03 / 19 / 93$

\section{$06 / 10 / 93$}

Top of Casin

$292.4 \mathrm{ft} \mathrm{msl}$

$\begin{array}{ll}1093 & \underline{2093} \\ 287.6 & 285.0 \\ 4.8 & 7.4 \\ 7.3 & 6.2 \\ 165 & 121 \\ 15.1 & 22.7 \\ 51 & 44 \\ 72 & 311 \\ 1.2 & 1.1 \\ x & x\end{array}$

$\mathbf{3 0 9 3}$
286.0
6.4
6.4
115
23.7
29
51
1.0
$x$

Cosing
4" PVC
(9)

ANALYTICAL DATA

A D $\quad$ Analyte
Actinium-228
Aluminum, total recoverable
Antimony-125
Arsenic, total recoverable
Barium, total rocoverable
Cadmium, total rocoverable
Calcium, total recoverable
Corium-144
Cosium-134
Cosium-137

\begin{tabular}{|c|c|c|c|c|c|c|c|}
\hline 1093 & 2093 & $\underline{3093}$ & 4093 & Mod & $\underline{\text { Unit }}$ & Lab & Flag \\
\hline 563 & & 335 & $<8.4 E+01$ & & $\begin{array}{l}\mathrm{pCi} / \mathrm{L} \\
\mu \mathrm{g} / \mathrm{L}\end{array}$ & CN & 0 \\
\hline$<2.5 E+01$ & $<2.5 E+01$ & $<2.5 E+01$ & $<2.5 E+01$ & & pCi/L & $\mathrm{CN}$ & 0 \\
\hline$<2.0$ & $<2.0$ & $<2.0$ & $<2.0$ & & $\mu \mathrm{g} / \mathrm{L}$ & GE & 0 \\
\hline 19 & 23 & 19 & 22 & & $\mu \mathrm{g} / \mathrm{L}$ & $\mathrm{GE}$ & 0 \\
\hline$<2.0$ & $<2.0$ & $<2.0$ & $<2.0$ & & $\mu g / L$ & $G E$ & 0 \\
\hline $\begin{array}{c}19,300 \\
<5.0 E+01\end{array}$ & $\begin{array}{c}19.800 \\
<5.0 E+01\end{array}$ & $\begin{aligned} & 13,500 \\
&<5,0 F+01\end{aligned}$ & $\begin{array}{c}18,900 \\
<5.0 F+01\end{array}$ & & $\mu \mathrm{g} / \mathrm{L}$ & GE & 0 \\
\hline$<1.0 E+01$ & $<1.0 E+01$ & $<1.0 E+01$ & $<1.0 E+01$ & & pCi/L & $\mathrm{CN}$ & 0 \\
\hline$<1.0 E+01$ & $<1.0 E+01$ & $<1.0 E+01$ & $<1.0 E+01$ & & $\mathrm{pCi} / \mathrm{L}$ & $\mathrm{CN}$ & 0 \\
\hline
\end{tabular}

Note: Flagging levels, modifiers, and laboratories are for 4th quarter 1993 data only. See Appendix B for flagging criteria. - = exceeded holding time for 4th quarter 1993.

- = exceeded screening level or final primary drinking water standard for 4th quarter 1993. 


\section{ANALYTICAL DATA}

H D Analyte

Chloride

Chromium, total recoverable

Cobalt-57

Cobalt-60

Copper, total recoverable

2,4-Dichlorophenoxyacetic acid

Endrin

Europium-152

Europium-154

Europium-155

Fluoride

Gross alpha

Iron, total recoverablo

Lead, total recoverable

Load-21 2

Lind ano

Magnesium, total recoverable

Manganeso, total recoverable

Manganese-54

Mercury, total recoverable

Methoxychlor

Nickel, total recovorable

Nitrate-nitrito as nitrogen

Nonvolatilo bota

Phenols.

Plutonium-238

Piutonium-239/240

Potassium, total recoverable

Potassium-40

Promethium-144

Promethium-146

Radium, total alpha-omitting

Ruthenium-106

Selonium, total recoverable

Silica, total rocoverable

Silver, total recoverable

Sodium, total recoverable

Sodium-22

Specific conductance

Strontium-90

Sulfato

Thorium-234

Total dissolved solids

Total organic carbon

Total organic halogens

Total phosphates (as P)

Totol suspended solids

Toxaphene

2,4,5-TP (Silvex)

Tritium

Yttrium-88

Zinc, total recoverable

Zinc-65

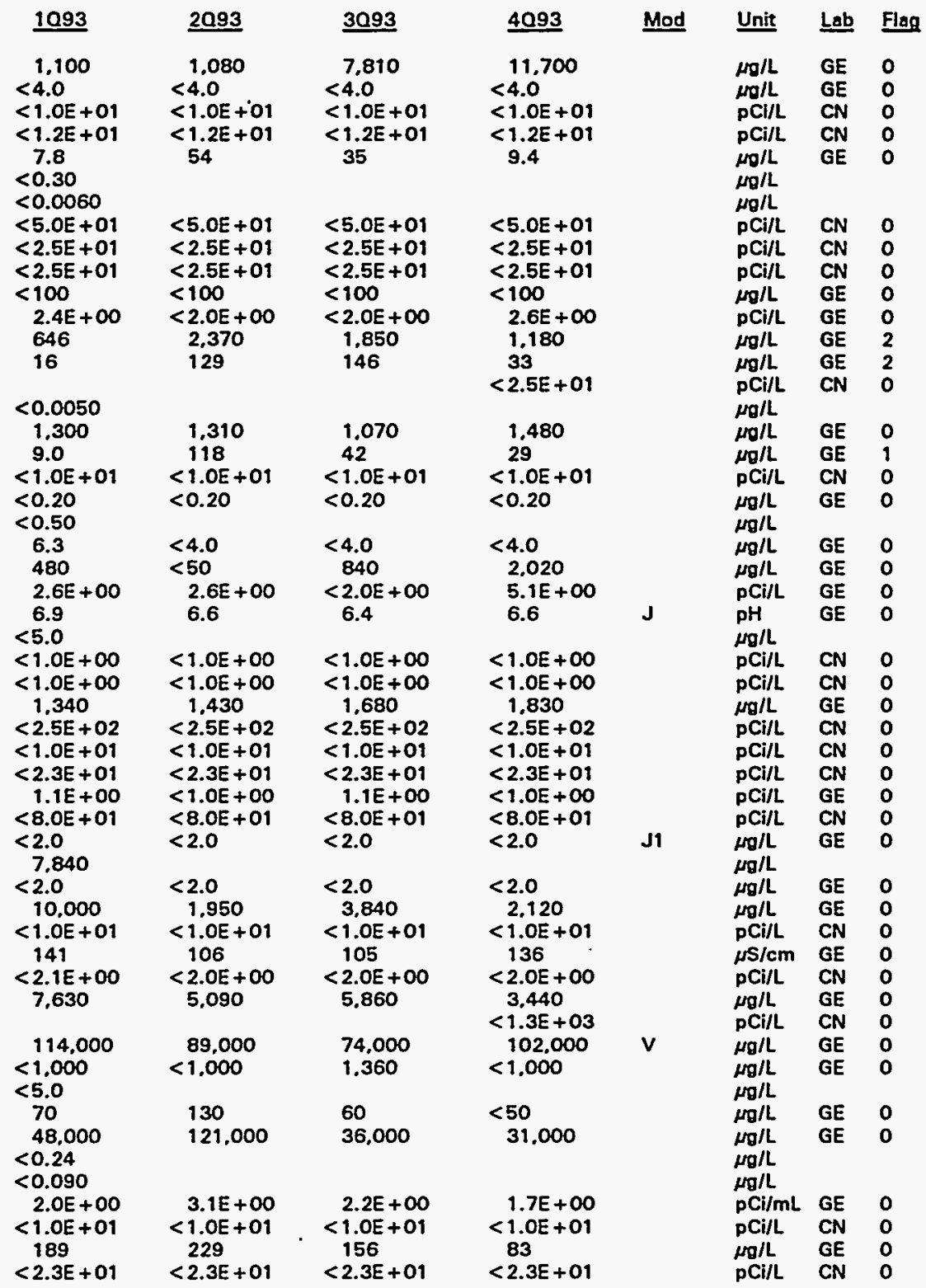

Note: Flagging levels, modifiers, and laboratories are for 4th quarter 1993 data only. See Appendix $B$ for flegging criteria.

- = exceeded holding time for 4th quarter 1993.

- = exceeded screening level or final primary drinking water standard for 4th quarter 1993. 


\section{WELL RDB 2D}

\begin{tabular}{|c|c|c|c|c|c|c|}
\hline SRS Coord. & Lat/Longitude & Screen Zone Elevation & Top of Casing & Casing & Pump & Screen Zone \\
\hline $\begin{array}{l}\text { N56879.8 } \\
\text { E74782.2 }\end{array}$ & $\begin{array}{l}33.273563 \text { o } \\
81.5803811^{\circ} \mathrm{W}\end{array}$ & 285.7-265.7 ft msl & $292.6 \mathrm{ft} \mathrm{msl}$ & 4" PVC & $\mathbf{s}$ & Water Table \\
\hline SAMPLE DA & & $03 / 19 / 93$ & $06 / 10 / 93$ & $09 / 09 / 93$ & $12 / 27 / 93$ & \\
\hline FIELD DATA & & & & & & \\
\hline & & 1093 & $\underline{2093}$ & $\underline{3093}$ & 4093 & Unit \\
\hline $\begin{array}{l}\text { u } \\
\text { D } \\
\text { p } \\
\text { S } \\
\text { u } \\
\text { A } \\
T \\
\text { V } \\
\text { S }\end{array}$ & $\begin{array}{l}\text { elovation } \\
\text { to water } \\
\text { onductance } \\
\text { temperature } \\
\text { nity as } \mathrm{CaCO} \text {, } \\
\text { lity } \\
\text { le purged } \\
\text { ling codo }\end{array}$ & $\begin{array}{l}286.5 \\
6.1 \\
6.9 \\
308 \\
16.0 \\
110 \\
68 \\
1.8 \\
x\end{array}$ & $\begin{array}{l}284.0 \\
8.6 \\
6.5 \\
365 \\
21.4 \\
169 \\
104 \\
1.5 \\
x\end{array}$ & $\begin{array}{l}285.2 \\
7.4 \\
6.6 \\
351 \\
23.7 \\
182 \\
249 \\
1.0 \\
\times\end{array}$ & $\begin{array}{l}284.9 \\
7.7 \\
6.7 \\
284 \\
18.8 \\
123 \\
71 \\
1.5 \\
x\end{array}$ & $\begin{array}{l}\text { ft msl } \\
\mathrm{ft} \\
\mathrm{pH} \\
\mu \mathrm{S} / \mathrm{cm} \\
{ }^{\circ} \mathrm{C} \\
\mathrm{mg} / \mathrm{L} \\
\mathrm{NTU} \\
\text { Woll vol. }\end{array}$ \\
\hline
\end{tabular}

\section{ANALYTICAL DATA}

H D Analyte

Actinium-228

Aluminum, total recoverable Antimony-125

Arsonic, total recoverable

Barium, total recoverable

Cadmium, total recoverable

Calcium, total recoverable

Cerium-144

Cosium-134

Cosium-137

Chloride

Chromium, total recoverable

Cobalt-57

Cobalt-60

Copper, total recoverable

2.4-Dichlorophenoxyacetic acid

Endrin

Europium-152

Europium-154

Europium-155

Fluorido

Gross alpha

Iron, tatal recoverable

Lead, total rocoverable

Lead-21 2

Lindane

Magnesium, total recoverable

Manganese, total recoverable

Manganese-54

Mercury, total recoverable

Methoxychlor

Nickal, total recoverable

Nitrate-nitrite as nitrogen

Nonvolatile bets

- $\quad \mathrm{pH}$

Phenols

Plutonium-238

Plutonium-239/240

Potassium, total recoverable $\underline{1093}$

168

$<2.5 \mathrm{E}+01$

$<2.0$

33

$<2.0$

46.500

$<5.0 E+01$

$<1.0 E+01$

$<1.0 E+01$

987

$<4.0$

$<1.0 E+01$

$<1.2 E+01$

$<4.0$

$<0.30$

$<0.0060$

$<5.0 E+01$

$<2.5 E+01$

$<2.5 E+01$

$<100$

$<2.0 E+\infty O$

12,600

$<3.0$

$<0.0050$

4.790

133

1.7E+01

$<0.20$

$<0.50$

$<4.0$

$<50$

$6.1 E+00$

6.7

$<5.0$

$<1.0 E+00$

$<1 . \mathrm{OE}+\mathrm{OO}$

1.460
2093

$\underline{3093}$

533

$<2.5 E+01$

$<2.0$

44

$<2.0$

60,700

$<5.0 E+01$

$<1.0 E+01$

$<1.0 E+01$

1,120

$<4.0$

$<1.0 E+01$

$<1.2 E+01$

9.3

$<1.2 \mathrm{E}+01$

$<4.0$

$<5.0 E+01$

$<2.5 E+01$

$<2.5 E+01$

$<100$

$2.7 E+\infty 0$

13,800

$<3.0$

$<5 . O E+01$

$<2.5 E+01$

$<2.5 E+01$

$<100$

$<2.0 E+00$

15,800

$<3.0$

\section{7,020}

$<1.0 E+01$

$<0.20$

$<4.0$

$<50$

$7.3 \mathrm{E}+00$

6.8

$<1.0 E+\infty 0$

$<1.0 E+\infty 0$

1,520
6.280

138

$<1.0 E+01$

$<0.20$

$<4.0$

$<50$

8.4E +00

6.6

$<1.0 E+\infty 0$

$<1.0 E+00$

1.890

\section{3 \\ $<8.4 E+01$}

$<2.5 E+01$

$<2.0$

31

$<2.0$

40,000

$<5.0 E+01$

$<1.0 E+01$

$<1.0 E+01$

1.380

$<4.0$

$<1.0 \mathrm{E}+01$

$<1.2 \mathrm{E}+01$

$<4.0$

$<5.0 E+01$

$<2.5 E+01$

$<2.5 \mathrm{E}+01$

$<100$

2. $2 \mathrm{E}+00$

12.300

$<3.0$

$<2.5 E+01$

4,060

126

$<1.0 E+01$

$<0.20$

$<4.0$

$<50$

$3.9 \mathrm{E}+00$

6.7

$<1.0 E+00$

$<1.0 E+00$

1.540
Mod

Unit Lab Flag

pCi/L CN 0

$\mu g / L$

$\mathrm{PCi} / \mathrm{L} \quad \mathrm{CN} \quad 0$

$\mu g / L$ GE $O$

$\mu Q / L \quad G E$

$\begin{array}{lll}\mu \mathrm{g} / \mathrm{L} & \mathrm{GE} & 0 \\ \mu \mathrm{g} / \mathrm{L} & \mathrm{GE} & 0\end{array}$

PCi/L CN $\quad$ C

$\mathrm{PCi} / \mathrm{L} \quad \mathrm{CN} \quad \mathrm{O}$

$\mathrm{PCi} / \mathrm{L} \quad \mathrm{CN} O$

$\mu \mathrm{g} / \mathrm{L}$ GE $\mathrm{O}$

$\mu g / L$ GE $O$

PCi/L CN 0

$\mathrm{PCI} / \mathrm{L}$ CN 0

$\mu \mathrm{g} / \mathrm{L}$ GE $O$

$\mu g / L$

$\mu g / L$

pCi/L CN 0

pCi/L CN 0

$\mathrm{pCi} / \mathrm{L} \quad \mathrm{CN} \quad 0$

$\mu \mathrm{g} / \mathrm{L}$ GE 0

$\mathrm{PCi} / \mathrm{L}$ GE 0

$\mu g / L$ GE 2

$\mu g / L \quad G E \quad 0$

$\mathrm{PCi} / \mathrm{L} \quad \mathrm{CN} \quad 0$

$\mu \mathrm{g} / \mathrm{L}$

$\mu g / L \quad$ GE 0

$\mu \mathrm{g} / \mathrm{L}$ GE

PCi/L CN

$\mu \mathrm{g} / \mathrm{L}$ GE $\mathrm{O}$

哟

$\mu \mathrm{g} / \mathrm{GE}$

$\mu \mathrm{g} / \mathrm{L}$ GE 0

PCi/l GE 0

$\mathrm{pH}$ GE

$\mu \mathrm{g} / \mathrm{L}$

PCill CN 0

$\mathrm{PCi} / \mathrm{L}$ CN 0

$\mu \mathrm{g} / \mathrm{L}$ GE 0

Note: Flagging levels, modifiers, and laboratories are for 4th quarter 1993 data only. See Appendix B for flagging criteria. - = exceeded holding time for 4th quarter 1993.

- = exceeded screening level or final primary drinking water stendard for 4th quarter 1993. 


$\begin{array}{ll}\text { D. } & \text { Analute } \\ \text { Potassium-40 } \\ \text { Promothium-144 } \\ \text { Promethium-146 } \\ \text { Radium, total alpha-omitting } \\ \text { Ruthenium-106 } \\ \text { Solenium, total recoverable } \\ \text { Silica, total recoverable } \\ \text { Silver, total recoverable } \\ \text { Sodium, total recoverable } \\ \text { Sodium-22 } \\ \text { Specific canductance } \\ \text { Strontium-90 } \\ \text { Sulfato } \\ \text { Thorium-234 } \\ \text { Total dissolved solids } \\ \text { Total organic carbon } \\ \text { Total organic halogens } \\ \text { Total phosphates (as P) } \\ \text { Total suspended solids } \\ \text { Toxaphone } \\ \text { 2,4,5-TP (Silvex) } \\ \text { Tritium } \\ \text { Yttrium-88 } \\ \text { Zinc, total recovorable } \\ \text { Zinc-65 }\end{array}$

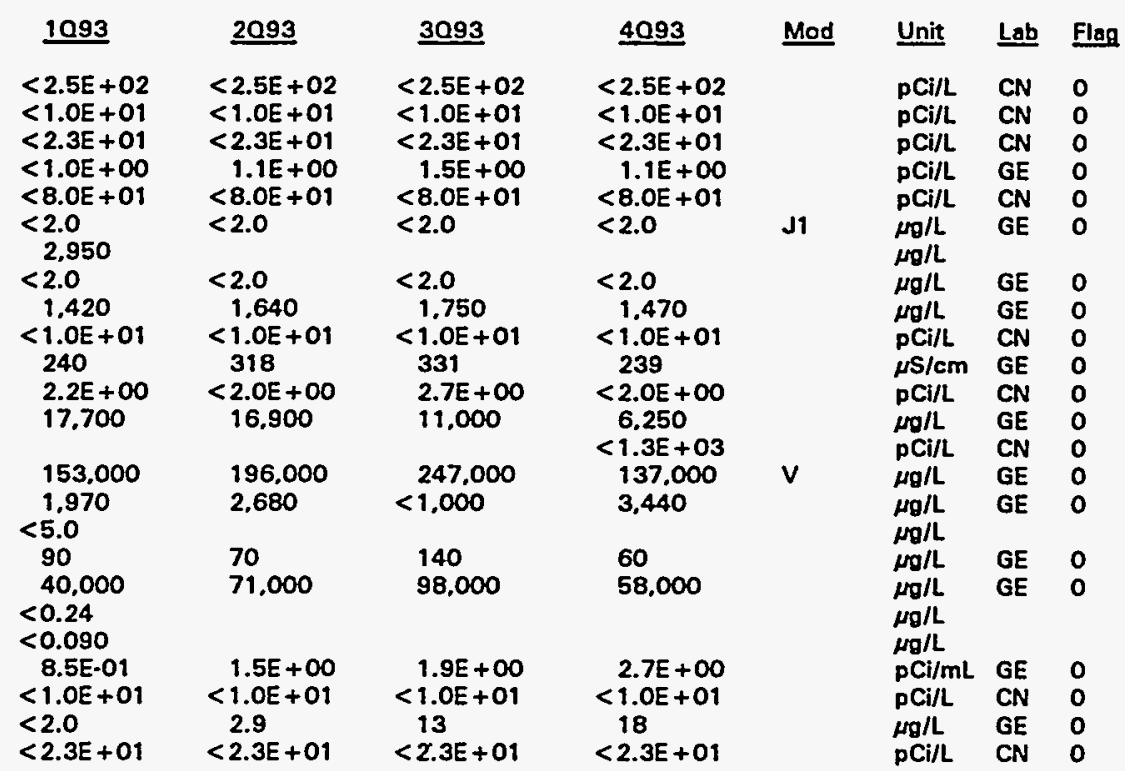

\section{WELL RDB 3D}

\begin{tabular}{|c|c|c|c|c|c|c|c|c|c|}
\hline \multirow{2}{*}{$\begin{array}{l}\text { SRS Coord. } \\
\text { N56881.9 } \\
\text { E74899,0 }\end{array}$} & Lat/Longitude & Screen Zone Elevation & Top of Casing & Casing & Pump & \multicolumn{4}{|c|}{ Screen Zone } \\
\hline & $\begin{array}{l}33.273758{ }^{\circ} \mathrm{N} \\
81.580077 \circ \mathrm{W}\end{array}$ & $285.8-265.8 \mathrm{ft} \mathrm{msl}$ & $292.7 \mathrm{ft} \mathrm{msl}$ & 4" PVC & $\mathbf{s}$ & \multicolumn{4}{|c|}{ Water Table } \\
\hline \multicolumn{2}{|c|}{ SAMPLE DATE } & $03 / 19 / 93$ & $06 / 10 / 93$ & $09 / 09 / 93$ & $12 / 27 / 93$ & & & & \\
\hline \multicolumn{10}{|c|}{ FIELD DATA } \\
\hline \multicolumn{2}{|r|}{ Analyto } & 1093 & $\underline{2093}$ & 3093 & 4093 & \multicolumn{4}{|l|}{ Unit } \\
\hline & $\begin{array}{l}\text { Water elovation } \\
\text { Depth to water } \\
\text { pH } \\
\text { Sp. conductance } \\
\text { Water temperature } \\
\text { Alkalinity as CaCO, } \\
\text { Turbidity } \\
\text { Volume purged } \\
\text { Sampling code }\end{array}$ & $\begin{array}{l}284.6 \\
8.1 \\
6.5 \\
97 \\
15.4 \\
12 \\
40 \\
1.5 \\
X\end{array}$ & $\begin{array}{l}282.5 \\
10.2 \\
5.9 \\
111 \\
21.7 \\
42 \\
103 \\
1.6 \\
\times\end{array}$ & $\begin{array}{l}282.3 \\
10.4 \\
6.2 \\
135 \\
21.0 \\
66 \\
269 \\
1.4 \\
X\end{array}$ & $\begin{array}{l}281.3 \\
11.4 \\
6.5 \\
206 \\
18.1 \\
77 \\
74 \\
1.4 \\
X\end{array}$ & \multicolumn{3}{|l|}{$\begin{array}{l}\text { ft msl } \\
\mathrm{ft} \\
\mathrm{pH} \\
\mu \mathrm{s} / \mathrm{cm} \\
{ }^{\circ} \mathrm{C} \\
\mathrm{mg} / \mathrm{L} \\
\text { NTU } \\
\text { Well vol. }\end{array}$} & \\
\hline \multicolumn{10}{|c|}{ ANALYTICAL DATA } \\
\hline \multirow[t]{2}{*}{$\underline{H}$ D } & Analyte & 1093 & $\underline{2093}$ & 3093 & 4093 & Mad & Unit & $\underline{\text { Lab }}$ & Flag \\
\hline & $\begin{array}{l}\text { Actinium-228 } \\
\text { Aluminum, total rocoverable } \\
\text { Antimony-125 } \\
\text { Arsenic, total recovorable } \\
\text { Barium, total recoverable } \\
\text { Cadmium, total recoverable } \\
\text { Calcium, total recoverable } \\
\text { Corium-144 } \\
\text { Cosium-134 } \\
\text { Cosium-137 }\end{array}$ & $\begin{aligned} & 109 \\
&< 2.5 E+01 \\
&< 2.0 \\
& 16 \\
&< 2.0 \\
& 10.100 \\
&< 5.0 E+01 \\
&<1.0 E+01 \\
&<1.0 E+01\end{aligned}$ & $\begin{aligned}< & 2.5 E+01 \\
< & 2.0 \\
& 26 \\
< & 2.0 \\
& 12.400 \\
< & 5.0 E+01 \\
< & 1.0 E+01 \\
< & 1.0 E+01\end{aligned}$ & $\begin{aligned} & 413 \\
&< 2.5 E+01 \\
&< 2.0 \\
& 29 \\
&< 2.0 \\
& 12,200 \\
&< 5.0 E+01 \\
&< 1.0 E+01 \\
&<1.0 E+01\end{aligned}$ & $\begin{aligned}<8.4 E+01 \\
<2.5 E+01 \\
<2.0 \\
29 \\
<2.0 \\
14.400 \\
<5.0 E+01 \\
<1.0 E+01 \\
<1.0 E+01\end{aligned}$ & & $\begin{array}{l}\mathrm{pCi} / \mathrm{L} \\
\mu \mathrm{g} / \mathrm{L} \\
\mathrm{pCi} / \mathrm{L} \\
\mu \mathrm{g} / \mathrm{L} \\
\mu \mathrm{g} / \mathrm{L} \\
\mu \mathrm{g} / \mathrm{L} \\
\mu \mathrm{g} / \mathrm{L} \\
\mathrm{pCi} / \mathrm{L} \\
\mathrm{pCi} / \mathrm{L} \\
\mathrm{pCi} / \mathrm{L}\end{array}$ & $\begin{array}{l}\text { CN } \\
\text { CN } \\
\text { GE } \\
\text { GE } \\
\text { GE } \\
\text { GE } \\
\text { CN } \\
\text { CN } \\
\text { CN }\end{array}$ & $\begin{array}{l}0 \\
0 \\
0 \\
0 \\
0 \\
0 \\
0 \\
0 \\
0\end{array}$ \\
\hline
\end{tabular}

Note: Flagging levels, modifiers, and laboratories are for 4th quarter 1993 data only. See Appendix $B$ for flagging criteria. - = exceeded holding time for 4th querter 1993.

= exceeded screening level or final primary drinking water standard for 4th quarter 1993. 


\begin{tabular}{|c|c|c|c|c|c|c|c|}
\hline 1093 & 2093 & 3093 & 4093 & Mod & Unit & Lab & Flas \\
\hline 705 & 1,070 & 924 & 1.070 & & $\mu \sigma / L$ & GE & 0 \\
\hline$<4.0$ & $<4.0$ & $<4.0$ & $<4.0$ & & $\mu g / L$ & GE & 0 \\
\hline$<1.0 E+01$ & $<1.0 E+01$ & $<1.0 E+01$ & $<1.0 E+01$ & & $\mathrm{pCi} / \mathrm{L}$ & $\mathrm{CN}$ & 0 \\
\hline$<1.2 E+01$ & $<1.2 \mathrm{E}+01$ & $<1.2 E+01$ & $<1.2 \mathrm{E}+01$ & & $\mathrm{PCI} / \mathrm{L}$ & $\mathrm{CN}$ & 0 \\
\hline $\begin{array}{c}32 \\
<0.30\end{array}$ & & 57 & 9.8 & & $\underset{\mu g / L}{\mu g / L}$ & GE & 0 \\
\hline$<0.0060$ & & & & & $\mu g / L$ & & \\
\hline$<5.0 E+01$ & $<5.0 E+01$ & $<5.0 E+01$ & $<5.0 E+01$ & & $\mathrm{pCi} / \mathrm{L}$ & $\mathrm{CN}$ & 0 \\
\hline$<2.5 E+01$ & $<2.5 E+01$ & $<2.5 E+01$ & $<2.5 E+01$ & & $\mathrm{pCi} / \mathrm{L}$ & $\mathrm{CN}$ & 0 \\
\hline$<2.5 E+01$ & $<2.5 \mathrm{E}+01$ & $<2.5 E+01$ & $<2.5 E+01$ & & $\mathrm{pCi} / \mathrm{L}$ & $\mathrm{CN}$ & 0 \\
\hline$<100$ & $<100$ & $<100$ & $<100$ & & $\mu g / L$ & GE & 0 \\
\hline$<2.0 E+00$ & $3.9 E+\infty 0$ & $<2.0 E+\infty O$ & $<2.0 E+\infty 0$ & & $\mathrm{pCi} / \mathrm{L}$ & GE & 0 \\
\hline $\begin{array}{l}1.050 \\
8.6\end{array}$ & $\begin{array}{l}8.890 \\
9.1\end{array}$ & $\begin{array}{l}18.600 \\
31\end{array}$ & $\begin{aligned} & 22.700 \\
& 8.4 \\
< & 2.5 E+01\end{aligned}$ & & $\begin{array}{l}\mu \mathrm{g} / \mathrm{L} \\
\mu \mathrm{g} / \mathrm{L} \\
\mathrm{pCCi/L}\end{array}$ & $\begin{array}{l}\mathrm{GE} \\
\mathrm{GE} \\
\mathrm{CN}\end{array}$ & $\begin{array}{l}2 \\
1 \\
0\end{array}$ \\
\hline$<0.0050$ & & & & & $\mu \mathrm{g} / \mathrm{L}$ & & \\
\hline 1.160 & 1.500 & 1.380 & 1.430 & & $\mu_{\mathrm{g} / \mathrm{L}}$ & GE & 0 \\
\hline $\begin{array}{l}61 \\
<1.0 E+01\end{array}$ & $\begin{array}{l}383 \\
<10 E+01\end{array}$ & 355 & 360 & & $\mu g / L$ & GE & 2 \\
\hline$<0.20$ & $<0.20$ & $<0.20$ & $\begin{array}{l}<1.0 E+01 \\
<0.20\end{array}$ & & $\begin{array}{l}\mathrm{PCi} / \mathrm{L} \\
\mu \mathrm{g} / \mathrm{L}\end{array}$ & $\begin{array}{l}\mathrm{CN} \\
\mathrm{GE}\end{array}$ & $\begin{array}{l}0 \\
0\end{array}$ \\
\hline$<0.50$ & & & & & $\mu \mathrm{g} / \mathrm{L}$ & & \\
\hline$<4.0$ & $\begin{array}{c}<4.0 \\
70\end{array}$ & $<4.0$ & 4.1 & & $\mu \mathrm{g} / \mathrm{L}$ & GE & 0 \\
\hline $\begin{array}{l}770 \\
2.2 E+\infty 0\end{array}$ & $\begin{array}{l}70 \\
2.6 E+\infty 0\end{array}$ & $<50$ & $<50$ & & $\mu \mathrm{g} / \mathrm{L}$ & GE & o \\
\hline $\begin{array}{l}2.2 \mathrm{E}+00 \\
6.4\end{array}$ & $\begin{array}{l}2.6 E+\infty 0 \\
6.2\end{array}$ & $2.1 E+00$ & $3.3 E+\infty$ & & $\mathrm{pCi} / \mathrm{L}$ & GE & 0 \\
\hline$<5.4$ & & 6.2 & 6.5 & $J$ & $\begin{array}{l}\mathrm{pH} \\
\mu \mathrm{g} / \mathrm{L}\end{array}$ & GE & 0 \\
\hline$<1.0 E+\infty$ & $<1.0 E+\infty 0$ & $<1.0 E+\infty 0$ & $<1.0 E+\infty 0$ & & $\mathrm{pCi} / \mathrm{L}$ & CN & 0 \\
\hline$<1.0 E+00$ & $<1.0 E+O 0$ & $<1.0 E+00$ & $<1.0 E+\infty O$ & & $\mathrm{pCi} / \mathrm{L}$ & CN & 0 \\
\hline 1.610 & 1.310 & 1.500 & 1.630 & & $\mu \mathrm{g} / \mathrm{L}$ & GE & 0 \\
\hline$<2.5 \mathrm{E}+02$ & $<2.5 E+02$ & $<2.5 E+02$ & $<2.5 E+02$ & & $\mathrm{pCi} / \mathrm{L}$ & CN & 0 \\
\hline$<1.0 E+01$ & $<1.0 E+01$ & $<1.0 E+01$ & $<1.0 E+01$ & & $\mathrm{pCj} / \mathrm{L}$ & $\mathrm{CN}$ & 0 \\
\hline$<2.3 E+01$ & $<2.3 E+01$ & $<2.3 E+01$ & $<2.3 E+01$ & & $\mathrm{PCi} / \mathrm{L}$ & $\mathrm{CN}$ & 0 \\
\hline$<1.0 E+\infty 0$ & $1.3 E+\infty 0$ & $<1.0 E+\infty 0$ & $<1.0 E+\infty 0$ & & $\mathrm{pCi} / \mathrm{L}$ & $\mathrm{GE}$ & 0 \\
\hline$<8.0 E+01$ & $<8.0 E+01$ & $<8.0 E+01$ & $\angle 8.0 E+01$ & & $\mathrm{pCi} / \mathrm{L}$ & $\mathrm{CN}$ & 0 \\
\hline $\begin{array}{l}<2.0 \\
3,880\end{array}$ & $<2.0$ & $<2.0$ & $<2.0$ & J1 & $\begin{array}{l}\mu \mathrm{g} / \mathrm{L} \\
\mu \mathrm{g} / \mathrm{L}\end{array}$ & GE & 0 \\
\hline$<2.0$ & $<2.0$ & $<2.0$ & $<2.0$ & & $\mu g / L$ & GE & 0 \\
\hline 4,740 & 1,580 & 1.070 & 1.090 & & $\mu \mathrm{g} / \mathrm{L}$ & GE & 0 \\
\hline$<i_{77} .0 E+01$ & $\begin{array}{l}<1.0 E+01 \\
80\end{array}$ & $\begin{array}{c}<i . O E+01 \\
90\end{array}$ & $\begin{array}{l}<1.0 E+01 \\
118\end{array}$ & & $\mathrm{PCi} / \mathrm{L}$ & $\mathrm{CN}$ & $\begin{array}{l}0 \\
0\end{array}$ \\
\hline $\begin{array}{c}<2.0 E+00 \\
6,880\end{array}$ & $\begin{array}{c}<2.0 E+O 0 \\
5.760\end{array}$ & $\begin{array}{c}<2,0 E+\infty 0 \\
3,190\end{array}$ & $\begin{array}{c}<2.0 E+00 \\
1.380 \\
<1.3 E+03\end{array}$ & & $\begin{array}{l}\mathrm{pCi} / \mathrm{L} \\
\mu \mathrm{g} / \mathrm{L}\end{array}$ & $\begin{array}{l}\mathrm{CN} \\
\mathrm{GE} \\
\mathrm{CN}\end{array}$ & $\begin{array}{l}0 \\
0 \\
0\end{array}$ \\
\hline 56,000 & 52.000 & 51,000 & 66,000 & v & $\begin{array}{l}\text { PCi/L } \\
\mu_{\theta} / L\end{array}$ & $\begin{array}{l}C N \\
G E\end{array}$ & $\begin{array}{l}0 \\
0\end{array}$ \\
\hline $\begin{array}{l}<1.000 \\
<5.0\end{array}$ & $<1.000$ & 4,930 & 2,430 & & $\mu \mathrm{g} / \mathrm{L}$ & GE & 0 \\
\hline 50 & $<50$ & $<50$ & $<50$ & & $\mu \mathrm{g} / \mathrm{L}$ & GE & 0 \\
\hline $\begin{aligned} & 21.000 \\
&<0.24\end{aligned}$ & 57.000 & 93.000 & 50.000 & & $\mu \mathrm{g} / \mathrm{L}$ & GE & 0 \\
\hline $\begin{array}{l}<0.24 \\
<0.090\end{array}$ & & & & & $\mu \mathrm{g} / \mathrm{L}$ & & \\
\hline 9.4E-01 & $2.4 E+00$ & $3.2 \mathrm{E}+00$ & $3.5 E+00$ & & $\mathrm{pCi} / \mathrm{mL}$ & GE & 0 \\
\hline$<1.0 E+01$ & $<1.0 E+01$ & $<1.0 E+01$ & $<1.0 E+01$ & & pCi/L & $\mathrm{CN}$ & 0 \\
\hline 133 & 173 & 236 & 615 & & $\mu \mathrm{g} / \mathrm{L}$ & GE & 0 \\
\hline$<2.3 E+01$ & $<2.3 E+01$ & $<2.3 E+01$ & $<2.3 E+01$ & & $\mathrm{pCi} / \mathrm{L}$ & $C N$ & 0 \\
\hline
\end{tabular}

Note: Flagging levels, modifiers, and laboratories are for 4th quarter 1993 data only. See Appendix B for flagging criteria. - = exceeded holding time for 4th quarter 1993.

- = exceeded screening level or final primary drinking water standard for 4th quarter 1993. 
WELL RRP 1

\begin{tabular}{|c|c|c|c|c|c|c|}
\hline SRS Coord. & Lat/Longitude & Screen Zone Elevation & Top of Casing & Casing & Pump & Screen Zone \\
\hline $\begin{array}{l}\text { N54563.5 } \\
\text { E75634.6 }\end{array}$ & $\begin{array}{l}33.2698266^{\circ} \mathrm{N} \\
81.573641^{\circ} \mathrm{W}\end{array}$ & $272.4-242.4 \mathrm{ft} \mathrm{msl}$ & $284.4 \mathrm{ft} \mathrm{msl}$ & $4^{-P V C}$ & $\mathbf{s}$ & Water Table \\
\hline SAMPLE DATE & & $03 / 18 / 93$ & $05 / 30 / 93$ & $09 / 09 / 93$ & $12 / 16 / 93$ & \\
\hline
\end{tabular}

FIELD DATA

Analyte
Water elovation
Depth to water
pH
Sp. conductance
Water temperature
Alkalinity as CaCO,
Turbidity
Volume purged
Sampling codo

ANALYTICAL DATA

1093
271.6
12.8
4.9
23
18.3
0
2
6.9

$\underline{1093}$

$<20$

$<2.0$

19

$<1.0$

$<1.0$

$<1.0$

$<1.0$

$<2.0$

409

$<1.0$

1,670

$<1.0$

$<1.0$

$<1.0$

$<1.0$

$<1.0$

$<1.0$

$<4.0$

$<4.0$

$<1.0$

$<1.0$

$<1.0$

$\begin{array}{ll}1,1-\text { Dichloroothylene } & <1.0 \\ \text { trans-1,2-Dichloroothylene } & <1.0\end{array}$

$\begin{array}{ll}1,1-\text { Dichloroethylene } & <1.0 \\ \text { trans-1,2-Dichloroothylene } & <1.0\end{array}$

Dichloromethane (Methylene chloride) $<1.0$

2.4-Dichlorophenoxyacetic acid $\quad<0.30$

1,2-Dichloropropane

cis-1.3-Dichloropropeno

trans-1,3-Dichloropropeno

Endrin

Ethylbenzene

Fluorido

Gross alpha

Iron, total recoverable

Load, total recoverable

Lindane

Magnesium, total recoverable

Manganeso, total recoverable

Mercury, total recoverable
$<1.0$

$<1.0$

$<1.0$

$<0.0060$

$<1.0$

$<100$

$<2.0 E+00$

108

14

$<0.0050$

743

2.1

$<0.20$

$\begin{array}{llll}2093 & \underline{3093} & \underline{4093} & \text { Unit } \\ 269.8 & 265.6 & 264.8 & \mathrm{ft} \mathrm{msl} \\ 14.6 & 18.8 & 19.6 & \mathrm{ft} \\ 4.6 & 4.9 & 4.6 & \mathrm{pH} \\ 23 & 25 & 25 & \mu \mathrm{s} / \mathrm{cm} \\ 18.5 & 18.6 & 18.9 & { }^{\circ} \mathrm{C} \\ 0 & 0 & 0 & \mathrm{mg} / \mathrm{L} \\ 2 & 1 & 1 & \mathrm{NTU} \\ 4.7 & 8.0 & 13.7 & \text { Well vol. }\end{array}$

$\underline{2093}$

$\underline{3093}$

4093

Mad Unit Lab Flag

$\mu \mathrm{rg} / \mathrm{L}$

$\mu g / L$

$\mu \sigma / L$

$\mu \mathrm{\mu g} / \mathrm{L}$

$\mu \mathrm{g} / \mathrm{L}$

$\mu g / L$

$\mu g / L$
$\mu g / L$

$\mu g / L$

$\mu \mathrm{g} / \mathrm{L}$

$\mu g / L$

$\mathrm{\mu g} / \mathrm{L}$

$\mu \mathrm{g} / \mathrm{L}$

$\mu / L$

$\mathrm{\mu g} / \mathrm{L}$

$\mu g / L$

$\mu g / L$

$\mu \mathrm{g} / \mathrm{L}$

$\mu g / L$

$\mu \mathrm{g} / \mathrm{L}$

$\mu g / L$

$\mu g / L$

$\mu g / L$

$\mu \mathrm{g} / \mathrm{L}$

$\mu g / L$

$\mu \mathrm{g} / \mathrm{L}$

$\mu g / L$

$\mathrm{pCi} / \mathrm{L}$
$\mu \mathrm{g} / \mathrm{L}$

150

$\mu \mathrm{g} / \mathrm{L}$

$\mu_{g} / L$

$\mu \mathrm{g} / \mathrm{L}$

$\mu g / L$
$\mu g / L$

Note: Flagging levels, modifiers, and laboratories are for 4th quarter 1993 data only. See Appendix B for flagging criteria.

- = exceeded holding time for 4th quarter 1993.

- = exceeded screening level or final primary drinking water standard for 4th quarter 1993. 
WSRC-TR-94-0354

Unclassified

Woll RRP 1 continued

ANALYTICAL DATA

H D Analyto

Methoxychlor

Nitrato-nitrite as nitrogen

Nonvalatila boto

pH

Phenols

Potassium, total recoverable

Radium, total alpha-emitting

Selenium, tatal recoverable

Silica, total rocoverable

Silver, total recoverable

Sodium, total recovorable

Specific conductence

Sulfate

1,1,2,2-Tetrachloroethane

Tetrachloroethylone

Toluene

Total dissolved solids

Total organic carbon

Total organic halogens

Total phosphates (as P)

Toxaphene

2,4,5-TP (Silvex)

1,1,1-Trichloroothane

1,1,2-Trichloroethane

Trichloroethylene

Trichlorofluoromethane

Tritium

1093
$<0.50$
1.070
$<2.0 \mathrm{E}+00$
5.0
$<5.0$
$<500$
$1.3 \mathrm{E}+00$
$<2.0$
5.160
$<2.0$
893
20
$<1.000$
$<1.0$
$<1.0$
$<1.0$
22.000
$<1.000$
$<5.0$
$<50$
$<0.24$
$<0.090$
$<1.0$
$<1.0$
$<1.0$
$<1.0$
$1.2 \mathrm{E}+00$

$\underline{2093}$

$\underline{3093}$

$\underline{4093}$

Mod

Unit Lab Flag

ra/L

$\mu \mathrm{g} / \mathrm{L}$

$\mathrm{pCi} / \mathrm{L}$
$\mathrm{pH}$
$\mu \mathrm{g} / \mathrm{L}$

ro/L

$\mathrm{pCi} / \mathrm{L}$

$\mu g / L$

$\mu g / L$

$\mu \mathrm{g} / \mathrm{L}$

$\mu \mathrm{mo}$

$\mu \mathrm{S} / \mathrm{cm}$

$\mu g / L$

$\mu \mathrm{g} / \mathrm{L}$

$\mu \mathrm{o} / \mathrm{L}$

$\mu \mathrm{g} / \mathrm{L}$

$\mu \mathrm{g} / \mathrm{L}$

$\mu g / L$

$\mu g / L$

$\mu g / L$

$\mu g / L$

$\mu g / L$

$\mu \mathrm{g} / \mathrm{L}$

$\mu \mathrm{g} / \mathrm{L}$



$\underset{\mathrm{pCi} / \mathrm{mL}}{\mu \mathrm{g} / \mathrm{L}}$

\section{WELL RRP 2}

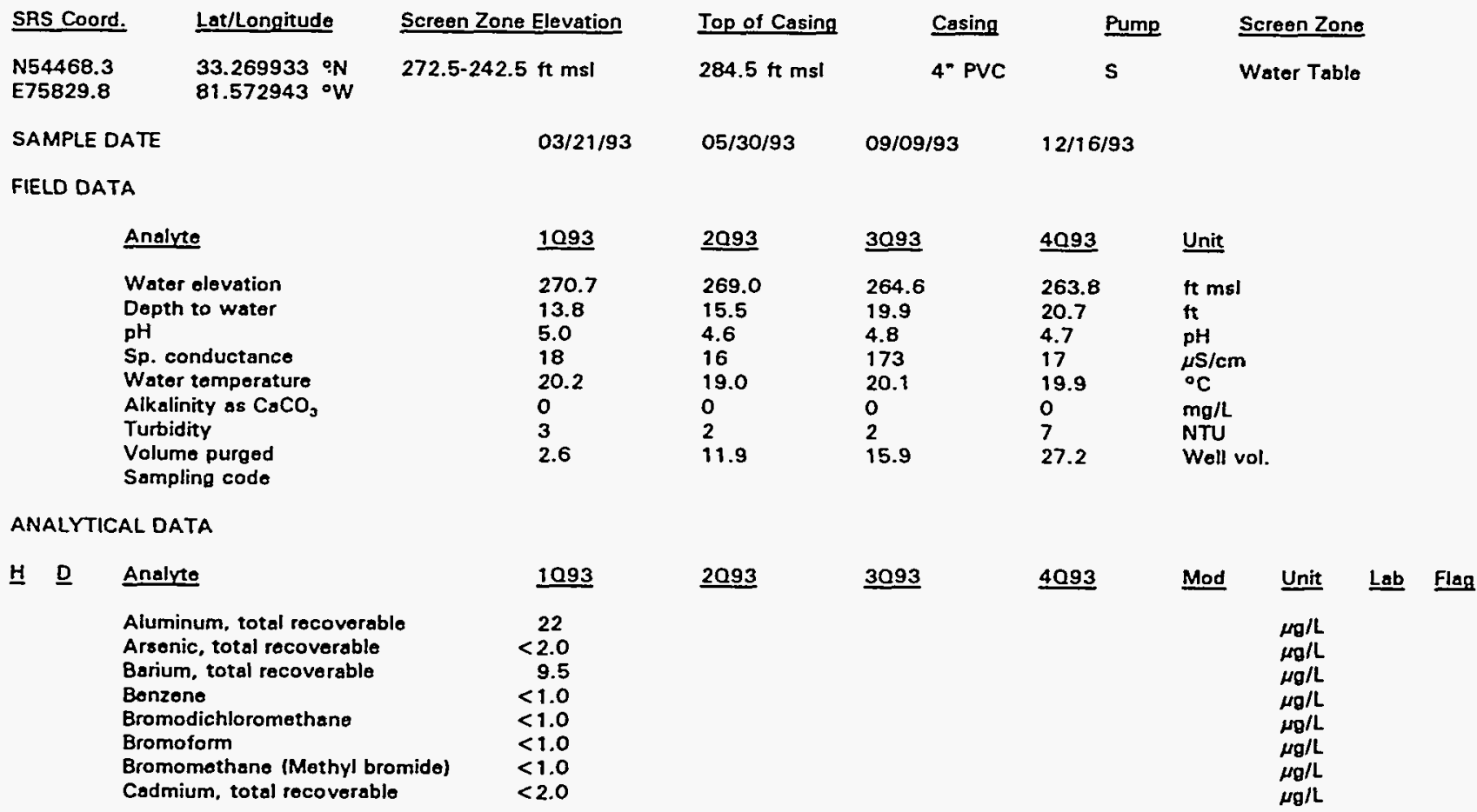

Note: Flagging levels, modifiers, and laboratories are for 4th quarter 1993 data only. See Appendix B for flagging criteria.

- = exceeded holding time for 4th quarter 1993.

- =xceeded screening level or final primary drinking water standard for 4th quarter 1993. 


\section{ANALYTICAL DATA}

H D Analyte

Calcium, total recoverable

Cabon totrachlorido

Chloride

Chlorobenzene

Chloroothano

Chloroethene (Vinyl chloride)

2-Chloroethyl vinyl other

Chloroform

Chloromethane (Methyl chloride)

Chromium, total recoverable

Copper, total recoverable

Dibromochloromethane

1.1-Dichloroethane

1,2-Dichloroethane

1.1-Dichloroethylene

trans-1,2-Dichloroothylene

Dichloromethane (Methylene chloride)

2,4-Dichlorophenoxyacetic acid

1.2-Dichloropropane

cie-1,3-Dichloropropene

trans-1,3-Dichloropropene

Endrin

Ethylbenzene

Fluoride

Gross alpha

Iron, total recoverable

Load, total recoverable

Lindane

Magnesium, total recoverable

Manganese, total recoverable

Mercury, total recoverable

Methoxychlor

Nitrate-nitrite as nitrogen

Nonvolatilo beta

pH

Phonols

Potassium, total rocoverable

Radium, total alpha-emitting

Selenium, total recoverable

Silica, total rocoverable

Silver, total recoverable

Sodium, total recoverable

Spocific conductance

Sulfate

1,1,2,2-Totrachloroethane

Totrachloroethylene

Toluene

Total dissolved solids

Total organic carbon

Total organic halogens

Total phosphates (as P

Toxaphene

2,4,5-TP (Silvex)

1,1,1-Trichloroethane

1.1.2-Trichloroethane

Trichloroethylene

Trichlorofluoromethane

Tritium $\underline{4093}$

Mod

Unit Lab Flag

254

$<1.0$

1.060

$<1.0$

$<1.0$

$<1.0$

$<1.0$

$<1.0$

$<1.0$

$<4.0$

15

$<1.0$

$<1.0$

$<1.0$

$<1.0$

$<1.0$

1.0

$<0.30$

$<1.0$

$<1.0$

$<1.0$

$<0.0060$

$<1.0$

$<100$

$<2.0 E+00$

121

9.0

$<0.0050$

177
6.7

$<0.20$

$<0.50$

440

$<2.0 \mathrm{E}+00$

5.1

$<5.0$

943

$1.0 E+00$

$<2.0$

4.530

$<2.0$

500

12

1.220

$<1.0$

$<1.0$

$<1.0$

13,000

$<1.000$

6.2

$<50$

$<0.24$

$<0.090$

$<1.0$

$<1.0$

$<1.0$

$<1.0$

$<7.0$ OE-01 $\mu g / L$

$\mathrm{mg} / \mathrm{L}$

$\mu / L$

$\mu g / L$

$\mu \mathrm{g} / \mathrm{L}$

$\mu g / L$



$\mu \mathrm{g} / \mathrm{L}$

$\mu \mathrm{g} / \mathrm{L}$

$\mu \mathrm{rg} / \mathrm{L}$

$\mu \mathrm{rg} / \mathrm{L}$

$\mu \mathrm{g} / \mathrm{L}$

$\mu \mathrm{g} / \mathrm{L}$

$\mu \mathrm{g} / \mathrm{L}$

$\mu \mathrm{g} / \mathrm{L}$

$\mu \mathrm{g} / \mathrm{L}$

$\mu \mathrm{O} / \mathrm{L}$

$\mu \mathrm{g} / \mathrm{L}$

moll

$\mu g / L$

pCi/L

$\mu \mathrm{g} / \mathrm{L}$

$\mu \mathrm{g} / \mathrm{L}$

$\mu \mathrm{g} / \mathrm{L}$

$\mu g / L$

$\mu \mathrm{g} / \mathrm{L}$

$\mu \mathrm{g} / \mathrm{L}$

p $\mathrm{Ci} / \mathrm{L}$

pH

$\boldsymbol{\mu g} / \mathrm{L}$.

$\mu \mathrm{g} / \mathrm{L}$

pCi/L

$\mu \mathrm{g} / \mathrm{L}$

$\mu g / L$

$\mu g / L$

$\mu g / L$

$\mu \mathrm{S} / \mathrm{cm}$

$\mu \mathrm{g} / L$

$\mu g / L$

$\mu \mathrm{g} / \mathrm{L}$

$\mu g / L$

$\mu \mathrm{g} / \mathrm{L}$

$\mu g / L$

$\mu g / L$

$\mu g / L$

$\mu g / L$

$\mu g / L$

$\mathrm{\mu g} / \mathrm{L}$

$\mu g / L$

$\mu \mathrm{g} / \mathrm{L}$

$\mu \mathrm{g} / \mathrm{L}$

pCi/mL

Note: Flagging levels, modifiers, and laboratories are for 4th quarter 1993 data only. See Appendix 8 for flagging criteria. - = exceeded holding time for 4th quarter 1993.

- = exceeded screening level or final primary drinking water standard for 4th quarter 1993. 
WELL RRP 3

\begin{tabular}{|c|c|c|c|c|c|c|}
\hline SRS Coord. & Lat/Longitude & Screen Zone Elevation & Top of Casing & Casing & Pump & Screen Zone \\
\hline $\begin{array}{l}\text { N54303.0 } \\
\text { E75853.0 }\end{array}$ & $\begin{array}{l}33.269605{ }^{\circ} \mathrm{N} \\
81.5725611^{\circ} \mathrm{W}\end{array}$ & 268.1-238.1 ft msl & $280.1 \mathrm{ft} \mathrm{msl}$ & 4" PVC & $\mathbf{S}$ & Water Table \\
\hline SAMPLE DATE & & $03 / 18 / 93$ & $05 / 30 / 93$ & $09 / 09 / 93$ & $12 / 17 / 93$ & \\
\hline
\end{tabular}

FIELD DATA

Analyte
Water elevation
Depth to water
pH
Sp. conductance
Water temperature
Alkalinity as $\mathrm{CaCO}_{3}$
Turbidity
Volume purged
Sampling code

ANALYTICAL DATA
H D Analyte

Aluminum, total recoverable Arsenic, total recoverable

Barium, total recoverable

Benzene

Bromodichloromethane

Bromoform

Bromomethane (Methyl bromide)

Cadmium, total recovorable

Calcium, total recoverable

Carbon tetrachloride

Chlorido

Chlorobenzene

Chloroethane

Chloroethene (Vinyl chloride)

2-Chloroethyl vinyl ether

Chloroform

Chloromethane (Methyl chloride)

Chromium, total recoverable

Copper, total recoverable

Dibromochloromethane

1.1-Dichloroethane

1,2-Dichloroethone

1.1-Dichloroethylene

trans-1,2-Dichloroethylene

Dichloromethane (Methylene chloride)

2,4-Dichlorophenoxyacetic acid

1,2-Dichloropropane

cis-1,3-Dichloropropene

trans-1,3-Dichloropropene

Endrin

Ethylbenzene

Fluoride

Gross alpha

Iron, total recoverable

Load, total recoverable

Lindane

Magnesium, total recoverable

Manganese, total recoverable

Mercury, total recoverable

1093
270.4
9.7
5.3
14
18.4
1
10
7.9

2093
268.4
11.7
5.1
14
18.3
1
3
2.6

2093

1093

23

$<2.0$

8.9

$<1.0$

$<1.0$

$<1.0$

$<1.0$

$<2.0$

586

$<1.0$

749

$<1.0$

$<1.0$

$<1.0$

$<1.0$

$<1.0$

$<1.0$

$<4.0$

68

$<1.0$

$<1.0$

$<1.0$

$<1.0$

$<1.0$

$<1.0$

$<0.30$

$<1.0$

$<1.0$

$<1.0$

$<0.0060$

$<1.0$

$<100$

$<2.0 E+00$

866

19

$<0.0050$

169

5.7

$<0.20$

\begin{tabular}{lll}
\hline 3093 & $\underline{4093}$ & Unit \\
263.6 & 262.9 & $\mathrm{ft} \mathrm{msl}$ \\
16.5 & 17.1 & $\mathrm{ft}$ \\
5.0 & 5.2 & $\mathrm{pH}$ \\
129 & 13 & $\mu \mathrm{S} / \mathrm{cm}$ \\
20.2 & 21.0 & ${ }^{\circ} \mathrm{C}$ \\
0 & 2 & $\mathrm{mg} / \mathrm{L}$ \\
2 & 3 & $\mathrm{NTU}$ \\
3.1 & 2.9 & Well vol.
\end{tabular}

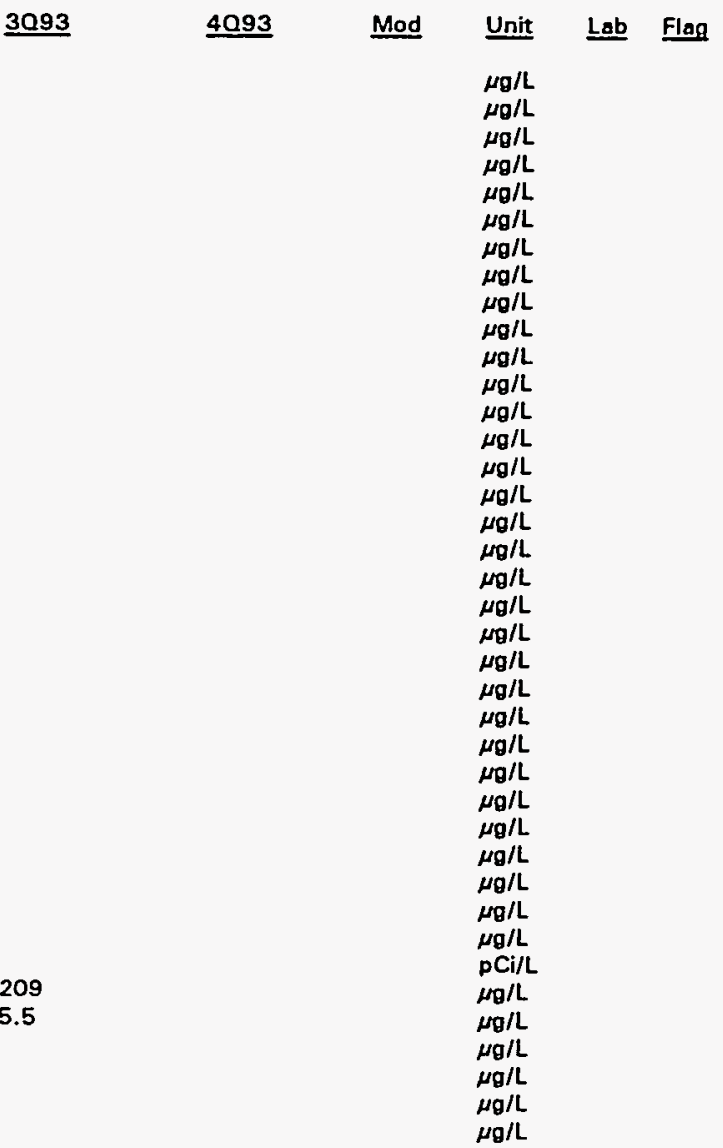

Note: Flagging levels, modifiers, and laboratories are for 4th quarter 1993 data only. See Appendix B for flagging criteria. - =xceeded holding time for 4th quarter 1993.

- =xceeded screening level or final primary drinking water standard for 4th quarter 1993. 


\section{ANALYTICAL DATA}

H $\underline{\text { Analyto }}$

Mothoxychlor

Nitrato-nitrite as nitrogen

Nonvolatile bota

$\mathrm{pH}$

Phenols

Potassium, total recoverable

Radium, total alpha-omitting

Selenium, total recoverable

Silica, total recovorable

Silver, total rocovereble

Sodium, total recoverable

Specific conductance

Sulfato

1,1,2,2-Totrachloroethane

Totrachloroethylene

Tolueno

Total diesolved colids

Total organic carbon

Total organic halogens

Total phosphatos (as P)

Toxaphene

2,4,5-TP (Silvox)

1,1,1-Trichloroethane

1,1,2-Trichloroethane

Trichloroethylene

Trichlorofluoromethane

Tritium

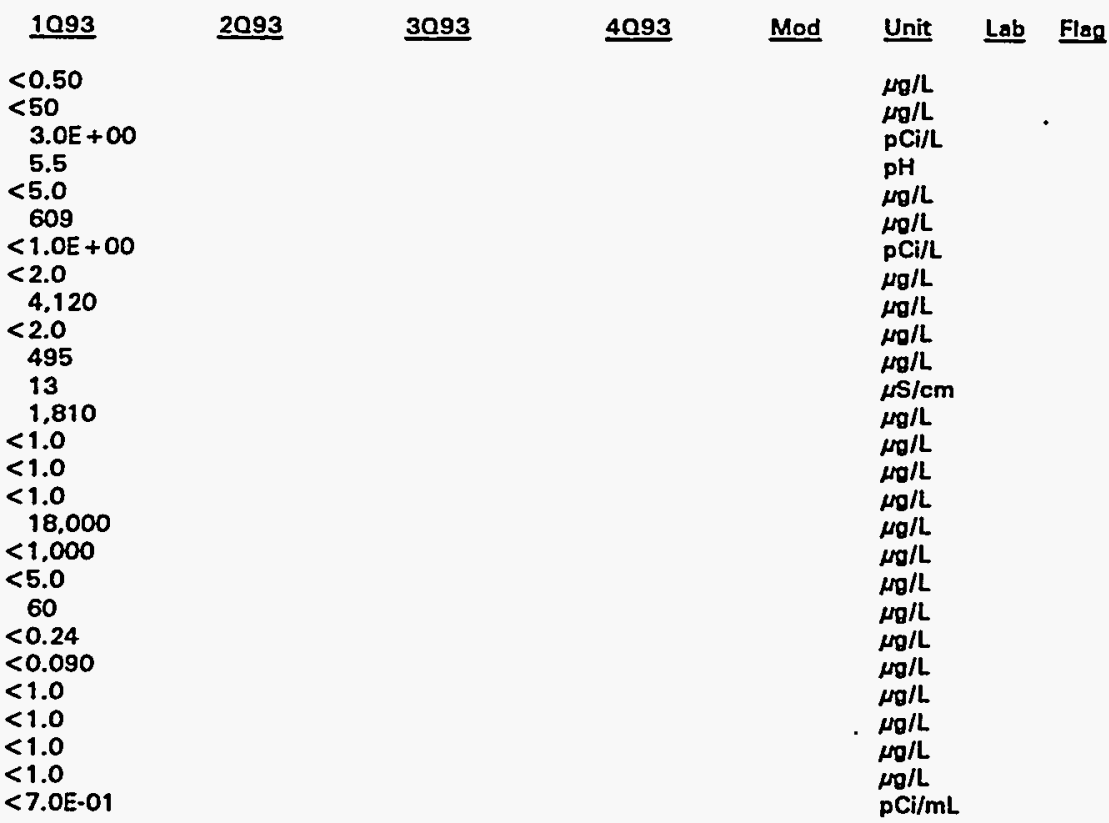

WELL RRP 4

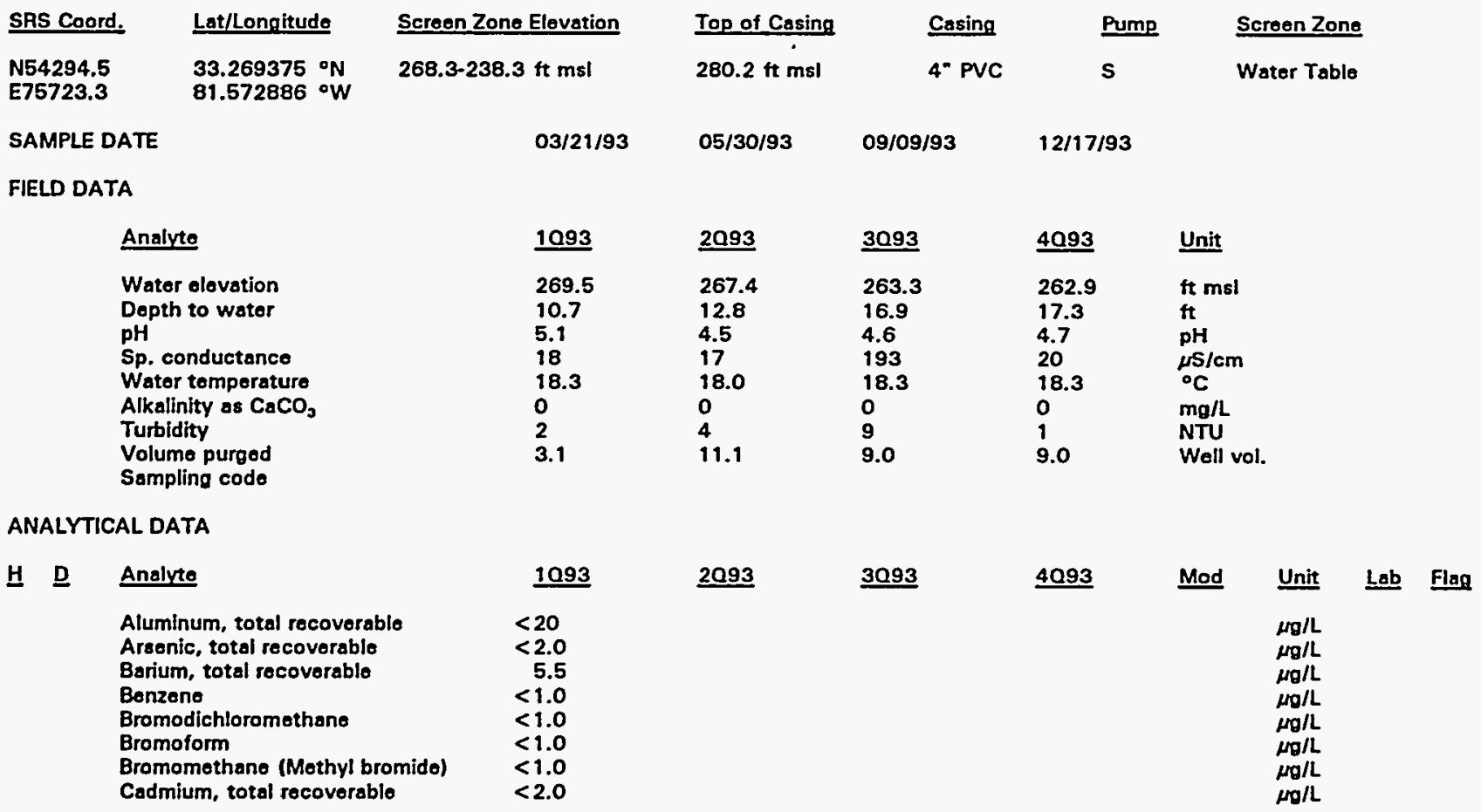

Note: Flagging levels, modifiers, and laboratories are for 4th quarter 1993 data only. See Appendix B for flagging criteria. - = exceeded holding time for 4th quarter 1993.

- = exceeded screening level or final primary drinking water standard for 4th quarter 1993. 
WSRC-TR-94-0354

\section{Unclassified}

Well RRP 4 continued

\section{ANALYTICAL DATA}

\section{H D Analyte}

Calcium, total recoverable

Carbon tetrachloride

Chloride

Chlorobenzene

Chloroethane

Chloroethene (Vinyl chloride)

2-Chloroothyl vinyl ether

Chloroform

Chloromothane (Methyl chloride)

Chromium. total recoverable

Copper, total recoverable

Dibromochloromethane

1,1-Dichloroethane

1,2-Dichloroethane

1.1-Dichloroethylene

trans-1,2-Dichloroe thylene

Dichloromethane (Methylene chloride)

2,4-Dichlorophenoxyacetic acid

1.2-Dichloropropane

cis-1,3-Dichloropropene

trans-1,3-Dichloropropene

Endrin

Ethylbenzene

Fluoride

Gross alpha

Iron, total recoverable

Lead, total recoverable

Lindane

Magnesium, total recoverable

Manganese, total recoverable

Mercury, total recoverable

Methoxychlor

Nitrate-nitrite as nitrogen

Nonvolatile beta

$\mathrm{pH}$

Phonols

Potassium, total recoverable

Radium, total alpha-emitting

Solenium, total recoverable

Silica, total recoverable

Silver, total recoverable

Sodium, total recoverable

Specific conductance

Sulfate

1,1,2,2-Tetrachloroethane

Tetrachloroethylene

Toluene

Total dissolved solids

Total organic carbon

Total organic halogens

Total phosphates (as P)

Toxaphene

2.4,5-TP (Silvex)

1,1,1-Trichloroethane

1,1,2-Trichloroethane

Trichloroethylene

Trichlorofluoromethane

Tritium
1093

$\underline{2093}$

$\underline{3093}$

$\underline{4093}$

Mod

Uni

Lab Flag

237

$<1.0$

1.610

$<1.0$

$<1.0$

$<1.0$

$<1.0$

$<1.0$

$<1.0$

$<4.0$

$<4.0$

$<1.0$

$<1.0$

$<1.0$

$<1.0$

$<1.0$

$<1.0$

$<0.30$

$<1.0$

$<1.0$

$<1.0$

$<0.0060$

$<1.0$

$<100$

$<2.0 E+00$

395

$<3.0$

$<0.0050$

193

2.1

$<0.20$

$<0.50$

$<50$

$<2.0 E+O 0$

5.1

$<5.0$

$<500$

1. $2 E+00$

$<2.0$

4,950

$<2.0$

934

12

1.000

$<1.0$

$<1.0$

$<1.0$

17.000

$<1.000$.

$<5.0$

$<50$

$<0.24$

$<0.090$

$<1.0$

$<1.0$

$<1.0$

$<1.0$

$<7.0$-O 01

$\mu g / L$

$\mu \mathrm{g} / \mathrm{L}$

$\mu \mathrm{g} / \mathrm{L}$

$\mu g / L$

$\mu g / L$

$\mu \mathrm{g} / \mathrm{L}$

pCi/L

pH

$\mu_{g} / L$

$\mu \mathrm{g} / \mathrm{L}$

PCi/L

$\mu \mathrm{g} / \mathrm{L}$

$\mu \mathrm{g} / \mathrm{L}$

$\mu \mathrm{g} / \mathrm{L}$

$\mu \mathrm{g} / \mathrm{L}$

$\mu \mathrm{S} / \mathrm{cm}$

$\mu \mathrm{g} / \mathrm{L}$

$\mu \mathrm{g} / \mathrm{L}$

$\mu \mathrm{g} / \mathrm{L}$

$\mu \mathrm{g} / \mathrm{L}$

$\mu \mathrm{g} / \mathrm{L}$

$\mu \mathrm{g} / \mathrm{L}$

$\mu g / L$

$\mu \mathrm{g} / \mathrm{L}$

$\mu \mathrm{g} / \mathrm{L}$

$\mu \mathrm{g} / \mathrm{L}$

$\mu \mathrm{g} / \mathrm{L}$

$\mu g / L$

$\mu_{\mathrm{g}} / \mathrm{L}$

$\mathrm{pCi} / \mathrm{mL}$

Note: Flagging levels, modifiers, and laboratories are for 4th quarter 1993 data only. See Appendix B for flagging criteria.

- = exceeded holding time for 4th quarter 1993.

- = exceeded screening level or final primary drinking water standard for 4th quarter 1993. 
WELL RSA 6

\begin{tabular}{|c|c|c|c|c|c|c|}
\hline SRS Coord. & Lat/Longitude & Screen Zone Elevation & Top of Casing & Casing & Pump & Screen Zone \\
\hline $\begin{array}{l}\text { N58159.0 } \\
\text { E75000.0 }\end{array}$ & $\begin{array}{l}33.276749 \circ \mathrm{oN} \\
81.582289 \circ \mathrm{W}\end{array}$ & 270.0-1) ft msl & $312.3 \mathrm{ft} \mathrm{msl}$ & $3^{n}$ Steel & B & Water Tablo \\
\hline SAMPLE DATE & & $02 / 23 / 93$ & & & & \\
\hline
\end{tabular}

FIELD DATA

Analvte

Wator olovation

Dopth to water

$\mathrm{pH}$

Sp. conductance

Water temperature

Alkalinity as $\mathrm{CaCO}_{3}$

Turbidity

Volumo purged

Sampling codo

ANALYTICAL DATA

H D Analyte

\section{WELL RSA 7}

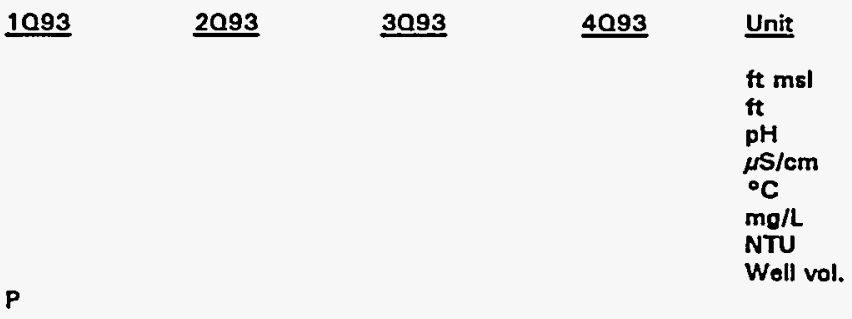

1093

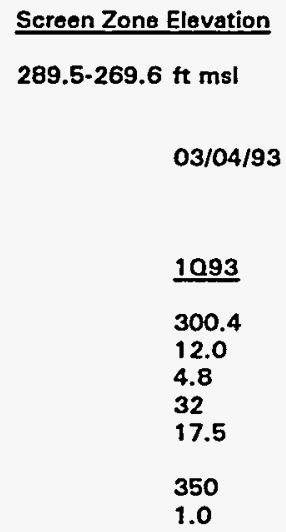

03/04/93

1093

300.4

12.0

4.8

32

17.5

350

1.0

Volume purged Sampling codo

ANALYTICAL DATA

ㅂ D

Analyte
Aluminum, total recoverable
Antimony-125
Arsenic, total recoverable
Barium, total recoverable
Cadmium, total recoverable
Calcium, total recoverable
Cerium-144
Cesium-134
Cesium-137
Chloride
Chromium, total recoverable
Cobalt-57

Analyte

Arsenic, total recoverable Barium, total recoverablo

Cadmium, total recoverable

Cerium-144

Cosium-134

Chloride

Cobalt-57

$\begin{array}{lrrrr}\text { Top of Cosing } & \text { Casing } & \text { Pump } & \text { Screen Zone } \\ 312.4 \mathrm{ft} \mathrm{msl} & \text { 4" PVC } & \text { S } & \text { Water Table } \\ 05 / 18 / 93 & 07 / 27 / 93 & 12 / 04 / 93 & \end{array}$

$\begin{array}{ll}\underline{2093} & \text { 3093 } \\ 294.9 & \quad 284.9 \\ 17.5 & 27.5 \\ 6.6 & 7.0 \\ 97 & 29 \\ 22.5 & 23.8 \\ 135 & 74 \\ 1.9 & 1.6 \\ \times & \end{array}$

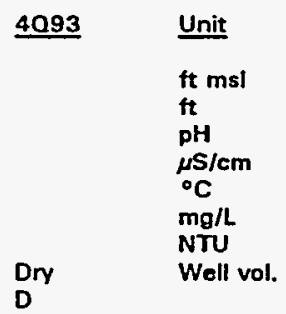

4093 Mod $\quad \begin{gathered}\text { Unit Lab Flag } \\ \mu \mathrm{g} / \mathrm{L} \\ \mathrm{pCi} / \mathrm{L} \\ \mu \mathrm{g} / \mathrm{L} \\ \mu \mathrm{g} / \mathrm{L} \\ \mu \mathrm{g} / \mathrm{L} \\ \mu \mathrm{g} / \mathrm{L} \\ \mathrm{pCi/L} \\ \mathrm{pCi/L} \\ \mathrm{pCi/L} \\ \mu \mathrm{g} / \mathrm{L} \\ \mu \mathrm{g} / \mathrm{L} \\ \mathrm{pCi} / \mathrm{L}\end{gathered}$

Note: Flagging levels, modifiers, and laboratories are for 4th quarter 1993 data only. See Appendix B for flagging criteria.

- = exceeded holding time for 4th quarter 1993.

- = exceeded screening level or final primary drinking water standard for 4th quarter 1993. 
WSRC-TR-94-0354

Unclassified

Woll RSA 7 continued

ANALYTICAL DATA

H D Analyte

Cobsit-60

Copper, total recoverable

2,4-Dichlorophenoxyacetic acid

Endrin

Europium-152

Europium-154

Europium-155

Fluoride

Gross alpha

Iron, total rocoverable

Lead, total recoverable

Lindane

Magnesium, total recoverable

Manganose, total recoverable

Manganese-54

Mercury, total recoverable

Methoxychlor

Nickol, total recoverable

Nitrate-nitrite as nitrogen

Nonvolatile bota

pH

Phenols

Putonium-238

Putonium-239/240

Potassium, total recoverable

Potassium 40

Promethium-144

Promethium-146

Radium, total alpha-emitting

Ruthenium-106

Solenium, total recoverable

Silica, total recoverable

Silver, total recoverable

Sodium, total recoverable

Sodium-22

Spocific conductance

Strontium-90

Sulfate

Total dissolved solids

Total organic carbon

Total organic halogens

Total phosphates (as P)

Total suspended solids

Toxaphene

2,4.5-TP (Silvex)

Tritium

Yttrium-88

Zinc, total recoverable

Zinc-65

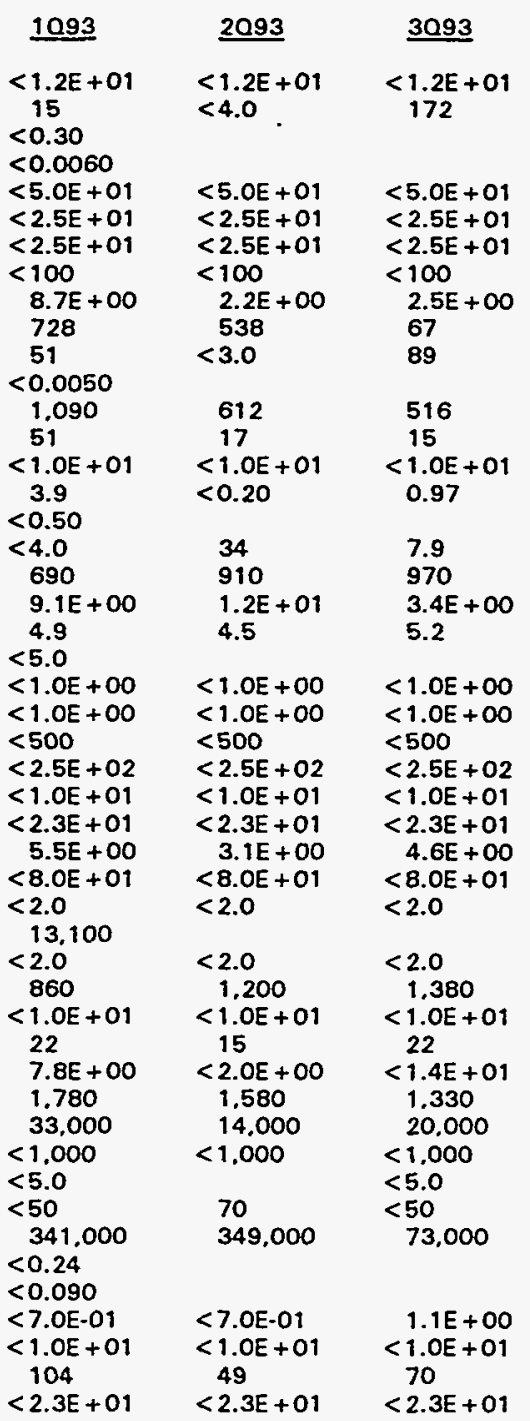

4093 Mod Unit Lab Flag

$\mathrm{pCi} / \mathrm{L}$

$\mu \mathrm{g} / \mathrm{L}$

$\mu g / L$

$\mu g / L$

$\mathrm{pCi} / \mathrm{L}$

$\mathrm{pCi} / \mathrm{L}$

$p C i / L$

$\mu g / L$

PCi/L

$\mu \mathrm{g} / \mathrm{L}$

$\mu g / \mathrm{L}$

$\mu g / L$

$\mu \mathrm{\mu g} / \mathrm{L}$

$\mathrm{pCi} / \mathrm{L}$

$\mu \mathrm{g} / \mathrm{L}$

$\mu_{\mathrm{g}} / \mathrm{L}$

$\mu \mathrm{g} / \mathrm{L}$

$\mu \mathrm{g} / \mathrm{L}$

pCi/L

$\mathrm{pH}$

$\mu \mathrm{pg} / \mathrm{L}$

pCi/l

$\mu \mathrm{g} / \mathrm{L}$

pCi/L

$\mathrm{pCi} / \mathrm{L}$

pCi/L

pCi/L

PCi/L

$\mu g / L$

$\mu \mathrm{g} / \mathrm{L}$

$\mu \mathrm{g} / \mathrm{L}$

$\mu \mathrm{g} / \mathrm{L}$

$\mathrm{pCi} / \mathrm{L}$

$\mu \mathrm{g} / \mathrm{L}$

$\mu g / L$

$\mu g / L$

$\mu \mathrm{g} / \mathrm{L}$

$\mu \mathrm{g} / \mathrm{L}$

$\mu g / L$

$\mu \mathrm{g} / \mathrm{L}$

$\mu \mathrm{g} / \mathrm{L}$

pCi $/ \mathrm{mL}$

$\mathrm{pCi} / \mathrm{L}$

$\mu \mathrm{g} / \mathrm{L}$

pCi/L

Note: Flagging levels, modifiers, and laboratories are for 4th quarter 1993 data only. See Appendix $B$ for flagging criteria. - = exceeded holding time for 4th quarter 1993.

- = exceeded screening level or final primary drinking water standard for 4th quarter 1993. 
WELL RSA 8

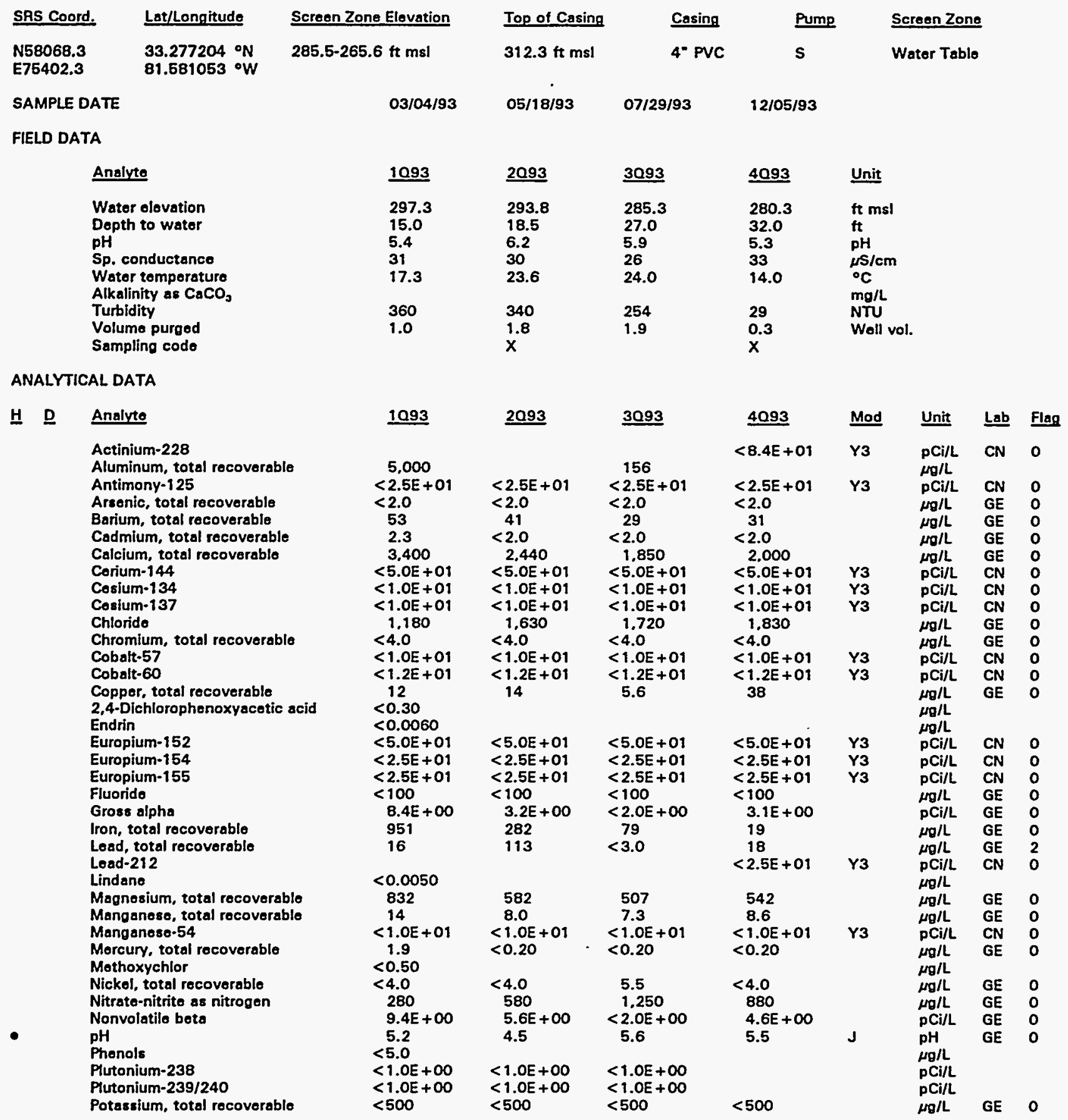

Note: Flagging levels, modifiers, and laboratories are for 4th quarter 1993 data only. See Appendix B for flagging criteria. - = exceeded holding time for 4th quarter 1993.

a = exceeded screening level or final primary drinking water standard for 4th quarter 1993. 


$\begin{array}{ll}\text { H. } & \text { Analyte } \\ \text { Potassium-40 } \\ \text { Promethium-144 } \\ \text { Promethium-146 } \\ \text { Radium, total alpha-emitting } \\ \text { Ruthenium-106 } \\ \text { Salonium, total recoverable } \\ \text { Silica, total recoverable } \\ \text { Silver, total recoverable } \\ \text { Sodium, total recoverable } \\ \text { Sodium-22 } \\ \text { Specific conductance } \\ \text { Strontium-89 } \\ \text { Strontium-90 } \\ \text { Sulfate } \\ \text { Thorium-234 } \\ \text { Total dissolved solids } \\ \text { Total organic carbon } \\ \text { Total organic halogens } \\ \text { Total phosphates (as P) } \\ \text { Total suspended solids } \\ \text { Toxaphene } \\ \text { 2,4,5-TP (Silvex) } \\ \text { Tritium } \\ \text { Yttrium-88 } \\ \text { Zine, total recoverable } \\ \text { Zinc-65 }\end{array}$

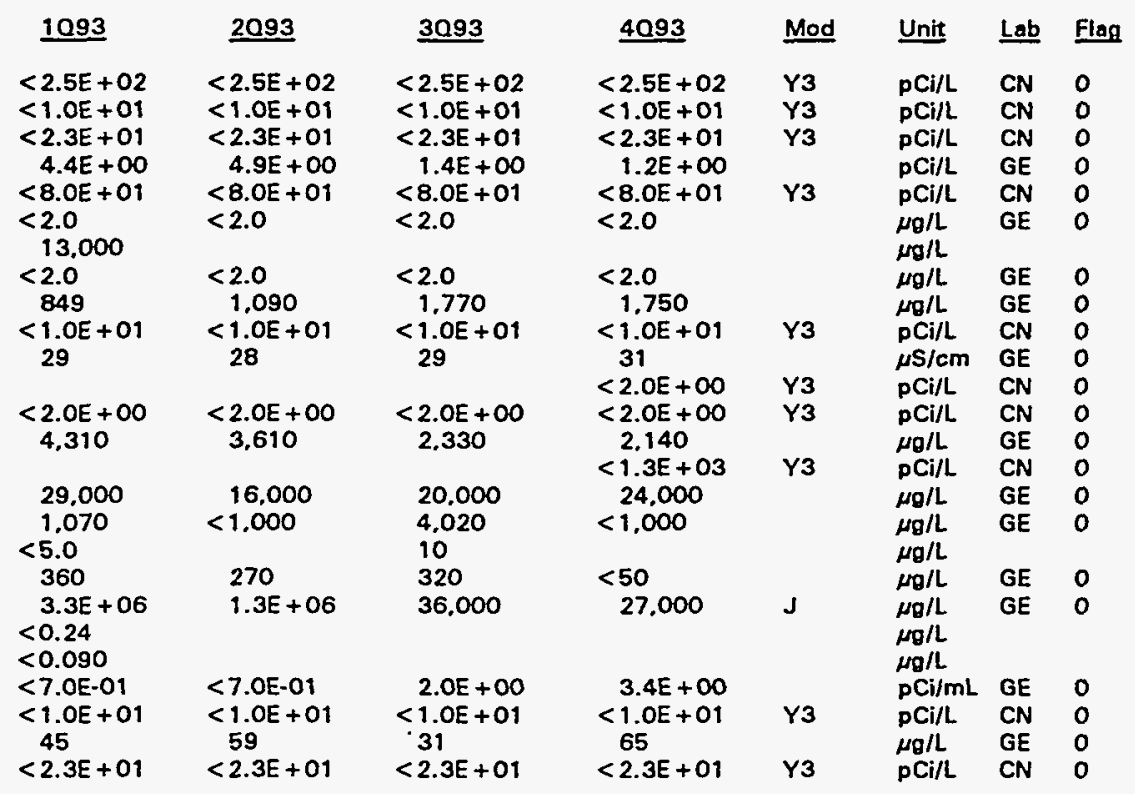

\section{WELL RSA 9}

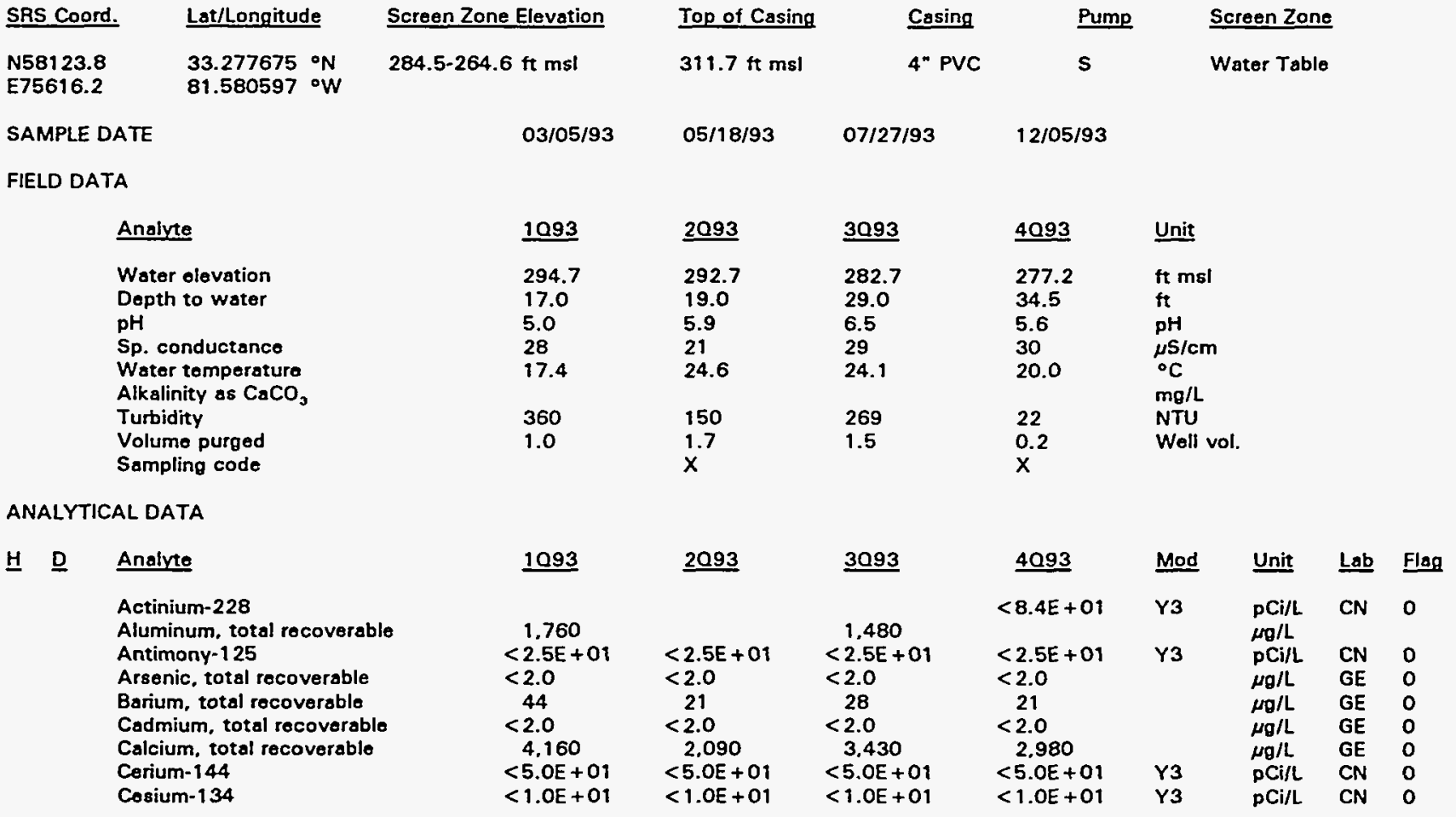

Note: Flagging lavels, modifiers, and laboratories are for 4th quarter 1993 data anly. See Appendix B for flagging criteria. - = exceeded holding time for 4th quarter 1993.

- = exceeded screening level or final primary drinking water standard for 4th quarter 1993. 


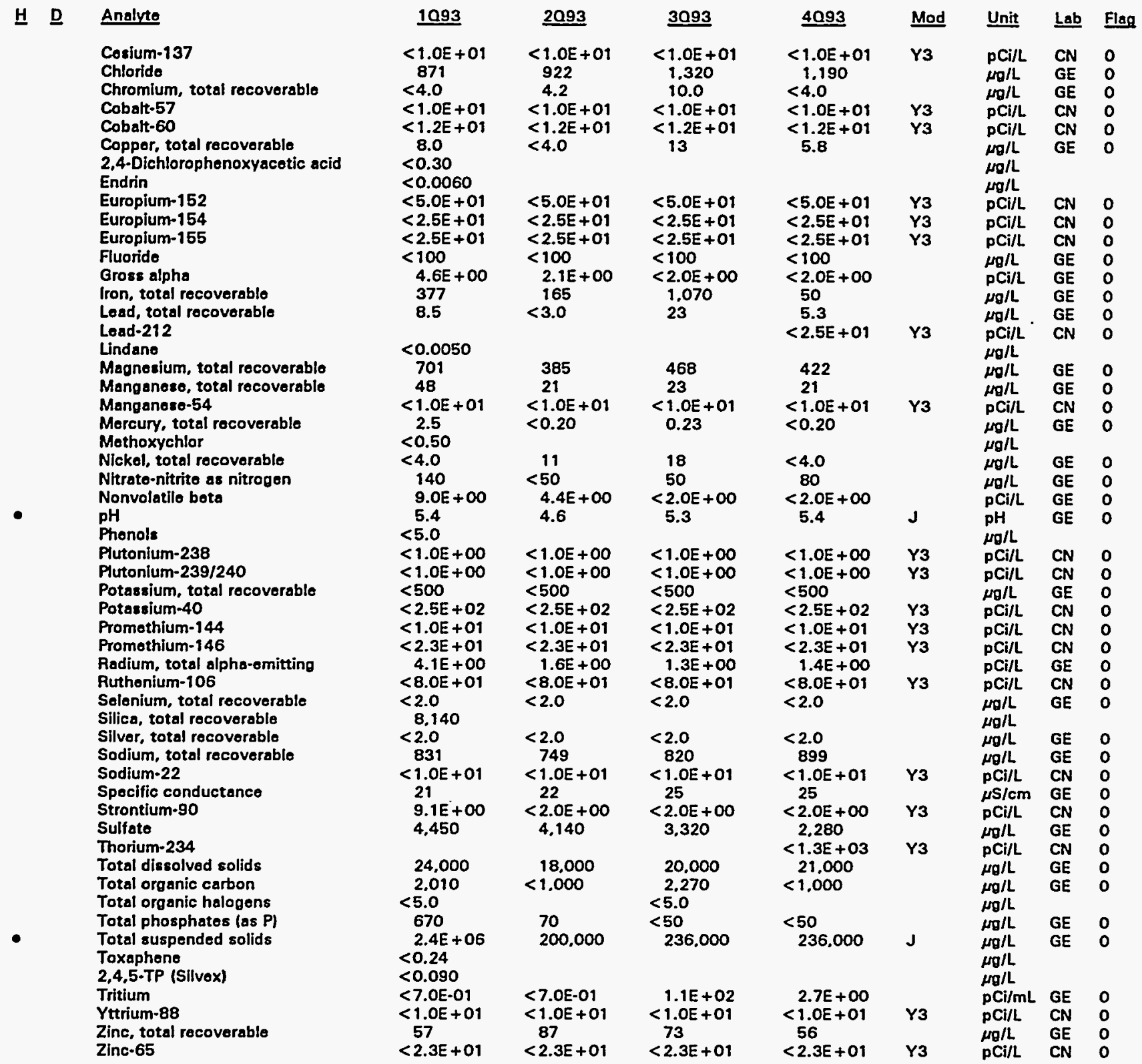

Note: Flagging levels, modifiers, and laboratories are for 4th quarter 1993 data only. See Appendix B for flagging criteria.

$-=$ exceeded holding time for 4th quarter 1993.

- = exceeded screening level or final primary drinking water standard for 4th quarter 1993. 


\section{WELL RSA 10}

$\begin{array}{lll}\text { SRS Coord. } & \text { Lat/Longitude } & \text { S } \\ \text { N58172.8 } & & 33.277414 \\ \text { E75389.3 } & 81.581290^{\circ} \mathrm{W}\end{array}$

SAMPLE DATE

FIELD DATA

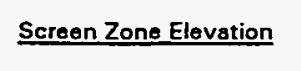

288.7-268.8 ft ms!

\section{Top of Casing}

$311.3 \mathrm{ft} \mathrm{msl}$

03/05/93

$\begin{array}{ll}\text { Cosing } & \text { Pump } \\ \text { 4" PVC } & \mathrm{s}\end{array}$

Screon Zone

Water Table

Analyte
Water elovation
Depth to water
pH
Sp. conductance
Water temperature
Alkalinity as $\mathrm{CaCO}_{3}$
Turbidity
Volume purged
Sampling codo

ANALYTICAL DATA

1093
289.3
22.0
4.9
31
16.0

210
1.0

\section{3}

Aluminum, total recoverable Antimony-125

Arsenic, total recoverable Barium, total recoverable Cadmium. total recoverable Calcium, total recoverable

Corium-144

Cosium-134

Cosium-137

Chloride

Chromium, total recoverable

Cobalt-57

Cobalt-60

Copper, total recoverable

2,4-Dichlorophenoxyacetic acid

Endrin

Europium-152

Europium-154

Europium-155

Fluorido

Gross alpha

Iron, total recoverable

Lead, total recoverable

Lindane

Magnesium, total recoverable

Manganese, total recoverable

Manganese- 54

Mercury, total recoverable

Methoxychlor

Nickel, total recoverable

Nitrato-nitrite as nitrogen

Nonvolatile beta

$\mathrm{pH}$

Phenols

Plutonium-238

Plutonium-239/240

Potassium, total recoverable

Potassium-40

Promethium-144

477
$<2.5$
$<+1$

$<2.5 \mathrm{E}+01$

$<2.0$

25

3.6

618

$<5.0 E+01$

$<1.0 E+01$

$<1.0 E+01$

1,570

$<4.0$

$<1.0 E+01$

$<1.2 E+01$

4.3

$<0.30$

$<0.0060$

$<5.0 E+01$

$<2.5 E+01$

$<2.5 E+01$

$<100$

$4.8 \mathrm{E}+00$

79

$<3.0$

$<0.0050$

1.100

25

$<1.0 E+01$

0.74

$<0.50$

$<4.0$

2.160

1. $1 E+01$

4.9

$<5.0$

$<1.6 E+00$

$<1.2 \mathrm{E}+00$

$<500$

$<2.5 \mathrm{E}+02$

$<1.0 E+01$

$05 / 18 / 93$

2093
287.3
24.0
6.0
30
24.6

21
1.4
$X$

2093

$<2.5 E+01$

$<2.0$

27

$<2.0$

$<5.0 E+01$

$<1.0 E+01$

$<1.0 E+01$

1.600

$<4.0$

$<1.0 E+01$

$<1.2 E+01$

5.5

$07 / 27 / 93$

$12 / 04 / 93$

$<5.0 E+01$

$<2.5 E+01$

$<2.5 E+01$

$<100$

$+00<2.0 E+00$

$195 \quad 81$

8.0

1.070

15

$<1.0 E+01$

0.27

12

1.930

$<2.0 E+00$

4.6

$\underline{3093}$

281.3

30.0

6.6

24.4

193

1.1

\section{$\underline{3093}$}

167

$<2.5 E+01$

$<2.0$

14

$<2.0$

1.470

$<5.0 E+01$

$<1.0 E+01$

$<1.0 E+01$

2.010

$<4.0$

$<1.0 E+01$

$<1.2 E+01$

8.3

$<1.0 E+00$

$<1.0 E+00$

$<500$

$<2.5 E+02$

$<1.0 E+01$
4093

Unit

ft msl

ft

pH

$\mu \mathrm{S} / \mathrm{cm}$

$\mathrm{mg} / \mathrm{L}$

NTU

Dry Well vol.

4093 Mod Unit Lab Flog

$\mu \mathrm{g} / \mathrm{L}$

$\mu \mathrm{g} / \mathrm{L}$

$\mu g / L$

$\mu g / L$

$\mu \mathrm{g} / \mathrm{L}$

pCi/L

$\mathrm{pCi} / \mathrm{L}$

$\mu \mathrm{g} / \mathrm{L}$

$\mu \mathrm{g} / \mathrm{L}$

pCi/L

PCi/L

$\mu \mathrm{g} / \mathrm{L}$

$\mu \mathrm{g} / \mathrm{L}$

$\mu \mathrm{g} / \mathrm{L}$

$\mathrm{pCi} / \mathrm{L}$

pCill

$\mu \mathrm{g} / \mathrm{L}$

$\mu \mathrm{g} / \mathrm{L}$

$\mu \mathrm{g} / \mathrm{L}$

$\mu \mathrm{g} / \mathrm{L}$

$\mu \mathrm{g} / \mathrm{L}$

$\mu \mathrm{g} / \mathrm{L}$

$\mathrm{pCi} / \mathrm{L}$

$\mu \mathrm{g} / \mathrm{L}$

$\mu g / L$

$\mu \mathrm{g} / \mathrm{L}$

$\mu \mathrm{g} / \mathrm{L}$

$\mathrm{pCi/L}$

$\mathrm{pCH} / \mathrm{L}$
$\mathrm{pH}$
$\mu \mathrm{g} / \mathrm{L}$

$\mu \mathrm{g} / \mathrm{L}$

$\mathrm{pCi/L}$

$\mathrm{pCi} / \mathrm{L}$

$\mu \mathrm{g} / \mathrm{L}$

$\mathrm{pCi} / \mathrm{L}$

$\mathrm{pCi} / \mathrm{L}$

Note: Flagging levels, modifiers, and laboratories are for 4th quarter 1993 data only. See Appendix B for flagging criteria.

$-=$ exceeded holding time for 4th quarter 1993.

- = exceeded screening level or final primary drinking water standard for 4th quarter 1993. 


\section{ANALYTICAL DATA}

H D Analyte

Promothium-146
Radium, total alpha-omitting
Ruthenium-106
Solenium, total recoverable
Silica, total recoverable
Silver, total recoverable
Sodium, total recoverable
Sodium-22
Specific conductance
Strontium-90
Sulfate
Total dissolved solids
Total oroanic carbon
Total oraanic halogons
Total phosphatos (as P)
Total susponded solids
Toxaphene
2,4,5-TP (Silvox)
Tritium
Yttrium-88
Zinc, total recoverable
Zinc-65

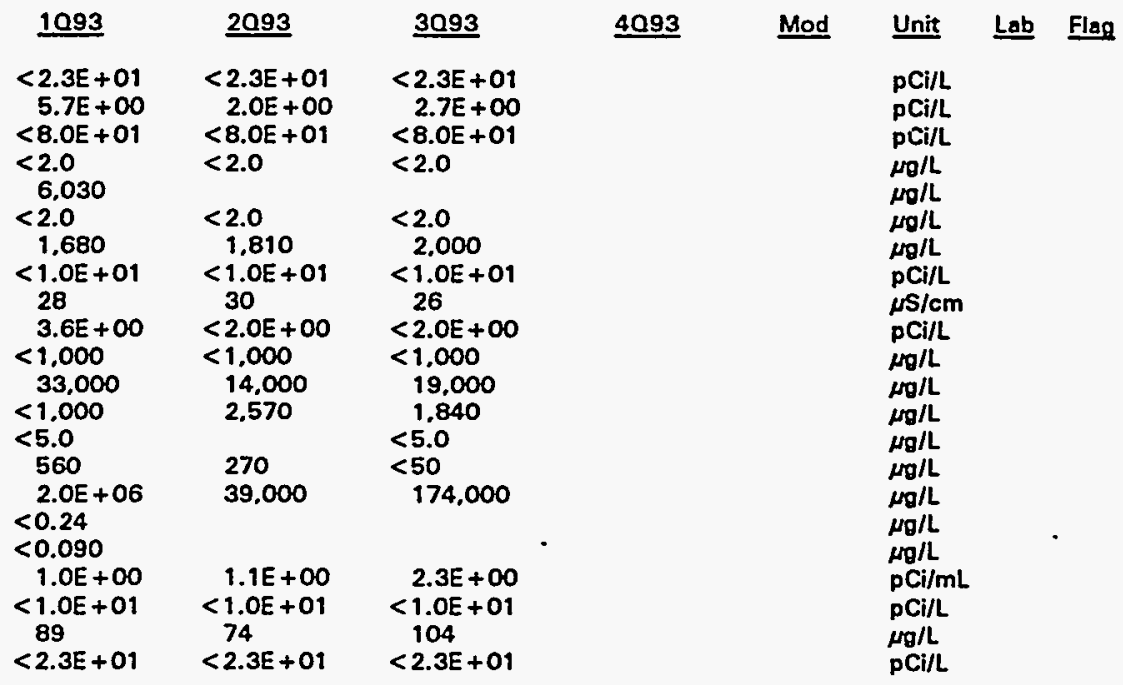

\section{WELL RSB 7}

\begin{tabular}{|c|c|c|c|c|c|c|}
\hline SAS Coord. & Lat/Longitude & Screen Zone Elevation & Top of Casing & Casing & Pump & Screen Zone \\
\hline $\begin{array}{l}\text { N57692.8 } \\
\text { E75044.3 }\end{array}$ & $\begin{array}{l}33.2757899^{\circ} \mathrm{N} \\
81.5812688^{\circ} \mathrm{W}\end{array}$ & 292.6-272.7 ft msl & $309 \mathrm{ft} \mathrm{msl}$ & 4" PVC & $\mathbf{s}$ & Water Table \\
\hline \multicolumn{2}{|c|}{ SAMPLE DATE } & $02 / 22 / 93$ & $05 / 21 / 93$ & $07 / 27 / 93$ & $12 / 05 / 93$ & \\
\hline \multicolumn{7}{|l|}{ FIELD DATA } \\
\hline \multicolumn{2}{|c|}{ Analvte } & 1093 & $\underline{2093}$ & 3093 & 4093 & Unit \\
\hline \multirow{2}{*}{\multicolumn{2}{|c|}{$\begin{array}{l}\text { Wator olevation } \\
\text { Dopth to water } \\
\text { pH } \\
\text { Sp. conductence } \\
\text { Water temperature } \\
\text { Alkalinity as } \mathrm{CaCO}_{3} \\
\text { Turbidity } \\
\text { Volume purged } \\
\text { Sampling code }\end{array}$}} & $\begin{array}{l}293.5 \\
15.5 \\
5.4 \\
23 \\
20.6\end{array}$ & $\begin{array}{l}291.0 \\
18.0 \\
6.3 \\
64 \\
23.9\end{array}$ & $\begin{array}{l}287.0 \\
22.0 \\
6.8 \\
31 \\
24.4\end{array}$ & $\begin{array}{l}283.0 \\
26.0 \\
5.7 \\
24 \\
23.0\end{array}$ & \multirow{2}{*}{$\begin{array}{l}\mathrm{ft} \mathrm{msl} \\
\mathrm{ft} \\
\mathrm{pH} \\
\mu \mathrm{S} / \mathrm{cm} \\
{ }^{\circ} \mathrm{C} \\
\mathrm{mg} / \mathrm{L} \\
\text { NTU } \\
\text { Wall vol. }\end{array}$} \\
\hline & & 1.9 & $\begin{array}{l}15 \\
1.2 \\
x\end{array}$ & $\begin{array}{l}64 \\
1.2\end{array}$ & $\begin{array}{l}303 \\
0.1 \\
\times\end{array}$ & \\
\hline
\end{tabular}

\section{ANALYTICAL DATA}

H $\underline{\text { Analyte }}$

Actinium-228

Aluminum, total recoverable Antimony-125

Arsenic, total recoverable

Barium, total recoverable

Cadmium, total recoverable

Calcium, total recoverable

Corium-144

Cosium-134

Cosium-137

Chlorido

Chromium, total recoverable

Cobalt-57

$\begin{array}{lll}\frac{1093}{} & \underline{2093} & \underline{3093} \\ 7.880 & & \\ <2.5 E+01 & <2.5 E+01 & <234 \\ <2.0 & <2.0 & <2.0 \\ 24 & 13 & 3.7 \\ 6.3 & 2.3 & <2.0 \\ 546 & 3.060 & 245 \\ <5.0 E+01 & <5.0 E+01 & <5.0 E+01 \\ <1.0 E+01 & <1.0 E+01 & <1.0 E+01 \\ <1.0 E+01 & <1.0 E+01 & <1.0 E+01 \\ 1.540 & 1.620 & 1.620 \\ <4.0 & 4.1 & <4.0 \\ <1.0 E+01 & <1.0 E+01 & <1.0 E+01\end{array}$

Note: Flagging levels, modifiers, and laboratories are for 4th quarter 1993 data only. See Appendix B for flagging criteria. - = exceeded holding time for 4th quarter 1993.

- = exceeded screening level or final primary drinking water standard for 4th quarter 1993. 


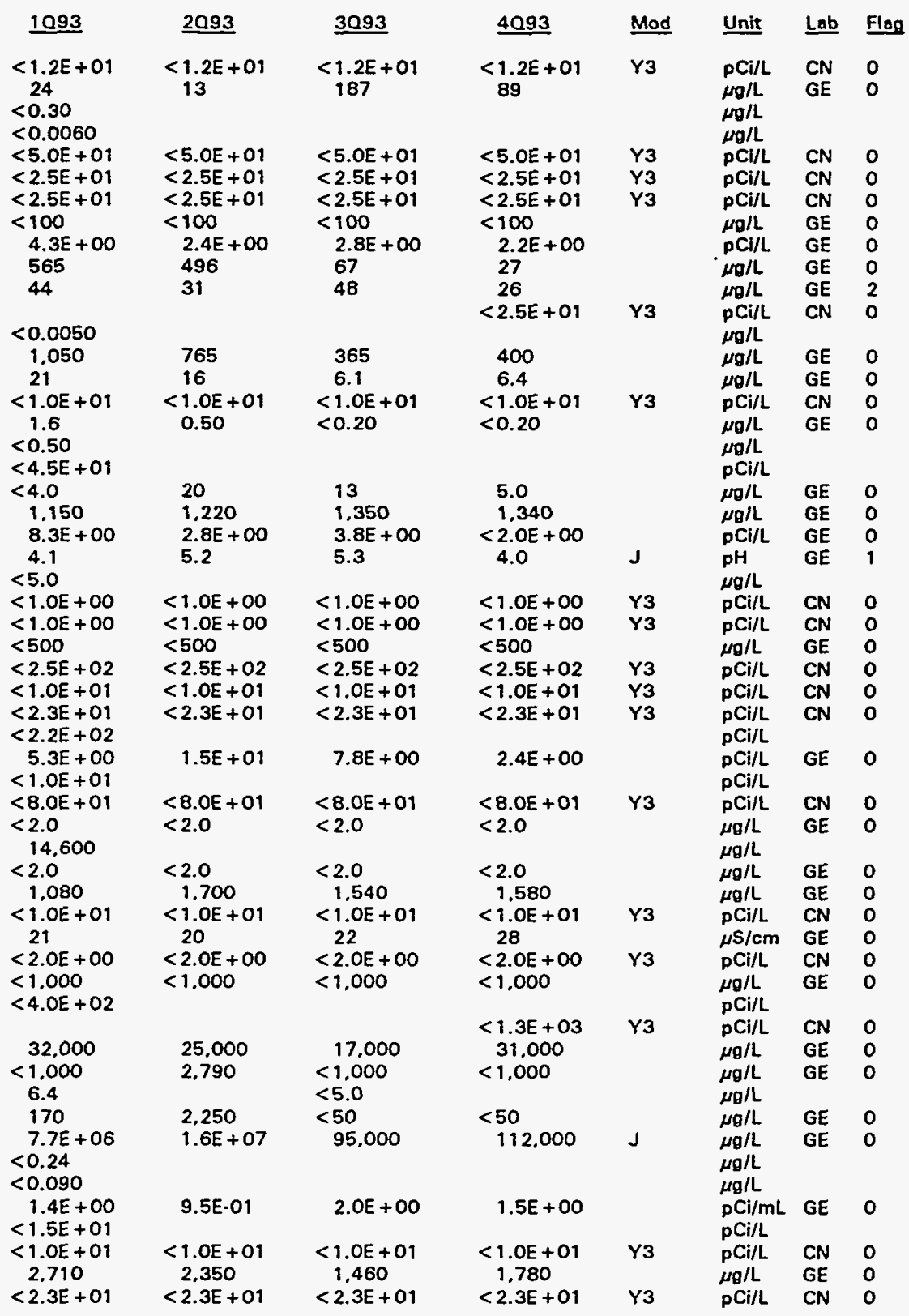

Note: Flagging levels, modifiers, and laboratories are for 4th quarter 1993 data only. See Appendix $B$ for flagging criteria. - = exceeded holding time for 4th quarter 1993.

- = exceeded screening level or final primary drinking water standard for 4th quarter 1993. 
WELL RSB 8

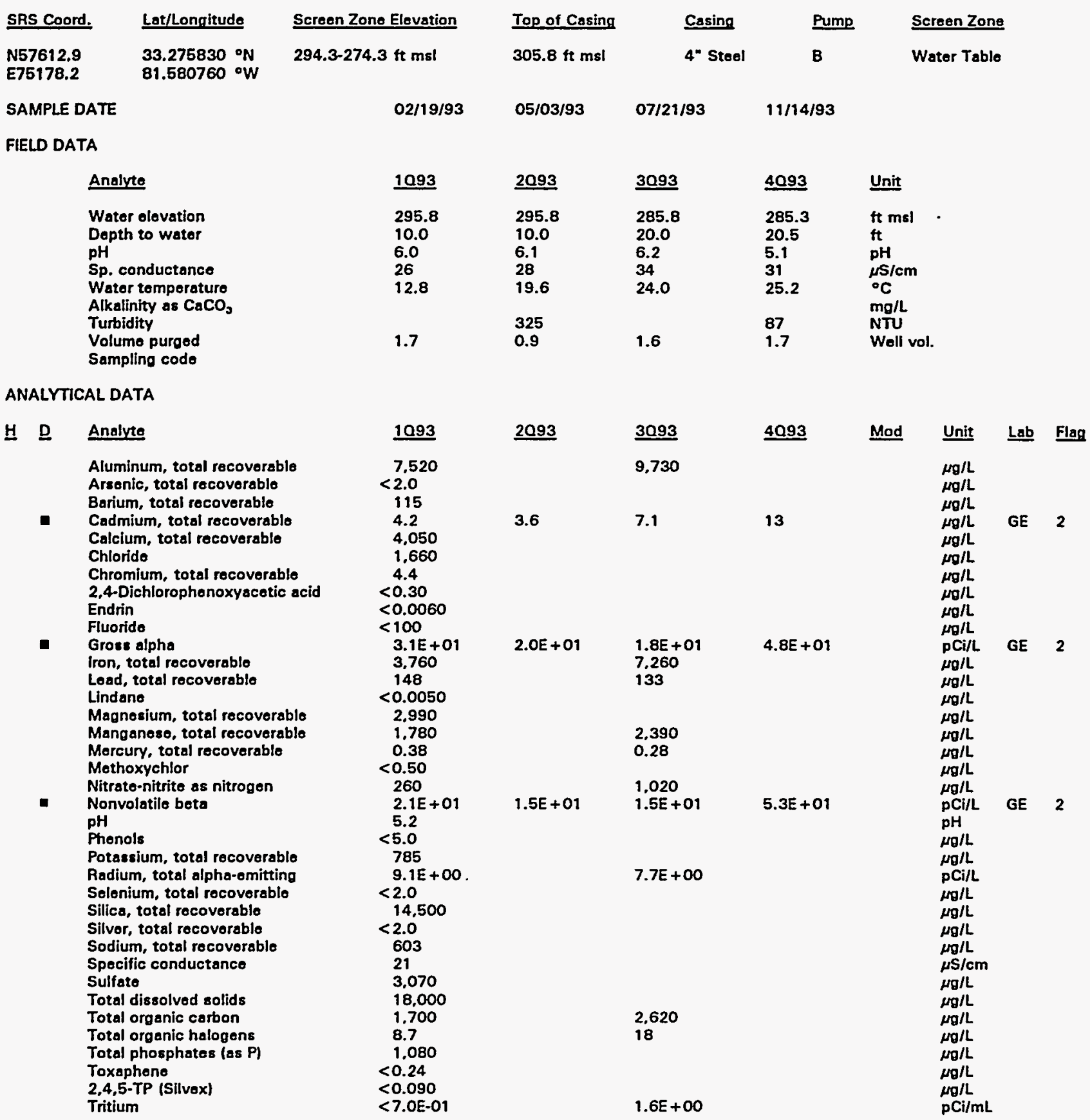

Note: Flagging levels, modifiers, and laboratories are for 4th quarter 1993 data only. See Appendix B for flagging criteria.

- = exceeded holding time for 4th quarter 1993.

- = exceeded screening level or final primary drinking water standard for 4th quarter 1993. 


\section{WELL RSB 9}

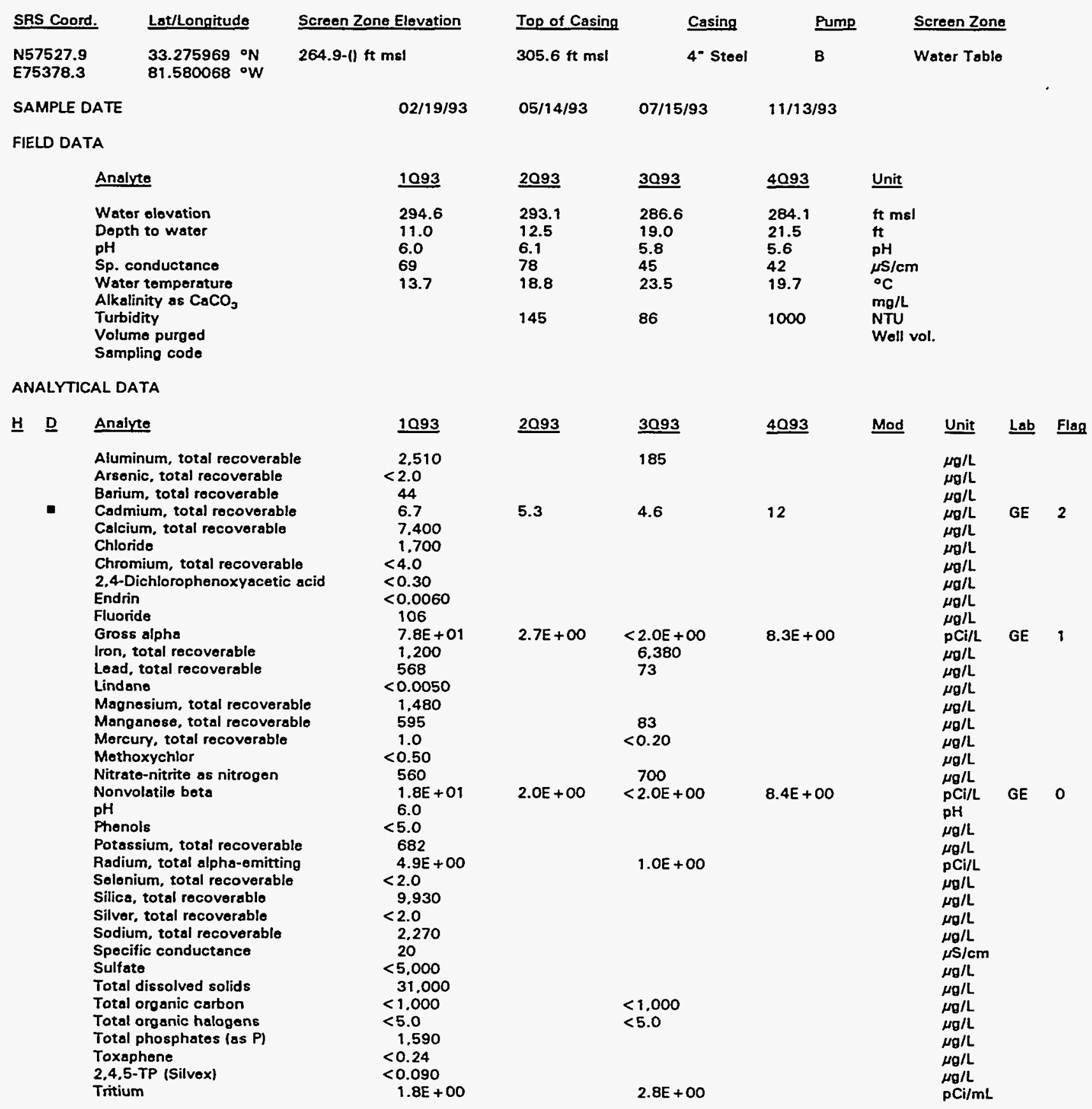

Note: Flagging levels, modifiers, and laboratories are for 4th quarter 1993 data only. See Appendix B for flagging criteria. - = exceeded holding time for 4th querter 1993.

- = exceeded screening level or final primary drinking water standard for 4th quarter 1993. 


\section{WELL RSC 1}

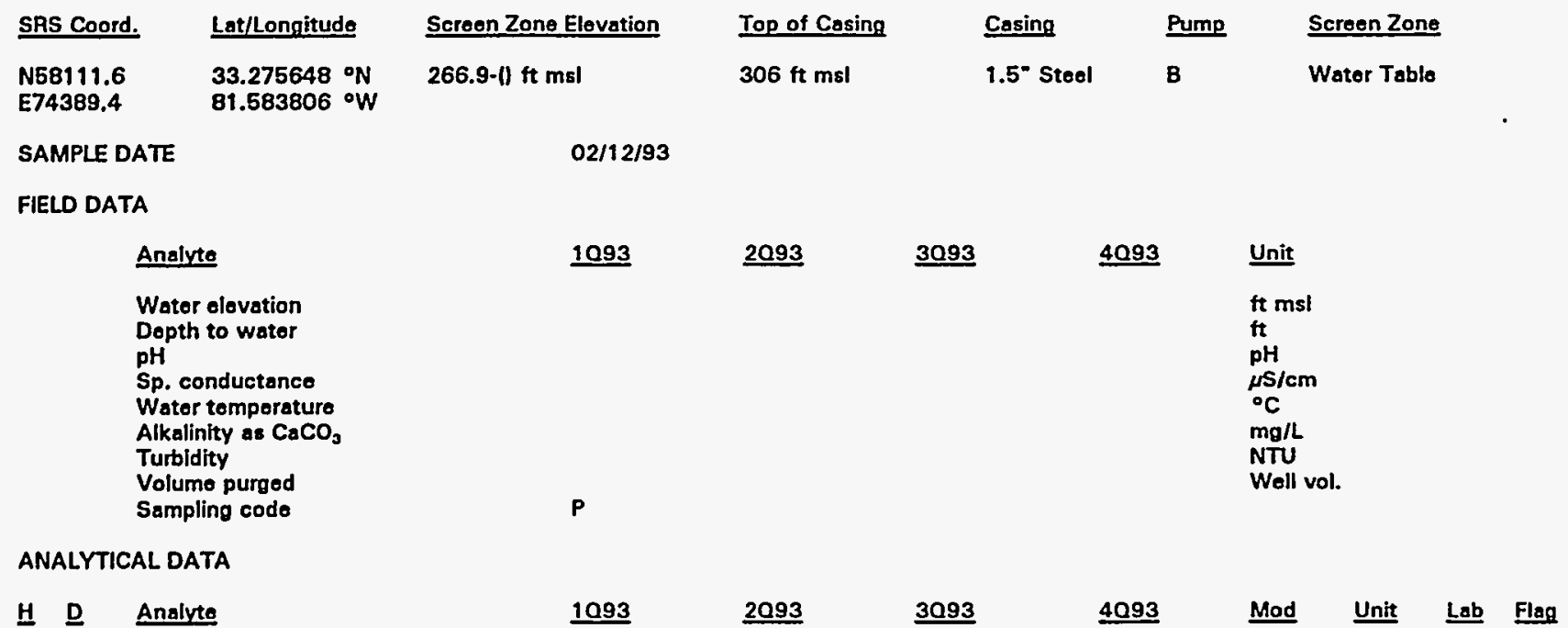

\section{WELL RSC 2}

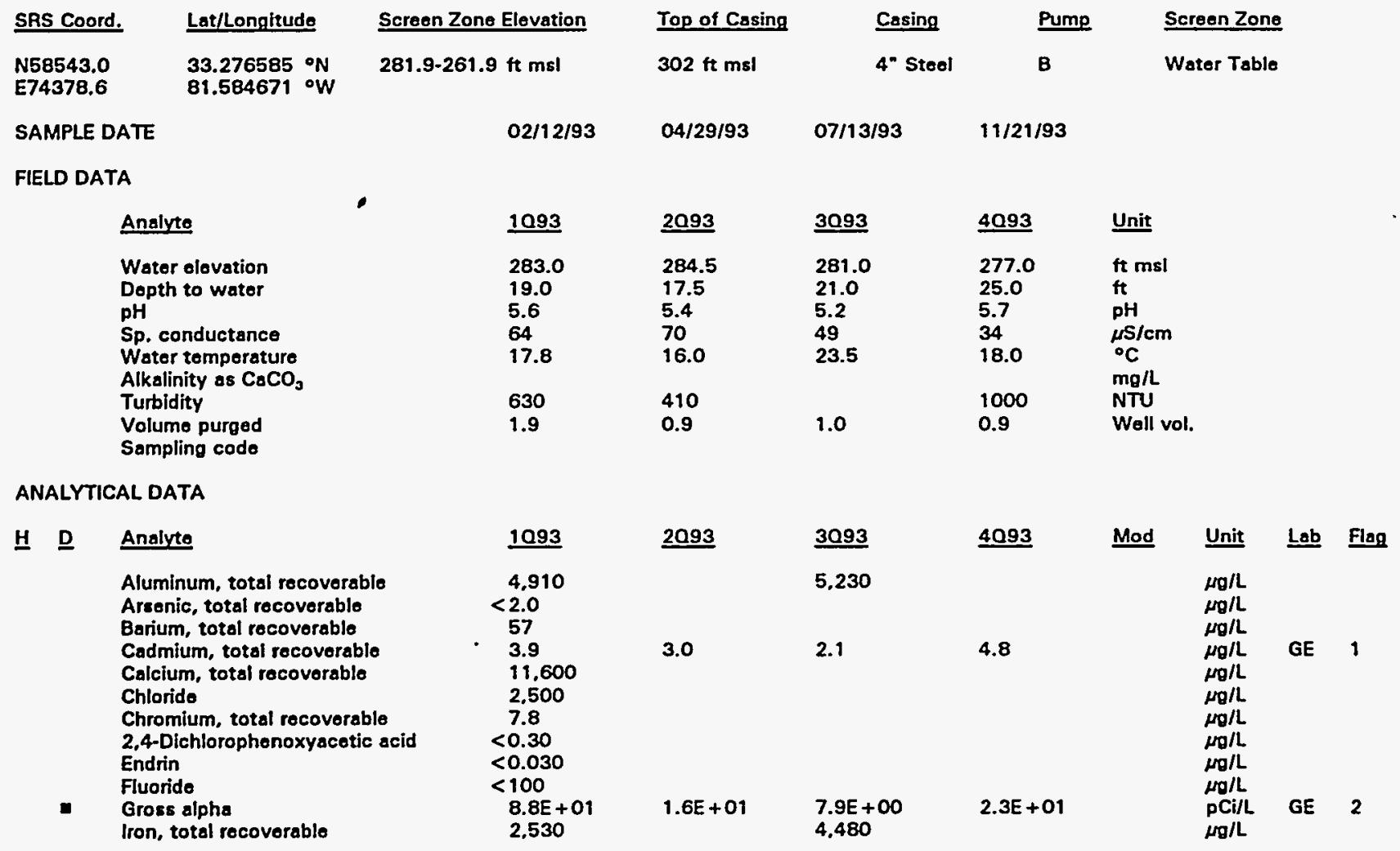

Note: Flagging levels, modifiers, and laboratories are for 4th quarter 1993 data only. See Appendix B for flagging criteria. - = exceeded holding time for 4th quarter 1993.

- = exceeded screening level or final primary drinking water standard for 4th quarter 1993. 


\section{ANALYTICAL DATA}

H D $\quad$ Analyte
Load, total recoverable
Lindane
Magnosium, total recoverable
Manganese, total recoverable
Mercury, total recoverable
Methoxychlor
Nitrate-nitrite as nitrogen
Nonvolatile beta
pH
Phenols
Potassium, total recoverable
Radium, total slpho-emitting
Solenium, total recoverable
Silica, total recoverable
Silver, total recoverable
Sodium, total recoverable
Specific conductance
Sulfate
Total dissolved solids
Total organic carbon
Total organic halogons
Total phosphates las P)
Toxaphene
2,4,5-TP (Silvex)
Tritium

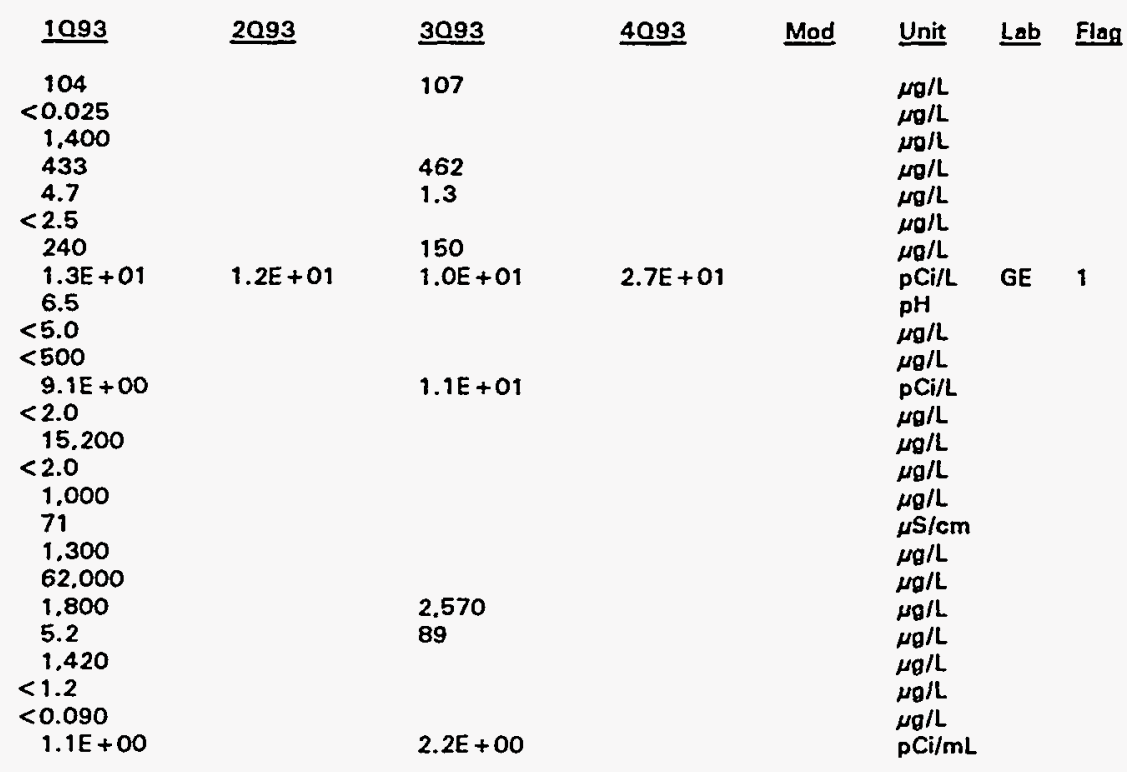

\section{WELL RSC 3}

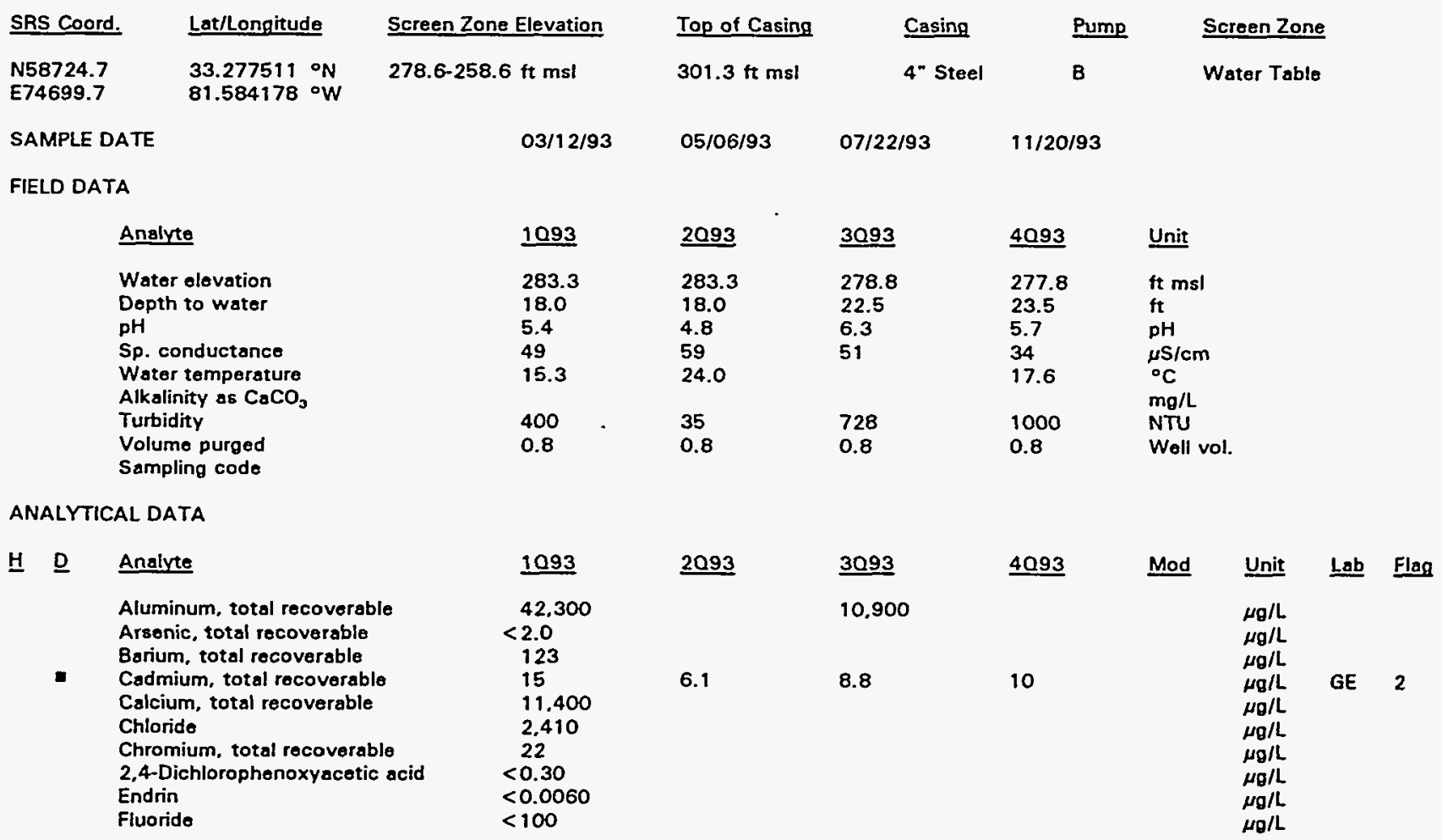

Note: Flagging levels, modifiers, and laboratories are for 4th quarter 1993 data only. See Appendix B for flagging criteria. - = exceeded holding time for 4th quarter 1993.

- =xceeded screening level or final primary drinking water standard for 4th quarter 1993. 
Well RSC 3 continued

\section{ANALYTICAL DATA}

H D Analyte

Groas alpha
Iron, total recoverable
Lead, total rocoverable
Lindane
Magnesium, total recoverable
Manganese, total recoverable
Mercury, total recoverable
Mothoxychlor
Nitrato-nitrite as nitrogen
Nonvolatile bota
pH
Phenols
Potassium, total recoverable
Radium, total alpho-emitting
Solenium, total rocoverablo
Silica, total recoverable
Silvor, total recoverablo
Sodium, total recoverable
Specific conductance
Sulfate
Total dissolved solids
Total organic carbon
Total organic halogens
Total phosphates (as P)
Toxapheno
2,4,5-TP (Silvex)
Tritium

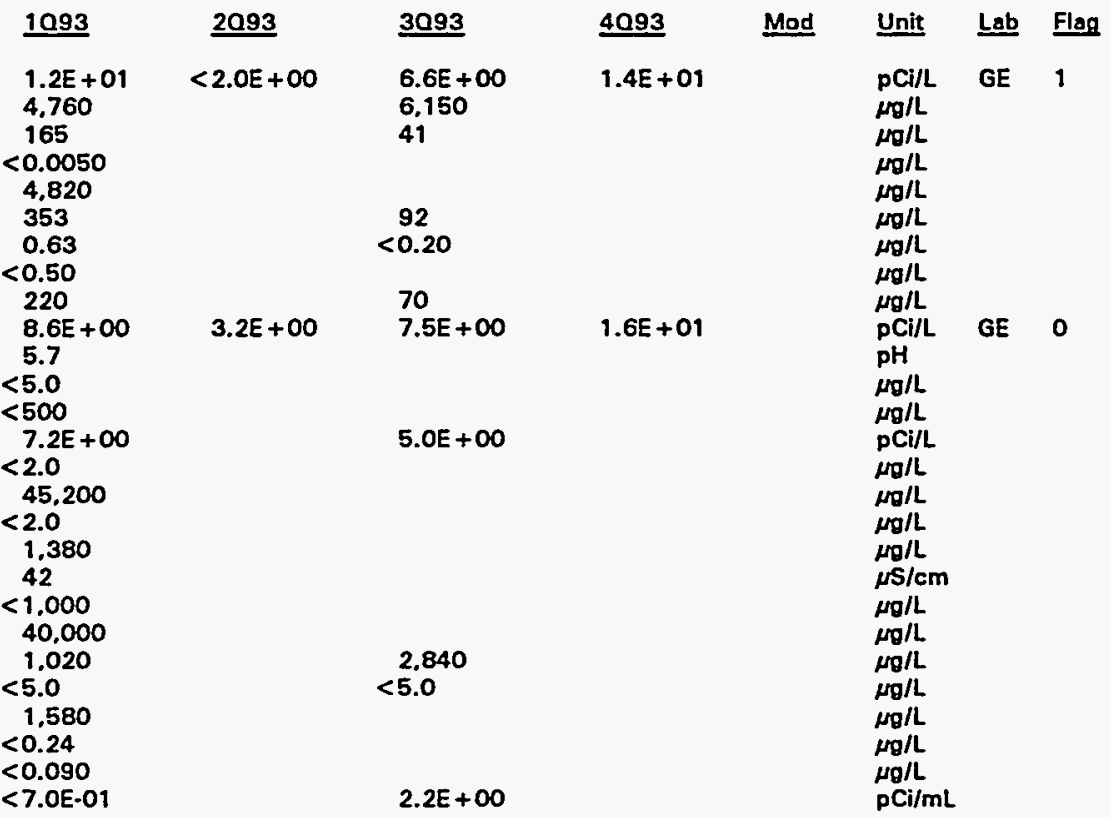

\section{WELL RSC 4}

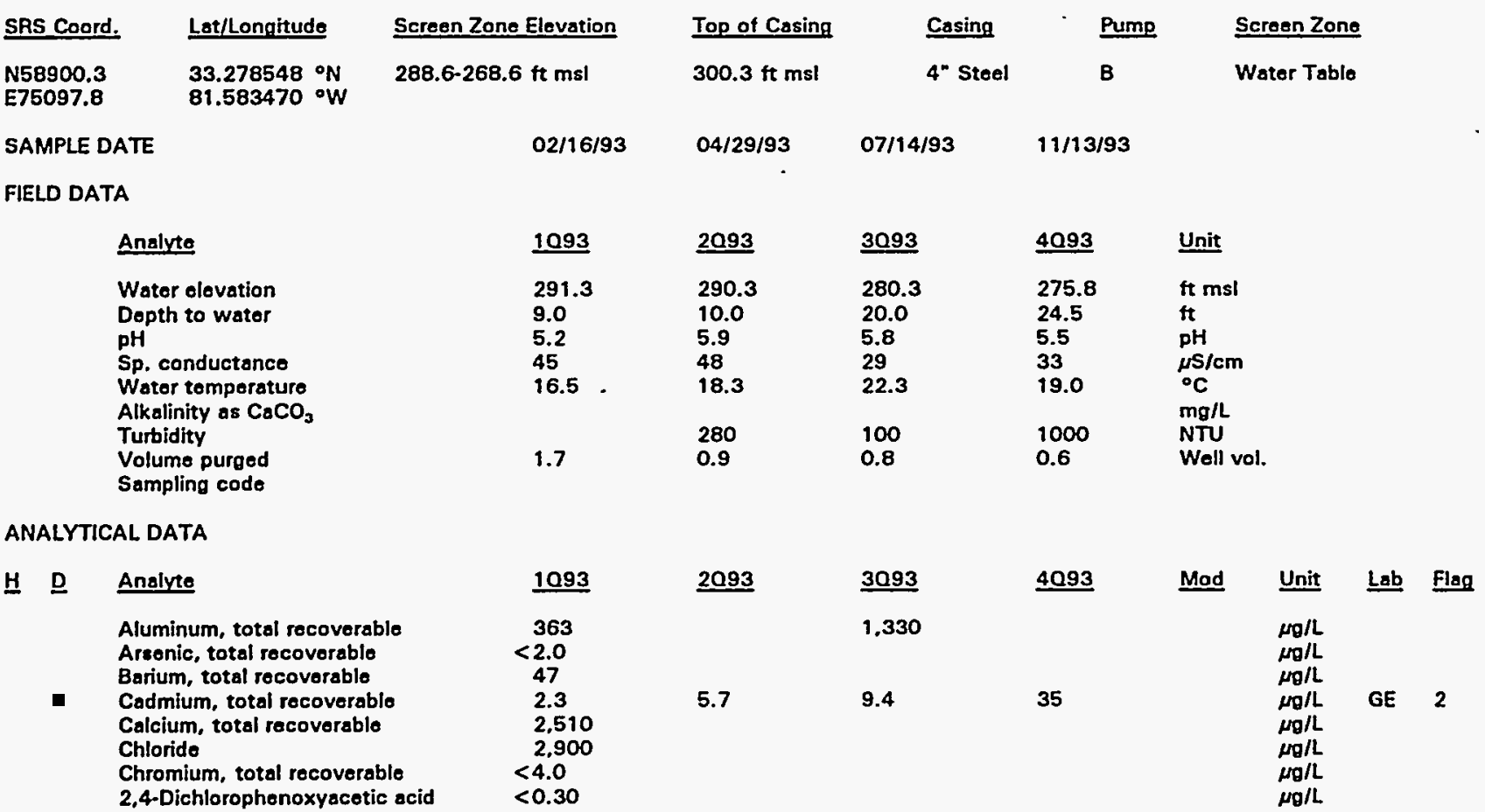

Note: Flagging levels, modifiers, and laboratories are for 4th quarter 1993 data only. See Appendix B for flagging criteria. - = exceeded holding time for 4th quarter 1993.

- = exceeded screening level or final primary drinking water standard for 4th quarter 1993. 
HE D Analyte

Endrin

Fluoride

- Gross alpha

Iron, total recoverable

Lead, total recovorable

Lindane

Manganese, total recoverable

Mercury, total recoverable

Methoxychlor

Nitrato-nitrite as nitrogen

- Nonvolatile bets

pH

Phenols

Potassium, total recoverable

Radium, total alpha-emitting

Solonium, total recoverable

Silica, total recovorable

Silver, total recoverable

Sodium, total recoverable

Specific conductance

Sulfate

Total dissolved solids

Total organic carbon

Total organic halogens

Total phosphates (as P)

Toxaphene

2,4,5-TP (Silvex)

Tritium
Magnesium, tatal recoverable
1093

$<0.0060$

$<100$

$2.6 E+01$

269

3.3

$<0.0050$

412

7.7

0.92

$<0.50$

210

1.9E + 01

5.3

$<5.0$

$<500$

$1.5 E+01$

$<2.0$

\begin{tabular}{l}
5.390 \\
\hline
\end{tabular}

$<2.0$

1,450

30

1.300

27,000

1.290

16

2.050

$<0.24$

$<0.090$

$1.1 E+\infty$

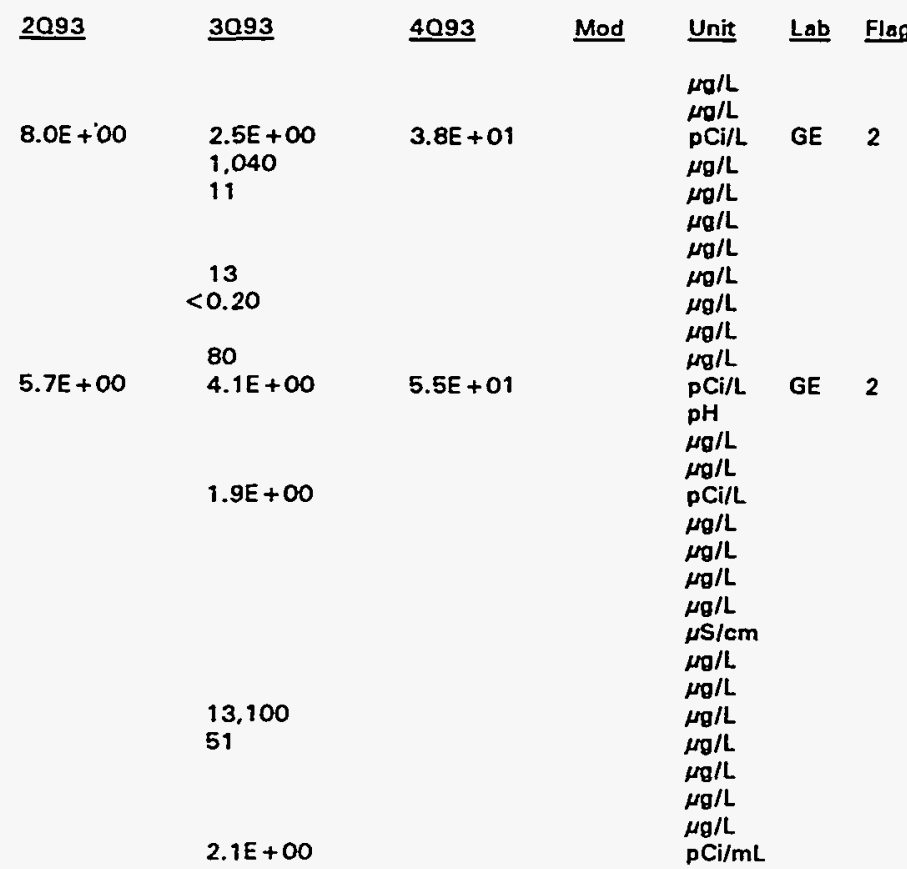

\section{WELL RSC 5}

\section{Screen Zone Elovation \\ 278.3-258.3 ft mst}

$02 / 16 / 93$

$04 / 29 / 93$

\section{$\underline{2093}$}

282.4

22.5

5.8

152

18.5

300

1.6

Volume purged

Sampling code

\section{ANALYTICAL DATA}

H $\underline{D}$

Analyte
Aluminum, total recoverable
Arsenic, total recoverable
Barium, total recoverable
Cadmium, total recoverable
Calcium, total recoverable
Chloride

1093

29,800

$<2.0$

141

8.4

41.400

5.400 $\underline{2093}$

3093

$\underline{4093}$

Mod

20,800

2.4

4.7

$<10$
Pump Screen Zone

B

Water Table 
H D Analyte

Chromium, total rocoverable 2,4-Dichlorophenoxyacetic acid Endrin Fluorido

Gross alphe

Iron, total recoverable

Loed, total recoverable

Lindano

Magnesium, total recoverable

Mercury, total recoverable

Mothoxychlor

Nitrate-nitrite as nitrogen

$\mathrm{pH}$

Phonols

Potassium, total recoverable

Radium, total alpha-omitting

Solenium, total recoverable

Silica, total recoverable

Silver, total recoverable

Specific conductance

Sulfato

Total organic carbon

Total organic halogons

Total phosphates (as P)

Toxaphene

2,4,5-TP (Silvex)

Tritium
Manganese, total recoverable

Nonvolatilo bota

Sodium, total recoverablo

Totol dissolved solids

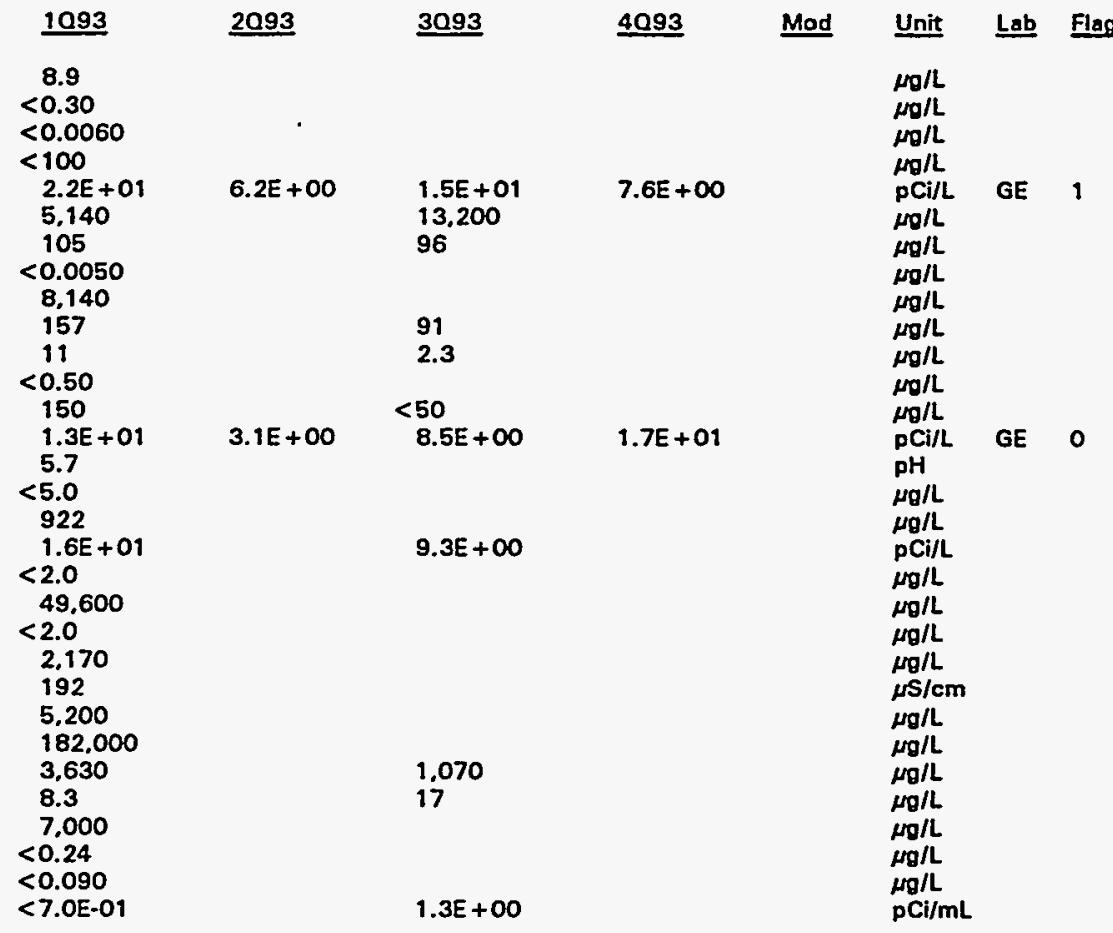

WELL RSC 6
Screon Zone Elevation

287.7-267.7 tt ms!

$03 / 15 / 93$

$05 / 14 / 93$

$07 / 20 / 93$

$\begin{array}{ll}\text { Casing } & \text { Pump } \\ 4^{\text {n Stoel }} & \text { B }\end{array}$

Scroen Zone

Water Table

SAMPLE DATE

\begin{tabular}{|c|c|c|c|c|}
\hline 1093 & 2093 & 3093 & 4093 & Unit \\
\hline $\begin{array}{l}296.1 \\
8.0 \\
5.5 \\
68 \\
13.1\end{array}$ & $\begin{array}{l}293.6 \\
10.5 \\
4.9 \\
57 \\
15.8\end{array}$ & $\begin{array}{l}288.1 \\
16.0 \\
5.4 \\
54 \\
22.1\end{array}$ & $\begin{array}{l}278.1 \\
26.0 \\
5.4 \\
44 \\
17.8\end{array}$ & $\begin{array}{l}\text { ft msl } \\
\text { ft } \\
\mathrm{pH} \\
\mu \mathrm{S} / \mathrm{cm} \\
{ }^{\circ} \mathrm{C}\end{array}$ \\
\hline $\begin{array}{l}75 \\
0.8\end{array}$ & $\begin{array}{l}105 \\
0.8\end{array}$ & $\begin{array}{l}273 \\
0.7\end{array}$ & $\begin{array}{l}1000 \\
0.4\end{array}$ & $\begin{array}{l}\text { NTU } \\
\text { Well vol. }\end{array}$ \\
\hline
\end{tabular}

ANALYTICAL DATA

H D Ansiyto

Aluminum, total recoverablo Arsenic, total recoverable

Barium, totsl recoverable

- Cadmium, total recoverablo
1093

1.410

$<2.0$

91

4.2 $\underline{2093}$

3093

1,310

6.2 $\underline{4093}$

Mod

Unit Lab Flag

$\mu \mathrm{g} / \mathrm{L}$

$\mu g / L$

$\mu g / L$

$\mu g / L$ GE 2

Note: Flagging levels, modifiers, and laboratories are for 4th quarter 1993 data only. See Appendix $B$ for flagging criteria. - = exceeded holding time for 4th quarter 1993.

a exceeded screening level or final primary drinking water standard for 4th quarter 1993. 
WSRC-TR-94-0354

Unclassified

Woll RSC 6 continued

ANALYTICAL DATA

H D Analvte

Calcium, total recoverable

Chloride

Chromium, total recoverable

2.4-Dichlorophenoxyacetic acid

Endrin

Fluoride

Grose alpha

Iron, total recoverable

Lead, total recoverable

Lindane

Magnosium, total recoverable

Manganese, total recoverable

Mercury, total recoverable

Methoxychlor

Nitrato-nitrite as nitrogen

Nonvolatile beta

pH

Phenols

Potassium, total recoverable

Radium, total alpha-emitting

Selenium, total recoverable

Silica, total recoverable

Silver, total recoverable

Sodium, total recoverable

Spocific conductance

Sulfate

Total dissolved solids

Total organic carbon

Total organic halogens

Total phosphates (as P)

Toxaphene

2,4,5-TP (Silvex)

Tritium

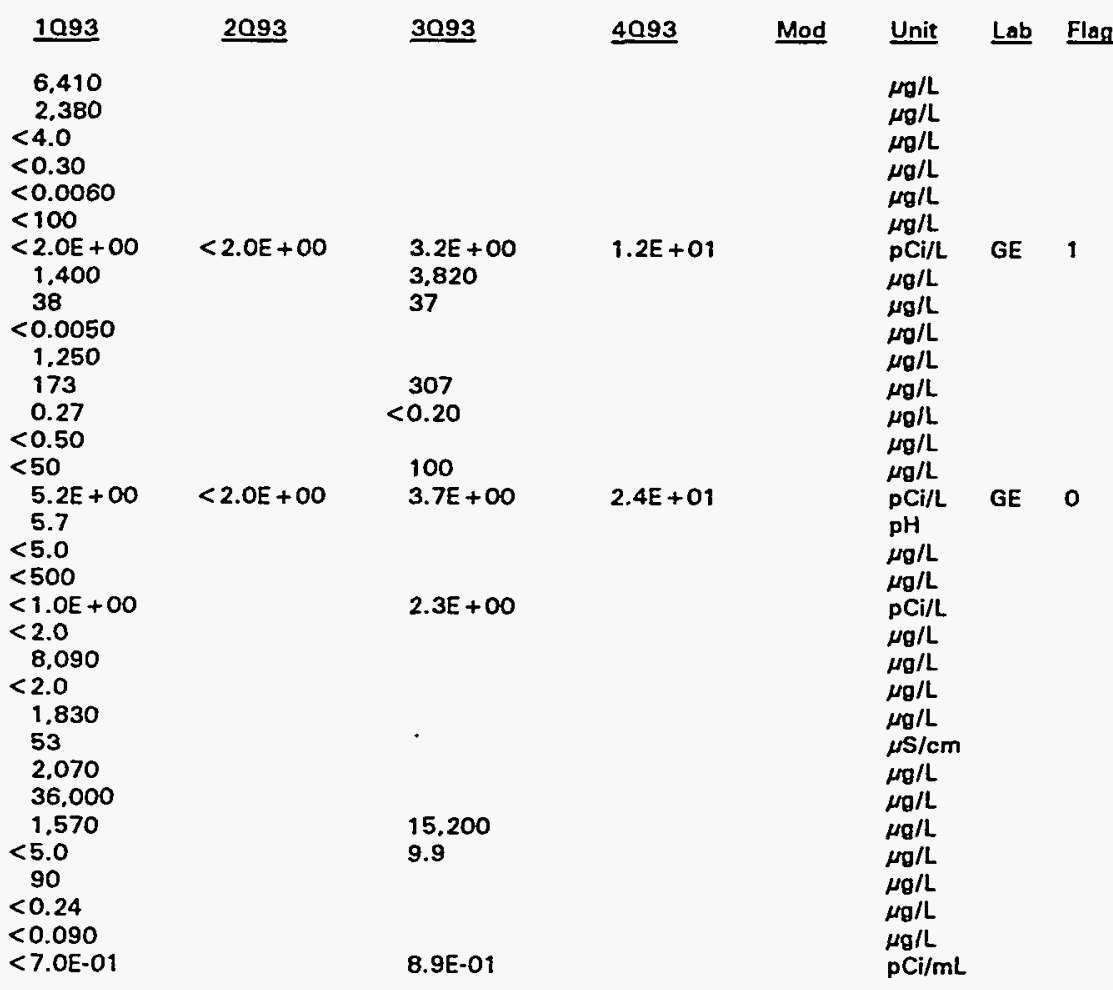

Note: Flagging levels, modifiers, and laboratories are for 4 th quarter 1993 data only. See Appendix B for flagging criteria.

- =xceeded holding time for 4th quarter 1993.

- =xceeded screening level or final primary drinking water standard for 4th quarter 1993. 
WELL RSC 7

$\begin{array}{ll}\text { SRS Coord. } & \text { Lat/Longitude } \\ \text { N58200.1 } & 33.277958{ }^{\circ} \mathrm{N} \\ \text { E75686.2 } & 81.580561^{\circ} \mathrm{W} \\ \text { SAMPLE DATE } & \end{array}$

$\begin{array}{lllll}\text { Screan Zone Elevation } & \text { Top of Casing } & \text { Casing } & \text { Pump } & \frac{\text { Screen Zone }}{283.4-263.4 \mathrm{ft} \mathrm{ms!}} \\ 307.8 \mathrm{ft} \mathrm{msl} & 4^{-} \text {Steel } & B & \text { Water Tablo }\end{array}$

SAMPLE DATE

$05 / 14 / 93 \quad 07 / 20 / 93 \quad 11 / 20 / 93$

FIELD DATA

Analyte
Water elevation
Depth to water
pH
Sp. conductance
Water temperature
Alkalinity as CaCO,
Turbidity
Volume purged
Sampling codo

\section{ANALYTICAL DATA}

\section{H D Analyte}

Aluminum, total recoverable

Arsenic, total recoverable

Barium, total recoverable

Cadmium, total recovorable

Calcium, total recoverable

Chlorido

Chromium, total recoverable

2,4-Dichlorophenoxyacetic acid

Endrin

Fluorido

Gross alpha

Iron, total recoverable

Lead, total recoverable

Lind ane

Magnesium, total recoverable

Manganoso, total recoverable

Mercury, total recoverable

Methoxychlor

Nitrato-nitrite as nitrogen

Nonvolatilo beta

pH

Phenols

Potassium, total recoverable

Radium, total alpha-omitting

Selenium, total recovorable

Silica, total recoverable

Silver, total rocoverable

Sodium, total recoverable

Specific conductance

Sulfate

Total dissolved solids

Total organic carbon

Total organic halogens

Total phosphates (as P)

Toxaphene

2,4,5-TP (Silvex)

Tritium

1093
292.8
15.0
6.4
133
13.5
140
0.9

$\underline{1093}$

7.140

$<2.0$

110

4.3

18,100

2.410

$<4.0$

$<0.30$

$<0.0060$

$<100$

$5.8 E+0$
14.500

365

$<0.0050$

1.380

201

0.56

$<0.50$

1,210

$1.7 E+01$

6.5

$<5.0$

1.920

$1.1 E+01$

$<2.0$

12.500

$<2.0$

2.120

108

2.110

109,000

$<1.000$

$<5.0$

800

$<0.24$

$<0.090$

$<7.0$-01

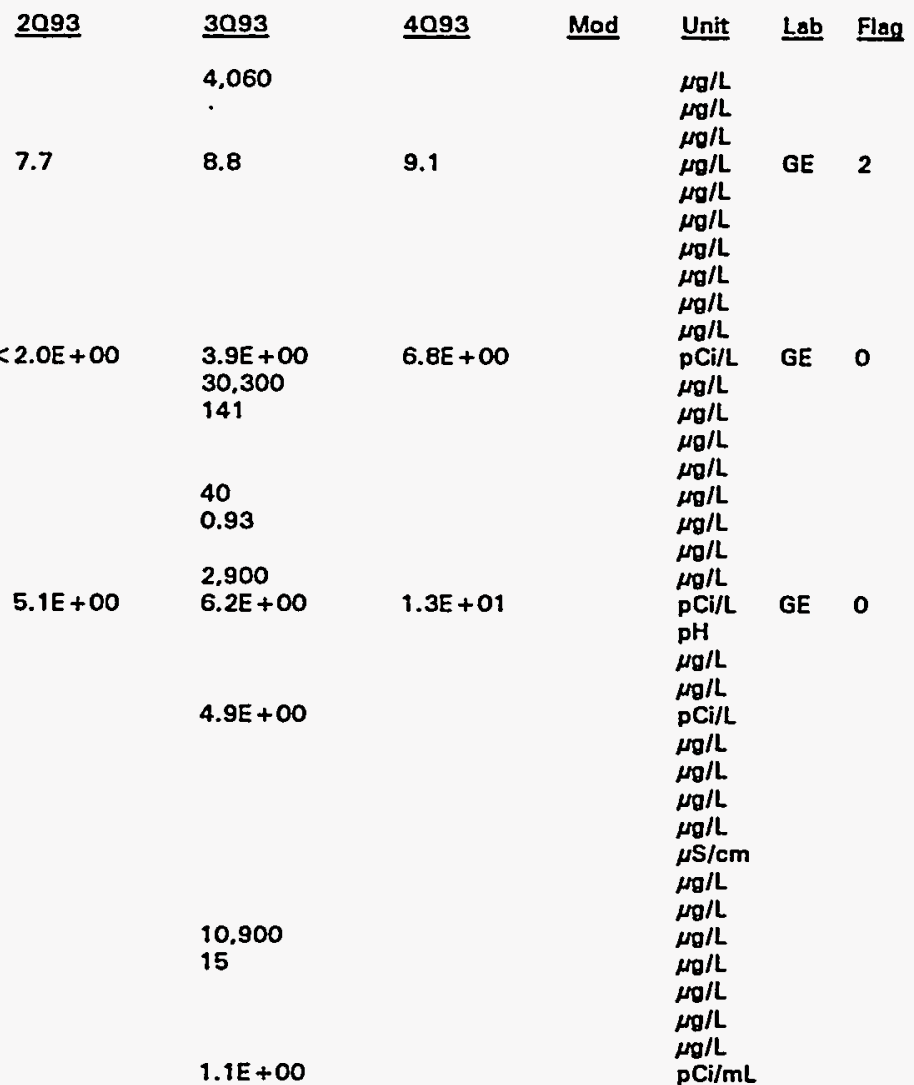

Note: Flagging lovels, modifiers, and laboratories are for 4th quarter 1993 data only. See Appendix B for flagging criteria. - = exceeded holding time for 4th quarter 1993.

- = exceeded screening level or finel primary drinking water standard for 4th quarter 1993. 


\section{WELL RSC 8}

\begin{tabular}{|c|c|c|c|c|c|c|}
\hline SRS Coord. & Lat/Longitude & Screen Zone Elevation & Top of Casing & Casing & Pump & Screen Zone \\
\hline $\begin{array}{l}\text { N57818.1 } \\
\text { E75684.0 }\end{array}$ & $\begin{array}{l}33.2771090^{\circ} \mathrm{N} \\
81.579826^{\circ} \mathrm{W}\end{array}$ & 299.3-271.3 ft msl & $308.8 \mathrm{ft} \mathrm{msl}$ & $4^{\prime \prime}$ Steel & B & Water Tablo \\
\hline SAMPLE & & $03 / 15 / 93$ & $05 / 14 / 93$ & $2 / 93$ & $0 / 93$ & \\
\hline
\end{tabular}

FIELD DATA

Analyte
Water olevation
Dopth to water
pH
Sp. conductance
Water temperature
Alkalinity as $\mathrm{CaCO}_{3}$
Turbidity
Volume purged
Sampling code

ANALYTICAL DATA

1093
301.8
7.0
5.8
53
13.5
150

2093
301.8
7.0
5.4
44
20.0
25

$\underline{3093}$

4093 Unit

291.8

17.0

6.4

33

20.0

5.8

35

18.4

822

1000

\section{tt msl}

tt

$\mathrm{pH}$

$\mu \mathrm{S} / \mathrm{cm}$

${ }^{\circ} \mathrm{C}$

$\mathrm{mg} / \mathrm{L}$

NTU

Well vol.

H D Analyte

Aluminum, total recoverable

Arsenic, total recoverable

Barium, total recoverable

- Cadmium, total recoverable

Calcium, total recoverable

Chloride

Chromium, total recoverable

2,4-Dichlorophenoxyacetic acid

Endrin

Fluoride

- Gross alpho

Iron, total recoverable

Lead, total recoverable

Lindane

Magnesium, total recoverable

Manganese, total recoverable

Mercury, total recoverable

Methoxychlor

Nitrate-nitrite as nitrogen

Nonvolatile bets

$\mathrm{pH}$

Phenols

Potassium, total recoverable

Radium, total alpha-emittin

Selenium. total recoverable

Silica, total recoverable

Silver, total recoverable

Sodium, total recoverable

Specific conductance

Sulfate

Total dissolved solids

Total organic carbon

Total organic halogens

Total phosphates (as P)

Toxaphone

2.4.5-TP [Silvex)

Tritium

\begin{tabular}{|c|c|c|c|}
\hline 1093 & 2093 & 3093 & 4093 \\
\hline 5.850 & & 6.100 & \\
\hline$<2.0$ & & & \\
\hline 108 & & & \\
\hline 12 & 2.6 & 4.5 & 8.5 \\
\hline 12,400 & & & \\
\hline 701 & & & \\
\hline$<4.0$ & & & \\
\hline$<0.30$ & & & \\
\hline$<0.0060$ & & & \\
\hline$<100$ & & & \\
\hline $2.8 E+01$ & $2.3 E+\infty 0$ & $8.3 E+\infty 0$ & $2.7 E+01$ \\
\hline $\begin{array}{l}8.350 \\
150\end{array}$ & & 29.900 & \\
\hline$<0.0050$ & & & \\
\hline 2.070 & & & \\
\hline 304 & & 85 & \\
\hline 0.48 & & 0.33 & \\
\hline$<0.50$ & & & \\
\hline$<50$ & & $<50$ & \\
\hline $9.0 E+O 0$ & $<2.0 E+00$ & $8.4 E+00$ & 3.7E + 01 \\
\hline 5.9 & & & \\
\hline $\begin{array}{r}<5.0 \\
510\end{array}$ & & & \\
\hline 510 & & & \\
\hline $\begin{aligned} & 5.6 E+00 \\
&<2.0\end{aligned}$ & & $5.7 E+00$ & \\
\hline$<2.0$ & & & \\
\hline 14,100 & & & \\
\hline$<2.0$ & & & \\
\hline 528 & & & \\
\hline 43 & & & \\
\hline 6.770 & & & \\
\hline 56,000 & & & \\
\hline 2,010 & & 3,710 & \\
\hline 8.4 & & 13 & \\
\hline 800 & & & \\
\hline$<0.24$ & & & \\
\hline$<0.090$ & & & \\
\hline & & $1.2 \mathrm{E}+00$ & \\
\hline
\end{tabular}

Note: Flagging levels, modifiers, and laboratories are for 4th quarter 1993 data only. See Appendix $B$ for flagging criteria. - = exceeded holding time for 4th quarter 1993.

- = exceeded screening level or final primary drinking water standard for 4th quarter 1993. 


\section{WELL.RSC 9}

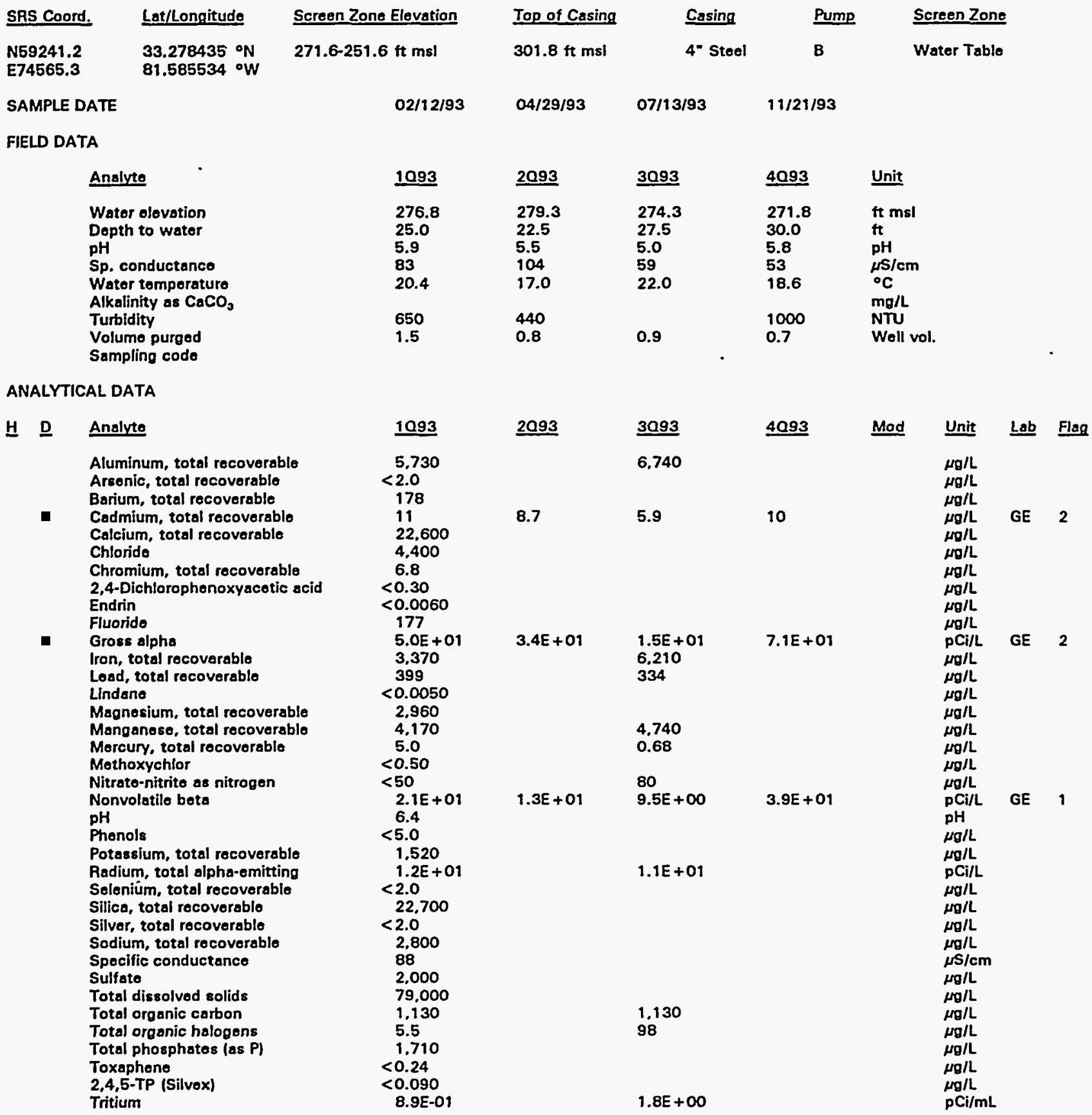

Note: Flagging levels, modifiers, and laboratories are for 4th quarter 1993 data only. See Appendix B for flagging criteria.

- = exceeded holding time for 4th quarter 1993.

I = exceeded screening level or final primary drinking water standard for 4th quarter 1993. 


\section{WELL RSC 10}

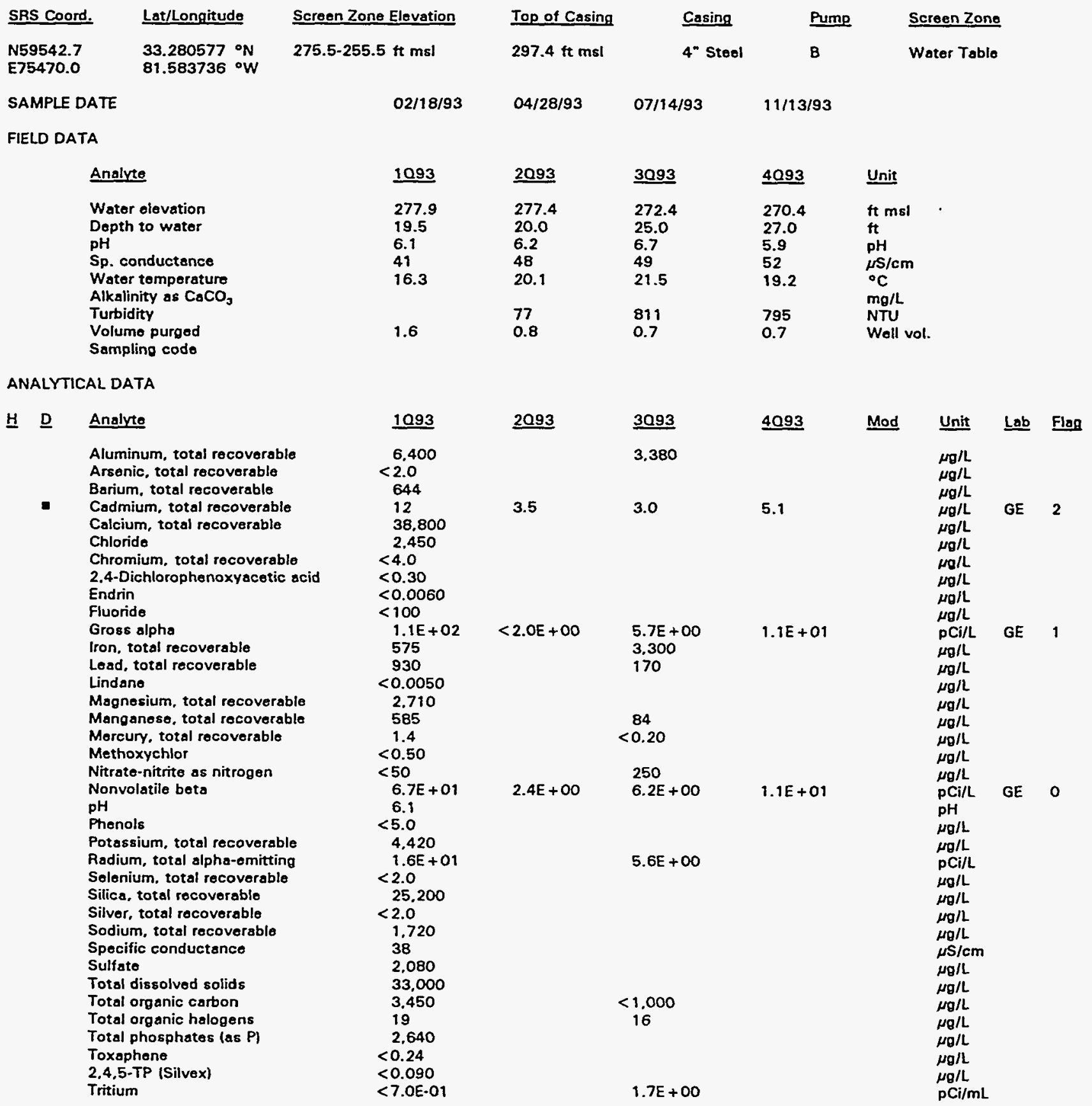

Note: Flagging levels, modifiers, and laboratories are for 4th quarter 1993 data only. See Appendix $B$ for flagging criteria. - = exceeded holding time for 4th quarter 1993.

- = exceeded screening level or final primary drinking water stendard for 4th quarter 1993.

\section{C-40 • R-Area Reactor 1993 Annual Report}


WELL RSD 1

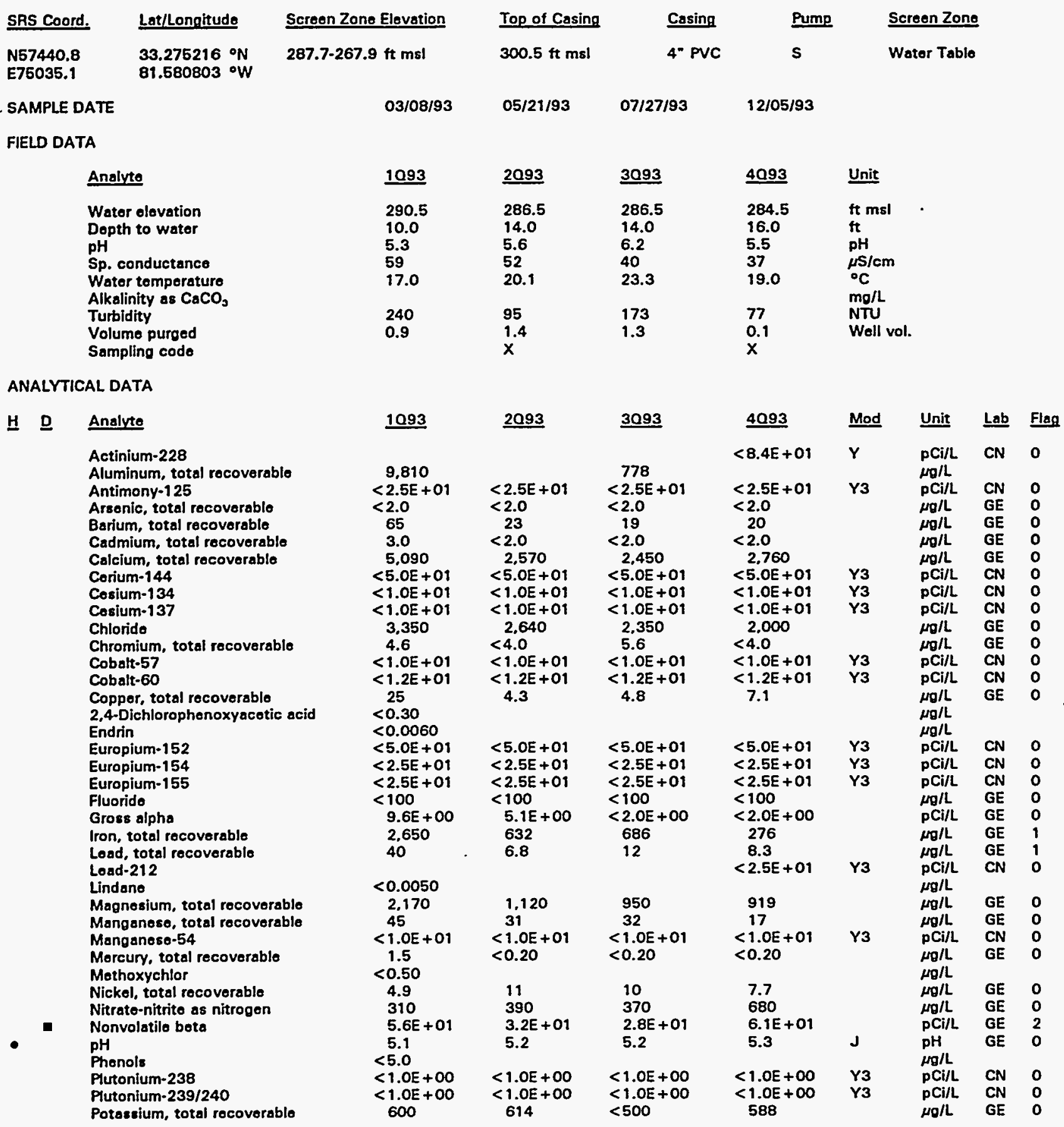

Note: Flegging levels, modifiers, and laboratories are for 4th quarter 1993 data only. See Appendix B for flagging criteria. - = exceeded holding time for 4th quarter 1993.

- =xceeded screening level or final primary drinking water standard for 4th quarter 1993. 


H. $\quad$ Analyte
Potassium-40
Promethium-144
Promethium-146
Radium, total alpha-emitting
Ruthenium-106
Solenium, total recoverable
Silica, total recovorable
Silvor, total recoverable
Sodium, total recoverable
Sodium-22
Specific conductance
Strontium-90
Sulfate
Thorium-234
Total dissolved solids
Total organic carbon
Total organic halogens
Total phosphates (as P)
Total suspended solids
Toxaphene
2,4,5-TP (Silvex)
Tritium
Yttrium-88
Zinc, total recoverable
Zinc-65

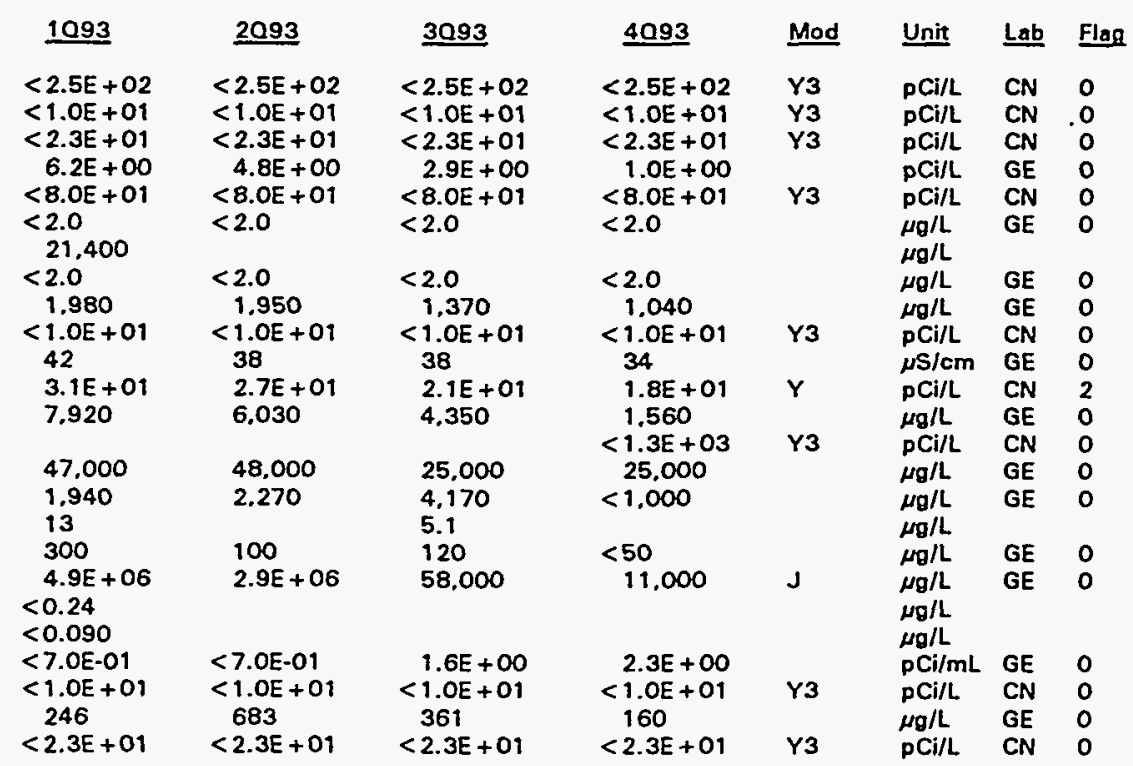

\section{WELL RSD 2A}

\begin{tabular}{|c|c|}
\hline SRS Coord. & Lat/Longitude \\
\hline $\begin{array}{l}\text { N57448.5 } \\
\text { E74811.5 }\end{array}$ & $\begin{array}{l}33.274869 \circ \mathrm{o} \\
81.581407 \circ \mathrm{W}\end{array}$ \\
\hline
\end{tabular}

SAMPLE DATE

FIELD DATA

\section{Screen Zone Elevation}

$02 / 23 / 93$

\section{Top of Casing}

$301.2 \mathrm{ft} \mathrm{msl}$

$05 / 05 / 93$

\section{Casing}

Steel

\section{Pump}

B
ScreenZone

Water Tablo

Analyte
Water elevation
Depth to water
pH
Sp. conductance
Water temperature
Alkalinity as CaCO
Turbidity
Volume purged
Sampling code

ANALYTICAL DATA

$\begin{array}{lll}\underline{1093} & \underline{2093} \\ 290.7 & & 290.2 \\ 10.5 & & 11.0 \\ 6.1 & 5.8 \\ 85 & 120 \\ 17.1 & 19.5 \\ 521 & 48 \\ x & \end{array}$

3093
286.2
15.0
6.3
102
23.3
160

$\begin{array}{ll}4093 & \text { Unit } \\ 286.2 & \mathrm{ft} \mathrm{msl} \\ 15.0 & \mathrm{ft} \\ 6.1 & \mathrm{pH} \\ 117 & \mu \mathrm{S} / \mathrm{cm} \\ 24.1 & { }^{\circ} \mathrm{C} \\ & \mathrm{mg} / \mathrm{L} \\ 371 & \text { NTU } \\ & \text { Well vol. }\end{array}$

$\underline{H} \quad \underline{D}$

Analyte

Aluminum, total recoverable Arsenic, total recoverable Barium, total recoverable

- Cadmium, total recoverable Calcium, total recoverable Chloride

Chromium, total recoverable 2.4-Dichlorophenoxyecetic acid Endrin Fluoride

2093

3093

69

12
4093

Mod

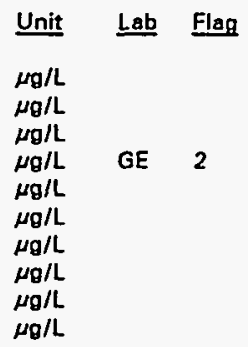

Note: Flagging levels, modifiers, and laboratories are for 4th quarter 1993 data only. See Appendix B for flagging criteria. - = exceeded holding time for 4 th quarter 1993.

- exceeded screening level or final primary drinking water standard for 4th quarter 1993. 
H D Analyte

Gross alphs

Iron, total recovorable

Lead, total recoverable

Lindano

Magnexium, total recoverable

Manganeso, total recovorable

Mercury, total recoverable

Mothoxychlor

Nitrato-nitrite as nitrogen

- Nonvolatilo bota

pH

Phonols

Potassium, total rocoverable

Radium, total alpha-emitting

Solonium, total recoverable

Silica, total recoverable

Silver, total recoverable

Sodium, total recoverable

Specific conductance

Sulfate

Total dissolved solids

Total oraanic carbon

Total organic halogens

Total phosphates (as PI

Toxapheno

2,4,5-TP (Silvox)

Tritium

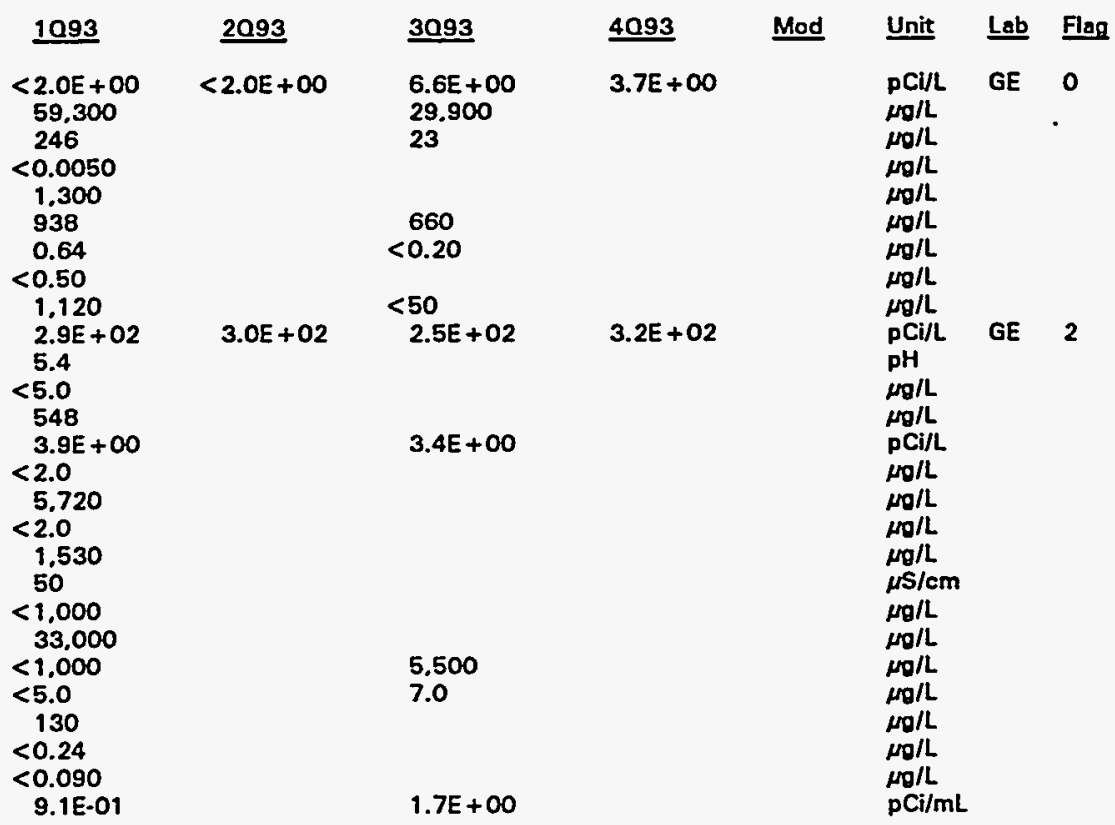

\section{WELL RSD 2B}

\begin{tabular}{|c|c|}
\hline SAS Caard. & Lat/Longitude \\
\hline $\begin{array}{l}\text { N57468.9 } \\
\text { E74810.7 }\end{array}$ & 81.274913 \\
\hline
\end{tabular}

SAMPLE DATE

FIELD DATA

Analvte

Water olovation

Depth to water

pH

Sp. conductance

Water tomperature

Alkalinity as $\mathrm{CaCO}_{3}$

Turbidity

Volume purged

Sampling code

\section{ANALYTICAL DATA}

H D Analyte

Aluminum, total recoverable Arsenic, total recoverable

Barium, total rocoverable

Cadmium, total recoverable

Calcium, total recoverable

Chloride

Chromium, total recoverable

Fluoride
Screen Zone Elevation

$02 / 23 / 93$

05/05/93

$07 / 21 / 93$

$11 / 14 / 93$

$\begin{array}{ll}1093 & \underline{2093} \\ 290.6 & 291.6 \\ 13.0 & 12.0 \\ 5.1 & 5.1 \\ 28 & 40 \\ 17.9 & 19.5 \\ & 30 \\ X & x\end{array}$

$\underline{3093}$

4093

Unit

tt msl

ft

$\mathrm{pH}$

$\mathrm{rS} / \mathrm{cm}$

$\mathrm{mg} / \mathrm{L}$.

NTU

Dry
D

Dry

Well vol.
Screen Zone

Water Table

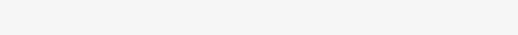

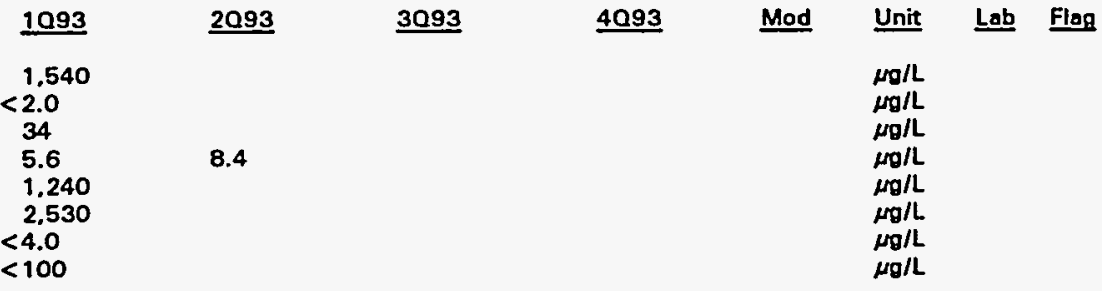

Note: Flagging levels, modifiers, and laboratories are for 4th quarter 1993 data only. See Appendix B for flagging criteria. - = exceeded holding time for 4th quarter 1993.

= exceeded screening level or final primery drinking water standard for 4th quarter 1993. 
WSRC-TR-94-0354

Unclassified

Well RSO 2B continued

\section{ANALYTICAL DATA}

\section{$\underline{H} \underline{D}$ \\ Analyte}

Iron, total recoverable

Lead, total recoverable

Magnesium, total recoverable

Manganese, total recoverable

Mercury, total recovorable

Nitrate-nitrite as nitrogen

Nonvolatila beto

pH

Phenols

Potassium, total recoverable

Radium, total alpha-emitting

Selenium, total recoverable

Silica, total recoverable

Silver, total recoverable

Sodium, total recoverable

Specific conductance

Sulfate

Total dissolved solids

Total organic carbon

Total organic halogens

Total phosphates (as P)

Tritium
Gross alpha

$\underline{3093}$

$\underline{4093}$

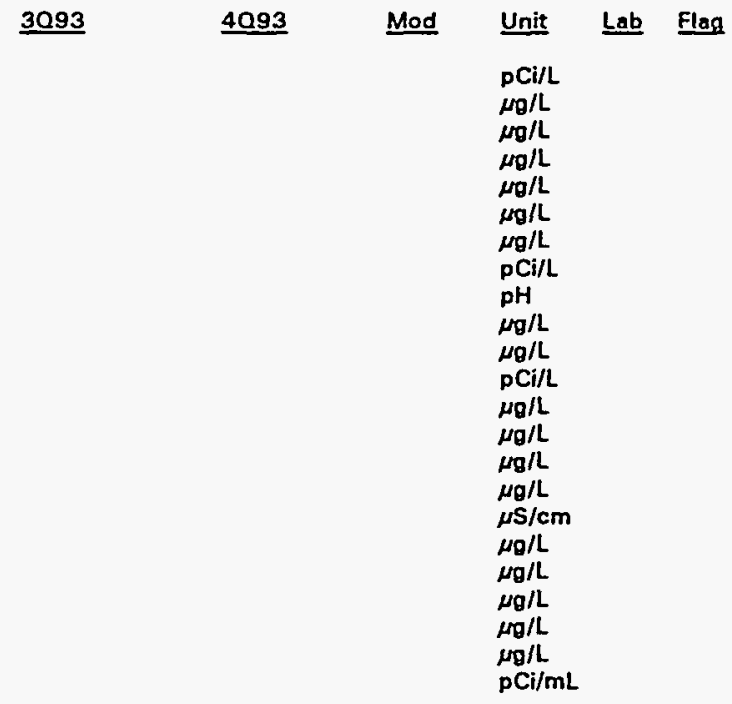

WELL RSD 2C

$6.6 E+00 \quad<2.0 E+00$
228
28
1.020
7.6
$<0.20$
1.520
$6.3 E+02$
5.0
$<5.0$
515
$1.0 E+01$
$<2.0$
6.550
$<2.0$
1.510
35
1.120
26.000
$<1.000$
12
120
$<7.0 E-01$

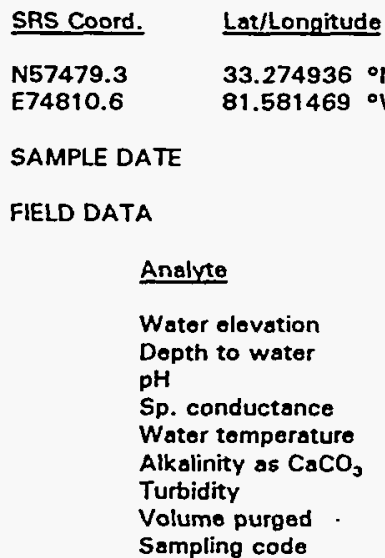

ANALYTICAL DATA
Scroen Zone Elevation

$02 / 23 / 93$

1093
290.7
11.0
5.6
63
18.8
595
$x$

20.93
290.7
11.0
5.9
70
19.5
18

886

$<2.0$

Aluminum, total recoverable Arsonic, total recoverable

Barium, total recoverable

- Cadmium, total recoverable Calcium, total recoverable Chlorido

Chromium, total recoverable 2,4-Dichlorophenoxyacetic acid Endrin

Fluoride

- Gross alpha

Iron, total recoverable

\section{$\underline{2093}$}

$\underline{3093}$

\begin{tabular}{|c|c|c|c|c|c|c|c|}
\hline Q93 & $\underline{2093}$ & $\underline{3093}$ & 40993 & Mod & Unit & $\underline{\text { Lab }}$ & Flag \\
\hline & & 4.260 & & & $\begin{array}{c}\mu \mathrm{g} / \mathrm{L} \\
\mu \mathrm{g} / \mathrm{L} \\
\mu \mathrm{g} / \mathrm{L}\end{array}$ & & \\
\hline $\begin{array}{l}.1 \\
.150 \\
.830 \\
.0 \\
.30 \\
.0060 \\
00\end{array}$ & 7.1 & 6.1 & 23 & & $\begin{array}{l}\mu g / L \\
\mu g / L \\
\mu g / L \\
\mu g / L \\
\mu g / L \\
\mu g / L \\
\mu g / L\end{array}$ & GE & 2 \\
\hline$+\infty$ & $4.5 E+01$ & $\begin{array}{c}<2.0 E+00 \\
184\end{array}$ & $7.3 E+01$ & & pCi/L & GE & 2 \\
\hline
\end{tabular}

Note: Flagging levels, modifiers, and laboratories are for 4th quarter 1993 data only. See Appendix B for flagging criteria. - exceeded holding time for 4th quarter 1993.

E = exceeded screening level or final primary drinking water standard for 4th quarter 1993. 
H $\underline{D}$

Lead, total recoverable
Lindane
Magnesium, total rocovorable
Manganese, total recoverable
Mercury, total recoverable
Mothoxychlor
Nitrate-nitrite as nitrogen
Nonvolatile beta
pH
Phenols
Potessium, total recoverable
Radium, total alpha-emitting
Solenium, total recoverable
Silica, total recoverable
Silvar, total recoverable
Sodium, total recoverable
Specific conductance
Sulfate
Total dissolvad solids
Total organic carbon
Total organic halogens
Total phosphates las P)
Toxapheno
$2,4,5-T P$ (Silvex)
Tritium

1093
$<3.0$
$<0.0050$
2.710
9.1
5.5
$<0.50$
4,800
$5.5 E+03$
4.3
$<5.0$
1.410
$5.8 E+01$
$<2.0$
5,250
$<2.0$
2,330
60
$<1.000$
49,000
2.480
$<5.0$
$<50$
$<0.24$
$<0.090$
$2.1 E+00$

$\underline{3093}$

3.5

10

0.22

$5.2 E+03$

4,250

$6.4 E+03$

$6.5 E+01$

2.960
$<5.0$

$2.9 E+00$

4093 Mod Unit Lab Flag

$3.4 E+03$

$\mu / \mathrm{m}$

$\mu \mathrm{m} / \mathrm{L}$

$\mu / L$

$\mu / L$

$\mu \mathrm{g} / \mathrm{L}$

pCi/L GE 2

pH

$\mu \mathrm{g} / \mathrm{L}$

$\mu \mathrm{g} / \mathrm{L}$

pCilL

$\mu / L$

$\mu \mathrm{g} / \mathrm{L}$

$\mu \mathrm{rg} / \mathrm{L}$

$\mu \mathrm{g} / \mathrm{L}$

$\mu / L$

$\mu \mathrm{g} / \mathrm{L}$

$\mu g / L$

$\mu \mathrm{g} / \mathrm{L}$

$\mu \mathrm{g} / \mathrm{L}$

$\mathrm{pCi} / \mathrm{mL}$

\section{WELL RSD 3}

\begin{tabular}{|c|c|c|c|c|c|c|c|c|c|}
\hline SRS Coord. & Lat/Longitude & Screen Zone Elevation & Top of Casing & Casing & Pump & \multicolumn{3}{|c|}{ Screen Zone } & \\
\hline $\begin{array}{l}\text { N57451.6 } \\
\text { E74702,3 }\end{array}$ & $\begin{array}{l}33.274698{ }^{\circ} \mathrm{N} \\
81.581701{ }^{\circ} \mathrm{W}\end{array}$ & 289.1-269.3 ft msl & $300.8 \mathrm{ft} \mathrm{msl}$ & 4" PVC & $\mathbf{S}$ & \multicolumn{3}{|c|}{ Water Table } & \\
\hline \multicolumn{2}{|c|}{ SAMPLE DATE } & $03 / 02 / 93$ & $05 / 21 / 93$ & $07 / 27 / 93$ & $12 / 05 / 93$ & & & & \\
\hline \multicolumn{10}{|l|}{ FIELD DATA } \\
\hline & Analyte & 1093 & $\underline{2093}$ & $\underline{3093}$ & 4093 & \multicolumn{4}{|l|}{ Unit } \\
\hline & $\begin{array}{l}\text { Water elevation } \\
\text { Depth to water } \\
\text { pH } \\
\text { Sp. conductance } \\
\text { Water temperature } \\
\text { Alkalinity as } \mathrm{CaCO}_{3} \\
\text { Turbidity } \\
\text { Volume purged } \\
\text { Sampling code }\end{array}$ & $\begin{array}{l}291.3 \\
9.5 \\
5.4 \\
57 \\
14.0 \\
90 \\
1.9\end{array}$ & $\begin{array}{l}290.8 \\
10.0 \\
5.9 \\
42 \\
19.6 \\
100 \\
1.8 \\
X\end{array}$ & $\begin{array}{l}6.4 \\
44 \\
26.0 \\
122\end{array}$ & $\begin{array}{l}285.8 \\
15.0 \\
5.6 \\
47 \\
24.0 \\
120 \\
0.1 \\
X\end{array}$ & \multicolumn{2}{|c|}{$\begin{array}{l}\text { ft msl } \\
\text { ft } \\
\text { pH } \\
\mu \text { s/cm } \\
{ }^{\circ} \mathrm{C} \\
\mathrm{mg} / \mathrm{L} \\
\text { NTU } \\
\text { Well vol. }\end{array}$} & & \\
\hline \multicolumn{10}{|c|}{ ANALYTICAL DATA } \\
\hline \multirow[t]{2}{*}{$\underline{H} \quad \underline{D}$} & Analyte & 1093 & $\underline{2093}$ & 3093 & 4093 & Mod & Unit & $\underline{L a b}$ & Flag \\
\hline & $\begin{array}{l}\text { Actinium-228 } \\
\text { Aluminum, total recoverable } \\
\text { Antimony-125 } \\
\text { Arsenic, total recoverable } \\
\text { Barium, total recoverable } \\
\text { Cadmium, total recoverable } \\
\text { Calcium, total recovorable } \\
\text { Corium-144 } \\
\text { Cosium-134 } \\
\text { Cosium-137 }\end{array}$ & $\begin{aligned} & 1.310 \\
&< 2.5 E+01 \\
&< 2.0 \\
& 23 \\
&< 2.0 \\
& 5.480 \\
&< 5.0 E+01 \\
&<1.0 E+01 \\
&<1.0 E+01\end{aligned}$ & $\begin{aligned}<2.5 E+01 \\
<2.0 \\
16 \\
<2.0 \\
3.970 \\
<5.0 E+01 \\
<1.0 E+01 \\
<1.0 E+01\end{aligned}$ & $\begin{aligned} & 754 \\
< & 2.5 E+01 \\
< & 2.0 \\
& 17 \\
< & 2.0 \\
& 4.200 \\
< & 5.0 E+01 \\
< & 1.0 E+01 \\
< & 1.0 E+01\end{aligned}$ & $\begin{aligned}<8.4 E+01 \\
\\
<2.5 E+01 \\
<2.0 \\
17 \\
<2.0 \\
3.630 \\
<5.0 E+01 \\
<1.0 E+01 \\
<1.0 E+01\end{aligned}$ & $\begin{array}{l}\text { Y3 } \\
\text { Y3 }\end{array}$ & $\begin{array}{l}\mathrm{pCi} / \mathrm{L} \\
\mu g / L \\
\mathrm{pCi} / \mathrm{L} \\
\mu g / L \\
\mu \mathrm{g} / \mathrm{L} \\
\mu g / \mathrm{L} \\
\mu g / L \\
\mathrm{pCi} / \mathrm{L} \\
\mathrm{pCi} / \mathrm{L} \\
\mathrm{pCi} / \mathrm{L}\end{array}$ & $\begin{array}{l}\text { CN } \\
\text { CN } \\
\text { GE } \\
\text { GE } \\
\text { GE } \\
\text { GE } \\
\text { CN } \\
\text { CN } \\
\text { CN }\end{array}$ & $\begin{array}{l}0 \\
0 \\
0 \\
0 \\
0 \\
0 \\
0 \\
0 \\
0\end{array}$ \\
\hline
\end{tabular}

Note: Flagging levels, modifiers, and laboratories are for 4th quarter 1993 data only. See Appendix B for flagging criteria.

- = exceeded holding time for 4th quarter 1993.

= exceeded screening level or final primary drinking water stendard for 4th querter 1993. 


\section{ANALYTICAL DATA}

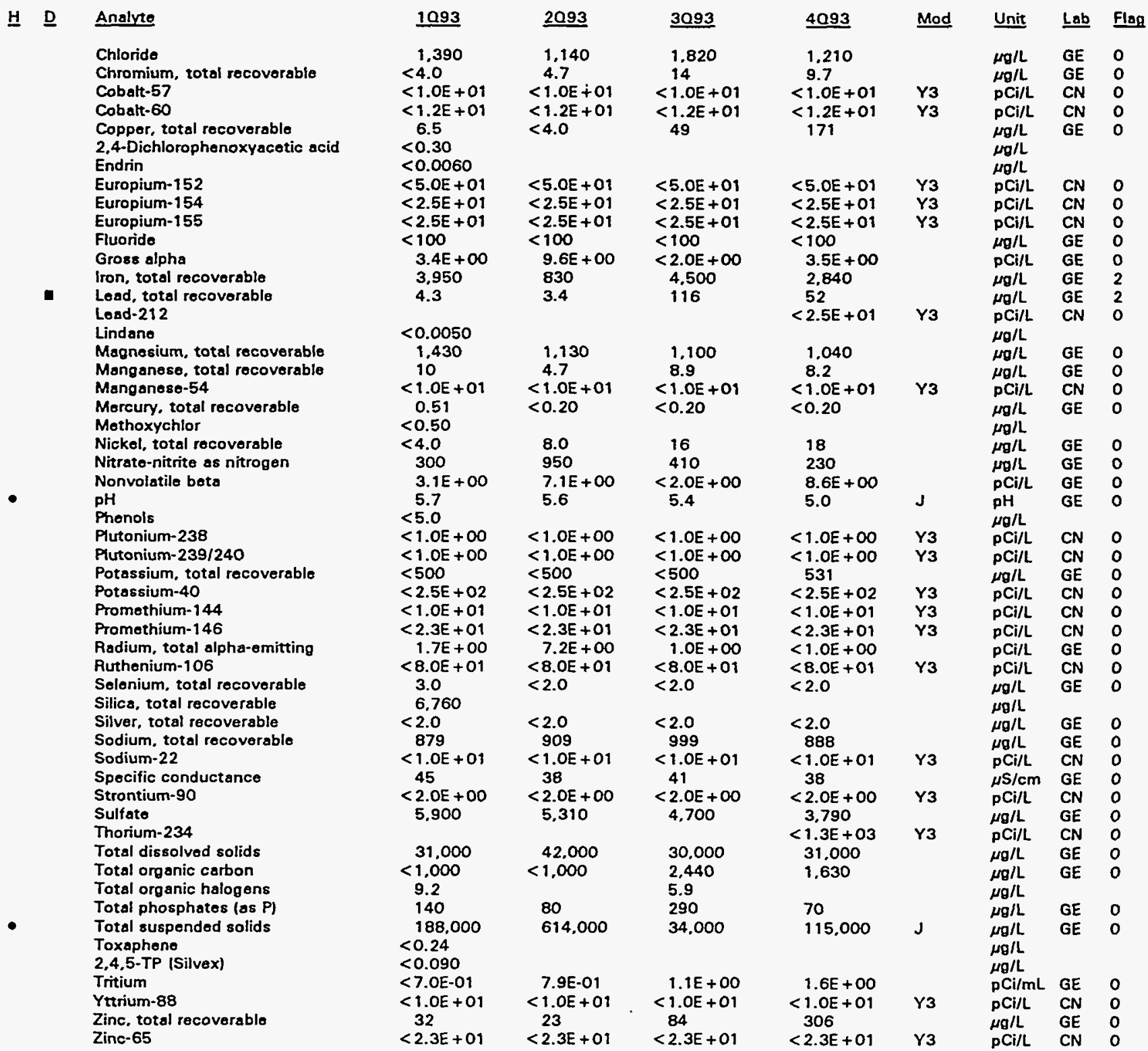

Note: Flagging levels, modifiers, and laboratories are for 4 th quarter 1993 data only. See Appendix B for flagging criterie. - exceeded holding time for 4th quarter 1993.

- = exceeded screening level or final primary drinking water standard for 4th quarter 1993. 
WELL RSD

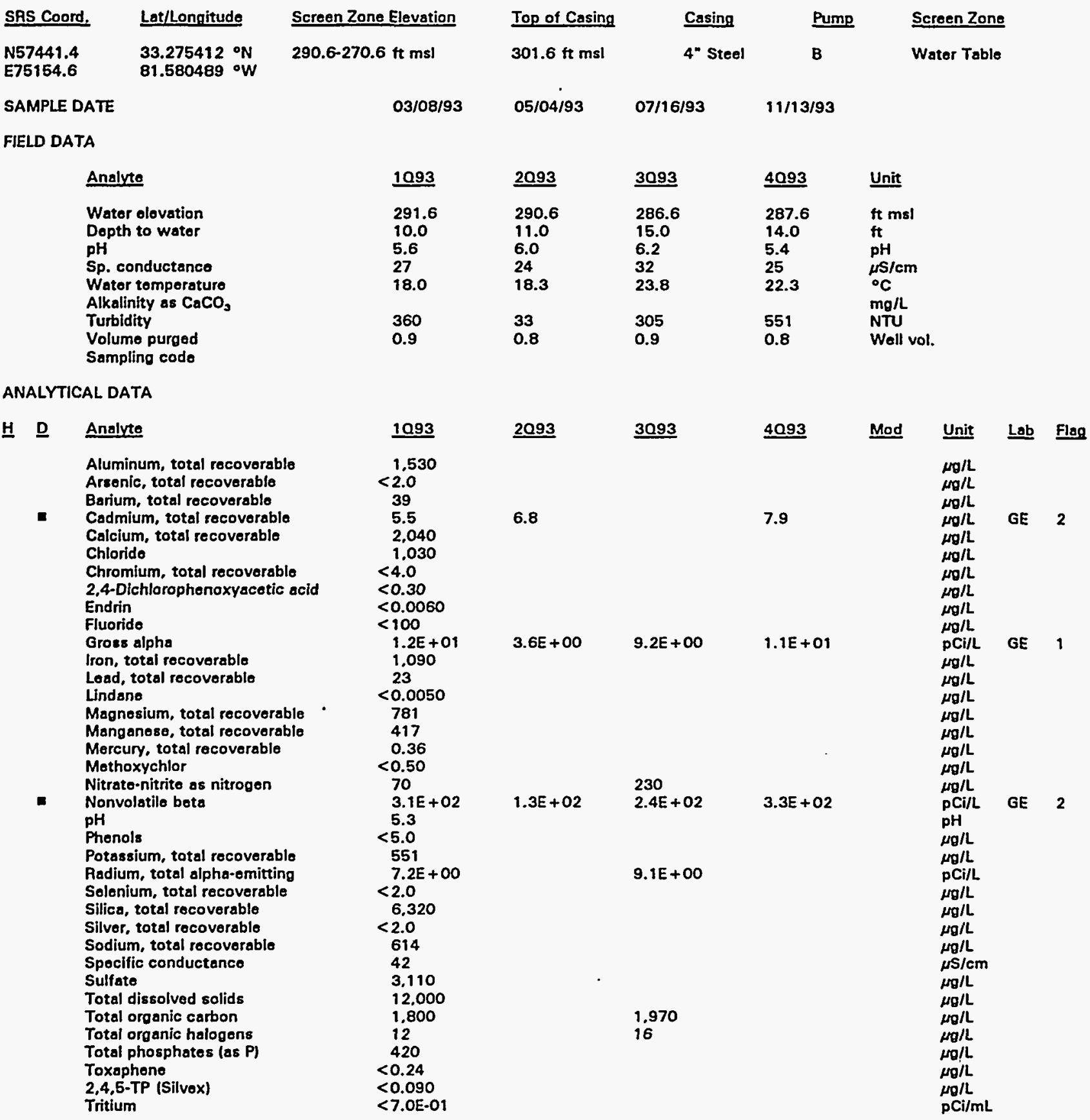

Note: Flagging levels, modifiers, and laboratories are for 4th quarter 1993 data only. See Appendix B for flagging criteria. - = exceeded holding time for 4th quarter 1993.

- = exceeded screening level or final primary drinking water standard for 4th quarter 1993. 


\section{WELL RSD 5}

\begin{tabular}{|c|c|c|c|c|c|c|}
\hline SRS Coord. & Lat/Longitude & Screen Zone Elovation & Top of Casing & Casing & Pump & Screen Zone \\
\hline $\begin{array}{l}\text { N57439.9 } \\
\text { E75207.0 }\end{array}$ & $\begin{array}{l}33.275495 \text { o } \\
81.580348 \text { oW }\end{array}$ & $289.6-269.6 \mathrm{ft} \mathrm{msl}$ & $301.7 \mathrm{ft} \mathrm{msl}$ & $4^{-}$Steel & B & Water Tablo \\
\hline SAMPLE DA & & $03 / 08 / 93$ & $05 / 04 / 93$ & $07 / 16 / 93$ & $11 / 13 / 93$ & \\
\hline FIELD DATA & & & & & & \\
\hline & & 1093 & $\underline{2093}$ & 3093 & 4093 & Unit \\
\hline & $\begin{array}{l}\text { olovation } \\
\text { to water } \\
\text { inductance } \\
\text { temperature } \\
\text { ity as } \mathrm{CaCO}\end{array}$ & $\begin{array}{l}291.2 \\
10.5 \\
5.5 \\
31 \\
18.6\end{array}$ & $\begin{array}{l}290.2 \\
11.5 \\
5.9 \\
30 \\
18.5\end{array}$ & $\begin{array}{l}285.7 \\
16.0 \\
4.6 \\
33 \\
23.2\end{array}$ & $\begin{array}{l}285.7 \\
16.0 \\
5.1 \\
29 \\
21.9\end{array}$ & $\begin{array}{l}\mathrm{ft} \mathrm{msl} \\
\mathrm{ft} \\
\mathrm{pH} \\
\mu \mathrm{H} / \mathrm{cm} \\
{ }^{\circ} \mathrm{C}\end{array}$ \\
\hline & o purged & $\begin{array}{l}370 \\
0.8\end{array}$ & $\begin{array}{l}70 \\
0.8\end{array}$ & $\begin{array}{l}243 \\
0.8\end{array}$ & $\begin{array}{l}1000 \\
0.8\end{array}$ & $\begin{array}{l}\text { NTU } \\
\text { Woll vol. }\end{array}$ \\
\hline
\end{tabular}

ANALYTICAL DATA

\section{H D Analyte}

Aluminum, total recoverable Arsenic, total recoverable

Barium, total recoverable

Cadmium, total recoverable Calcium, total recoverable

Chloride

Chromium, total recoverable

2.4-Dichlorophenoxyacetic acid Endrin

Fluoride

- Gross alpha

iron, total recoverable

Lead, total recoverable

Lindane

Magnesium, total recovorable

Manganese, total recoverable

Mercury, total recoverabie

Mothoxychlor

Nitrate-nitrite as nitrogen

- Nonvolatile beta

pH

Phenols

Potessium, total recoverable

Radium, total alpha-omitting

Selonium, total recoverable

Silica, total recoverable

Silver, total recoverable

Sodium, total recoverable

Specific conductance

Sulfate

Total dissolved solids

Total organic carbon

Total organic halogens

Total phosphates (as P)

Toxaphene

2,4,5-TP (Silvex)

Tritium

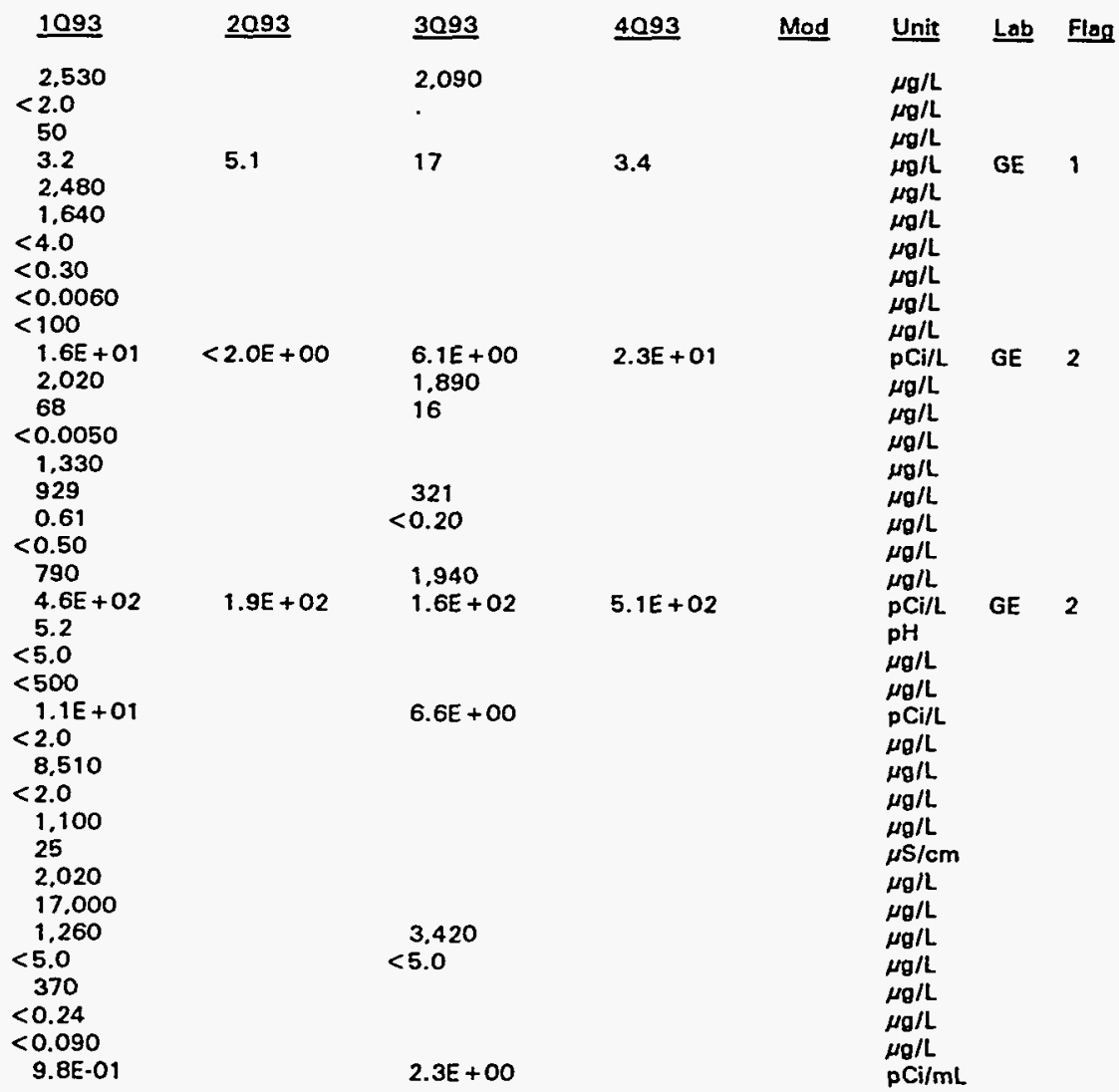

Note: Flagging levels, modifiers, and laboratories are for 4th quarter 1993 data only. See Appendix B for flagging criteria.

- exceeded holding time for 4 th quarter 1993.

a = exceeded screening level or final primary drinking water standard for 4th quarter 1993. 
WELL RSD 6

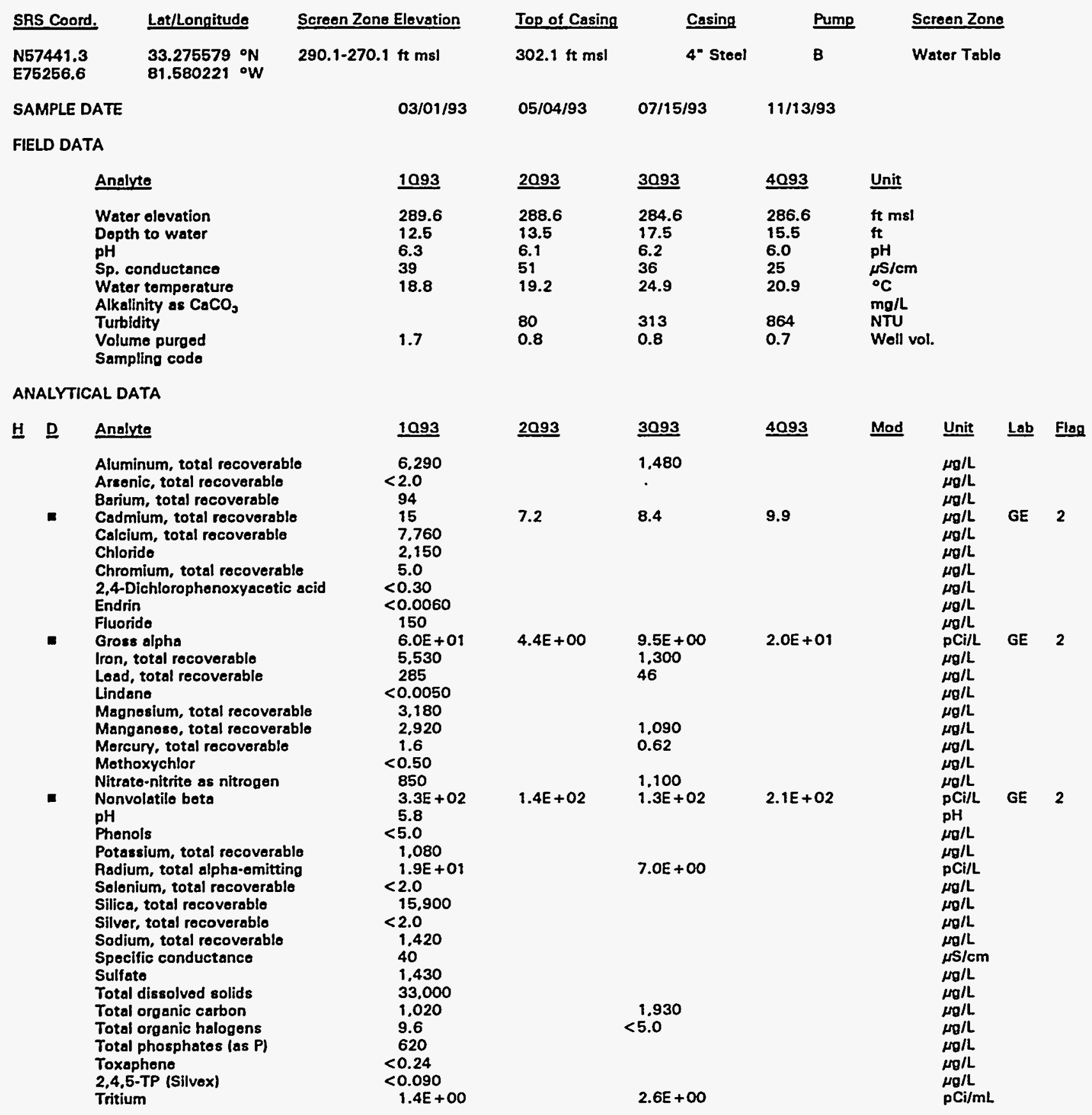

Note: Flagging levels, modifiers, and laboratories are for 4th quarter 1993 data only. See Appendix B for flagging criteria. - = exceeded holding time for 4th quarter 1993.

- = exceeded screening level or final primary drinking water standard for 4th quarter 1993. 
WELL RSD 7

\begin{tabular}{|c|c|c|c|c|c|c|}
\hline SAS Coord. & Lat/Longitude & Screen Zone Elevation & Top of Casing & Casing & Pump & Scroon Zone \\
\hline $\begin{array}{l}N 57394.3 \\
\text { E75178.4 }\end{array}$ & $\begin{array}{l}33.275347^{\circ} \mathrm{N} \\
81.580335 \circ \mathrm{W}\end{array}$ & $287.3-267.3 \mathrm{ft} \mathrm{msl}$ & $293.4 \mathrm{ft} \mathrm{msl}$ & $4^{-}$Steel & B & Water Table \\
\hline SAMPLE DATE & & $02 / 25 / 93$ & $05 / 04 / 93$ & $3 / 93$ & $3 / 93$ & \\
\hline
\end{tabular}

FIELD DATA

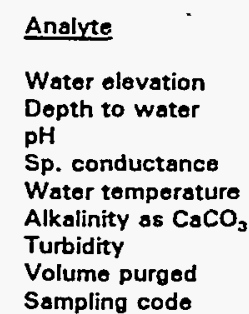

ANALYTICAL DATA

\section{H D Analvte}

Aluminum, total recoverable Arsenic, total recoverable

Barium, total recoverable

Cadmium, total recoverable

Colcium, total rocoverable

Chloride

Chromium, total rocoverable

2.4-Dichlorophenoxyacetic acid

Endrin

Fluoride

Gross alpha

Iron, total recoverable

Lead, total recoverable

Lindane

Magnesium, total recoverable

Manoanese. total recoverable

Mercury, total recoverable

Methoxychlor

Nitrate-nitrite as nitrogen

Nonvolatile beta

$\mathrm{pH}$

Phenols

Potassium, total recoverable

Radium. total alpha-emitting

Selenium, total recoverable

Silica, total recoverable

Silver, total recoverable

Sodium, total recoverable

Spocific conductanco

Sulfato

Total dissolved solids

Total organic carbon

Total organic halogens

Total phosphates (as P)

Toxaphene

2,4,5-TP (Silvex)

Tritium

1093
287.9
5.5
5.8
22
13.1
334
1.7

2093
287.4
6.0
5.9
23
20.2
180
0.8

3093

283.4

10.0

10.0
2.9

22.0

400

0.9

2093

3093

3,370

2,540

2,5
$<2.0$
26

26

$<2.0$

1.430
1.500

$<4.0$

$<4.0$
$<0.30$

$<0.0060$

$<100$

$5.8 E+00$
1.240

1.240
62

$<0.0050$

819

509

0.85

$<0.50$

650

1. $3 E+02$

5.3

$<5.0$

$<500$

$1.0 \mathrm{E}+01$

$<2.0$

11.800

$<2.0$

1.310

21

1.130

29.000

1.550

5.7

210

$<0.24$

$<0.090$

$1.4 E+00$

\begin{tabular}{|c|c|c|}
\hline \multicolumn{3}{|c|}{3,370} \\
\hline$<2.0$ & $<2.0$ & 2.5 \\
\hline $1.8 E+01$ & $\begin{array}{l}4.5 E+00 \\
6.360 \\
78\end{array}$ & $7.2 E+00$ \\
\hline & $\begin{array}{l}1.490 \\
<0.20\end{array}$ & \\
\hline $9.0 E+01$ & $\begin{array}{l}1.070 \\
8.8 E+01\end{array}$ & $1.7 E+02$ \\
\hline
\end{tabular}

Mod

Unit

Lab Flag

$\mu g / L$

$\mu g / L$

$\mu \mathrm{NG}$

$\mu g / L$

$\mu g / L$

$\mu g / L$
$\mu g / L$

$\mu g / L$

$\mu \mathrm{g} / \mathrm{L}$

$\mu g / L$

$\mathrm{pCi} / \mathrm{L}$

$\mu g / L$

$\mu_{g} / \mathrm{L}$

$\mu g / L$

$\mu g / L$

$\mu g / L$

$\mu \mathrm{g} / \mathrm{L}$

$\mu_{g} / \mathrm{L}$

PCi/L GE 2

GE $O$

GE 0

pH

$\mu g / L$

$\begin{array}{ll}4.5 E+00 & \mu g / L \\ & \mathrm{pCi} / \mathrm{L}\end{array}$

$\mu g / L$

$\mu_{\mathrm{g}} / \mathrm{L}$

$\mu \mathrm{g} / \mathrm{L}$

$\mu \mathrm{S} / \mathrm{cm}$

$\mu \mathrm{g} / \mathrm{h}$

$\mu_{\mathrm{g}} / \mathrm{L}$

$\mu \mathrm{g} / \mathrm{l}$

$\mu \mathrm{g} / \mathrm{L}$

$\mu \mathrm{g} / \mathrm{L}$

$\mu \rho / \mathrm{L}$

$\mu \mathrm{g} / \mathrm{L}$

$\mathrm{pCi} / \mathrm{mL}$

Note: Flagging levels, modifiers, and laboratories are for 4th quarter 1993 data only. See Appendix B for flagging criteria. - = exceeded holding time for 4th quarter 1993.

- = exceeded screening level or final primary drinking water standard for 4th quarter 1993. 
WELL.RSD 8

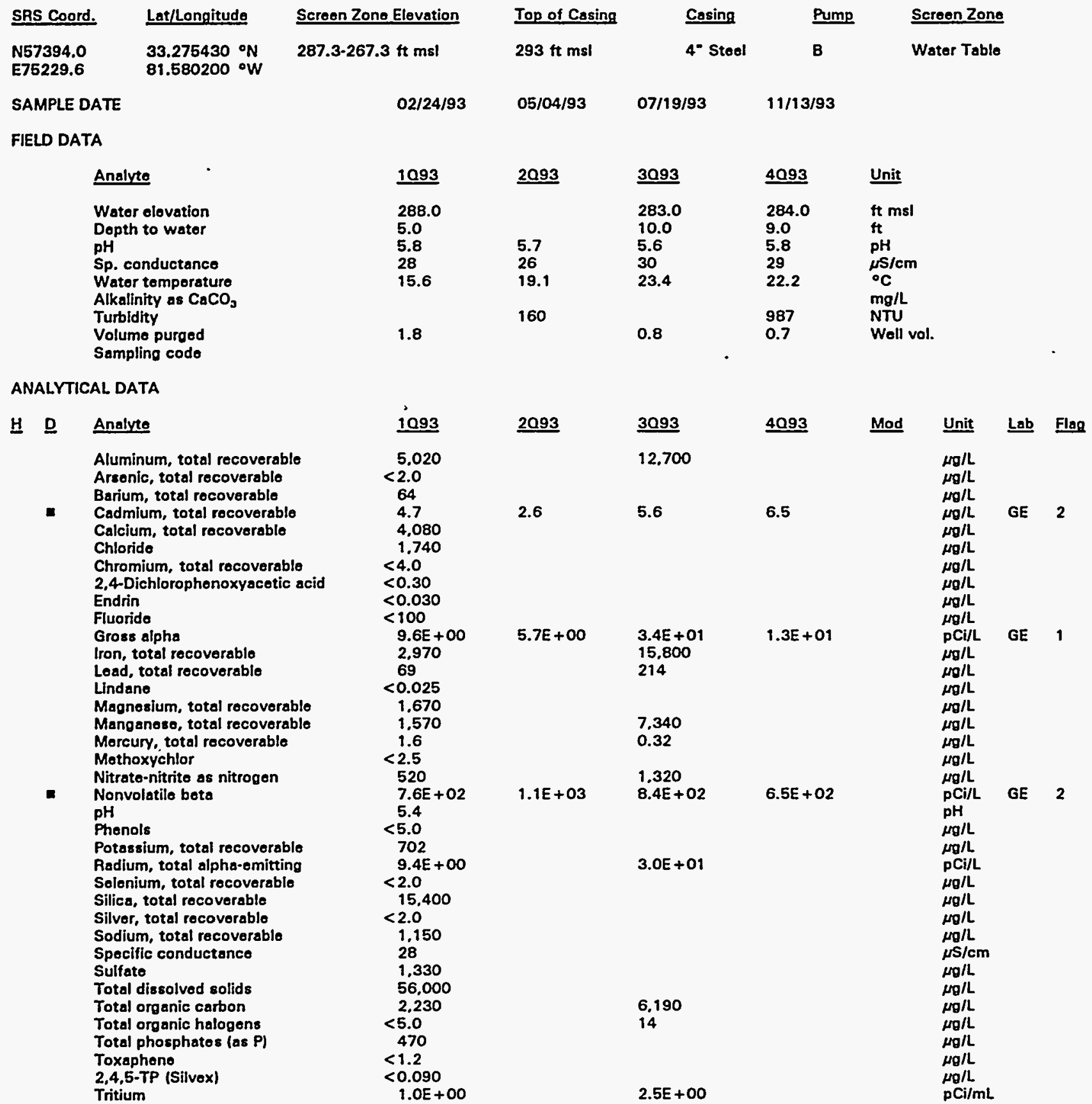

Note: Flagging levels, modifiers, and laboratories are for 4th quarter 1993 data only. See Appendix B for flagging criteria.

- = exceeded holding time for 4th quarter 1993.

- = exceeded screening level or final primary drinking water standard for 4th quarter 1993. 


\section{WELL RSD 9}

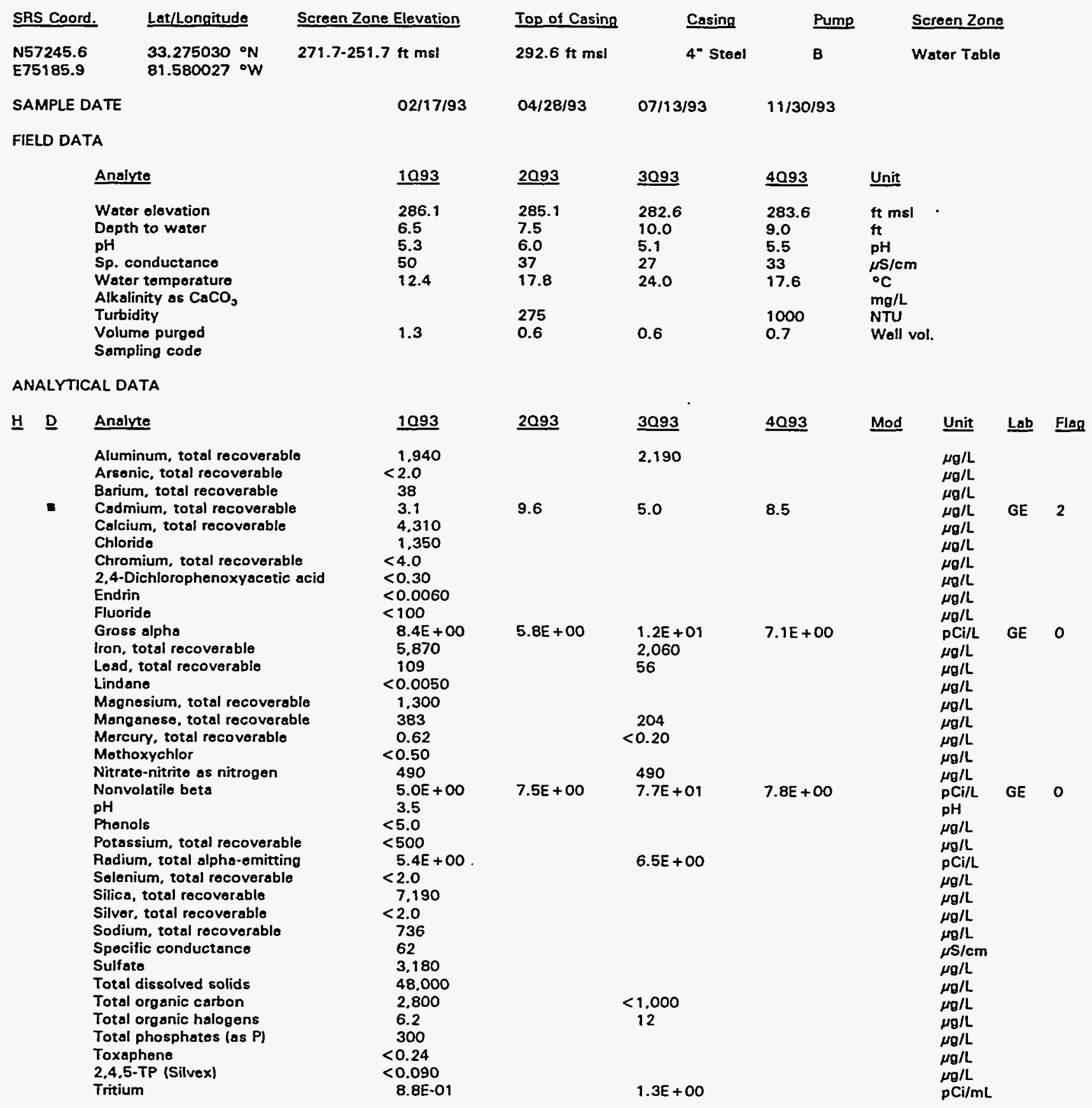

Note: Flagging levels, modifiers, and laboratories are for 4th quarter 1993 data only. See Appendix B for flagging criteria. - = exceeded holding time for 4th quarter 1993.

- = exceeded screening level or final primary drinking water standard for 4th quarter 1993. 
WELL RSD 10

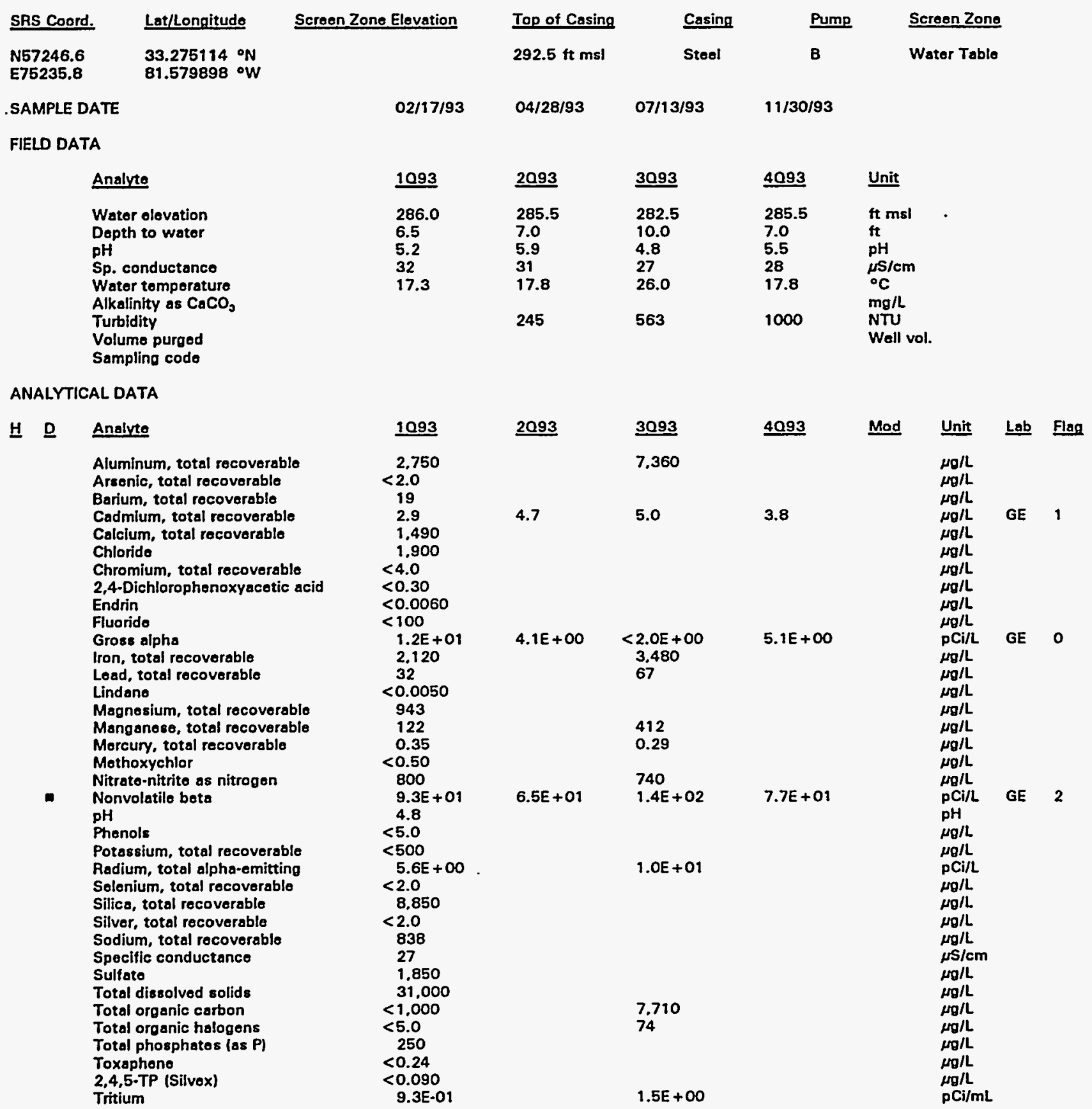

Note: Flagging levels, modifiers, and laboratories are for 4th quarter 1993 data only. See Appendix B for flagging criteria. - = exceeded holding time for 4th quarter 1993.

- =xceeded screening level or final primary drinking water standard for 4th quarter 1993. 


\section{WELL RSD 11}

$\begin{array}{lllllll}\text { SRS Coord. } & \text { Lat/Longitude } & \text { Screen Zone Elevation } & \text { Top of Casing } & \text { Casing } & \text { Pump } & \text { Screen Zone } \\ \text { N57249.1 } & \begin{array}{l}33.275200 \text { o } \\ \text { E75285.6 }\end{array} & 81.579771 \text { oW } & 292.3 \mathrm{ft} \mathrm{msl} & \text { Steel } & \text { B } & \text { Water Table }\end{array}$

$02 / 18 / 93 \quad 04 / 28 / 93 \quad 07 / 73 / 93 \quad 11 / 30 / 93$

FIELD DATA

\section{Analyte}

Water olevation Depth to water $\mathrm{pH}$

$\mathrm{Sp}$. conductance

Water temperature

Alkalinity as $\mathrm{CaCO}_{3}$

Turbidity

Volume purged

Sampling code

ANALYTICAL DATA

H D Analvte

Aluminum, total recoverable Arsenic, total recoverable Barium, total recoverable Cadmium, total recoverable Calcium, total recoverable Chlorido

Chromium, total recoverable 2.4-Dichlorophenoxyacetic acid Endrin Fluorido

Gross siphs

Iron total recoverable

Lead, total recoverabio

Lindano

Magnesium, total recoverable

Monganese, total recoverable

Mercury, total rocoverable

Methoxychlor

Nitrate-nitrite as nitrogen

Nonvolatile beta

pH

Phenols

Potassium, total recoverable

Radium, total alpha-emitting

Solenium, total recoverable

Silica, total recoverable

Silver, total recoverable

Sodium, total recoverable

Specific conductance

Sulfate

Total dissolved solids

Total organic carbon

Total organic halogens

Total phosphates (as P)

Toxaphene

2.4,5-TP (Silvex)

Tritium

$\begin{array}{ll}\underline{1093} & \underline{2093} \\ 285.3 & 285.3 \\ 7.0 & 7.0 \\ 5.6 & 6.0 \\ 51 & 39 \\ 13.0 & 18.6 \\ 308 & 145\end{array}$

30.93
282.3
10.0
5.0
28
24.2

$\underline{2093}$

8.4

$<2.0$

32

3.5

2,370

1.840

$<4.0$

$<0.30$

$<0.0060$

$<100$

$7.7 E+O 0$
3.930

3.930
78

$<0.0050$

979

179

0.35

$<0.50$

760

$1.7 \mathrm{E}+01$

4.8

$<5.0$

$<500$

$1.8 \mathrm{E}+\infty$

$<2.0$

7.360

$<2.0$
68

36

2.830

46,000

2.990

$<5.0$

220

$<0.24$

$<0.090$

8.0E-01

\begin{tabular}{|c|c|c|}
\hline \multirow[t]{2}{*}{2093} & $\underline{3093}$ & 4093 \\
\hline & \multicolumn{2}{|l|}{3,660} \\
\hline 8.4 & 4.7 & 3.9 \\
\hline \multirow[t]{2}{*}{$2.9 E+00$} & $\begin{array}{l}2.4 E+00 \\
7.110 \\
57\end{array}$ & $2.8 E+00$ \\
\hline & $\begin{array}{r}519 \\
<0.20\end{array}$ & \\
\hline \multirow[t]{3}{*}{$1.5 E+01$} & $\begin{array}{l}710 \\
1.0 E+01\end{array}$ & $2.1 E+01$ \\
\hline & $7.7 E+\infty 0$ & \\
\hline & $\begin{array}{l}5.250 \\
12\end{array}$ & \\
\hline & $1.2 \mathrm{E}+00$ & \\
\hline
\end{tabular}

$\underline{\text { Unit }}$

ft msl

$\mathrm{ft}$

pH

$\mu \mathrm{S} / \mathrm{cm}$

$51 \quad$ $5 \mathrm{~S} / \mathrm{C}$

$112 \mathrm{mg} / \mathrm{L}$

WTU

Mod Unit Lab Flag

$\mu \mathrm{g} / \mathrm{L}$

$\mu g / L$

$\mu \mathrm{g} / \mathrm{L}$ GE 1

$\mu g / L$

$\mu \mathrm{g} / \mathrm{L}$

$\mu g / L$

$\mu \mathrm{\mu g} / \mathrm{L}$

$\mu \mathrm{g} / \mathrm{L}$

pCi/L GE O

$\mu \mathrm{g} / \mathrm{L}$

$\mu_{g} / \mathrm{L}$

$\mu g / L$

$\mu \mathrm{g} / \mathrm{L}$

$\mu g / L$

$\mu g / L$

$\mu \mathrm{g} / \mathrm{L}$

$\begin{array}{lll}\mu \mathrm{g} / \mathrm{L} & \\ \mathrm{pCi} / \mathrm{L} & \mathrm{GE} & 0\end{array}$

$\mathrm{pH}$

$\mu g / L$

$\mu \mathrm{g} / \mathrm{L}$

PCill

$\mu g / L$

$\mu g / L$

$\mu \mathrm{g} / \mathrm{L}$

$\stackrel{\mu \mathrm{g} / \mathrm{L}}{\mu \mathrm{S} / \mathrm{cm}}$

$\mu g / L$

$\mu g / L$

$\mu g / L$

$\mu \mathrm{g} / \mathrm{L}$

$\mu g / L$

$\mu \mathrm{g} / \mathrm{L}$

$\mathrm{pCi} / \mathrm{mL}$

Note: Flagging levels, modifiers, and laboratories are for 4th quarter 1993 data only. See Appendix B for flagging criteria. - = exceeded holding time for 4th quarter 1993.

- = exceeded screening level or final primary drinking water standard for 4th quarter 1993. 
WELL RSE 1 A

\begin{tabular}{|c|c|c|c|c|c|c|}
\hline SAS Coord. & Lat/Longitude & Screen Zone Elovation & Top of Casing & Casing & Pump & Screen Zone \\
\hline $\begin{array}{l}\text { N57734.5 } \\
\text { E74712.7 }\end{array}$ & $\begin{array}{l}33.275341{ }^{\circ} \mathrm{N} \\
81.582222\end{array}$ & $294.8-274.8 \mathrm{ft} \mathrm{msl}$ & $304.2 \mathrm{ft} \mathrm{msl}$ & 4" PVC & $\mathbf{s}$ & Water Table \\
\hline SAMPLE DA & & $03 / 09 / 93$ & $05 / 19 / 93$ & $07 / 29 / 93$ & $12 / 05 / 93$ & \\
\hline FIELD DATA & & & & & & \\
\hline & & 1093 & 2093 & $\underline{3093}$ & 4093 & $\underline{\text { Unit }}$ \\
\hline & $\begin{array}{l}\text { olovation } \\
\text { to water } \\
\text { inductance } \\
\text { tomperature } \\
\text { ity as } \mathrm{CaCO} \text {, }\end{array}$ & $\begin{array}{l}295.2 \\
9.0 \\
5.0 \\
37 \\
18.9\end{array}$ & $\begin{array}{l}293.7 \\
10.5 \\
5.7 \\
45 \\
21.7\end{array}$ & $\begin{array}{l}288.2 \\
16.0 \\
5.7 \\
35 \\
25.1\end{array}$ & $\begin{array}{l}285.7 \\
18.5 \\
5.5 \\
26 \\
23.0\end{array}$ & $\begin{array}{l}\mathrm{ft} \mathrm{msl} \\
\mathrm{ft} \\
\mathrm{pH} \\
\mathrm{fS}^{\prime} / \mathrm{cm} \\
{ }^{\circ} \mathrm{C} \\
\mathrm{mg} / \mathrm{L}\end{array}$ \\
\hline & $\begin{array}{l}\text { ity } \\
\text { ie purged } \\
\text { ing code }\end{array}$ & $\begin{array}{l}240 \\
1.0\end{array}$ & $\begin{array}{l}290 \\
1.9 \\
x\end{array}$ & $\begin{array}{l}116 \\
1.9\end{array}$ & $\begin{array}{l}52 \\
0.1 \\
x\end{array}$ & $\begin{array}{l}\text { NTU } \\
\text { Well vol. }\end{array}$ \\
\hline
\end{tabular}

\section{ANALYTICAL DATA}

H D Analyte

Actinium-228

Aluminum, total recoverable Antimony-125

Arsenic, total rocovorablo

Barium, total recoverable

Cadmium, total recoverable

Calcium, total recoverable

Corium-144

Cosium-134

Cosium-137

Chloride

Chromium, total recoverablo

Cobalt-57

Cobalt-60

Copper total recoverablo

2,4-Dichlorophenoxyacetic acid

Endrin

Europium-152

Europium-154

Europium-155

Fluorido

Grose alpha

Iron, total recoverable

Lead, total recoverable

Lead-212

Lindano

Magnesium, total recoverable

Manganeso-54

Mercury, total recoverable

Mothoxychlor

Nickol, total recoverable

Nitrate-nitrite as nitrogen

Nonvolatile bota

$\mathrm{pH}$

Phenols

Plutonium-238

Plutonium-239/240

Potassium, total recoverable

\begin{tabular}{|c|c|c|}
\hline 1093 & 2093 & $\underline{3093}$ \\
\hline 5.530 & & 2,890 \\
\hline & $<2.5 E+01$ & $<2.5 E+01$ \\
\hline $\begin{array}{r}<2.0 \\
58\end{array}$ & $<2.0$ & $<2.0$ \\
\hline 58 & 33 & 21 \\
\hline$<2.0$ & $<2.0$ & $<2.0$ \\
\hline 2,400 & $\begin{aligned} & 2.500 \\
< & 5.0 E+01 \\
< & 1.0 E+01 \\
< & 1.0 E+01\end{aligned}$ & $\begin{aligned} & 1.210 \\
&< 5.0 E+01 \\
&<1.0 E+01 \\
&<1.0 E+01\end{aligned}$ \\
\hline $\begin{array}{r}1.710 \\
<4.0^{\circ}\end{array}$ & $\begin{aligned} & 1.890 \\
< & 11 \\
< & 1.0 E+01 \\
< & 1.2 E+01\end{aligned}$ & $\begin{array}{l}1.630 \\
22 \\
<1.0 E+01 \\
<1.2 E+01\end{array}$ \\
\hline \multirow{2}{*}{$\begin{aligned} & 203 \\
< & 0.30 \\
< & 0.0060\end{aligned}$} & 392 & 435 \\
\hline & $\begin{array}{l}<5.0 E+01 \\
<2.5 E+01 \\
<2.5 E+01\end{array}$ & $\begin{array}{l}<5.0 E+01 \\
<2.5 E+01 \\
<2.5 E+01\end{array}$ \\
\hline $\begin{array}{l}<100 \\
6.0 E+\infty 0 \\
444 \\
11\end{array}$ & $\begin{array}{l}<100 \\
4.6 E+00 \\
2.330 \\
4.6\end{array}$ & $\begin{array}{l}<100 \\
4.2 E+00 \\
805 \\
9.8\end{array}$ \\
\hline \multicolumn{3}{|l|}{$<0.0050$} \\
\hline $\begin{array}{l}1.140 \\
91\end{array}$ & $\begin{aligned} & 1.300 \\
< & 18 \\
< & 1.0 E+01\end{aligned}$ & $\begin{array}{l}1.030 \\
14 \\
<1.0 E+01\end{array}$ \\
\hline $\begin{array}{r}0.43 \\
<0.50\end{array}$ & $<0.20$ & $<0.20$ \\
\hline $\begin{array}{l}<4.0 \\
300 \\
2.1 E+01 \\
5.2\end{array}$ & $\begin{array}{l}19 \\
890 \\
5.3 E+02 \\
5.3\end{array}$ & $\begin{array}{l}39 \\
1.030 \\
3.0 E+02 \\
5.1\end{array}$ \\
\hline & $\begin{array}{l}<1.0 E+00 \\
<1.0 E+00\end{array}$ & $\begin{array}{l}<1.0 E+00 \\
<1.0 E+00\end{array}$ \\
\hline$<500$ & 672 & $<500$ \\
\hline
\end{tabular}

\begin{tabular}{|c|c|c|}
\hline Unit & $\underline{\text { Lab }}$ & \\
\hline $\begin{array}{l}\mathrm{pCi} / \mathrm{L} \\
\mu \mathrm{g} / \mathrm{L}\end{array}$ & $\mathrm{CN}$ & 0 \\
\hline pCi/L & $\mathrm{CN}$ & 0 \\
\hline$\mu \mathrm{g} / \mathrm{L}$ & GE & \\
\hline$\mu g / L$ & $\mathrm{GE}$ & \\
\hline$\mu \mathrm{g} / \mathrm{L}$ & & \\
\hline $\mathrm{\mu g} / \mathrm{L}$ & GE & 0 \\
\hline $\begin{array}{l}\text { pCi/L } \\
\text { pCi/L }\end{array}$ & $\begin{array}{l}\mathrm{CN} \\
\mathrm{CN}\end{array}$ & \\
\hline $\mathrm{pCi} / \mathrm{l}$ & $\mathrm{CN}$ & 0 \\
\hline$\mu_{g} / L$ & GE & 0 \\
\hline$\mu \mathrm{g} / \mathrm{L}$ & GE & 0 \\
\hline $\mathrm{pCi} / \mathrm{L}$ & $\mathrm{CN}$ & \\
\hline $\mathrm{pCi} / \mathrm{L}$ & $\mathrm{CN}$ & 0 \\
\hline$\mu g / L$ & GE & \\
\hline$\mu \mathrm{\mu g} / \mathrm{L}$ & & \\
\hline $\mathrm{pCi} / \mathrm{L}$ & $\mathrm{CN}$ & 0 \\
\hline pCi/L & $\mathrm{CN}$ & 0 \\
\hline $\mathrm{pCi} / \mathrm{L}$ & $\mathrm{CN}$ & 0 \\
\hline$\mu g / L$ & GE & 0 \\
\hline $\mathrm{pCi} / \mathrm{L}$ & GE & 0 \\
\hline$\mu g / L$ & GE & 0 \\
\hline $\mathrm{\mu g} / \mathrm{L}$ & GE & 0 \\
\hline pCi/L & $\mathrm{CN}$ & 0 \\
\hline$\mu \mathrm{g} / \mathrm{L}$ & & \\
\hline$\mu \mathrm{g} / \mathrm{L}$ & GE & 0 \\
\hline $\mathrm{rg} / \mathrm{L}$ & GE & \\
\hline$\mu g / L$ & GE & 0 \\
\hline$\mu \mathrm{g} / \mathrm{L}$ & & \\
\hline$\mu \mathrm{g} / \mathrm{L}$ & GE & 0 \\
\hline$\mu \mathrm{g} / \mathrm{L}$ & GE & \\
\hline $\mathrm{pCi} / \mathrm{L}$ & GE & . \\
\hline pH & GE & \\
\hline $\begin{array}{l}\mu g / L \\
\mathrm{pC} / \mathrm{L} / \mathrm{s}\end{array}$ & & \\
\hline $\mathrm{pCi/L}$ & $\mathrm{CN}$ & \\
\hline$\mu \mathrm{g} / \mathrm{L}$ & GE & \\
\hline
\end{tabular}

Note: Flagging levels, modifiers, and laboratories are for 4th quarter 1993 data only. See Appendix B for flagging criteria. - = exceeded holding time for 4th quarter 1993.

- = exceeded screening level or final primary drinking water standard for 4th quarter 1993. 
Woll RSE IA continued

ANALYTICAL DATA

$\begin{array}{ll}\text { H. } & \text { Analyte } \\ \text { Potassium-40 } \\ \text { Promethium-144 } \\ \text { Promothium-146 } \\ \text { Radium, total alpha-emitting } \\ \text { Ruthonium-106 } \\ \text { Selenium, total recoverable } \\ \text { Silica, total recoverable } \\ \text { Silvor, total recoverable } \\ \text { Sodium, total recoverable } \\ \text { Sodium-22 } \\ \text { Specific conductance } \\ \text { Strontium-90 } \\ \text { Sulfate } \\ \text { Thorium-234 } \\ \text { Total activity } \\ \text { Total dissolved solids } \\ \text { Total organic carbon } \\ \text { Total organic halogens } \\ \text { Total phosphates (as P) } \\ \text { Total suspended solids } \\ \text { Toxaphene } \\ 2,4,5-T P \text { (Silvox) } \\ \text { Tritium } \\ \text { Yttrium-88 } \\ \text { Zinc, total recoverable } \\ \text { Zinc-65 }\end{array}$

\begin{tabular}{|c|c|c|c|c|c|c|c|}
\hline 1093 & 2093 & $\underline{3093}$ & 4093 & Mod & Unit & $\underline{\text { Lab }}$ & Flag \\
\hline & $<2.5 E+02$ & $<2.5 E+02$ & $<2.5 E+02$ & Y3 & $\mathrm{pCi} / \mathrm{L}$ & $\mathrm{CN}$ & 0 \\
\hline & $<1.0 E+01$ & $<1.0 E+01$ & $<1.0 E+01$ & Y3 & $\mathrm{pCi} / \mathrm{L}$ & $\mathrm{CN}$ & 0 \\
\hline & $<2.3 E+01$ & $<2.3 E+01$ & $<2.3 E+01$ & Y3 & $\mathrm{pCi} / \mathrm{L}$ & $\mathrm{CN}$ & 0 \\
\hline $6.5 E+00$ & $9.3 E+00$ & $2.5 E+\infty 0$ & $1.0 E+\infty$ & & $\mathrm{pCill}$ & GE & 0 \\
\hline & $<8.0 E+01$ & $<8.0 E+01$ & $<8.0 E+01$ & Y3 & $\mathrm{pCi} / \mathrm{L}$ & $\mathrm{CN}$ & \\
\hline $\begin{aligned}<2.0 \\
7.940\end{aligned}$ & $<2.0$ & $<2.0$ & $<2.0$ & & $\begin{array}{l}\mu g / L \\
\mu g / L\end{array}$ & GE & 0 \\
\hline$<2.0$ & $<2.0$ & $<2.0$ & $<2.0$ & & $\mu \mathrm{g} / \mathrm{L}$ & GE & \\
\hline 1.120 & 1.450 & 960 & 920 & & $\mu \mathrm{g} / \mathrm{L}$ & $G E$ & \\
\hline 32 & & $\begin{array}{c}<1.0 E+01 \\
29\end{array}$ & $\begin{array}{c}<1.0 E+01 \\
20\end{array}$ & Y3 & $\begin{array}{l}\text { pCi/L } \\
\mu \mathrm{S} / \mathrm{cm}\end{array}$ & $\begin{array}{l}C N \\
G E\end{array}$ & $\begin{array}{l}0 \\
0\end{array}$ \\
\hline & $2.5 E+02$ & $1.3 E+02$ & $4.9 E+01$ & $\mathbf{Y}$ & $\mathrm{pCi} / \mathrm{L}$ & CN & \\
\hline 5,330 & 4,500 & $<1.000$ & $\begin{array}{l}<1.000 \\
<1.3 E+03 \\
4.1 E+03\end{array}$ & Y3 & $\begin{array}{l}\mu \mathrm{g} / \mathrm{L} \\
\mathrm{pC \textrm {C } / \mathrm { L }} \\
\mathrm{pC \textrm {Ci } / \mathrm { L }}\end{array}$ & $\begin{array}{l}\text { GE } \\
C N \\
\text { EM }\end{array}$ & $\begin{array}{l}0 \\
0 \\
0\end{array}$ \\
\hline 22,000 & 30,000 & 21,000 & 18,000 & & $\mu \mathrm{g} / \mathrm{L}$ & GE & 0 \\
\hline $\begin{array}{c}<1.000 \\
26\end{array}$ & $<1,000$ & $\begin{aligned} & 2.380 \\
< & 5.0\end{aligned}$ & $<1.000$ & & $\underset{\mu g / L}{\mu g / L}$ & GE & \\
\hline 310 & 100 & 70 & $<50$ & & $\mu \mathrm{g} / \mathrm{L}$ & GE & \\
\hline 912,000 & 710,000 & 450,000 & 32.000 & $J$ & $\mu / L$ & GE & \\
\hline $\begin{array}{l}<0.24 \\
<0.090\end{array}$ & & & & & $\begin{array}{l}\mu g / L \\
\mu g / L\end{array}$ & & \\
\hline$<7.0 \mathrm{E}-01$ & $\begin{array}{r}2.1 \mathrm{E}+02 \\
10 \mathrm{O}\end{array}$ & $\begin{array}{c}9.4 \mathrm{E}-01 \\
\end{array}$ & $\begin{array}{r}1.8 E+00 \\
.0 E+01\end{array}$ & $Y_{3}$ & $\mathrm{pCi} / \mathrm{mL}$ & GE & $\begin{array}{l}0 \\
0\end{array}$ \\
\hline 18 & $\begin{array}{l}40 \\
<2.3 E+01\end{array}$ & $\begin{array}{l}81 \\
<2.3 E+01\end{array}$ & $\begin{array}{l}22 \\
<2.3 E+01\end{array}$ & Y3 & $\begin{array}{l}\mu \mathrm{g} / \mathrm{L} \\
\mathrm{pCi} / \mathrm{L}\end{array}$ & $\begin{array}{l}\mathrm{GE} \\
\mathrm{CN}\end{array}$ & $\begin{array}{l}0 \\
0\end{array}$ \\
\hline
\end{tabular}

\section{WELL RSE 1B}

\section{SRS Coord. \\ N57731.4 \\ E74698.1

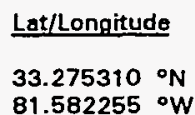

SAMPLE DATE

FIELD DATA

Analyte
Water olevation
Depth to water
pH
Sp. conductance
Water tomperature
Alkalinity as $\mathrm{CaCO}_{3}$
Turbidity
Volume purged
Sampling code

\section{Screen Zone Elevation \\ 295.7-275.7 ft msl}

$03 / 09 / 93$

\begin{tabular}{|c|c|}
\hline 1093 & 2093 \\
\hline $\begin{array}{l}296.3 \\
7.0 \\
4.9 \\
26 \\
18.2\end{array}$ & $\begin{array}{l}293.8 \\
9.5 \\
5.8 \\
27 \\
21.2\end{array}$ \\
\hline $\begin{array}{l}390 \\
1.0\end{array}$ & $\begin{array}{l}180 \\
1.5 \\
X\end{array}$ \\
\hline
\end{tabular}

$\begin{aligned} & 1093 \\ & 6.590 \\ < & 2.0 \\ & 41 \\ < & 2.0 \\ & 2.010\end{aligned}$

ANALYTICAL DATA

Analyte
Actinium-228
Aluminum, total recoverable
Antimony-125
Arsenic, total rocoverable
Barium, total recoverable
Cadmium, total recoverable
Calcium, total recoverable
Corium-144
Casium-134

브 $\underline{D}$

\section{Top of Casing}

$303.3 \mathrm{ft} \mathrm{msl}$

$05 / 19 / 93$

$07 / 29 / 93$

\section{Casing}

4- PVC

Pump

s

\section{Screen Zone}

Water Toble

$\begin{array}{ll}\underline{2093} & \underline{3093} \\ & \\ <2.5 E+01 & <2.5 E+01 \\ <2.0 & <2.0 \\ 15 & 10 \\ <2.0 & <2.0 \\ 1.160 & 1.150 \\ <5.0 E+01 & <5.0 E+01 \\ <1.0 E+01 & <1.0 E+01\end{array}$

\begin{tabular}{|c|c|c|c|}
\hline 4093 & Mod & Unit & Lab \\
\hline$<8.4 E+01$ & $\gamma 3$ & $\begin{array}{l}\mathrm{pCi} / \mathrm{L} \\
\mu_{g} / \mathrm{L}\end{array}$ & CN \\
\hline $\begin{array}{l}<2.5 E+01 \\
<2.0\end{array}$ & Y3 & $\mathrm{pCi} / \mathrm{L}$ & $\begin{array}{l}\text { CN } \\
\text { GE }\end{array}$ \\
\hline $\begin{array}{r}10 \\
<2.0 \\
\end{array}$ & & $\begin{array}{l}\mu g / L \\
\mu g / L\end{array}$ & GE \\
\hline $\begin{array}{l}878 \\
<5.0 E+01\end{array}$ & $\begin{array}{l}Y 3 \\
Y 3\end{array}$ & $\begin{array}{l}\mu \mathrm{g} / \mathrm{L} \\
\mathrm{pCi} / \mathrm{L}\end{array}$ & GE \\
\hline
\end{tabular}

Note: Flegging levels, modifiers, and laboratories are for 4 th quarter 1993 data only. See Appendix B for flagging criteria. - = exceeded holding time for 4th quarter 1993.

- exceeded screening level or final primary drinking water standard for 4th quarter 1993. 
H D Analyte

Cosium-137

Chloride

Chromium, total recoverable

Cobalt-57

Cobalt-60

Copper, total recoverable

2,4-Dichlorophenoxyacetic acid

Endrin

Europium-152

Europium-154

Europium-155

Fluoride

Gross alpha

Iron, total recoverable

Load, total recoverable

Load-21 2

Lindane

Magnesium, total recoverable

Manganese, total recoverable

Manganose-54

Mercury, total rocoverable

Methoxychlor

Nickel, total rocoverable

Nitrato-nitrite as nitrogen

- Nonvolatile beto

pH

Phonols

Piutonium-238

Plutonium-239/240

Potassium, total recoverablo

Potassium- 40

Promethium-144

Promothium-146

Radium, total alpha-emitting

Ruthenium-106

Solenium, total recovorable

Silica, total recoverable

Silver, total recoverablo

Sodium, total recoverablo

Sodium-22

Specific conductance

Strontium-90

Sulfato

Thorium-234

Total dissolved solids

Total organic carbon

Total organic halogens

Total phosphates (as P)

Total suspended solids

Toxaphene

2,4,5-TP (Silvex)

Tritium

Yttrium-88

Zinc, total recoverable

Zinc. 65

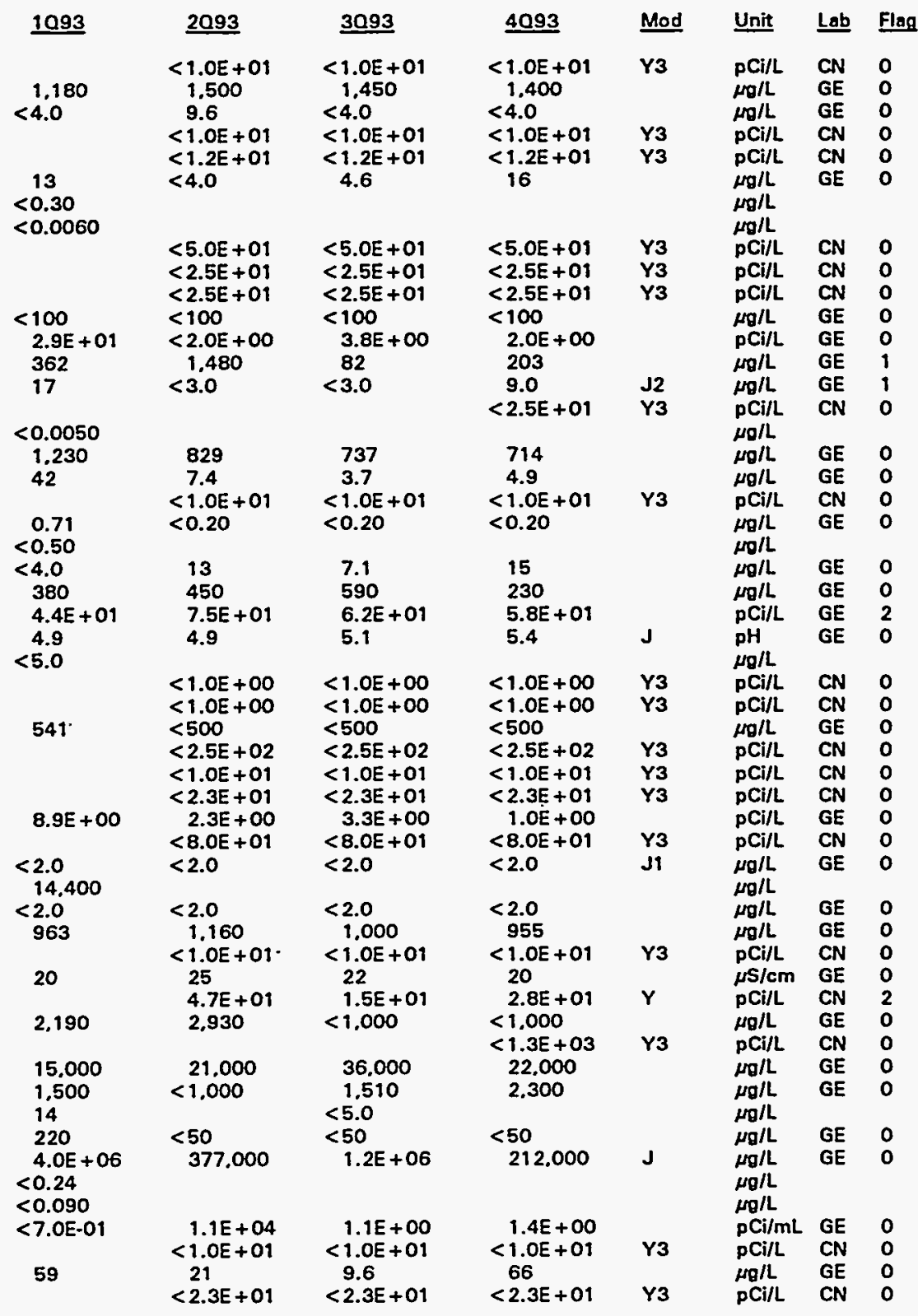

Note: Flagging levels, modifiers, and laboratories are for 4th quarter 1993 data only. See Appendix B for flagging criteria. - = exceeded holding time for 4th quarter 1993.

- =xceeded screening level or final primary drinking water standard for 4th quarter 1993. 


\section{WELL RSE 1C}

\begin{tabular}{|c|c|c|c|c|c|c|}
\hline SRS Coord. & Lat/Longitude & Screen Zone Elevation & Top of Casing & Casing & Pump & Screen Zone \\
\hline $\begin{array}{l}N 57730.8 \\
\text { E74684.1 }\end{array}$ & $\begin{array}{l}33.2752866^{\circ} \mathrm{N} \\
81.582290^{\circ} \mathrm{W}\end{array}$ & 288.5-268.5 $\mathrm{ft} \mathrm{msl}$ & $303.3 \mathrm{ft} \mathrm{msl}$ & 4" PVC & $\mathbf{s}$ & Water Table \\
\hline SAMPLE DA7 & & $03 / 09 / 93$ & $05 / 19 / 93$ & $07 / 29 / 93$ & $12 / 05 / 93$ & \\
\hline FIELD DATA & & & & & & \\
\hline & & 1093 & $\underline{2093}$ & 3093 & 4093 & Unit \\
\hline $\begin{array}{l}W \\
D \\
\text { pl } \\
\text { S } \\
W \\
A\end{array}$ & $\begin{array}{l}\text { olevation } \\
\text { to water } \\
\text { inductance } \\
\text { temperature } \\
\text { ity as } \mathrm{CaCO}_{3}\end{array}$ & $\begin{array}{l}295.3 \\
8.0 \\
5.3 \\
34 \\
16.4\end{array}$ & $\begin{array}{l}293.8 \\
9.5 \\
5.8 \\
31 \\
21.9\end{array}$ & $\begin{array}{l}288.3 \\
15.0 \\
5.8 \\
27 \\
24.7\end{array}$ & $\begin{array}{l}286.3 \\
17.0 \\
5.4 \\
22 \\
22.0\end{array}$ & $\begin{array}{l}\text { ft msl } \\
\mathrm{ft} \\
\mathrm{pH} \\
\mu \mathrm{S} / \mathrm{cm} \\
{ }^{\circ} \mathrm{C} \\
\mathrm{mg} / \mathrm{L}\end{array}$ \\
\hline & $\begin{array}{l}\text { ity } \\
\text { o purged } \\
\text { ing codo }\end{array}$ & $\begin{array}{l}80 \\
0.9\end{array}$ & $\begin{array}{l}90 \\
1.1 \\
\times\end{array}$ & $\begin{array}{l}28 \\
1.8\end{array}$ & $\begin{array}{l}26 \\
0.1 \\
x\end{array}$ & $\begin{array}{l}\text { NTU } \\
\text { Woll vol. }\end{array}$ \\
\hline
\end{tabular}

\section{ANALYTICAL DATA}$$
\text { H D Analye }
$$

Actinium-228

Aluminum, total racoverable

Antimony-125

Arsenic, total recoverable

Barium, total recoverable

Cadmium, total recoverable

Calcium, total recoverable

Corium-144

Cesium-134

Cosium-137

Chloride

Chromium, total recoverable

Cobalt-57

Cobalt-60

Copper, total recoverable

2,4-Dichlorophenoxyacetic acid

Endrin

Europium-152

Europium-154

Europium-155

Fluoride

Gross alpha

Iron, total recoverable

Lead, total recoverable

Lead-212

Lindane

Magnesium, total recoverable

Manganese, total recoverable

Manganose-54

Mercury, total recoverable

Mathoxychlor

Nickel, total recoverable

Nitrato-nitrite as nitrogen

Nonvolatile beta

- $\quad \mathrm{pH}$

pH

Plutonium-238

Plutonium-239/240

Potassium, total recoverable

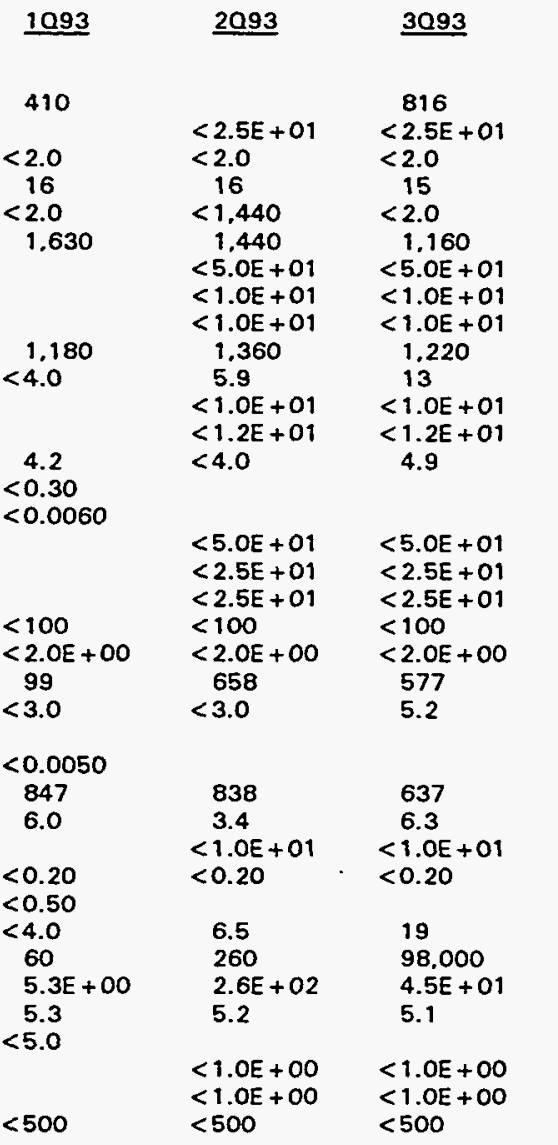

\begin{tabular}{|c|c|c|c|}
\hline 4093 & Mod & $\underline{\text { Unit }}$ & $\underline{\text { Lab }}$ \\
\hline$<8.4 E+01$ & Y3 & $\begin{array}{l}\mathrm{pCi} / \mathrm{L} \\
\mu g / \mathrm{L}\end{array}$ & CN \\
\hline $\begin{array}{l}<2.5 E+01 \\
<2.0\end{array}$ & Y3 & $\begin{array}{l}\mathrm{pCi} / \mathrm{L} \\
\mu \mathrm{g} / \mathrm{L}\end{array}$ & $\begin{array}{l}\text { CN } \\
\text { GE }\end{array}$ \\
\hline 9.2 & & $\mu \mathrm{g} / \mathrm{L}$ & GE \\
\hline$<2.0$ & & $\mu g / L$ & GE \\
\hline 822 & & $\mu g / L$ & GE \\
\hline$<5.0 E+01$ & Y3 & pCi/L & $\mathrm{CN}$ \\
\hline$<1.0 E+01$ & Y3 & $\mathrm{pCi} / \mathrm{L}$ & $\mathrm{CN}$ \\
\hline$<1.0 E+01$ & Y3 & $\mathrm{pCi} / \mathrm{L}$ & $\mathrm{CN}$ \\
\hline $\begin{array}{l}1.420 \\
5.4\end{array}$ & & $\begin{array}{l}\mu g / L \\
\mu g / L\end{array}$ & $\begin{array}{l}\text { GE } \\
\text { GE }\end{array}$ \\
\hline$<1.0 E+01$ & Y3 & $\mathrm{pCi} / \mathrm{L}$ & $\mathrm{CN}$ \\
\hline$<1.2 E+01$ & Y3 & $\mathrm{pCi} / \mathrm{L}$ & $\mathrm{CN}$ \\
\hline$<4.0$ & & $\begin{array}{l}\mu \mathrm{g} / \mathrm{L} \\
\mu \mathrm{g} / \mathrm{L} \\
\mu_{\mathrm{g}} / \mathrm{L}\end{array}$ & GE \\
\hline$<5.0 E+01$ & Y3 & $\mathrm{pCi} / \mathrm{L}$ & $\mathrm{CN}$ \\
\hline$<2.5 E+01$ & Y3 & $\mathrm{pCi} / \mathrm{L}$ & $\mathrm{CN}$ \\
\hline$<2.5 E+01$ & Y3 & $p C i / L$ & $\mathrm{CN}$ \\
\hline 23 & & $\mu g / L$ & GE \\
\hline $\begin{array}{l}2.1 E+00 \\
176\end{array}$ & & $\begin{array}{l}\mathrm{pCi} / \mathrm{L} \\
\mathrm{eg} / \mathrm{L}\end{array}$ & $\begin{array}{l}G E \\
G E\end{array}$ \\
\hline$<3.0$ & & $\mu \mathrm{g} / \mathrm{L}$ & $\overline{G E}$ \\
\hline$<2.5 E+01$ & Y3 & $\begin{array}{l}\mathrm{pCi} / \mathrm{L} \\
\mu_{\mathrm{g}} / \mathrm{L}\end{array}$ & $\mathrm{CN}$ \\
\hline $\begin{array}{l}540 \\
3.7\end{array}$ & & $\begin{array}{l}\mu g / L \\
\mu g / L\end{array}$ & $\begin{array}{l}\text { GE } \\
\text { GE }\end{array}$ \\
\hline$<1.0 E+01$ & Y3 & $\mathrm{pCi} / \mathrm{L}$ & $\mathrm{CN}$ \\
\hline$<0.20$ & & $\begin{array}{l}\mu \mathrm{g} / \mathrm{L} \\
\mu \mathrm{g} / \mathrm{L}\end{array}$ & $\mathbf{G E}$ \\
\hline 12 & & $\mu \theta / L$ & GE \\
\hline $\begin{array}{l}70 \\
4.2 E+00\end{array}$ & & $\mu g / L$ & $\begin{array}{l}\text { GE } \\
\text { GE }\end{array}$ \\
\hline 5.1 & $J$ & $\begin{array}{l}\mathrm{pH} \\
\mu \mathrm{g} / \mathrm{L}\end{array}$ & $\overrightarrow{\mathrm{GE}}$ \\
\hline$<1.0 E+00$ & Y3 & $\mathrm{pCi} / \mathrm{L}$ & $\mathrm{CN}$ \\
\hline$<1.0 E+00$ & Y3 & $\mathrm{pCi} / \mathrm{L}$ & $\mathrm{CN}$ \\
\hline$<500$ & & $\mu g / L$ & GE \\
\hline
\end{tabular}

Note: Flagging levels, modifiers, and laboratories are for 4th quarter 1993 data only. See Appendix B for flagging criteria.

- = exceeded holding time for 4th quarter 1993.

- =xceeded screening level or final primary drinking water standard for 4th quarter 1993. 
Woll RSE $1 \mathrm{C}$ continued

ANALYTICAL DATA

H D Analyto

Potassium-40

Promothium-144

Promethium-146

Radium, total alpha-emitting

Ruthenium-106

Selenium, total recoverable

Silica, total recoverable

Silver, total recoverablo

Sodium, total recoverable

Sodium-22

Specific conductance

Strontium-90

Sulfate

Thorium-234

Total activity

Total dissolved solids

Total organic carbon

Total orosnic halogons

Total phosphates (as P)

Total suspended solids

Toxaphene

2,4,5-TP (Silvex)

Tritium

Yttrium-88

Zinc, total recoverable

Zinc-65

\begin{tabular}{|c|c|c|c|c|c|c|}
\hline 1093 & $\underline{2093}$ & $\underline{3093}$ & 4093 & Mod & Unit & Lab \\
\hline & $\begin{array}{l}<2.5 E+02 \\
<1.0 E+01 \\
<2.3 E+01\end{array}$ & $\begin{array}{l}<2.5 E+02 \\
<1.0 E+01 \\
<2.3 E+01\end{array}$ & $\begin{array}{l}<2.5 E+02 \\
<1.0 E+01 \\
<2.3 E+01\end{array}$ & $\begin{array}{l}\text { Y3 } \\
\text { Y3 } \\
\text { Y3 }\end{array}$ & $\begin{array}{l}\text { pCi/L } \\
\text { pCi/L } \\
\text { pCi/L }\end{array}$ & $\begin{array}{l}\mathrm{CN} \\
\mathrm{CN} \\
\mathrm{CN}\end{array}$ \\
\hline $3.5 E+\infty$ & $\begin{array}{r}3.4 E+\infty 0 \\
<8.0 E+01\end{array}$ & $\begin{array}{l}<1.0 E+00 \\
<8.0 E+01\end{array}$ & $\begin{array}{l}<1.0 E+00 \\
<8.0 E+01\end{array}$ & Y3 & $\begin{array}{l}\mathrm{pCi} / \mathrm{L} \\
\mathrm{pCi} / \mathrm{L}\end{array}$ & $\begin{array}{l}\mathrm{GE} \\
\mathrm{CN}\end{array}$ \\
\hline $\begin{array}{l}<2.0 \\
5.310\end{array}$ & $<2.0$ & $<2.0$ & $<2.0$ & & $\begin{array}{l}\mu g / L \\
\mu g / L\end{array}$ & GE \\
\hline $\begin{array}{c}<2.0 \\
918\end{array}$ & $\begin{array}{c}<2.0 \\
1.030 \\
<1.0 \mathrm{O}+01\end{array}$ & $\begin{array}{c}<2.0 \\
911 \\
<1.0 E+01\end{array}$ & $\begin{array}{l}<2.0 \\
841 \\
<1.0 E+01\end{array}$ & Y3 & $\begin{array}{l}\mu g / L \\
\mu g / L \\
p C i / L\end{array}$ & $\begin{array}{l}\text { GE } \\
\text { GE } \\
\mathrm{CN}\end{array}$ \\
\hline 21 & 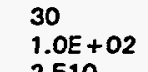 & 18 & $\begin{array}{l}32 \\
<2.0 E+\infty 0\end{array}$ & Y3 & $\begin{array}{l}\mu \mathrm{S} / \mathrm{cm} \\
\mathrm{PCj/L}\end{array}$ & GE \\
\hline 2.440 & 2,510 & $<1,000$ & $\begin{array}{r}4.470 \\
<1.3 E+03 \\
3.5 E+03\end{array}$ & Y3 & $\begin{array}{l}\mu \rho / L \\
p C i / L \\
p C i / L\end{array}$ & $\begin{array}{l}\text { GE } \\
\text { CN } \\
\text { EM }\end{array}$ \\
\hline $\begin{aligned} & 11,000 \\
< & 1.000 \\
< & 5.0\end{aligned}$ & $\begin{aligned} & 19,000 \\
< & 1,000\end{aligned}$ & $\begin{aligned} & 16.000 \\
< & 1.000 \\
< & 5.0\end{aligned}$ & $\begin{array}{l}24.000 \\
2.600\end{array}$ & & $\begin{array}{l}\mu \sigma / L \\
\mu g / L \\
\mu g / L\end{array}$ & $\begin{array}{l}\mathrm{GE} \\
\mathrm{GE}\end{array}$ \\
\hline $\begin{array}{l}120 \\
316,000\end{array}$ & 250 & $<50$ & $<50$ & & $\mu \mathrm{g} / \mathrm{L}$ & GE \\
\hline $\begin{array}{l}<0.24 \\
<0.090\end{array}$ & 166,000 & 40.000 & 166,000 & $J$ & $\begin{array}{l}\mu \mathrm{g} / \mathrm{L} \\
\mu \mathrm{g} / \mathrm{L} \\
\mu \mathrm{g} / \mathrm{L}\end{array}$ & GE \\
\hline $\begin{array}{l}<7.0 E-01 \\
30\end{array}$ & $\begin{array}{r}3.2 E+03 \\
<1.0 E+01 \\
2.9 \\
<2.3 E+01\end{array}$ & $\begin{array}{l}<7.0 E-01 \\
<1.0 E+01 \\
33 \\
<2.3 E+01\end{array}$ & $\begin{array}{r}2.1 E+00 \\
<1.0 E+01 \\
30 \\
<2.3 E+01\end{array}$ & Y3 & $\begin{array}{l}\mathrm{pCi} / \mathrm{mL} \\
\mathrm{pCi/L} \\
\mu \mathrm{g} / \mathrm{L} \\
\mathrm{pCi} / \mathrm{L}\end{array}$ & $\begin{array}{l}\mathrm{GE} \\
\mathrm{CN} \\
\mathrm{GE} \\
\mathrm{CN}\end{array}$ \\
\hline
\end{tabular}

\section{WELL RSE 2}

\begin{tabular}{|c|c|c|c|c|c|c|}
\hline SRS Coord. & Lat/Longitude & Screen Zone Elevation & Top of Casing & Casing & Pump & Screen Zone \\
\hline $\begin{array}{l}\text { N57594.9 } \\
\text { E74743.5 }\end{array}$ & $\begin{array}{l}33.275082 \circ \mathrm{N} \\
81.581870^{\circ} \mathrm{W}\end{array}$ & 289.5-269.7 ft msl & $302.5 \mathrm{ft} \mathrm{msl}$ & 4" PVC & $\mathbf{s}$ & Water Table \\
\hline SAMPLE DATE & & $03 / 10 / 93$ & $05 / 21 / 93$ & $07 / 27 / 93$ & $12 / 05 / 93$ & \\
\hline
\end{tabular}

FIELD DATA

Analyte

Water elevation

Dopth to water

pH

Sp. conductance

Water temperature

Alkalinity as $\mathrm{CaCO}_{3}$

Turbidity

Volume purged

Sampling codo

ANALYTICAL DATA

Actinium-228

Aluminum, total recoverable

Antimony-125

Arsenic, total recoverable

Barium, total recoverable

Cadmium, total recoverable

Calcium, total rocoverable

Corium-144

Cosium.134

\begin{tabular}{ll}
\hline 1093 & $\underline{2093}$ \\
293.5 & 292.5 \\
9.0 & 10.0 \\
5.4 & 5.6 \\
46 & 44 \\
19.7 & 21.5 \\
360 & 190 \\
1.0 & 1.5 \\
& $x$
\end{tabular}

$\underline{3093}$

4093 Unir

$\begin{array}{ll} & 283.5 \\ 5.8 & 19.0 \\ 38 & 5.7 \\ 28.0 & 28 \\ 34 & 22.0 \\ & 255 \\ & 0.2 \\ & x\end{array}$

ft msl

ft

$\mathrm{pH}$

$\mu \mathrm{S} / \mathrm{cm}$

${ }^{\circ} \mathrm{C}$

$\mathrm{mg} / \mathrm{L}$

NTU

X.2 Well vol.

\begin{tabular}{|c|c|c|c|c|c|c|c|}
\hline 1093 & $\underline{2093}$ & 30993 & 4093 & Mod & Unit & $\underline{\text { Lab }}$ & Flas \\
\hline 1.940 & & 640 & $<8.4 \mathrm{E}+01$ & Y3 & $\begin{array}{l}\mathrm{pCi} / \mathrm{L} \\
\mu_{\mathrm{N} / \mathrm{L}}\end{array}$ & CN & 0 \\
\hline$<20$ & $<2.5 E+01$ & $<2.5 E+01$ & $<2.5 E+01$ & Y3 & $\mathrm{pCi} / \mathrm{L}$ & $\begin{array}{l}\text { CN } \\
\text { GE }\end{array}$ & $\begin{array}{l}0 \\
0\end{array}$ \\
\hline 41 & 30 & 23 & 15 & & $\mu \mathrm{g} / \mathrm{L}$ & GE & \\
\hline$<2.0$ & $<2.0$ & $<2.0$ & $<2.0$ & & $\mu \mathrm{g} / \mathrm{L}$ & GE & 0 \\
\hline 1.830 & $\begin{aligned} & 1.800 \\
< & 5.0 E+01\end{aligned}$ & $\begin{array}{c}1.220 \\
<5.0 E+01\end{array}$ & $\begin{array}{l}954 \\
<5.0 E+01\end{array}$ & & $\mu \mathrm{g} / \mathrm{L}$ & $\begin{array}{l}\text { GE } \\
\text { CN }\end{array}$ & 0 \\
\hline & $<1.0 E+01$ & $<1.0 E+01$ & $<1.0 E+01$ & Y3 & $\mathrm{pCi} / \mathrm{L}$ & $\mathrm{CN}$ & \\
\hline
\end{tabular}

Note: Flagging levels, modifiers, and laboratories are for 4th quarter 1993 data only. See Appendix B for flagging criteria.

- = exceeded holding time for 4th quarter 1993.

a = excoeded screening level or final primary drinking water standard for 4th quarter 1993. 


\section{Unclassified}

Woll RSE 2 continuad

ANALYTICAL DATA

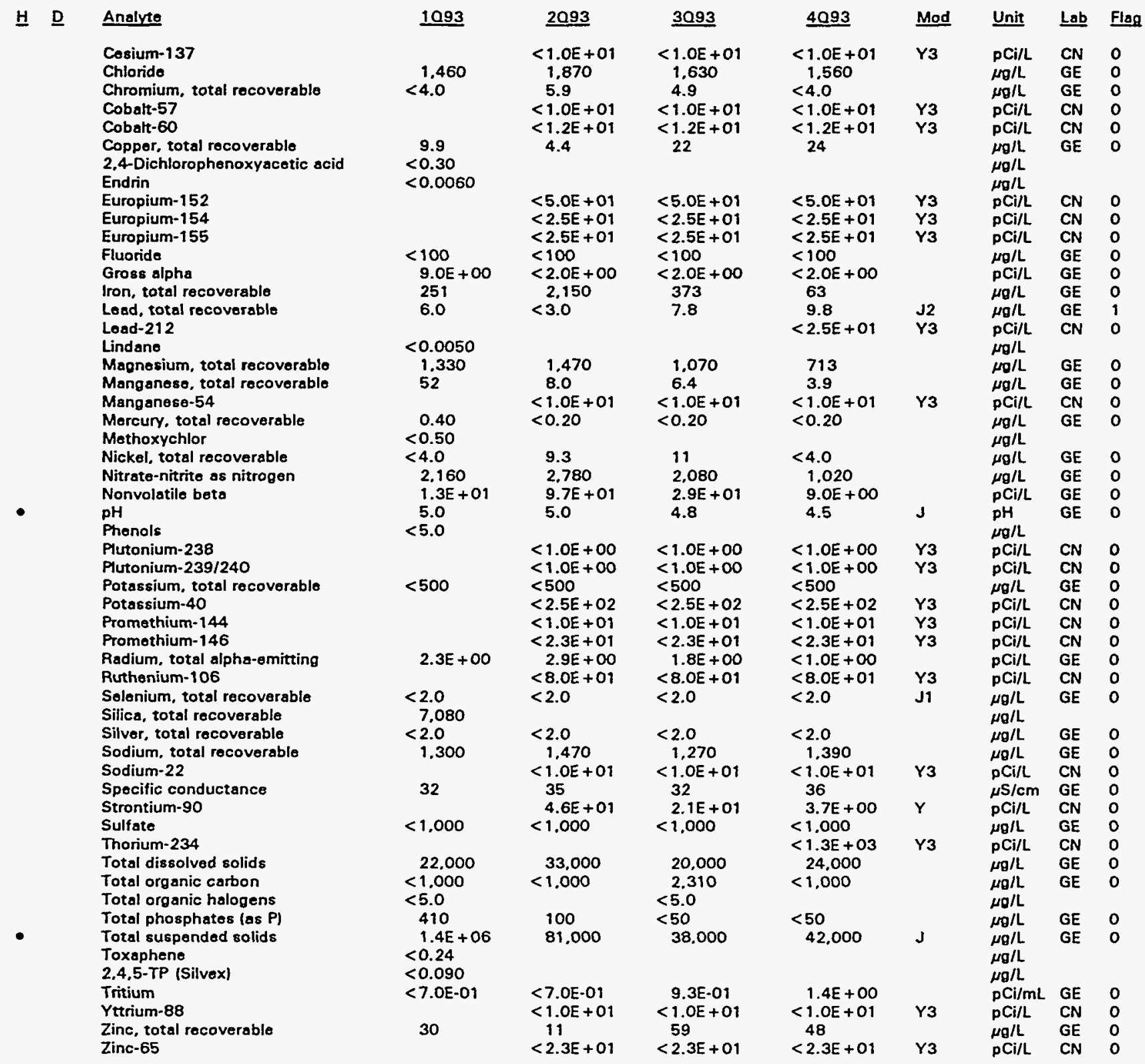

Note: Flagging levels, modifiers, and laboratories are for 4 th quarter 1993 data only. See Appendix $B$ for flagging criteria. - = exceeded holding time for 4th quarter 1993.

- = exceeded screening level or final primary drinking water standard for 4th quarter 1993. 
WELL RSE 3A

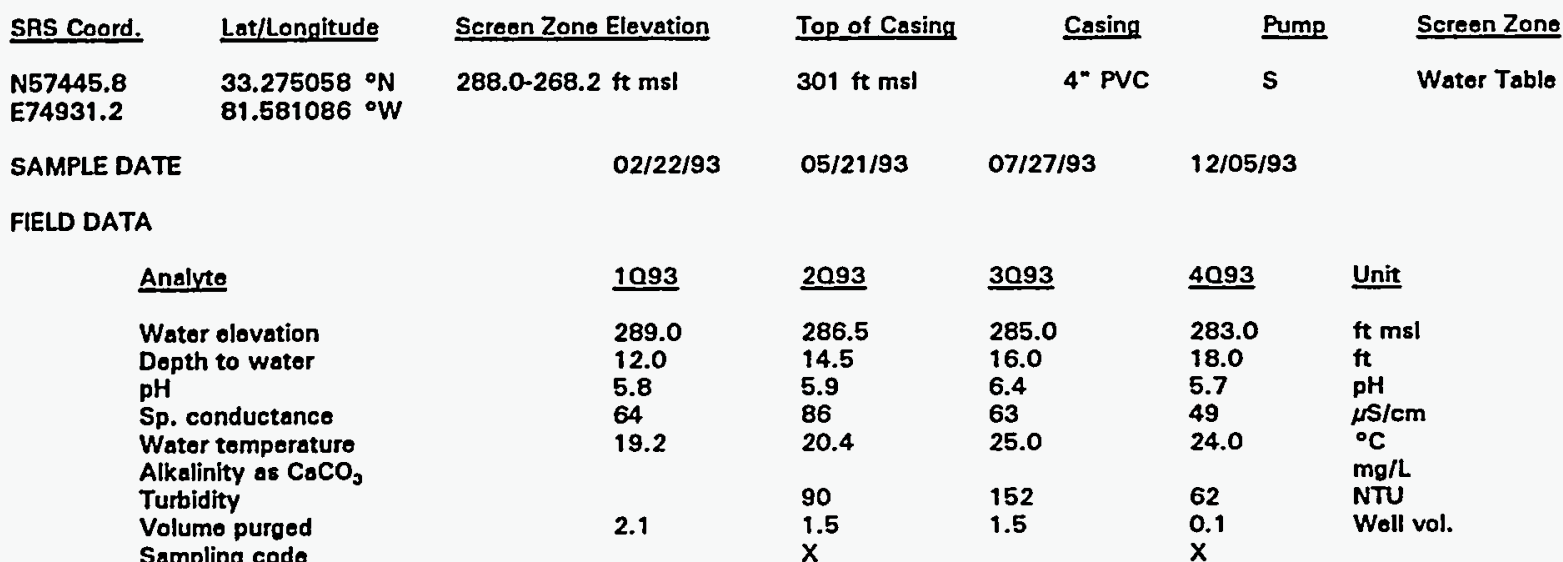

\section{ANALYTICAL DATA}

\section{브 \\ Analyte}

Actinium-228

Aluminum, total recoverable Antimony-125

Arsenic, total recoverable

Barium, total recoverable

Cadmium, total recoverable

Calcium, total recoverable

Cerium-144

Cesium-134

Cosium-137

Chloride

Chromium, total recoverable

Cobalt-57

Cobalt 60

Copper, total recoverable

2,4-Dichlorophenoxyacetic acid

Endrin

Europium-152

Europium-154

Europium-155

Fluoride

- Grose alpha

Iron, total recoverable

Load, total recoverablo

Load-212

Lindane

Magnesium, total recoverable

Manganese, total recoverable

Manganese-54

Mereury, total recoverable

Mothoxychlor

Nickel, total recoverable

Nitrate-nitrite as nitrogen

- Nonvolatilo beto

pH

Phenols

Plutonium-238

Plutonium-239/240

Potassium, total recoverable

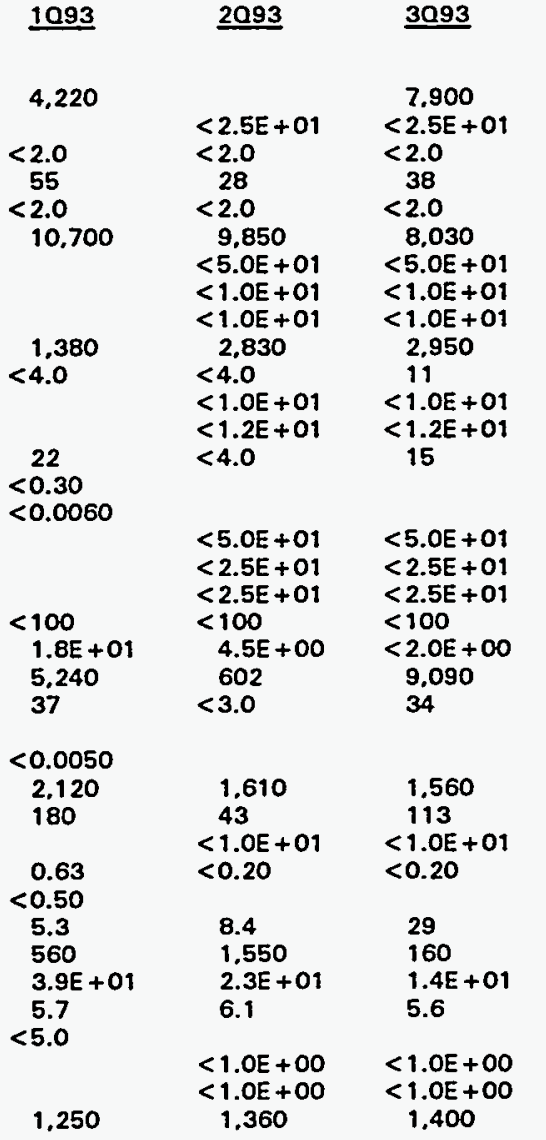

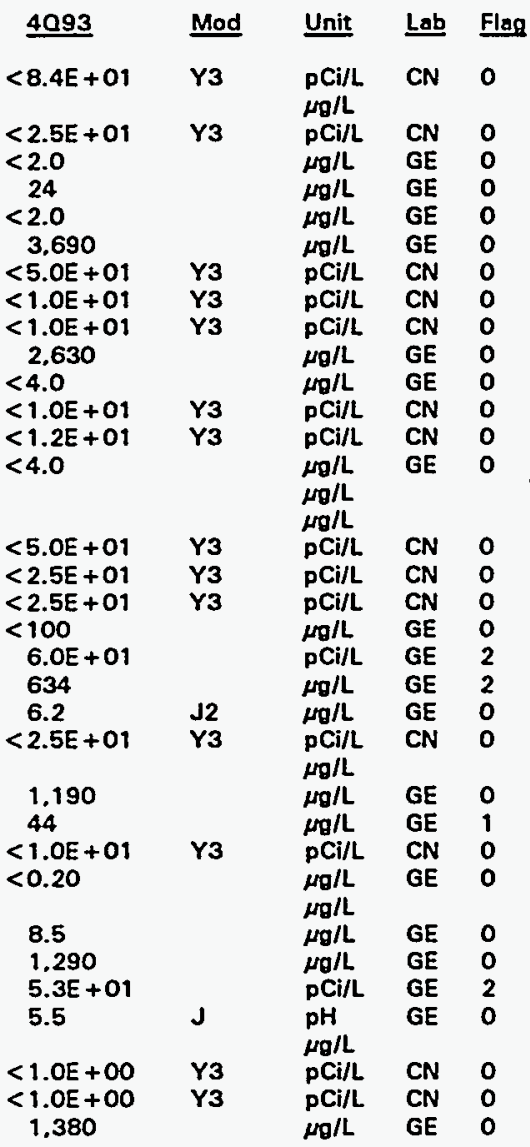

Note: Flagging levels, modifiers, and laboratories are for 4th quarter 1993 data only. See Appendix B for flagging criteria. - = exceeded holding time for 4th quarter 1993.

- = exceeded screening level or final primary drinking water standard for 4th quarter 1993. 


\section{ANALYTICAL DATA}

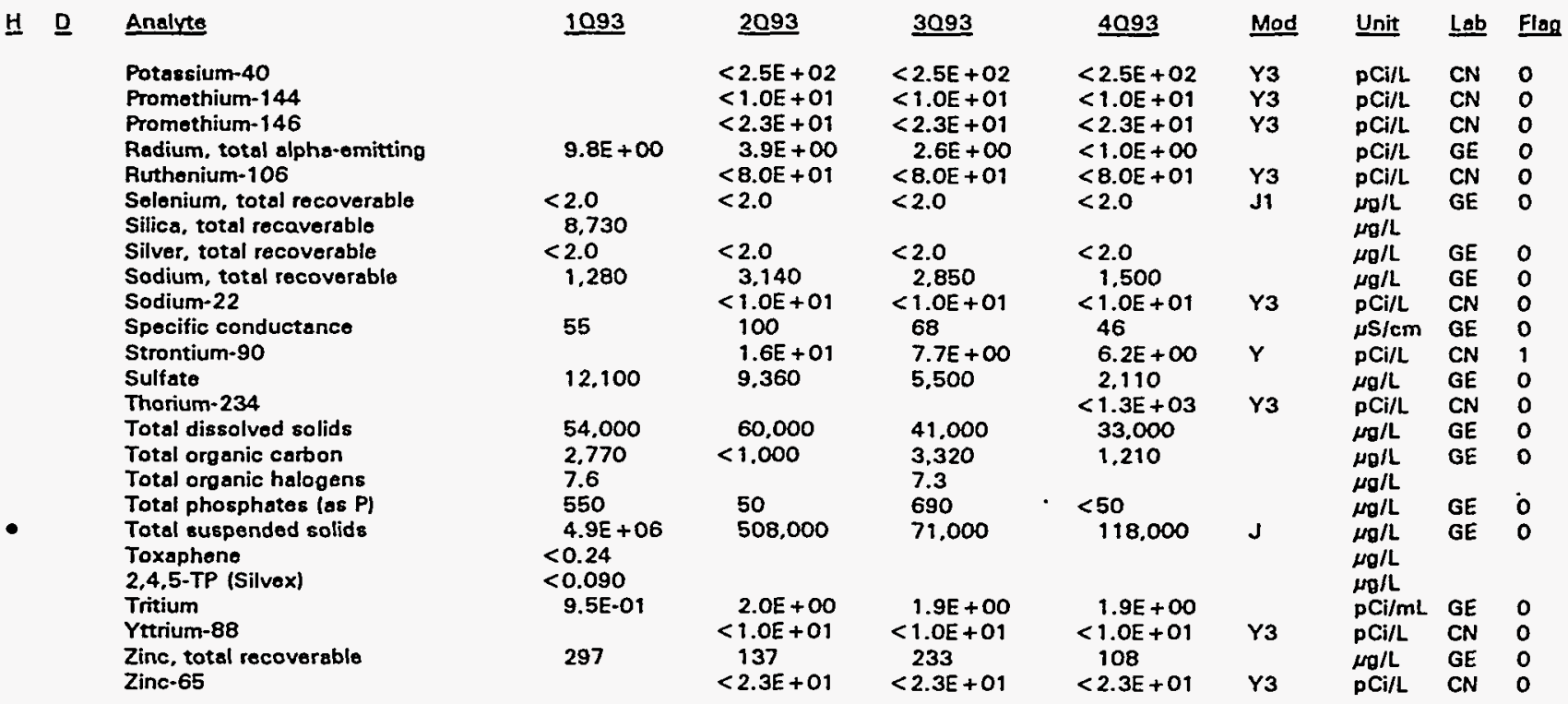

\section{WELL RSE 4A}

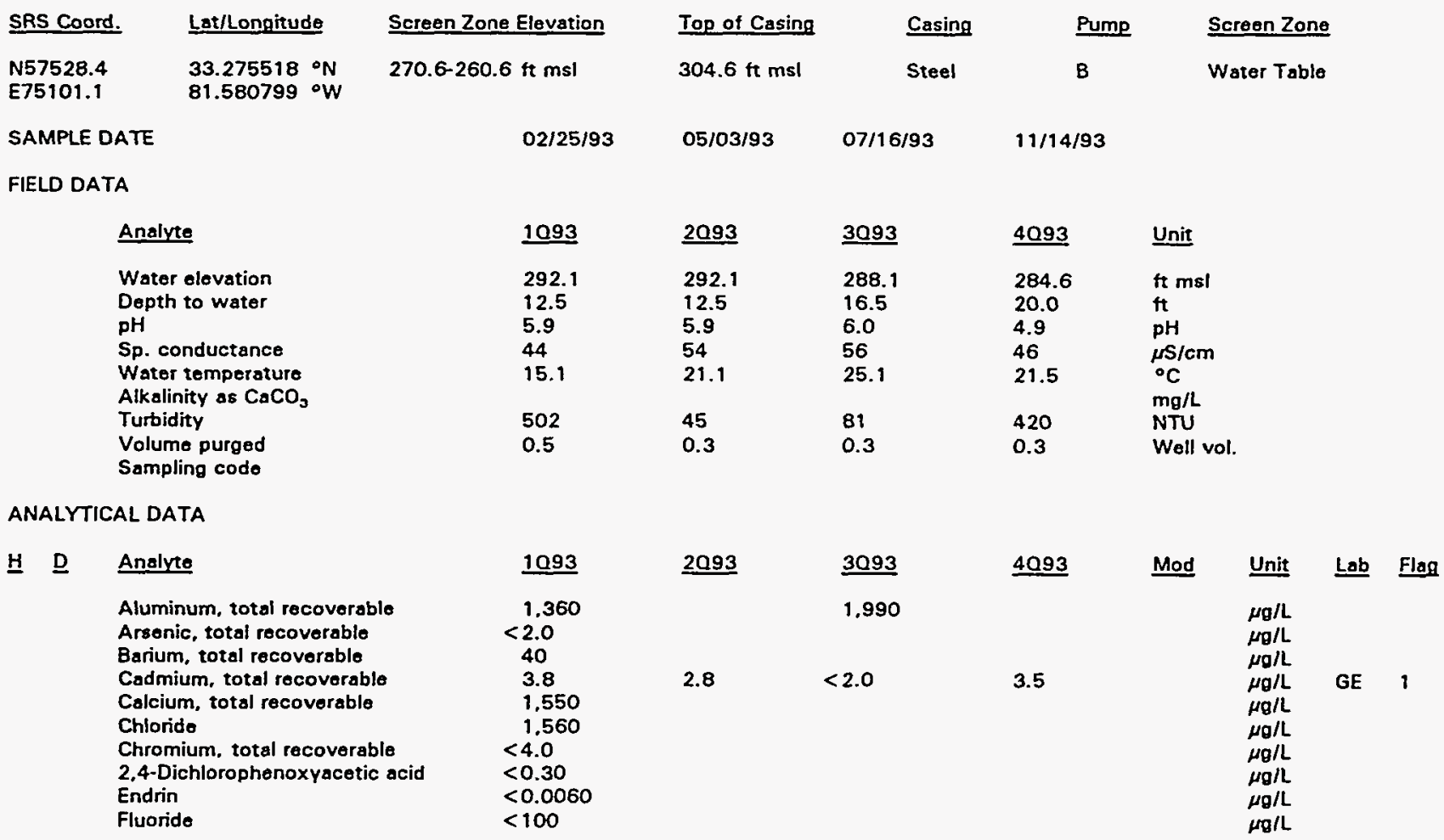

Note: Flagging levels, modifiers, and laboratories are for 4th quarter 1993 data only. See Appendix $B$ for flagging criteria. - = exceeded holding time for 4th quarter 1993.

- = exceeded screening level or final primary drinking water standard for 4th quarter 1993. 


\section{ANALYTICAL DATA}

H D Analyte

Grose alpha

Iron, total recoverable

Lead, total recoverable

Lindano

Magnesium, total recoverable

Manganese, total recoverablo

Mercury, total recovorable

Mothoxychlor

Nitrato-nitrito as nitrogen

- Nonvolatile bota

pH

Phenole

Potossium, total recoverable

Radium, total alpha-omitting

Solenium, total recoverable

Silice, total rocoverable

Silver, total recoverable

Sodium, total recoverable

Spocific conductence

Sulfate

Total dissolved solids

Total organic carbon

Total organic halogens

Total phosphates (as PI

Toxaphene

2,4,5-TP (Silvex)

Tritium

1093
$<2.0 \mathrm{E}+0 \mathrm{O}$
286
$<3.0$
$<0.0050$
1.740
16
2.8
$<0.50$
3.350
$8.2 \mathrm{E}+02$
5.0
$<5.0$
$<500$
$4.4 \mathrm{E}+00$
$<2.0$
7.480
$<2.0$
1.760
45
1,580
28.000
$<1.000$
$<5.0$
$<50$
$<0.24$
$<0.090$
$1.3 \mathrm{E}+00$

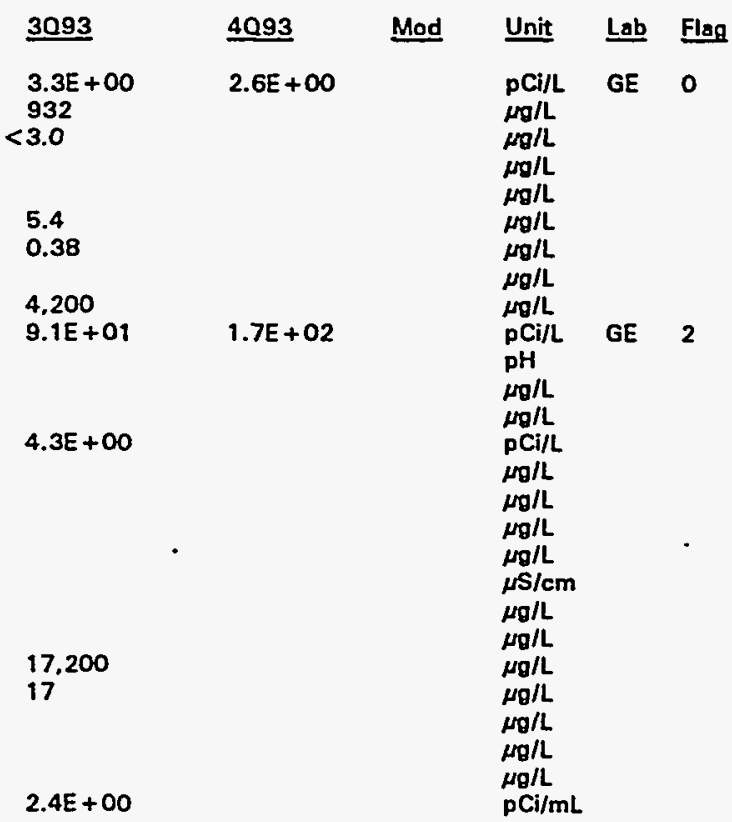

\section{WELL RSE 4B}

SRS Coord.

N57531.0

E75096.2

Lat/Longitude

$33.275516 \circ \mathrm{N}$ 81.580817 oW

SAMPLE DATE

FIELD DATA

\section{Analyte}

Water olevation Dopth to water

$\mathrm{pH}$

Sp. conductence

Water temperature

Alkalinity as $\mathrm{CaCO}_{3}$

Turbidity

Volume purged

Sampling code

ANALYTICAL DATA

H D Analyte

Aluminum, total recoverable

Arsenic, total recoverable

Barium, total recoverable

Cadmium, total recoverable

Calcium, total recoverable

Chloride

Chromium, total rocoverablo

Fluoride
Screen Zone Elevation

$02 / 25 / 93$

Top of Casing

305.9 ft msl

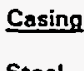

Pump

$\mathbf{B}$

Screen Zone

Water Table
2093

292.4

13.5

6.4

24

20.8

260

$\mathrm{x}$

2093

3093

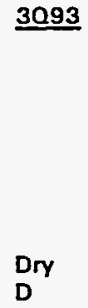

D
Dry
D

$\begin{array}{ll}\text { 4093 Unit } \\ & \mathrm{ft} \text { msl } \\ & \mathrm{ft} \\ & \mathrm{pH} \\ & \mu \mathrm{S} / \mathrm{cm} \\ & { }^{\circ} \mathrm{C} \\ & \mathrm{mg} / \mathrm{L} \\ & \mathrm{NTU} \\ & \text { Well vol. } \\ \text { Dry } & \end{array}$

8.3

4093 Mod Unit Lab Flag

Note: Flagging levels, modifiers, and laboratories are for 4th quarter 1993 data only. See Appendix 8 for flagging criteria.

- =xceeded holding time for 4th quarter 1993.

* =xceeded screening level or final primary drinking water standard for 4th quarter 1993. 
ANALYTICAL DATA

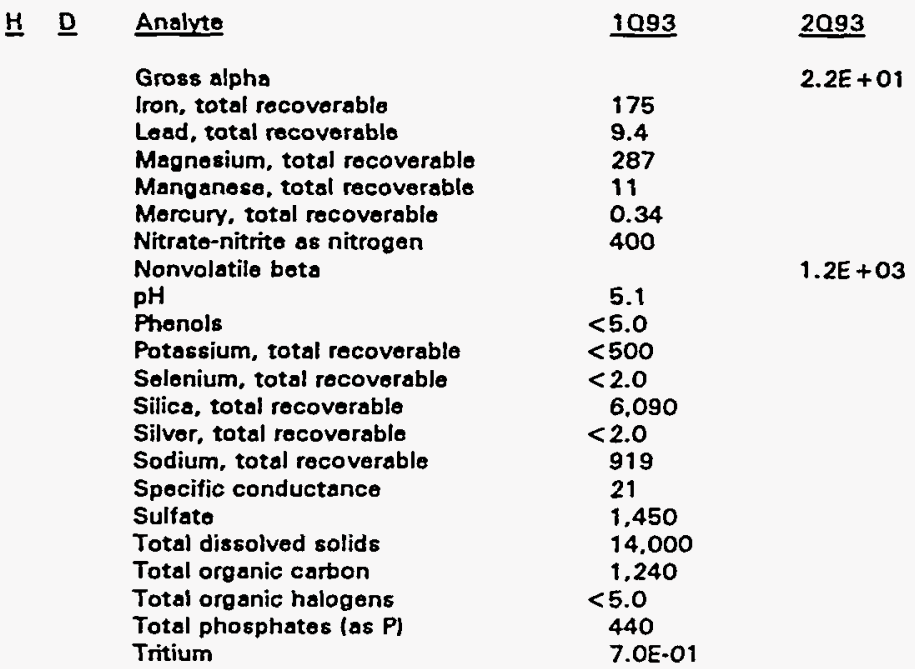

$\underline{3093}$

4093

Mod

Unit

Lab Flag

pCi/L

$\mu g / L$

$\mu g / L$

$\mathrm{moll}$

$\mu g / L$

roll

$\mathrm{pCi} / \mathrm{L}$.

pH

inglL

$\mu g / L$

$\mu g / L$

$\mu \mathrm{glL}$

ugll

$\mu \mathrm{S} / \mathrm{cm}$

$\mu \mathrm{g} / \mathrm{L}$

$\mu g / L$

$\mu g / L$

$\mu g / L$

$\mu g / L$

$\mathrm{pCi} / \mathrm{mL}$

\section{WELL RSE 4C}

$\begin{array}{ll}\text { SRS Coord. } & \text { Lat/Longitude } \\ \text { N57533.6 } & 33.275515{ }^{\circ} \mathrm{N} \\ \text { E75092.6 } & 81.580832{ }^{\circ} \mathrm{W} \\ & \end{array}$

$304.7 \mathrm{ft} \mathrm{ms}$

Casing

Pump

Screen Zone

SAMPLE DATE

$02 / 25 / 93$

05/03/93

07/16/93

$11 / 14 / 93$

FIELD DATA

Analyte
Water olevation
Dopth to water
pH
Sp. conductance
Water tomperature
Alkalinity as $\mathrm{CaCO}_{3}$
Turbidity
Volume purged
Sampling code

$\frac{1093}{292.2}$

12.5

5.8

39

15.6

144

2093
292.2
12.5
6.1
37
20.0
100

3093
287.7
17.0
5.9
41
24.1

4093

Unit

284.7 ft msl

$20.0 \quad \mathrm{ft}$

$5.1 \quad \mathrm{pH}$

$34 \quad \mu \mathrm{S} / \mathrm{cm}$

21.8

${ }^{\circ} \mathrm{C}$

77

$\mathrm{mg} / \mathrm{L}$

Woll vol.

Water Table

\section{ANALYTICAL DATA}

\section{H D Analvte}

Aluminum, total recoverable Arsenic, total recoverable Barium, total recoverable

Cadmium, total recoverable

Calcium, total racoverable

Chloride

Chromium, total recoverable

2,4-Dichlorophenoxyacetic acid

Endrin

Fluoride

Gross alpha

Iron, total recoverable

Lead, total recoverable
1093

1.400

$<2.0$

23

578

2,320

$<4.0$

$<0.30$

$<0.0060$

$<100$

$<2.0 E+O O<2.0 E+O O<2.0 E+$

618

$<3.0$

2093

3.7

$<2.0$

$<3.0$
3093

1,070

7.5

120
4093

$\underline{\text { Mod }}$

$\underline{\text { Unit }}$ Lab Flag

$\mu \mathrm{g} / \mathrm{L}$

$\mu \mathrm{g} / \mathrm{L}$

$\mu \mathrm{g} / \mathrm{L}$

$\mu g / L$

$\mu \mathrm{g} / \mathrm{h}$

$\mu \mathrm{g} / \mathrm{l}$

$\mu \mathrm{g} / \mathrm{L}$

$\mu \mathrm{g} / \mathrm{L}$

$\mu_{\mathrm{g}} / \mathrm{L}$

$\mathrm{pCi} / \mathrm{L}$.

$\mu g / L$

$\mu \mathrm{g} / \mathrm{L}$
GE 2

GE $\quad 0$

Note: Flagging levels, modifiers, and laboratories are for 4th quarter 1993 data only. See Appendix B for flagging criteria. - = exceeded holding time for 4th quarter 1993.

- =xceeded screening level or final primary drinking water standard for 4th quarter 1993. 
Well RSE $4 \mathrm{C}$ continued

ANALYTICAL DATA

H

Analyte

Lindane

Magnesium, total recoverable

Manganese, total recoverable

Mercury, total recoverable

Mothoxychlor

Nitrate-nitrite as nitrogen

- Nonvolatile beta

pH

Phenole

Potassium, total recoverable

Radium, total alpha-omitting

Selenium, total recoverable

Silica, total recoverable

Silvor, total recoverable

Sodium, total recoverable

Specific conductanco

Sulfato

Total dissolved solids

Total oroanic carbon

Total organic halogens

Total phosphates (as P)

Toxaphene

2,4,5-TP (Silvox)

Tritium

1093
$<0.0050$
1.380
18
0.31
$<0.50$
2.700
$3.5 \mathrm{E}+02$
5.1
$<5.0$
$<500$
$2.3 \mathrm{E}+00$
$<2.0$
7.150
$<2.0$
1,900
38
$<1,000$
35,000
$<1.000$
$<5.0$
$<50$
$<0.24$
$<0.090$
$1.5 \mathrm{E}+00$

2093

3093

4093

Mod

Unit

Lab Flag

\begin{tabular}{|c|c|c|}
\hline & $\begin{aligned} & 3.8 \\
< & 0.20\end{aligned}$ & \\
\hline $8.2 E+02$ & $\begin{array}{l}3.550 \\
1.6 E+02\end{array}$ & $1.6 E+02$ \\
\hline
\end{tabular}

4.OE +00

1.070

42

$1.7 E+00$ $\mu a / 2$

$\mu / 2$

$\mu g / L$

$\mu \mathrm{g} / \mathrm{L}$

$\mu g / L$

$\mu \mathrm{g} / \mathrm{L}$

$\mathrm{pCi} /$

po/L

$\stackrel{\mu \rho}{\mu g} / L$

PCi/L

$\mu \mathrm{g} / \mathrm{L}$

$\mu g / L$

$\mu \mathrm{g} / \mathrm{L}$

$\mathrm{\mu g} / \mathrm{L}$

$\mu \mathrm{S} / \mathrm{cm}$

$\mu \mathrm{g} / \mathrm{L}$

$\mu \mathrm{g} / \mathrm{L}$

$\mu g / L$

$\mu \mathrm{g} / \mathrm{L}$

$\mu \mathrm{g} / \mathrm{L}$

$\mu \mathrm{g} / \mathrm{L}$

$\mathrm{mo} / \mathrm{L}$

$\mathrm{pCi} / \mathrm{mL}$

\section{WELL RSE 5}

\begin{tabular}{|c|c|}
\hline SRS Coord & Lat/Longitude \\
\hline $\begin{array}{l}\text { N57588.9 } \\
\text { E74969.6 }\end{array}$ & $\begin{array}{l}33.275437 \\
81.581263\end{array}$ \\
\hline \multicolumn{2}{|c|}{ SAMPLE DATE } \\
\hline \multicolumn{2}{|c|}{ FIELD DATA } \\
\hline \multicolumn{2}{|r|}{ Analyte } \\
\hline & $\begin{array}{l}\text { Water elevation } \\
\text { Dopth to water } \\
\text { pH } \\
\text { Sp. conductance } \\
\text { Water temperature } \\
\text { Alkalinity as } \mathrm{CaCO}_{3} \\
\text { Turbidity } \\
\text { Volume purged } \\
\text { Sampling code }\end{array}$ \\
\hline
\end{tabular}

ANALYTICAL DATA

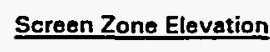

03/01/93

\section{Top of Casing}

$306 \mathrm{ft} \mathrm{msl}$

05/05/93

$\underline{2093}$

292.0

14.0

5.4

60

21.0

19.0

24

\begin{tabular}{ll}
1093 & $\underline{2093}$ \\
\hline 291.5 & 292.0 \\
14.5 & 14.0 \\
5.7 & 5.4 \\
47 & 60 \\
19.0 & 21.0 \\
24 & 12
\end{tabular}

12

Dry
D

$\frac{\text { Casing }}{\text { Steel }}$

$07 / 21 / 93$

$11 / 20 / 93$

\section{Pump Scroen Zone \\ Water Table}

H $\underline{D}$

Analyte

Aluminum, total recoverable

Arsenic, total recoverable

Barium, total recoverable

Cadmium, total recovorable

Calcium, total recoverable

Chlorido

Chromium, total recoverable

2,4-Dichlorophenoxyacetic acid

Endrin

Fluoride

Gross alpho
1093

911

$<2.0$

11
27

654

4,030

$<4.0$

$<0.30$

$<0.0060$

$<100$

$<2.0 E+O O<2.0 E+O O$

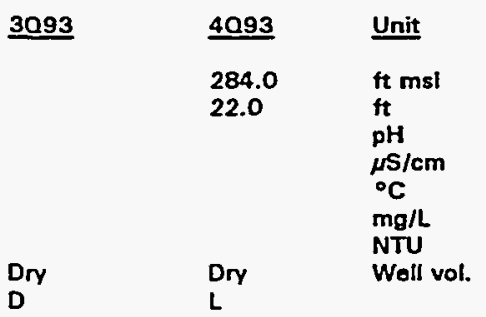

$\underline{3093}$

$\underline{4093}$

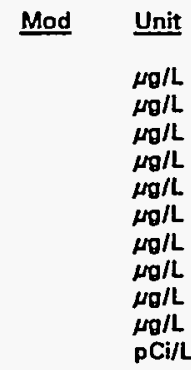




\section{ANALYTICAL DATA}

\section{브 D Analyte}

Iron, total recoverable Lead, total recoverable Lindane

Magnosium, total recoverable

Manganese, total recoverable

Mercury, total recoverable

Methoxychlor

Nitrate-nitrite as nitrogen

Nonvolatile beta

pH

Phenols

Potassium, total recoverable

Radium, total alpha-amitting

Selenium, total recoverable

Silica, total recoverable

Silver, total recoverable

Sodium, total recavorable

Specific conductance

Sulfate

Total dissolved solids

Total organic carbon

Total organic halogens

Total phosphates (as P)

Toxaphene

2,4,5-TP (Silvex)

Tritium

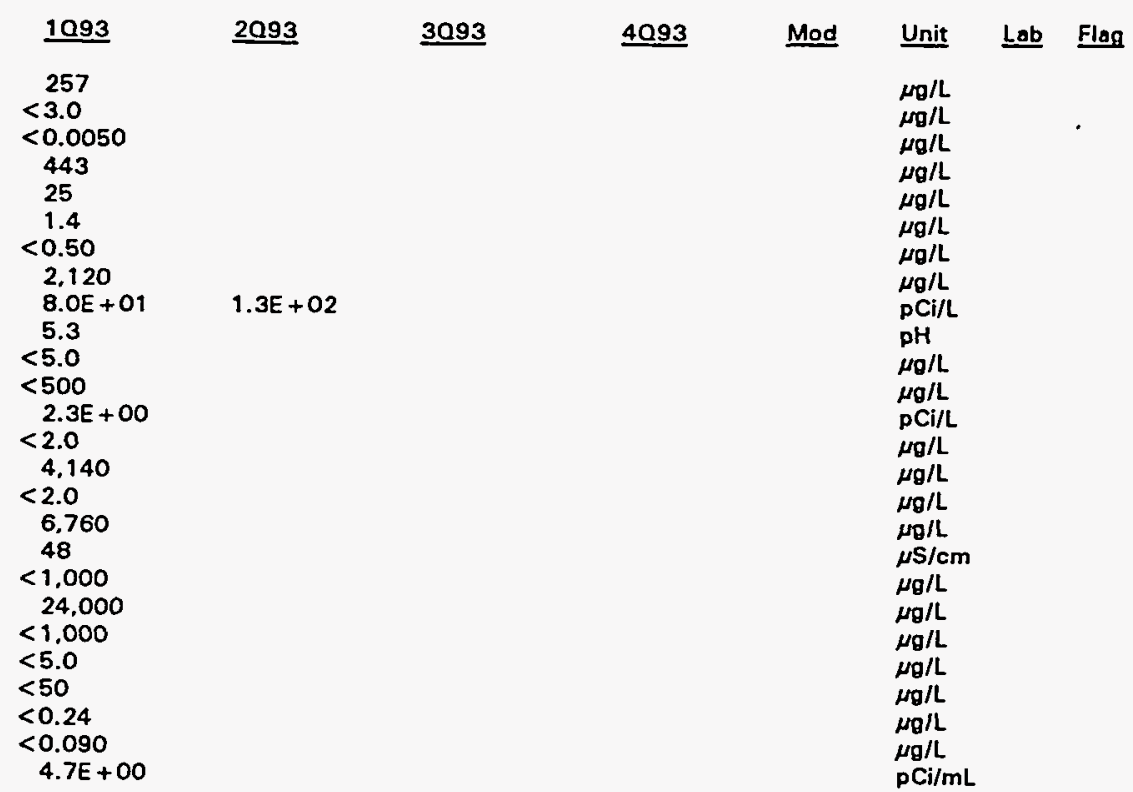

\section{WELL RSE 6}

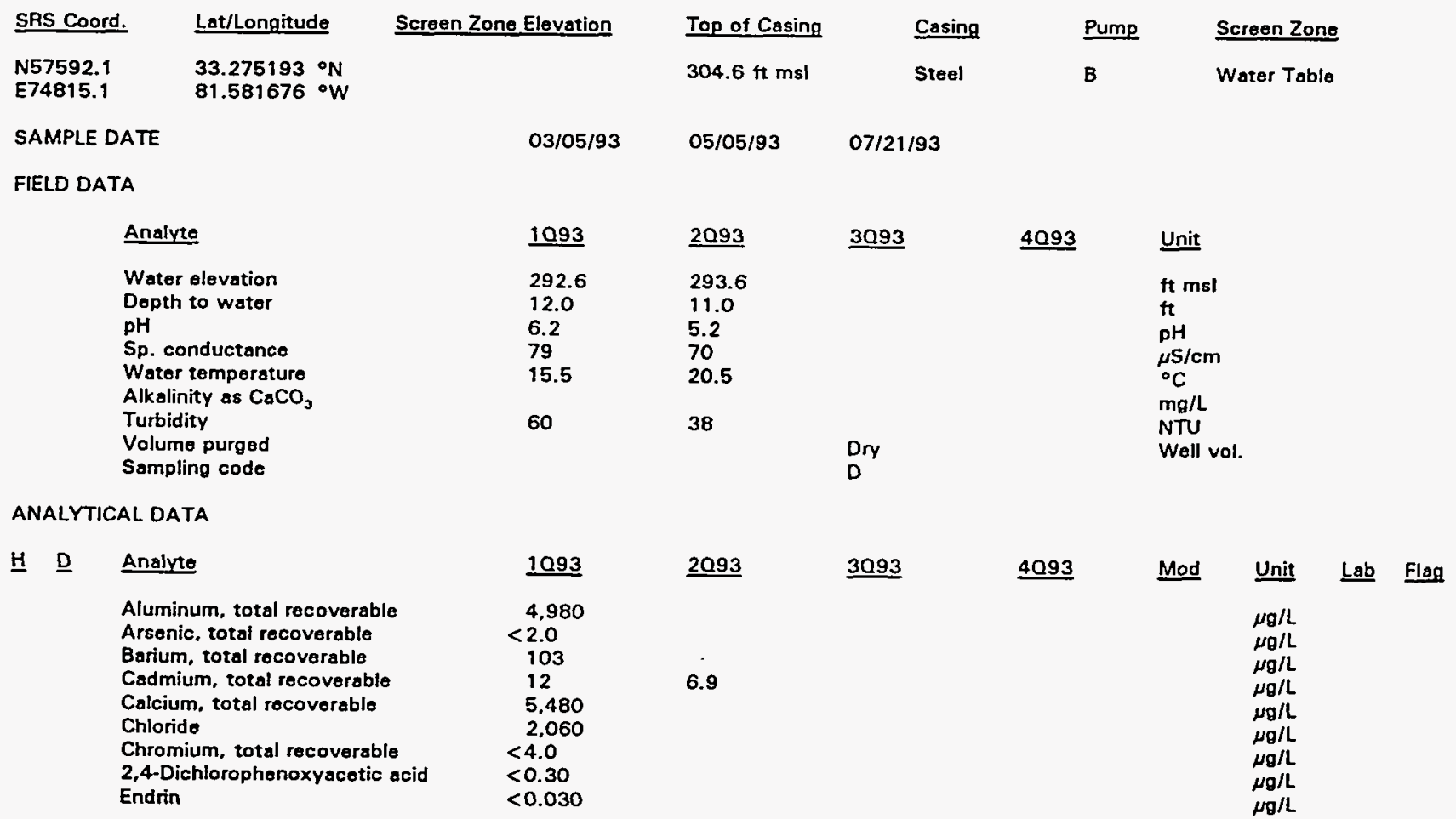

Note: Flagging levels, modifiers, and laboratories are for 4th quarter 1993 data only. See Appendix B for flagging criteria. - = exceeded holding time for 4th quarter 1993.

- = exceeded screening level or final primary drinking water standard for 4th quarter 1993. 
Woll RSE 6 continued

ANALYTICAL DATA

H D Analyte

Fluorido

Gross alpha

Iron, total recovarable

Lead, total recoverable

Lindano

Magnosium, total recovorable

Manganese, total recoverable

Mercury, total recoverable

Mothoxychlor

Nitrato-nitrite as nitrogon

Nonvolatile beta

pH

Phonols

Potassium, total recoverable

Radium, total alpha.omitting

Solenium, total recoveroble

Sillca, total recoverable

Silver, total recoverable

Sodium, total recovorable

Specific conductance

Sulfato

Total dissolved solids

Total organic carbon

Total organic halogens

Total phosphates (as P

Toxapheno

2,4,5-TP (Silvex)

Tritium

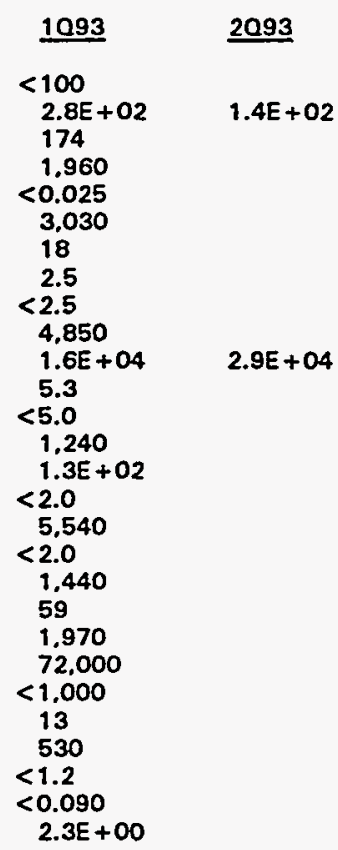

$\underline{3093}$

$\underline{4093}$

Mod

Unit Lab Flag

$\mu g / L$

pCill

$\mu \mathrm{g} / \mathrm{L}$

$\mu g / L$

$\mu g / L$

$\mathrm{Ng} / \mathrm{L}$

$\mu \mathrm{g} / \mathrm{L}$

$\mu \mathrm{\mu g} / \mathrm{L}$

$\mu \mathrm{Ml}$

$\mathrm{pCi} / \mathrm{L}$

$\mathrm{pH}$

$\mu \mathrm{mol}$

$m a / L$

$\mathrm{PCi} / \mathrm{L}$

$\mu \mathrm{g} / \mathrm{L}$

$\mu \mathrm{g} / \mathrm{L}$

$\mu g / L$

$\mu \mathrm{rg} / \mathrm{L}$

$\mu \mathrm{S} / \mathrm{cm}$

$\mu \mathrm{g} / L$

$\mu \mathrm{g} / \mathrm{L}$

$\mu / L$

$\mu g / L$

$\mu g / L$

$\mu g / L$

$\mathrm{\mu g} / \mathrm{L}$

$\mathrm{pCi} / \mathrm{mL}$

\section{WELL RSE 7}

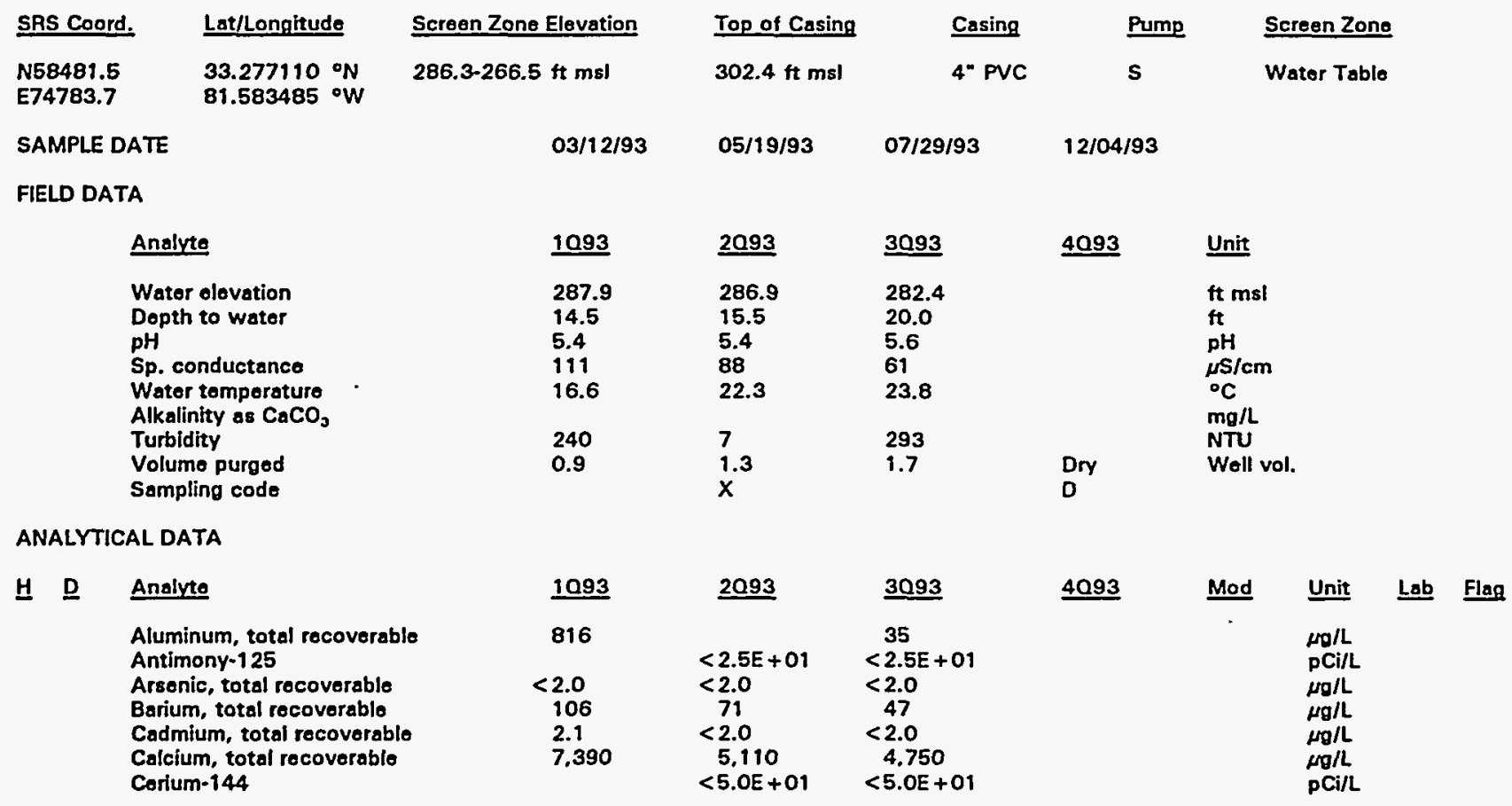

Note: Flagging levels, modifiers, and laboratories are for 4th quarter 1993 data only. See Appendix $B$ for flagging criteria.

- = exceeded holding time for 4th quarter 1993.

- = exceeded screening level or final primery drinking water standard for 4th quarter 1993. 
WSRC-TR-94-0354

Unclassified

Well RSE 7 continued

ANALYTICAL DATA

H D Analyte

Cesium-134

Cosium-137

Chlorido

Chromium, total recoverable $\quad<4.0$

Cobatt-57

Cobalt-60

Copper, total recoverable

2,4-Dichlorophenoxyacetic acid

Endrin

Europium-152

Europium-154

Europium-155

Fluorido

Gross alpha

Iron, total recoverable

Lead, total recoverable

Lindane

Magnesium, total recoverable

Manganese, total recoverable

Manganese-54

Mercury, tatal recoverable

Mothoxychlor

Nickol, total recoverable

Nitrate-nitrite as nitrogen

Nonvolatile beta

pH

Phenols

Plutonium-238

Plutonium-239/240

Potassium, total recoverable

Potassium-40

Promethium-144

Promethium-146

Radium, total alpha-emitting

Ruthenium-106

Selenium, total recoverable

Silica, total recoverable

Silver, total recoverable

Sodium, total recoverable

Sodium-22

Specific conductance

Strontium-90

Sulfate

Total dissolved solids

Total organic carbon

Total organic halogens

Total phosphates (as P)

Total suspended solids

Toxaphene

2,4,5-TP (Silvex)

Tritium

Yttrium-88

Zinc, total recoverable

Zine- 65

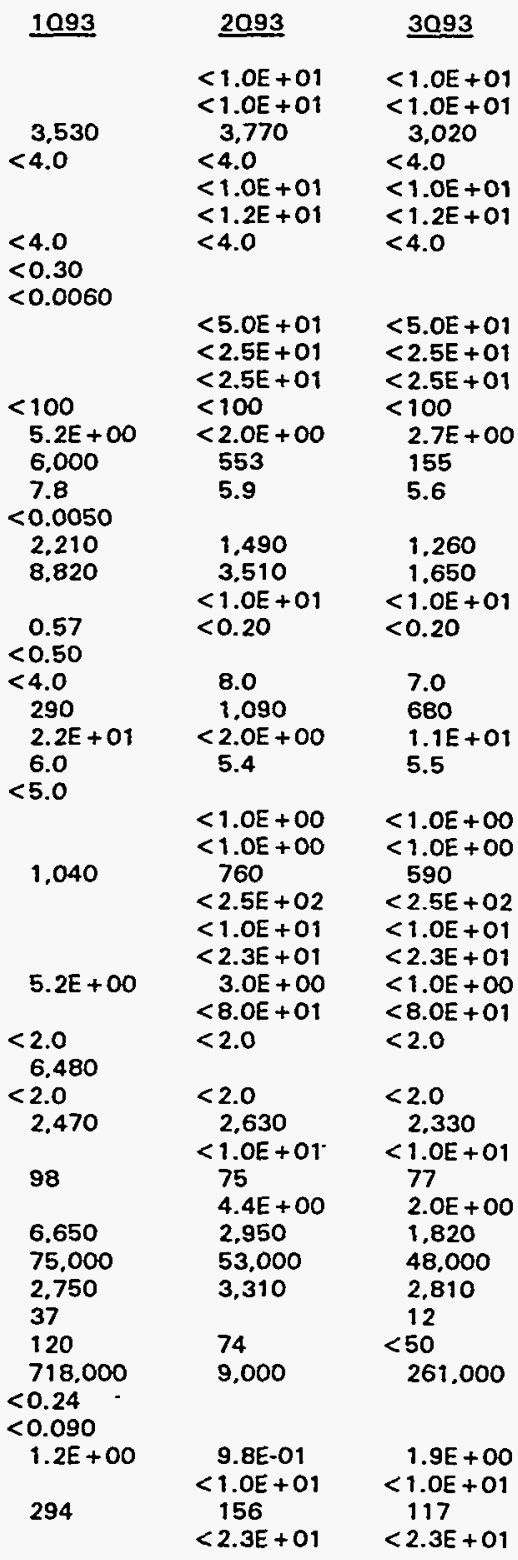

4093 Mod Unit Lab Flag

$\mathrm{pCi} / \mathrm{L}$

$\mathrm{pCi} / \mathrm{L}$

$\mu \mathrm{g} / \mathrm{L}$

$\mu \mathrm{g} / \mathrm{L}$

pCi/L

$\mathrm{pCi} / \mathrm{L}$

$\mu \mathrm{g} / \mathrm{L}$

$\mu_{\mathrm{g} / \mathrm{L}}$

$\mu \mathrm{g} / \mathrm{L}$

$\mathrm{pCi} / \mathrm{L}$

pCilL

pCi/L

$\mu \mathrm{g} / \mathrm{L}$

$\mathrm{pCi} / \mathrm{L}$

$\mu \mathrm{g} / \mathrm{L}$

$\mu \mathrm{g} / \mathrm{L}$

$\mu g / L$

$\mu \mathrm{g} / \mathrm{L}$

$\mathrm{\mu g} / \mathrm{L}$

$\mathrm{pCi} / \mathrm{L}$

$\mu \mathrm{g} / \mathrm{L}$

$\mu g / L$

$\mu \mathrm{g} / \mathrm{L}$

$\mu \mathrm{g} / \mathrm{L}$

pCi/L

$\mathrm{pH}$

$\mu \mathrm{g} / \mathrm{L}$

pCi/l

pCi/L

$\mu \mathrm{g} / \mathrm{L}$

pCi/L

pCi/L

pCi/L

pCi/L

pCi/L

$\mu g / L$

$\mu \mathrm{g} / \mathrm{L}$

$\mu \mathrm{g} / \mathrm{L}$

$\mu \mathrm{g} / \mathrm{L}$

pCi/L

$\mu \mathrm{S} / \mathrm{cm}$

$\mathrm{PCi} / \mathrm{L}$

$\mu \mathrm{g} / \mathrm{L}$

$\mu g / L$

$\mu \mathrm{g} / \mathrm{L}$

$\mu \mathrm{g} / \mathrm{L}$

$\mu g / L$

$\mu g / L$

$\mu \mathrm{g} / \mathrm{L}$

$\mu_{g} / L$

$\mathrm{pCi} / \mathrm{mL}$

pCill

$\mu g / L$

pCi/L

Note: Flagging levels, modifiers, and laboratories are for 4th quarter 1993 data only. See Appendix B for flagging criteria. - = exceeded holding time for 4th quarter 1993.

- = exceeded screening level or final primary drinking water standard for 4th quarter 1993. 
WELL RSE 8

\section{SRS Coord. \\ N58538.8 \\ E74869.4 \\ $33.277376 \circ \mathrm{N}$ 81.583370 o $W$}

SAMPLE DATE

FIELD DATA

Screen Zone Elovation

291.0-271.2 ft ms!
Analyto

Water olevation

Depth to water

pH

Sp. conductance

Water temperaturo

Alkalinity as $\mathrm{CaCO}_{3}$

Turbidity

Volume purged

Sampling codo
$03 / 12 / 93$

Top of Casing

$302.2 \mathrm{ft}$ msl

$05 / 19 / 93$

$07 / 29 / 93$

$12 / 04 / 93$

4093

Unit

$\begin{array}{ll}1093 & \underline{2093} \\ 294.2 & 292.7 \\ 8.0 & 9.5 \\ 5.2 & 5.5 \\ 84 & 85 \\ 15.4 & 21.4 \\ 350 & 110 \\ 1.0 & 2.1 \\ & x\end{array}$

3093

289.2

13.0

5.5

72

24.6

12

2.0

$\mathrm{x}$

Aluminum, total recoverable Antimony-125

Arsenic, total recoverable

Barium, total recoverable

Cadmium, total recoverable

Caleium, total recoverablo

Cerium-144

Cesium-134

Cosium-137

Chloride

Chromium, total recoverable

Cobalt-57

Cobalt-60

Copper, total recovorable

2,4-Dichlorophenoxyacetic acid

Endrin

Europium-152

Europium-154

Europium-155

Fluorido

Grose alph.

Iron, total recoverable

Lead, total recoverable

Lindano

Magnesium, total.recoverable

Manganese, total recoverable

Manganese-54

Mercury, total recoverable

Methoxychlor

Nickol, total recoverable

Nitrato-nitrite as nitrogen

Nonvolatile beta

pH

Phenols

Plutonium-238

Piutonium-239/240

Potassium, total recoverable

Potassium-40

Promothium-144

1093
992
$<2.0$
99
$<2.0$
6,140

\section{3}

3,960

$<4.0$

4.8

$<0.30$

$<0.0060$

$<2.5 E+01$

$<2.0$

99

$<2.0$

4,390

$<5.0 E+01$

$<1.0 E+01$

$<1.0 E+01$

4,280

$<4.0$

$<1.0 E+01$

$<1.2 E+01$

$<4.0$

\section{3}

125

$<2.5 E+01$

$<2.0$

95
$<2.0$

3,420

$<5.0 E+01$

$<1.0 E+01$

$<1.0 E+01$

4,510

$<4.0$

$<1$. OE + 01

$<1.2 E+01$

$<4.0$

$<5.0 E+01 . \quad<5.0 E+01$

$<2.5 E+01<2.5 E+01$

$<2.5 E+01<2.5 E+01$

$<100<100$

$\begin{array}{ll}<100 & <100 \\ 5.7 E+00 & 3.3 E+00\end{array}$

$2.4 \mathrm{E}+00$

$2.4 \mathrm{E}$
269
3.0

$\begin{array}{rrr}159 & 294 & 269 \\ <3.0 & <3.0 & <3.0\end{array}$

$<0.0050$

2,100

427

0.43

$<0.50$

$<4.0$

4,550

$4.2 E+00$

5.3

$<5.0$

$<500$
2.110

438

$<1.0 E+01$

$<0.20$

$<4.0$

4,400

4.3E + 00

5.1

$<1.0 E+00$

$<1.0 E+00$

$<500$

$<2.5 E+02$

$<1.0 E+01$
2.020

477

$<1.0 \mathrm{E}+01$

$<0.20$

$<4.0$

4.100

$5.6 E+00$

5.6

$<1.0 E+00$

$<1.0 E+00$

$<500$

$<2.5 \mathrm{E}+02$

$<1.0 E+01$
4093 Mod Unit Lab Flag

$\mu g / L$

$\mathrm{pCi} / \mathrm{L}$

$\mu \mathrm{g} / \mathrm{L}$

$\mu \mathrm{m} / \mathrm{L}$

$\mu \mathrm{g} / \mathrm{L}$

pCi/L

pCi/L

pCi/L

$\mu \mathrm{g} / \mathrm{L}$

$\mathrm{mg} / \mathrm{L}$

pCi/L

pCi/L

$\mathrm{mg/L}$

$\mu \mathrm{g} / \mathrm{L}$

$\mu \mathrm{g} / \mathrm{L}$

pCi/L.

pCi/L

pCi/L

pCill

$\mu \mathrm{g} / \mathrm{L}$

$\mu \mathrm{g} / \mathrm{L}$.

$\mu g / L$

$\mu \mathrm{g} / \mathrm{L}$

$\mathrm{\mu g} / \mathrm{L}$

$\mu_{0} / \mathrm{L}$

$\mu_{g} / \mathrm{L}$

$\mu \theta / L$

$\mu \mathrm{g} / \mathrm{L}$

pCi/L

$\mathrm{pH}$
$\mathrm{pg} / \mathrm{L}$

pCi/L

pCi/L

$\mu \mathrm{g} / \mathrm{L}$

pCill

pCi/L

Note: Flagging levels, modifiers, and laboratories are for 4th quarter 1993 data only. See Appendix B for flagging criteria.

- = exceeded holding time for 4th quarter 1993.

- = exceeded screening level or final primary drinking water standard for 4th quarter 1993. 


\section{ANALYTICAL DATA}

\section{H}

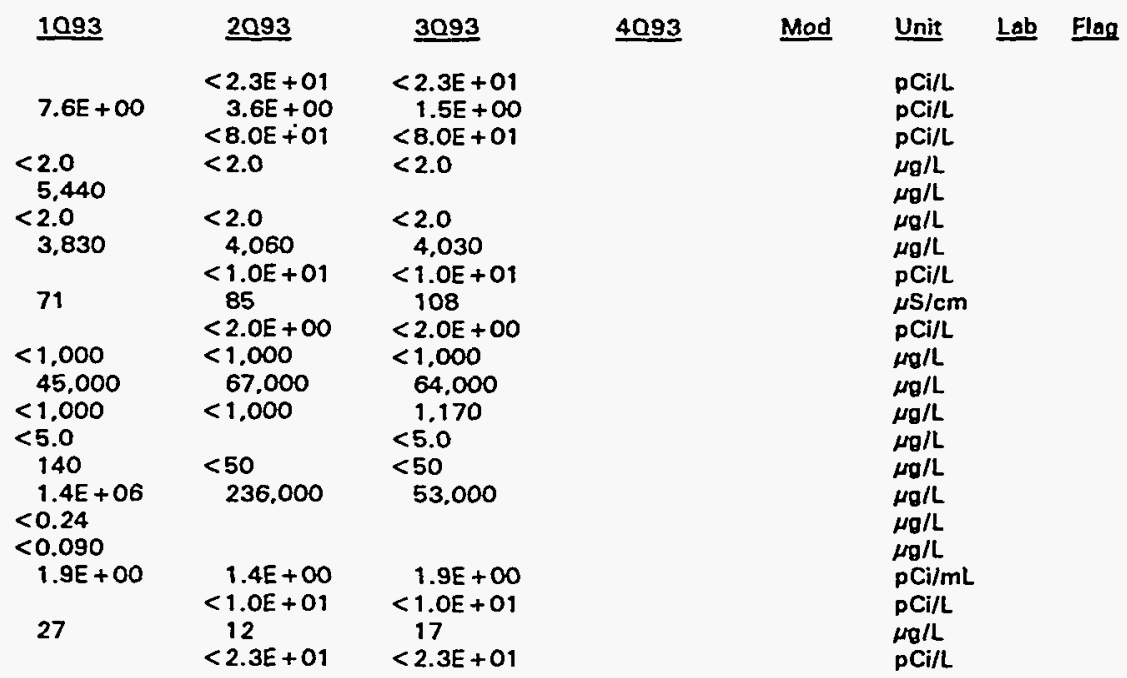

\section{WELL RSE 9}

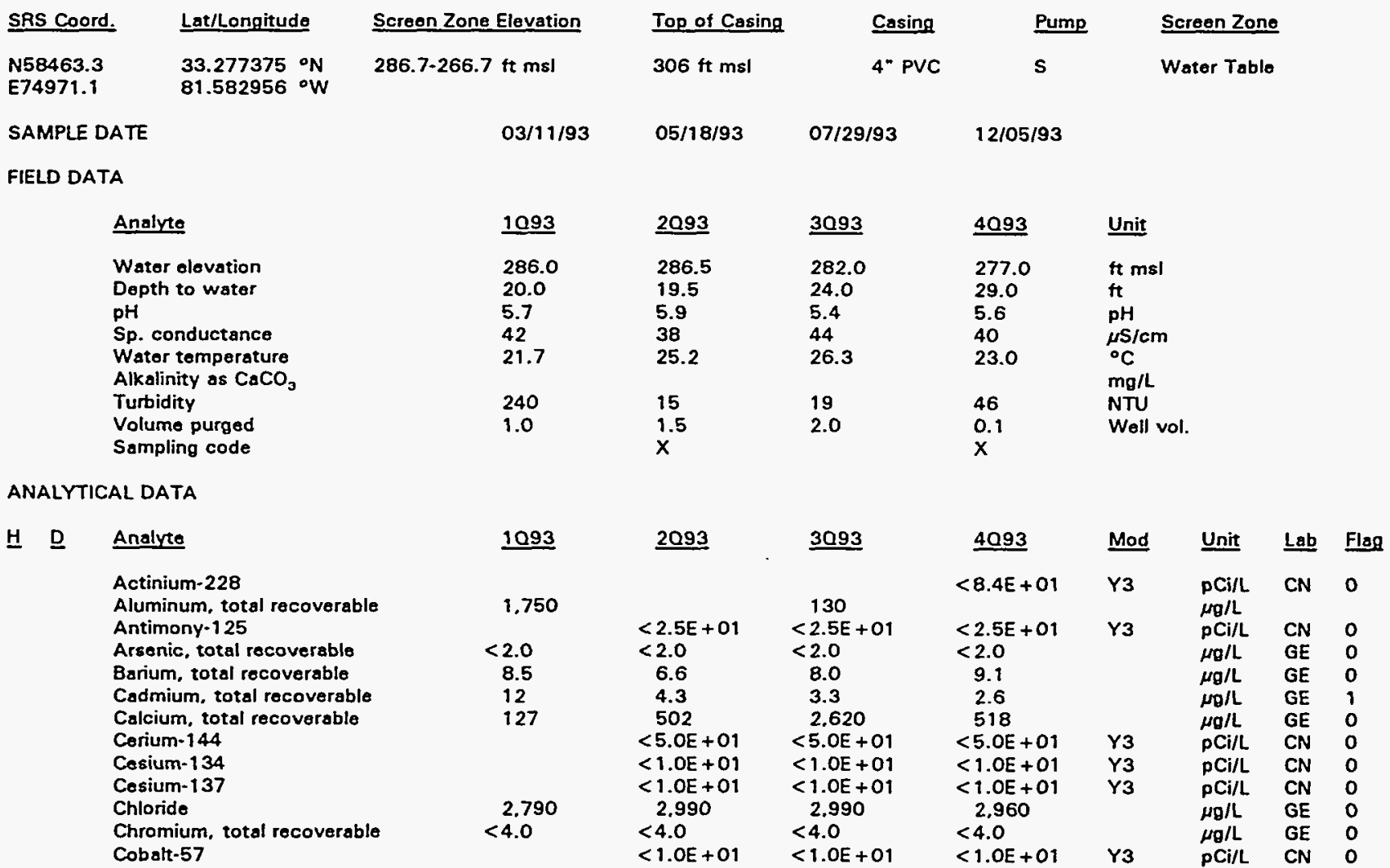

Note: Flagging levels, modifiers, and laboratories are for 4th quarter 1993 data only. See Appendix B for flagging criteria.

- = exceeded holding time for 4th quarter 1993.

- = exceeded screening level or final primary drinking water standard for 4th quarter 1993. 
Woll RSE 9 continued

\section{ANALYTICAL DATA}

H D Anslyte

Cobalt-60

Copper, total recoverable

2,4-Dichlorophenoxyacotic acid

Endrin

Europium-152

Europium-154

Europium-155

Fluorido

Gross alpho

Iron, total recoverable

Load, total recoverable

Load-212

Lindane

Magnesium, total recoverable

Manganeso, total recoverable

Manganeso-54

Mercury, total recoverable

Mothoxychlor

Nickel, total recoverable

Nitrato-nitrite as nitrogen

Nonvolatilo bets

- $\quad$ PH

Phenols

Plutonium-238

Plutonium-239/240

Potassium, total recoverable

Potassium-40

Promothium-144

Promothium-146

Radium, total alpha-omitting

Ruthenium-106

Solenium, total recoverable

Silica, total recoverable

Silver, total recoverable

Sodium, total recoverable

Sodium-22

Specific conductance

Strontium-90

Sulfate

Thorium-234

Total dissolved solids

Total organic carbon

Total organic halogens

Total phosphates (as P)

Total suspended solids

Toxaphene

2,4,5-TP (Silvex)

Tritium

Yttrium-88

Zinc, total recoverable

Zinc-65

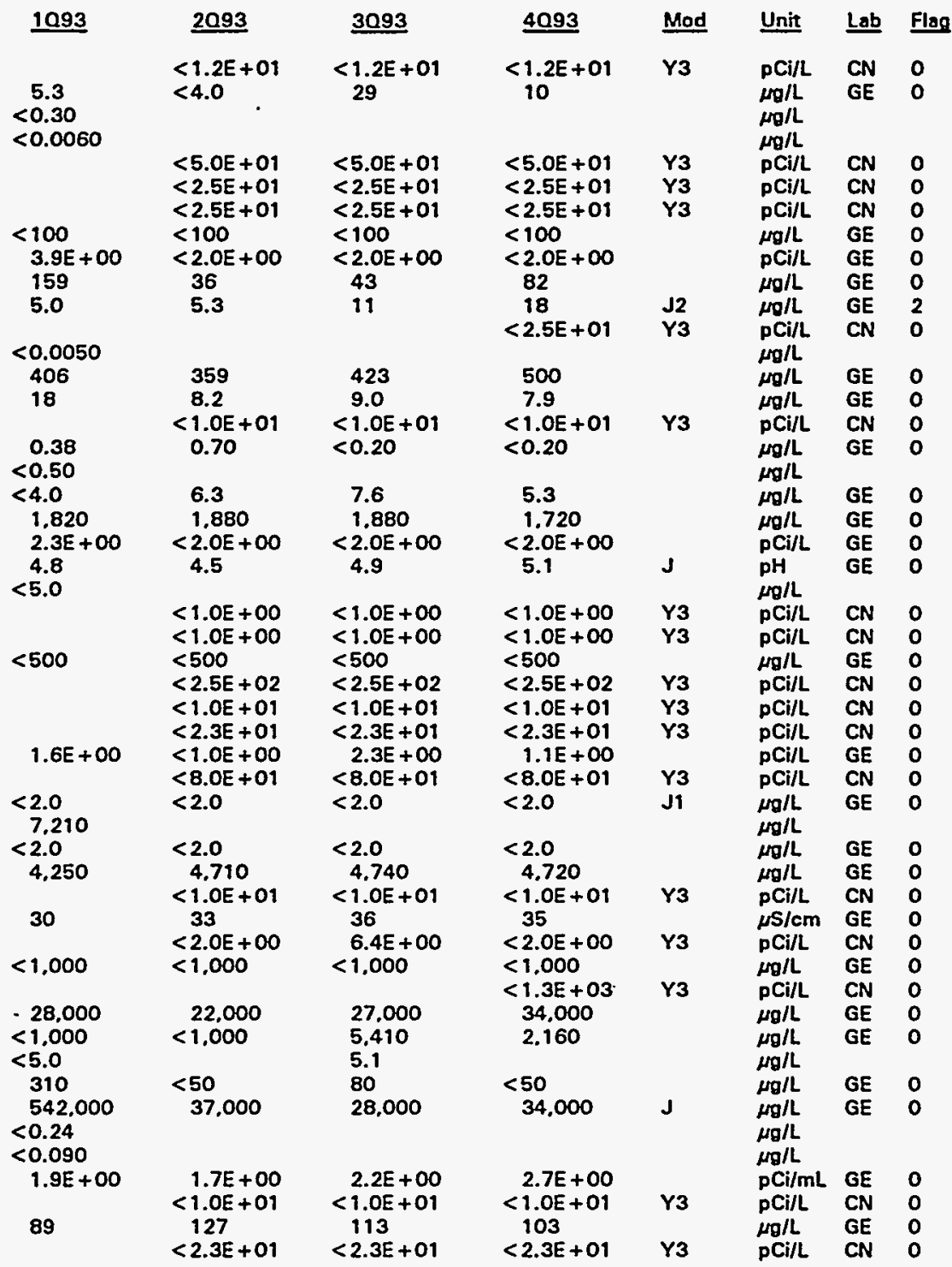

Note: Flagging levels, modifiers, and laboratories are for 4th quarter 1993 data only. See Appendix B for flagging criteria. - = exceeded holding time for 4th quarter 1993.

- = exceeded screening level or final primary drinking water standard for 4th quarter 1993. 


\section{WELL RSE 10}

\begin{tabular}{|c|c|c|c|c|c|c|}
\hline SRS Coord. & Lat/Longitude & Screen Zone Elevation & Top of Casing & Casing & Pump & $\underline{\mathrm{Sc}}$ \\
\hline $\begin{array}{l}\text { N58420.7 } \\
\text { E74848.3 }\end{array}$ & $\begin{array}{l}33.2770800^{\circ} \mathrm{N} \\
81.583196{ }^{\circ} \mathrm{W}\end{array}$ & 290.5-270.7 ft msl & $304.7 \mathrm{ft} \mathrm{msl}$ & 4" PVC & $\mathbf{s}$ & \\
\hline \multicolumn{2}{|c|}{ SAMPLE DATE } & $03 / 11 / 93$ & $05 / 18 / 93$ & $07 / 29 / 93$ & $12104 / 93$ & \\
\hline \multicolumn{7}{|l|}{ FIELD DATA } \\
\hline \multicolumn{2}{|c|}{ Anslyte } & 1093 & 2093 & $\underline{3093}$ & 4093 & Unit \\
\hline \multicolumn{2}{|c|}{$\begin{array}{l}\text { Water olovation } \\
\text { Depth to water } \\
\text { pH } \\
\text { Sp. conductance } \\
\text { Water temperature } \\
\text { Alkalinity as CaCO, } \\
\text { Turbidity } \\
\text { Volume purged } \\
\text { Sampling code }\end{array}$} & $\begin{array}{l}290.7 \\
14.0 \\
5.5 \\
40 \\
20.3 \\
360 \\
1.0\end{array}$ & $\begin{array}{l}292.7 \\
12.0 \\
5.5 \\
162 \\
25.9 \\
12 \\
1.9 \\
X\end{array}$ & $\begin{array}{l}285.7 \\
19.0 \\
5.7 \\
97 \\
26.8\end{array}$ & $\begin{array}{l}\text { Dry } \\
\text { D }\end{array}$ & $\begin{array}{l}\text { ft msl } \\
\mathrm{tt} \\
\mathrm{pH} \\
\mu \mathrm{S} / \mathrm{cm} \\
{ }^{\circ} \mathrm{C} \\
\mathrm{mg} / \mathrm{L} \\
\mathrm{NTU} \\
\text { Woll vol. }\end{array}$ \\
\hline
\end{tabular}

\section{ANALYTICAL DATA}

\section{H D \\ Analyte}

Aluminum, total recoverable Antimony-1 25

Arsenic, total recoverable

Barium, total recoverable

Cadmium, total recoverable

Colcium, total recoverable

Corium-144

Cosium-134

Cesium-137

Chloride

Chromium, total recoverable

Cobalt-57

Cobalt-60

Copper, total recoverable

2,4-Dichlorophenoxyacetic acid

Endrin

Europium-152

Europium-154

Eurapium-155

Fluorido

Gross alpha

Iron, total recoverable

Lead, total recoverable

Lindane

Magnesium, total recoverable

Manganese, total recoverable

Manganese-54

Mercury, total recoverable

Methoxychlor

Nickal, total recoverable

Nitrate-nitrite as nitrogen

Nonvolatile beta

pH

Phenols

Plutonium-238

Plutonium-239/240

Potassium, total recoverable

Potassium-40

Promethium-144

\begin{tabular}{|c|c|c|}
\hline 1093 & 2093 & 3093 \\
\hline 4,990 & & 401 \\
\hline & $<2.5 E+01$ & $<2.5 E+01$ \\
\hline$<2.0$ & $<2.0$ & $<2.0$ \\
\hline 56 & 208 & 94 \\
\hline 4.0 & $<2.0$ & $<2.0$ \\
\hline 4.970 & $\begin{aligned} & 19.700 \\
< & 5.0 E+01 \\
< & 1.0 E+01 \\
< & 1.0 E+01\end{aligned}$ & $\begin{aligned} & 9.850 \\
< & 5.0 E+01 \\
< & 1.0 E+01 \\
< & 1.0 E+01\end{aligned}$ \\
\hline 2,380 & 5.150 & 4,270 \\
\hline$<4.0$ & $\begin{aligned} & 4.7 \\
< & 1.0 E+01 \\
< & 1.2 E+01\end{aligned}$ & $\begin{array}{l}<4.0 \\
<1.0 E+01 \\
<1.2 E+01\end{array}$ \\
\hline 13 & $<4.0$ & 5.2 \\
\hline & $\begin{array}{l}<5.0 E+01 \\
<2.5 E+01 \\
<2.5 E+01\end{array}$ & $\begin{array}{l}<5.0 E+01 \\
<2.5 E+01 \\
<2.5 E+01\end{array}$ \\
\hline$<100$ & $<100$ & $<100$ \\
\hline $\begin{array}{l}1.7 E+01 \\
615\end{array}$ & $2.8 E+00$ & $\begin{array}{l}<2.0 \mathrm{E}+00 \\
116\end{array}$ \\
\hline 13 & 5.1 & 6.2 \\
\hline$<0.0050$ & & \\
\hline $\begin{array}{l}574 \\
98\end{array}$ & $\begin{aligned} & 2.770 \\
& 239 \\
< & 1.0 E+01\end{aligned}$ & $\begin{aligned} & 1.530 \\
& 99 \\
< & 1.0 E+01\end{aligned}$ \\
\hline $\begin{array}{r}0.61 \\
<0.50\end{array}$ & $<0.20$ & $<0.20$ \\
\hline$<4.0$ & 7.3 & $<4.0$ \\
\hline $\begin{array}{l}1.510 \\
5.5 E+01 \\
5.3 \\
<5.0\end{array}$ & $\begin{array}{l}25.600 \\
1.7 E+04 \\
4.8\end{array}$ & $\begin{array}{l}5.100 \\
6.0 E+03 \\
5.3\end{array}$ \\
\hline & $\begin{array}{l}<1 . O E+O O \\
<1 . O E+O O\end{array}$ & $\begin{array}{l}<1.0 E+00 \\
<1.0 E+00\end{array}$ \\
\hline 517 & $\begin{aligned} & 3,000 \\
&< 2.5 E+02 \\
&<1.0 E+01\end{aligned}$ & $\begin{aligned} & 1.800 \\
< & 2.5 E+02 \\
< & 1.0 E+01\end{aligned}$ \\
\hline
\end{tabular}

4093 Mod Unit Lab Flag

$\mu \mathrm{g} / \mathrm{L}$

$\mathrm{pCi} / \mathrm{L}$

$\mu g / \mathrm{L}$

$\mu g / L$

$\mu \mathrm{g} / \mathrm{L}$

$\mathrm{pCi} / \mathrm{L}$

pCill

pCi/L

$\mu g / L$

$\mu g / L$
$p C i / L$

pCi/L

$\mu \mathrm{g} / \mathrm{L}$

$\mu g / L$

$\mu g / L$

pCi/L

pCi/L

pCi/L

$\mu \mathrm{g} / \mathrm{L}$

pCi/l

$\mu g / L$

$\mu g / L$

$\mu g / L$

$\mu g / L$
$p C i / L$
$\mu g / L$

$\mu \mathrm{g} / \mathrm{L}$

$\mu_{\mathrm{g}} / \mathrm{L}$

$\mu \mathrm{g} / \mathrm{L}$

pCi/L

$\mathrm{pH}$
$\mu \mathrm{g} / \mathrm{L}$

$\mu \mathrm{g} / \mathrm{L}$
$\mathrm{pCi} / \mathrm{L}$

pCi/L

$\mu \mathrm{g} / \mathrm{L}$

$\mathrm{pCi} / \mathrm{L}$

PCi/L

Note: Flagging levels, modifiers, and laboratories are for 4th quarter 1993 data only. See Appendix B for flagging criteria. - = exceeded holding time for 4th quarter 1993.

- = exceeded screening level or final primary drinking water standard for 4th quarter 1993. 


\section{ANALYTICAL DATA}

\section{H D Analyto}

Promathium-146

Radlum, total alpha-emitting

Ruthenium-106

Selenium, total recoverable

Silica, total recoverable

Silver, total rocovorablo

Sodium, total recoverable

Sodium-22

Specific conductance

Strontium-90

Sulfato

Total dissolved solids

Total organic carbon

Total organic halogens

Total phosphates (as P)

Total suspended solids

Toxaphene

2,4,5-TP (Silvex)

Tritium

Yttrium-88

Zine, total recoverablo

Zinc-65

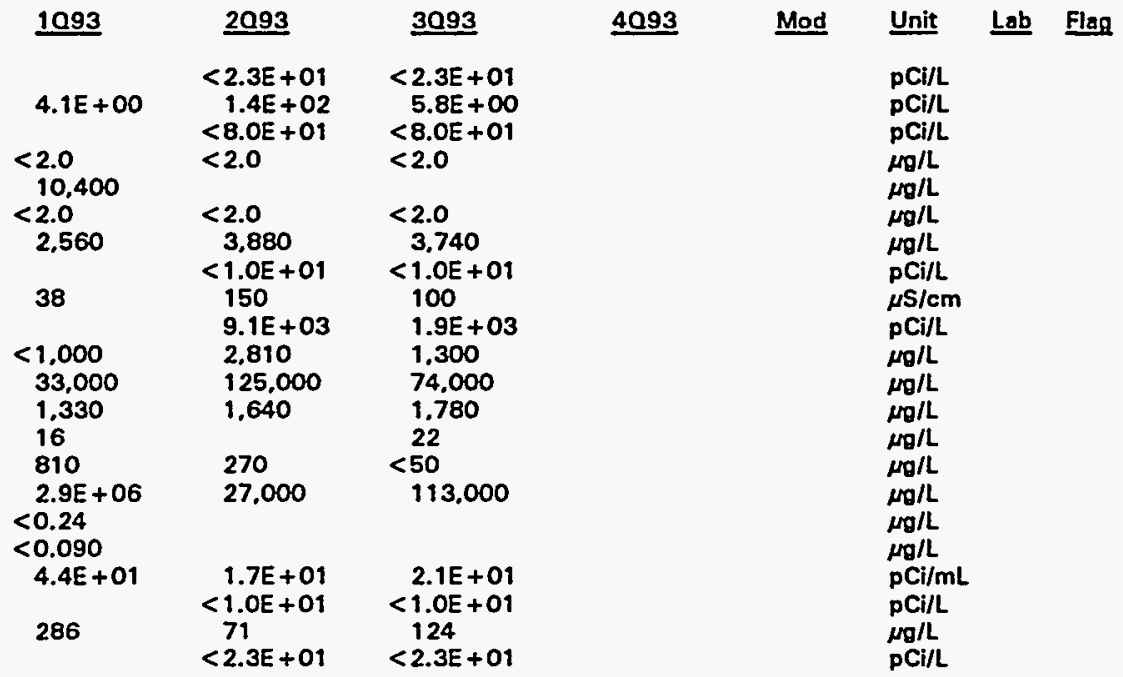

\section{WELL RSE 11}

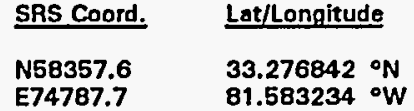

SAMPLE DATE

FIELD DATA

\section{Analyte}

Water elovation

Depth to water

$\mathrm{pH}$

Sp, conductance

Water temperature

Alkalinity as $\mathrm{CaCO}_{2}$

Turbidity

Volume purged

Sampling code

\section{ANALYTICAL DATA}

브

Analyte
Aluminum, total recoverable
Arsenic, total recoverable
Barium, total rocoverable
Cadmium, total recoverable
Calcium, total recoverable
Chlorido
Chromium, total recoverable
2,4-Dichlorophenoxyacetic acid
Endrin
Fluoride
Gross alpha
Iron, total recoverable
Lead, total recoverable

\section{Screen Zone Elevation}

$03 / 10 / 93$

1093

294.8

9.0

5.3

71

19.6

120

1093
2.240
$<2.0$
84
5.3
5,120
2,870
$<4.0$
$<0.30$
$<0.0060$
$<100$
$1.8 E+01$
455
4.8

\section{Top of Casing \\ $303.8 \mathrm{ft} \mathrm{msl}$

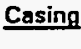 \\ Steel}

05/06/93

$07 / 22 / 93$

\section{Pump \\ B}

Screen Zone

Water Table

$11 / 20 / 93$

$\begin{array}{llll}\underline{2093} & \underline{3093} & \underline{4093} & \text { Unit } \\ 293.8 & 288.8 & 280.8 & \mathrm{ft} \mathrm{msl} \\ 10.0 & 15.0 & 23.0 & \mathrm{ft} \\ 4.7 & 6.2 & 5.2 & \mathrm{pH} \\ 68 & 59 & 60 & \mu \mathrm{HS} / \mathrm{cm} \\ 22.0 & & 17.4 & { }^{\circ} \mathrm{C} \\ 90 & 75 & 533 & \mathrm{mg} / \mathrm{L} \\ \mathrm{NTU} \\ \text { Well vol. }\end{array}$

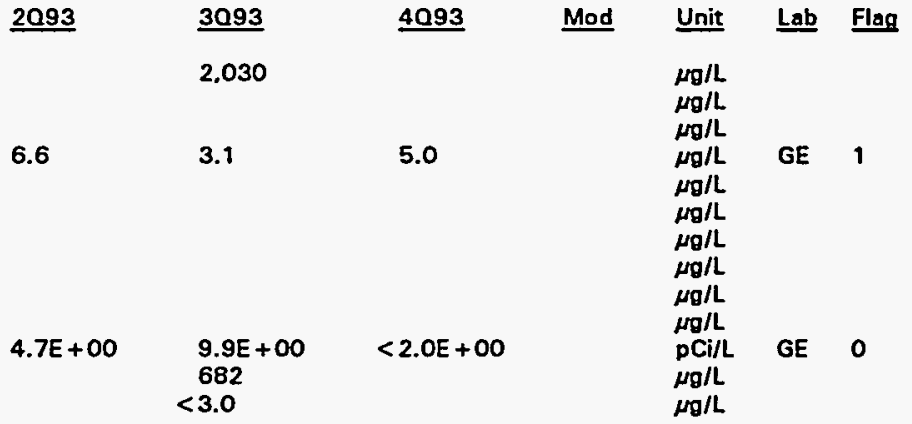

Note: Flagging levels, modifiers, and laboratories are for 4th quarter 1993 data only. See Appendix B for flagging criteria. - = exceeded holding time for 4th quarter 1993.

- = exceeded screening level or final primary drinking water standard for 4th quarter 1993. 
WSRC-TR-94-0354

Unclassified

Well RSE 11 continued

ANALYTICAL DATA

H D Analvte

\section{Lindane}

Magnesium, total recoverable

Manganese, total recoverable

Mercury, total recoverable

Mathoxychlor

Nitrate-nitrite as nitrogen

- Nonvolatilo beta

$\mathrm{pH}$

Phenols

Potassium, total recoverable

Radium, total alpha-emitting

Selenium, total recoverable

Silica, total recoverable

Silver, total recoverable

Sodium, total recoverable

Specific conductance

Sulfato

Total dissolved solids

Total organic carbon

Total organic halogens

Total phosphates (as P)

Toxaphone

2.4.5-TP (Silvex)

Tritium
$<0.0050$

1.530

186

0.27

$<0.50$

1.640

1. $O E+03$

5.3

$<5.0$

1,060

$5.5 E+00$

$<2.0$

5,800

$<2.0$

2,040

60

6,090

33,000

2,160

$<5.0$

220

$<0.24$

$<0.090$

$<7.0 \mathrm{E}-01$ $\underline{2093}$

$\begin{array}{lll} & 110 & \\ & <0.20 & \\ & & \\ & 1.820 & \\ 8.4 E+02 & 8.7 E+02 & 8.2 E+02\end{array}$

$\underline{3093} \underline{4093}$ Mod Unit Lab Flog

$\mu \mathrm{g} / \mathrm{L}$

$\mu \mathrm{g} / \mathrm{L}$

roll

$\mu g / L$

$\mu g / L$

PCi/L GE 2

$\mathrm{pH}$

$\mu \mathrm{g} / \mathrm{L}$

$\mu \mathrm{g} / \mathrm{L}$
$\mathrm{pCi} / \mathrm{L}$

$9.3 E+\infty$

$\mu g / L$

$\mu g / \mathrm{L}$

$\mu g / L$

$\mu g / L$

$\mu \mathrm{S} / \mathrm{cm}$

$\mu g / L$

$\mu \mathrm{g} / \mathrm{L}$

$\mu \mathrm{g} / \mathrm{L}$

$\mu \mathrm{g} / \mathrm{L}$

$\mu g / L$

$\mu g / L$

$\mu \mathrm{g} / \mathrm{L}$

$1.4 E+00$

\section{WELL RSE 12}

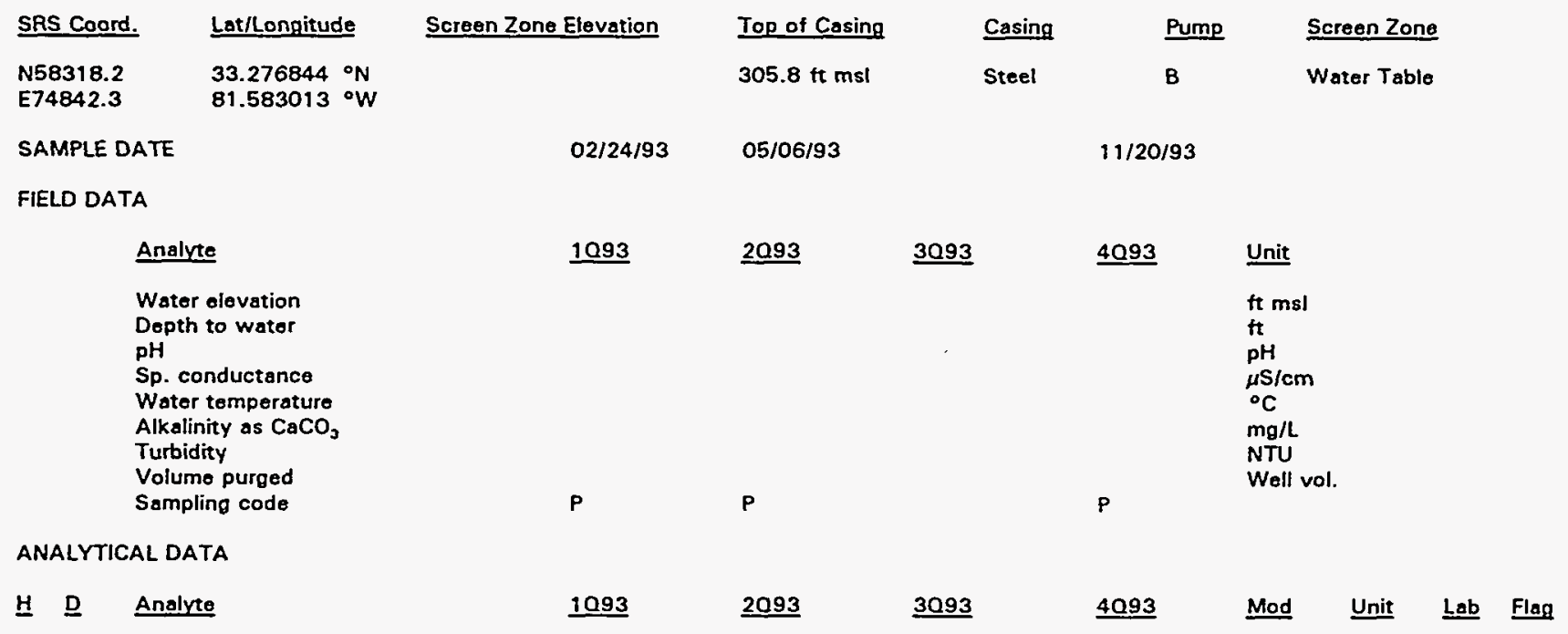

Note: Flagging levels, modifiers, and laboratories are for 4 th quarter 1993 data only. See Appendix B for flagging criterie. - exceeded holding time for 4th quarter 1993.

- = exceeded screening level or final primary drinking water standard for 4th quarter 1993. 
WELL.RSE 13

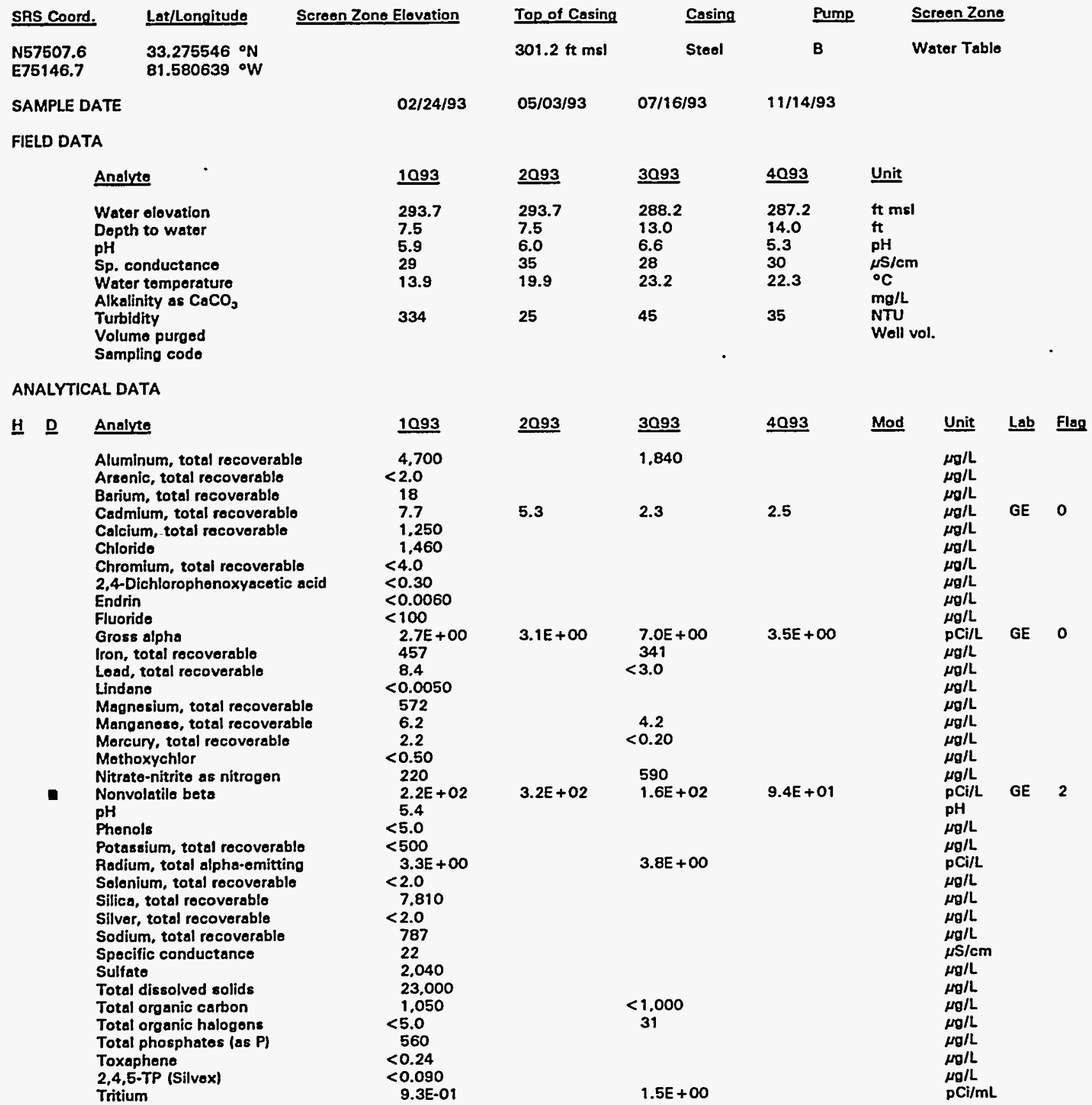

Note: Flagging levels, modifiers, and laboratories are for 4th quarter 1993 data only. See Appendix B for flagging criterie.

- = exceeded holding time for 4th quarter 1993.

= exceeded screening level or final primary drinking water standard for 4th quarter 1993. 


\section{WELL RSE 18}

\begin{tabular}{|c|c|c|c|c|c|c|}
\hline SRS Coord. & Lat/Longitude & Screen Zone Elevation & Top of Casing & Casing & Pump & Screen Zone \\
\hline $\begin{array}{l}\text { N58247.2 } \\
\text { E74839.5 }\end{array}$ & $\begin{array}{l}33.276682{ }^{\circ} \mathrm{N} \\
81.582883\end{array}$ & 288.1-268.1 ft msl & $307.1 \mathrm{ft} \mathrm{msl}$ & 4" Steel & B & Water Table \\
\hline \multicolumn{2}{|c|}{ SAMPLE DATE } & $03 / 11 / 93$ & $05 / 06 / 93$ & $07 / 21 / 93$ & \multicolumn{2}{|l|}{$11 / 20 / 93$} \\
\hline \multicolumn{7}{|l|}{ FIELD DATA } \\
\hline \multicolumn{2}{|c|}{ Analyte } & 1093 & $\underline{2093}$ & 3093 & 4093 & Unit \\
\hline \multicolumn{2}{|c|}{$\begin{array}{l}\text { Water olevation } \\
\text { Depth to water } \\
\text { pH } \\
\text { Sp. conductance } \\
\text { Water temperature } \\
\text { Alkalinity as CaCO } \\
\text { Turbidity } \\
\text { Volume purged } \\
\text { Sampling code }\end{array}$} & $\begin{array}{l}286.6 \\
20.5 \\
6.2 \\
107 \\
20.0 \\
175 \\
0.9\end{array}$ & $\begin{array}{l}287.1 \\
20.0 \\
6.0 \\
121 \\
20.7 \\
\\
300 \\
0.8\end{array}$ & $\begin{array}{l}282.1 \\
25.0 \\
5.3 \\
88 \\
24.7\end{array}$ & $\begin{array}{l}279.1 \\
28.0 \\
5.9 \\
70 \\
19.0 \\
1000 \\
0.8\end{array}$ & $\begin{array}{l}\text { ft msl } \\
\mathrm{ft} \\
\mathrm{pH} \\
\mu \mathrm{S} / \mathrm{cm} \\
{ }^{\circ} \mathrm{C} \\
\mathrm{mg} / \mathrm{L} \\
\mathrm{NTU} \\
\text { Well vol. }\end{array}$ \\
\hline
\end{tabular}

\section{ANALYTICAL DATA}

\section{H D Analyte}

Aluminum, total recoverable Arsenic, total recoverable

Barium, total recoverable

- Cadmium, total recoverable Calcium, total recoverable Chloride

Chromium, total recoverable 2.4-Dichlorophenoxyacetic acid Endrin

Fluoride

- Gross alpha

Iron, total recoverable

Lead, total recoverable

Lindano

Magnesium, total recoverable

Manganese, total recoverable

Mercury, total recoverable

Methoxychlor

Nitrate-nitrite as nitrogen

- Nonvolatilo beta

pH

Phenols

Potassium, total recoverable

Radium, total alpha-emitting

Solenium, total recoverable

Silica, total recoverable

Silver, total recoverable

Sodium, total recoverable

Specific conductance

Sulfate

Total dissolved solids

Total organic carbon

Total organic halogens

Total phosphates (as P)

Toxaphene

2,4,5-TP (Silvex)

Tritium

\section{3}

7.210

$<2.0$

162

17

20,600

3,740

$<4.0$

$<0.30$

$<0.0060$

130

4. $2 E+01$

4,340

124

$<0.0050$

4,060

3,840

1.5

$<0.50$

1,150

$2.4 E+01$

6.5

$<5.0$

1,550

1.1E + 01

$<2.0$

17.400

$<2.0$

2.560

95

1.620

64,000

$<1,000$

12

390

$<0.24$

$<0.090$

$<7.0 E-01$ $\underline{2093}$

$\underline{3093}$

2.110

9.4

17

48

$\underline{4093}$

Mod Unit Lab Flag

$3.0 E+01$

$1.9 \mathrm{E}+0$
1.870
51

2.040

0.72

1.020

$1.5 E+01$

$9.1 E+\infty 0$

$5.5 E+01$

5.7E +00

8. $. \mathrm{EE}+01$

$5.5 E+01$

2.170

8.1

$<7.0 \mathrm{E}-01$

Note: Flagging levels, modifiers, and laboratories are for 4th quarter 1993 data only. See Appendix B for flagging criteria. - = exceeded holding time for 4th quarter 1993.

- = exceeded screening level ar finel primary drinking water standard for 4th quarter 1993. 
WELL RSE 19

\begin{tabular}{|c|c|c|c|c|c|c|}
\hline SRS Coord. & Lot/Longitude & Screen Zone Elevation & Top of Casing & Casing & Pump & Screen Zone \\
\hline $\begin{array}{l}\text { N58318.4 } \\
\text { E74791.2 }\end{array}$ & $\begin{array}{l}33.276761 \text { oN } \\
81.583148 \text { oW }\end{array}$ & 282.5-262.5 ft msl & $304.8 \mathrm{ft} \mathrm{msl}$ & $4^{-}$Steel & B & Water Table \\
\hline \multicolumn{2}{|c|}{ SAMPLE DATE } & $03 / 10 / 93$ & $05 / 06 / 93$ & $07 / 22 / 93$ & $11 / 20 / 93$ & \\
\hline \multicolumn{7}{|l|}{ FIELD DATA } \\
\hline \multicolumn{2}{|c|}{ Analvte } & $\underline{1093}$ & 2093 & $\underline{3093}$ & $\underline{4093}$ & $\underline{\text { Unit }}$ \\
\hline \multicolumn{2}{|c|}{$\begin{array}{l}\text { Water olovation } \\
\text { Depth to water } \\
\text { pH } \\
\text { Sp. conductance } \\
\text { Water temperature } \\
\text { Alkalinity as } \mathrm{CaCO} \text {, } \\
\text { Turbidity } \\
\text { Volume purged }\end{array}$} & $\begin{array}{l}293.8 \\
11.0 \\
5.3 \\
76 \\
20.2\end{array}$ & $\begin{array}{l}275.8 \\
29.0 \\
4.7 \\
81 \\
21.9 \\
\\
35 \\
2.2\end{array}$ & $\begin{array}{l}285.8 \\
19.0 \\
6.1 \\
61\end{array}$ & $\begin{array}{l}279.8 \\
25.0 \\
5.3 \\
62 \\
17.4 \\
1000 \\
1.0\end{array}$ & $\begin{array}{l}\text { ft msl } \\
\text { ft } \\
\text { pH } \\
\mu S / c m \\
{ }^{\circ} \mathrm{C} \\
\mathrm{mg} / \mathrm{L} \\
\mathrm{NTU} \\
\text { Well vol. }\end{array}$ \\
\hline
\end{tabular}

\section{ANALYTICAL DATA}

\section{H D Analyte}

Aluminum, total rocoverable

Arsenic, total recoverable

Barium, total recoverable

Cadmium, total recovorable

Calcium, total recoverable

Chlorido

Chromium, total recoverable

2,4-Dichlorophenoxyscetic acid

Endrin

Fluoride

- Gross alpha

Iron, total recoverable

Load, total recoverablo

Lindane

Magnosium, total recoverable

Manganese. total recoverable

Mercury, total recoverable

Methoxychlor

Nitrato-nitrite as nitrogen

Nonvolatile beta

pH

Phenols

Potassium, total recoverable

Radium, total alpha-omitting

Selenium, total recoverable

Silica, total recoverable

Silver, total recoverable

Sodium, total recoverable

Specific conductance

Sulfate

Total dissolved solids

Total organic carbon

Total organic halogens

Total phosphates (as P)

Toxaphene

2,4.5-TP (Silvex)

Tritium

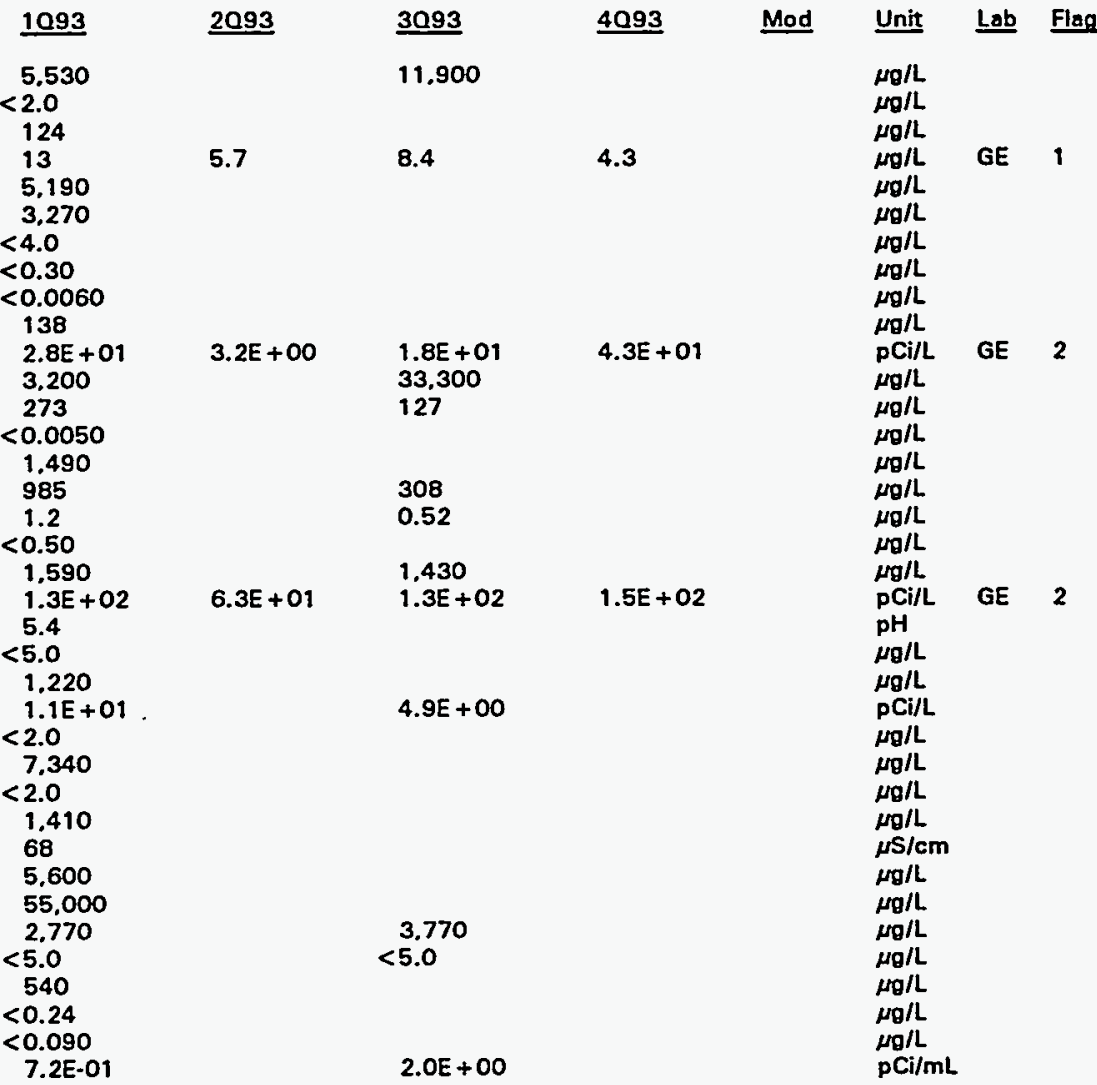

Note: Flagging levels, modifiers, and laboratories are for 4th quarter 1993 data only. See Appendix B for flagging criteria. - = exceeded holding time for 4th quarter 1993.

- = exceeded screening level or final primary drinking water standard for 4th quarter 1993. 


\section{WELL RSE 24}

\begin{tabular}{|c|c|c|c|c|c|c|}
\hline SRS Coord. & Lat/Longitude & Screen Zone Elevation & Top of Casing & Casing & Pump & $\underline{\text { Sc }}$ \\
\hline $\begin{array}{l}\text { N57370.4 } \\
\text { E74638.9 }\end{array}$ & $\begin{array}{l}33.2744155^{\circ} \mathrm{N} \\
81.581710^{\circ} \mathrm{W}\end{array}$ & $257.6-237.6 \mathrm{ft} \mathrm{msl}$ & $294.1 \mathrm{ft} \mathrm{ms!}$ & $4^{n}$ PVC & $\mathbf{S}$ & \\
\hline SAMPLE DA & & $03 / 19 / 93$ & $06 / 10 / 93$ & $09 / 09 / 93$ & $12 / 27 / 93$ & \\
\hline FIELD DATA & & & & & & \\
\hline & & 1093 & 2093 & 3093 & 4093 & Unit \\
\hline 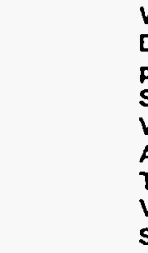 & $\begin{array}{l}\text { olevation } \\
\text { to water } \\
\text { onductance } \\
\text { tomperature } \\
\text { hity as } \mathrm{CaCO}_{3} \\
\text { ity } \\
\text { le purged } \\
\text { ing code }\end{array}$ & $\begin{array}{l}285.1 \\
9.0 \\
6.0 \\
37 \\
17.9 \\
3 \\
16 \\
1.3 \\
x\end{array}$ & $\begin{array}{l}283.9 \\
10.2 \\
5.3 \\
30 \\
22.3 \\
2 \\
18 \\
1.3 \\
x\end{array}$ & $\begin{array}{l}280.7 \\
13.4 \\
5.1 \\
29 \\
23.0 \\
1 \\
5 \\
1.3 \\
x\end{array}$ & $\begin{array}{l}278.8 \\
15.3 \\
5.2 \\
40 \\
18.3 \\
2 \\
7 \\
1.3 \\
x\end{array}$ & $\begin{array}{l}\mathrm{ft} \text { msl } \\
\mathrm{ft} \\
\mathrm{pH} \\
\mu \mathrm{S} / \mathrm{cm} \\
{ }^{\circ} \mathrm{C} \\
\mathrm{mg} / \mathrm{h} \\
\mathrm{NTU} \\
\text { Well vol. }\end{array}$ \\
\hline
\end{tabular}

ANALYTICAL DATA

H $\quad$ Analyte

Actinium-228

Aluminum, total recoverable

Antimony-125

Arsenic, total recoverable

Barium, total recoverable

Cadmium, total recoverable

Calcium, total recoverable

Corium-144

Cosium-134

Cesium-137

Chloride

Chromium, total recoverable

Cobatt-57

Cobatt-60

Copper, total recoverable

2,4-Dichlorophenoxyacetic acid

Endrin

Europium-152

Europium-154

Europium-155

Fluoride

Gross alpha

Iron, total recoverable

Load, total recoverable

Load-212

Lindane

Magnesium, total recoverable

Manganese, total recoverable

Manganese 54

Mercury, total recoverable

Methoxychlor

Nickel, total recoverable

Nitrate-nitrite as nitrogen

Nonvolatile beta

pH

Phenols

Plutonium-238

Piutonium-239/240

Potassium, total rocoverable

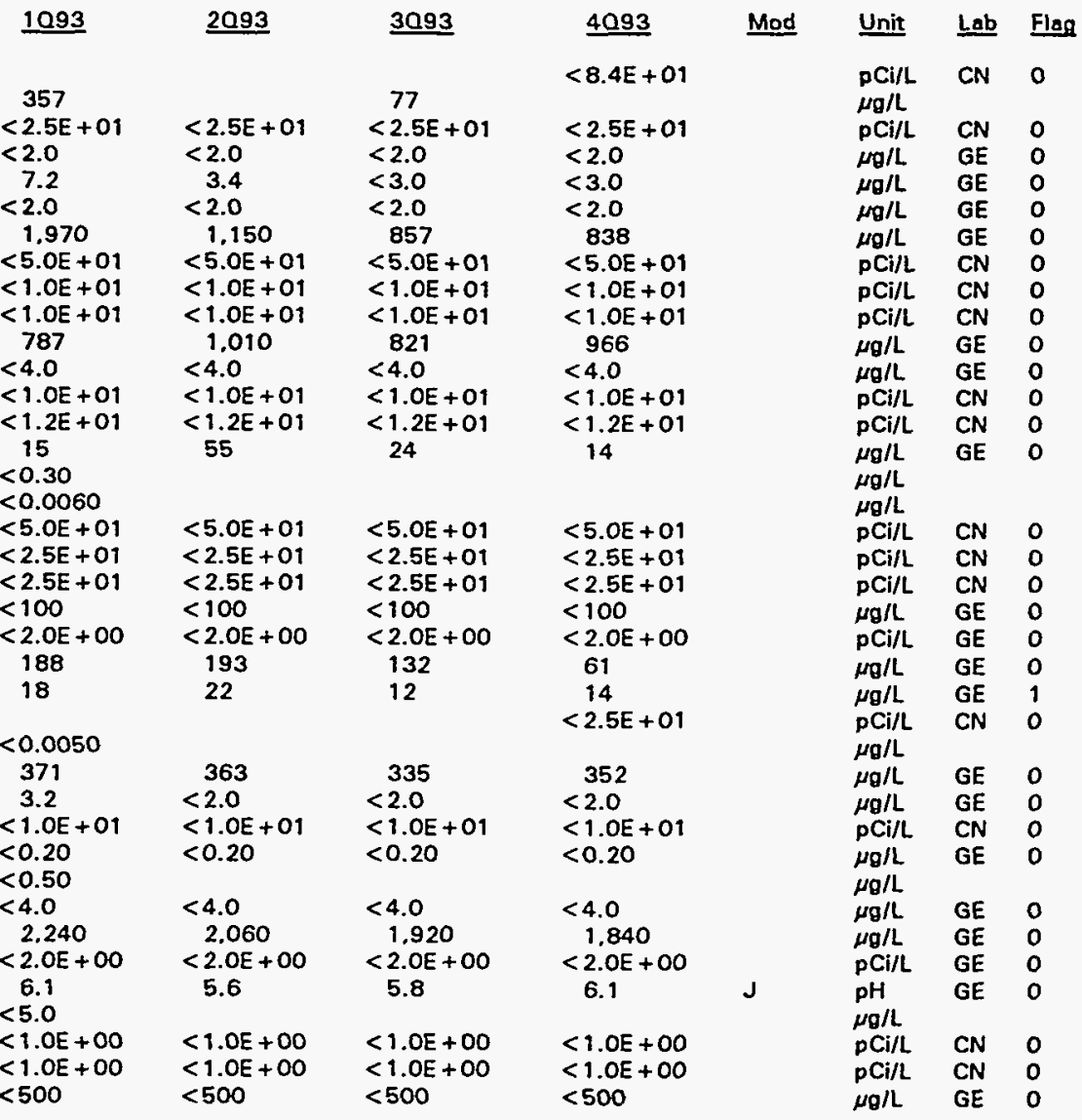

Note: Flagging levels, modifiers, and laboratories are for 4th quarter 1993 data only. See Appendix B for flagging criteria. - = exceeded holding time for 4th quarter 1993.

- = exceeded screening level or final primary drinking water standard for 4th quarter 1993. 
Anslyto

Potassium-40

Promethium-144

Promethium-146

Padium, total alpha-emitting

Ruthenium-106

Solonium, total recoverable

Silica, total recovorable

Silver, total recoverable

Sodium, total recoverable

Sodium-22

Specific conductance

Strontium.90

Sulfate

Thorium-234

Total dissolved solids

Total organic carbon

Total organic halogens

Total phosphates (as P)

Total suspended solids

Toxapheno

2,4,5-TP (Silvox)

Tritium

Yttrium-88

Zinc, total recoverable

Zinc-65

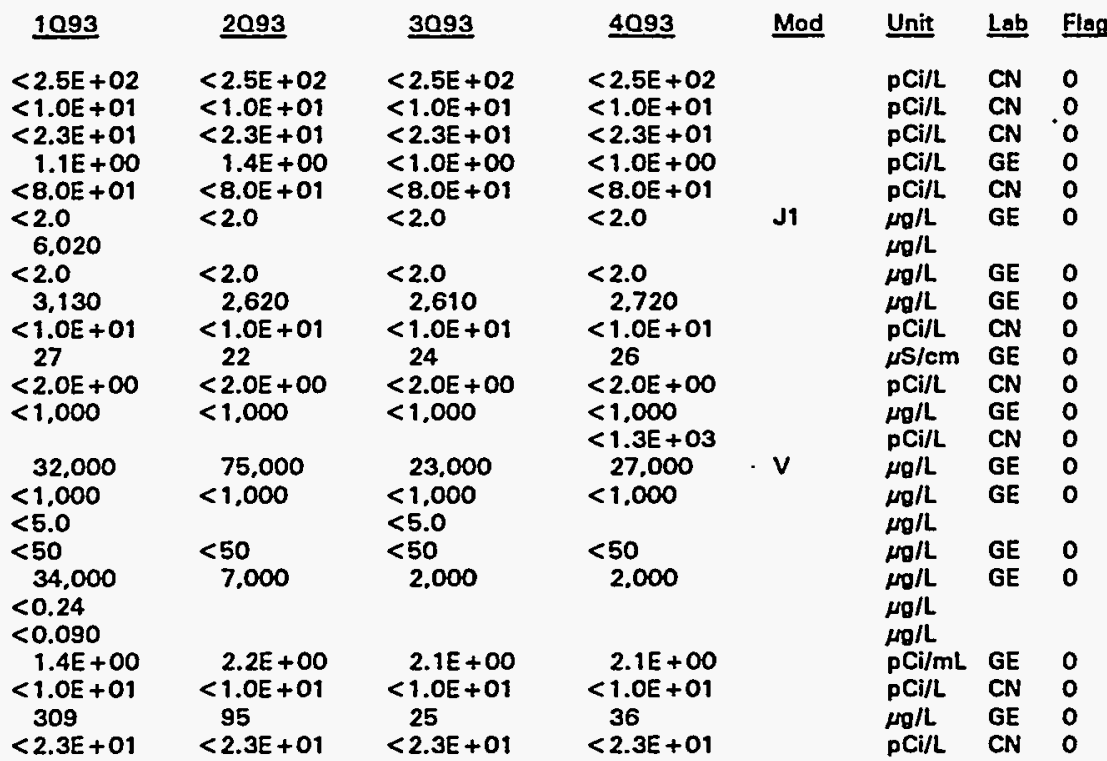

\section{WELL RSE 25}

\section{SRS Coord. \\ N55824.5 \\ E74544.5

Lat/Longitude
$33.270840^{\circ} \mathrm{N}$
$81.578960^{\circ} \mathrm{W}$

Screen Zone Elevation
257.5-237.5 $\mathrm{ft}$ msl
03/19/9

1093
279.6
14.3
6.1
119
17.2
21
39
1.2
$\times$

ANALYTICAL DATA

H

Analyte

Actinium-228

Aluminum, total recoverable

Antimany-125

Arsonic, total recoverable

Barium, total recoverable

Cadmium, total recoverable

Calcium, total recoverable

Corium-144

Cesium-134

Cesium-137

$\begin{array}{lll}1093 & \underline{2093} & \underline{3093} \\ 91 & & \\ <2.5 E+01 & <2.5 E+01 & <2.5 E+01 \\ <2.0 & <2.0 & <2.0 \\ 60 & 50 & 40 \\ <2.0 & <2.0 & <2.0 \\ 11.100 & 10.500 & 7.720 \\ <5.0 E+01 & <5.0 E+01 & <5.0 E+01 \\ <1.0 E+01 & <1.0 E+01 & <1.0 E+01 \\ <1.0 E+01 & <1.0 E+01 & <1.0 E+01\end{array}$

$\underline{3093}$
275.8
18.1
5.7
100
22.6
16
11
1.2
$x$

275.8

15.7

5.6

106

21

$\stackrel{1.1}{x}$

\begin{tabular}{l} 
Top of \\
293.9 \\
$06 / 10$ \\
\\
2093 \\
\hline 278.2 \\
15.7 \\
5.6 \\
106 \\
22.9 \\
21 \\
21 \\
1.1 \\
$\times$
\end{tabular}

Cosing

Pump

$\mathbf{s}$

Sereen Zone

Water Tablo

09/09/93

$12 / 27 / 93$

$\begin{array}{lll}4093 & & \text { Unit } \\ & & \\ 275.2 & & \mathrm{ft} \mathrm{msl} \\ 18.7 & & \mathrm{ft} \\ 5.5 & & \mathrm{pH} \\ 107 & & \mu \mathrm{S} / \mathrm{cm} \\ 18.0 & & { }^{\circ} \mathrm{C} \\ 14 & & \mathrm{mg} / \mathrm{L} \\ 11 & & \mathrm{NTU} \\ 1.2 & & \text { Well vol. } \\ \mathrm{X} & & \end{array}$

\begin{tabular}{|c|c|c|c|}
\hline 4093 & Mad & Unit & Lab \\
\hline$<8.4 E+01$ & & $\mathrm{pCi} / \mathrm{L}$ & $\mathrm{CN}$ \\
\hline $\begin{array}{l}<2.5 E+01 \\
<2.0 \\
40\end{array}$ & & $\begin{array}{l}\mu \mathrm{Ci} / \mathrm{L} \\
\mu \mathrm{g} / \mathrm{L} \\
\mu \mathrm{g} / \mathrm{L}\end{array}$ & $\begin{array}{l}\mathrm{CN} \\
\mathrm{GE} \\
\mathrm{GE}\end{array}$ \\
\hline $\begin{array}{l}<2.0 \\
7.300 \\
<5.0 E+01 \\
<1.0 E+01 \\
<1.0 E+01\end{array}$ & & $\begin{array}{l}\mu \mathrm{g} / \mathrm{L} \\
\mu \mathrm{g} / \mathrm{L} \\
\mathrm{pCi} / \mathrm{L} \\
\mathrm{pCi} / \mathrm{L} \\
\mathrm{pCi} / \mathrm{L}\end{array}$ & $\begin{array}{l}\mathrm{GE} \\
\mathrm{GE} \\
\mathrm{CN} \\
\mathrm{CN} \\
\mathrm{CN}\end{array}$ \\
\hline
\end{tabular}

Note: Flagging levels, modifiers, and laboratories are for 4th quarter 1993 data only. See Appendix $B$ for flagging criteria.

- = exceeded holding time for 4th quarter 1993.

- = exceeded screening level or final primary drinking water standard for 4th quarter 1993. 
WSRC-TR-94-0354

Unclassified

Well RSE 25 continued

ANALYTICAL DATA

H D Analyte

Chlorido

Chromium, total recoverable

Cobalt-57

Cobalt 60

Copper, total recoverable

2,4-Dichlorophenoxyacotic acid

Endrin

Europium-152

Europium-154

Europium-155

Fluoride

Gross alpha

Iron, total recoverable

Lead, total recoverable

Lead-212

Lindane

Magnesium, total recoverable

Menganese, total recoverable

Manganese-54

Mercury. total recoverable

Methoxychlor

Nickel, total recoverable

Nitrate-nitrite as nitrogen

Nonvolatile bets

pH

Phenols

Plutonium-238

Plutonium-239/240

Potassium, total recoverable

Potassium-40

Promethium-144

Promothium-146

Radium, total alphs-emitting

Puthenium-106

Selenium, total recoverable

Silica, total recoverable

Silver, total recoverable

Sodium, total recoverable

Sodium-22

Specific conductance

Strontium-90

Sulfate

Thorium-234

Total dissolved solids

Total arganic carbon

Total organic halogens

Total phosphates (as P)

Total suspended solids

Toxaphene

2,4,5-TP (Silvex)

Tritium

Yttrium-88

Zinc, total recoverable

Zinc-65

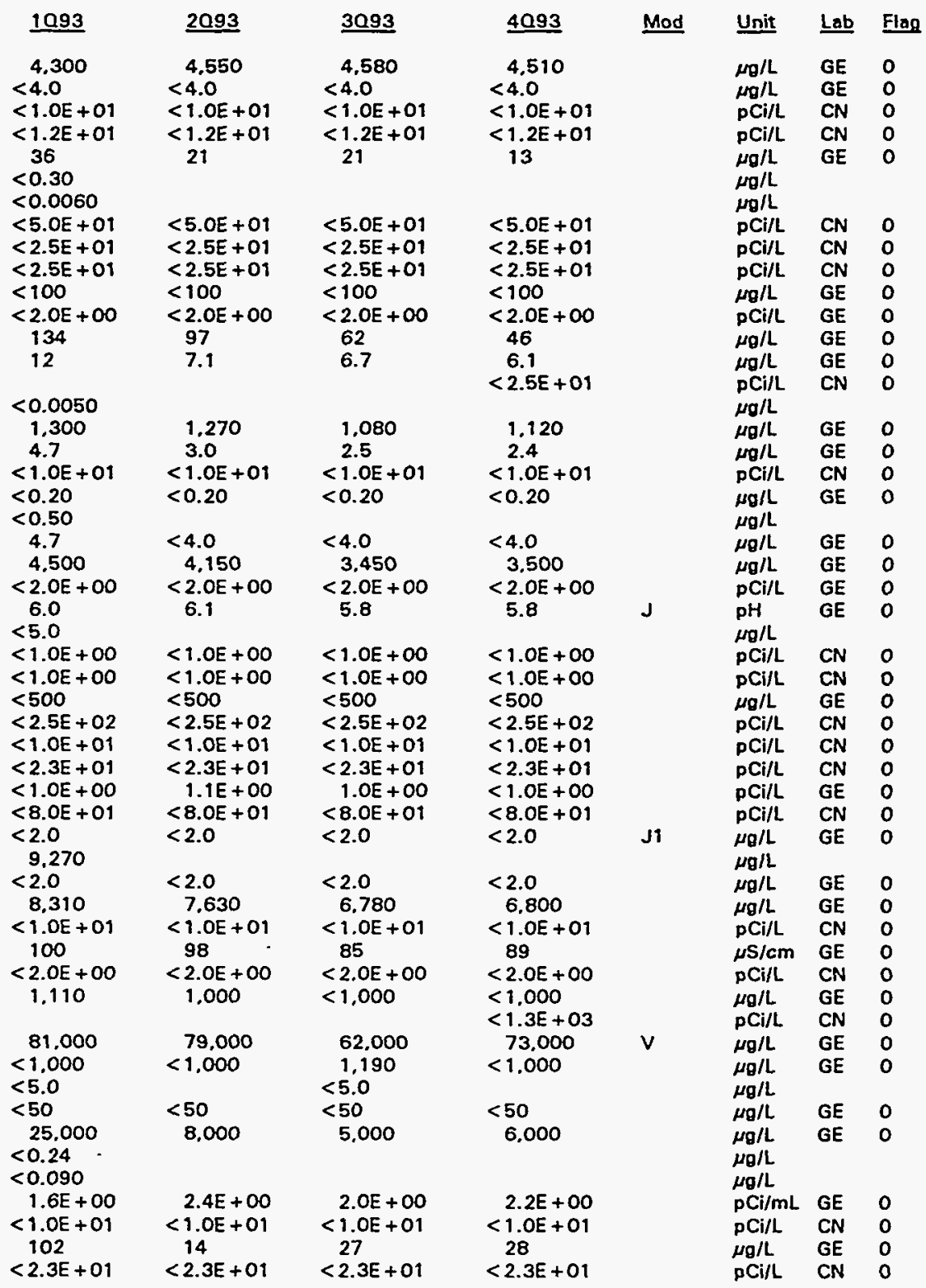

Note: Flagging levels, modifiers, and laboratories are for 4th quarter 1993 data only. See Appendix B for flagging criteria.

- = exceeded holding time for 4th quarter 1993.

- = exceeded screening level or final primary drinking water standard for 4th quarter 1993. 
WELL RSF 1

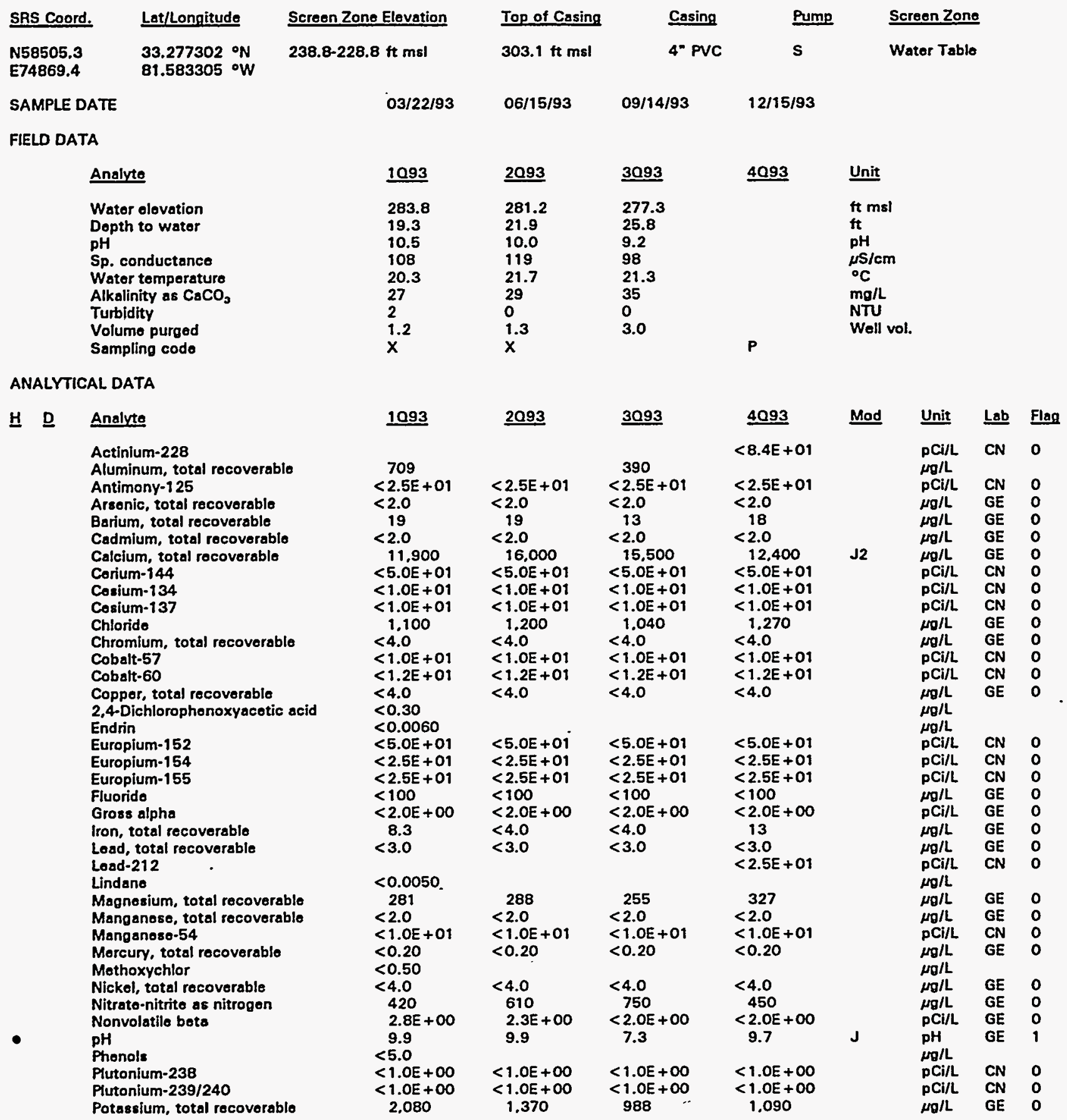

Note: Flagging levels, modifiers, and laboratories are for 4th quarter 1993 data only. See Appendix B for flagging criteria.

- = exceeded holding time for 4th quarter 1993.

- = exceeded screening level or final primary drinking water standard for 4th quarter 1993. 


\section{H D Analvte}

Potassium-40

Promothium-144

Promothium-146

Radium, total alpha-emitting

Ruthenium-106

Selenium, total recoverable

Silica, total recoverable

Silver, total recoverable

Sodium, total recoverable

Sodium-22

Specific conductance

Strontium-90

Sulfate

Thorium-234

Total dissolved solids

Total organic carbon

Total organic halogens

Total phosphates (as P)

Total suspended solids

Toxaphene

2,4,5-TP (Silvex)

Tritium

Yttrium-88

Zinc, total recoverable

Zinc-65

\begin{tabular}{|c|c|c|c|c|c|c|}
\hline 1093 & 2093 & 3093 & 4093 & Mod & Unit & $\underline{\text { Lab }}$ \\
\hline $\begin{aligned}<2.5 E+02 \\
<1.0 E+01 \\
<2.3 E+01 \\
1.2 E+00 \\
<8.0 E+01 \\
<2.0 \\
10.800\end{aligned}$ & $\begin{array}{l}<2.5 E+02 \\
<1.0 E+01 \\
<2.3 E+01 \\
<1.0 E+00 \\
<8.0 E+01 \\
<2.0\end{array}$ & $\begin{aligned} &<2.5 E+02 \\
&<1.0 E+01 \\
&<2.3 E+01 \\
& 1.3 E+00 \\
&<8.0 E+01 \\
&<2.0\end{aligned}$ & $\begin{array}{l}<2.5 E+02 \\
<1.0 E+01 \\
<2.3 E+01 \\
<1.0 E+00 \\
<8.0 E+01 \\
<2.0\end{array}$ & J1 & $\begin{array}{l}\mathrm{pCi} / \mathrm{L} \\
\mathrm{pCi} / \mathrm{L} \\
\mathrm{pCi} / \mathrm{L} \\
\mathrm{pCi} / \mathrm{L} \\
\mathrm{pCi} / \mathrm{L} \\
\mu \mathrm{g} / \mathrm{L} \\
\mu \mathrm{g} / \mathrm{L}\end{array}$ & $\begin{array}{l}\text { CN } \\
\text { CN } \\
\text { CN } \\
\text { GE } \\
\text { CN } \\
\text { GE }\end{array}$ \\
\hline $\begin{array}{l}<2.0 \\
3.230 \\
<1.0 E+01 \\
84\end{array}$ & $\begin{aligned} &<2.0 \\
& 2.510 \\
&<1.0 E+01 \\
& 102\end{aligned}$ & $\begin{array}{l}<2.0 \\
1.930 \\
<1.0 E+01 \\
91\end{array}$ & $\begin{aligned} &<2.0 \\
& 2.200 \\
&< 1.0 E+01 \\
& 68\end{aligned}$ & & $\begin{array}{l}\mu \mathrm{g} / \mathrm{L} \\
\mu \mathrm{g} / \mathrm{L} \\
\mathrm{pCi} / \mathrm{L} \\
\mu \mathrm{S} / \mathrm{cm}\end{array}$ & $\begin{array}{l}\text { GE } \\
\text { GE } \\
\text { CN } \\
\text { GE }\end{array}$ \\
\hline $\begin{array}{c}<2.0 E+00 \\
2.300\end{array}$ & $\begin{array}{c}<2.0 E+00 \\
2,460\end{array}$ & $\begin{array}{c}<2.0 E+00 \\
1.490\end{array}$ & $\begin{aligned}<2.0 E+00 \\
1.510 \\
<1.3 E+03\end{aligned}$ & & $\begin{array}{l}\mathrm{pCi} / \mathrm{L} \\
\mu \mathrm{g} / \mathrm{L} \\
\mathrm{pCi} / \mathrm{L}\end{array}$ & $\begin{array}{l}\text { CN } \\
\text { GE } \\
\text { CN }\end{array}$ \\
\hline 58,000 & 61,000 & 59,000 & 44,000 & & $\mu g / L$ & GE \\
\hline $\begin{array}{l}1.340 \\
<5.0\end{array}$ & $<1,000$ & $\begin{array}{l}<1.000 \\
<5.0\end{array}$ & $<1,000$ & & $\begin{array}{l}\mu g / L \\
\mu g / L\end{array}$ & GE \\
\hline $\begin{array}{l}<50 \\
4,000\end{array}$ & $\begin{array}{l}<50 \\
<1,000\end{array}$ & $\begin{array}{l}<50 \\
<1.000\end{array}$ & $\begin{array}{l}<50 \\
<1.000\end{array}$ & & $\begin{array}{l}\mu \sigma / L \\
\mu g / L\end{array}$ & $\begin{array}{l}\text { GE } \\
\text { GE }\end{array}$ \\
\hline $\begin{array}{l}<0.24 \\
<0.090\end{array}$ & & & & & $\begin{array}{l}\mu \mathrm{g} / \mathrm{L} \\
\mu \mathrm{g} / \mathrm{L}\end{array}$ & \\
\hline $\begin{array}{r}1.5 E+01 \\
<1.0 E+01\end{array}$ & $\begin{array}{r}2.3 E+01 \\
<1.0 E+01\end{array}$ & $\begin{array}{r}3.3 E+01 \\
<1.0 E+01\end{array}$ & $\begin{array}{r}1.7 E+01 \\
<1.0 E+01\end{array}$ & & $\begin{array}{l}\mathrm{pCi} / \mathrm{mL} \\
\mathrm{pCi} / \mathrm{L}\end{array}$ & $\begin{array}{l}\text { GE } \\
\mathrm{CN}\end{array}$ \\
\hline $\begin{array}{l}<2.0 \\
<2.3 E+01\end{array}$ & $\begin{array}{l}<2.0 \\
<2.3 E+01\end{array}$ & $\begin{array}{l}<2.0 \\
<2.3 E+01\end{array}$ & $<22.3 E+01$ & & $\begin{array}{l}\mu \mathrm{g} / \mathrm{L} \\
\mathrm{pCi} / \mathrm{L}\end{array}$ & $\begin{array}{l}\mathrm{GE} \\
\mathrm{CN}\end{array}$ \\
\hline
\end{tabular}

\section{WELL RSF 2}

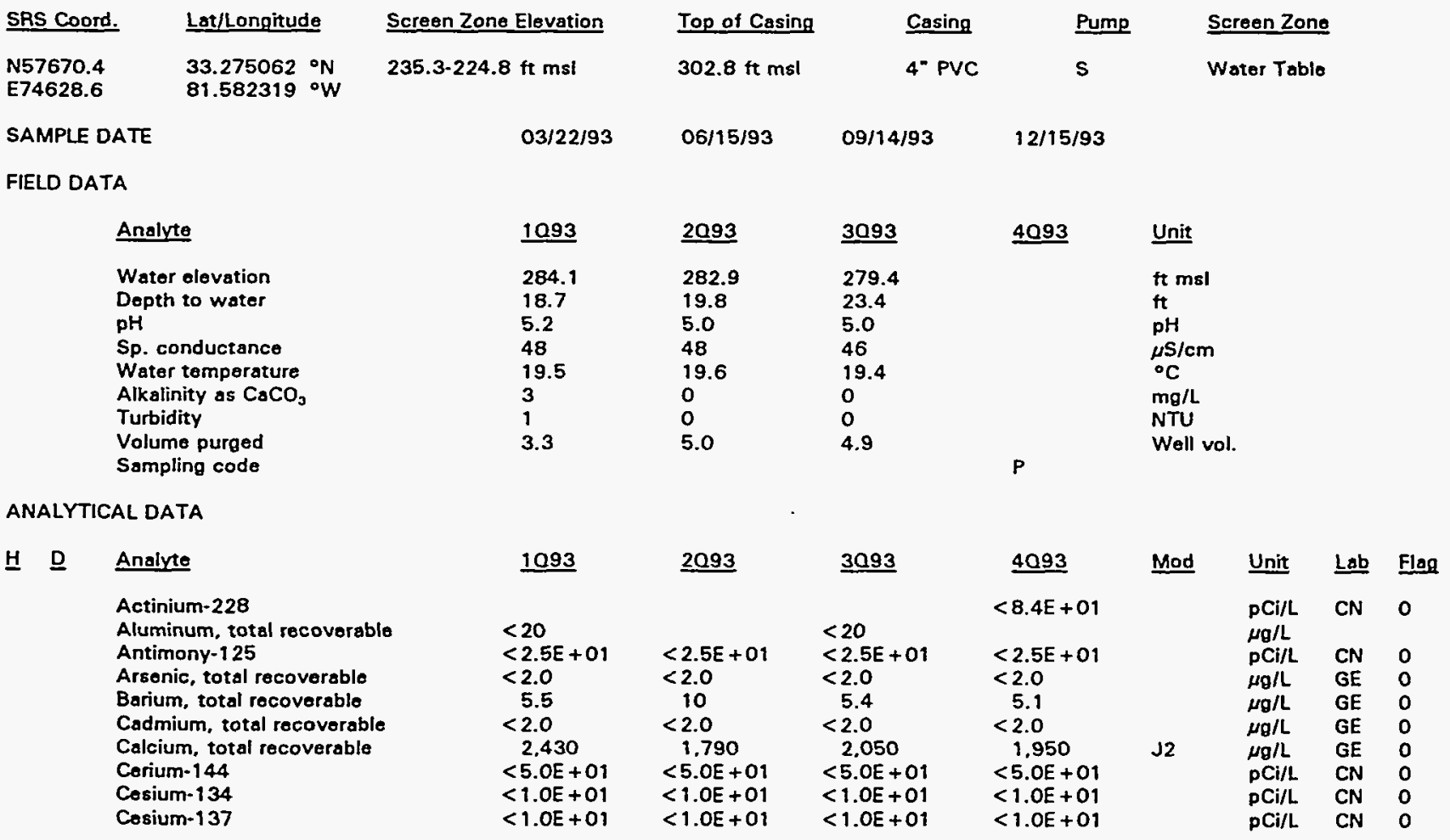

Note: Flagging levels, modifiers, and laboratories are for 4 th quarter 1993 data only. See Appendix B for flagging criteria. - =xceeded holding time for 4th quarter 1993.

- =xceeded screening level or final primary drinking water standard for 4th quarter 1993. 
Chlorido

Chromium, total recoverable

Cobalt-57

Cobalt-60

Copper, total recoverable

2,4-Dichlorophenoxyacetic acid

Endrin

Europium-152

Europium-154

Europium-155

Fluoride

Gross alpho

Iron, total recovorable

Lead, total recovorablo

Load-212

Lindano

Magnesium, total recoverable

Manganoso, total rocoverable

Manganese-54

Mercury, total recoverable

Methoxychlor

Nickol, total recoverable

Nitrato-nitrite as nitrogen

Nonvolatile bets

$\mathrm{pH}$

Phenols

Plutonium-238

Plutonium-239/240

Potassium, total recoverable

Potassium-40

Promethium-144

Promethium-146

Radium, total alpho-omitting

Ruthenium-106

Solenium, total recoverable

Silica, total recoverable

Silver, total recoverable

Sodium, total recovarablo

Sodium-22

Specific conductance

Strontium-90

Sulfate

Thorium-234

Total dissolved solids

Total organic carbon

Total organic halogens

Total phosphates las P

Total suspended solids

Toxaphene

2,4,5-TP (Silvex)

Tritium

Yttrium-88

Zinc, total recoverable

Zinc-65

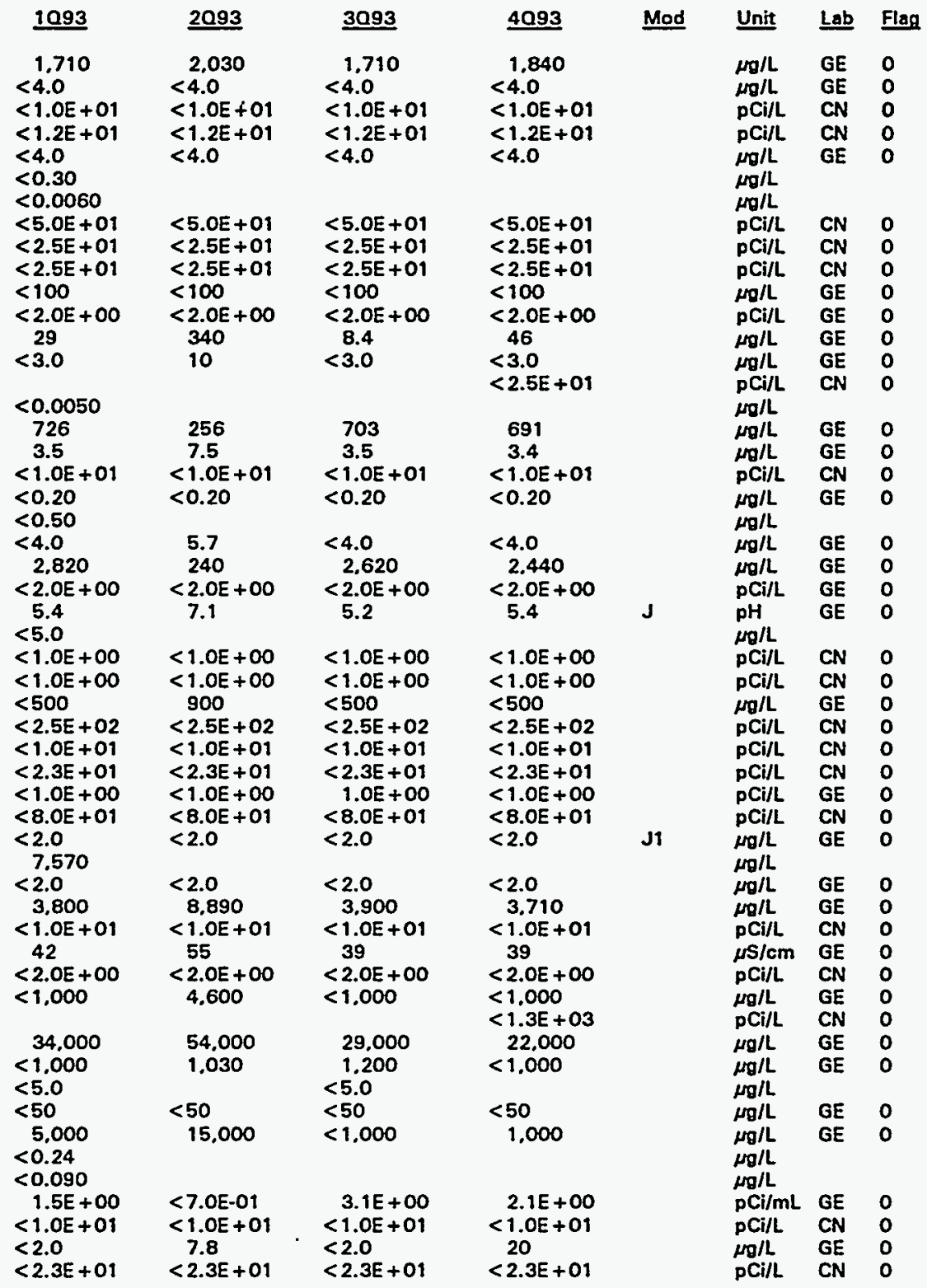

Note: Flagging levels, modifiers, and laboratories are for 4th quarter 1993 data only. See Appendix B for flagging criteria.

- = exceeded holding time for 4th quarter 1993.

- = exceeded screening level or final primary drinking water standard for 4th quarter 1993. 


\section{WELL RSF 3}

\begin{tabular}{|c|c|c|c|c|c|c|}
\hline SRS Coord. & Lat/Longitude & Screen Zone Elevation & Top of Casing & Casing & Pump & Screen Zone \\
\hline $\begin{array}{l}\text { N57621.4 } \\
\text { E75206.7 }\end{array}$ & $\begin{array}{l}33.275896{ }^{\circ} \mathrm{N} \\
81.5807011^{\circ} \mathrm{W}\end{array}$ & 239.8-229.8 ft msl & $307.1 \mathrm{ft} \mathrm{msl}$ & 4* PVC & $\mathbf{s}$ & Water Table \\
\hline SAMPLE DATE & & $03 / 22 / 93$ & $06 / 15 / 93$ & $09 / 14 / 93$ & $5 / 93$ & \\
\hline
\end{tabular}

FIELD DATA

\section{Analyte \\ Water elevation Depth to water pH \\ Sp. conductance \\ Water temperaturo \\ Alkalinity as $\mathrm{CaCO}_{3}$ \\ Turbidity \\ Volume purged \\ Sampling code}

ANALYTICAL DATA

1093
286.0
21.1
6.4
60
19.9
12
12
1.0
$x$

H D Analyte

Actinium-228

Aluminum, total recoverable Antimony-125

Arsenic, total recoverable

Barium, total recoverable

Cadmium, total rocoverable

Calcium, total recoverable

Cerium-144

Cesium-134

Cosium-137

Chlorido

Chromium, tozal recoverable

Cobalt-57

Cobatt-60

Copper, total recoverable

2,4-Dichlorophenoxyacetic acid

Endrin

Europium-152

Europium-154

Europium-155

Fluoride

Gross alpha

Iron, total recoverable

Lead, total recoverable

Lead-212

Lindane

Magnesium, total recoverable

Manganese, total recoverable

Manganese-54

Mercury, total recoverable

Methoxychlor

Nickel, total recoverable

Nitrato-nitrite as nitrogen

Nonvolatile bot.

pH

Phonols

Putonium-238

Pistonium-239/240

Potassium, total recoverable

\begin{tabular}{|c|c|c|c|c|c|c|c|}
\hline 1093 & $\underline{2093}$ & 3093 & 4093 & Mod & Unit & $\underline{\text { Lab }}$ & Flo \\
\hline 2,320 & & 261 & $<8.4 E+01$ & & $\begin{array}{l}\mathrm{pCi} / \mathrm{L} \\
\mu_{g} / \mathrm{L}\end{array}$ & $\mathrm{CN}$ & 0 \\
\hline$<2.5 E+01$ & $<2.5 E+01$ & $<2.5 E+01$ & $<2.5 E+01$ & & $\mathrm{pCi} / \mathrm{L}$ & $\mathrm{CN}$ & 0 \\
\hline$<2.0$ & $<2.0$ & $<2.0$ & $<2.0$ & & $\mu \mathrm{g} / \mathrm{L}$ & GE & 0 \\
\hline 12 & 5.4 & 10.0 & 6.6 & & $\mu g / L$ & GE & 0 \\
\hline$<2.0$ & $<2.0$ & $<2.0$ & $<2.0$ & & $\mu \mathrm{g} / \mathrm{L}$ & GE & 0 \\
\hline 1,610 & 2,230 & 1.620 & 980 & $\mathrm{~J} 2$ & $\mu g / L$ & GE & 0 \\
\hline$<5.0 E+01$ & $<5.0 E+01$ & $<5.0 E+01$ & $<5.0 E+01$ & & $\mathrm{pCi} / \mathrm{L}$ & $\mathrm{CN}$ & 0 \\
\hline$<1.0 E+01$ & $<1.0 E+01$ & $<1.0 E+01$ & $<1.0 E+01$ & & $\mathrm{pCi} / \mathrm{L}$ & $\mathrm{CN}$ & 0 \\
\hline$<1.0 E+01$ & $<1.0 E+01$ & $<1.0 E+01$ & $<1.0 E+01$ & & $\mathrm{pCi} / \mathrm{L}$ & $\mathrm{CN}$ & 0 \\
\hline 2.220 & 2,060 & 1.640 & 1.900 & & $\mu g / L$ & $\mathrm{GE}$ & 0 \\
\hline$<4.0$ & $<4.0$ & $<4.0$ & $<4.0$ & & $\mu g / L$ & GE & 0 \\
\hline$<1.0 E+01$ & $<1.0 E+01$ & $<1.0 E+01$ & $<1.0 E+01$ & & $\mathrm{pCi} / \mathrm{L}$ & $\mathrm{CN}$ & 0 \\
\hline$<1.2 E+01$ & $<1.2 E+01$ & $<1.2 E+01$ & $<1.2 \mathrm{E}+01$ & & $\mathrm{pCi} / \mathrm{L}$ & $\mathrm{CN}$ & 0 \\
\hline$<4.0$ & $<4.0$ & $<4.0$ & $<4.0$ & & $\mu \mathrm{g} / \mathrm{L}$ & GE & 0 \\
\hline $\begin{array}{l}<0.30 \\
<0.0060\end{array}$ & & & & & $\mu g / L$ & & \\
\hline$<5.0 E+01$ & $<5.0 E+01$ & $<5.0 E+01$ & $<5.0 E+01$ & & $\mathrm{PCi} / \mathrm{L}$ & $\mathrm{CN}$ & 0 \\
\hline$<2.5 E+01$ & $<2.5 E+01$ & $<2.5 E+01$ & $<2.5 E+01$ & & $\mathrm{pCi} / \mathrm{L}$ & $\mathrm{CN}$ & 0 \\
\hline$<2.5 E+01$ & $<2.5 E+01$ & $<2.5 E+01$ & $<2.5 E+01$ & & $\mathrm{pCi} / \mathrm{L}$ & $\mathrm{CN}$ & 0 \\
\hline 169 & $<100$ & $<100$ & 110 & & $\mu \mathrm{g} / \mathrm{L}$ & GE & 0 \\
\hline $4.3 E+00$ & $<2.0 E+00$ & $<2.0 E+00$ & $<2.0 E+00$ & & $\mathrm{pCi} / \mathrm{L}$ & $\overrightarrow{\mathrm{GE}}$ & 0 \\
\hline 827 & 15 & 319 & 98 & & $\mu_{g} / L$ & GE & 0 \\
\hline 6.2 & $<3.0$ & 11 & 9.5 & & $\mu \mathrm{g} / \mathrm{L}$ & GE & 1 \\
\hline & & & $<2.5 E+01$ & & $\mathrm{pCi} / \mathrm{L}$ & $\mathrm{CN}$ & 0 \\
\hline$<0.0050$ & & & & & $\mu g / L$ & & \\
\hline 503 & 725 & 241 & 170 & & $\mu g / L$ & GE & 0 \\
\hline 12 & 3.7 & 7.1 & 6.3 & & $\mu g / L$ & GE & 0 \\
\hline$<1.0 E+01$ & $<1.0 E+01$ & $<1.0 E+01$ & $<1.0 E+01$ & & $\mathrm{pCi} / \mathrm{L}$ & $\mathrm{CN}$ & 0 \\
\hline$<0.20$ & $<0.20$ & 2.8 & $<0.20$ & & $\mu g / L$ & GE & 0 \\
\hline$<0.50$ & & & & & $\mu_{g} / L$ & & \\
\hline 7.9 & $<4.0$ & 9.0 & 6.0 & & $\mu \mathrm{g} / \mathrm{L}$ & GE & 0 \\
\hline 180 & 2,700 & 190 & 140 & & $\mu_{g} / L$ & GE & 0 \\
\hline $3.8 \mathrm{E}+00$ & $<2.0 E+00$ & $<2.0 E+00$ & $3.1 E+\infty 0$ & & $\mathrm{pCi} / \mathrm{L}$ & GE & 0 \\
\hline 7.2 & 5.4 & 6.1 & 6.9 & $J$ & $\mathrm{pH}$ & GE & 0 \\
\hline$<5.0$ & & & & & $\mu \mathrm{g} / \mathrm{L}$ & & \\
\hline$<1.0 E+00$ & $<1.0 E+00$ & $<1.0 E+00$ & $<1.0 E+00$ & & $\mathrm{PCi} / \mathrm{L}$ & $\mathrm{CN}$ & 0 \\
\hline$<1.0 E+00$ & $<1 . \mathrm{OE}+00$ & $<1.0 E+00$ & $<1.0 E+00$ & & $\mathrm{pCi} / \mathrm{L}$ & $\mathrm{CN}$ & 0 \\
\hline 1.340 & $<500$ & 901 & 886 & & $\mu g / L$ & GE & 0 \\
\hline
\end{tabular}

Note: Flagging levels, modifiers, and laboratories are for 4th quarter 1993 data only. See Appendix B for flagging criteria. - = exceeded holding time for 4th quarter 1993

a = exceeded screening level or final primary drinking water standard for 4th quarter 1993. 


\section{ANALYTICAL DATA}

H D Analyte

Potasxium-40

Promothium-144

Promethium-146

Radium, total alpha-emitting

Ruthenium-106

Selonium, total recoverable

Silica, total recoverable

Silver, total recoverable

Sodium, total rocovorable

Sodium-22

Spocific conductanco

Strontium-90

Sulfato

Thorium-234

Total dissolved solids

Total organic carbon

Total organic halogens

Total phosphates (as P)

Total suepended solids

Toxapheno

2,4,5-TP (Silvex)

Tritium

Yttrium-88

Zinc, total recovorable

Zine-65

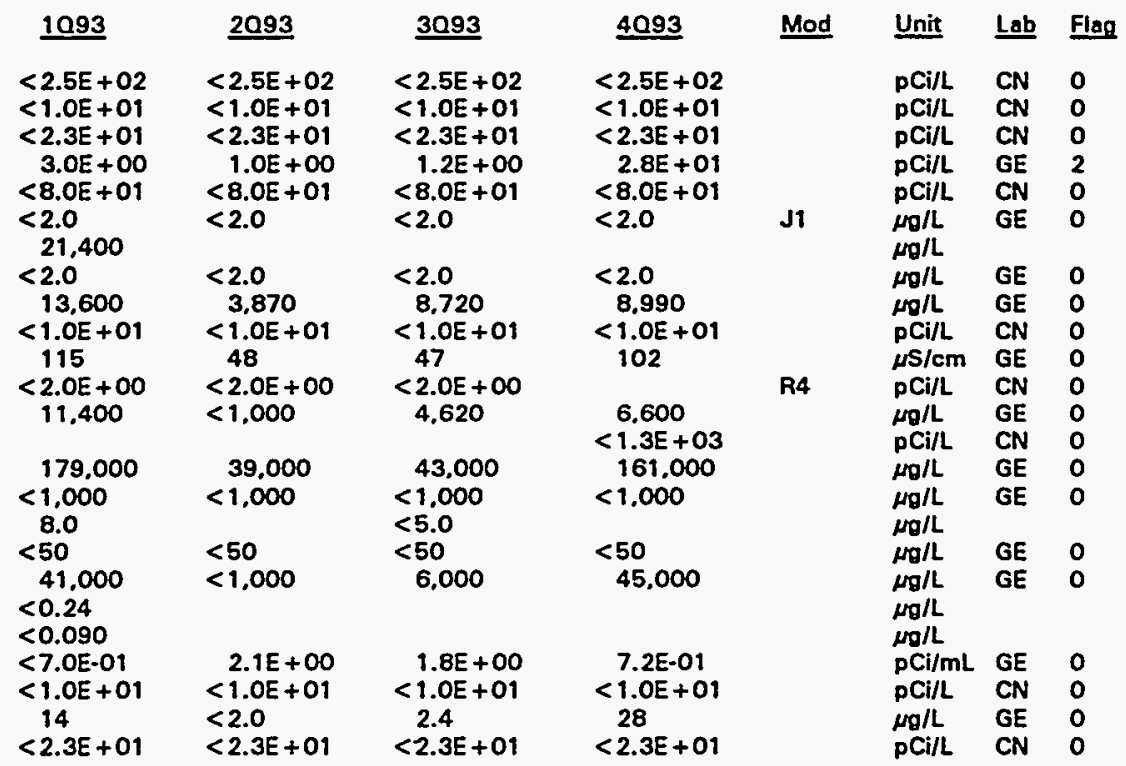

Note: Flagging levels, modifiers, and laboratories are for 4th quarter 1993 data only. See Appendix B for flagging criteria. - = exceeded holding time for 4th quarter 1993.

- = exceeded screening level or final primary drinking water standard for 4th quarter 1993. 
WSRC-TR-940354

Unclassified 


\section{Appendix D. Time Series Plots}




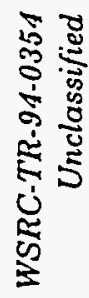
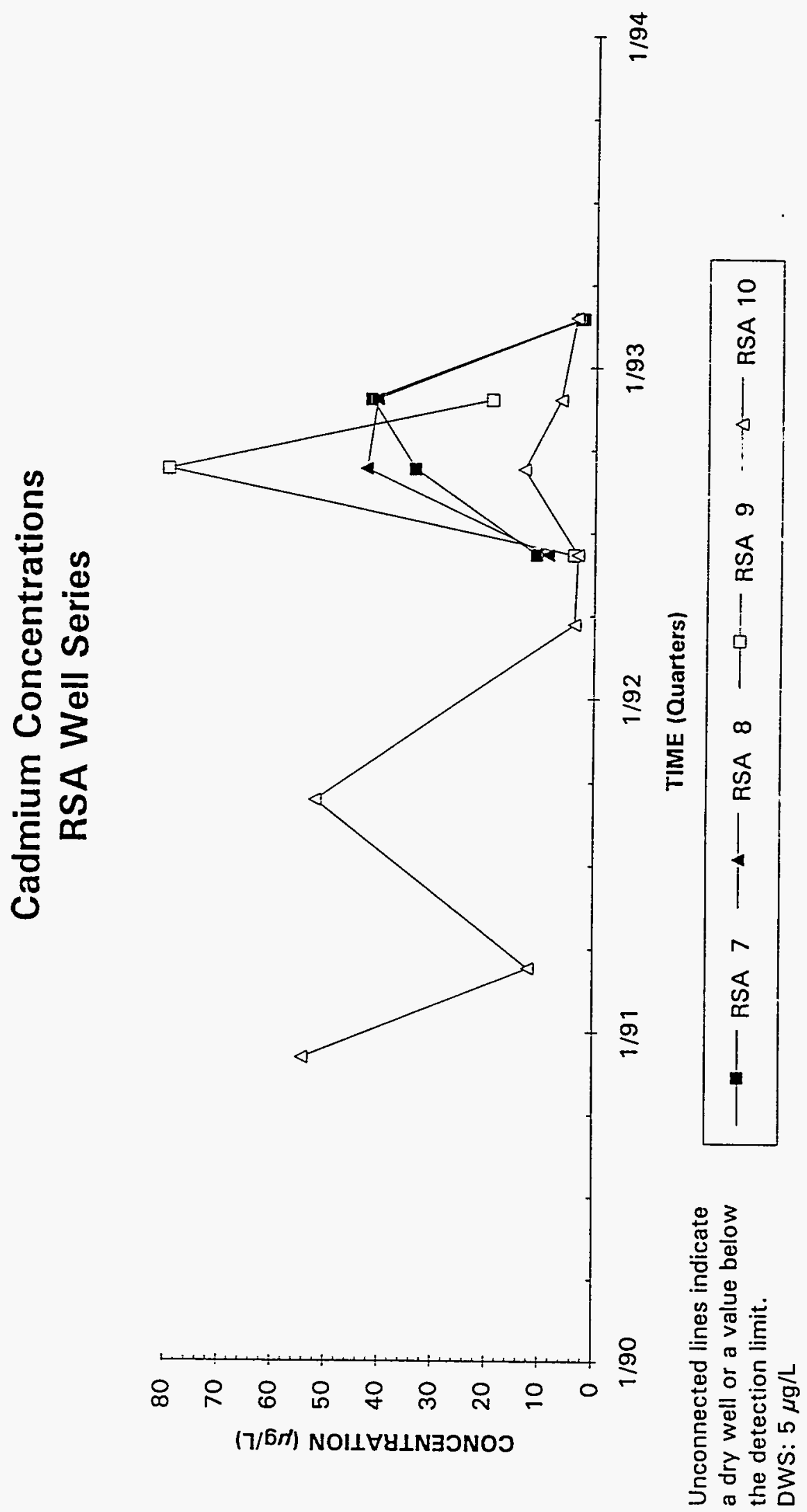

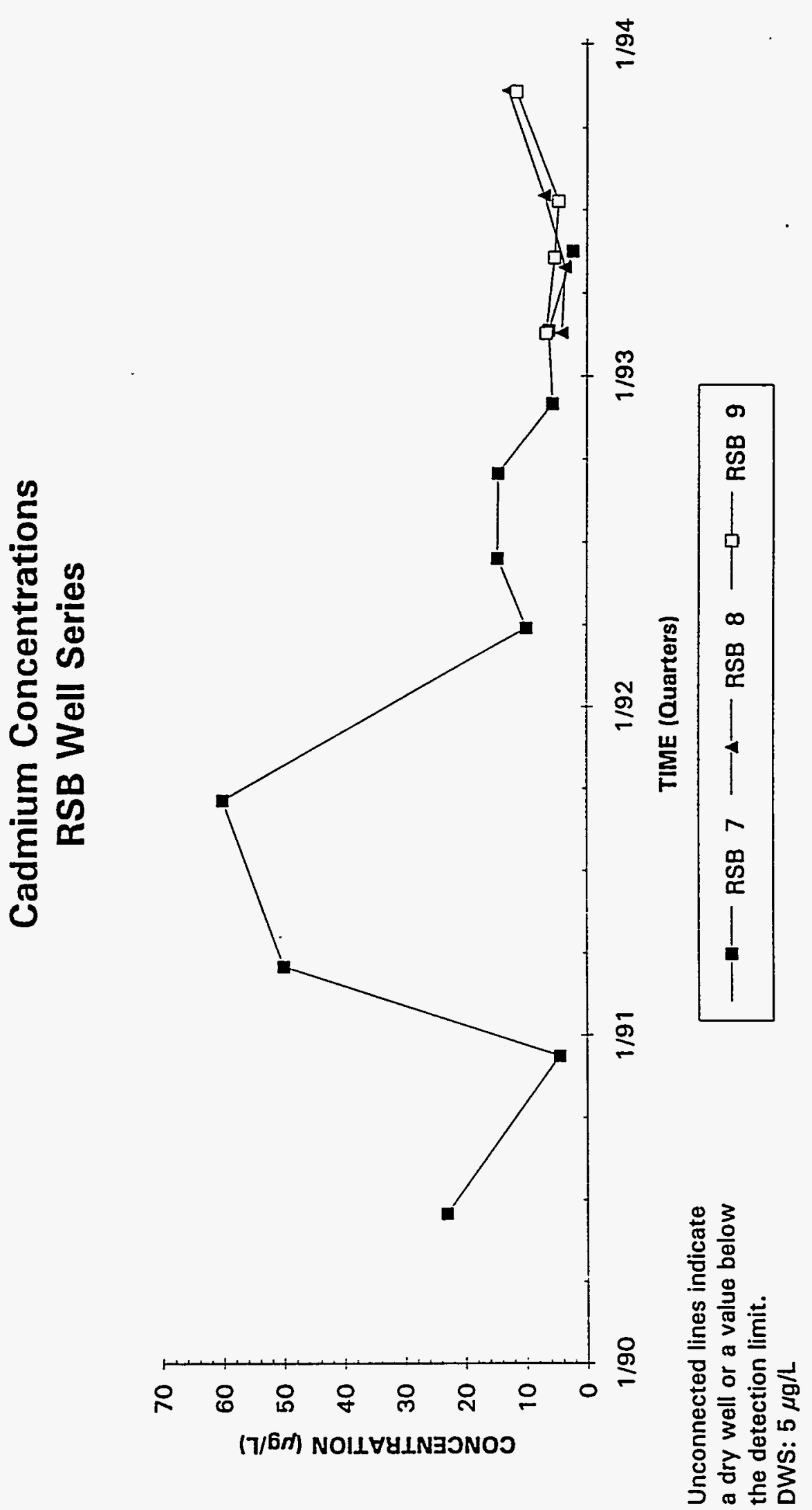


\section{Cadmium Concentrations \\ RSC Well Series}

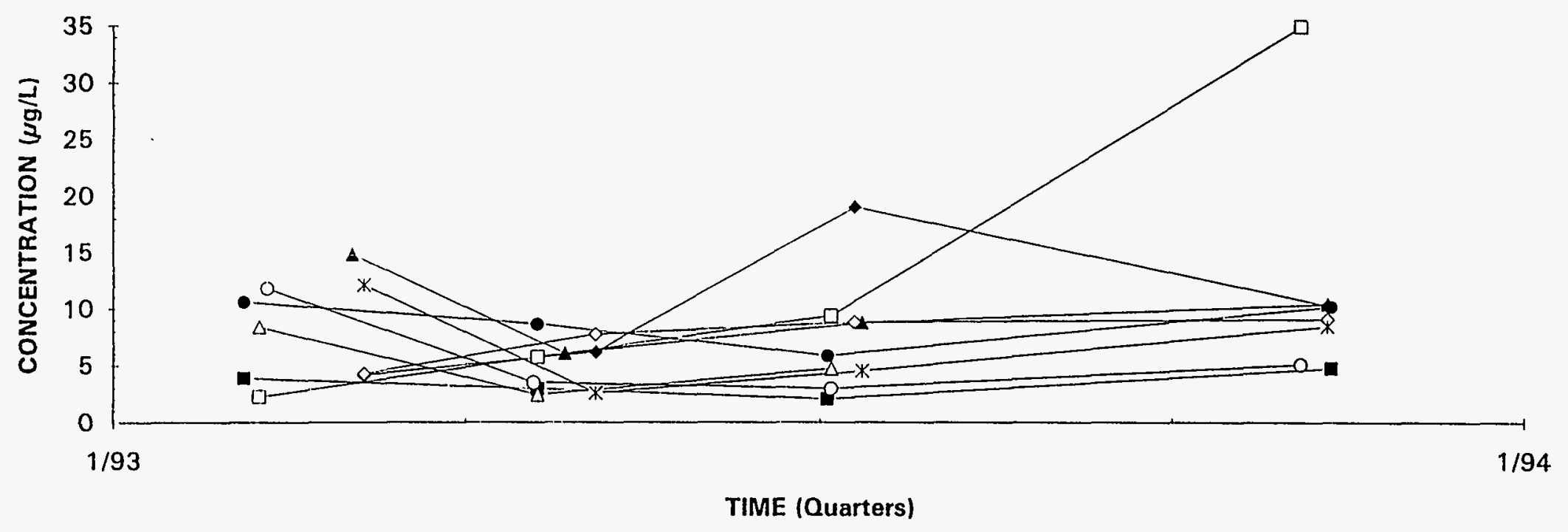

列

a dry well or a value below

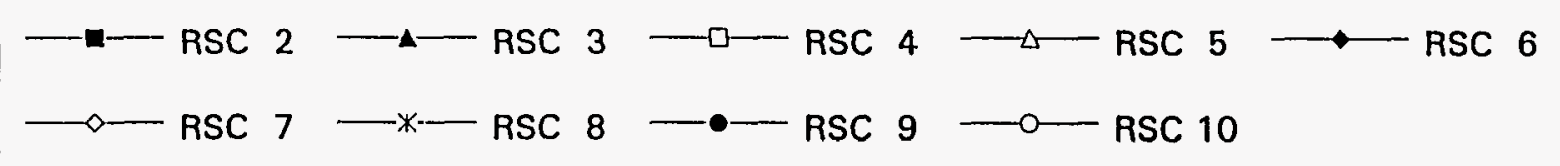

the detection limit.

DWS: $5 \mu \mathrm{g} / \mathrm{L}$ 


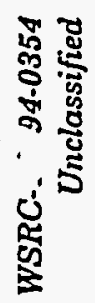
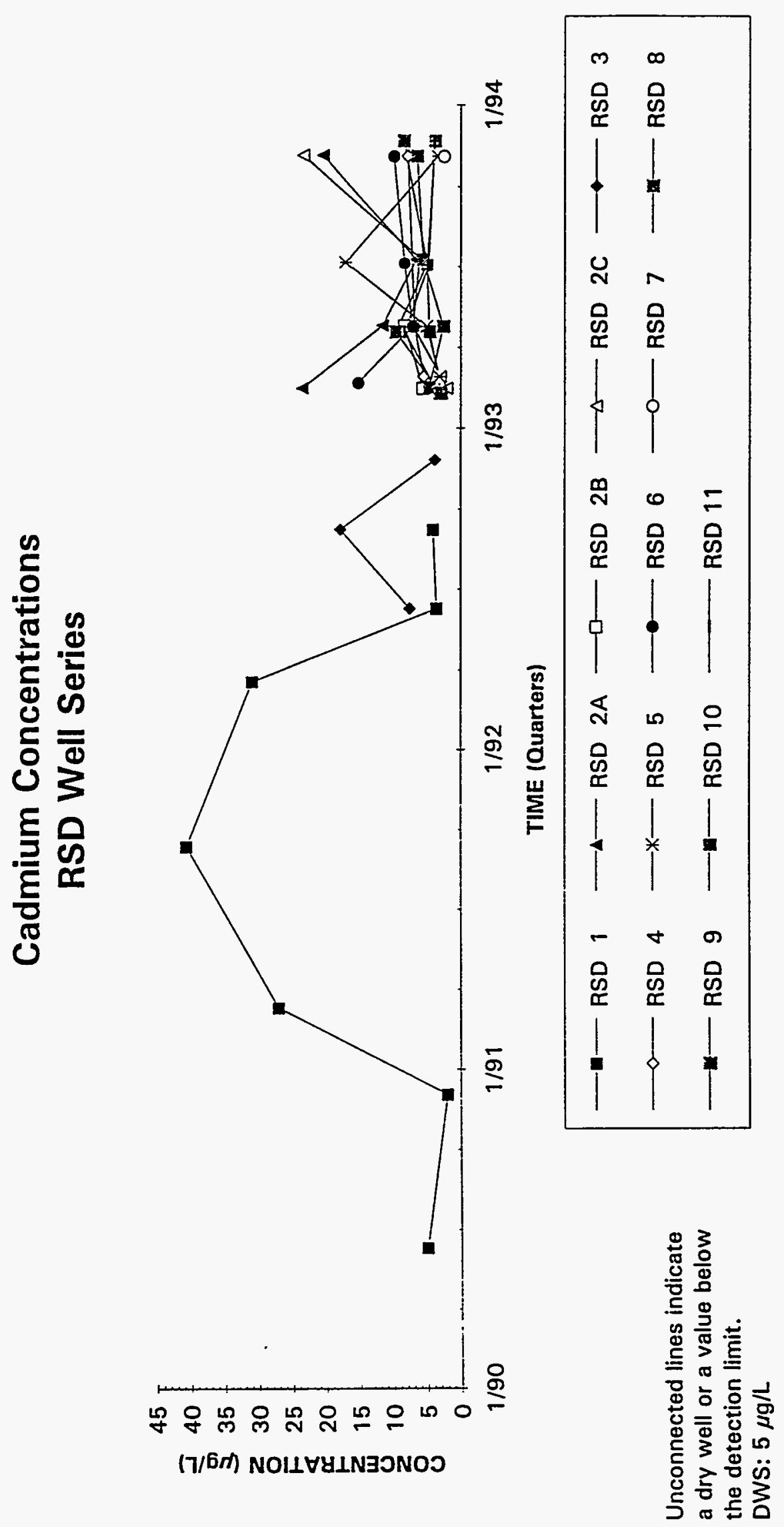


\section{Cadmium Concentrations \\ RSE Well Series (1A through 6)}
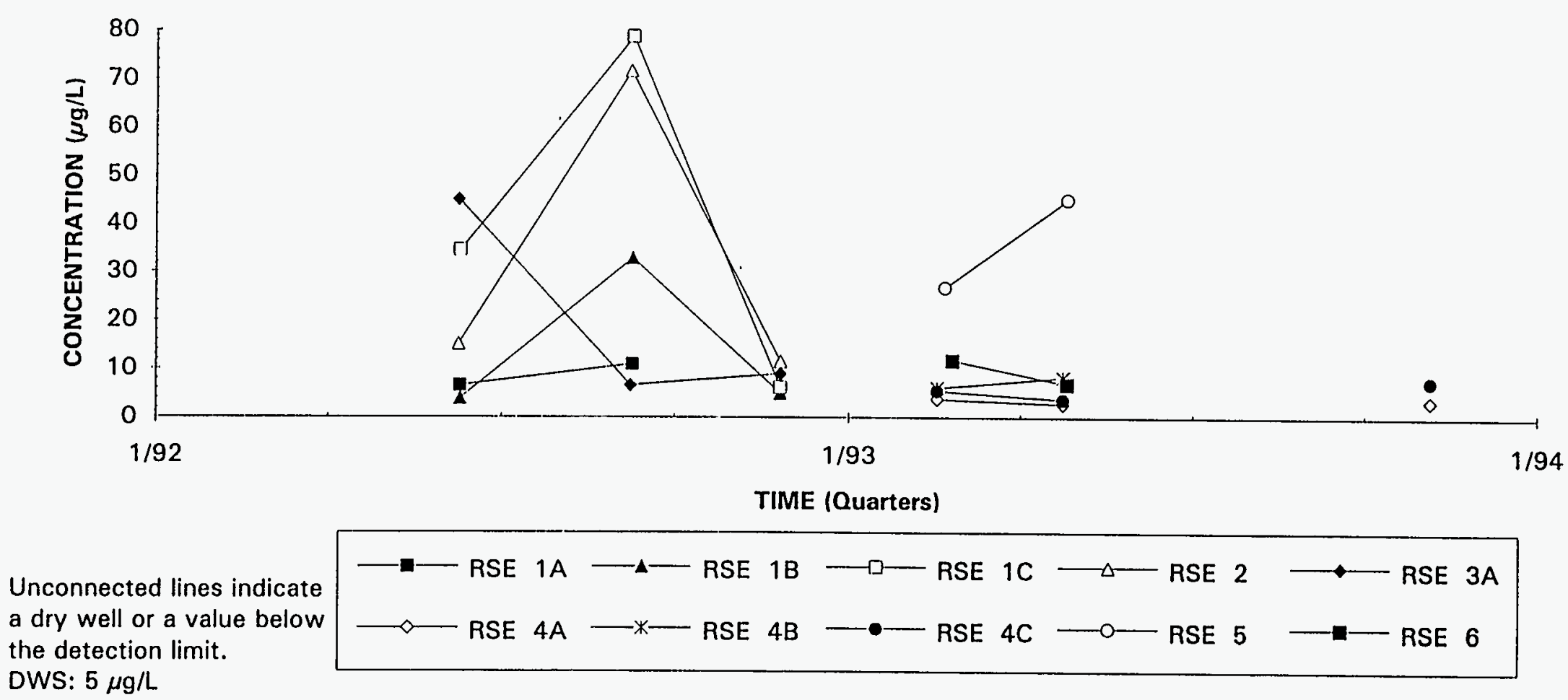

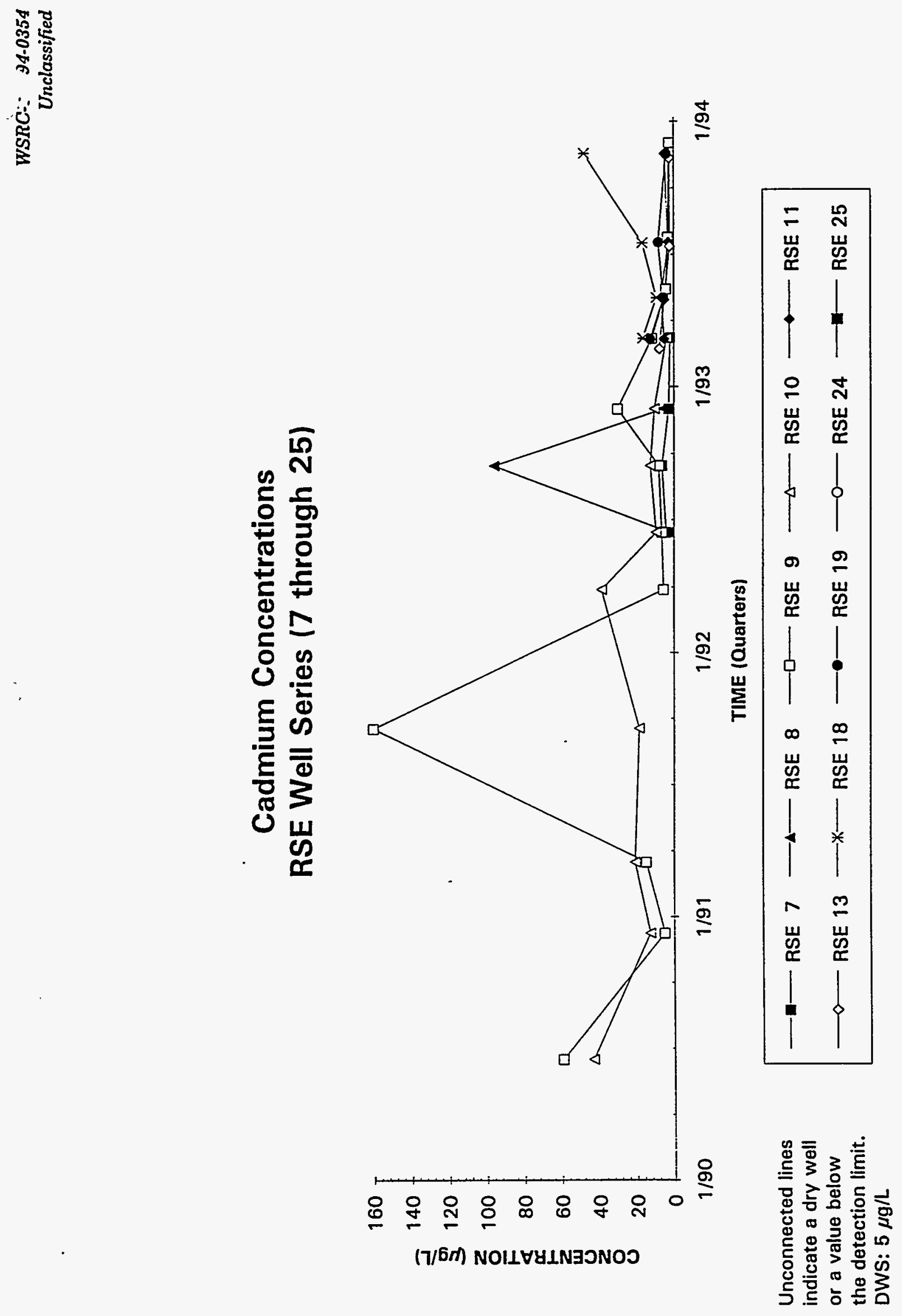


\section{Conductivity}

RAC Well Series

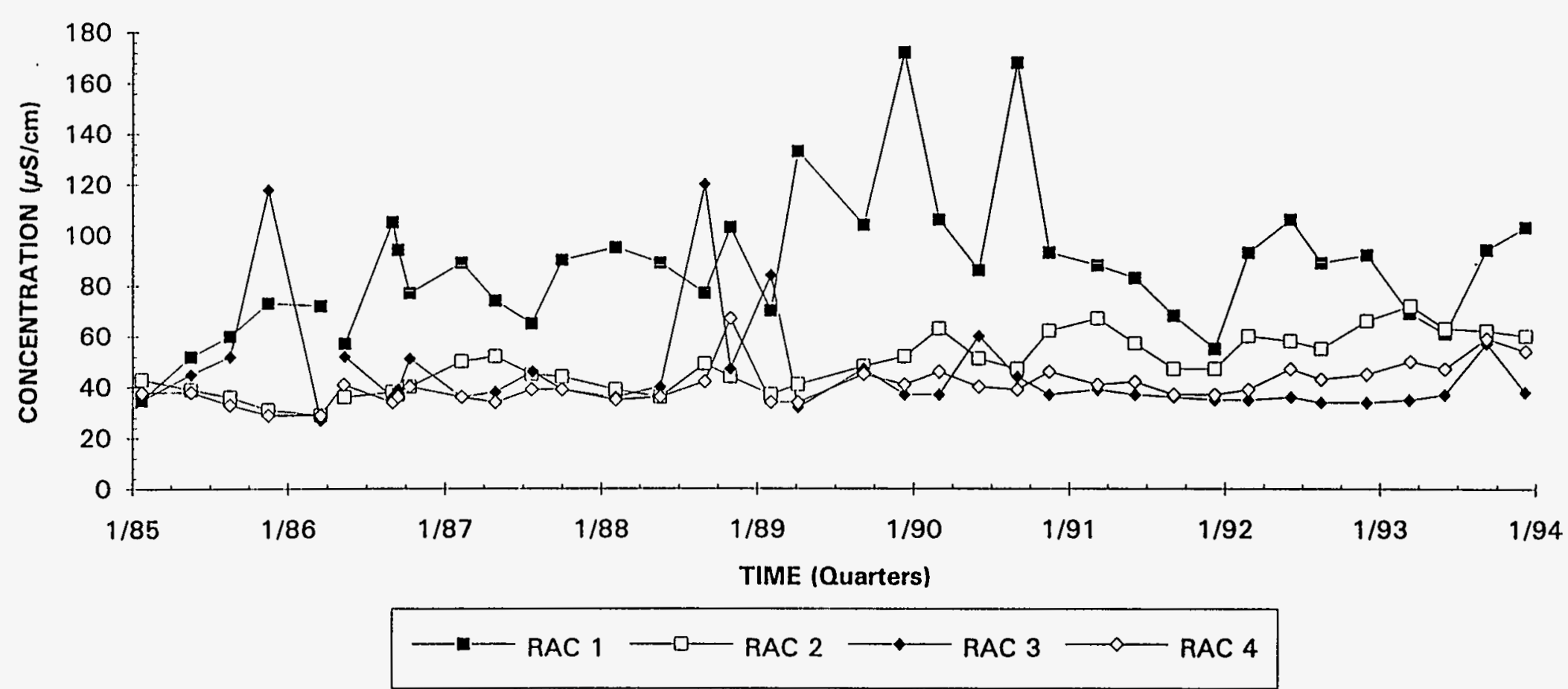



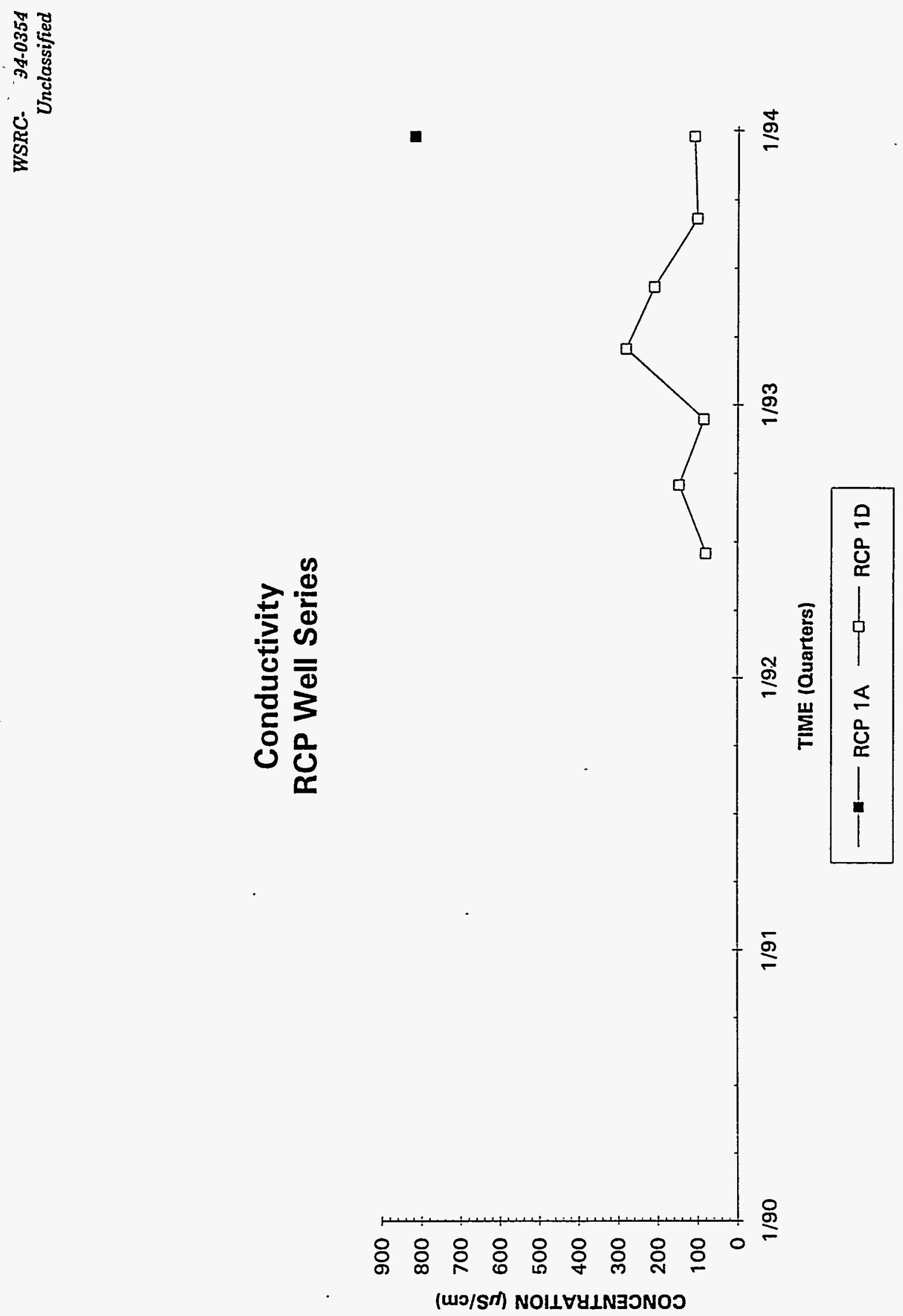

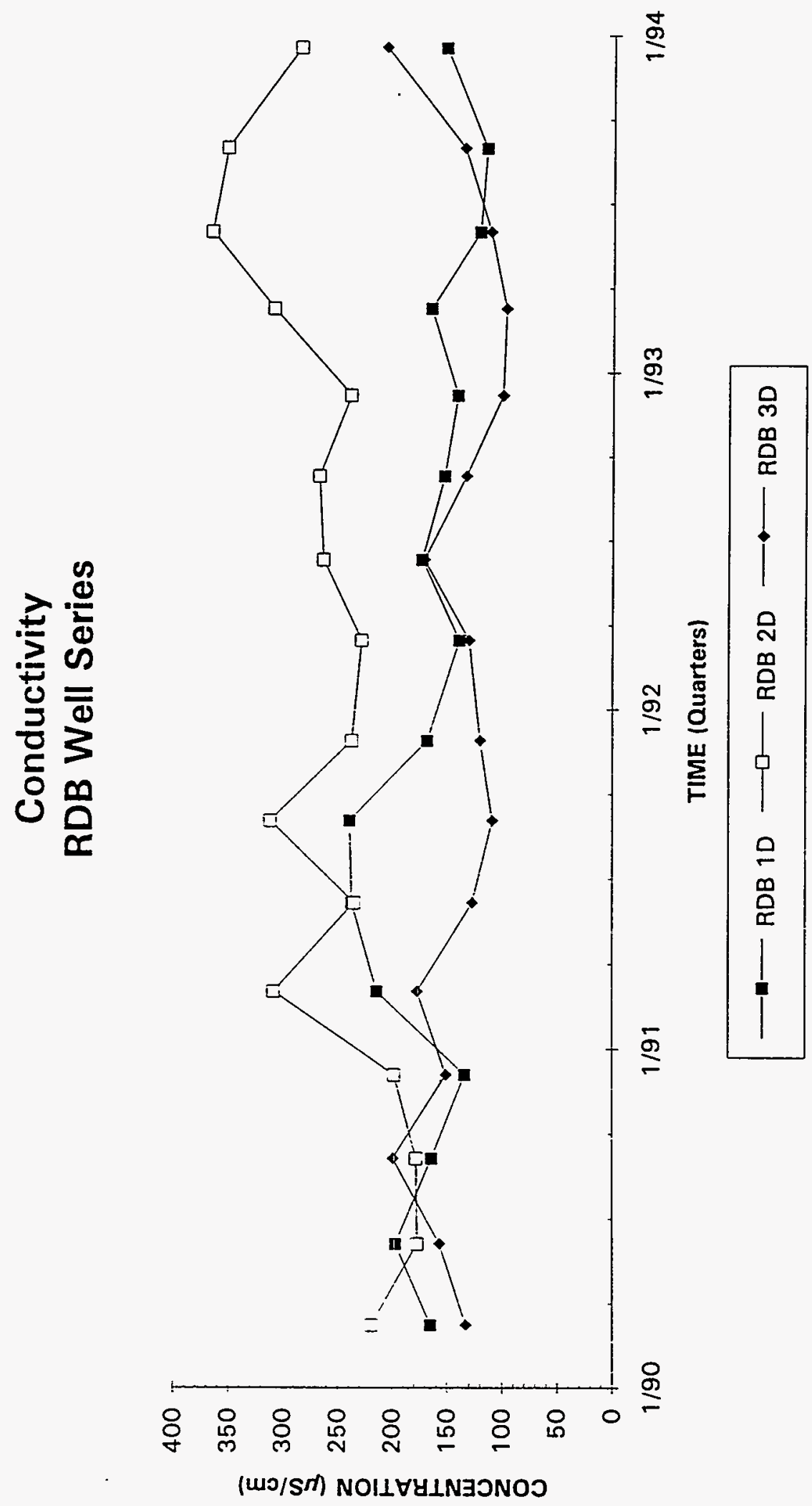


\section{Conductivity RRP Well Series}

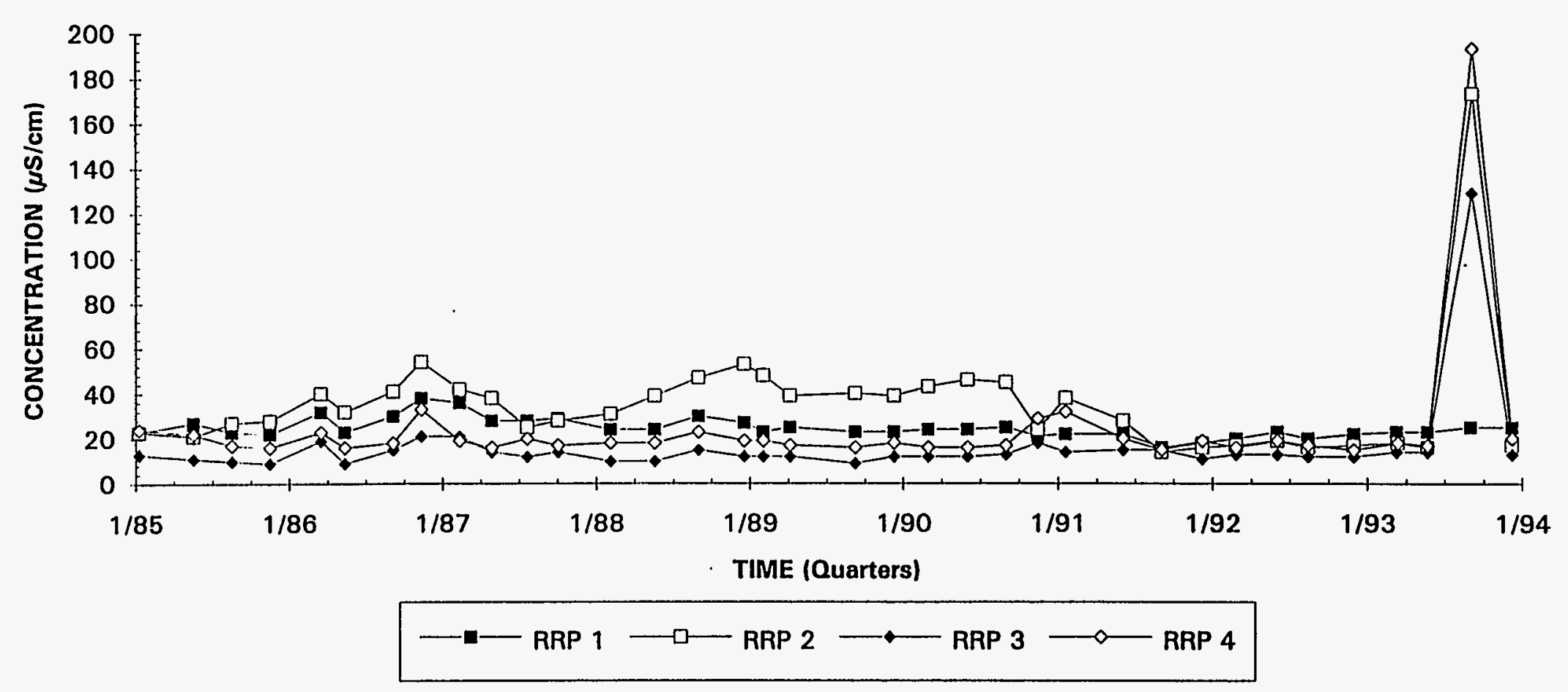




\section{Conductivity RSA Well Series}

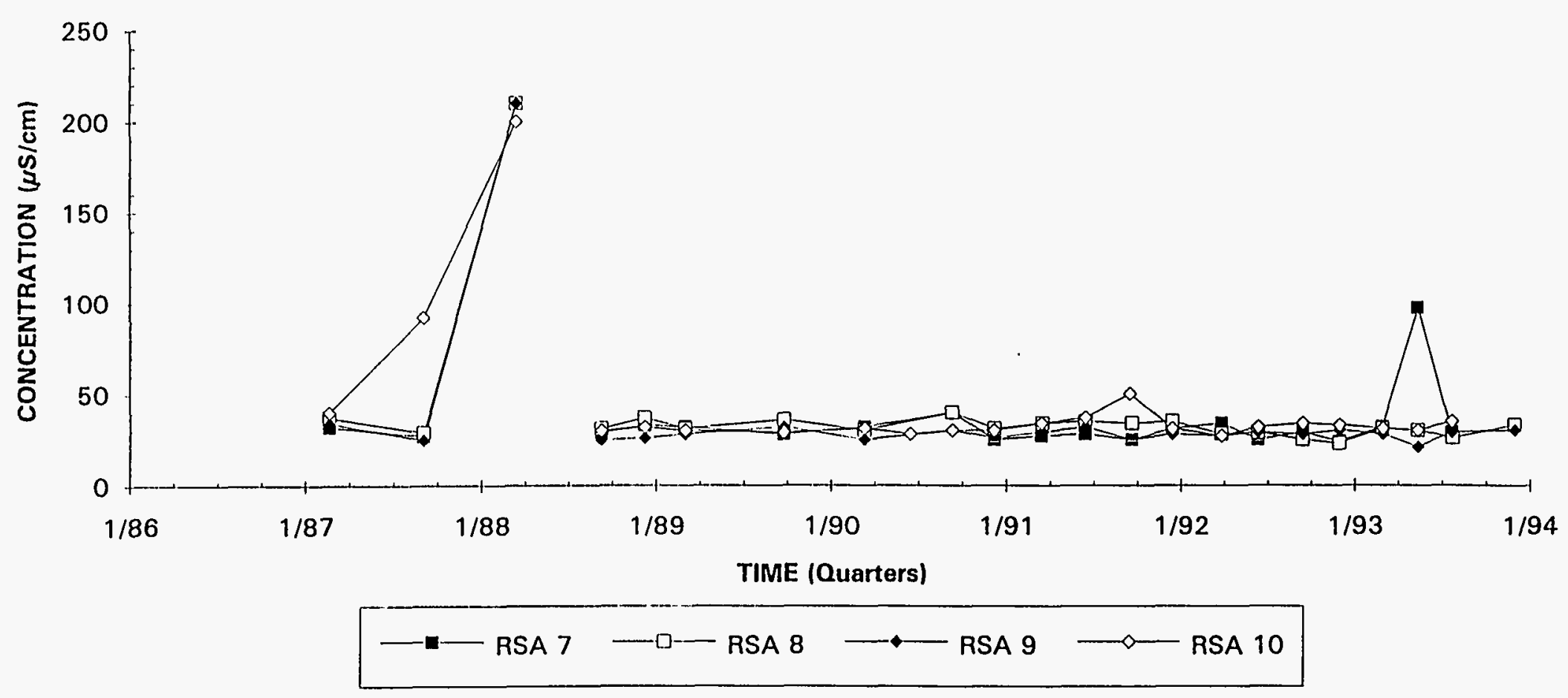




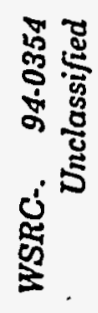
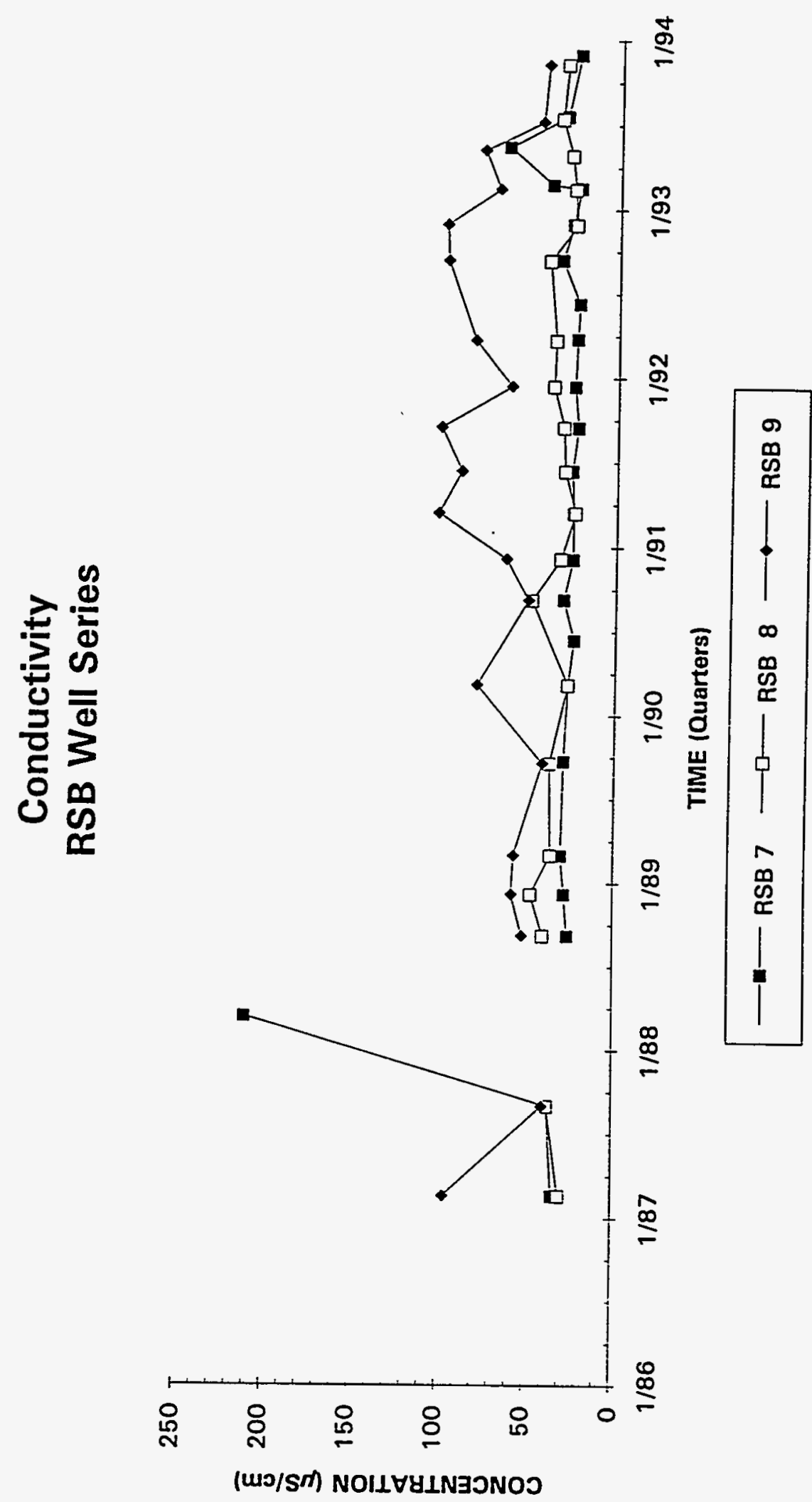

ปั. 


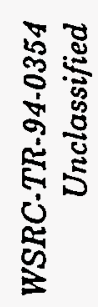
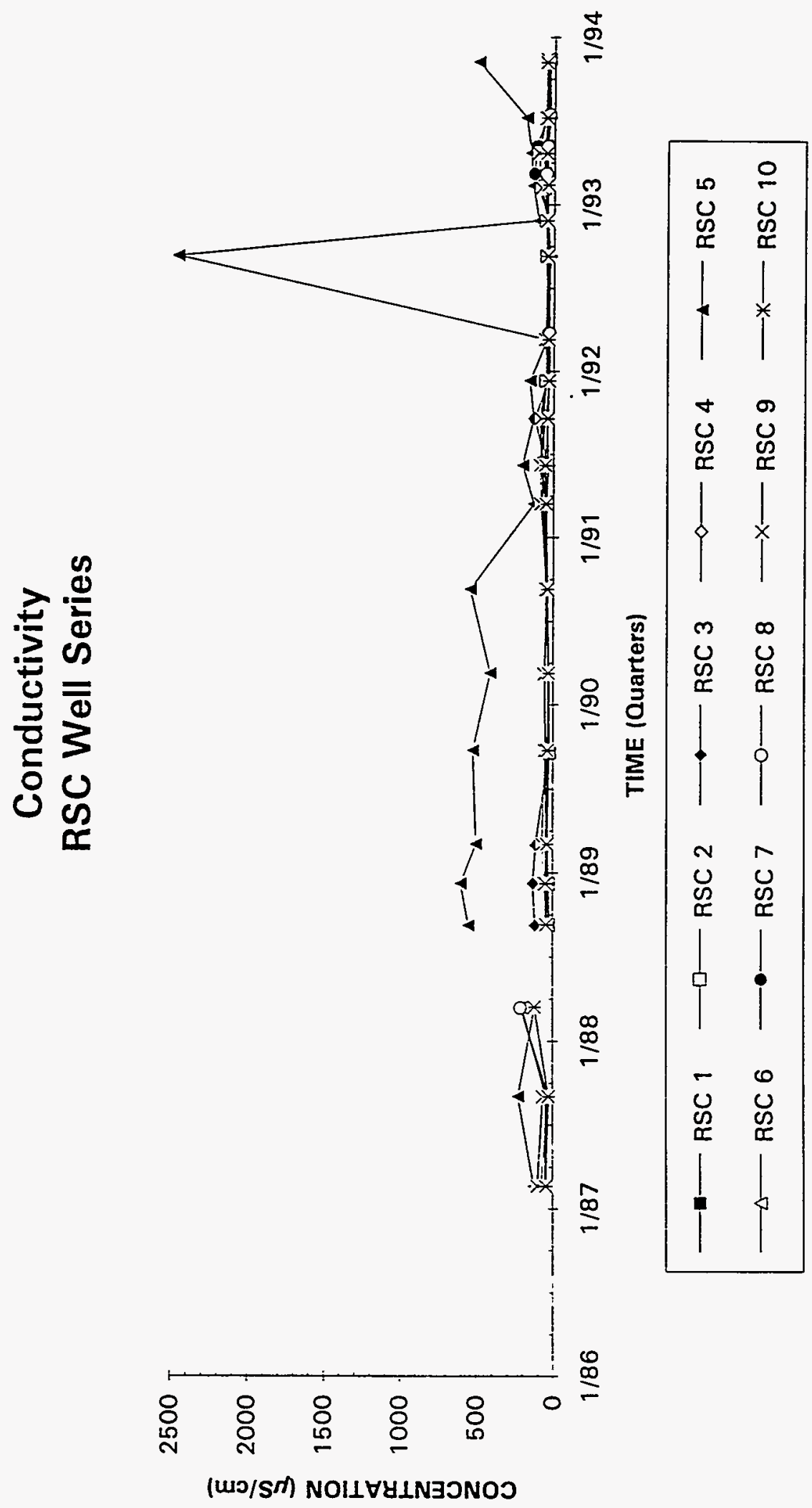

है 


\section{Conductivity RSD Well Series}

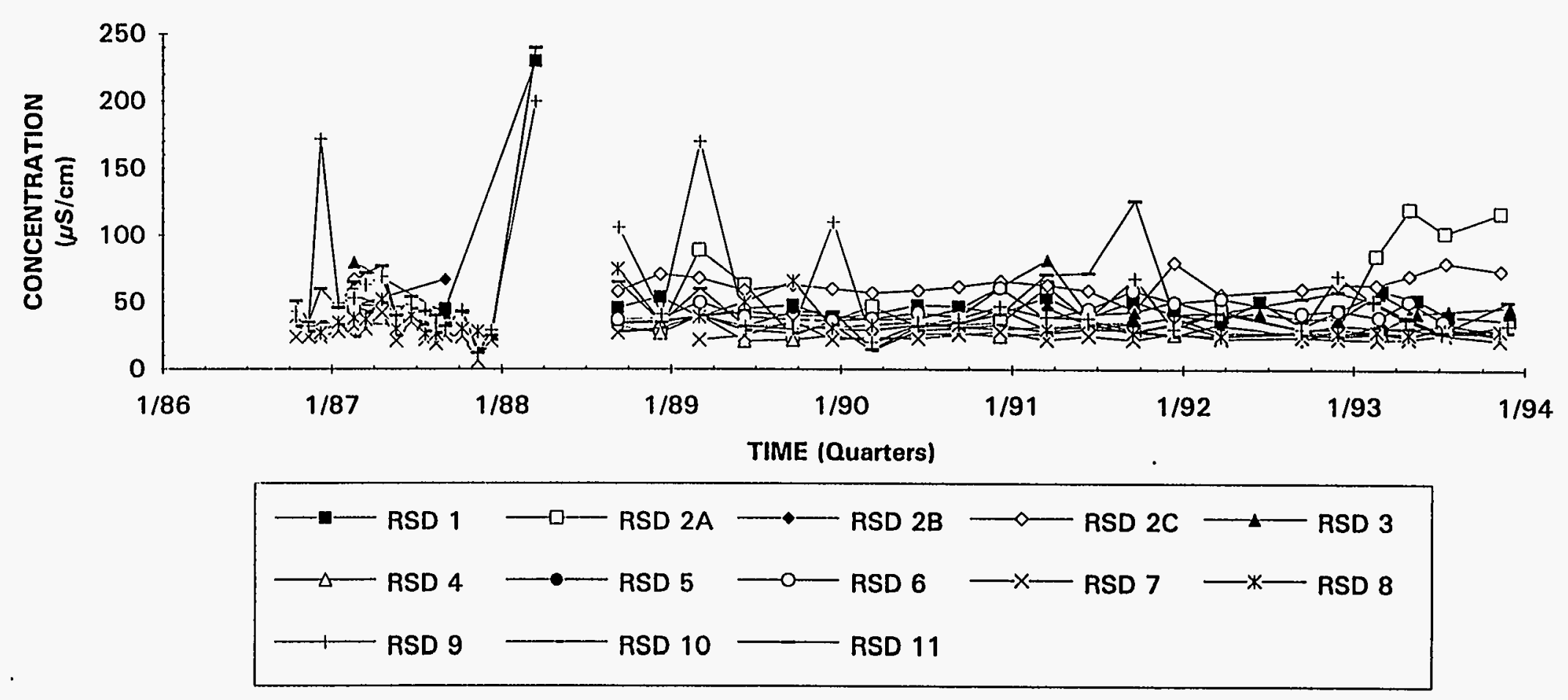


硧

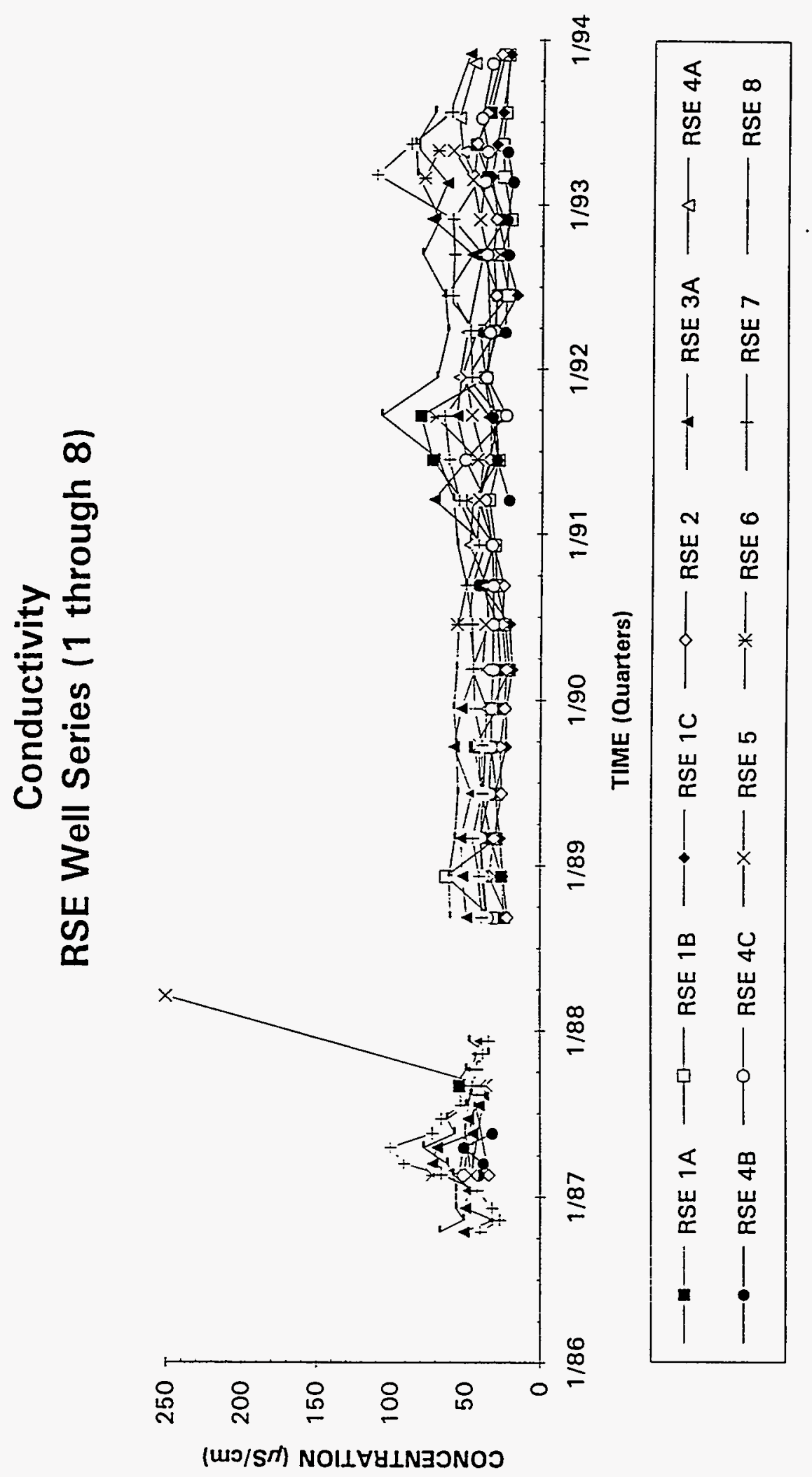

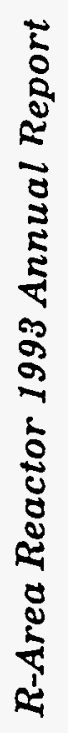




\section{Conductivity \\ RSE Well Series (9 through 25)}

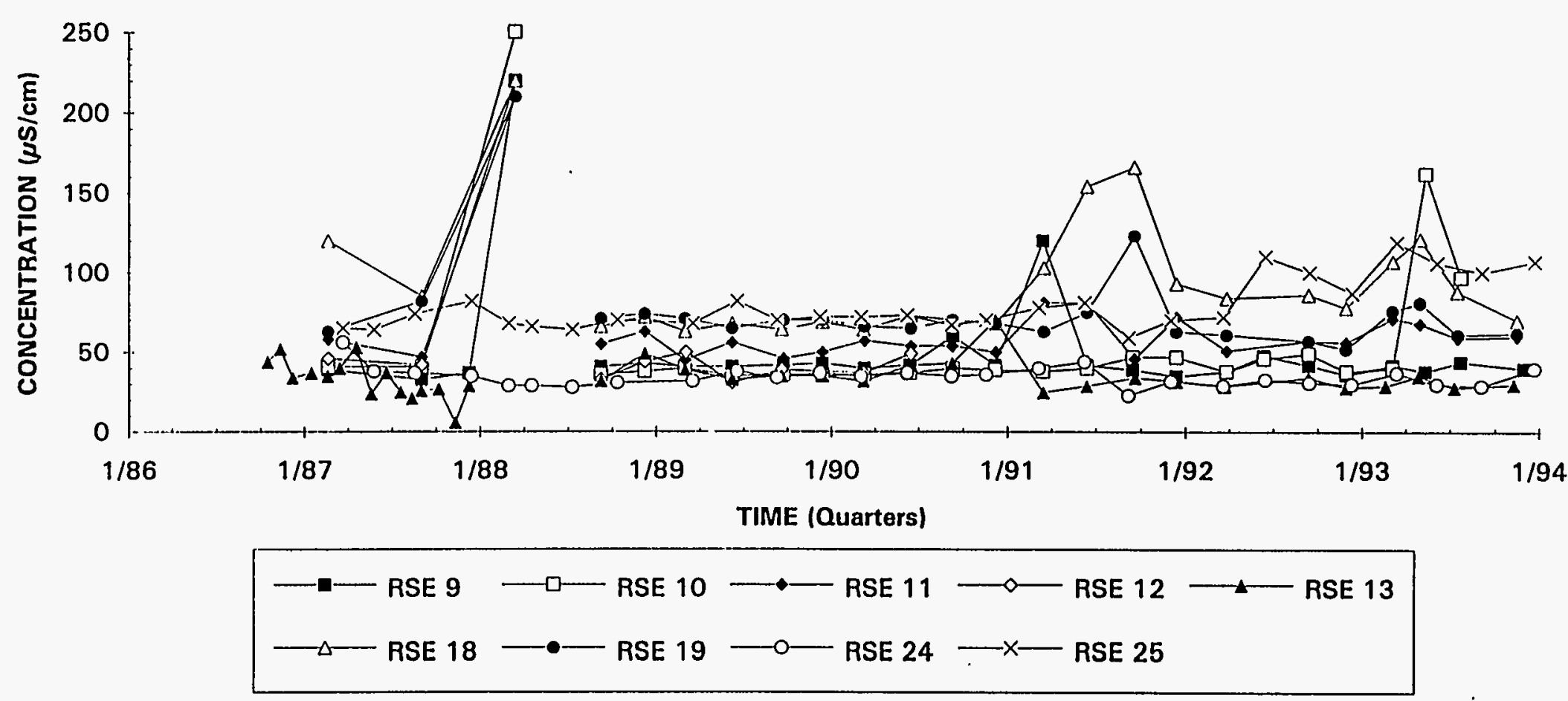



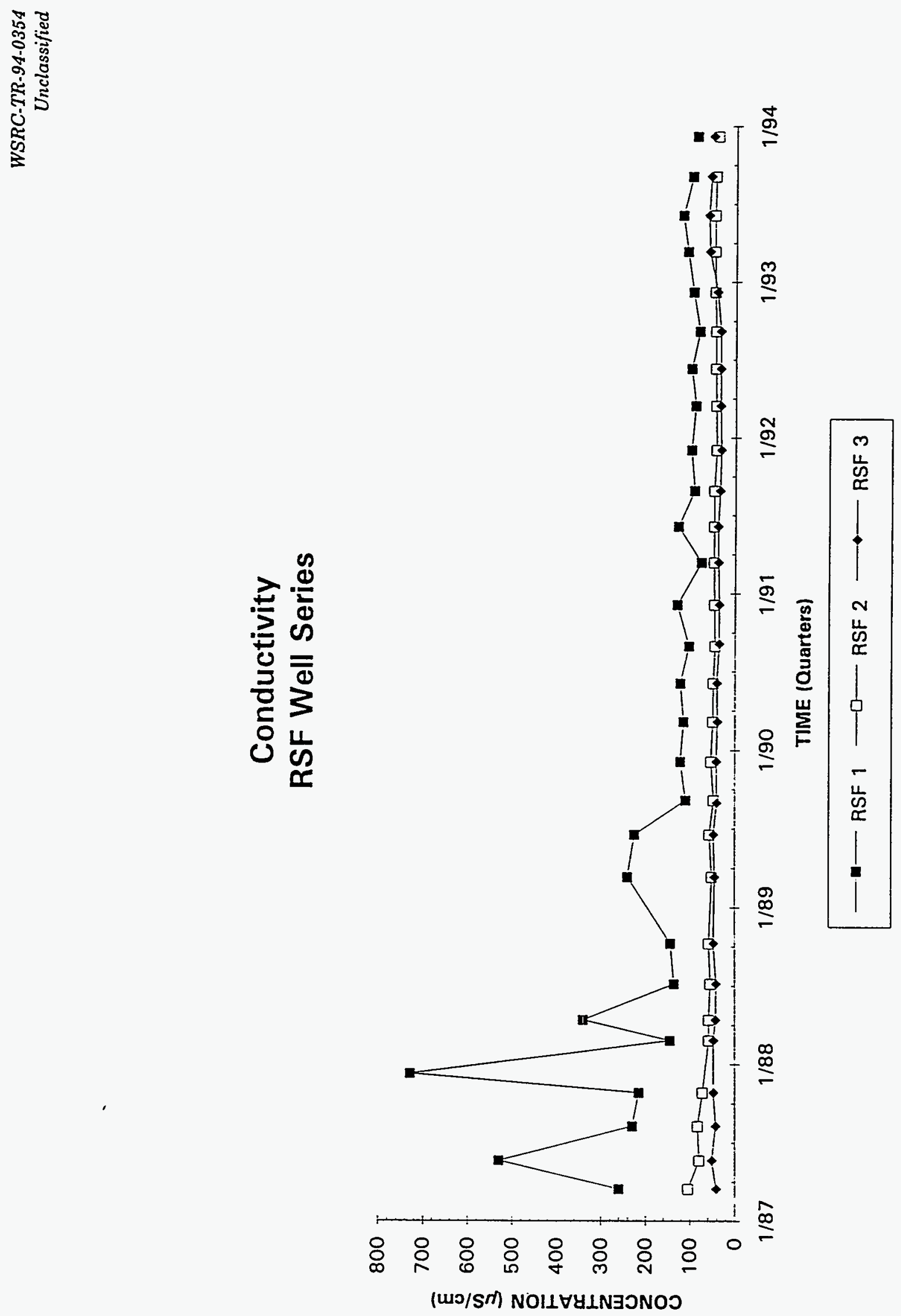


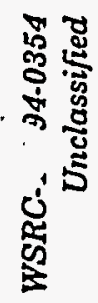
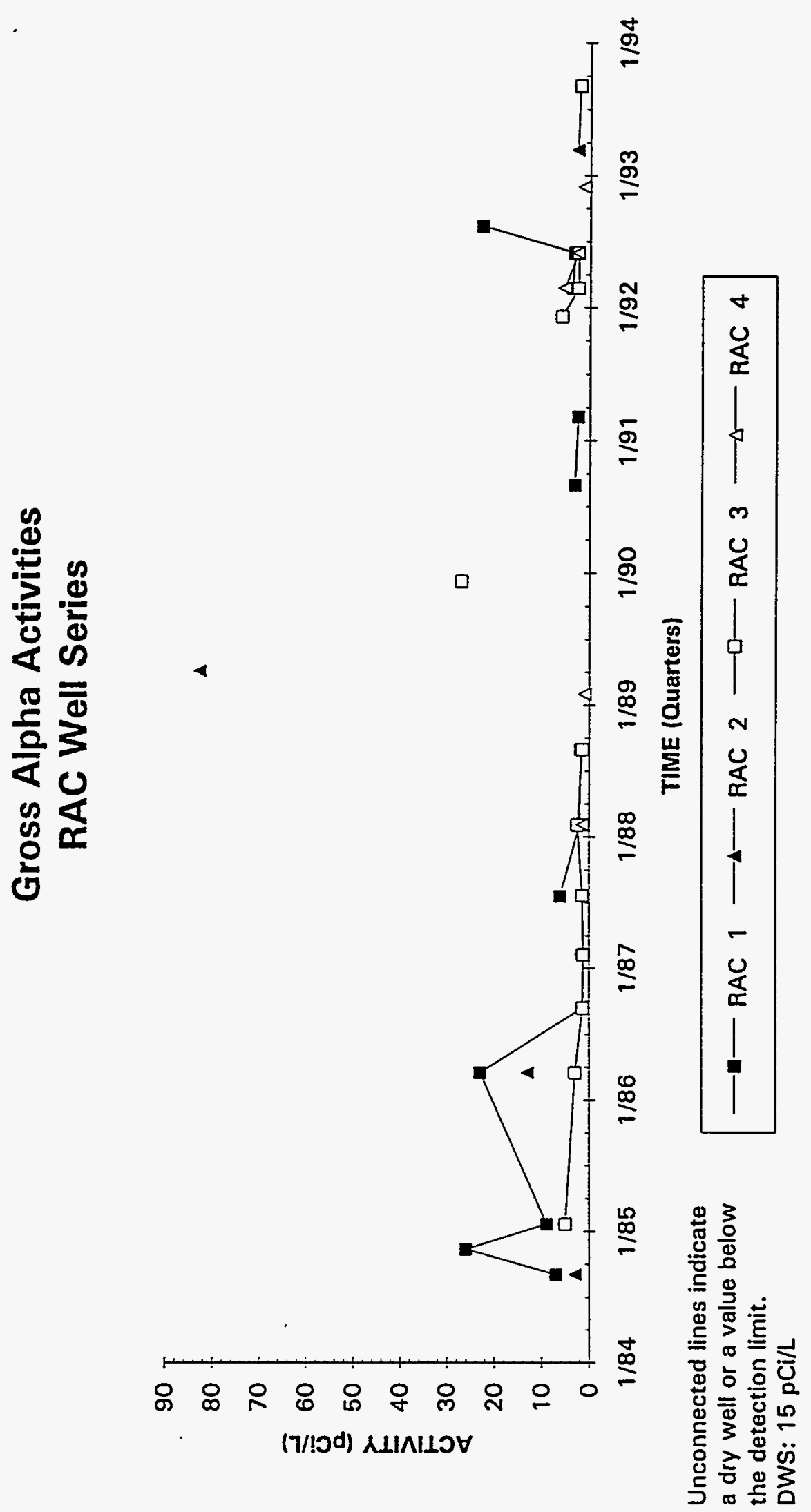

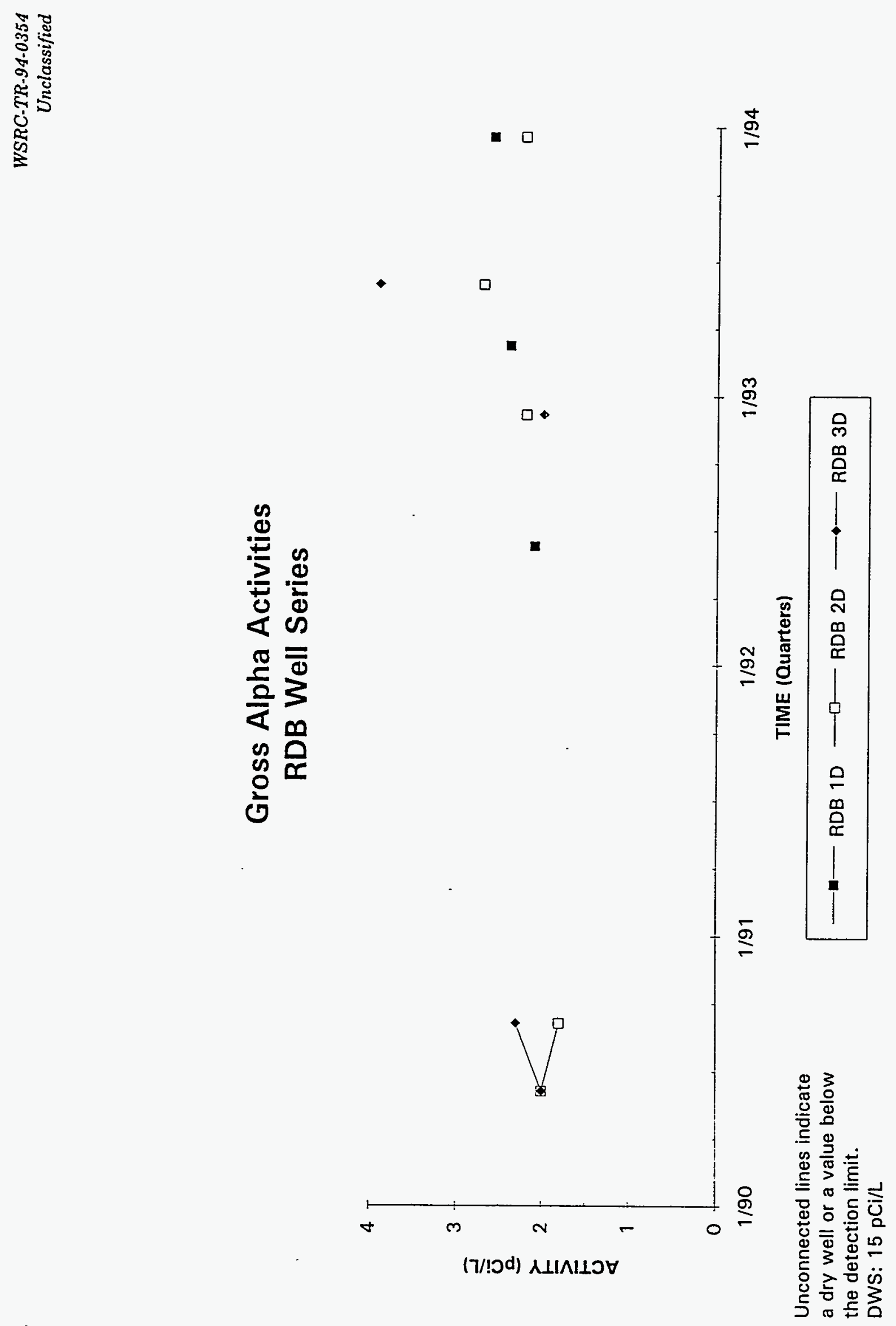


\section{Gross Alpha Activities RSA Well Series}

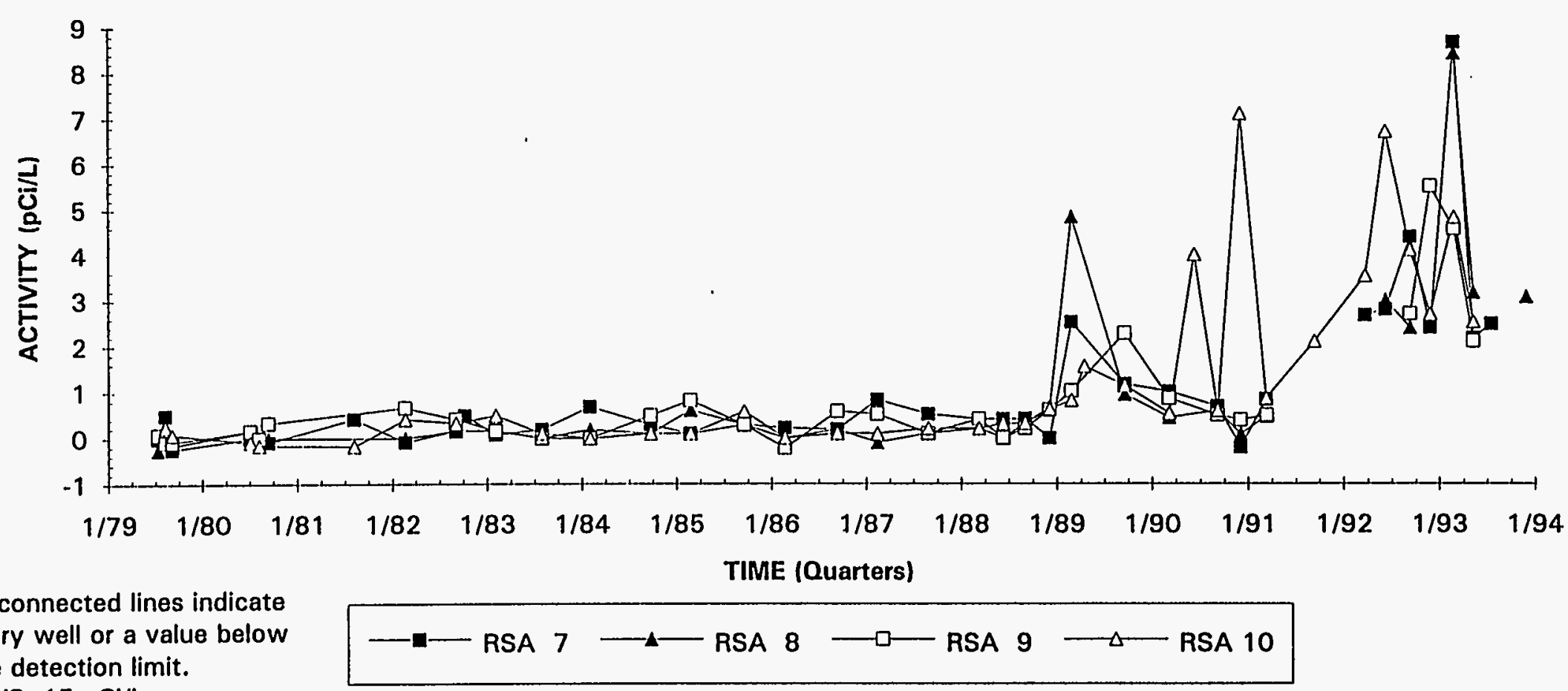

DWS: $15 \mathrm{pCi} / \mathrm{L}$ 
Gross Alpha Activities

RSB Well Series

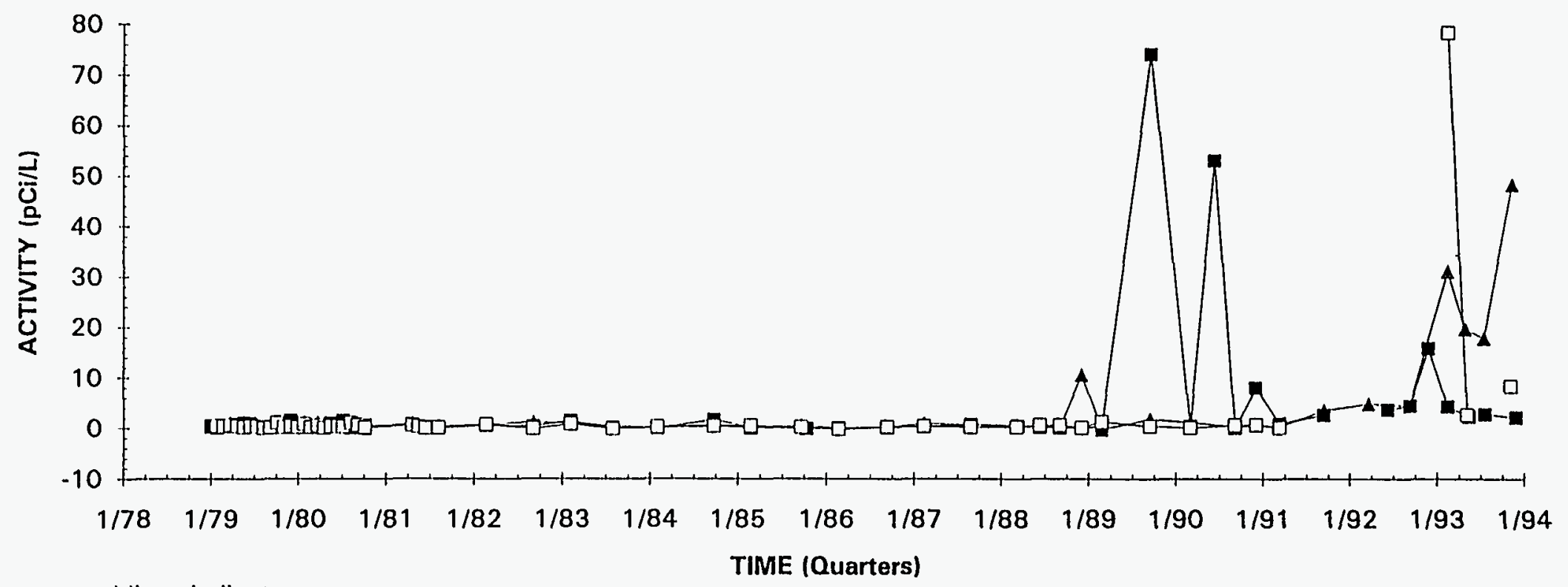

Unconnected lines indicate a dry well or a value below the detection limit.

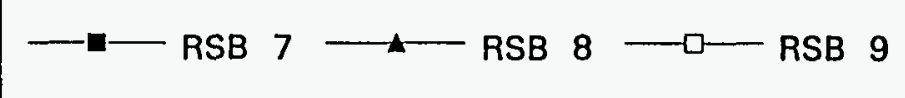

DWS: $15 \mathrm{pCi} / \mathrm{L}$ 


\section{Gross Alpha Activities RSC Well Series}

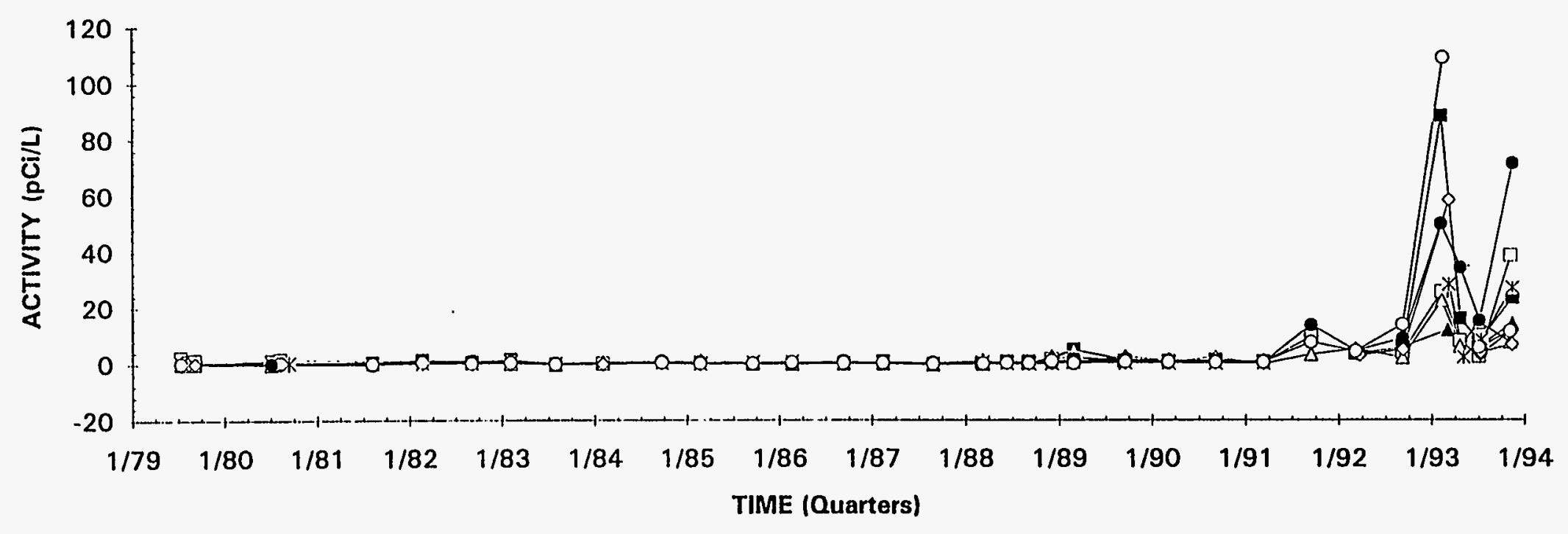

Unconnected lines indicate a dry well or a value below

the detection limit.

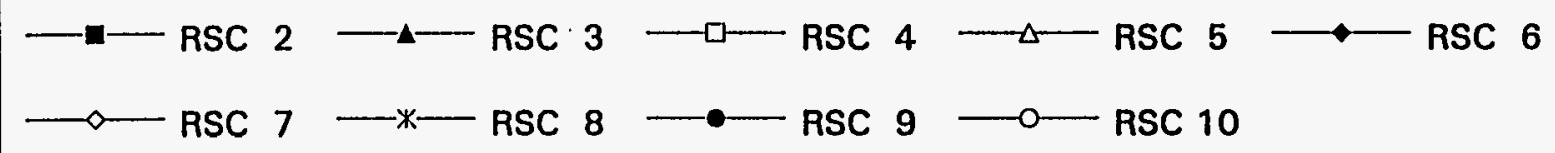

DWS: $15 \mathrm{pCi} / \mathrm{L}$ 


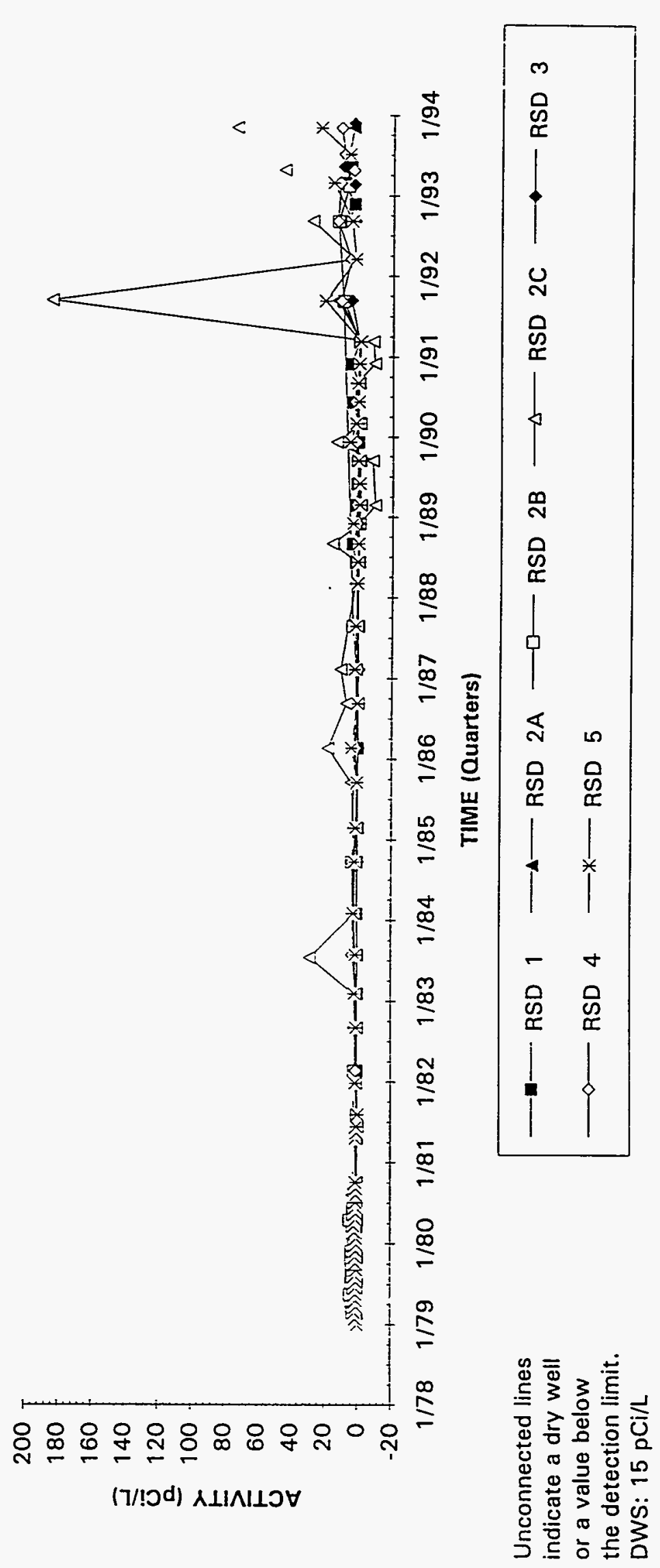




\section{Gross Alpha Activities \\ RSD Well Series (6 through 11)}

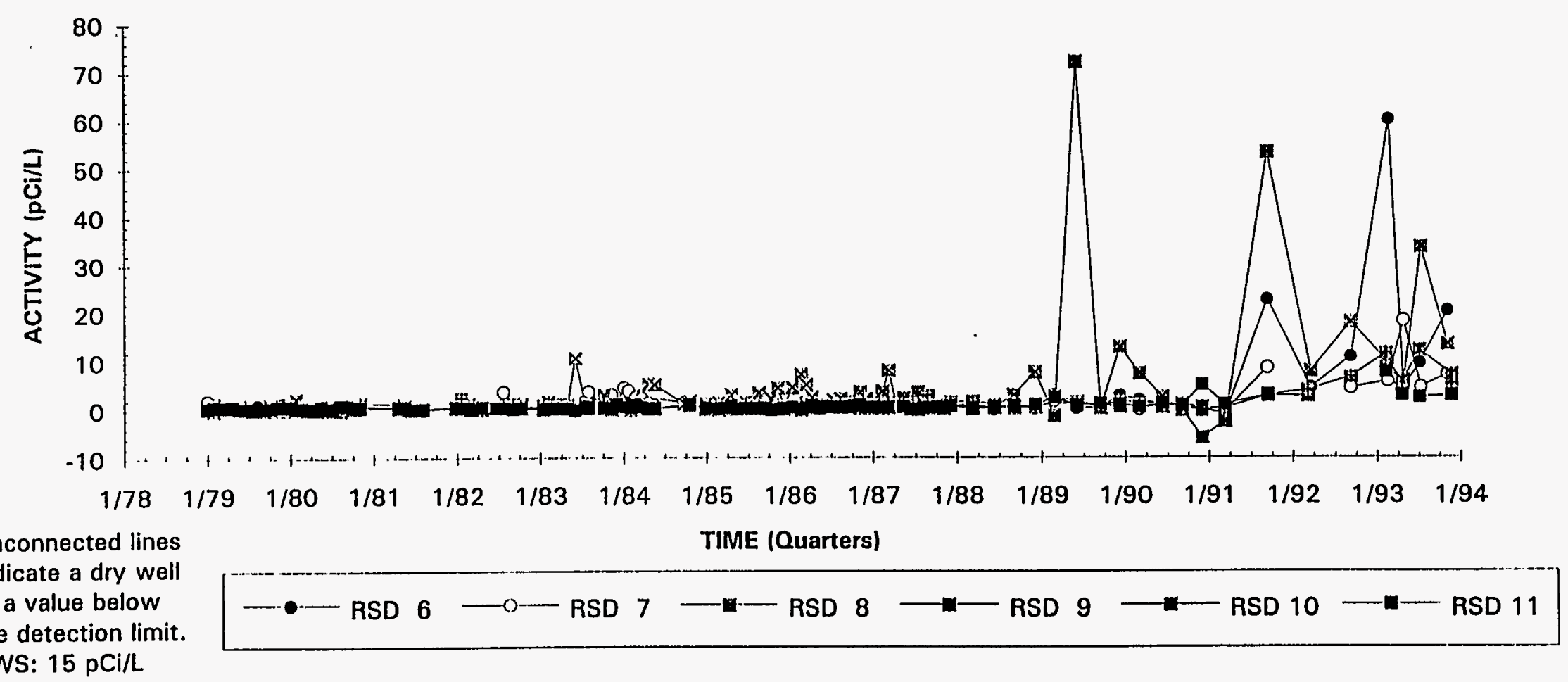



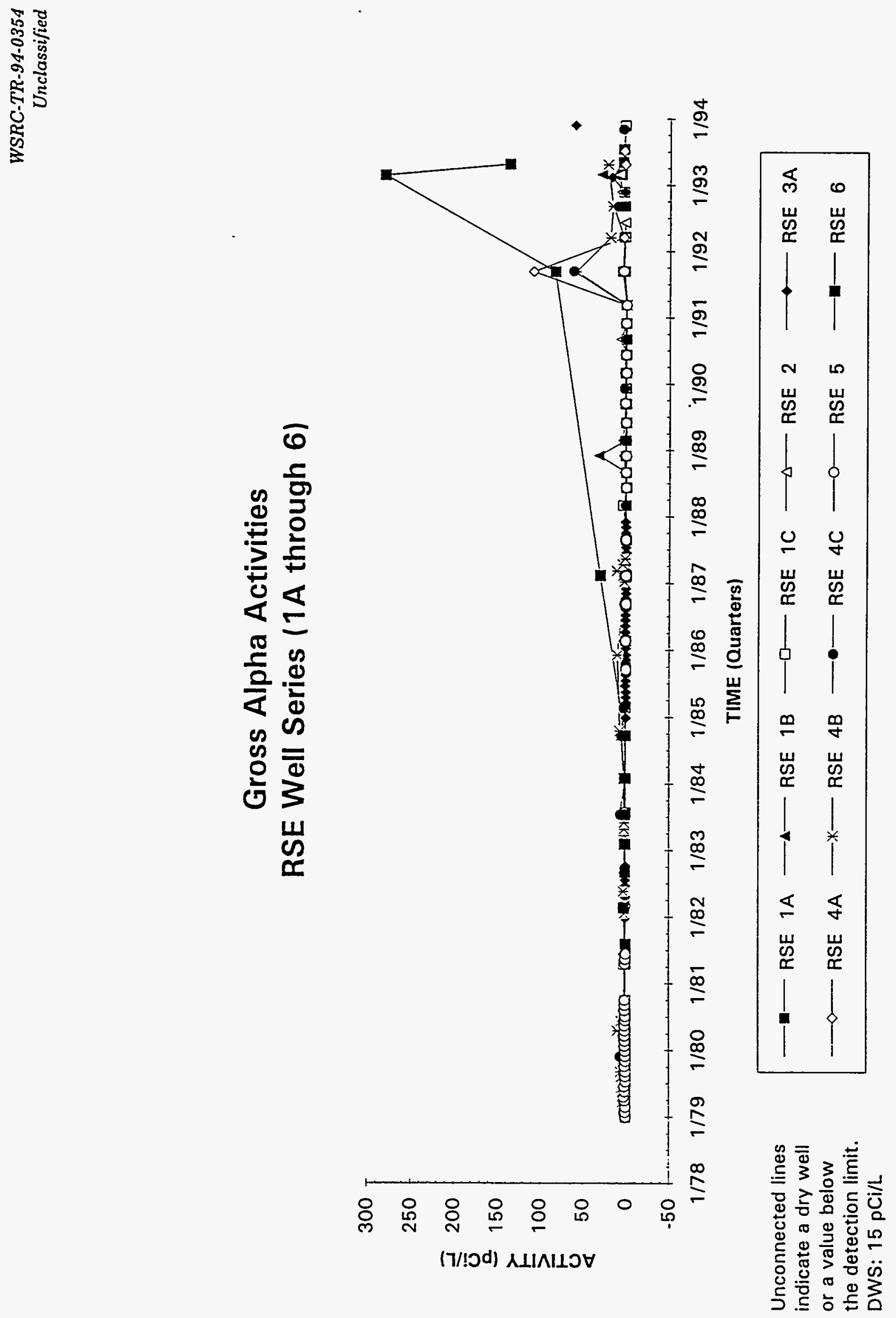


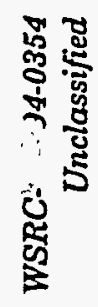
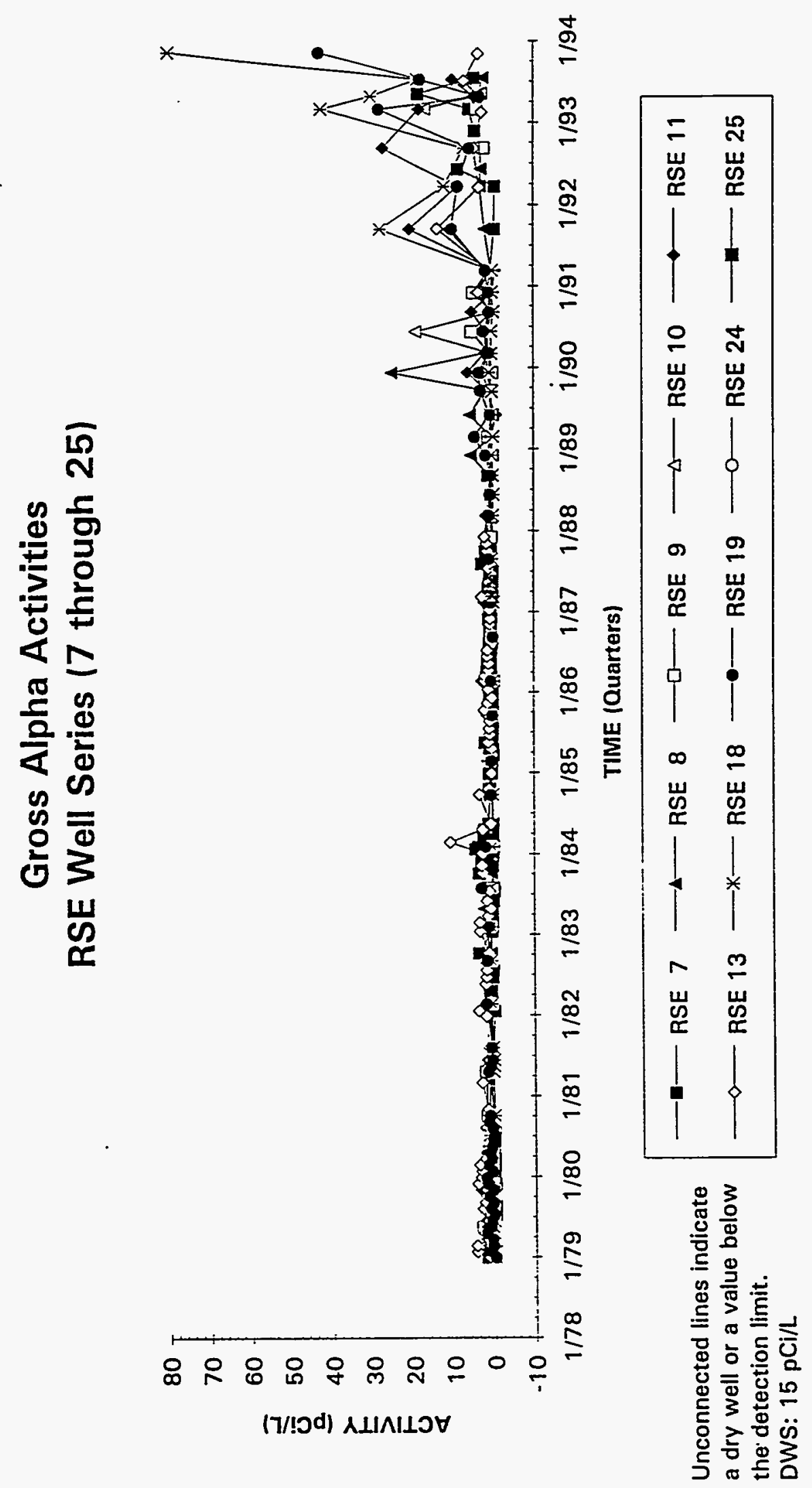


\section{Gross Alpha Activities}

RSF Well Series

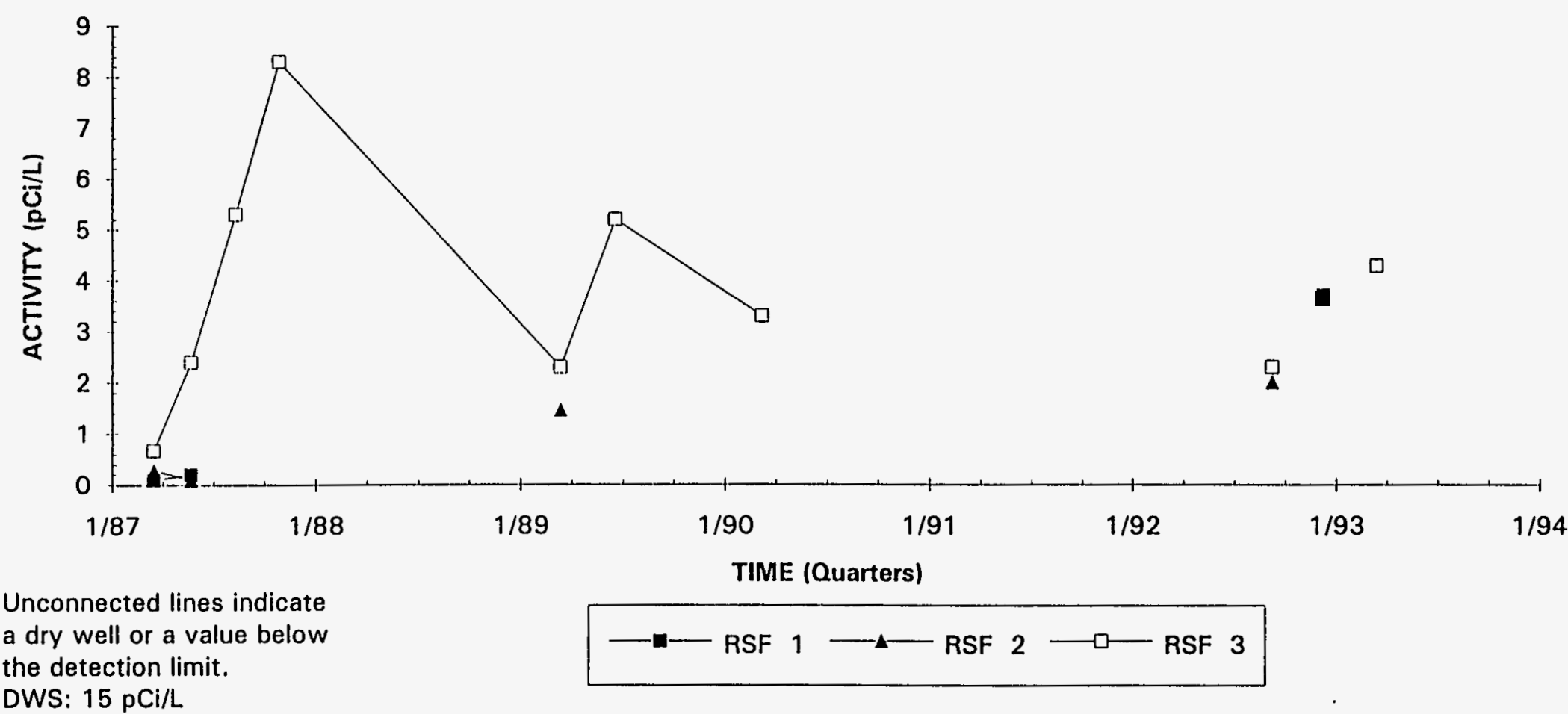



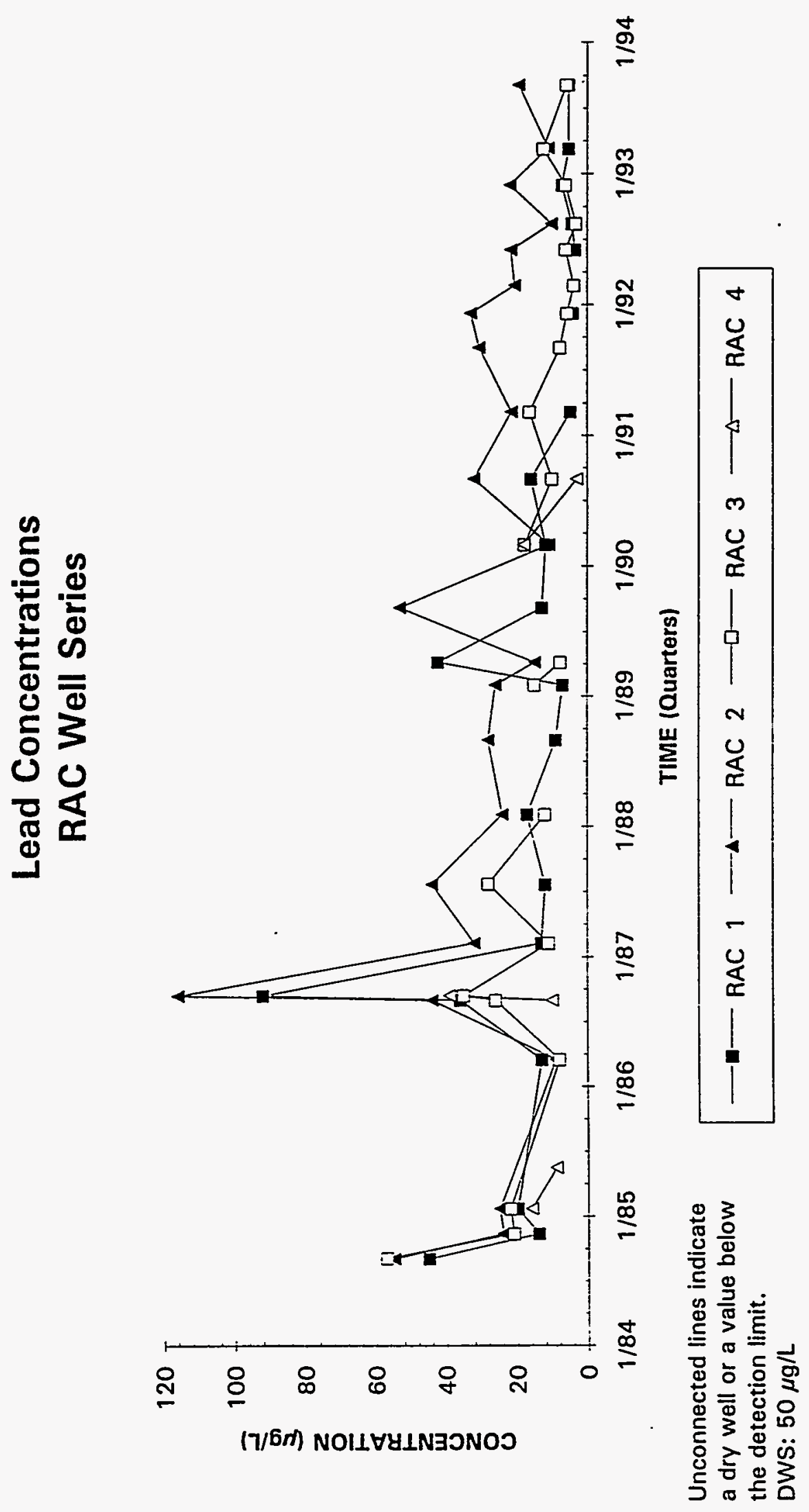

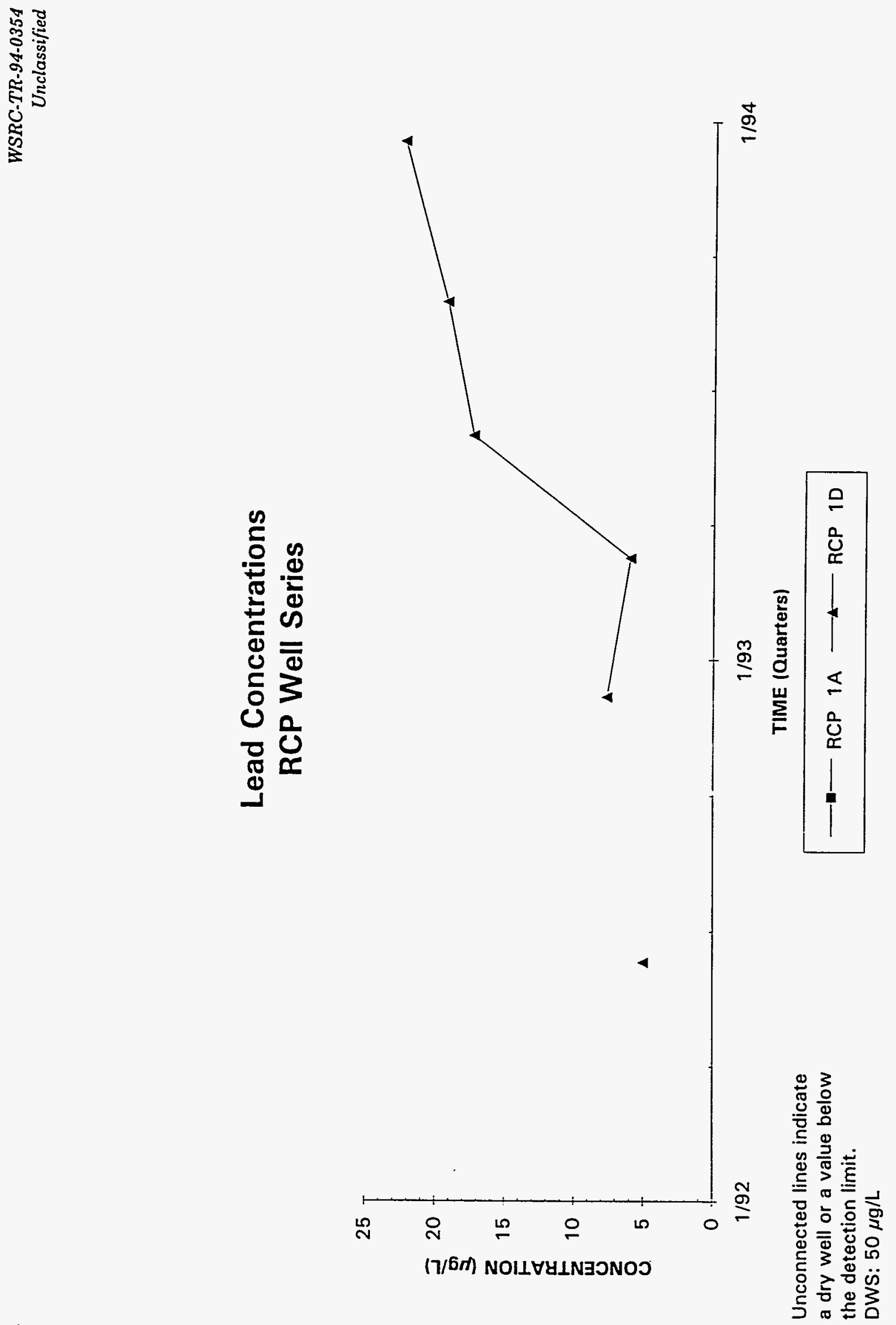


\section{Lead Concentrations RRP Well Series}

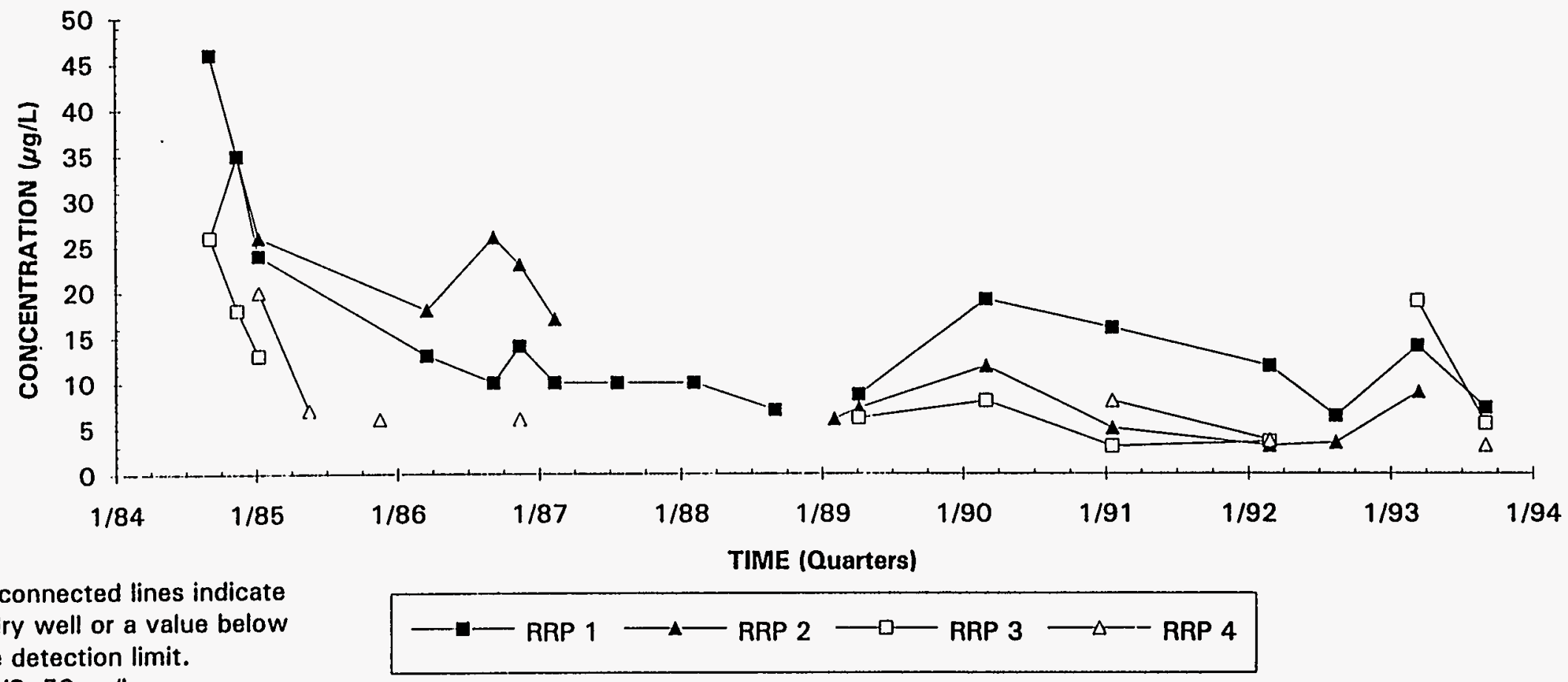

the detection limit.

DWS: $50 \mu \mathrm{g} / \mathrm{L}$ 

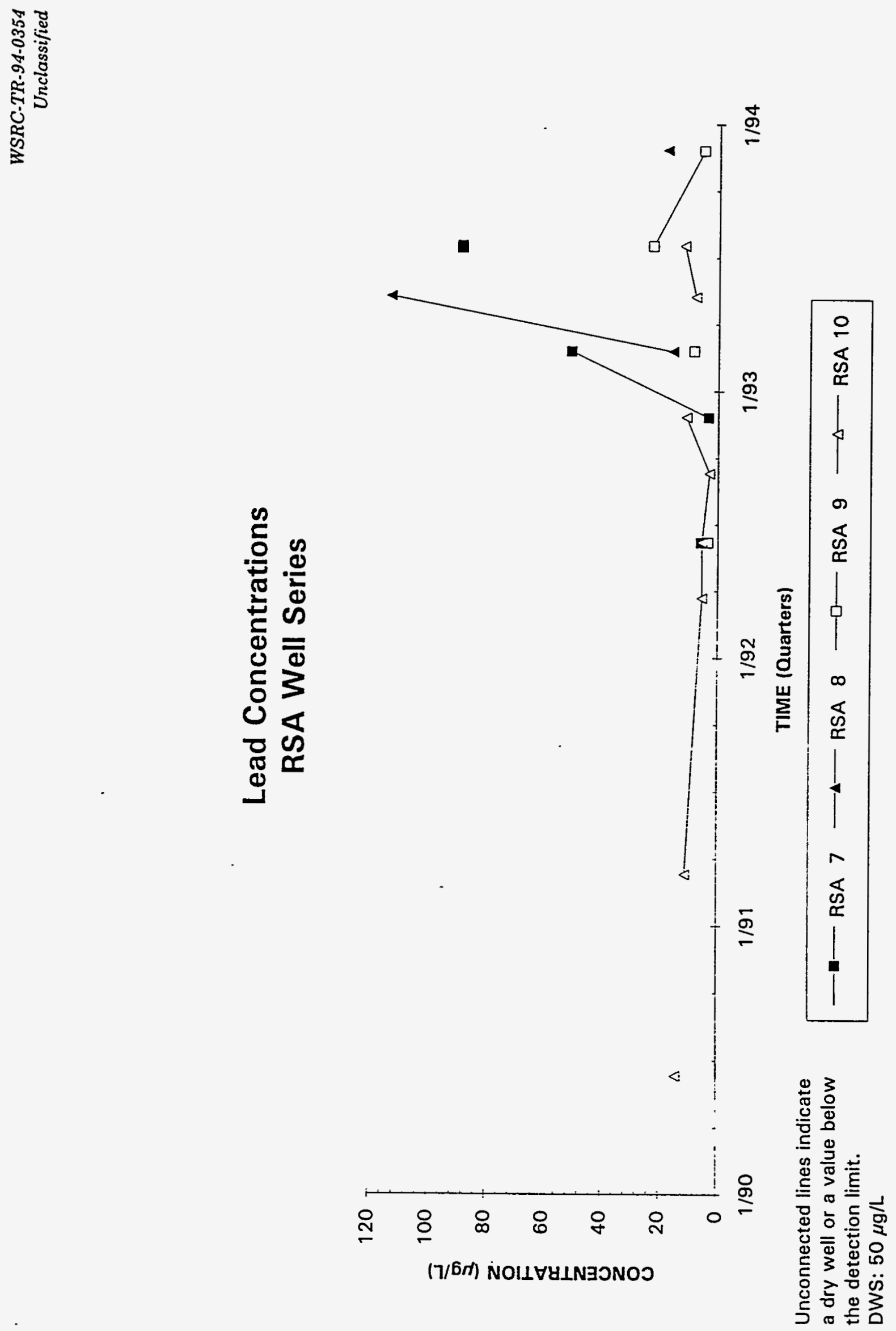


\section{Lead Concentrations RSB Well Series}

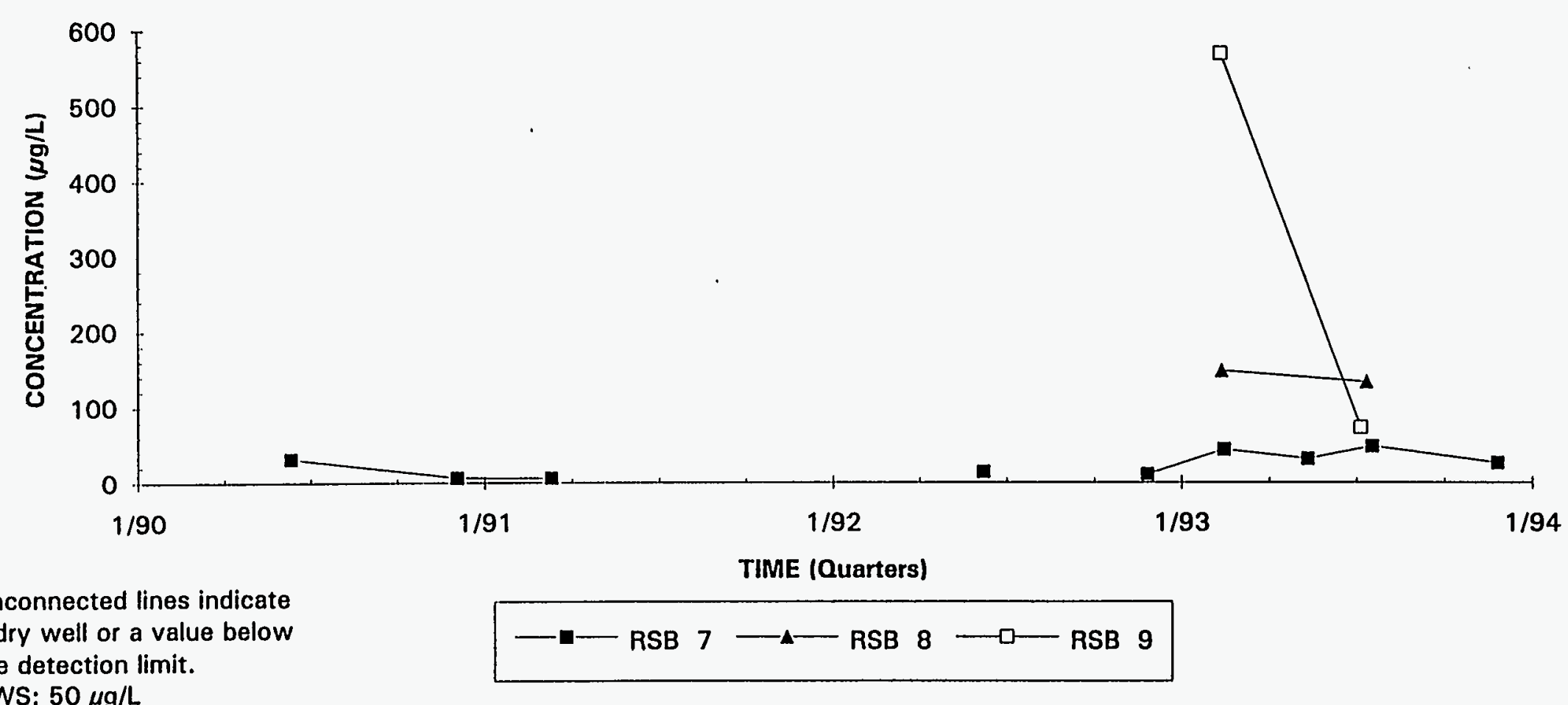

DWS: $50 \mu \mathrm{g} / \mathrm{L}$ 

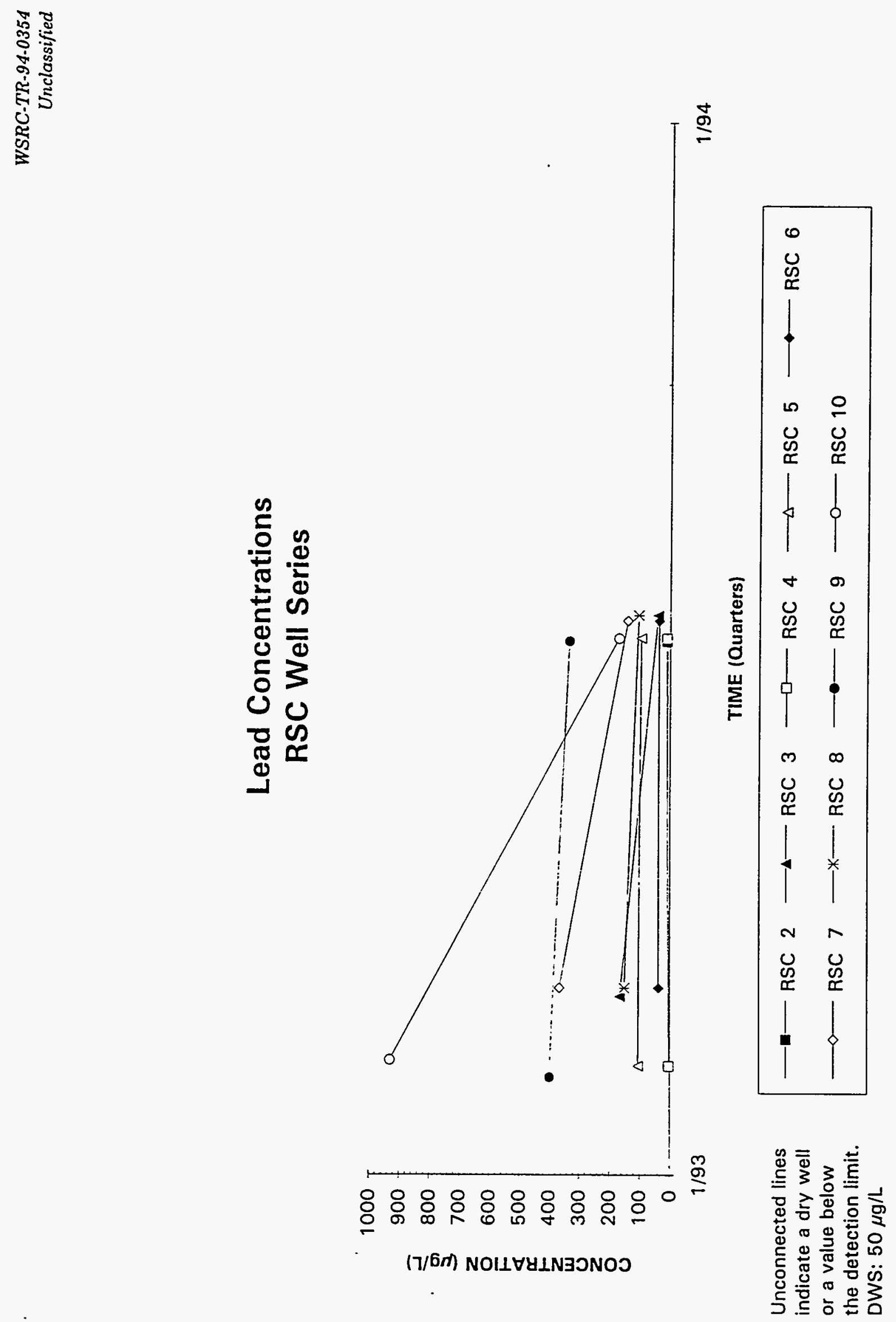

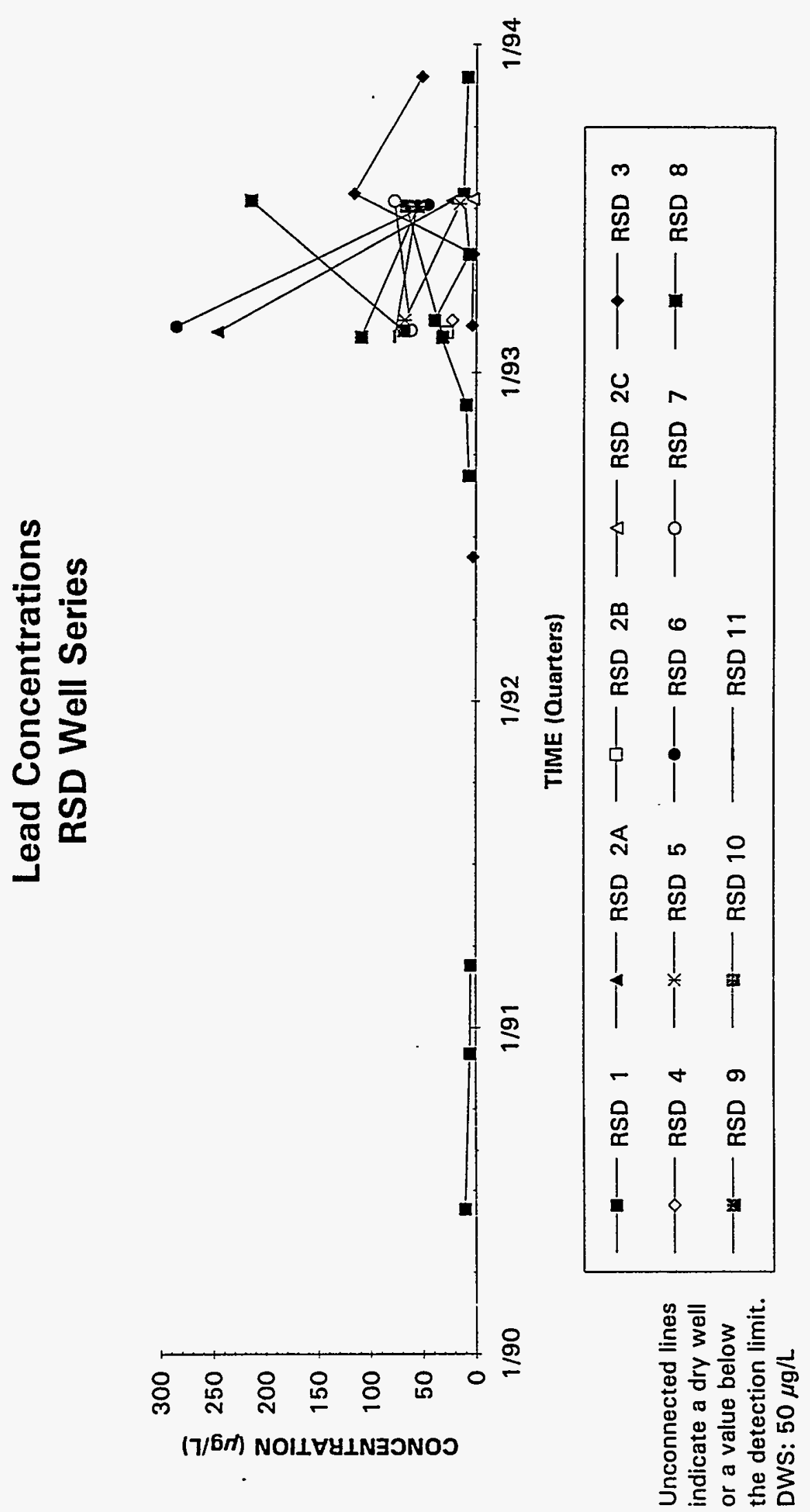


\section{Lead Concentrations \\ RSE Well Series (1A through 6)}

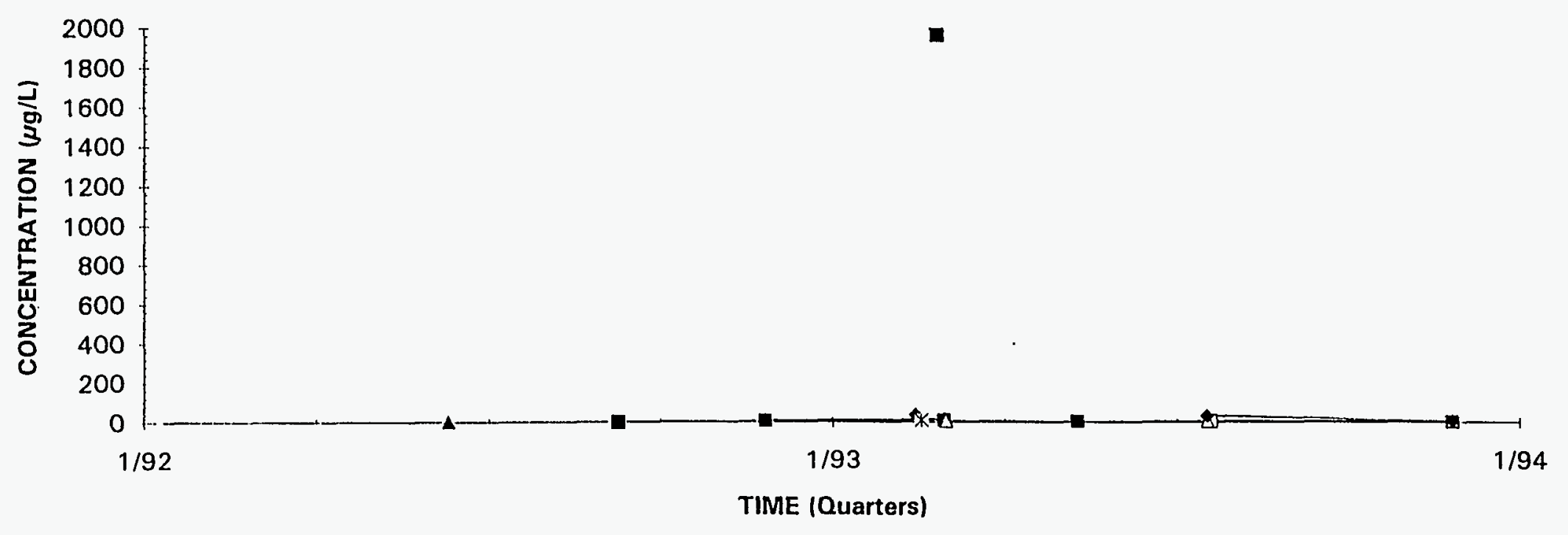

Unconnected lines indicate a dry well or a value below the detection limit. DWS: $50 \mu \mathrm{g} / \mathrm{L}$

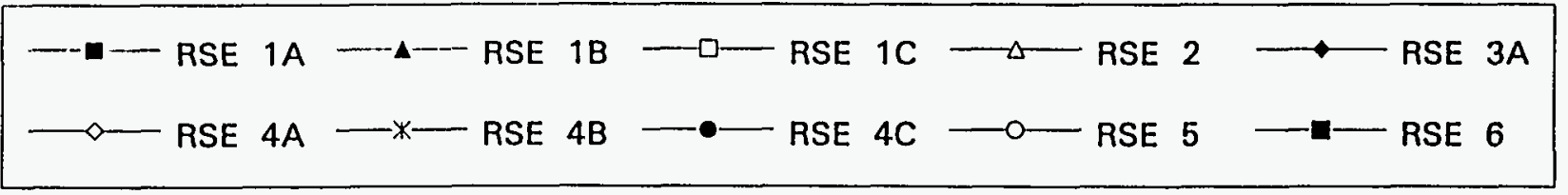




\section{Lead Concentrations \\ RSE Well Series (7 through 25)}

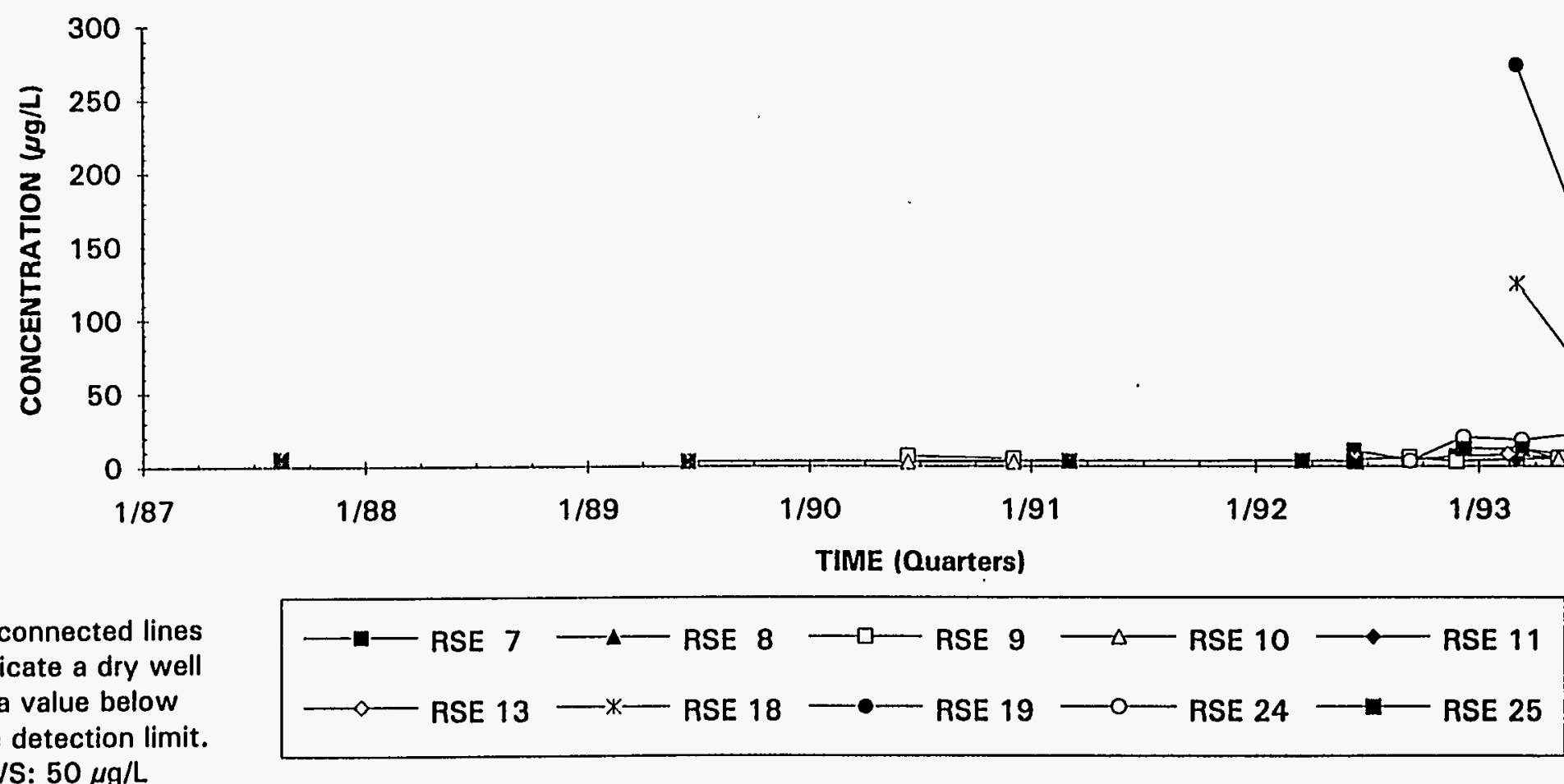

detection

DWS: $50 \mu \mathrm{g} / \mathrm{L}$ 


\section{Mercury Concentrations \\ RCP Well Series}

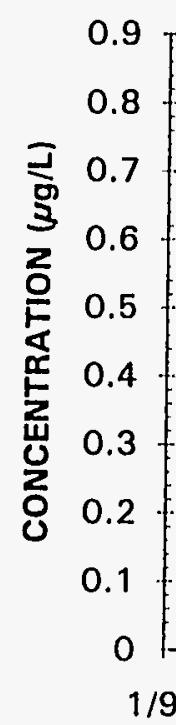

Unconnected lines indicate a dry well or a value below the detection limit. DWS: $2 \mu \mathrm{g} / \mathrm{L}$

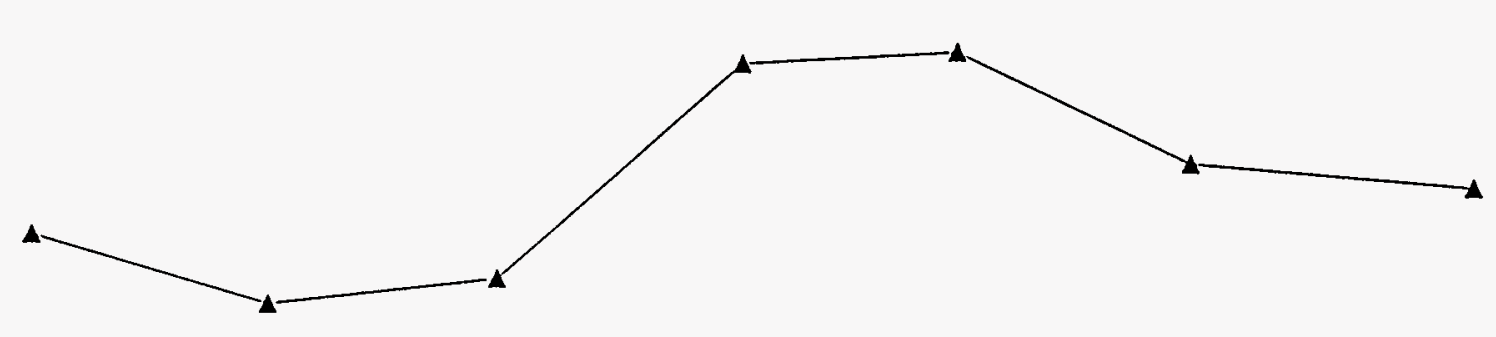

193

TIME (Quarters)

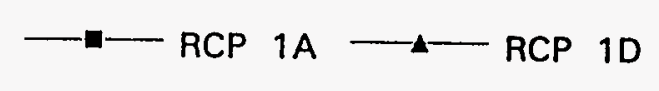




\section{Mercury Concentrations}

RSA Well Series

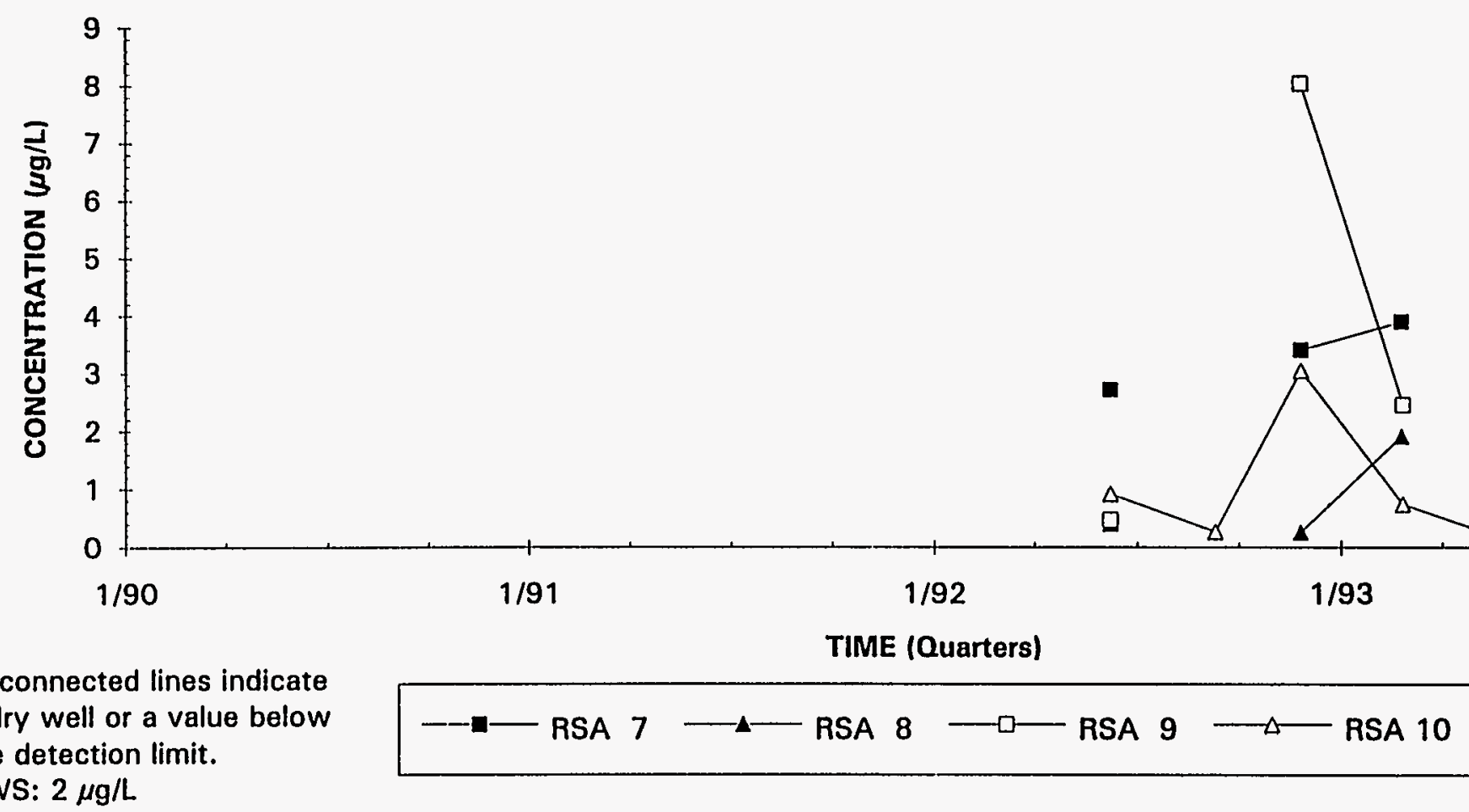

R-Area Reactor 1993 Annual Report 


\section{Mercury Concentrations}

RSB Well Series

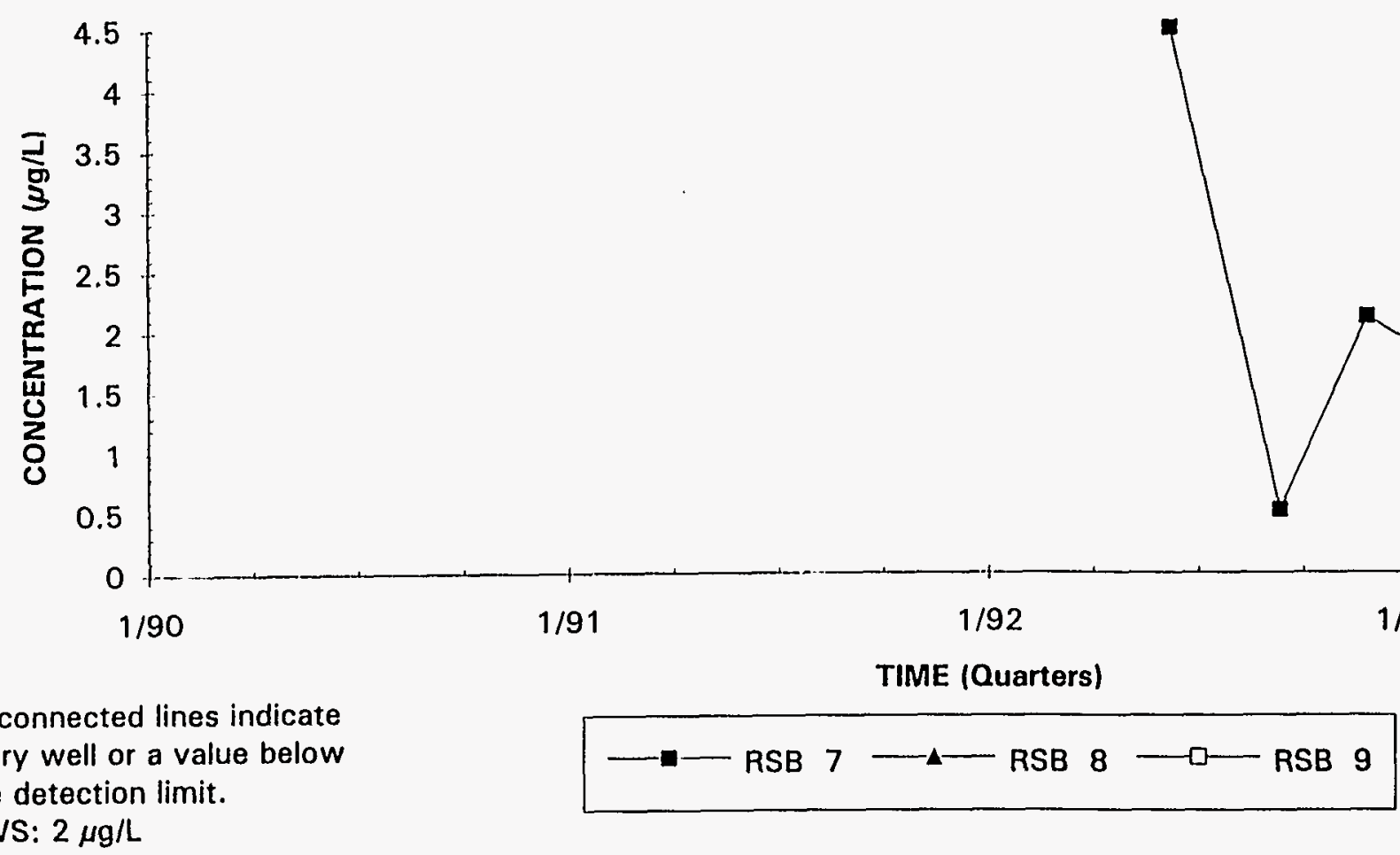




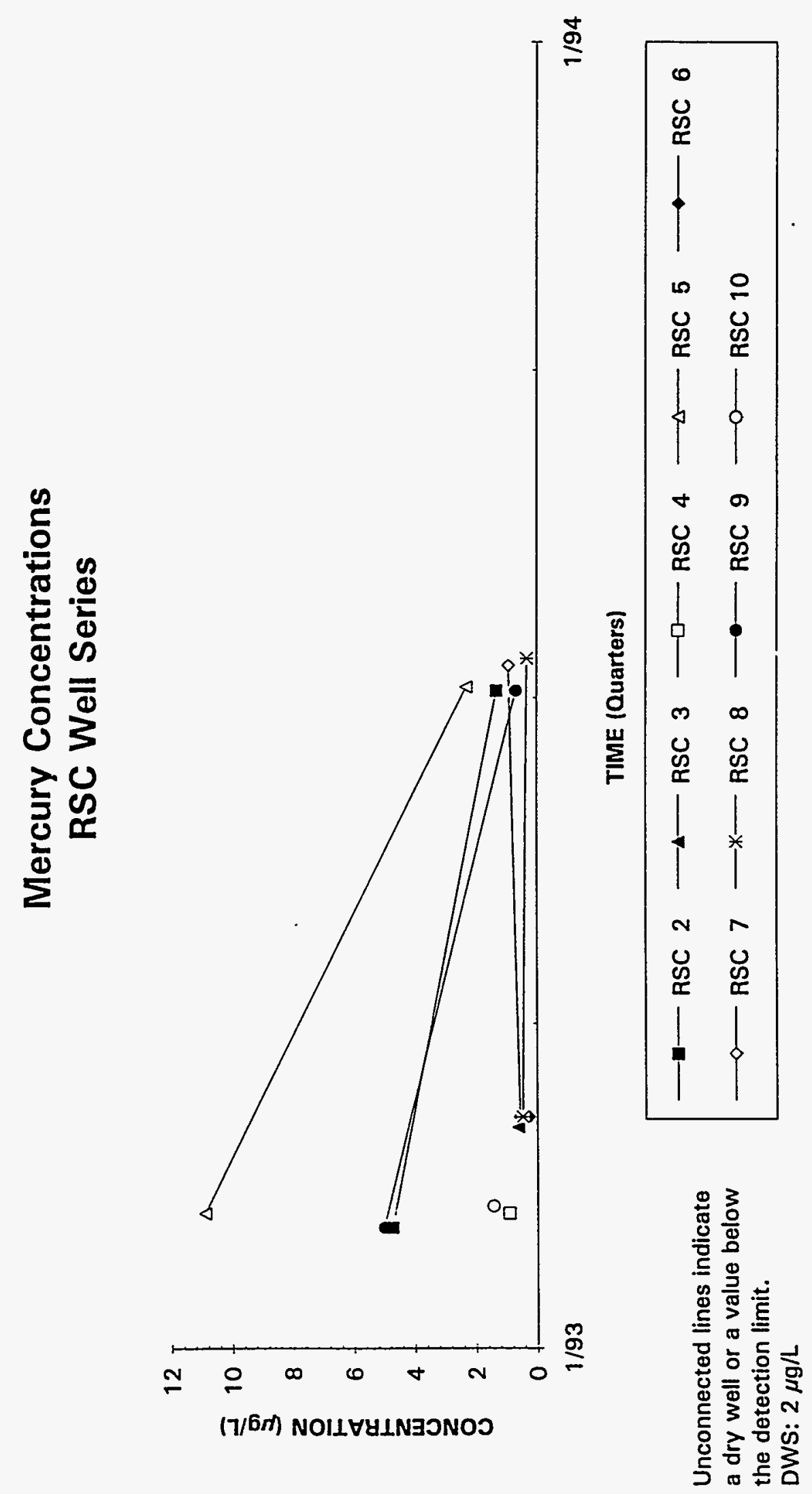



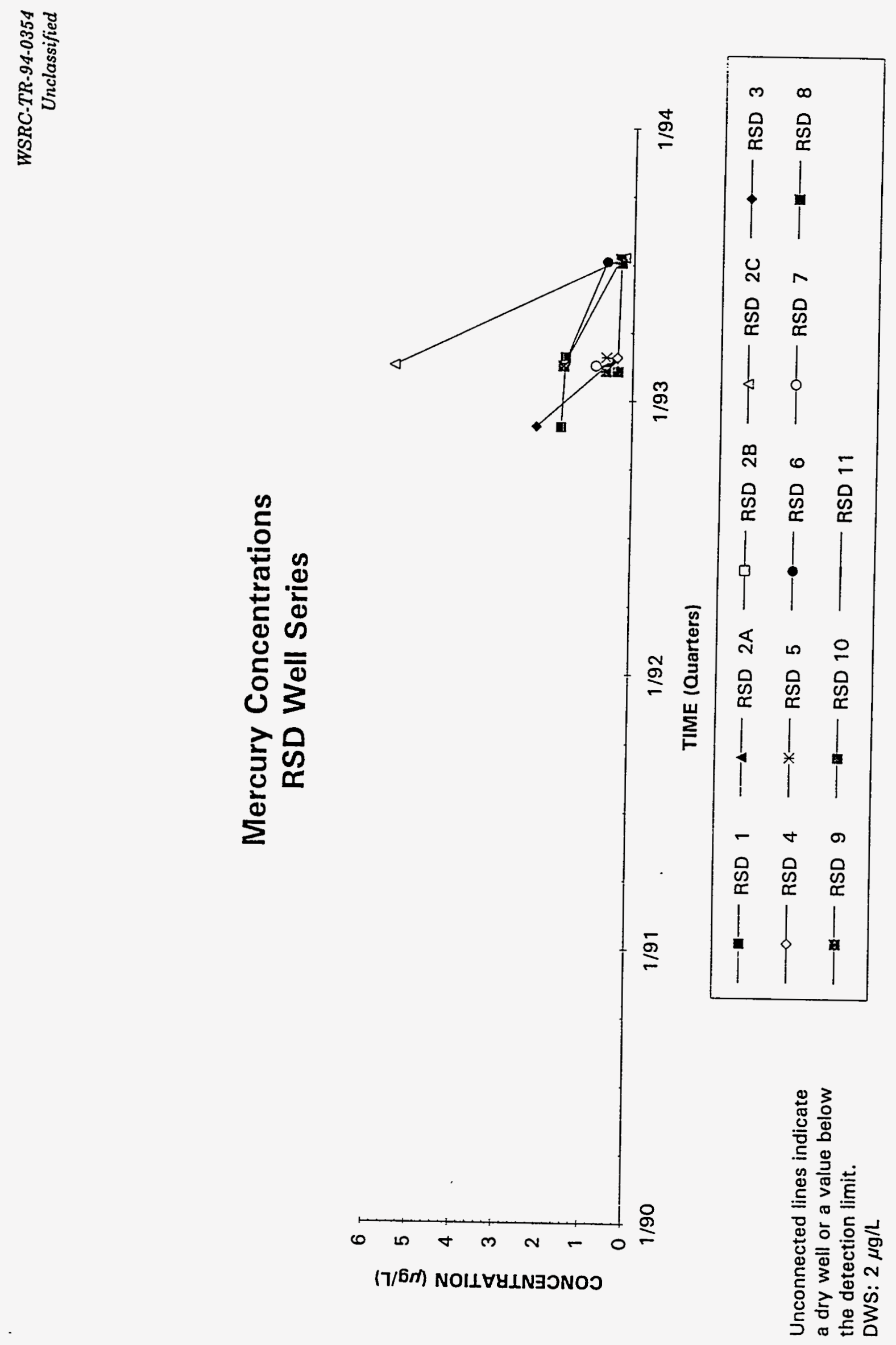


\section{Mercury Concentrations \\ RSE Well Series (1A through 6)}

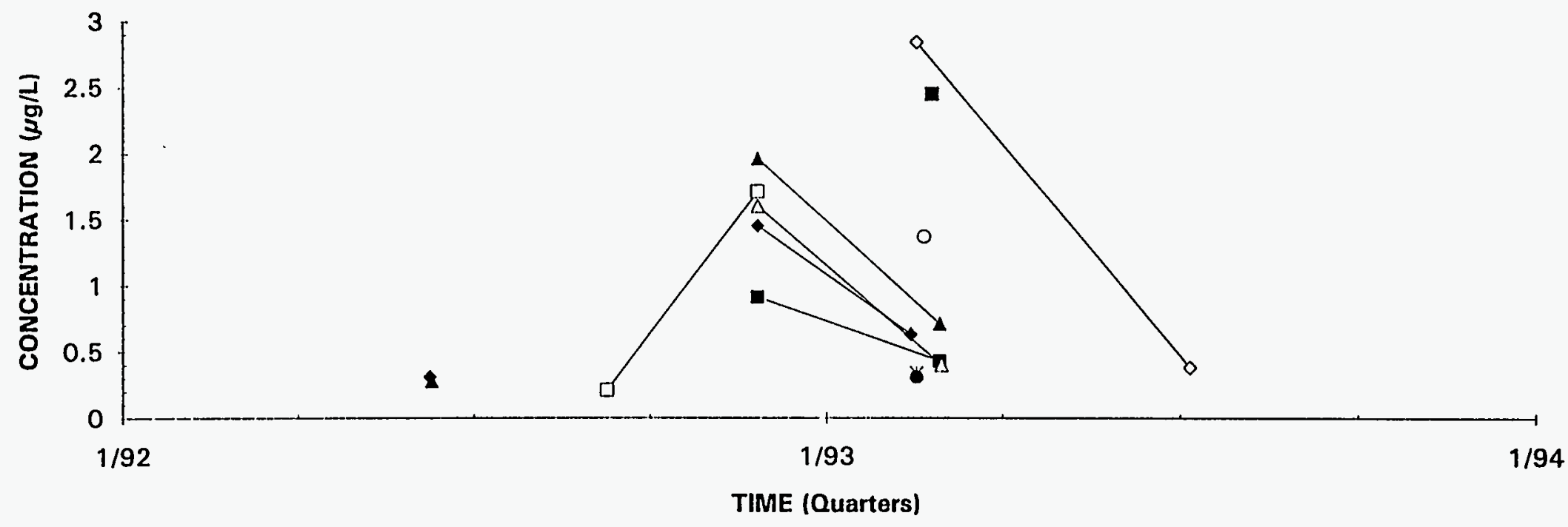

Unconnected lines indicate a dry well or a value below the detection limit. DWS: $2 \mu \mathrm{g} / \mathrm{L}$ 

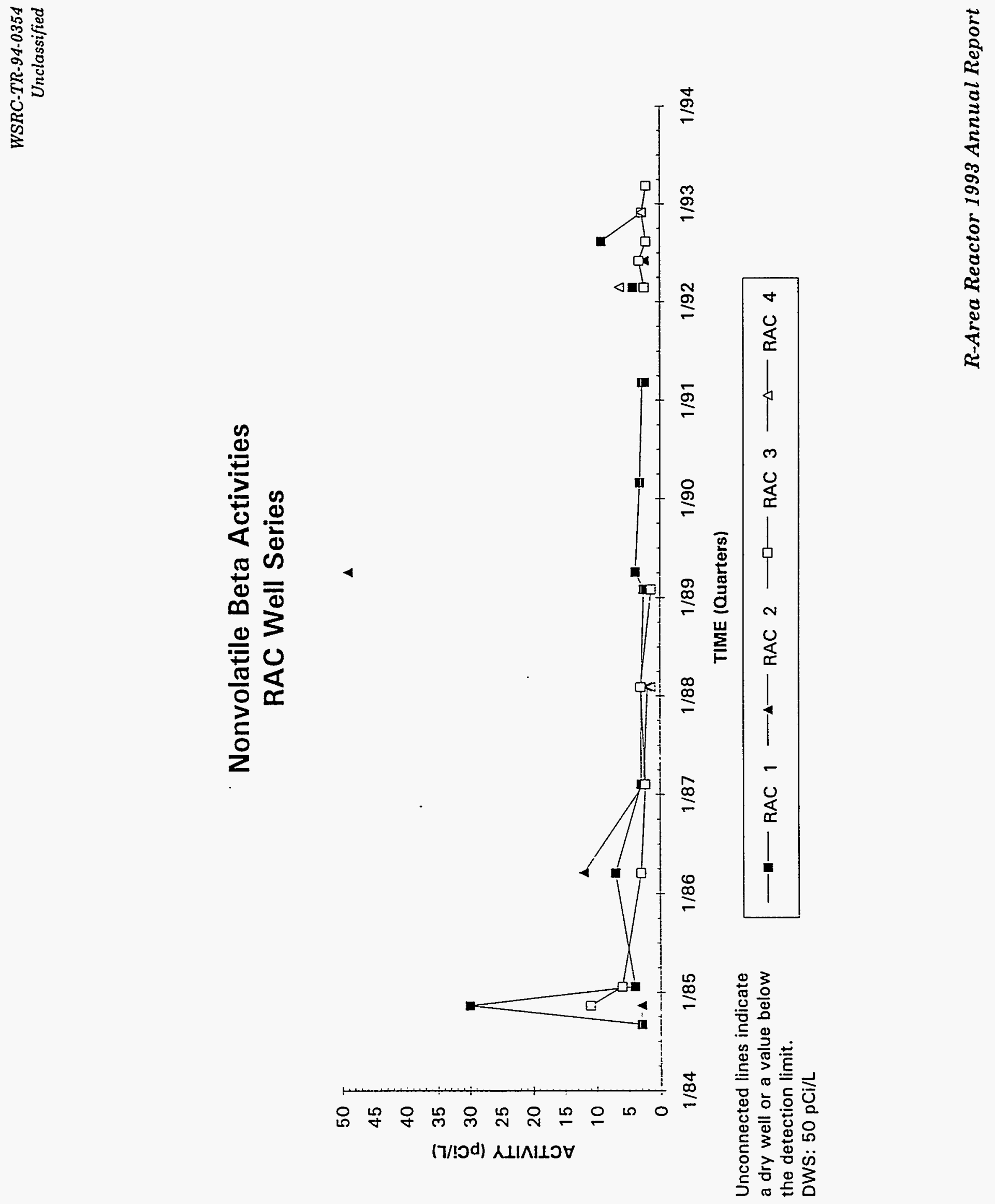


\section{Nonvolatile Beta Activities \\ RDB Well Series}

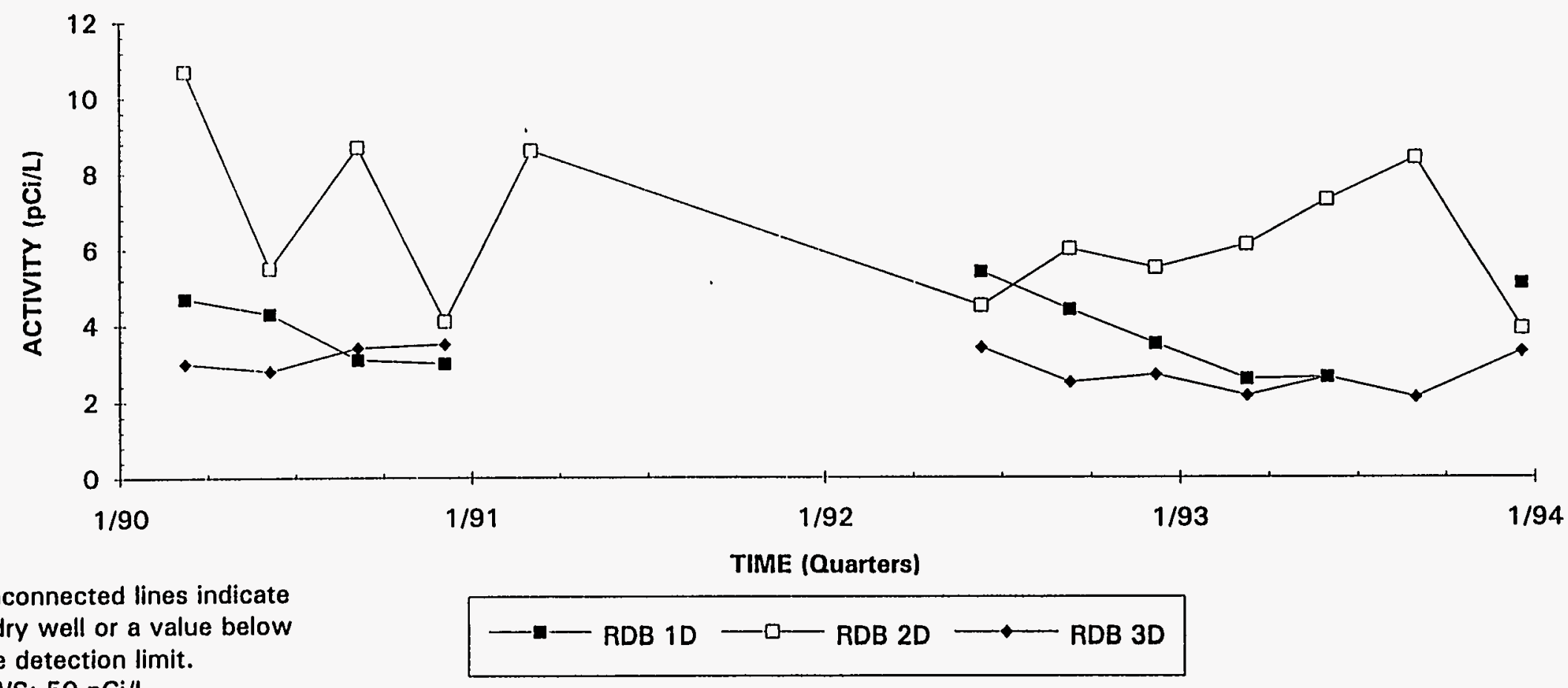

DWS: $50 \mathrm{pCi} / \mathrm{L}$ 

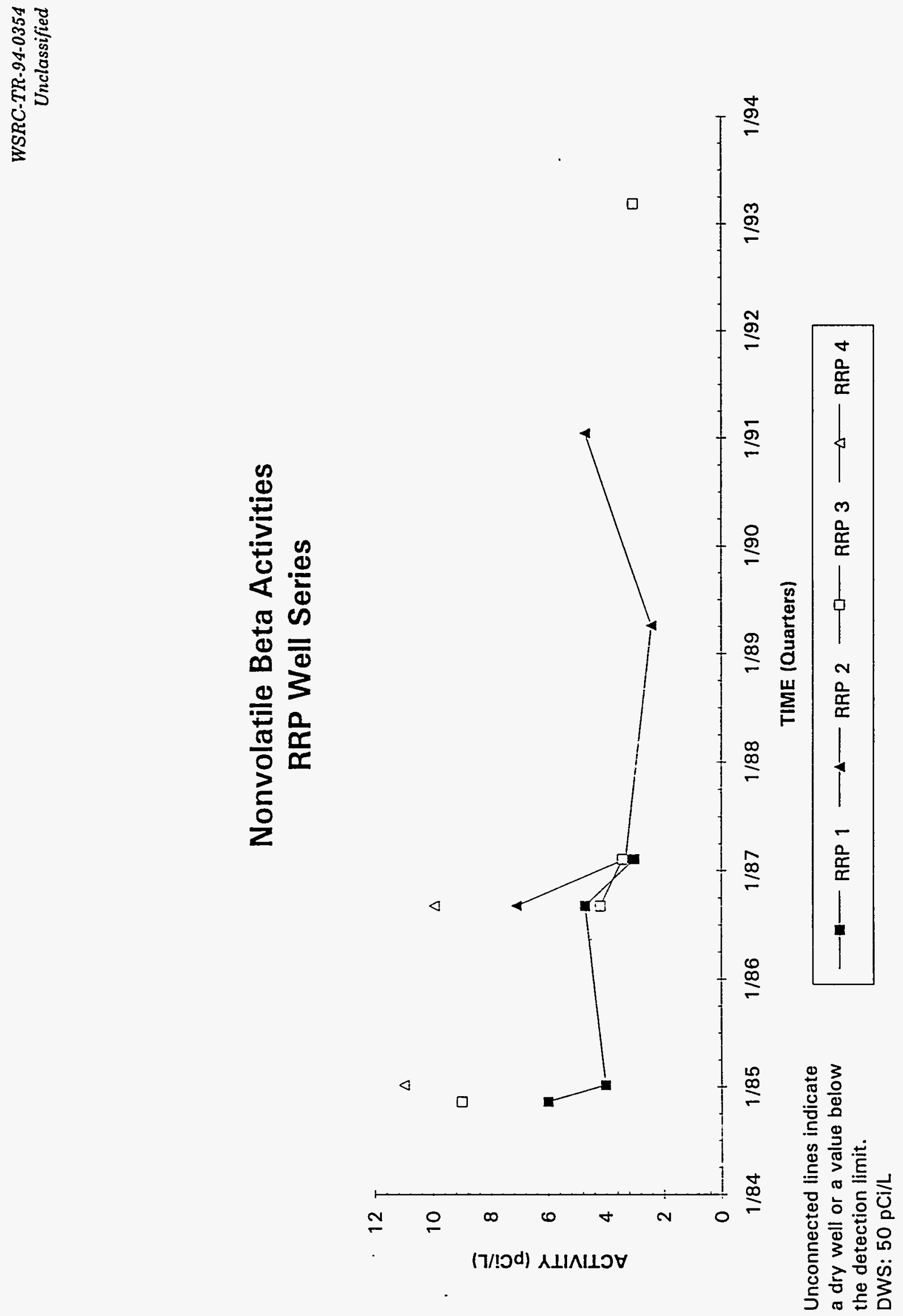


\section{Nonvolatile Beta Activities}

RSA Well Series

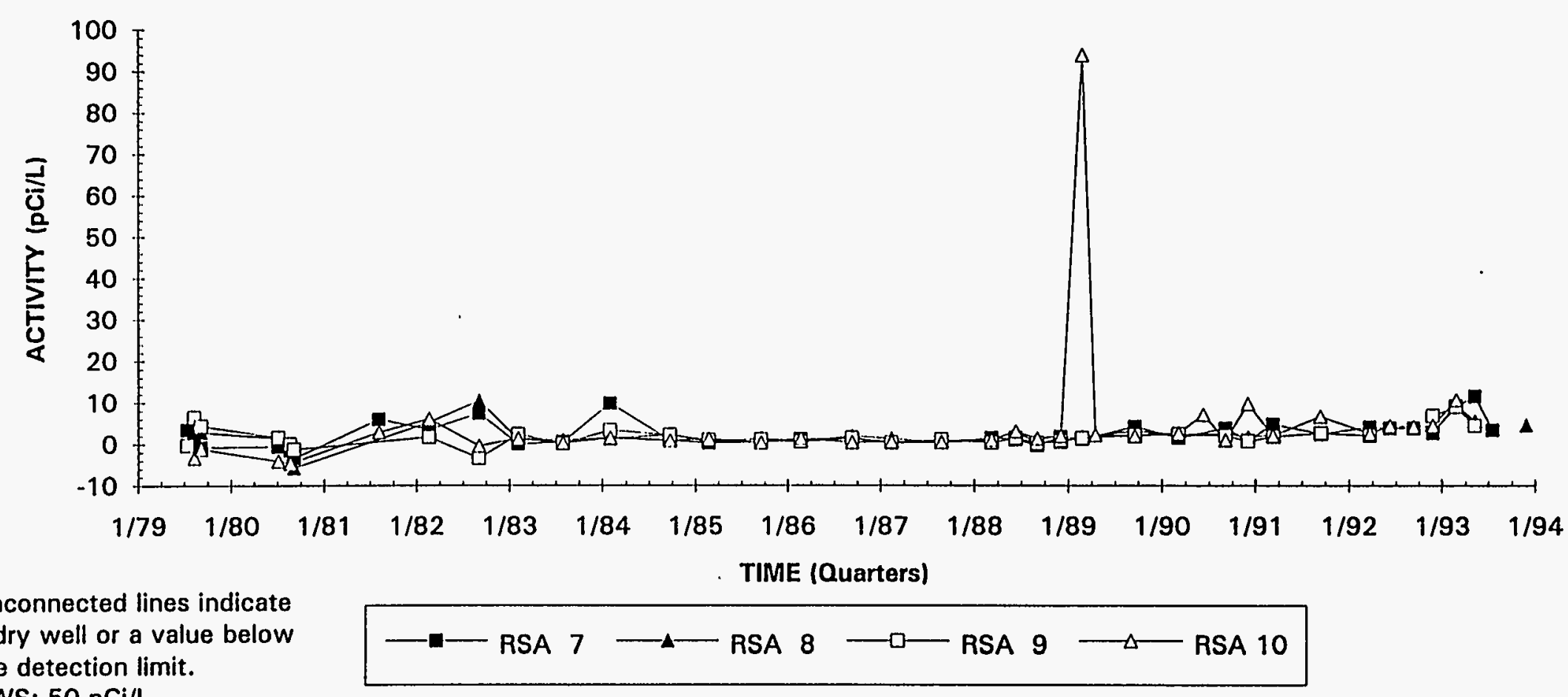

DWS: $50 \mathrm{pCi} / \mathrm{L}$ 


\section{Nonvolatile Beta Activities \\ RSB Well Series}

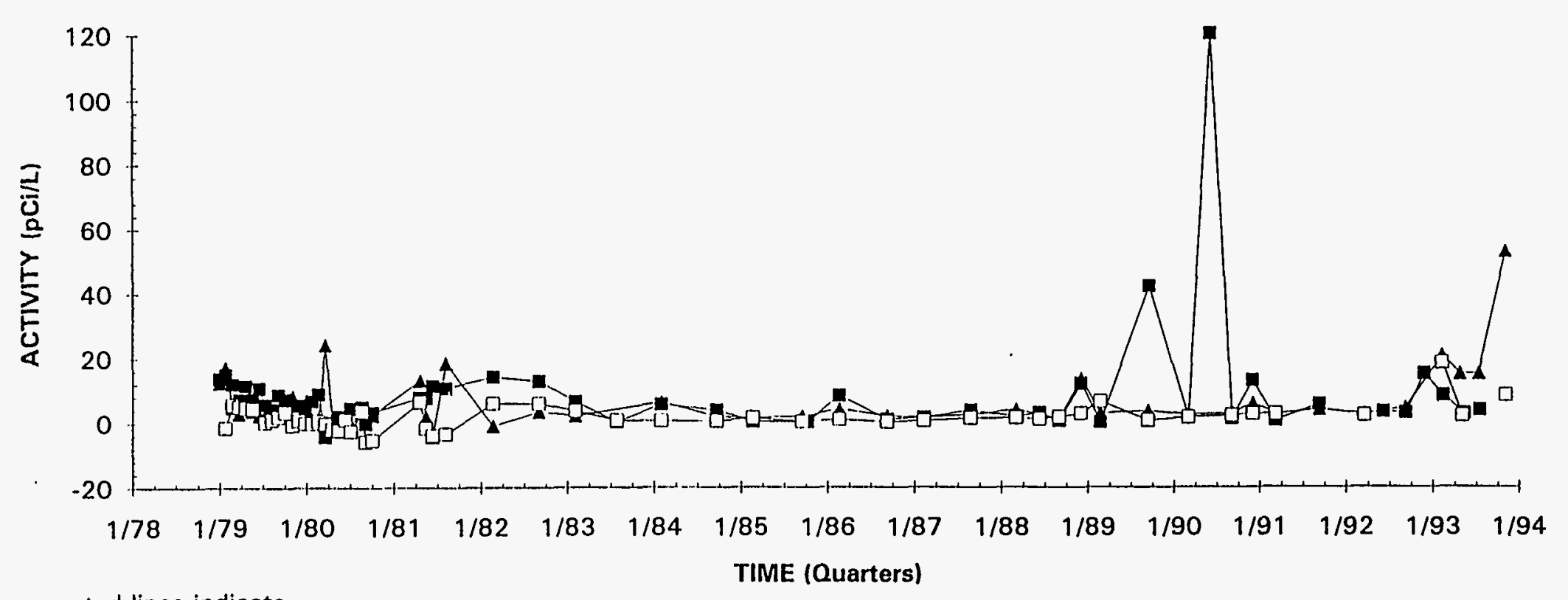

Unconnected lines indicate a dry well or a value below the detection limit.

$$
\text { - RSB } 7-\text { RSB } 8-\square-R S B 9
$$




\section{Nonvolatile Beta Activities \\ RSC Well Series (2 through 5)}

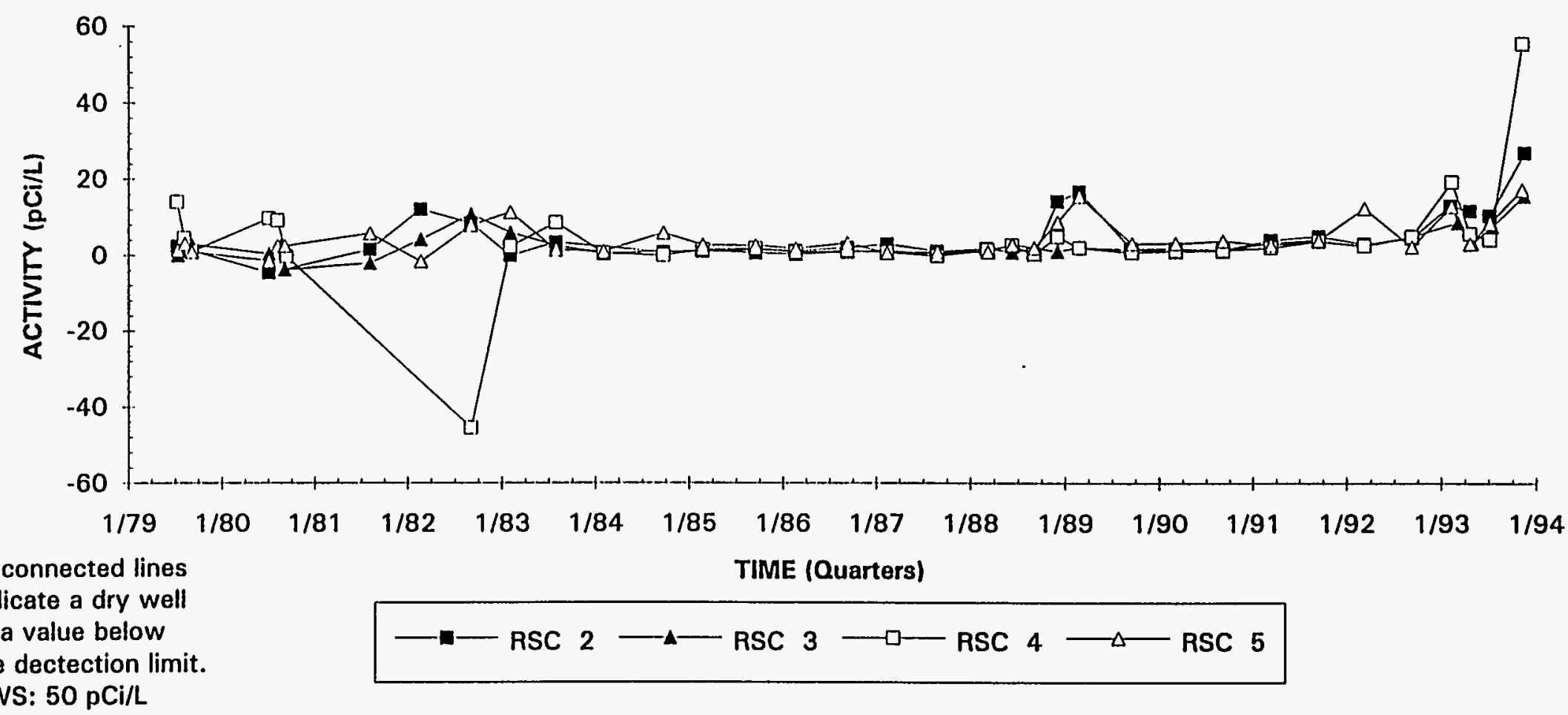




\section{Nonvolatile Beta Activities RSC Well Series (6 through 10)}

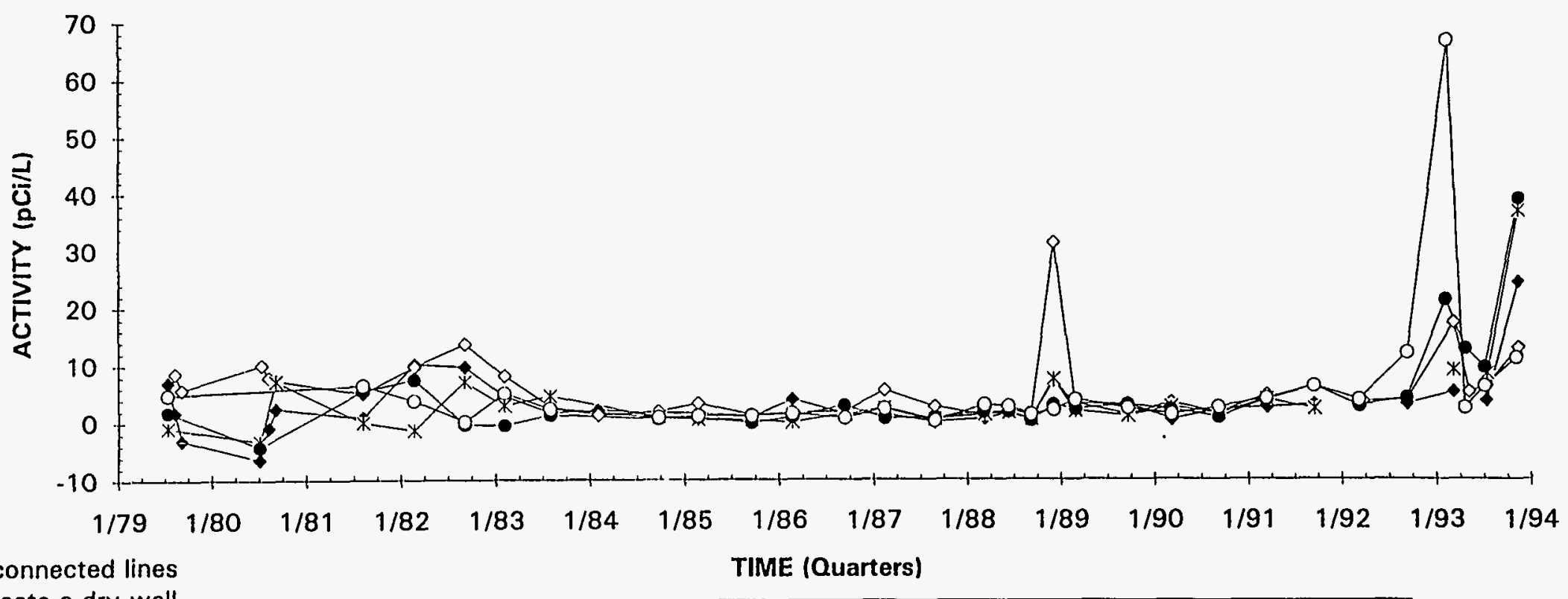

indicate a dry well or a value below the dectection limit. DWS: $50 \mathrm{pCi} / \mathrm{L}$ 


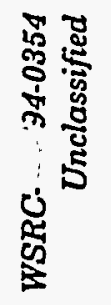

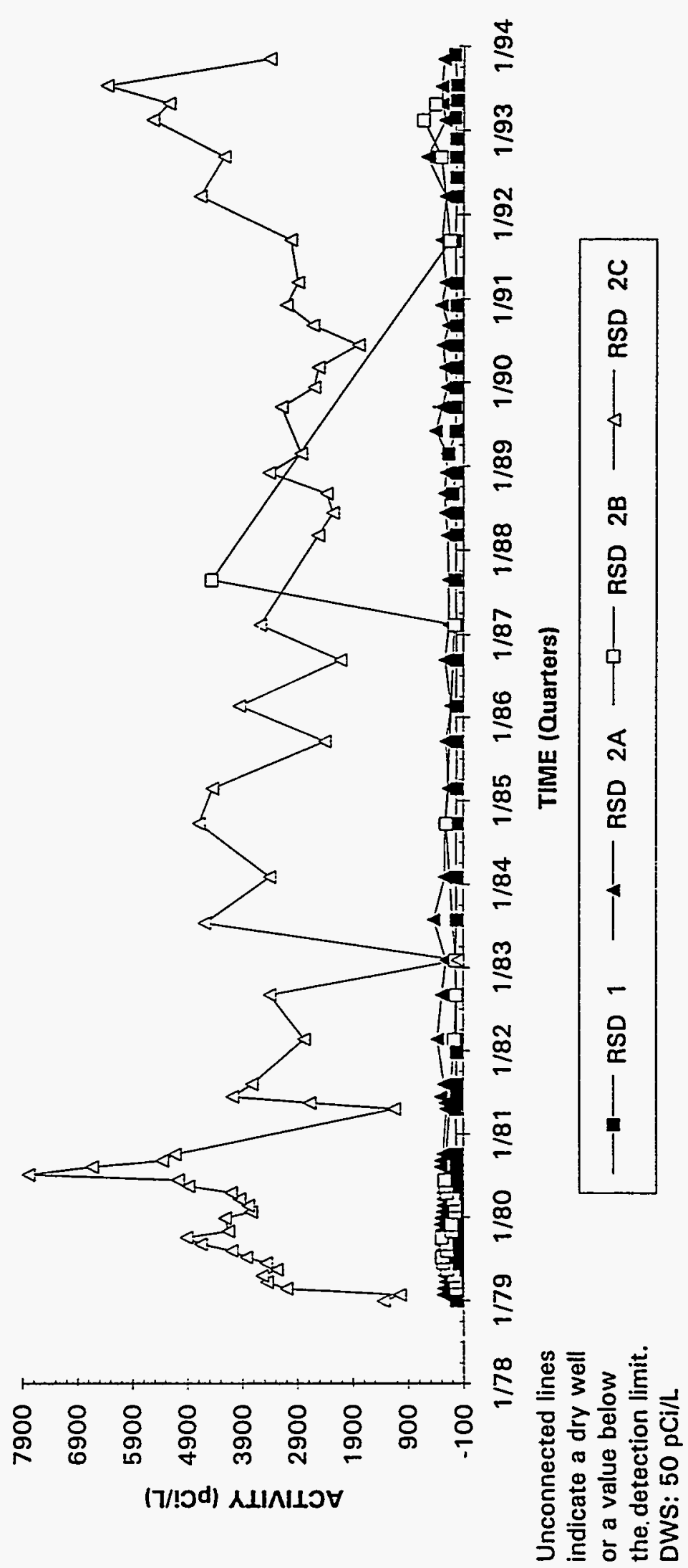




\section{Nonvolatile Beta Activities \\ RSD Well Series (3 through 5)}

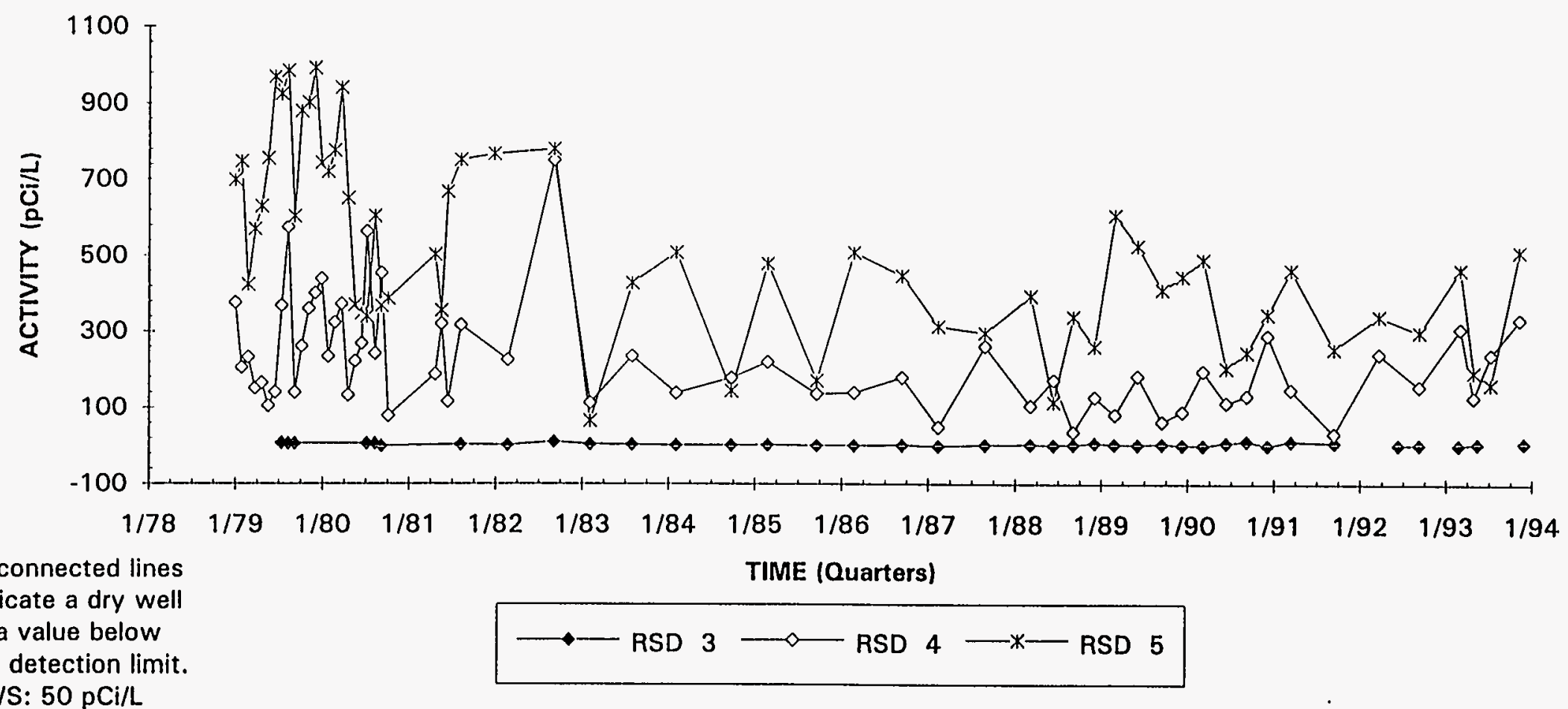



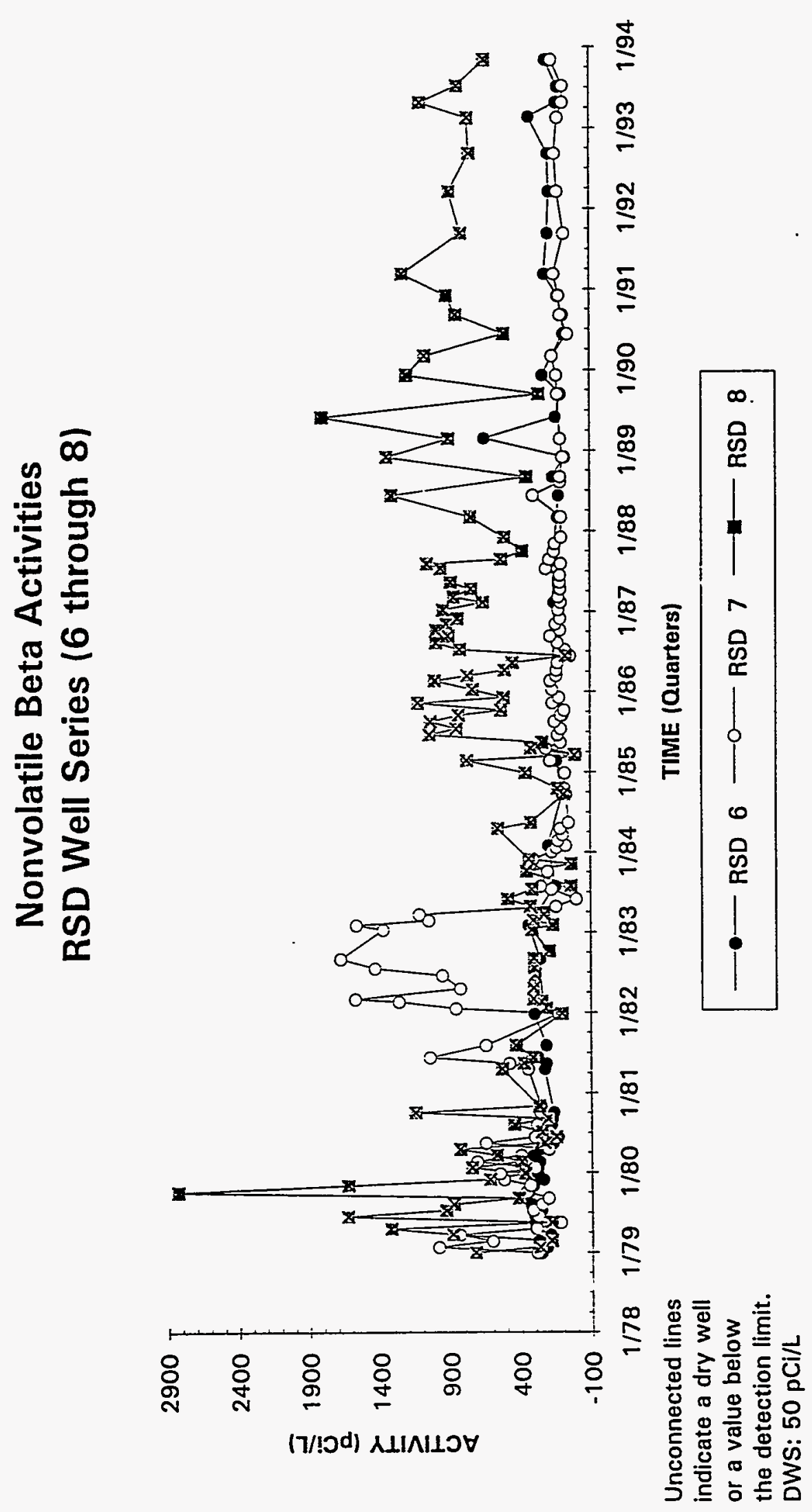


\section{Nonvolatile Beta Activities}

\section{RSD Well Series (9 through 11)}

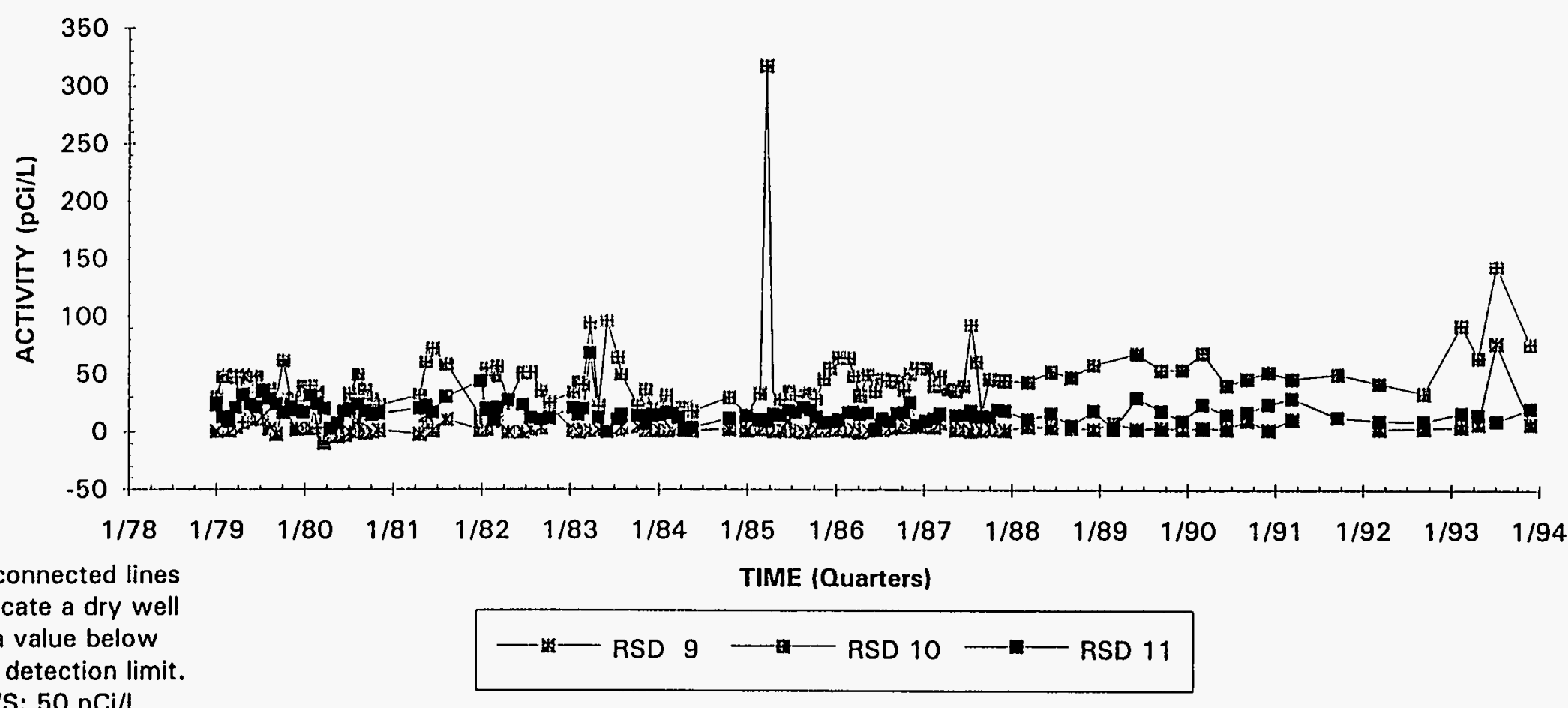




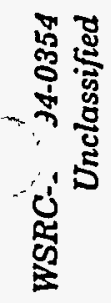

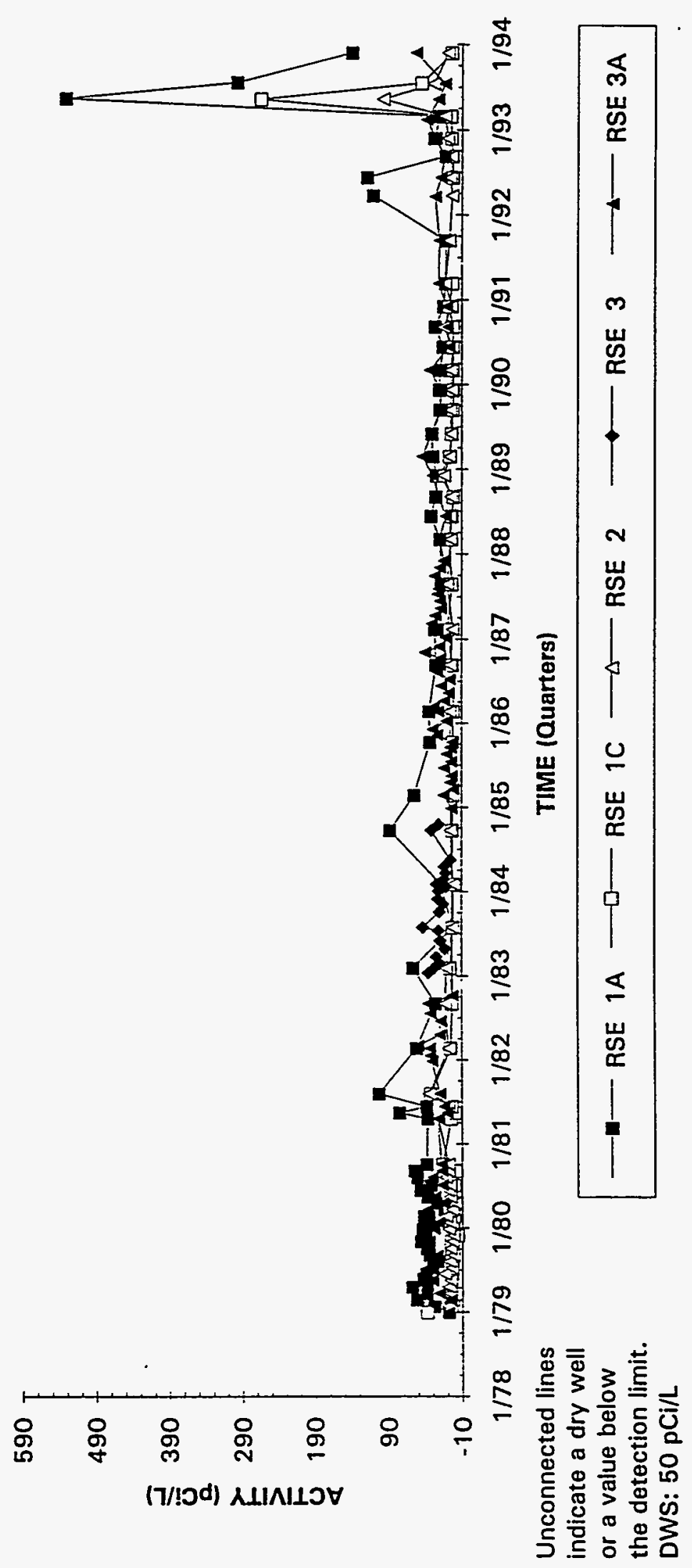



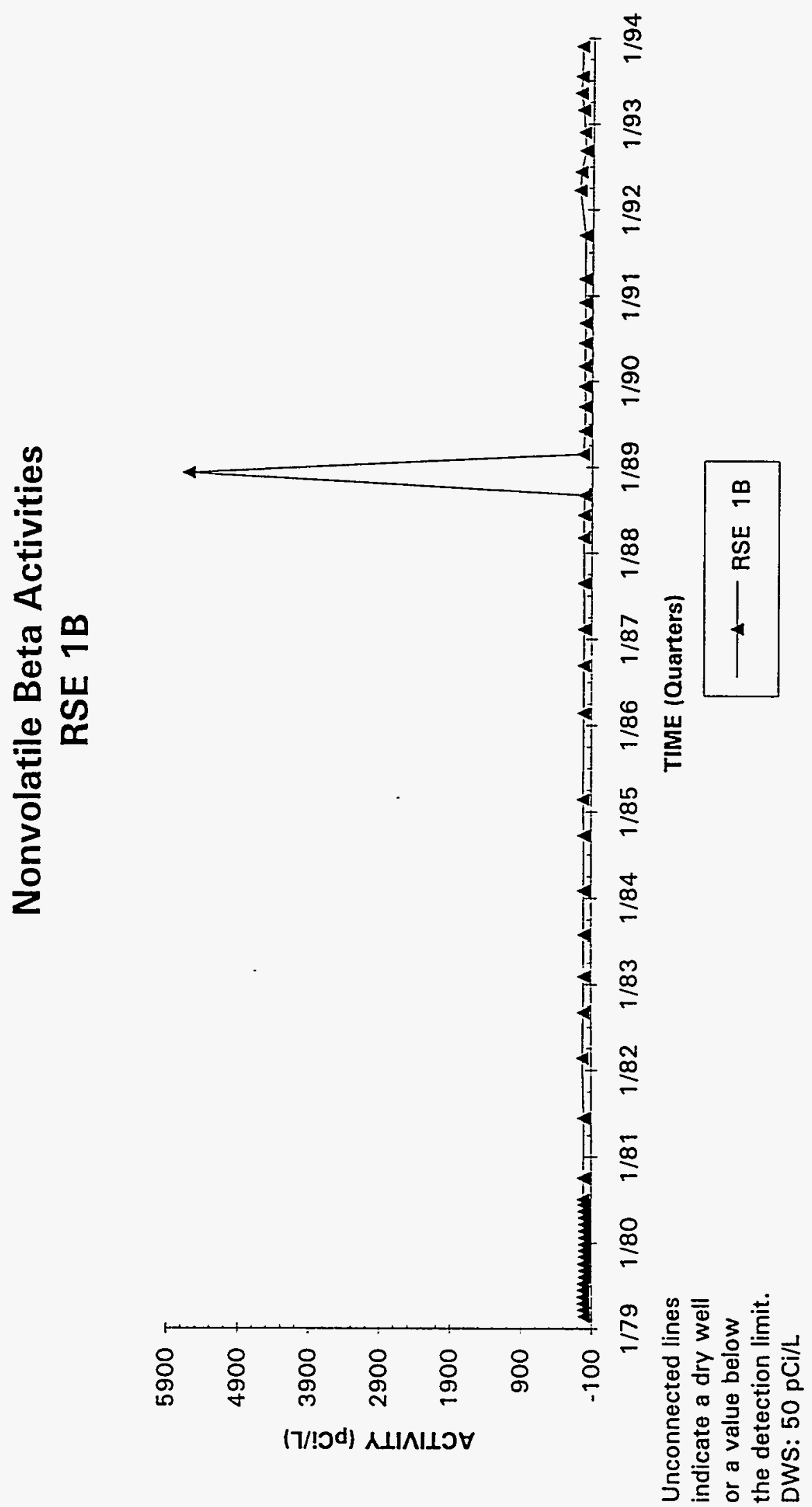


\section{Nonvolatile Beta Activities RSE Well Series (4A through 5)}

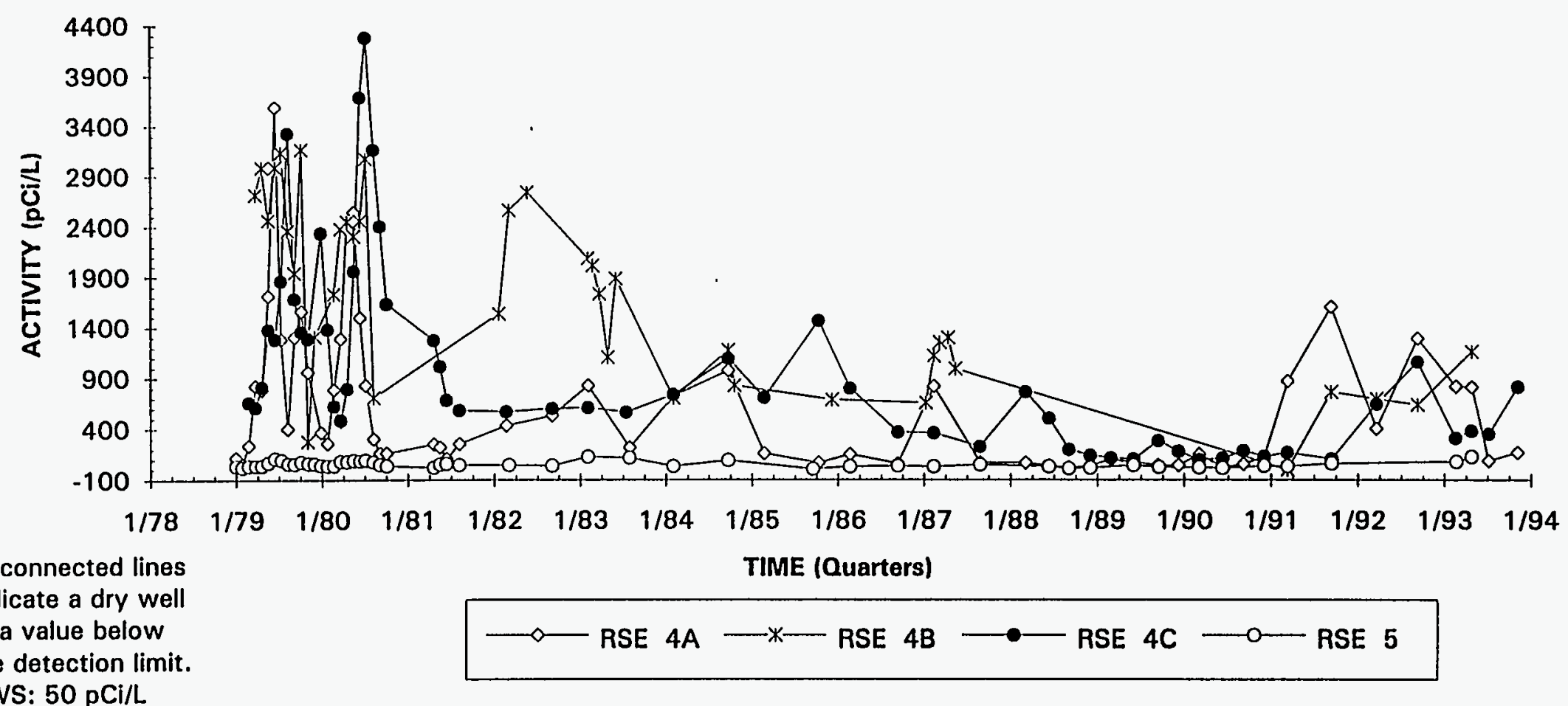



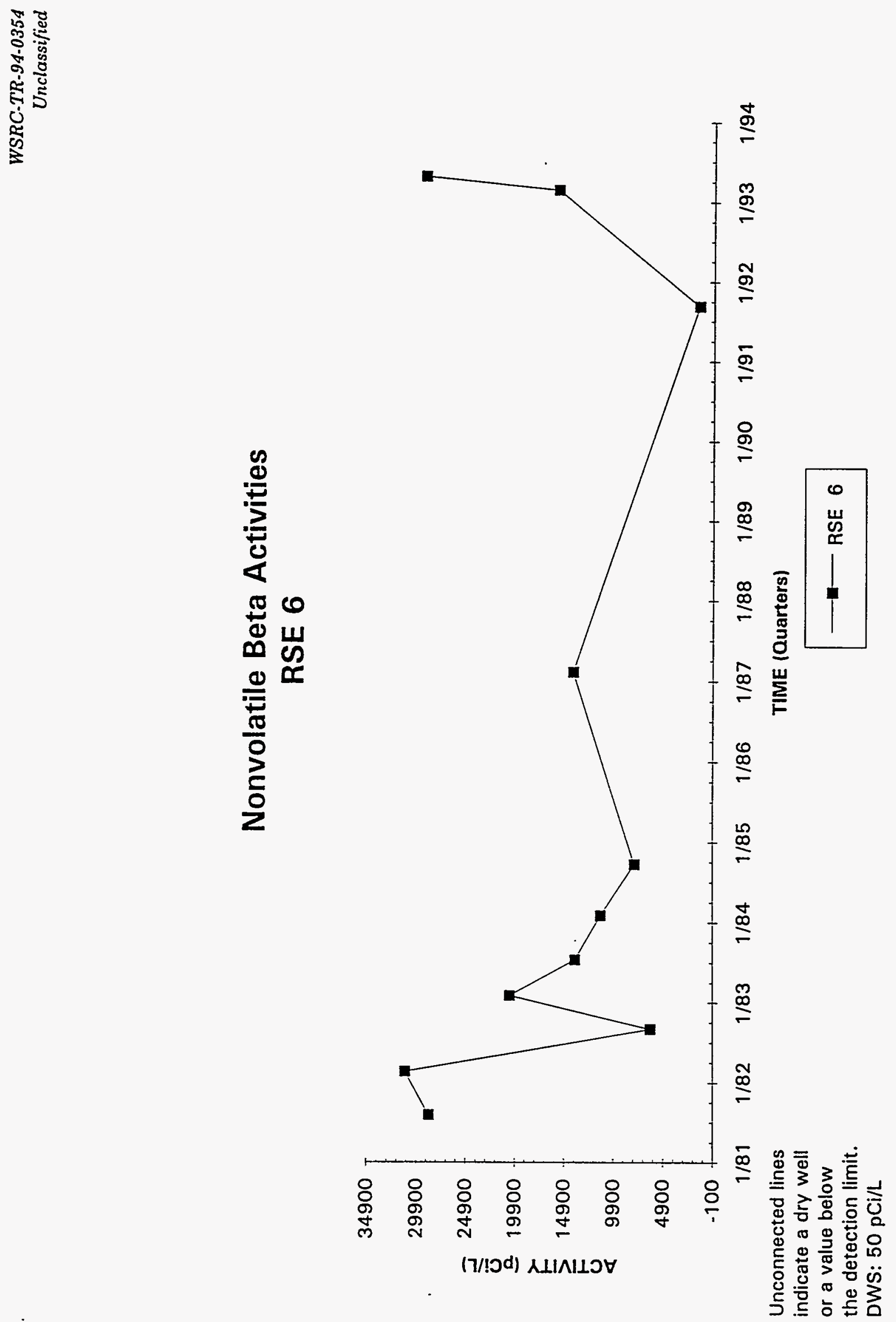

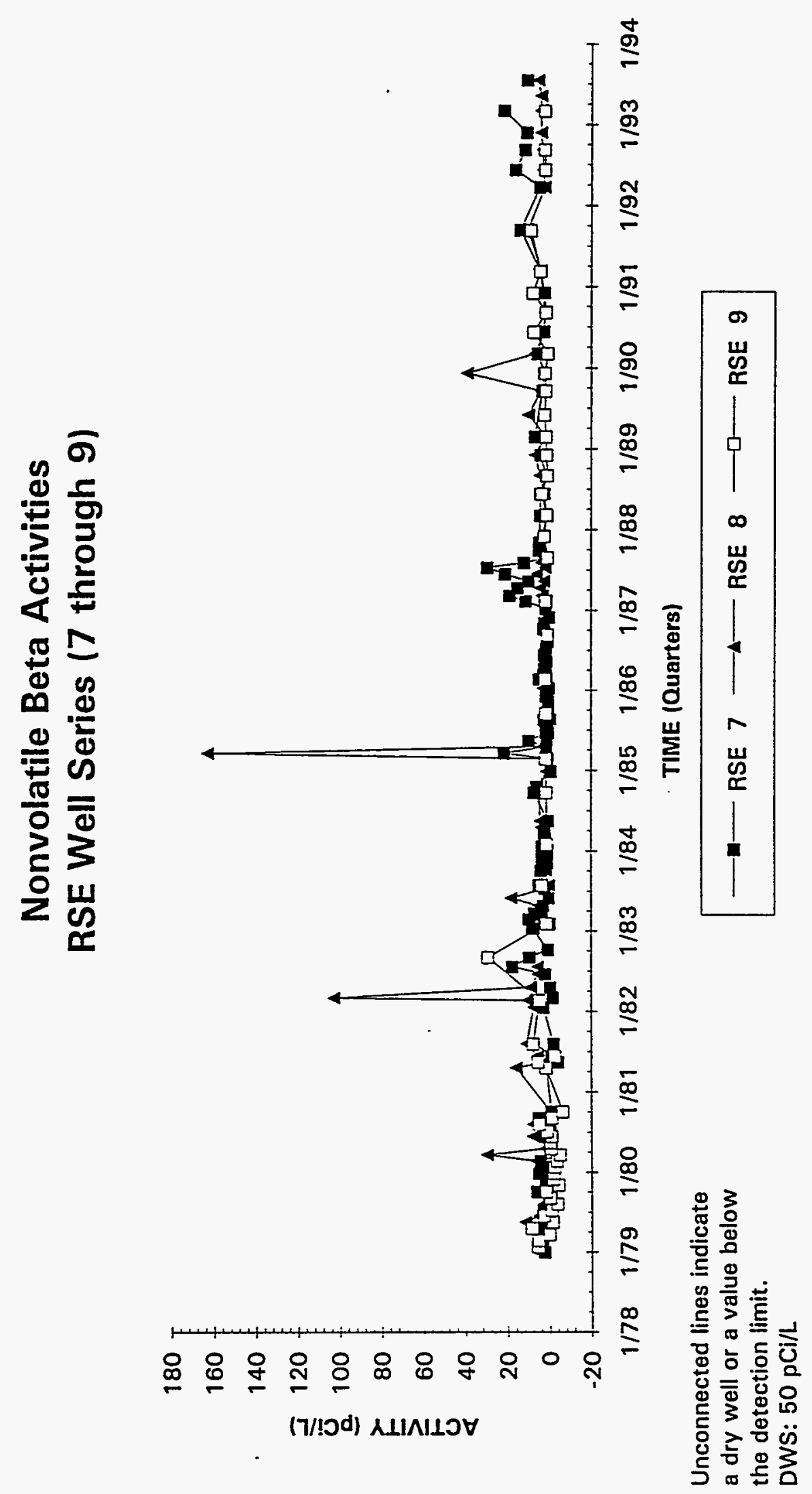


\section{Nonvolatile Beta Activities \\ RSE 10}

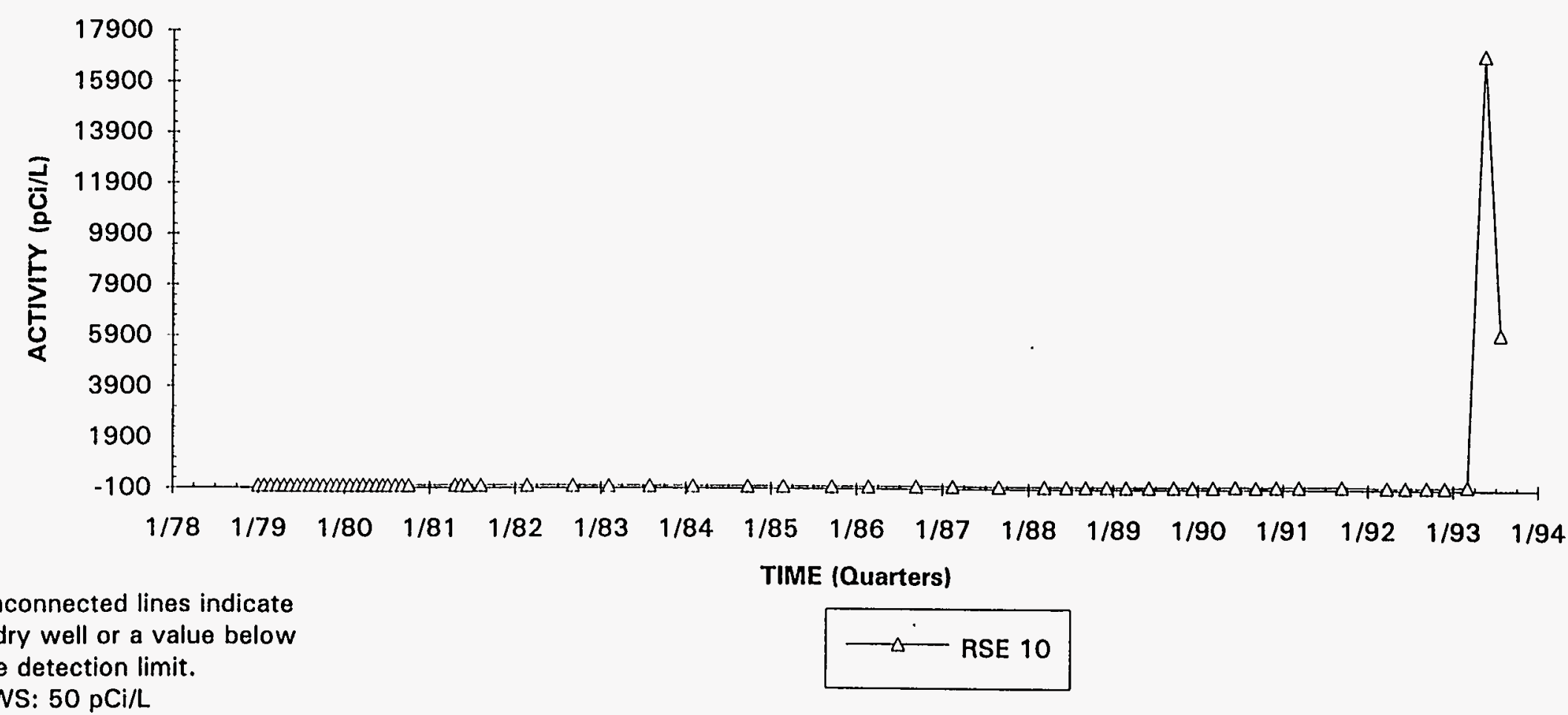




\section{Nonvolatile Beta Activities}

RSE 11

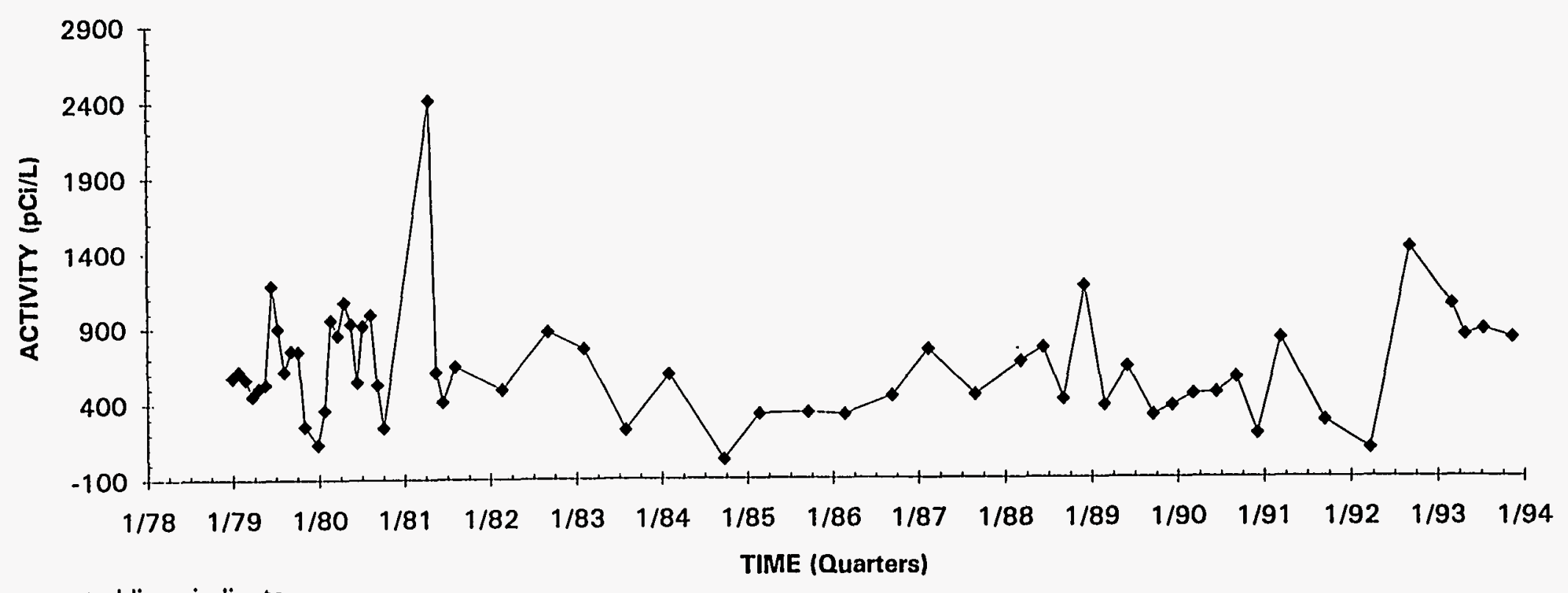

Unconnected lines indicate a dry well or a value below the detection limit.

DWS: $50 \mathrm{pCi} / \mathrm{L}$ 
हี

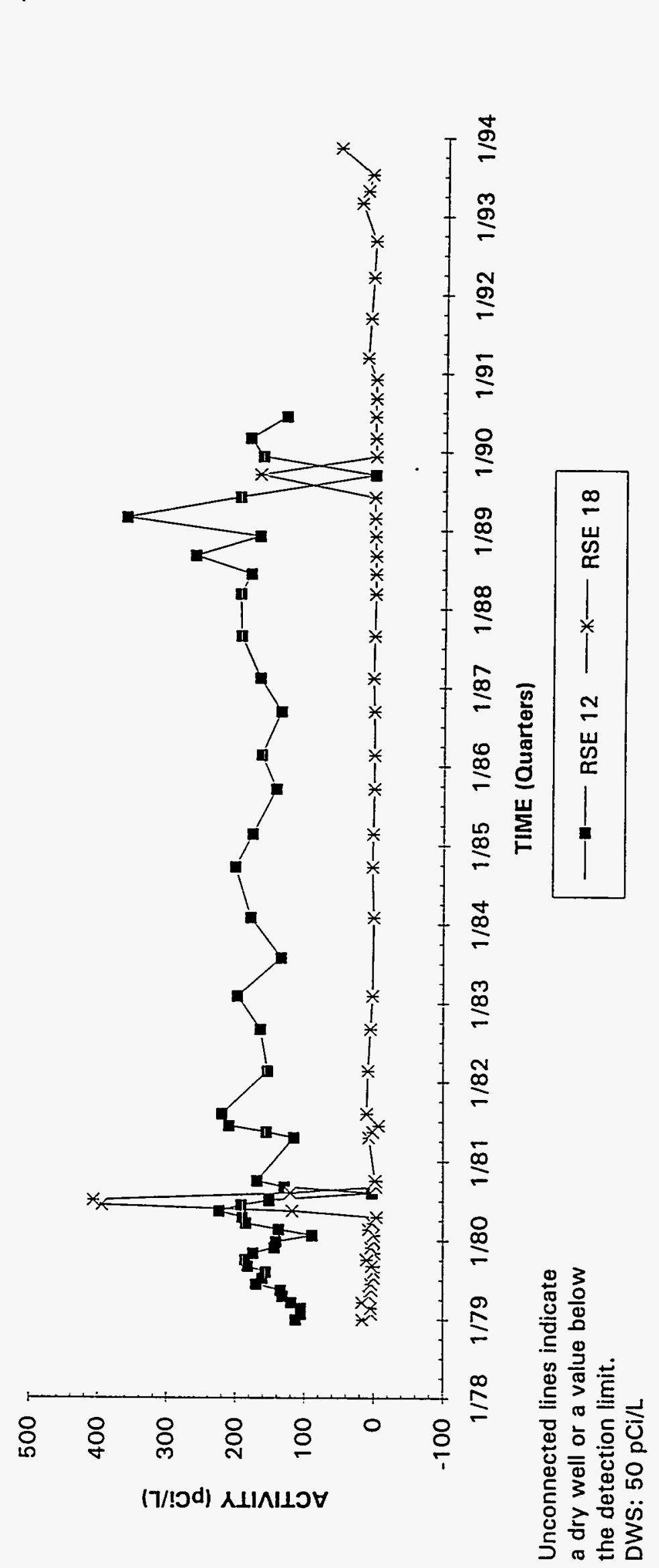




\section{Nonvolatile Beta Activities}

RSE 13

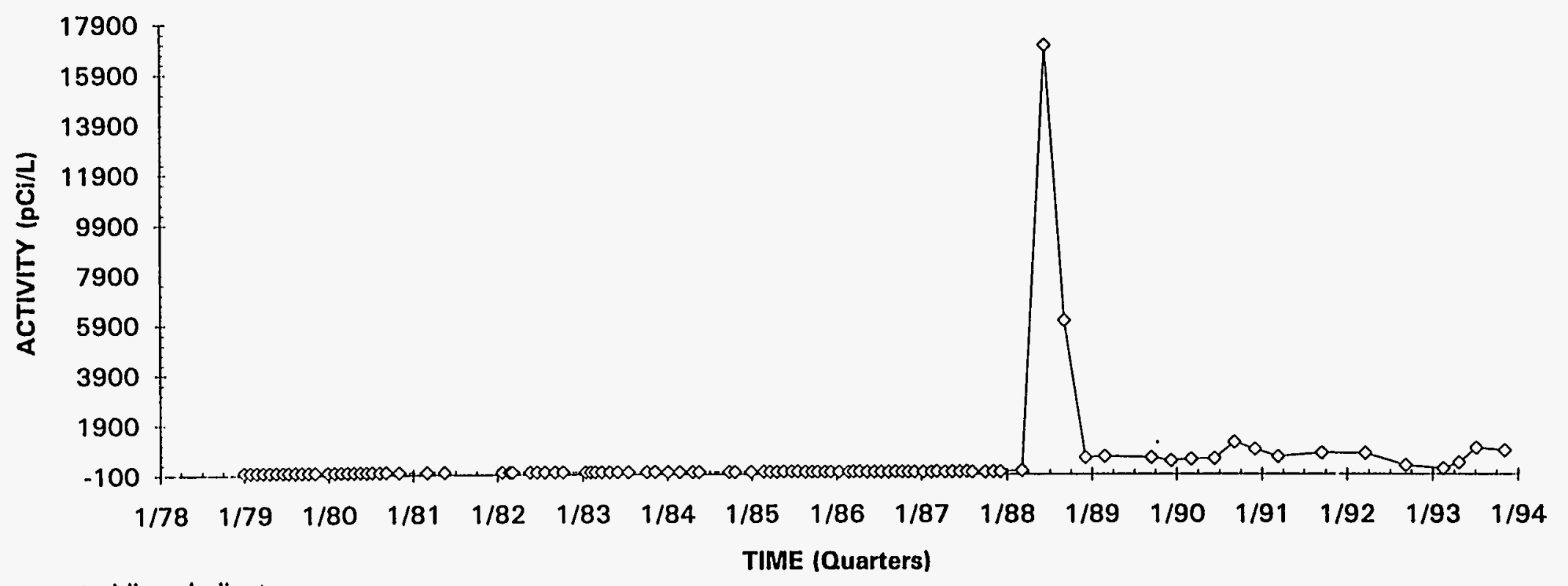

Unconnected lines indicate a dry well or a value below the detection limit. 

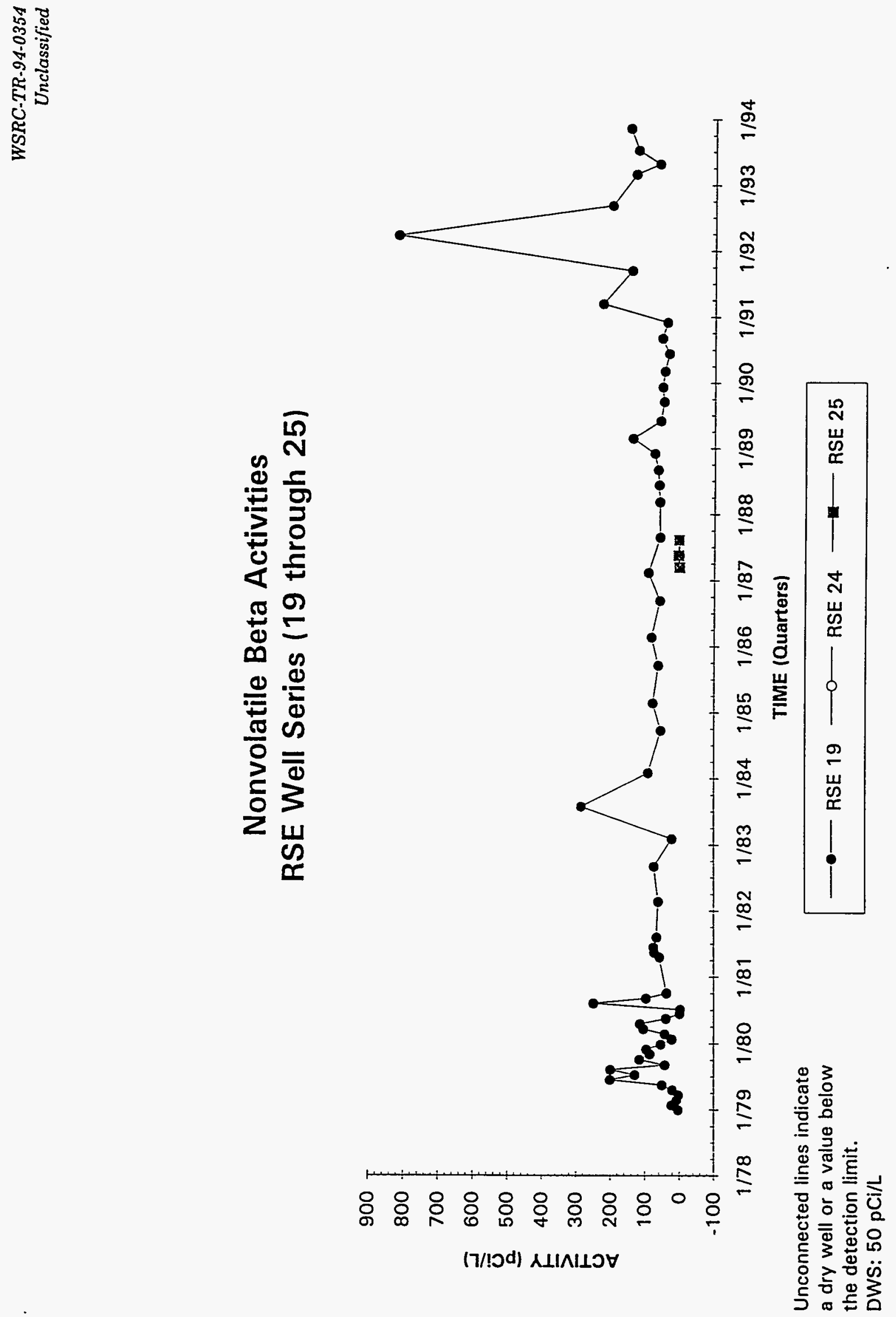


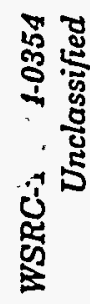

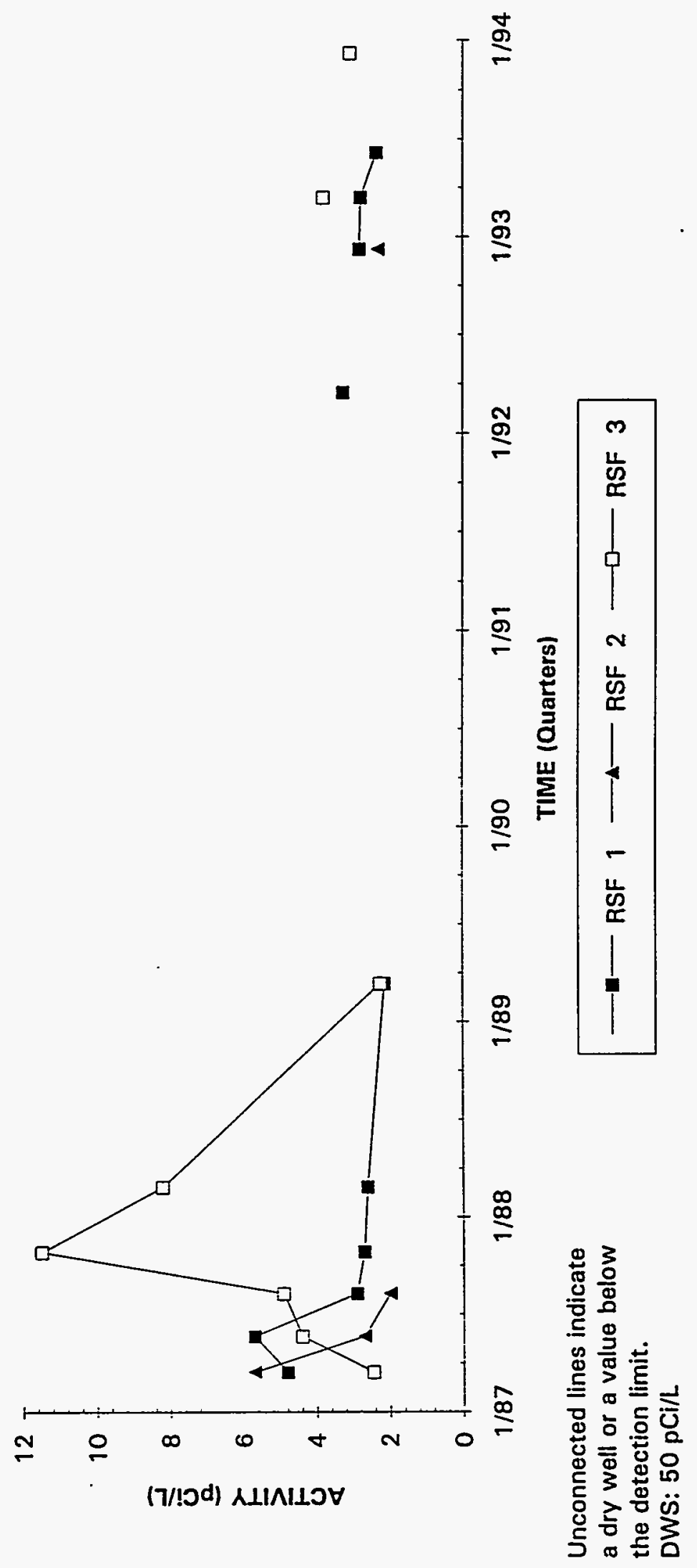


pH

RAC Well Series

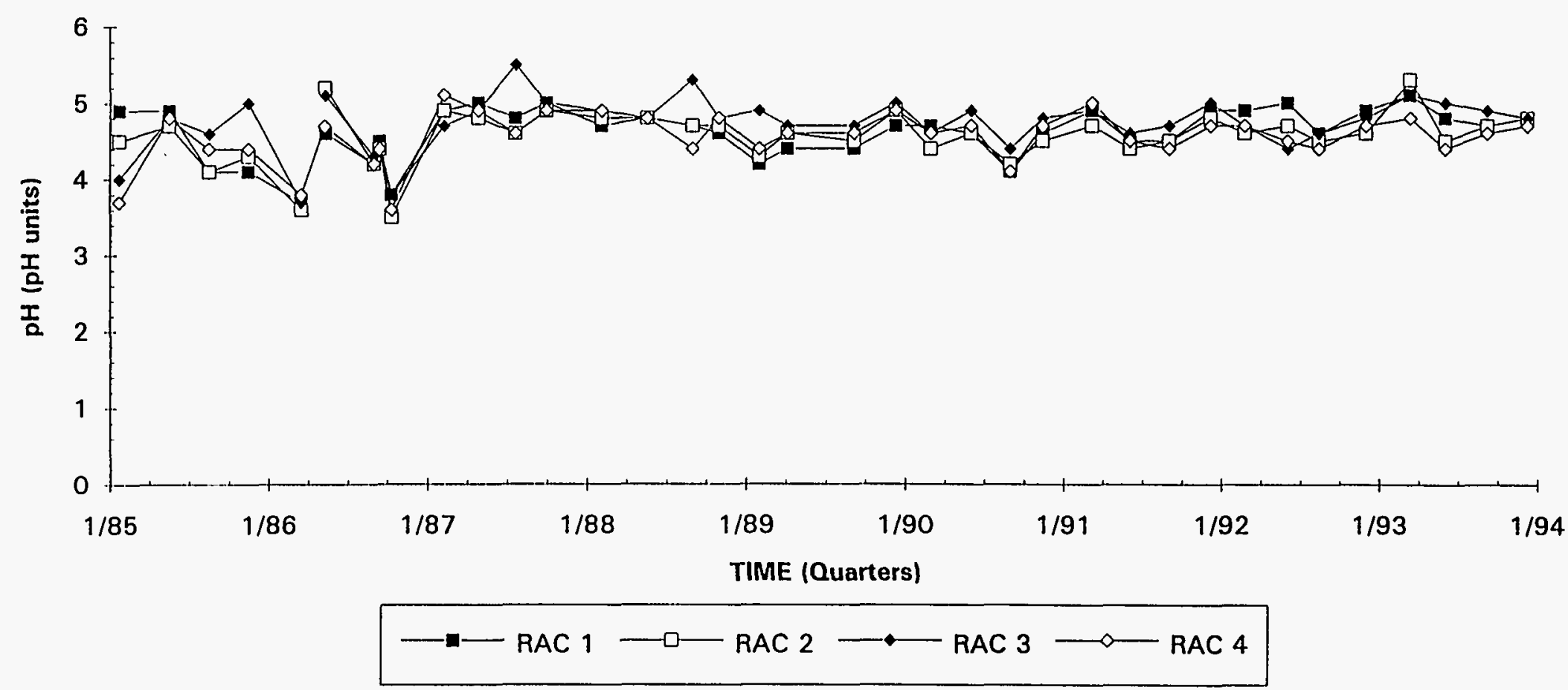



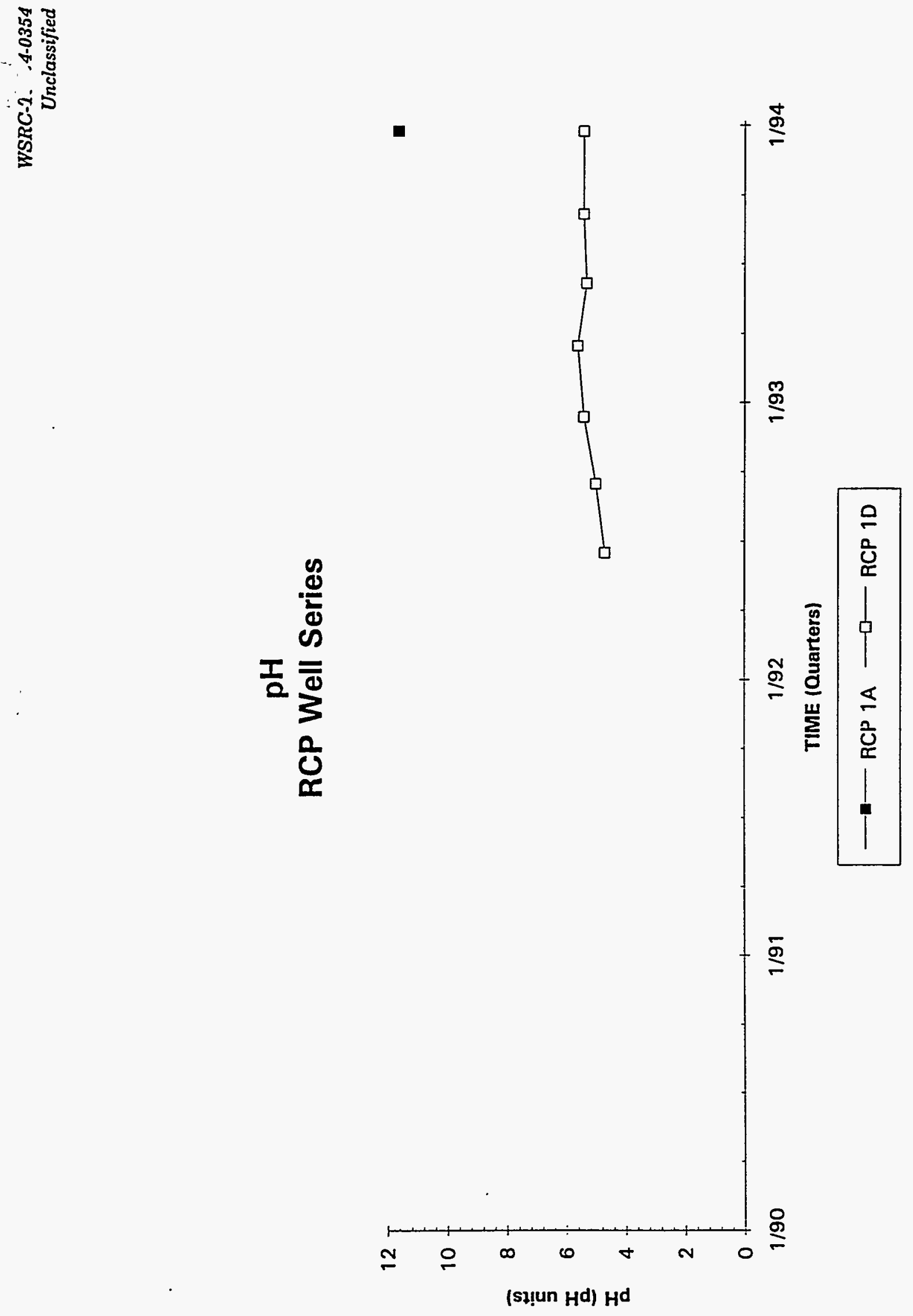
硧

$k$
0

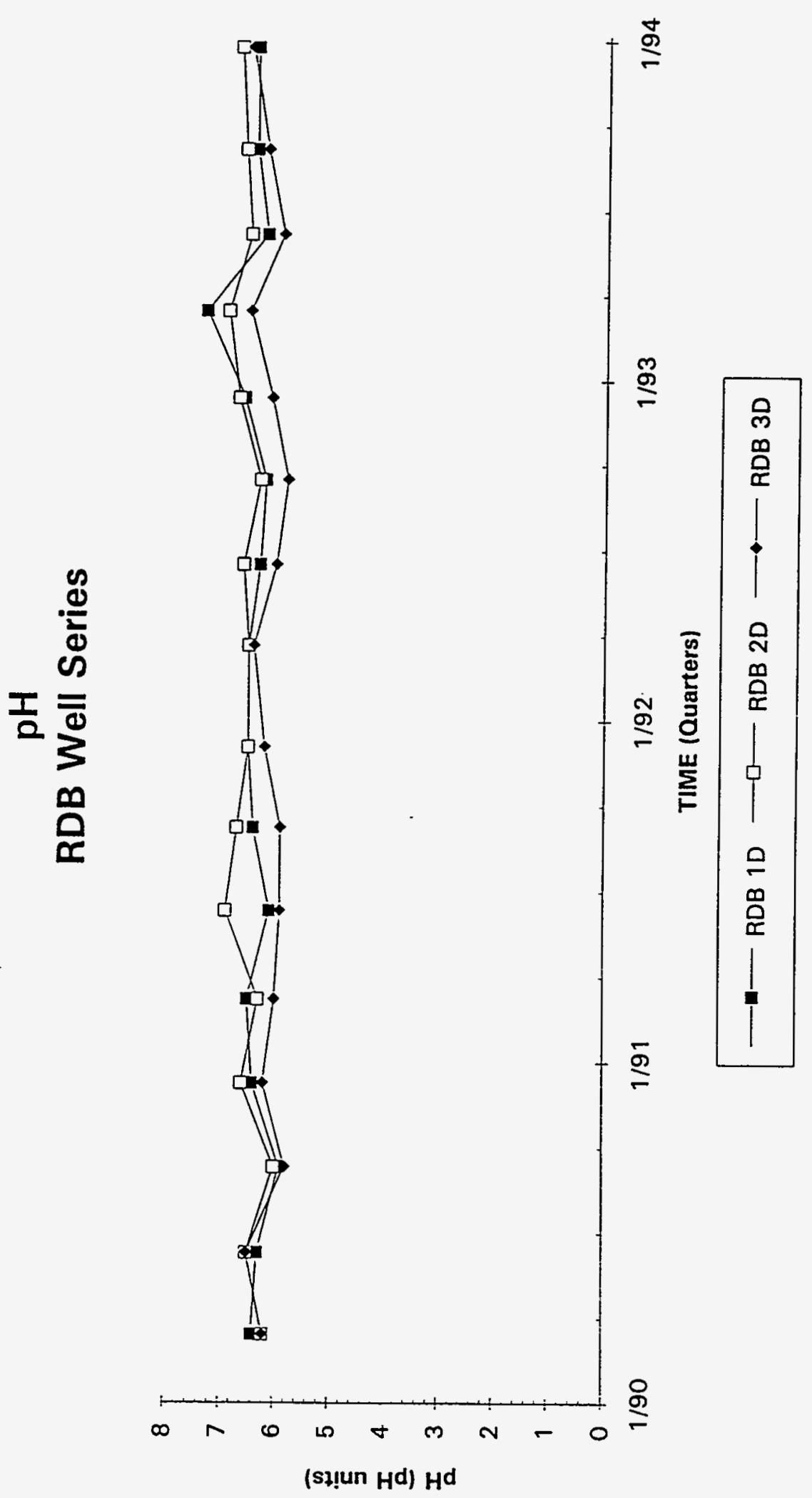

 


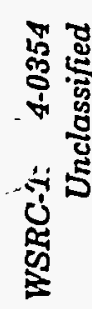

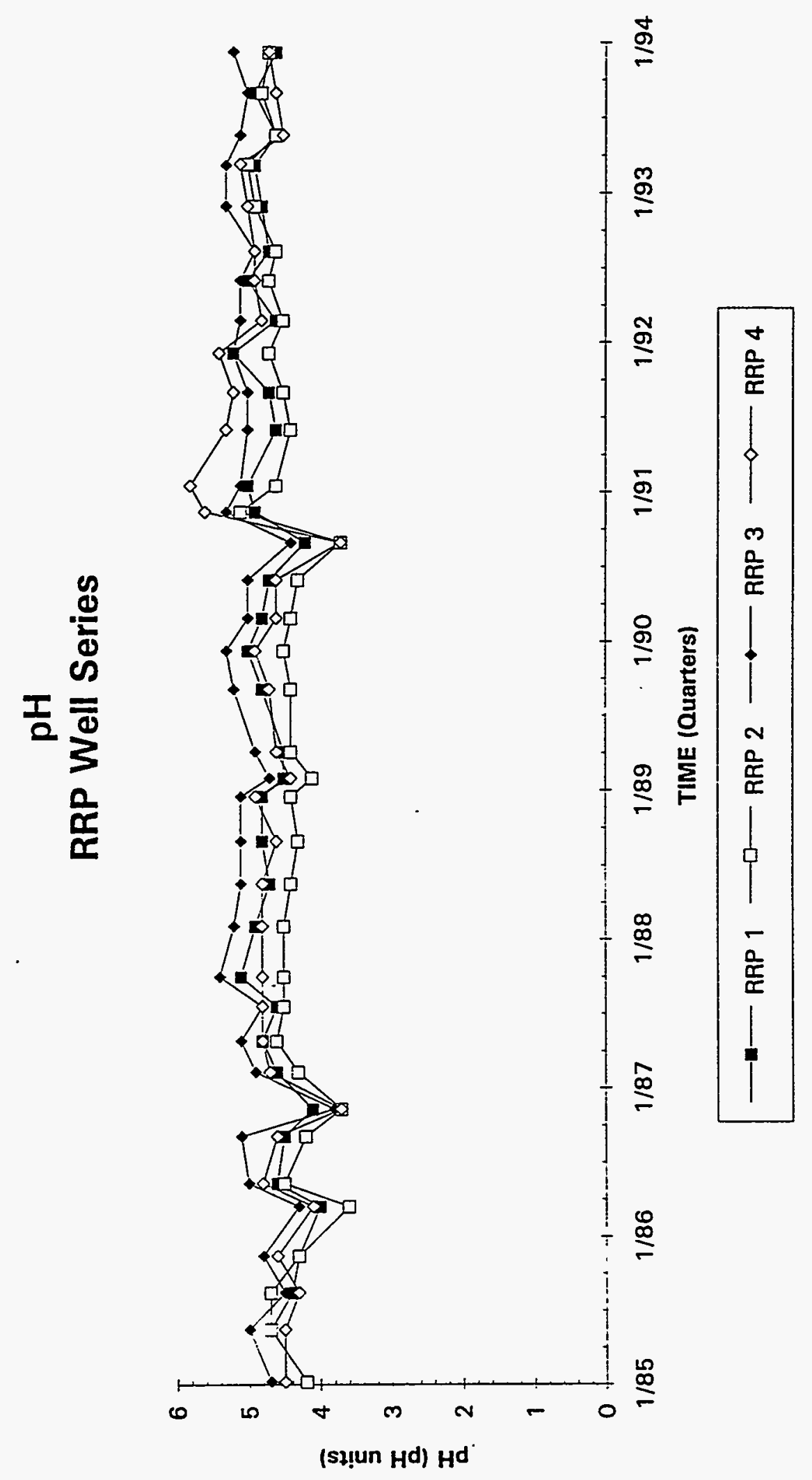




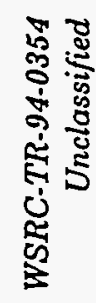

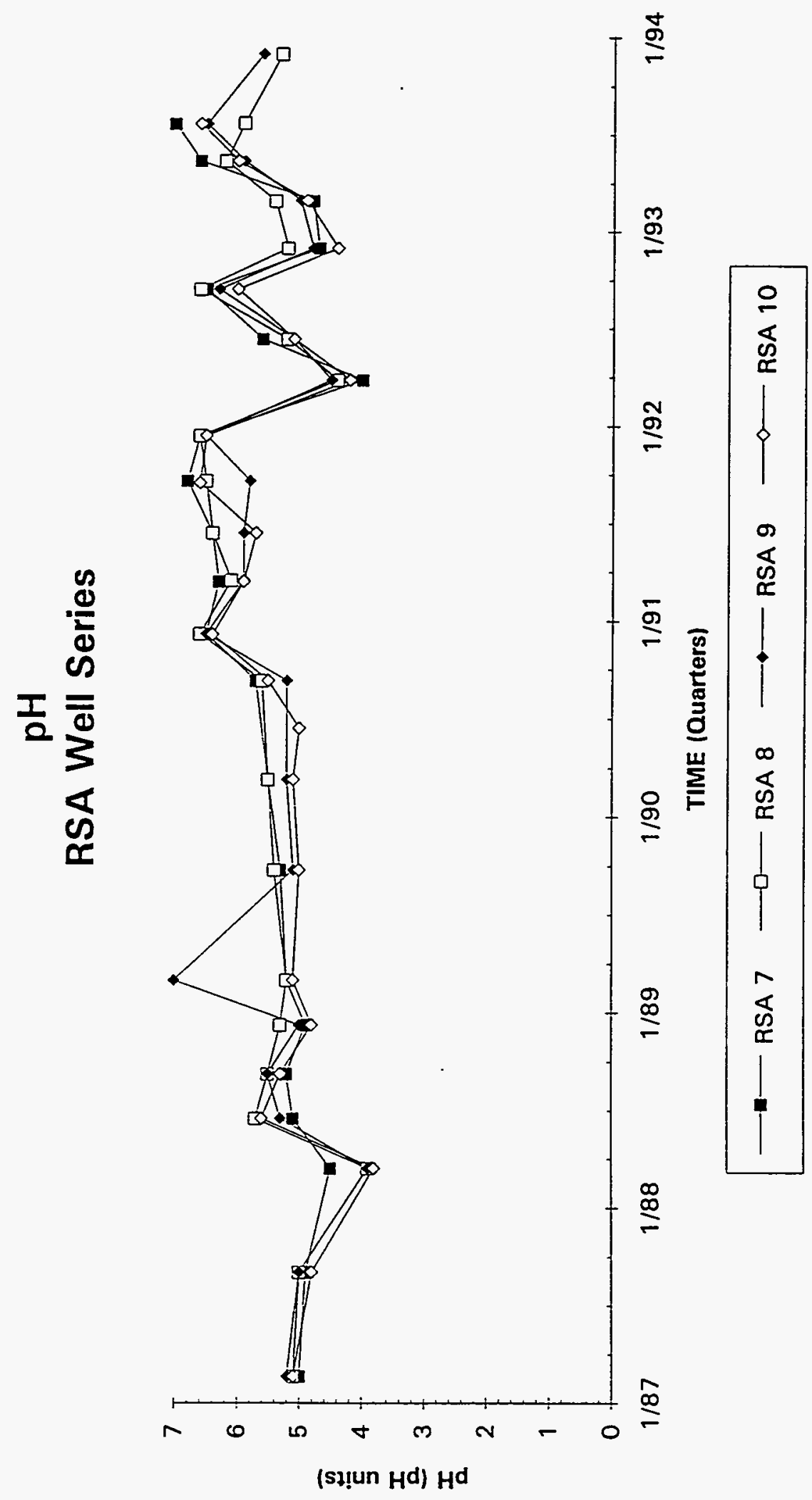




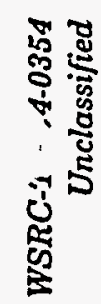

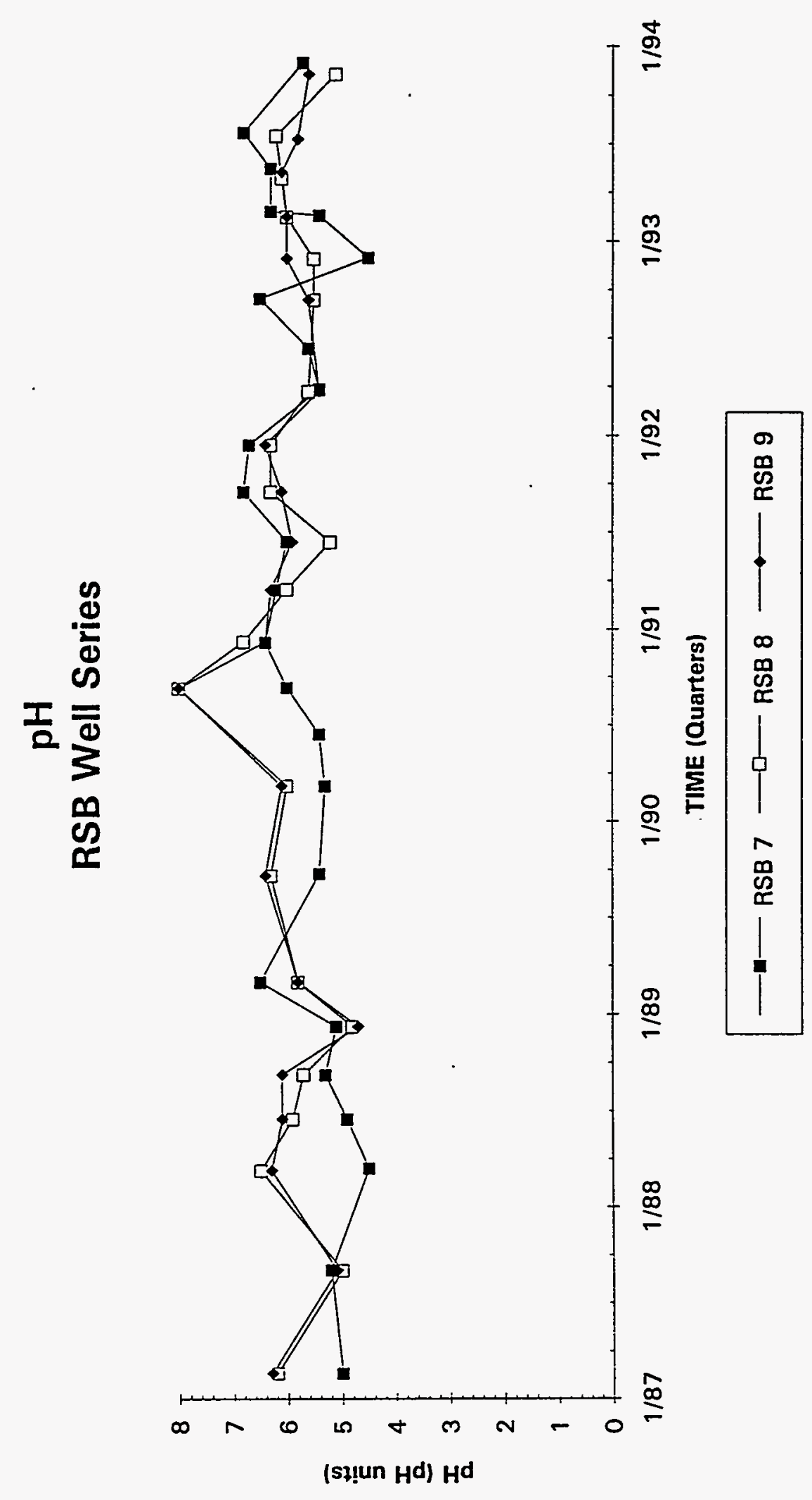




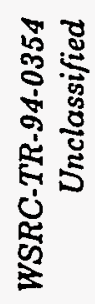

$\frac{\Phi}{2}$

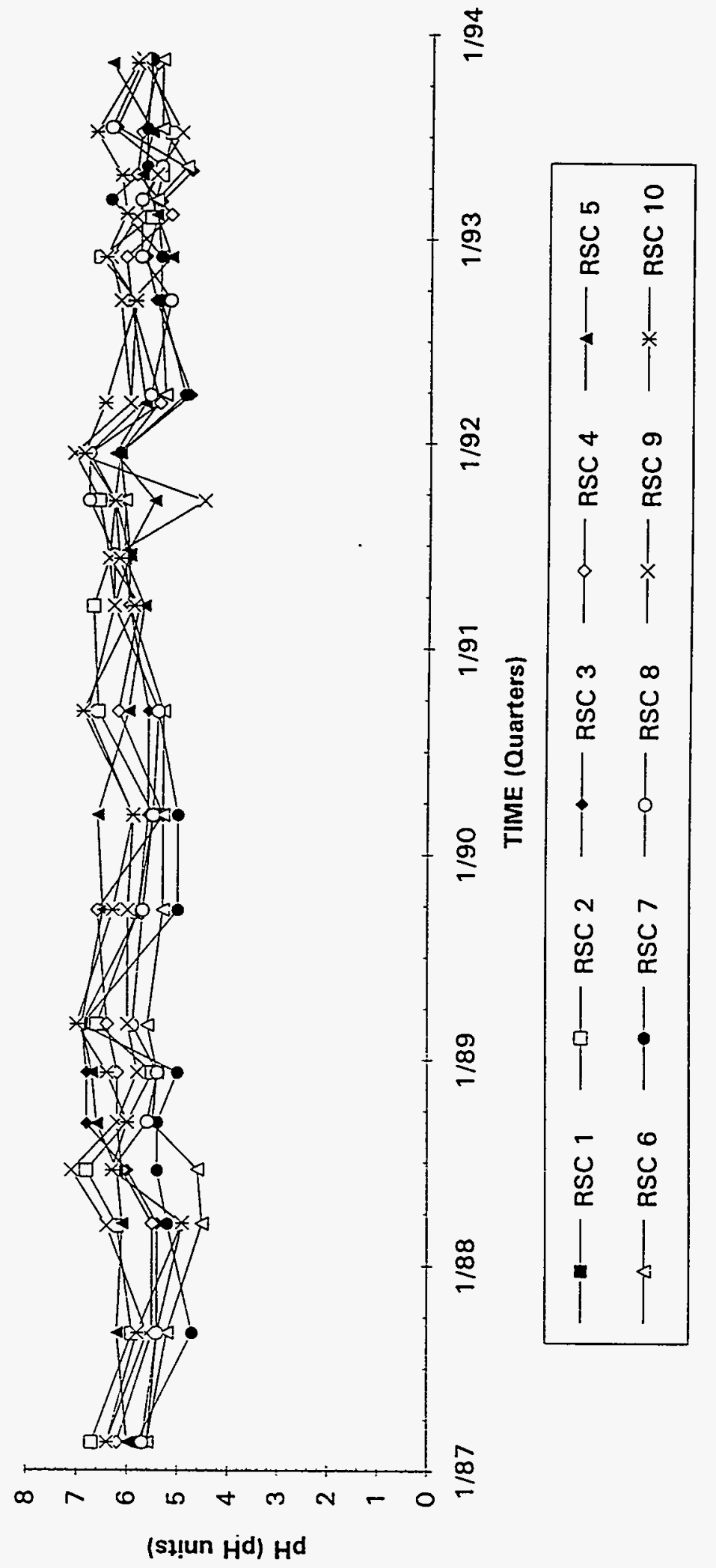




\section{$\mathrm{pH}$ \\ RSD Well Series}

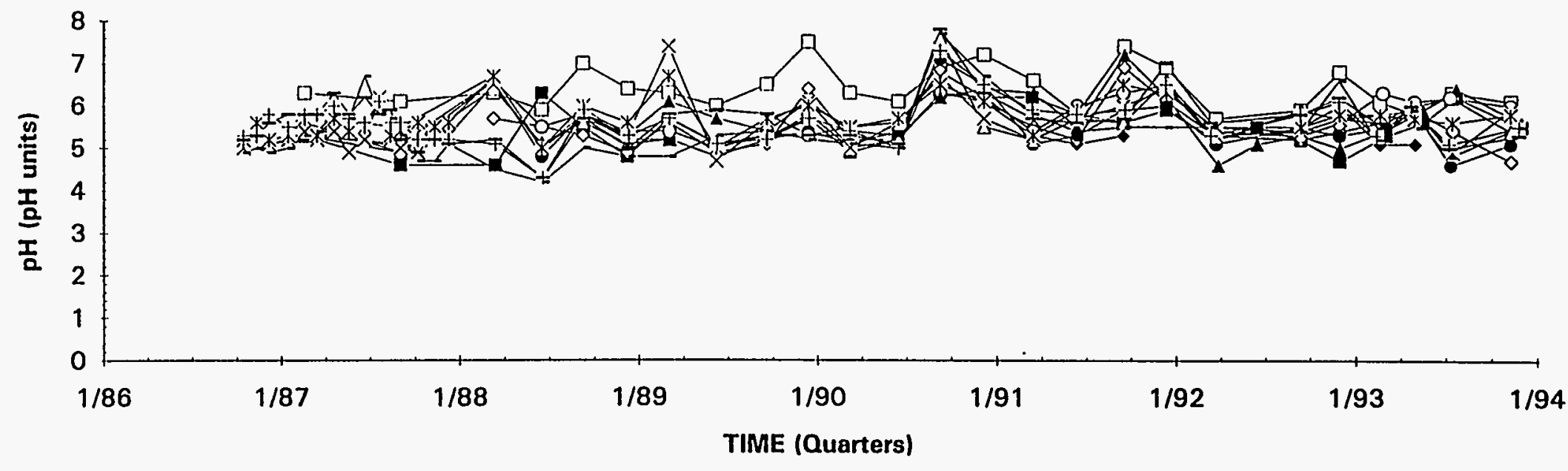

$$
\begin{aligned}
& \longrightarrow \text { RSD } 1 \longrightarrow \text { RSD 2A } \longrightarrow \text { RSD 2B } \longrightarrow \text { RSD 2C } \longrightarrow \text { RSD } 3 \\
& \longrightarrow \text { RSD } 4-\longrightarrow \text { RSD } 5-0 \text { RSD } 6-x-\operatorname{RSD} 7-\% \text { RSD } 8 \\
& \longrightarrow \text { RSD } 9 \longrightarrow \text { RSD } 10 \longrightarrow \text { RSD } 11
\end{aligned}
$$




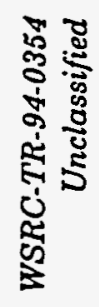

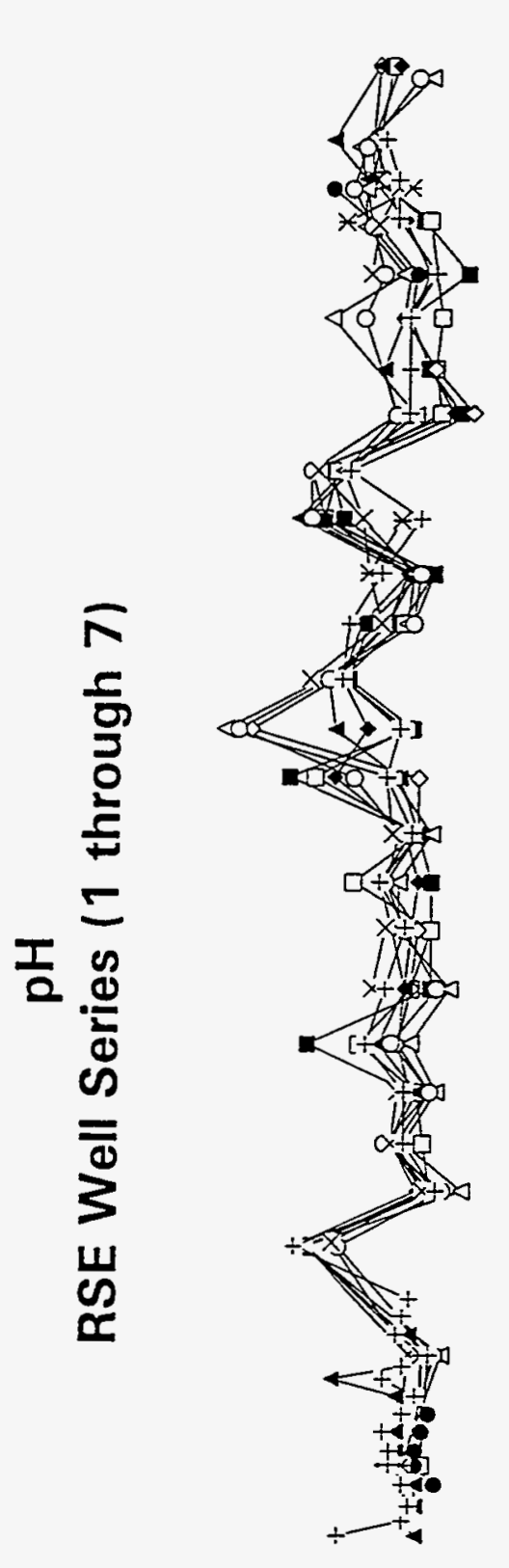

है

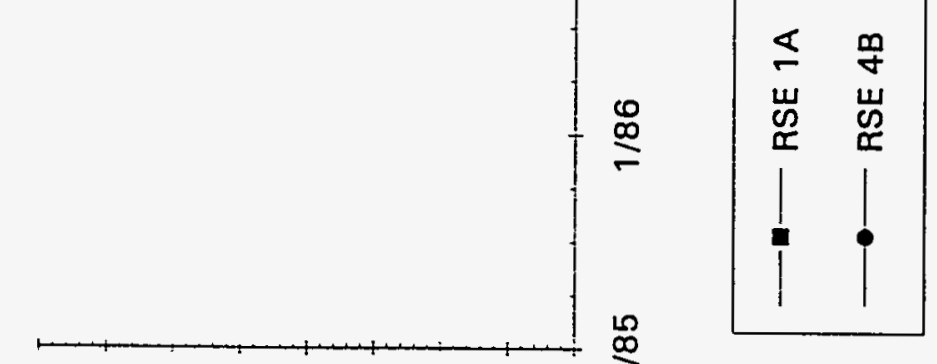

(sł!̣un $\mathrm{Hd}^{d} \mathrm{Hd}^{d}$ 


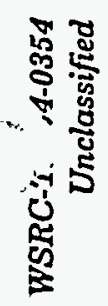

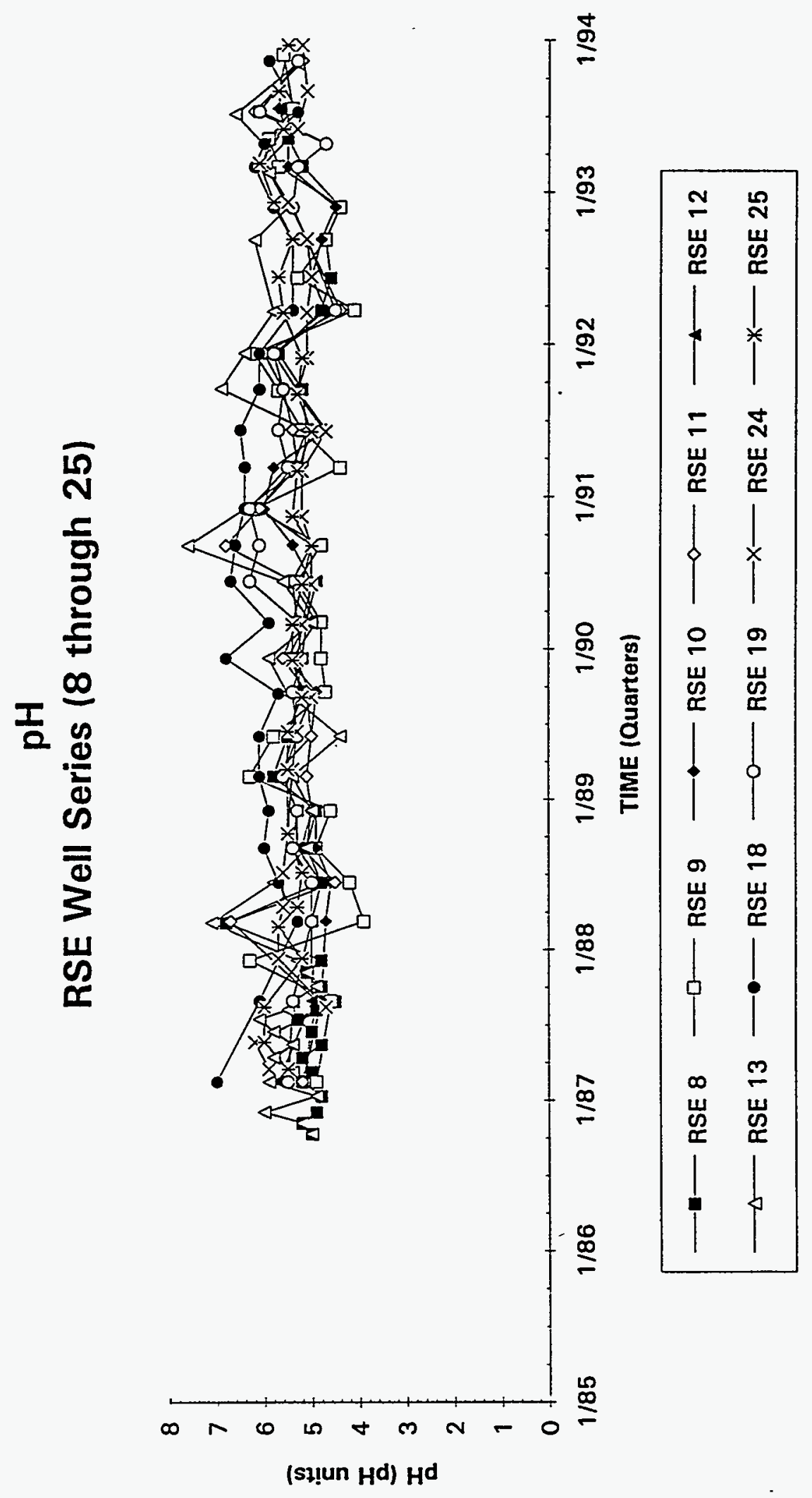


令

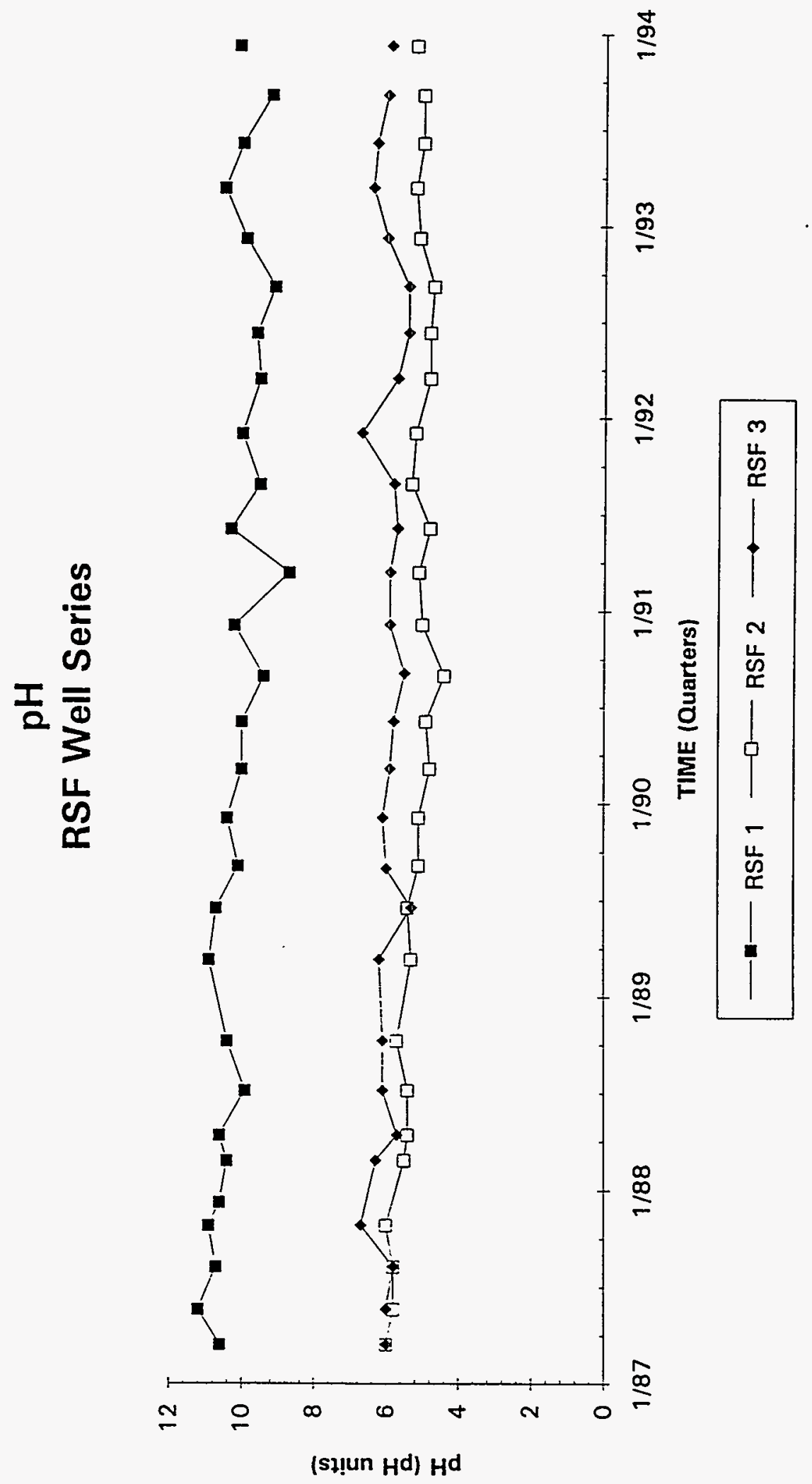




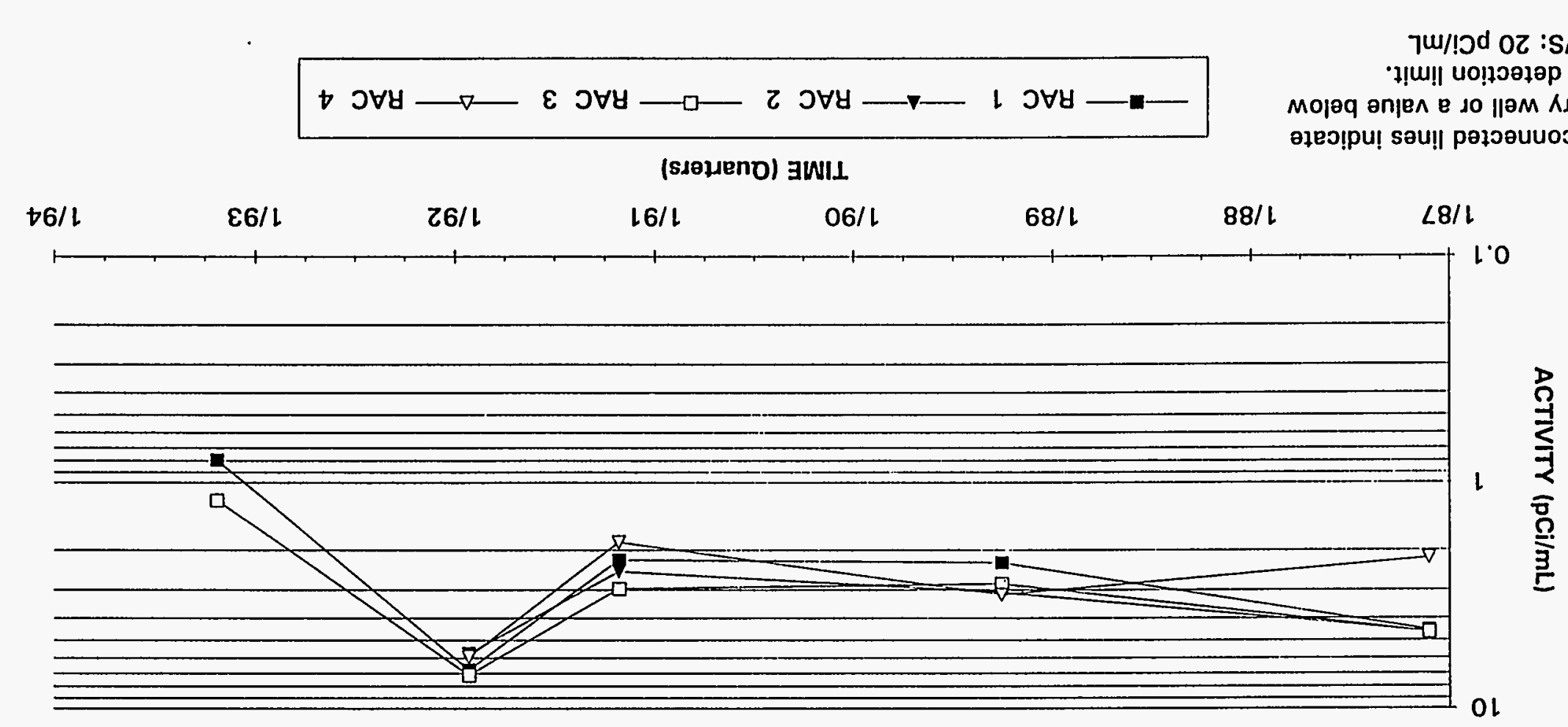

\section{Sə!นəS \|әM

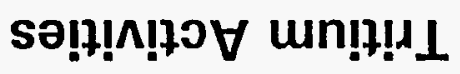




\section{Tritium Activities}

\section{RCP Well Series}

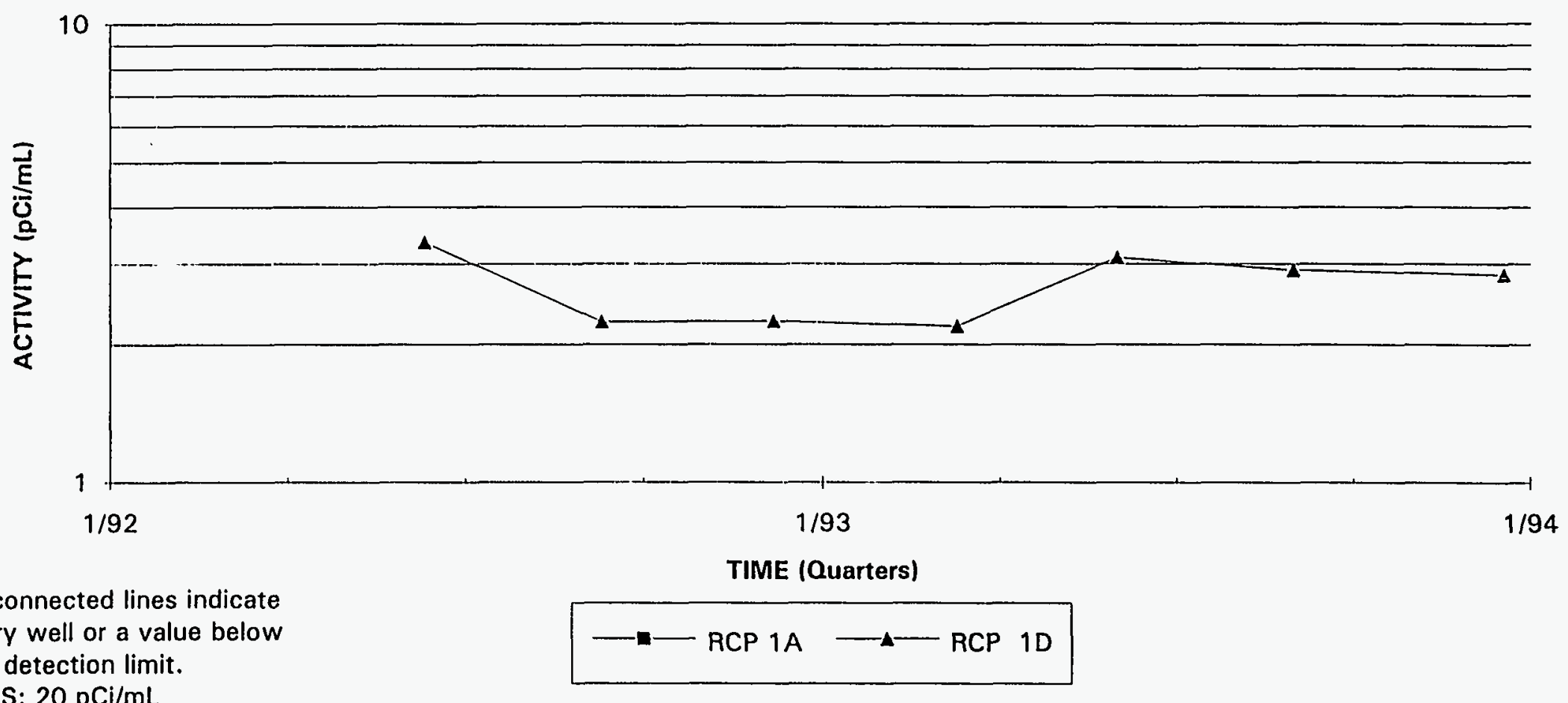

the detection limit.

DWS: $20 \mathrm{pCi} / \mathrm{mL}$ 


\section{Tritium Activities \\ RRP Well Series}

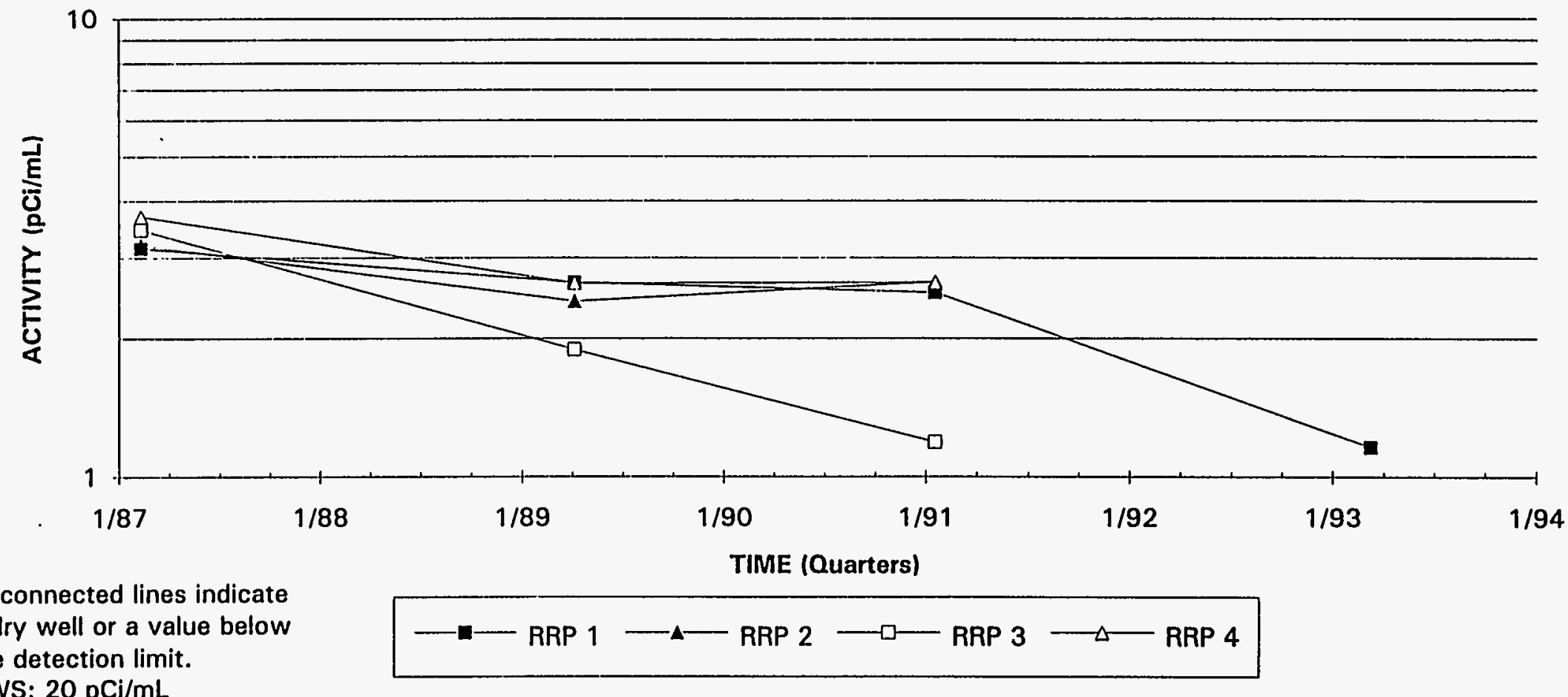

DWS: $20 \mathrm{pCi} / \mathrm{mL}$ 


\section{Tritium Activities}

\section{RSA Well Series}

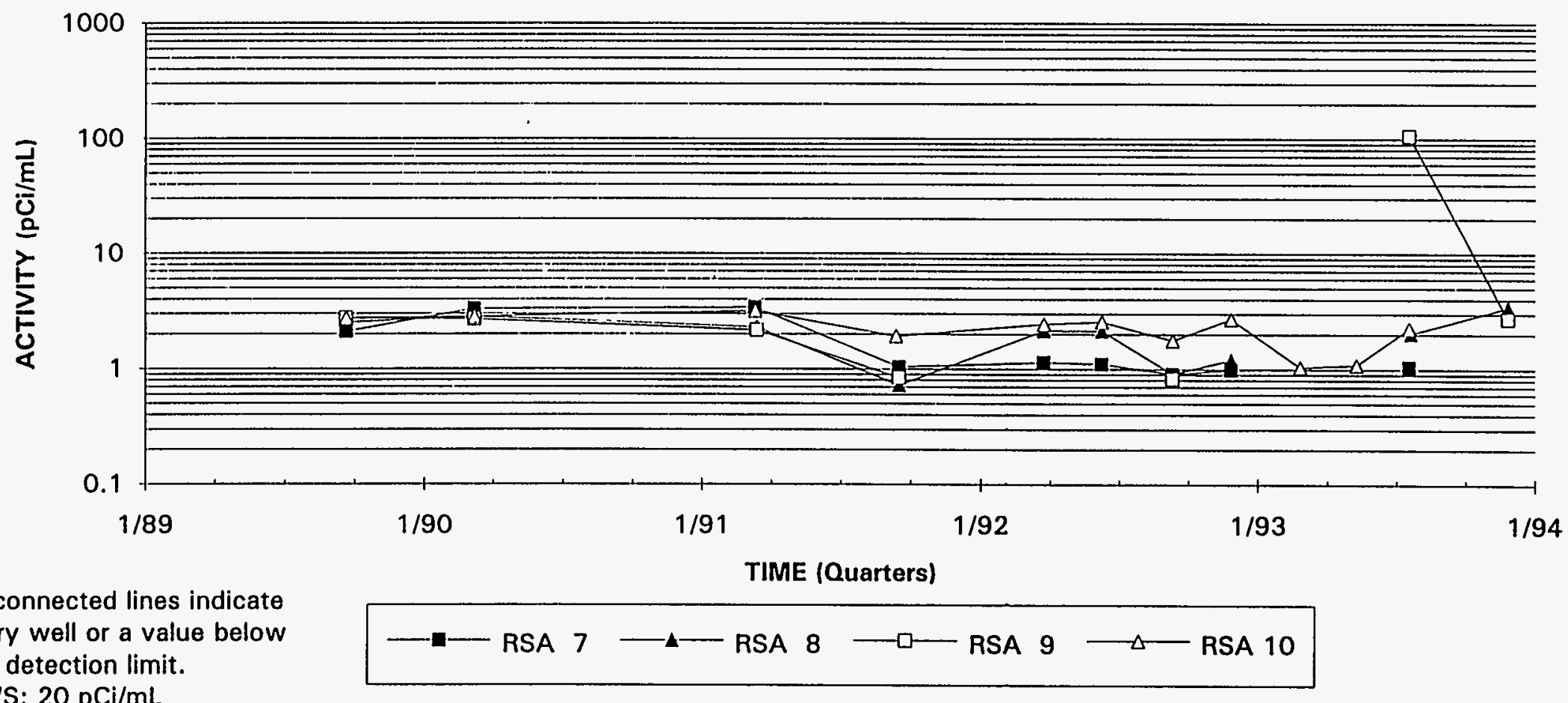

the detection limit.

DWS: $20 \mathrm{pCi} / \mathrm{mL}$ 


\section{Tritium Activities \\ RSB Well Series}

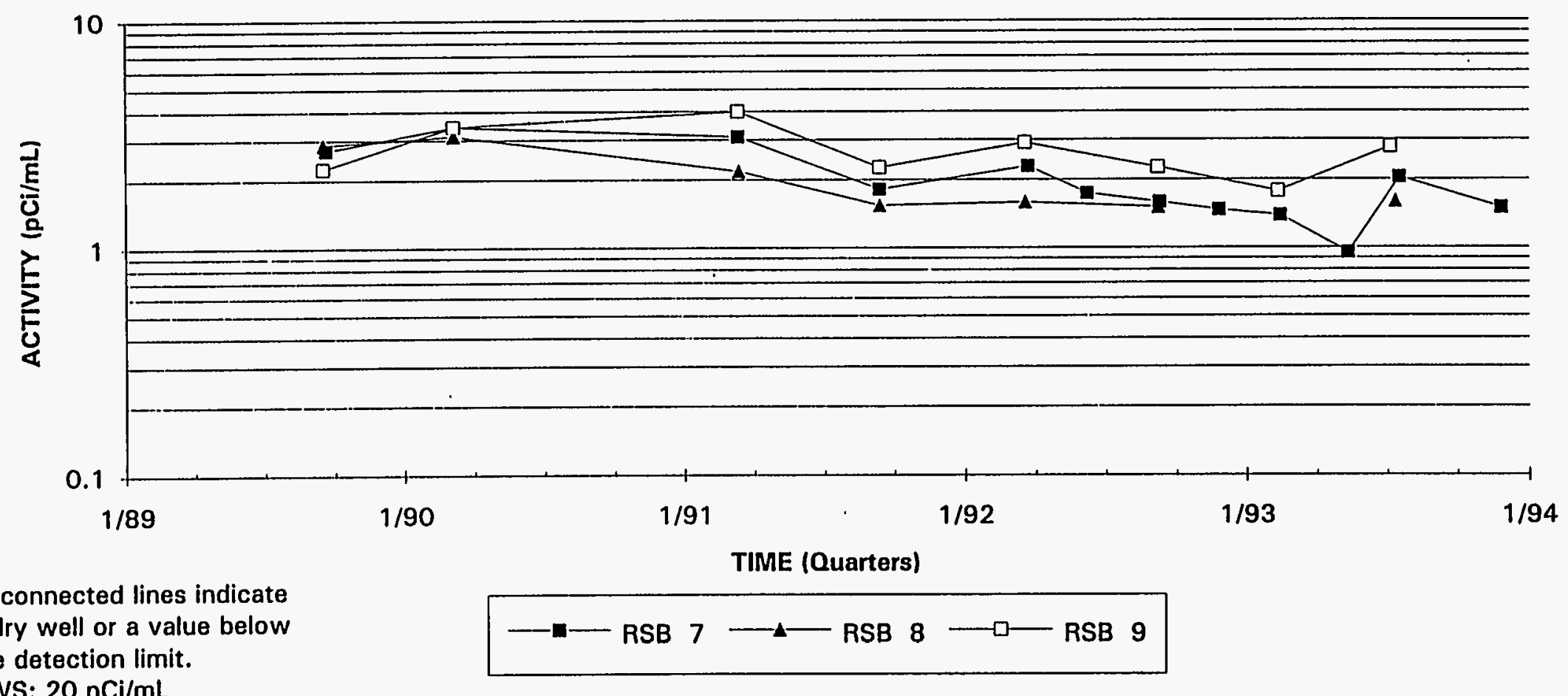




\section{Tritium Activities \\ RSC Well Series (2 through 5)}

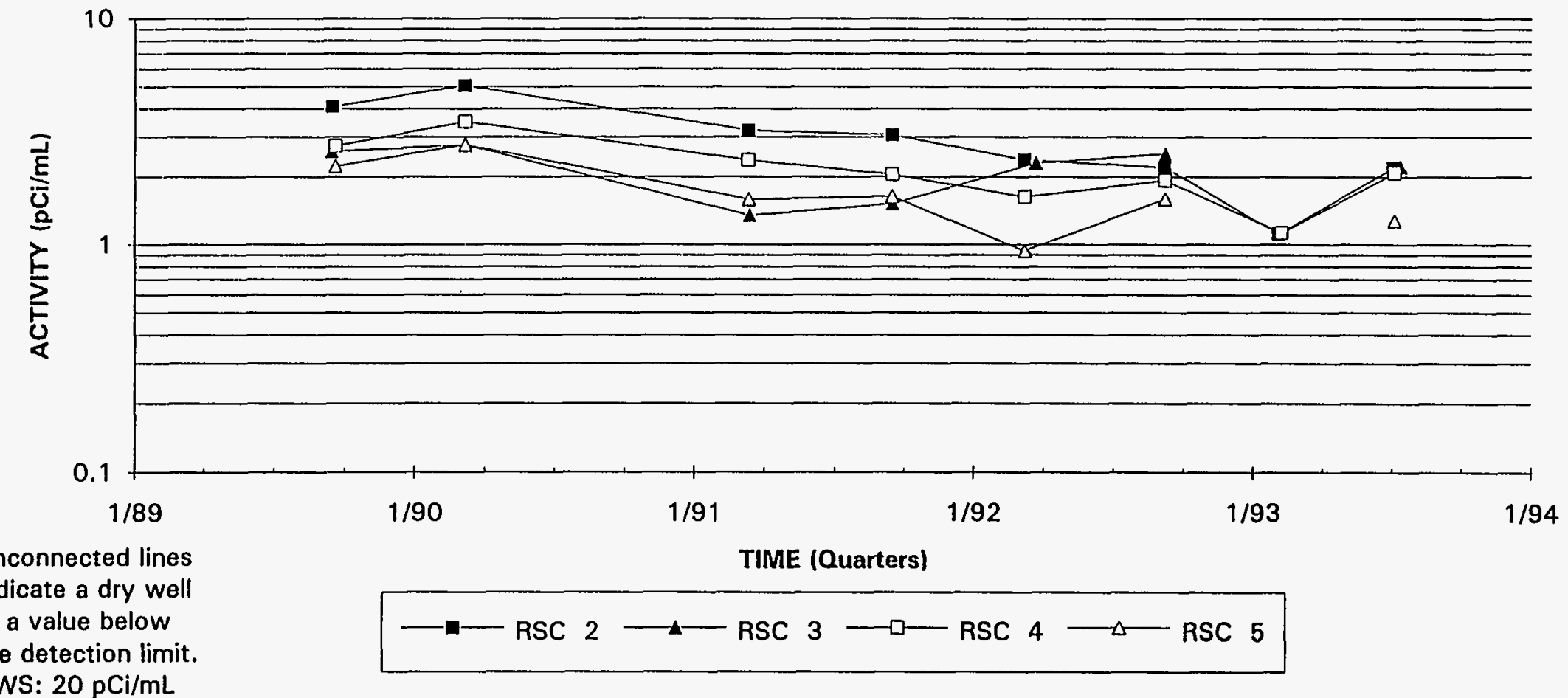




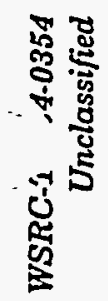

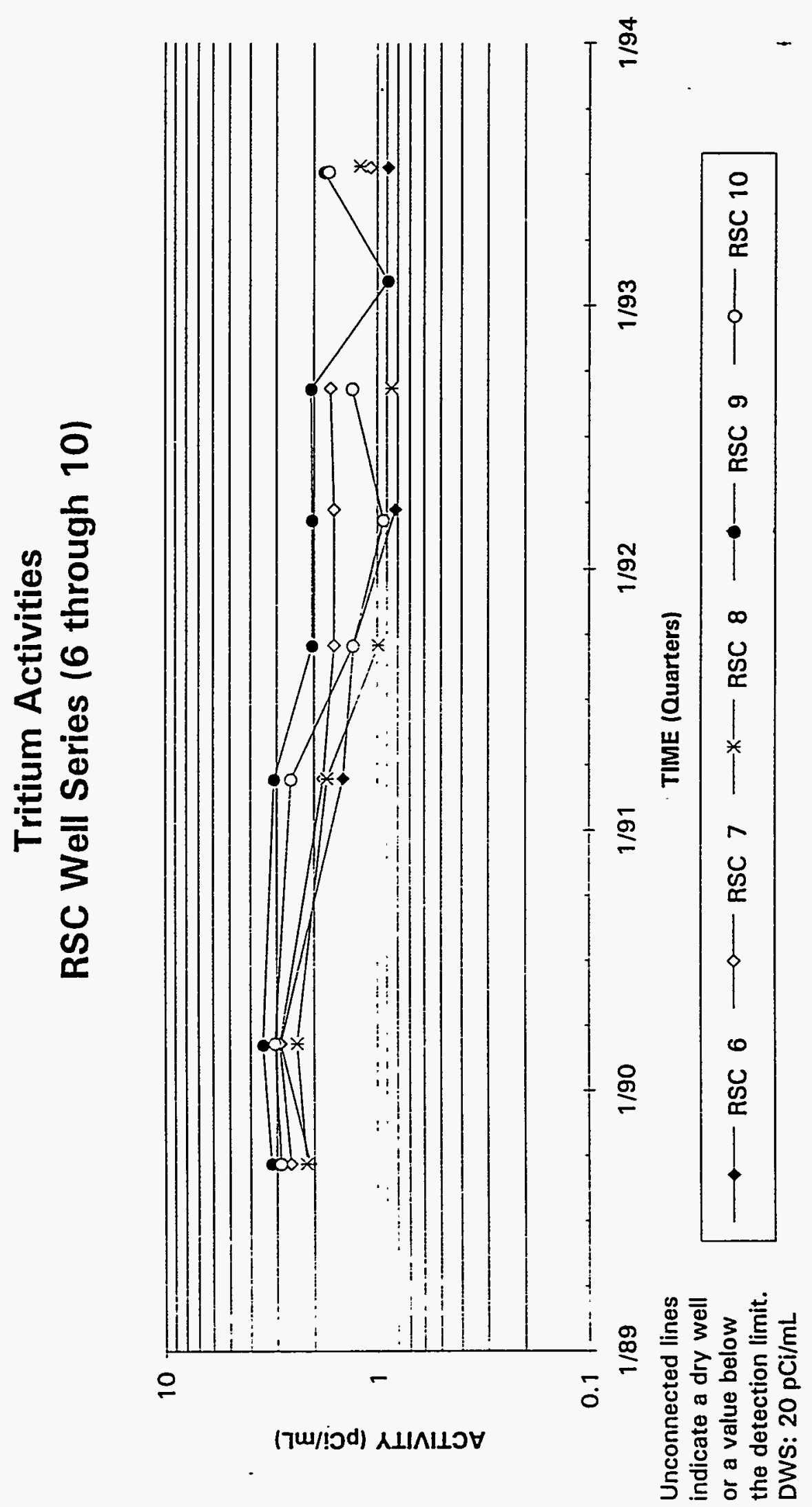




\section{Tritium Activities \\ RSD Well Series (1 through 5)}

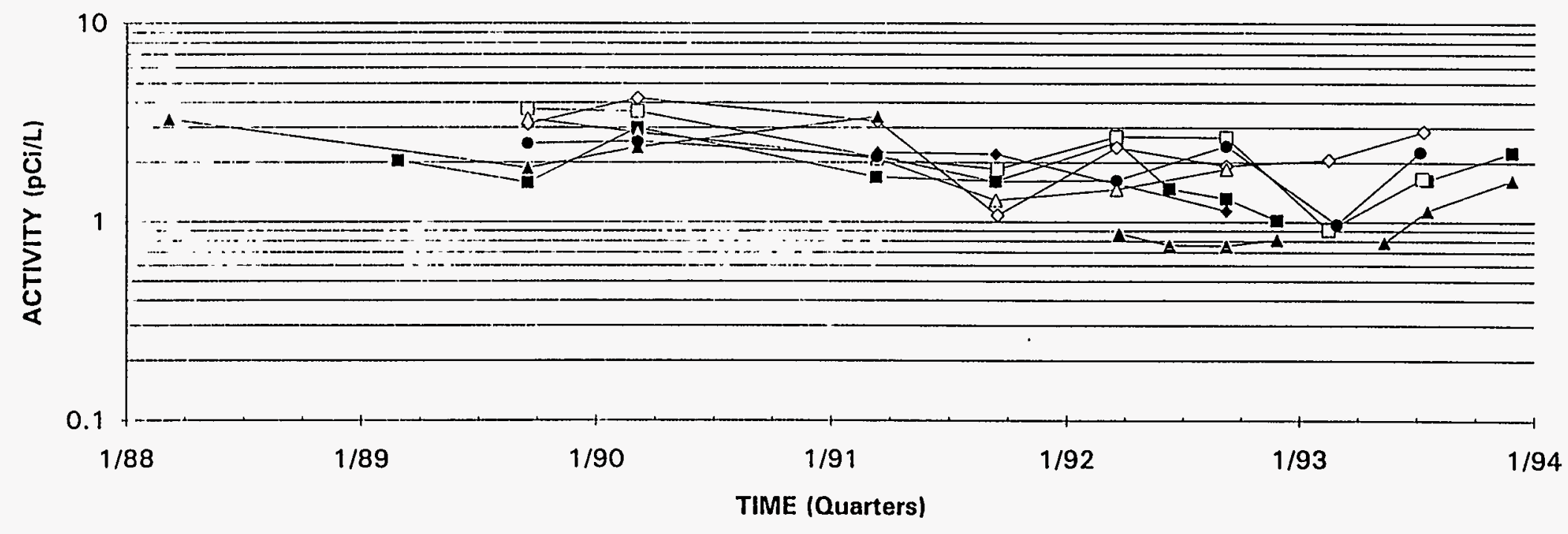

Unconnected lines indicate a dry well or a value below

the detection limit.

$\longrightarrow$ RSD $1-$ RSD 2A RSD 2B $\longrightarrow$ RSD 2C $\longrightarrow$ RSD 3
$\square$ RSD $4 \longrightarrow$ RSD 5

DWS: $20 \mathrm{pCi} / \mathrm{mL}$ 


\section{Tritium Activities \\ RSD Well Series (6 through 11)}

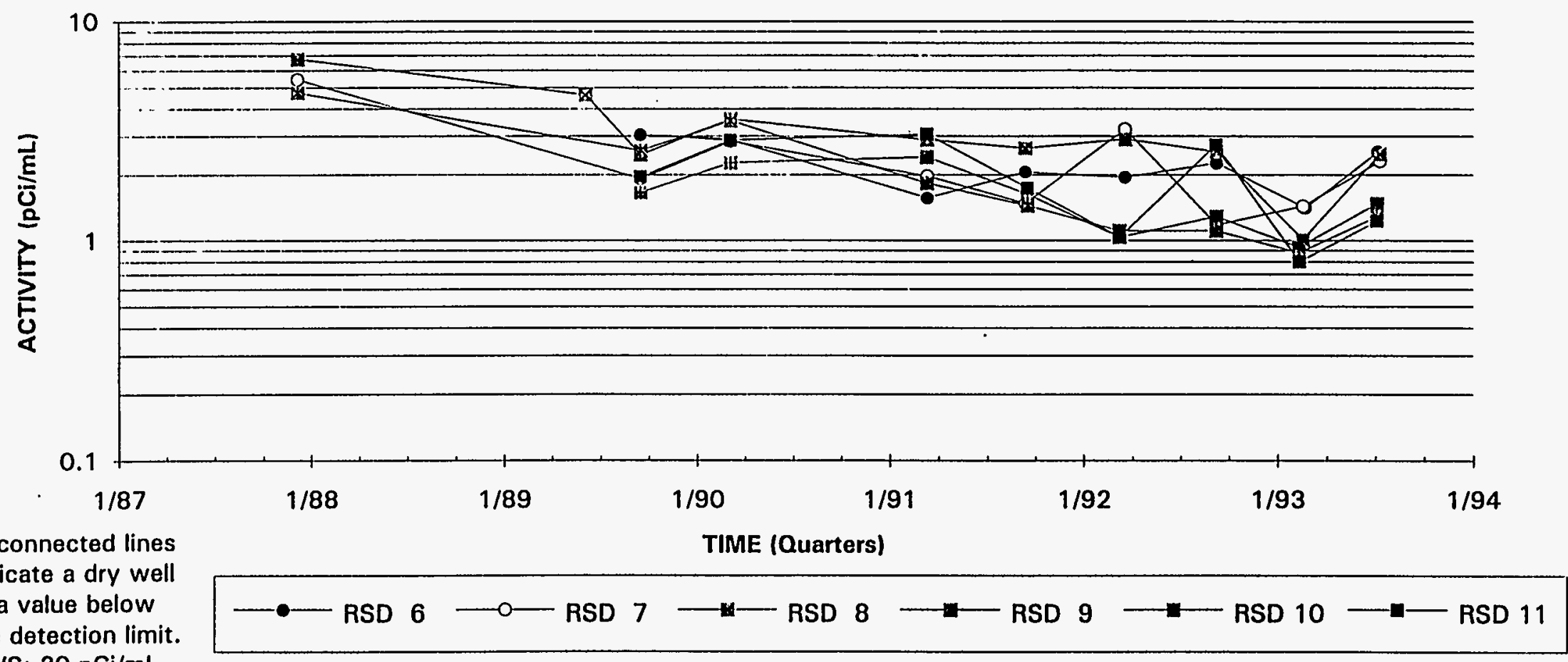
DWS: $20 \mathrm{pCi} / \mathrm{mL}$ 


\section{Tritium Activities \\ RSE Well Series (1A through 3A)}

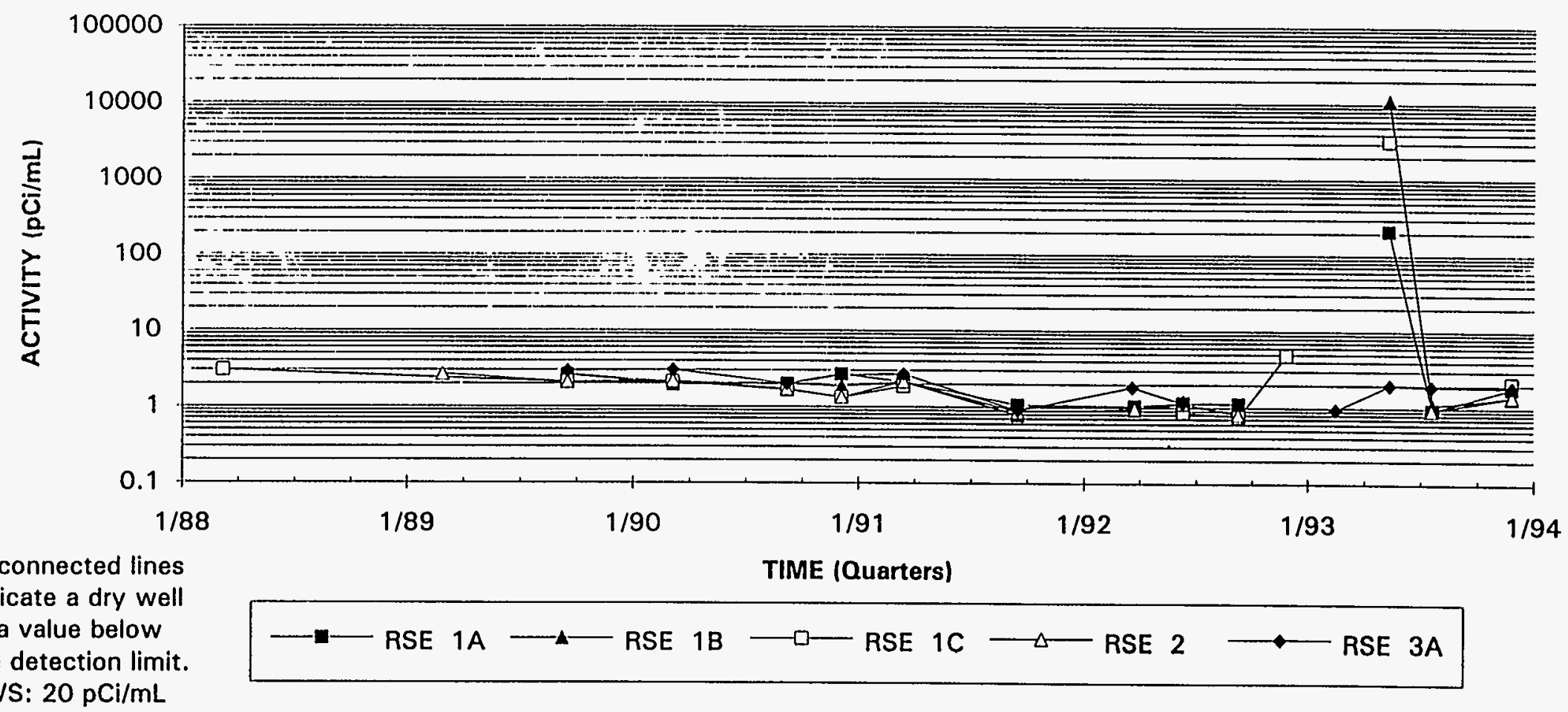




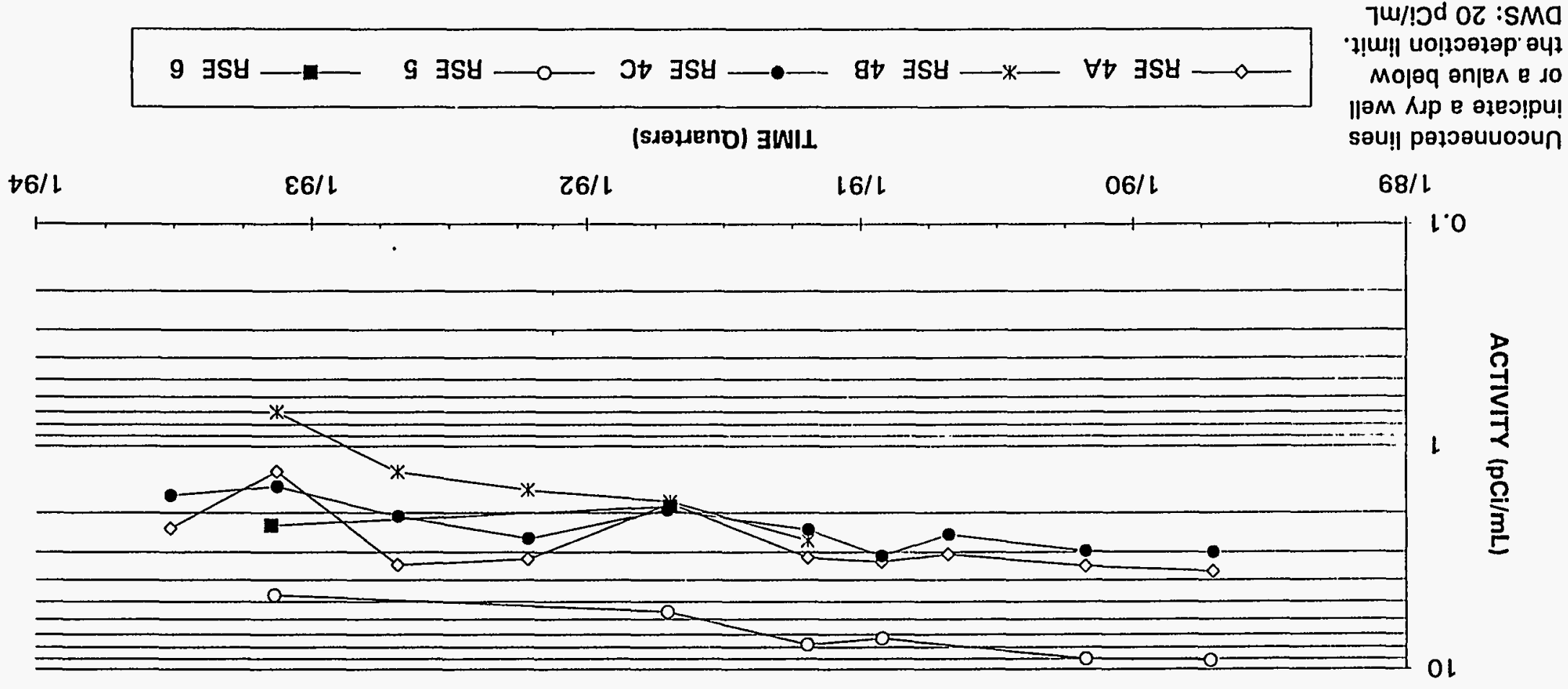

(9 46noגчl $\forall t$ ) sə!ıәS ॥әM $\exists S y$

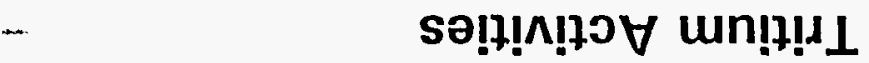


Tritium Activities

RSE Well Series (7 through 11)

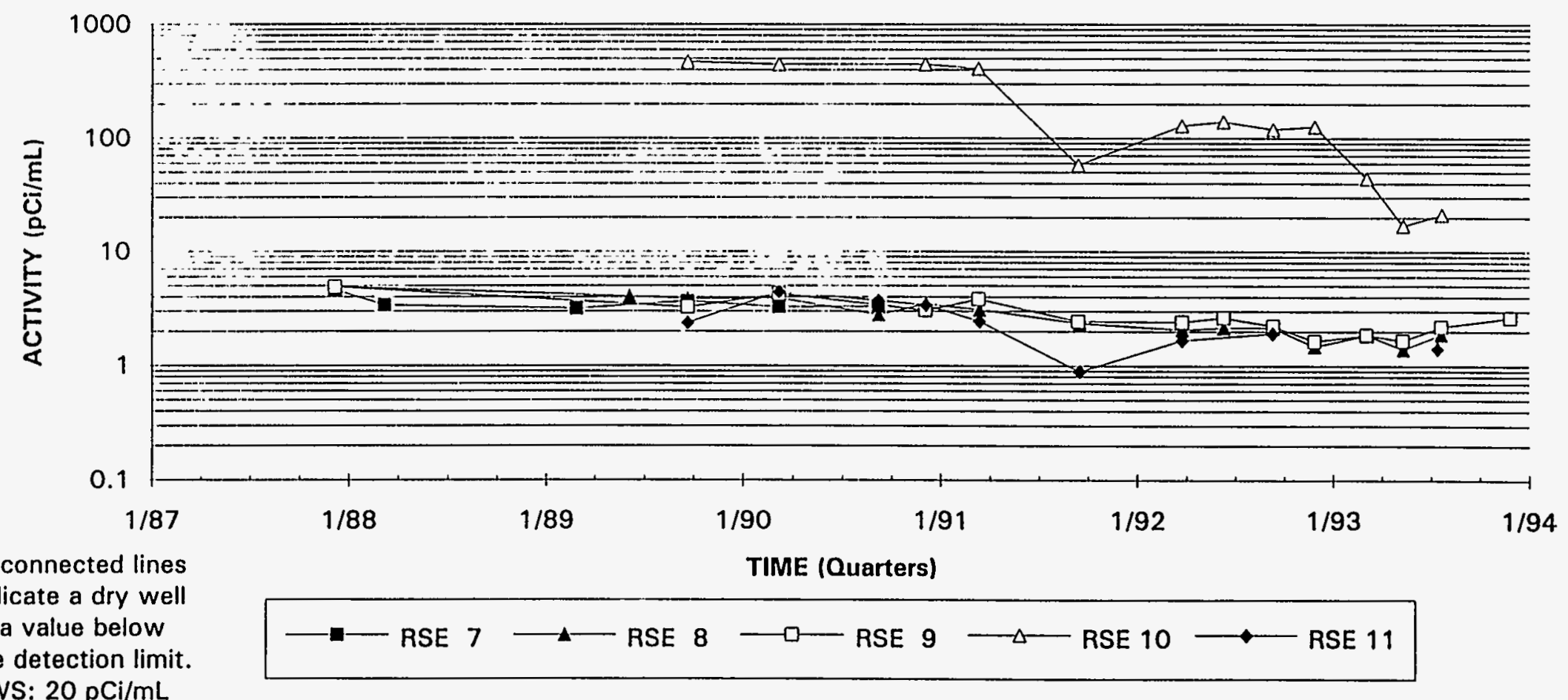

the detection limit. 


\section{Tritium Activities \\ RSE Well Series (12 through 25)}

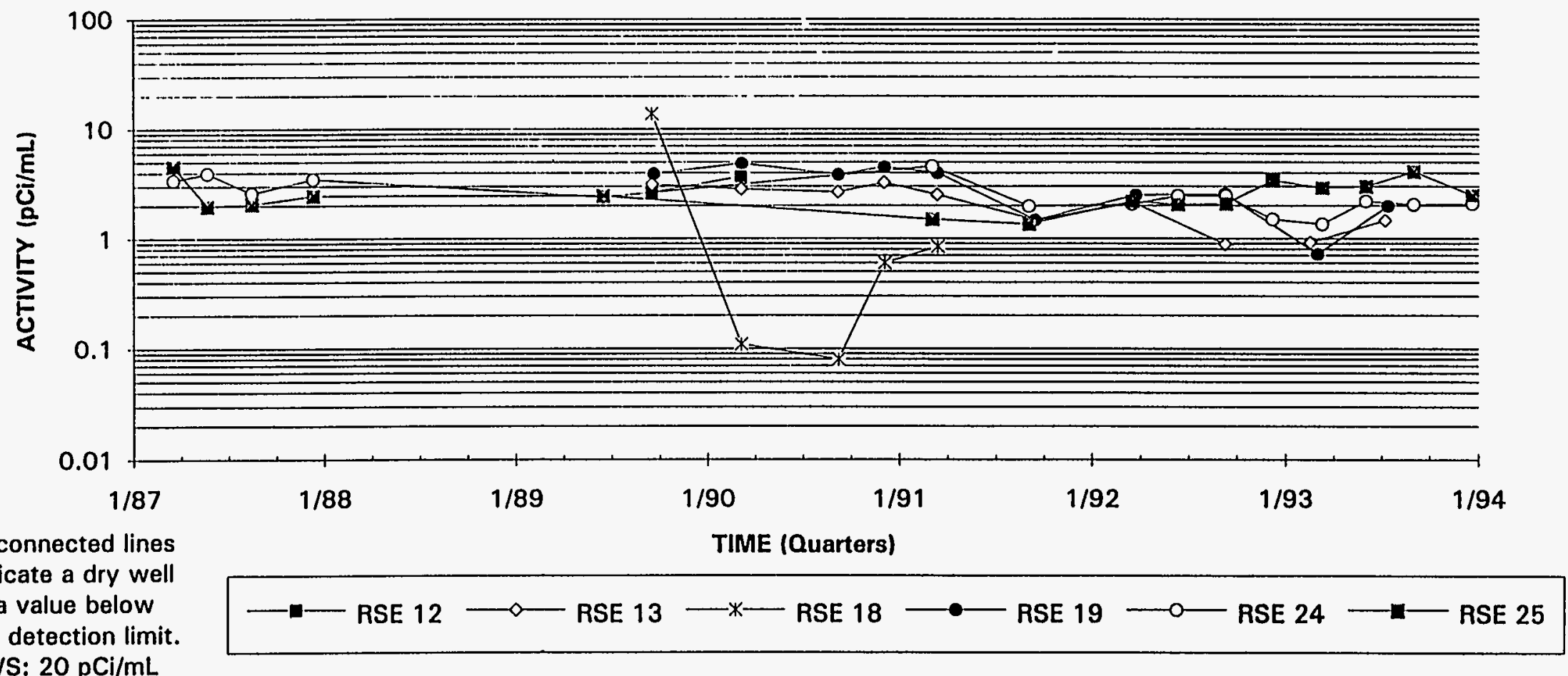




\section{Tritium Activities}

\section{RSF Well Series}

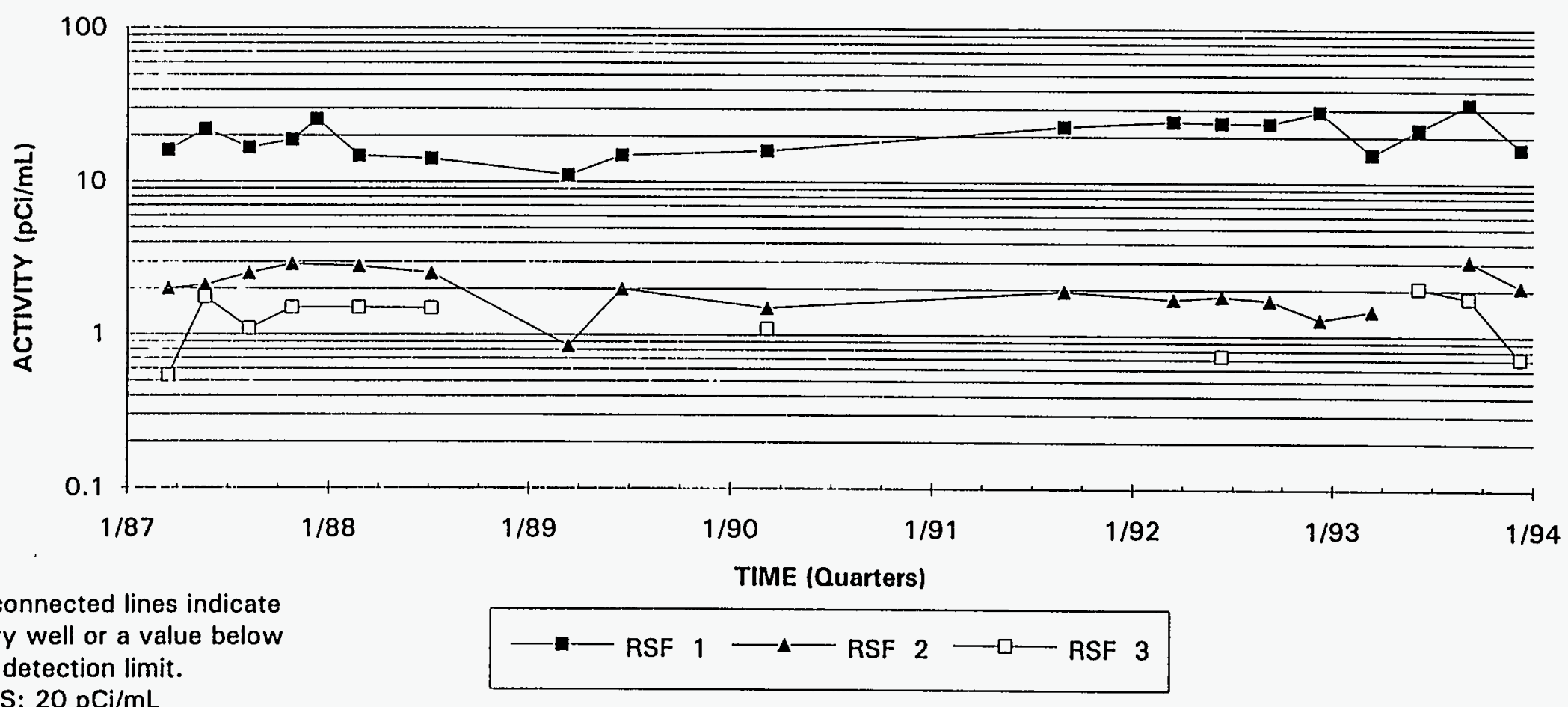




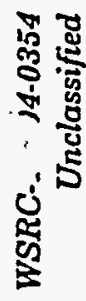
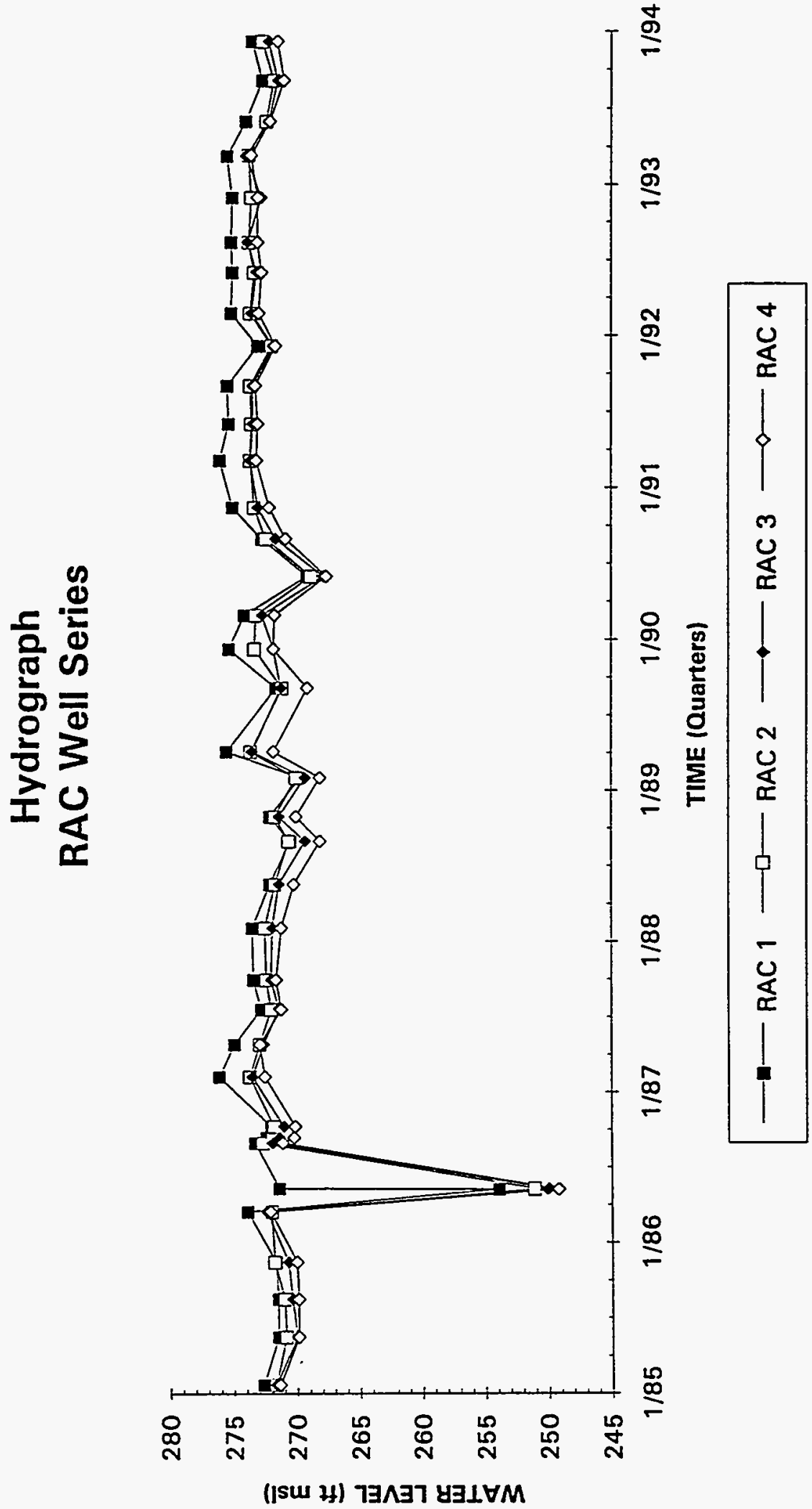

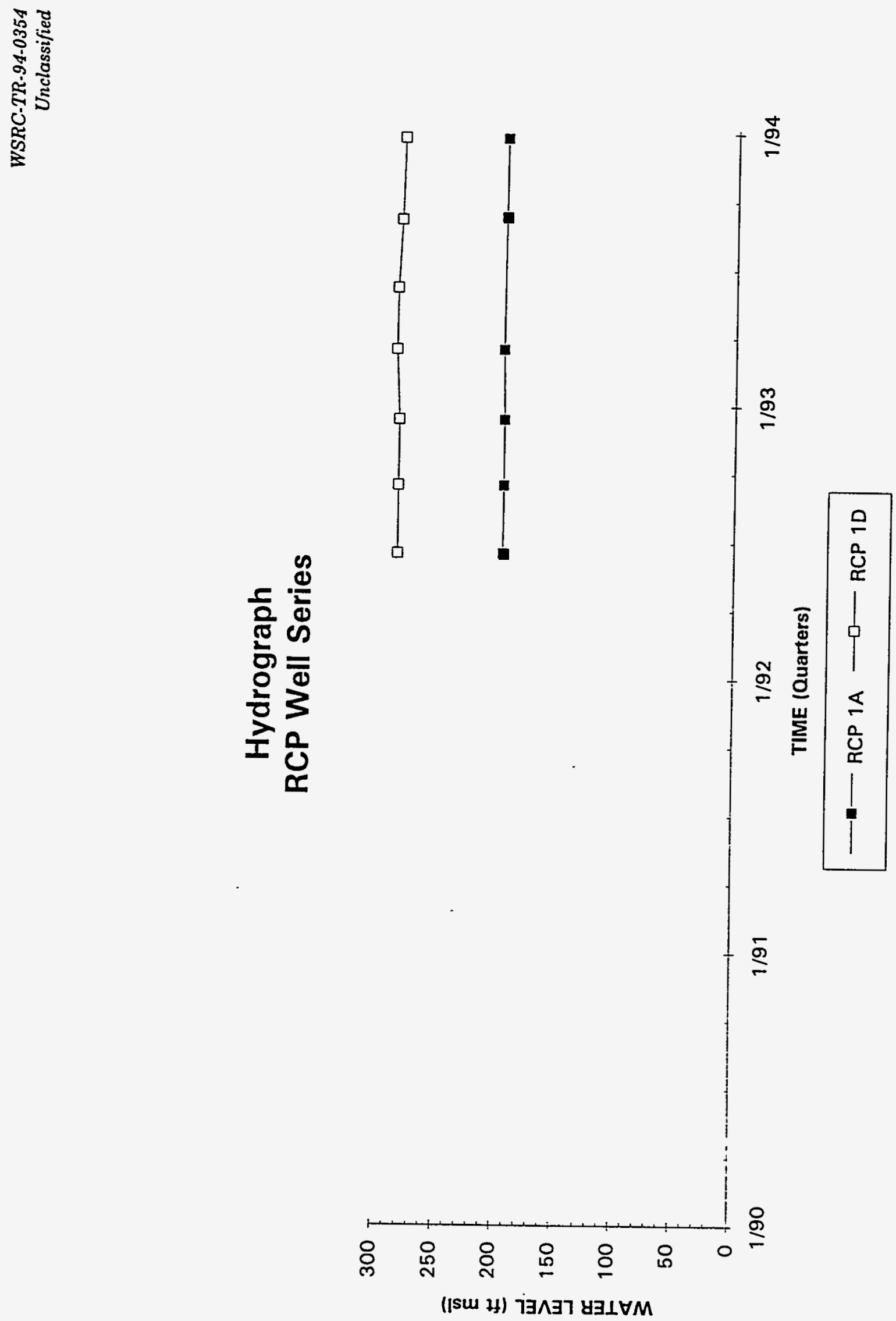


\section{Hydrograph}

\section{RDB Well Series}

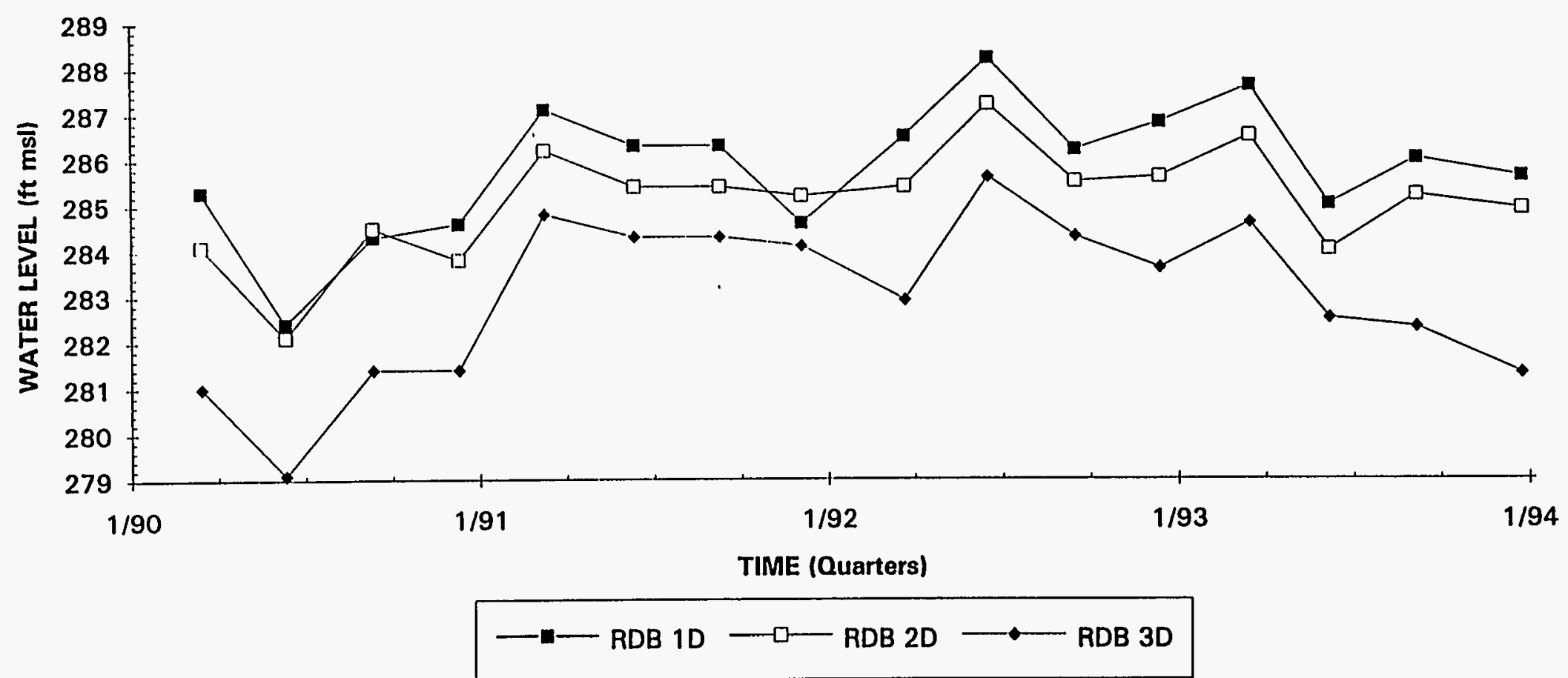




\section{Hydrograph \\ RRP Well Series}

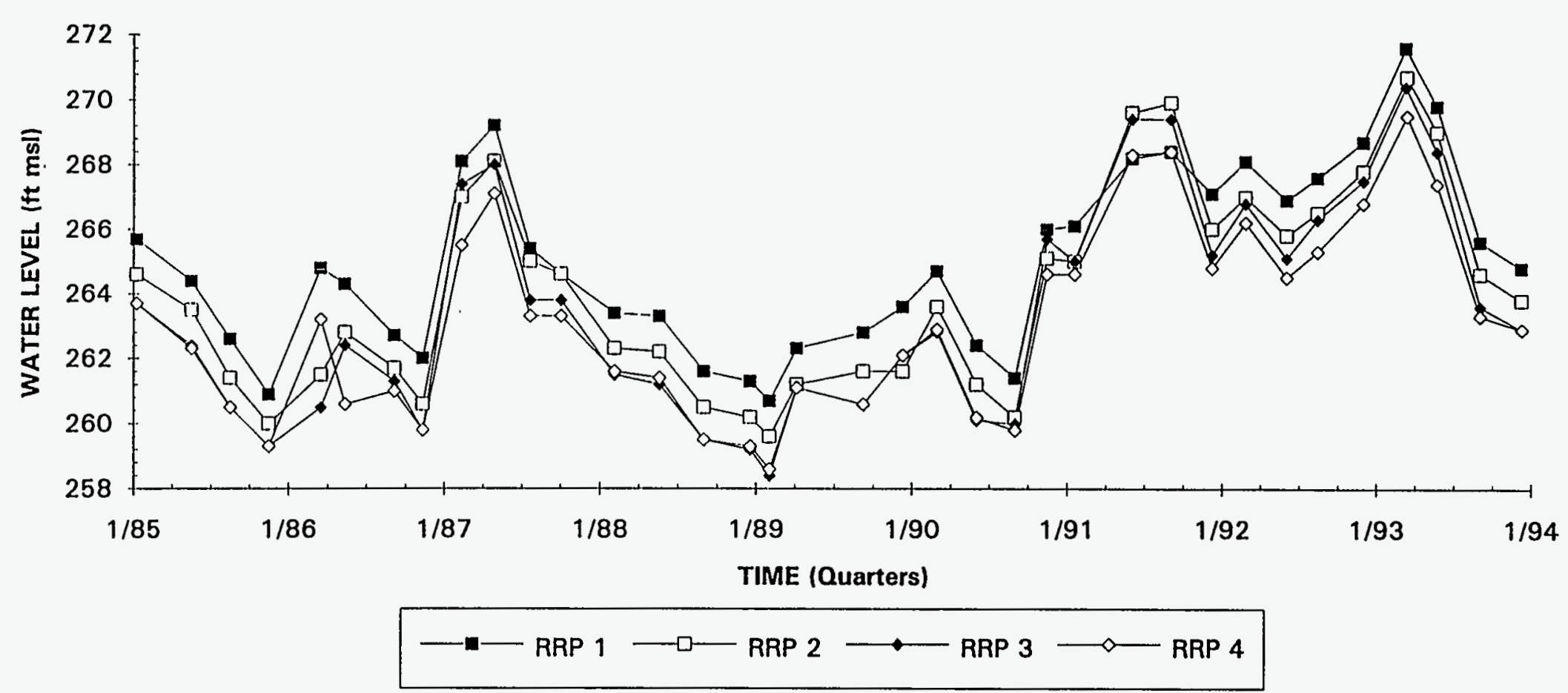




\section{Hydrograph \\ RSA Well Series}

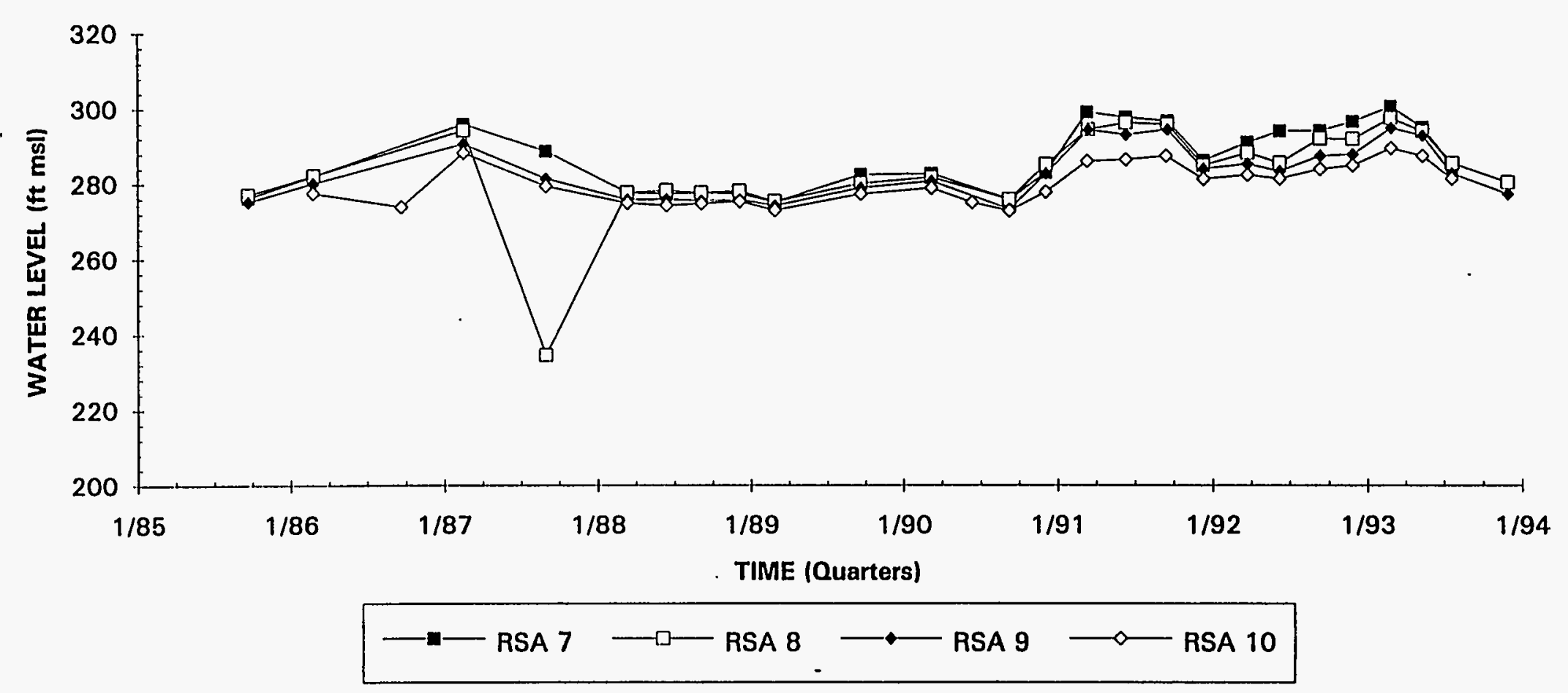

R-Area Reactor 1993 Annual Report 


\section{Hydrograph \\ RSB Well Series}

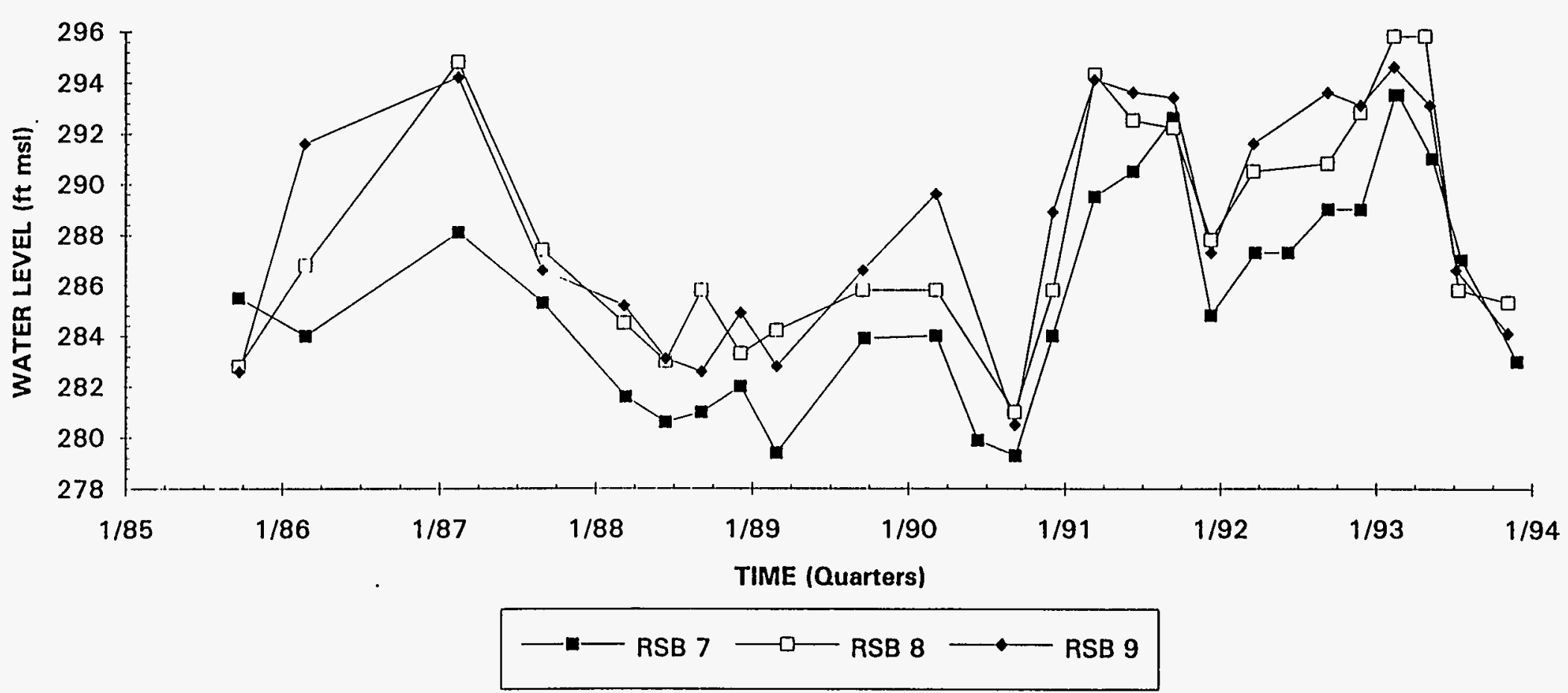




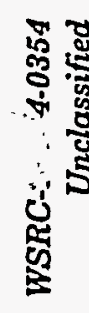

تี
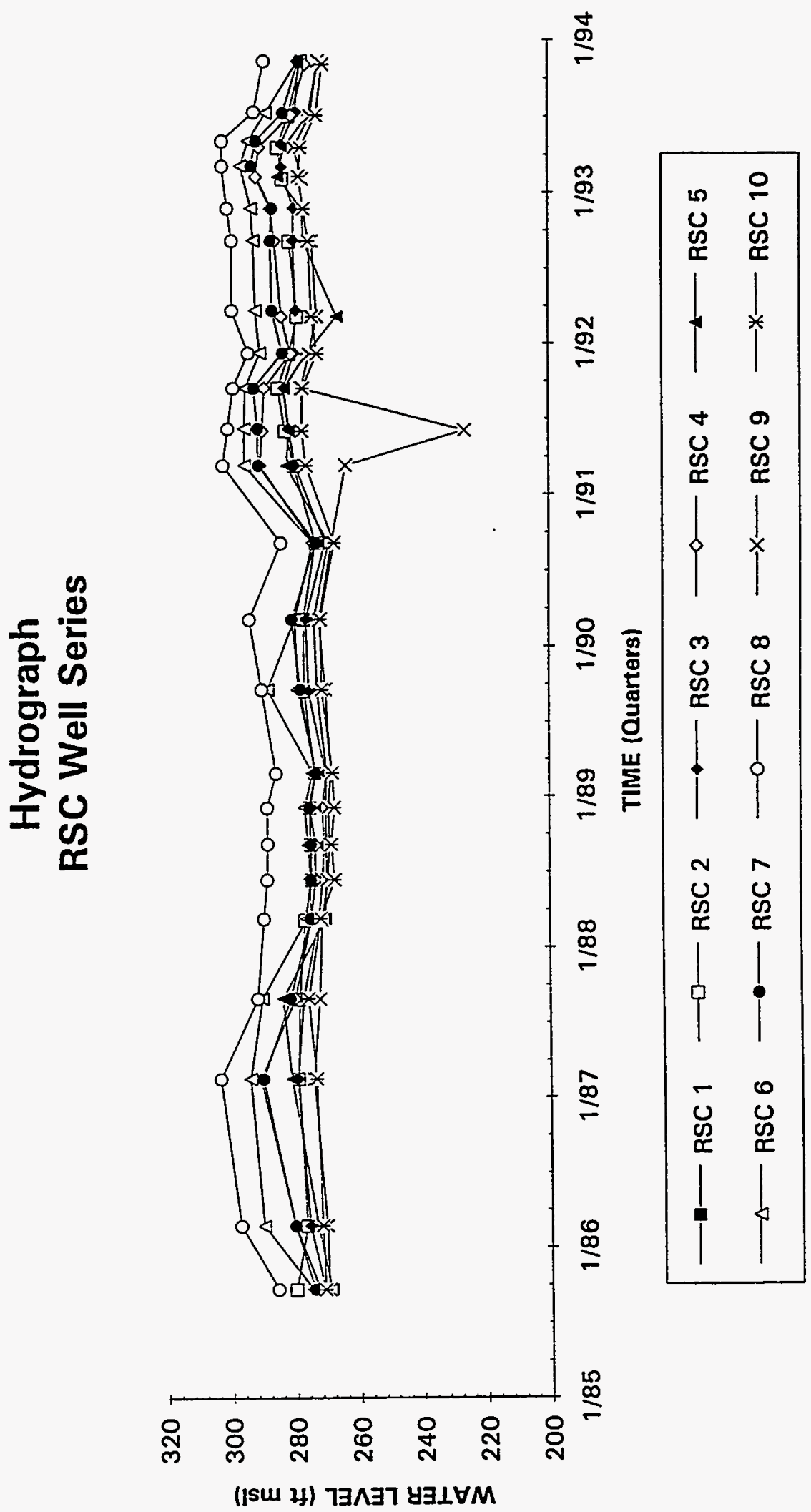


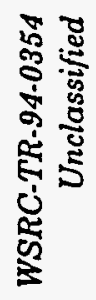
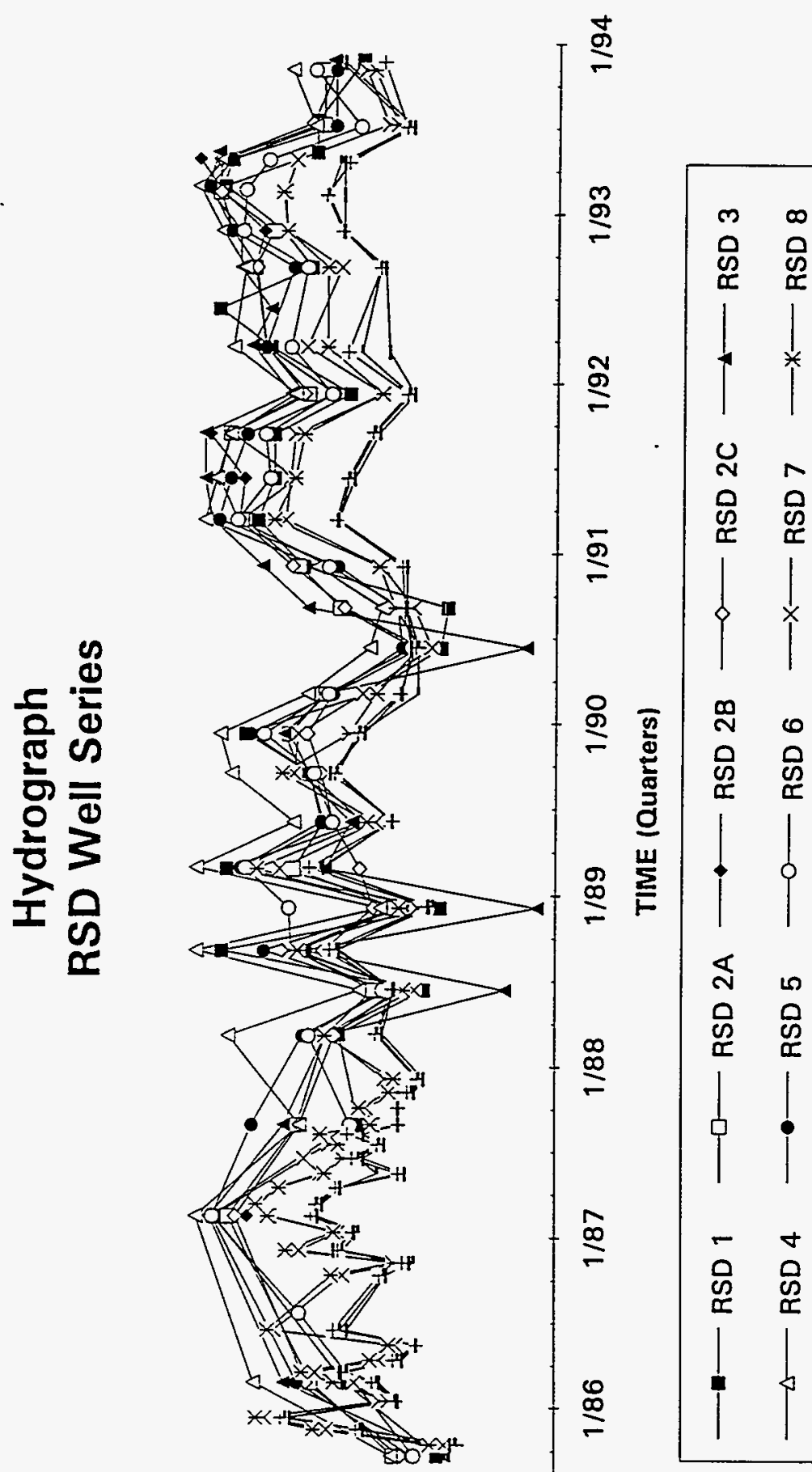

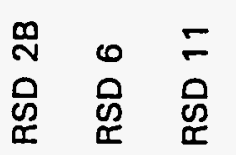
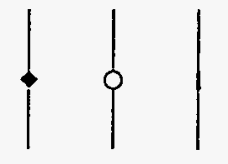

ㅈํ 10 음

邑

$\stackrel{\infty}{\stackrel{\infty}{\sim}}$

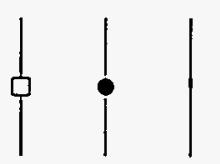

$\stackrel{م}{\sigma}$

$\begin{array}{lll}5 & + & 0 \\ 0 & 0 & 0 \\ 0 & 0 & 0 \\ \pi & 0\end{array}$

$\stackrel{\mathscr{\infty}}{\underline{2}}$

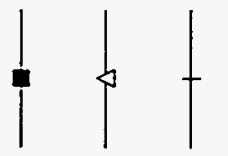

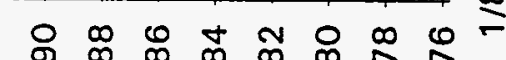

จิ

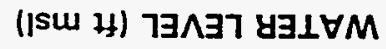




\section{Hydrograph \\ RSE Well Series (1 through 7)}

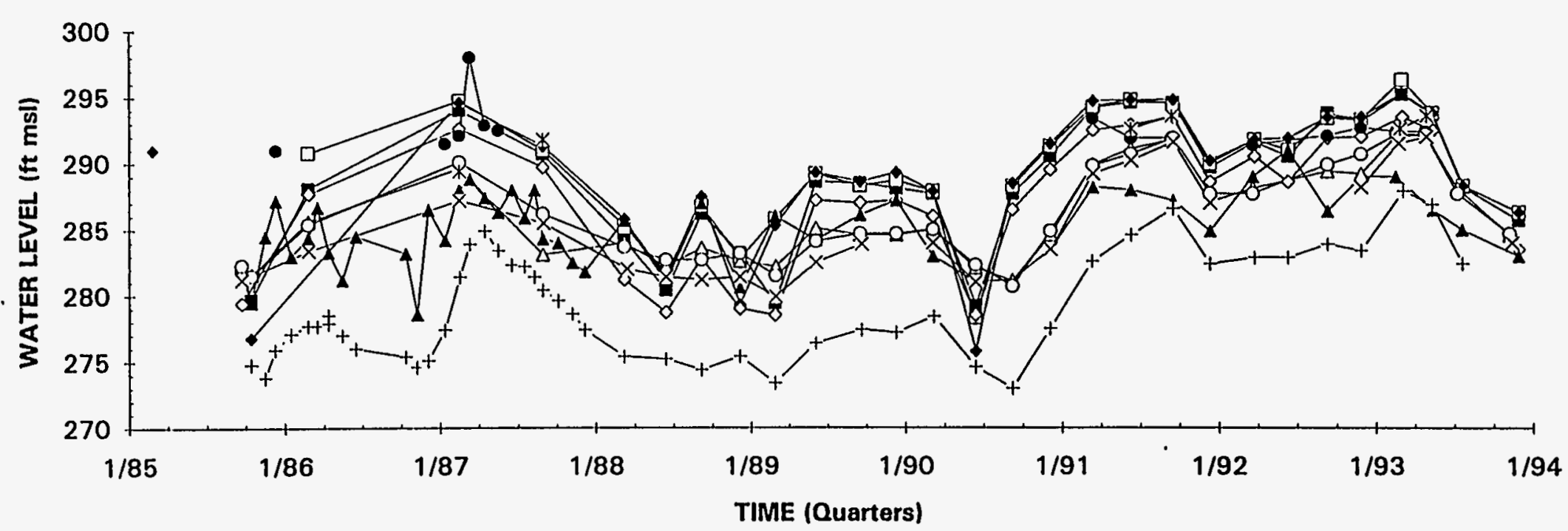

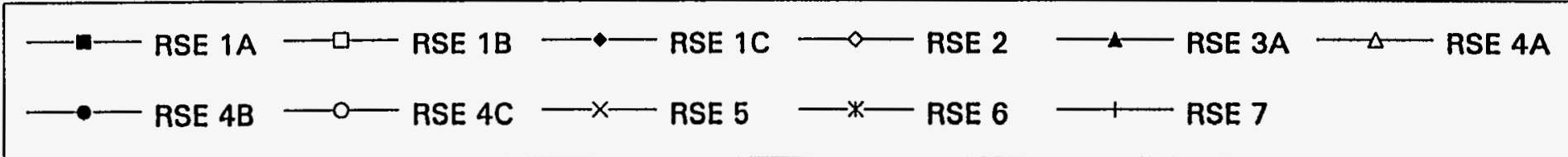




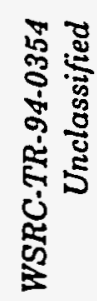

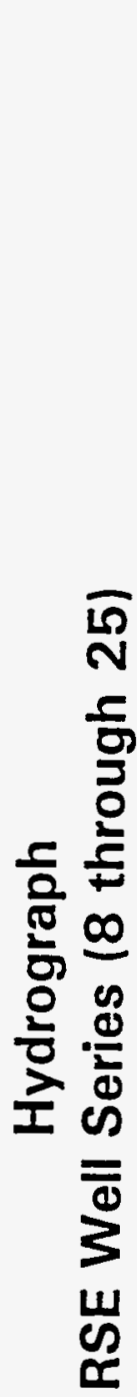

בัّ 


\section{Hydrograph}

\section{RSF Well Series}

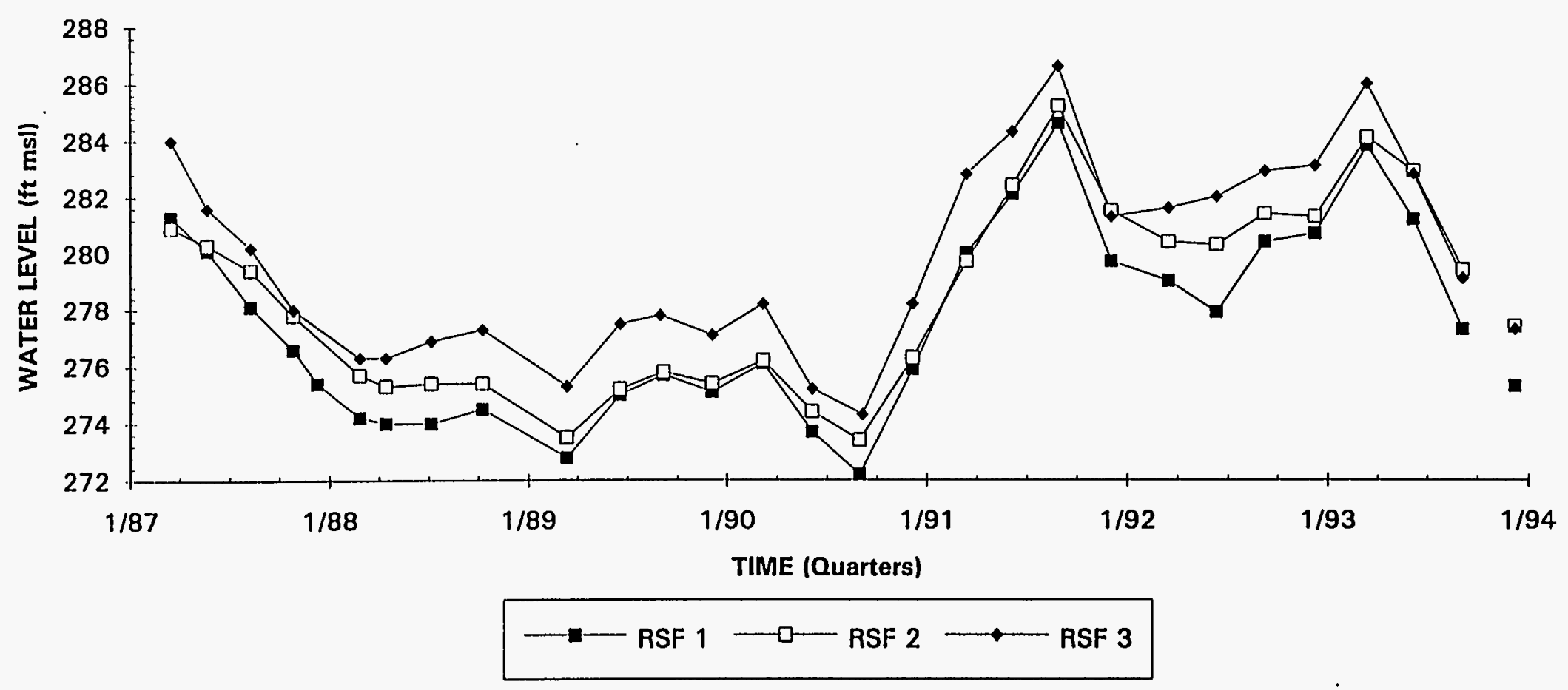




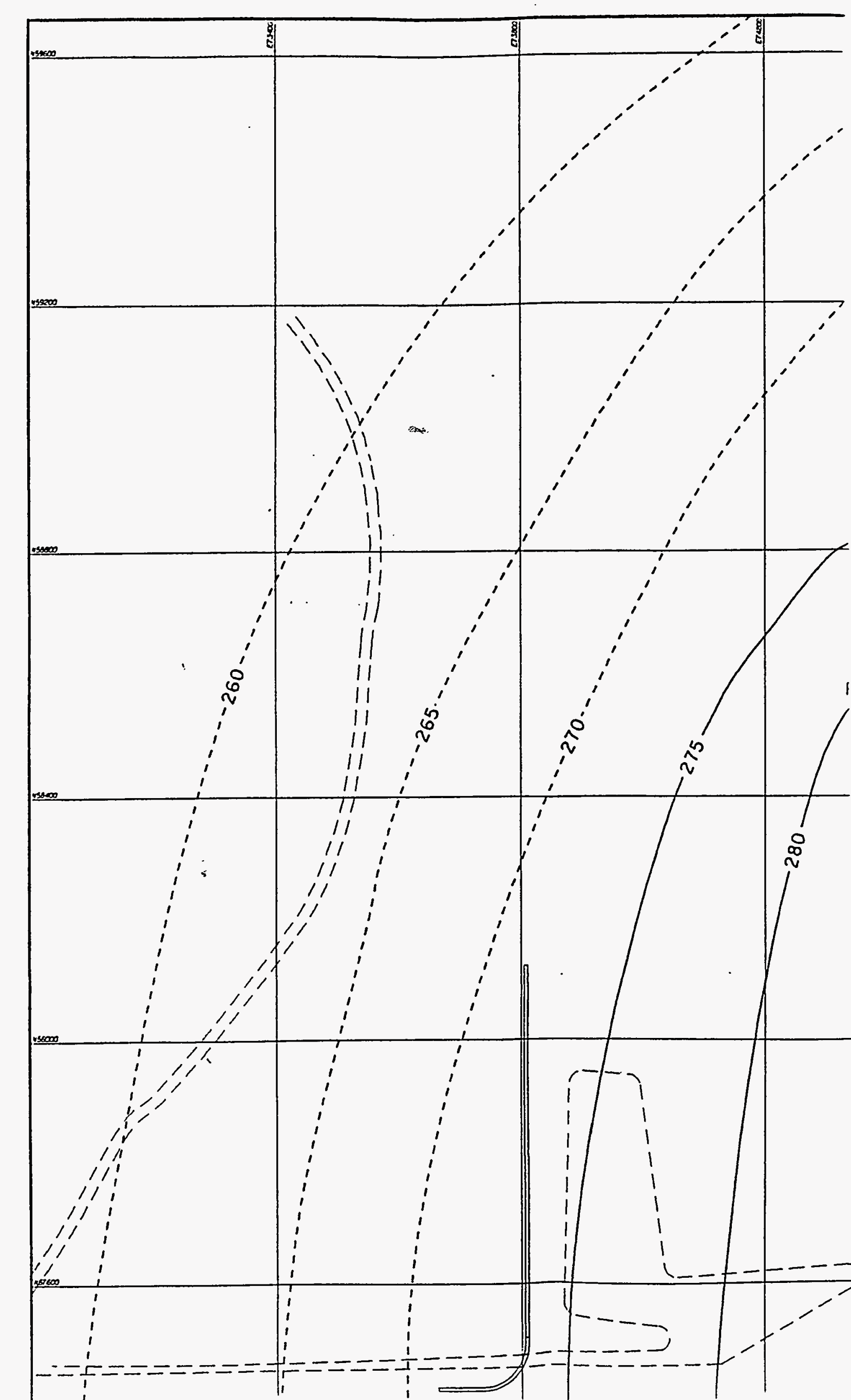




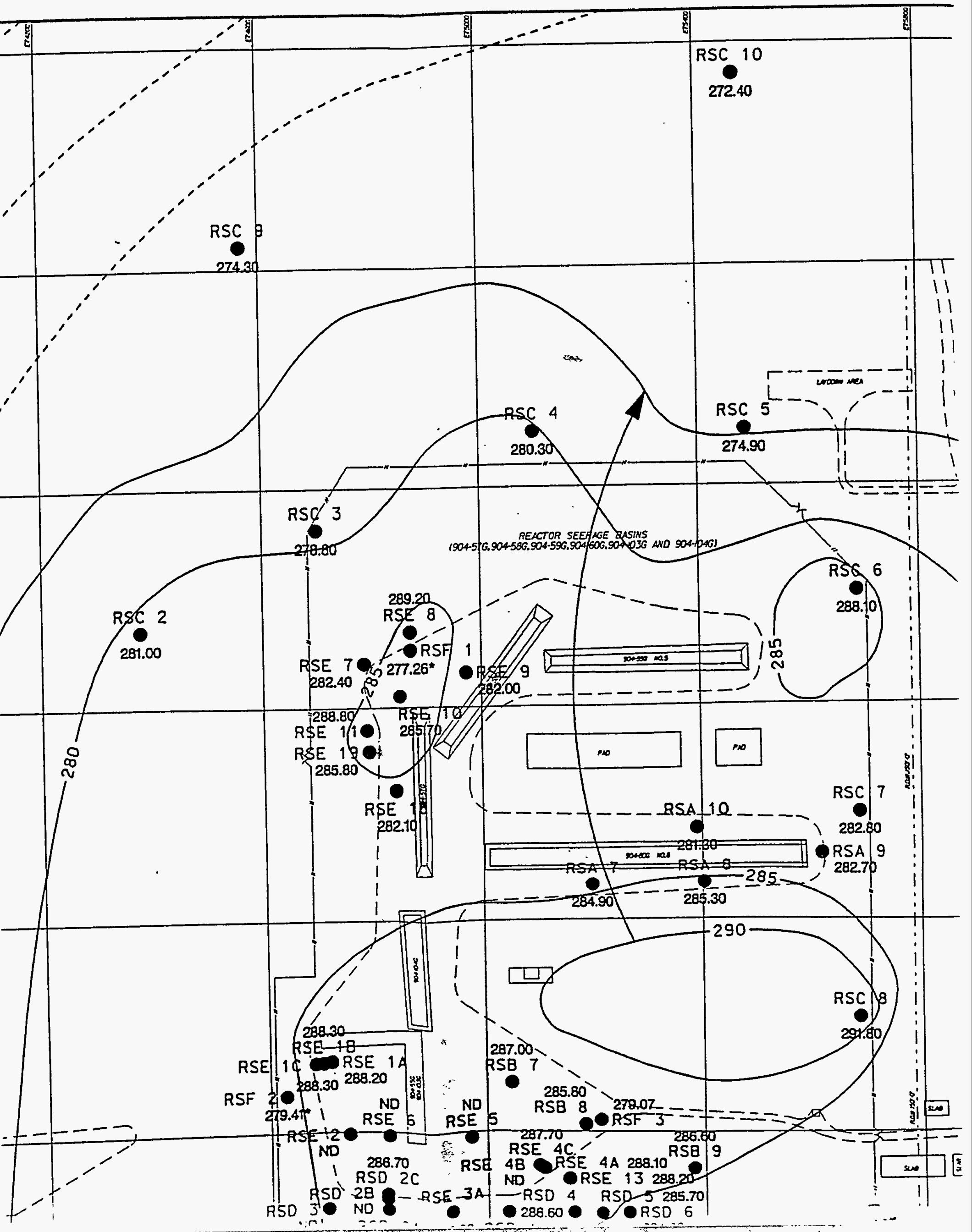




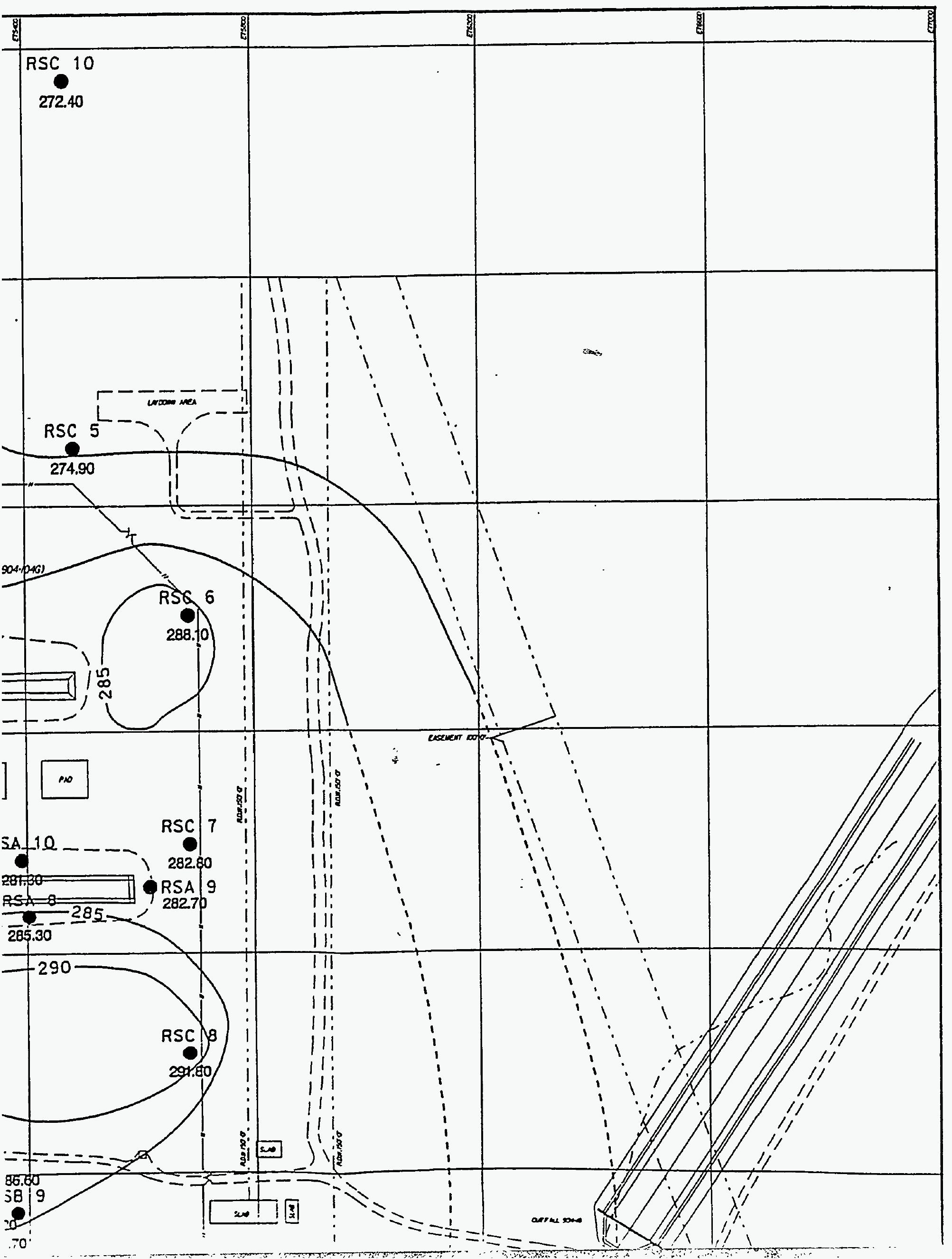




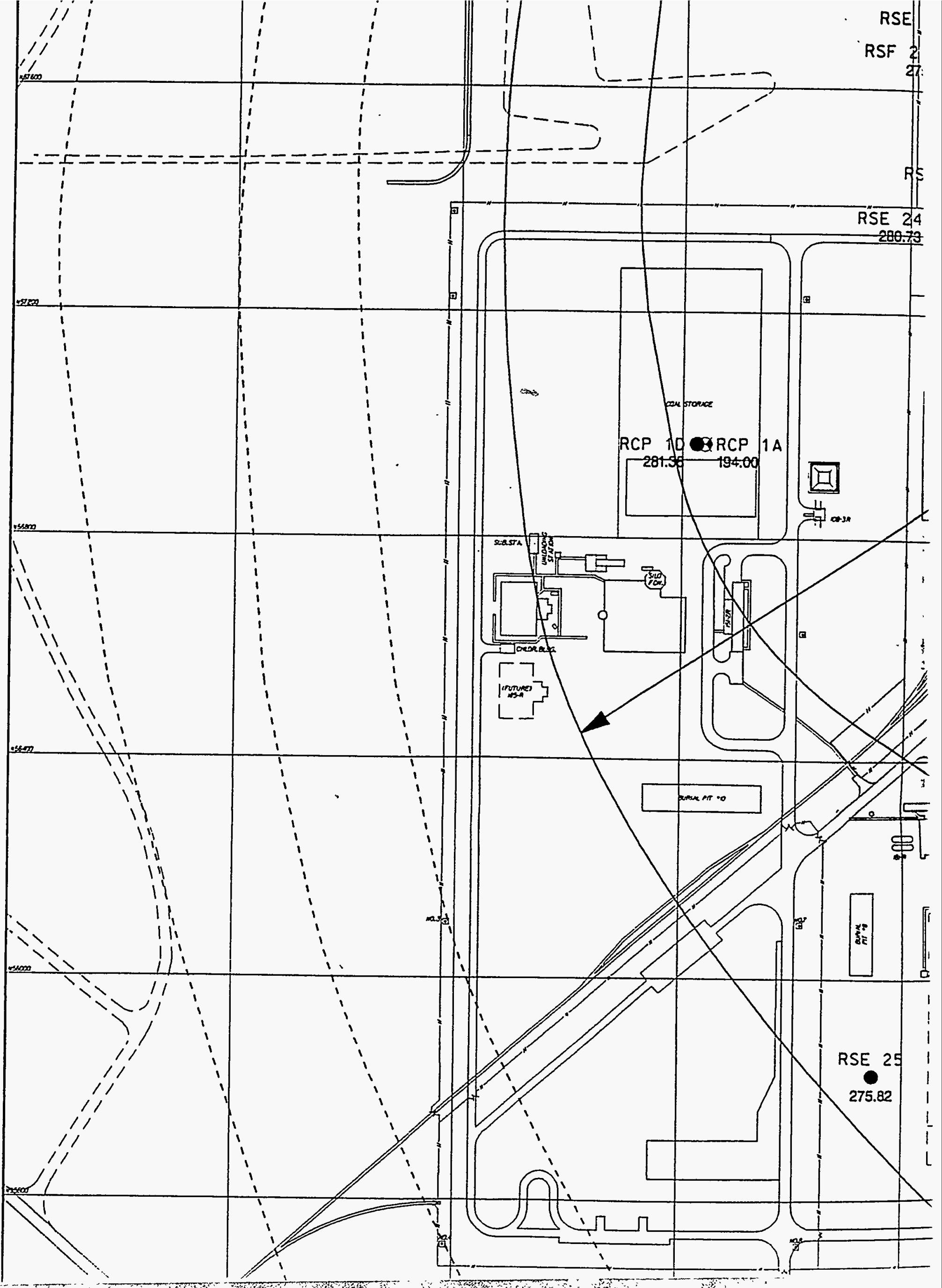




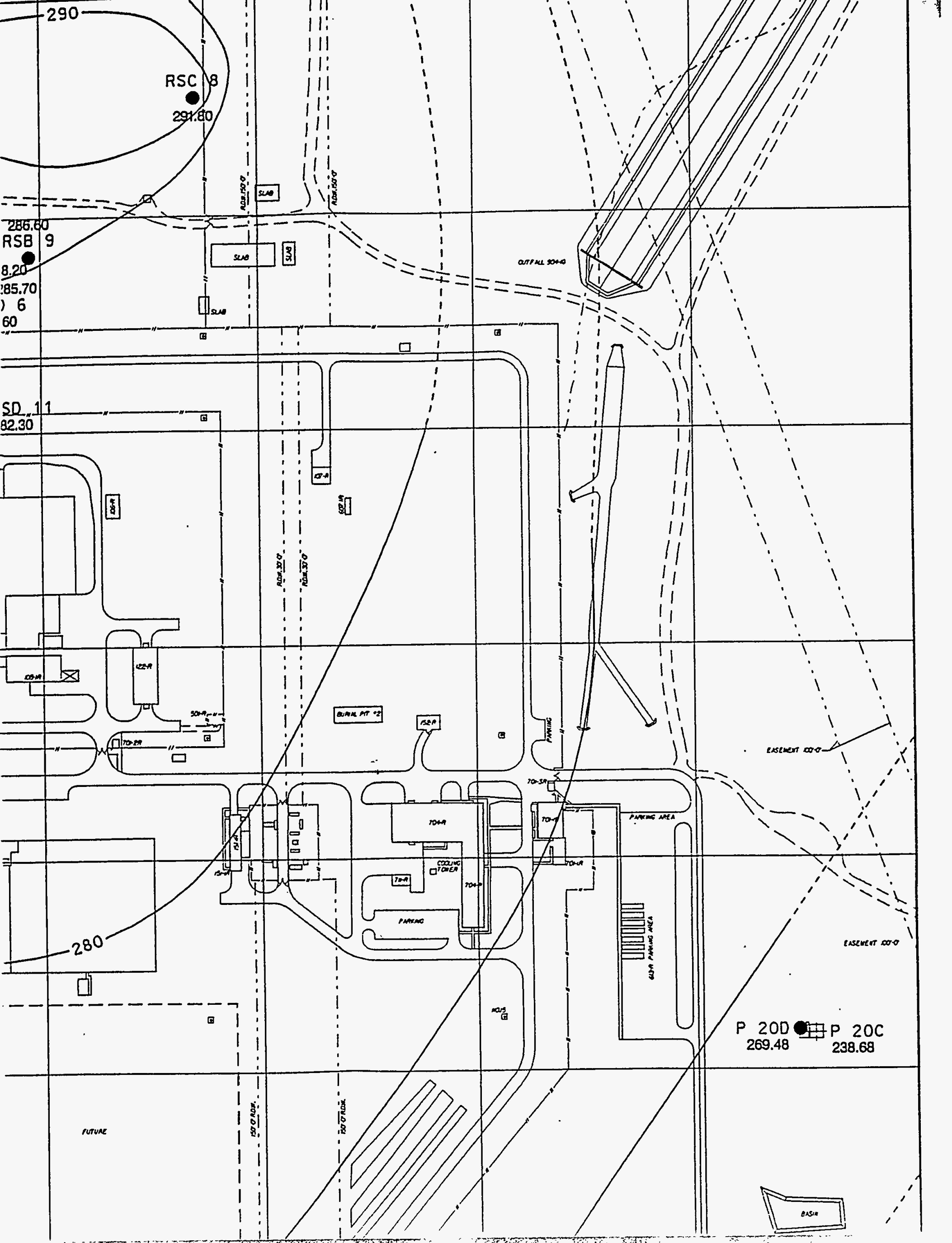




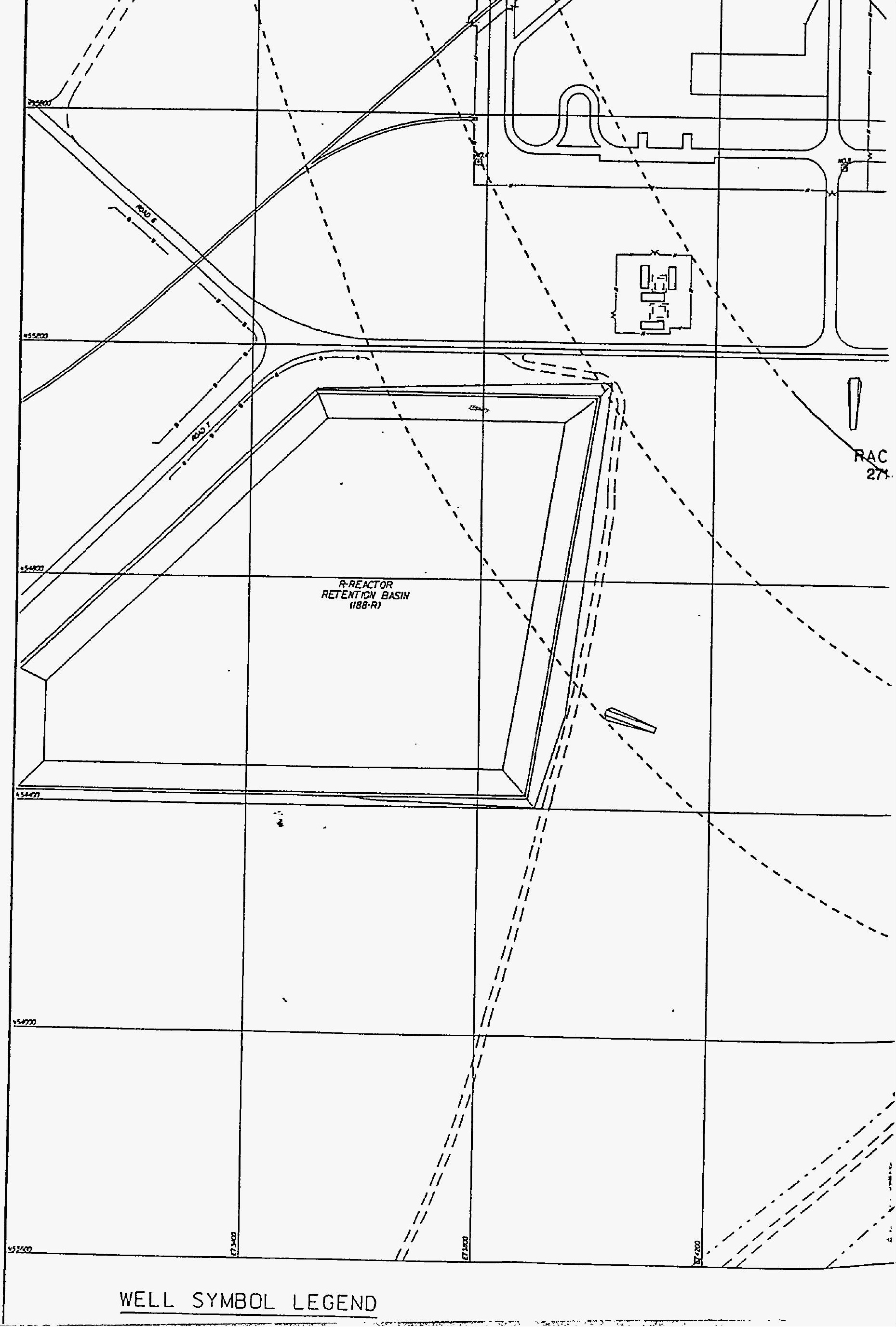




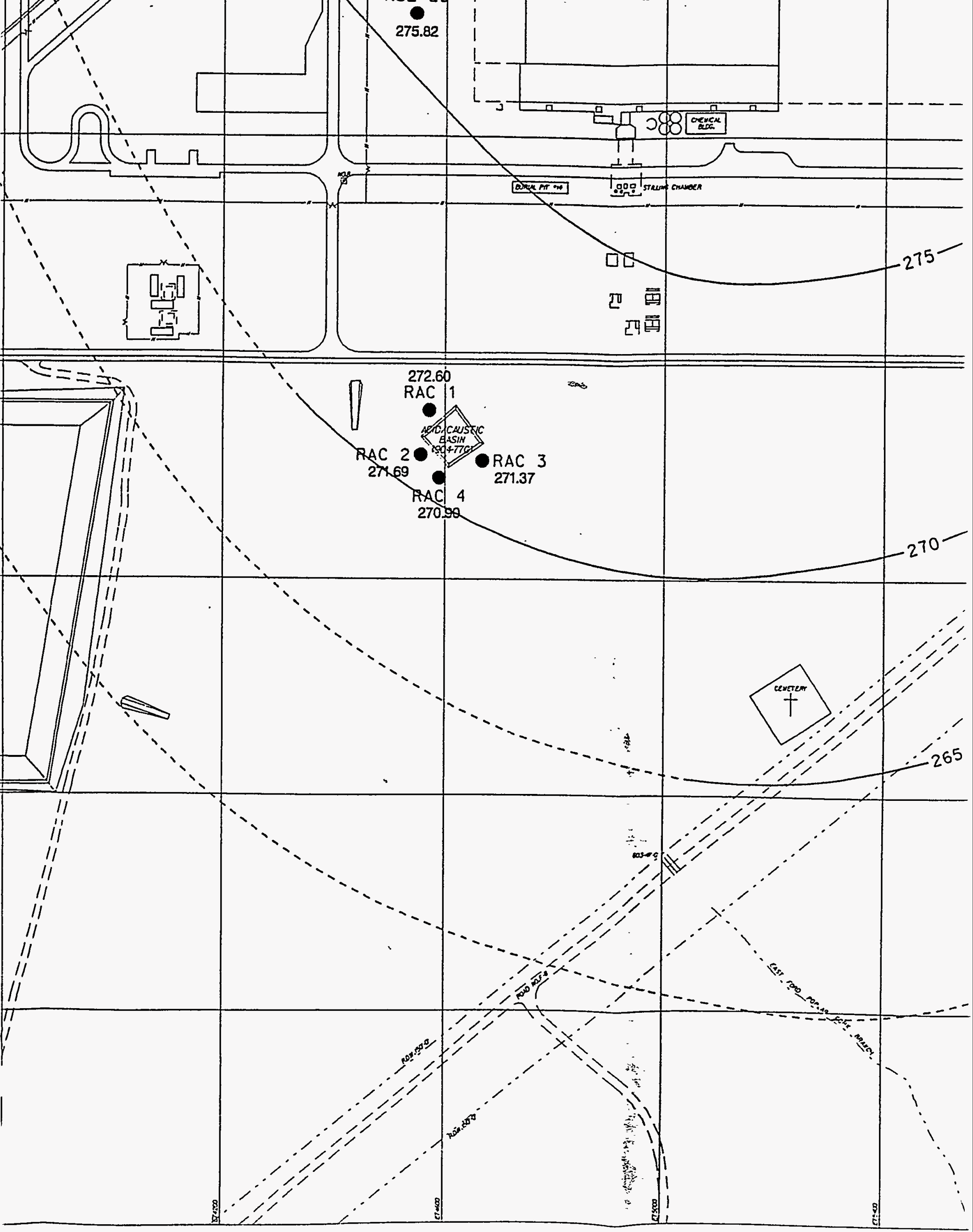




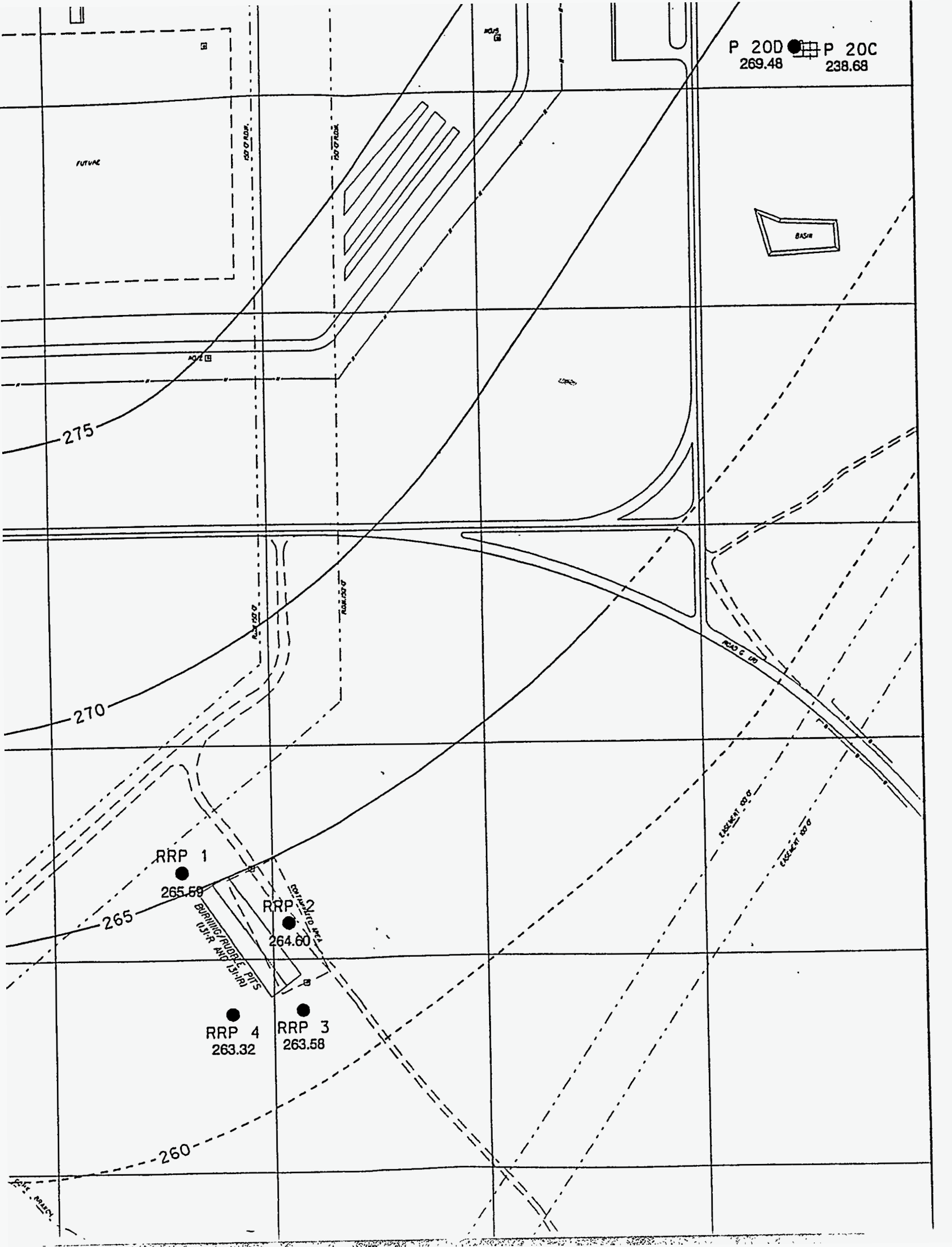




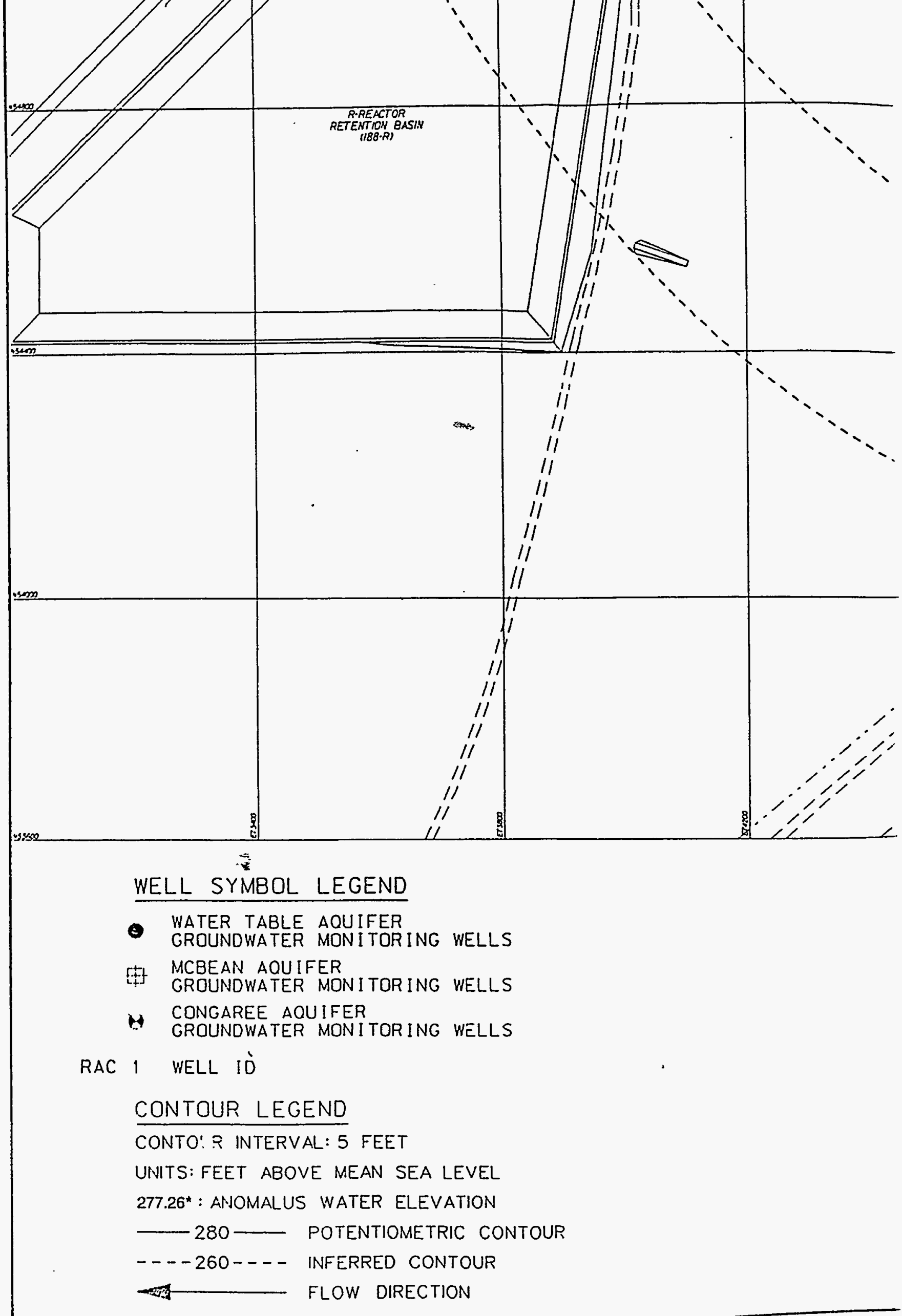




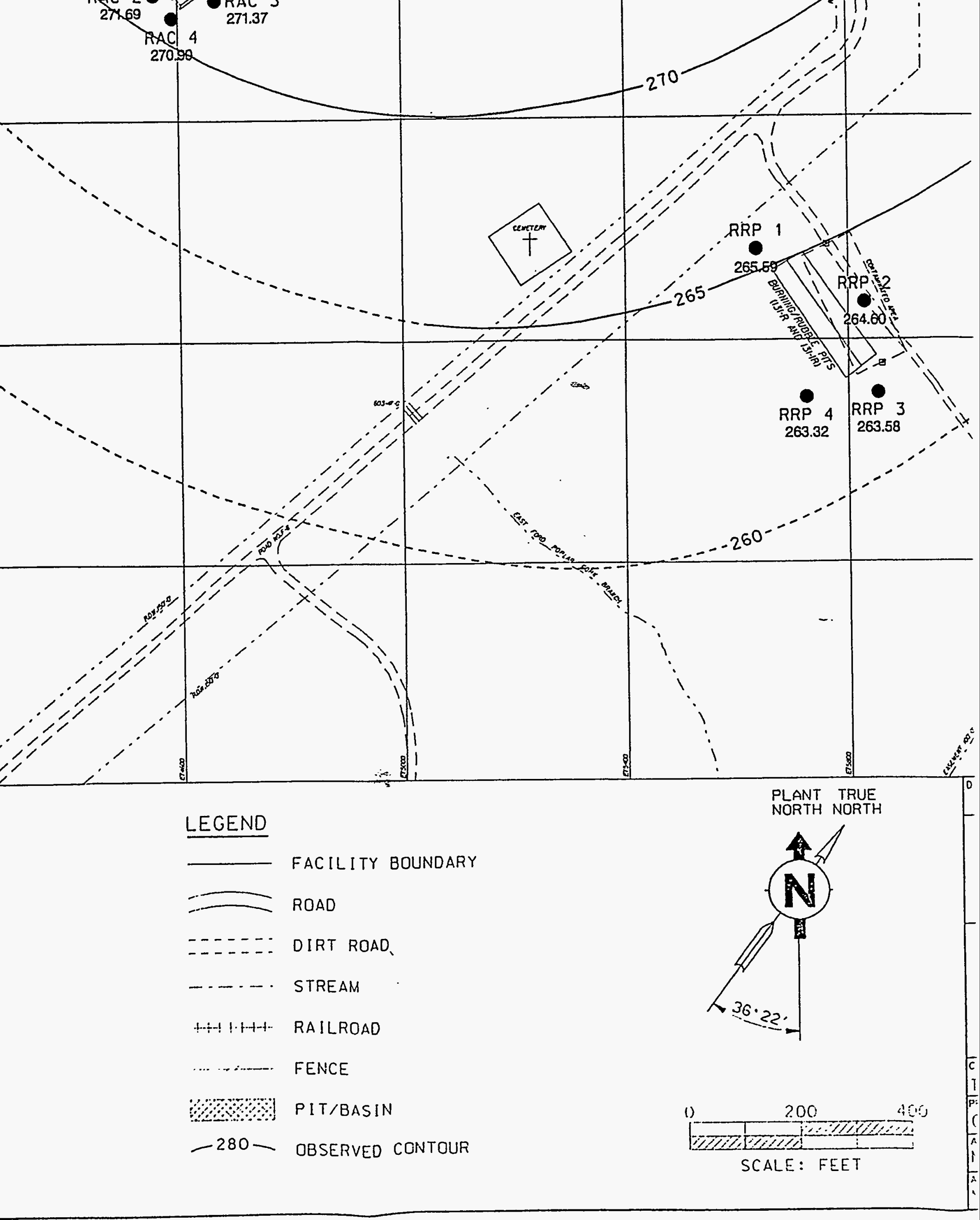




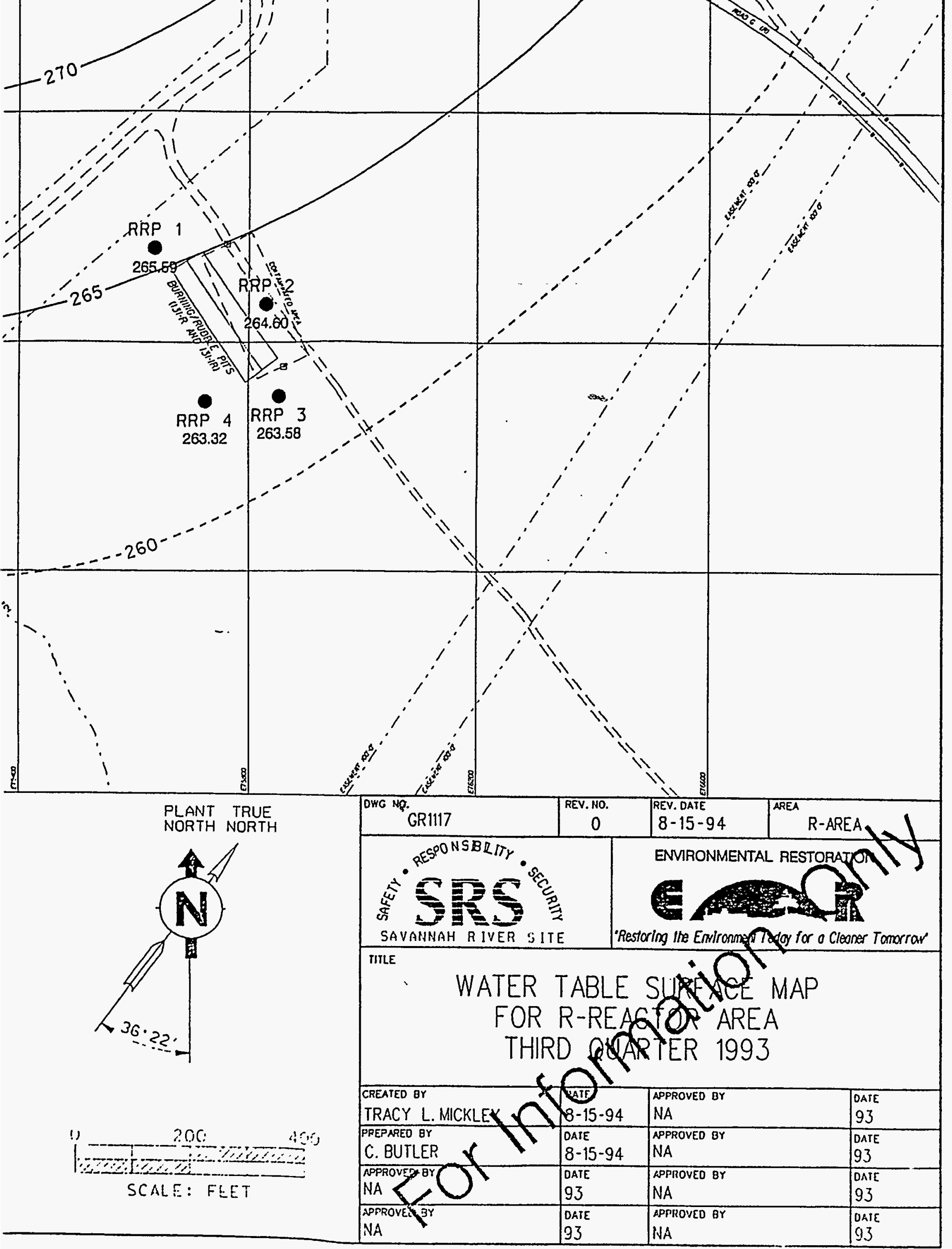




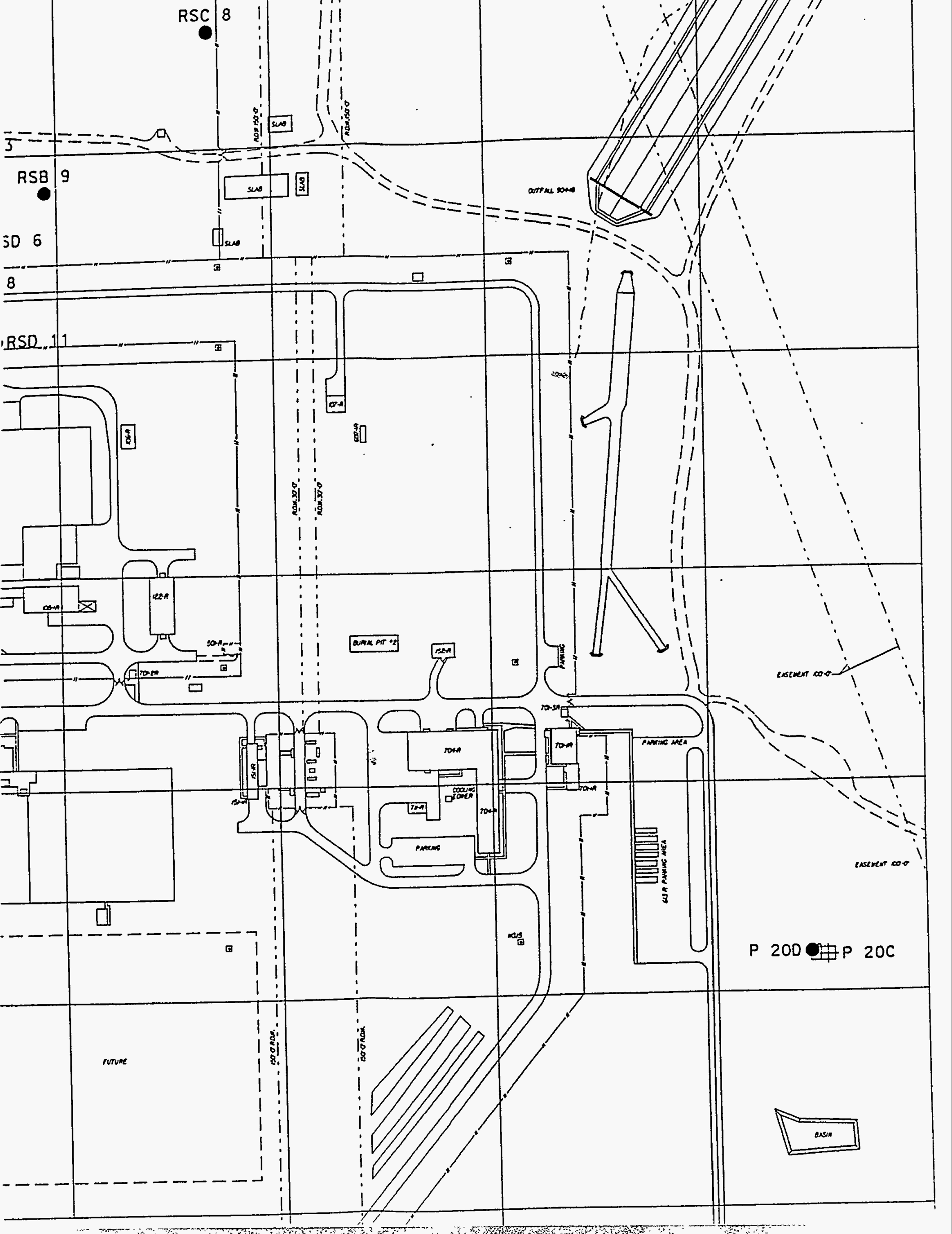




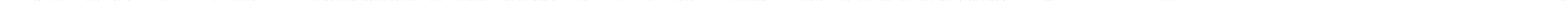




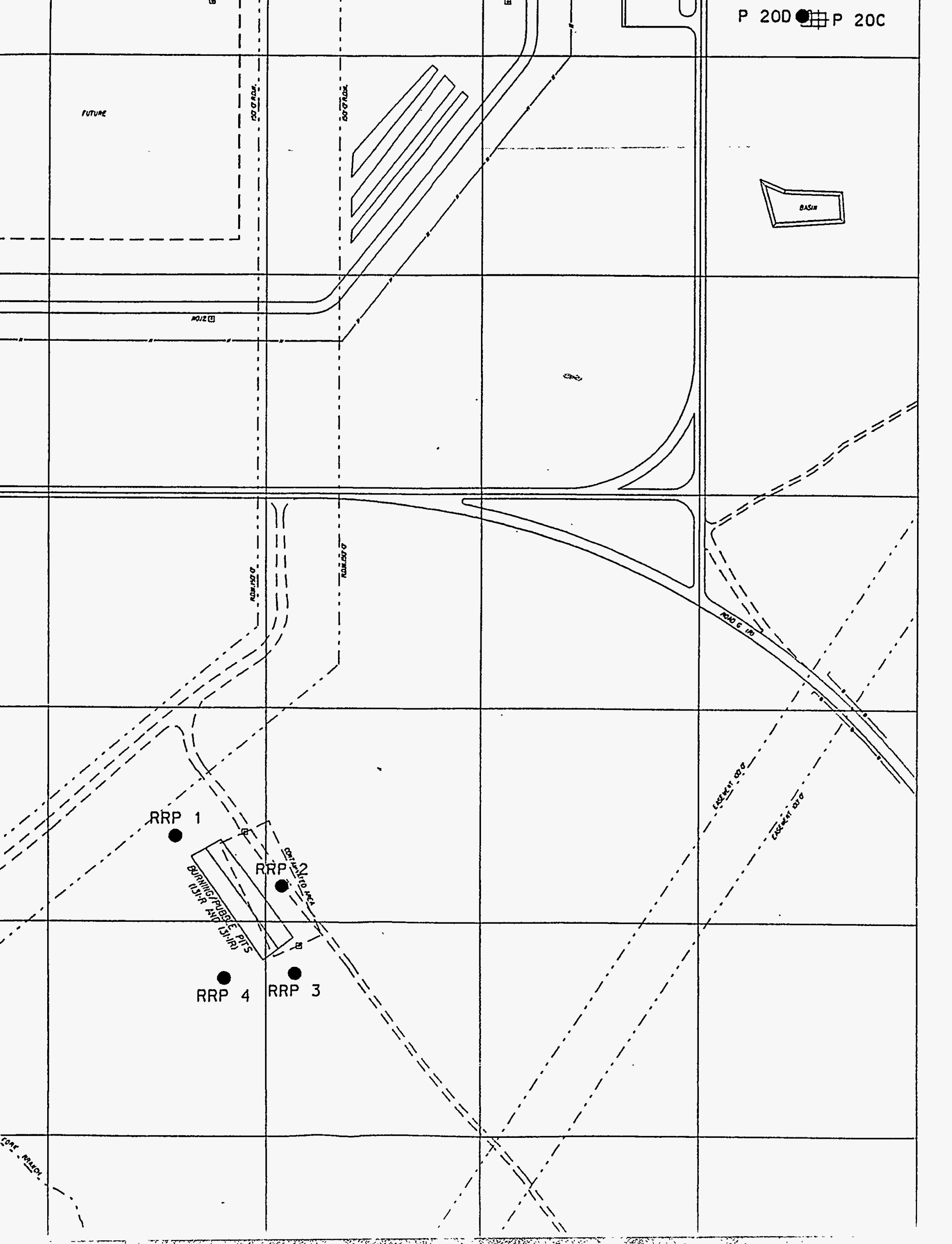




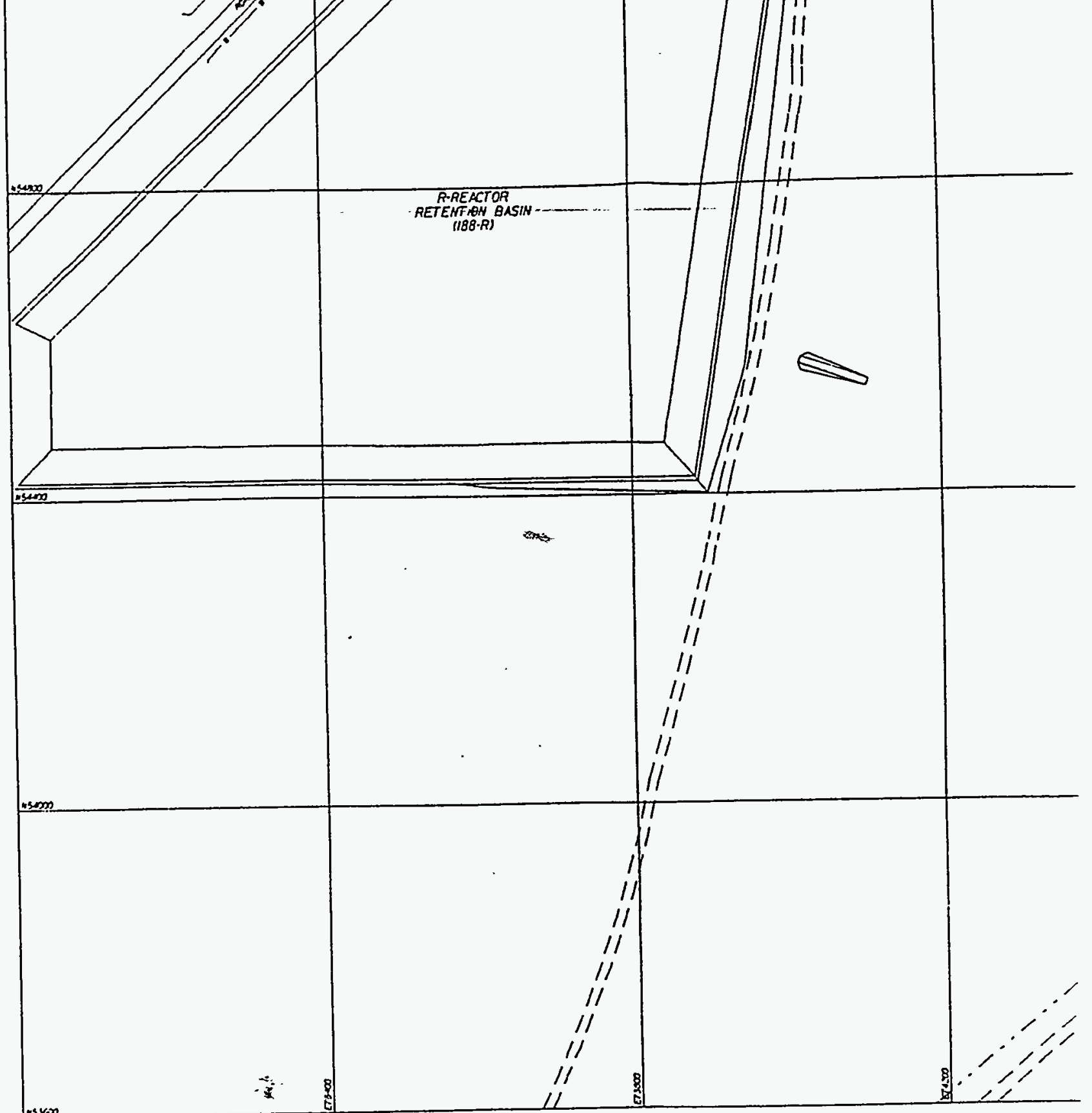

\section{WELL SYMBOL LEGEND}

- water table aouifer

- GROUNDWATER MONITORING WELLS

II MCBEAN AOUIFER

fit GROUNDWATER MONITORING WELLS

- congaree aquifer

GROUNDWATER MONITORING WELLS

RAC 1 WELL ID 


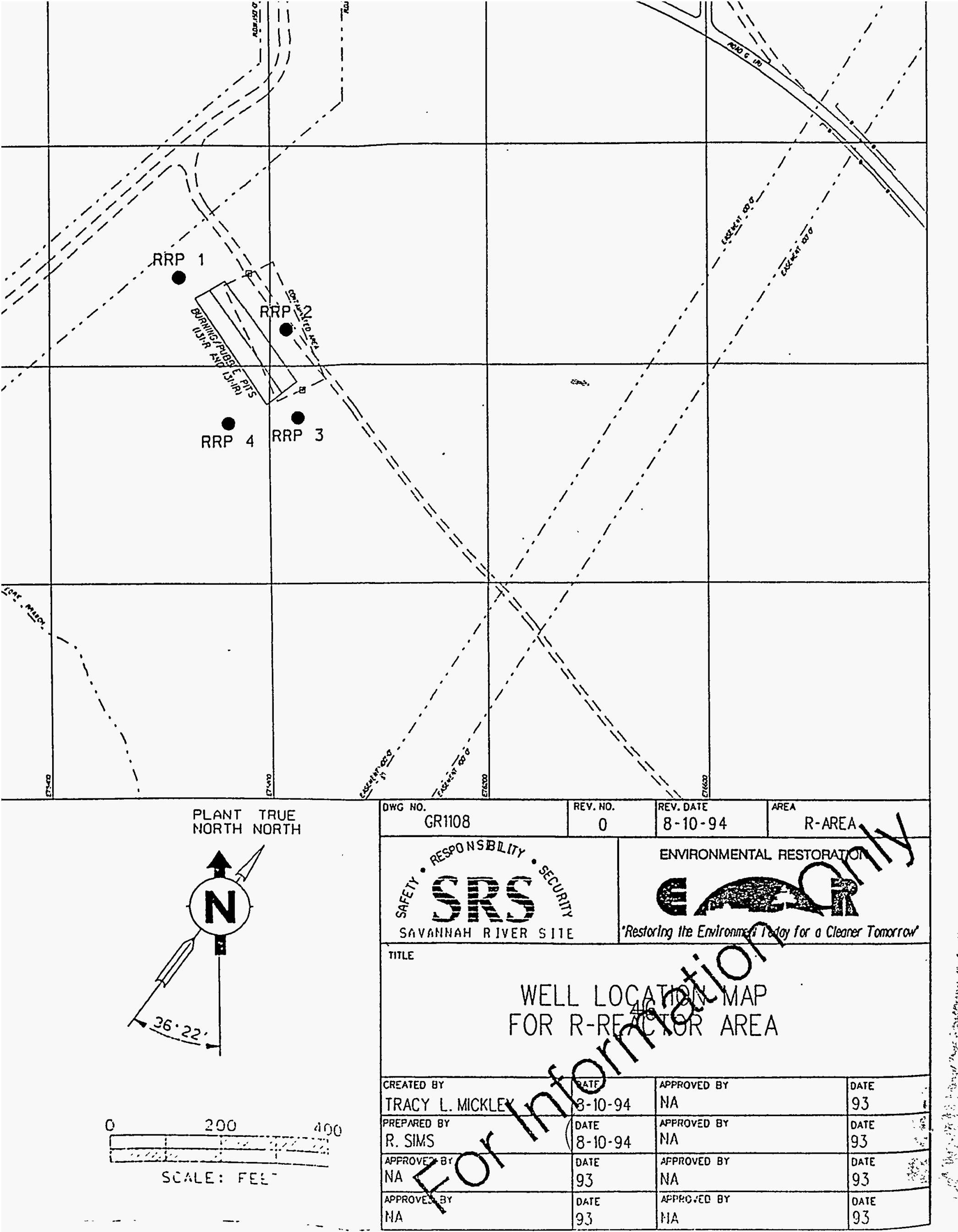




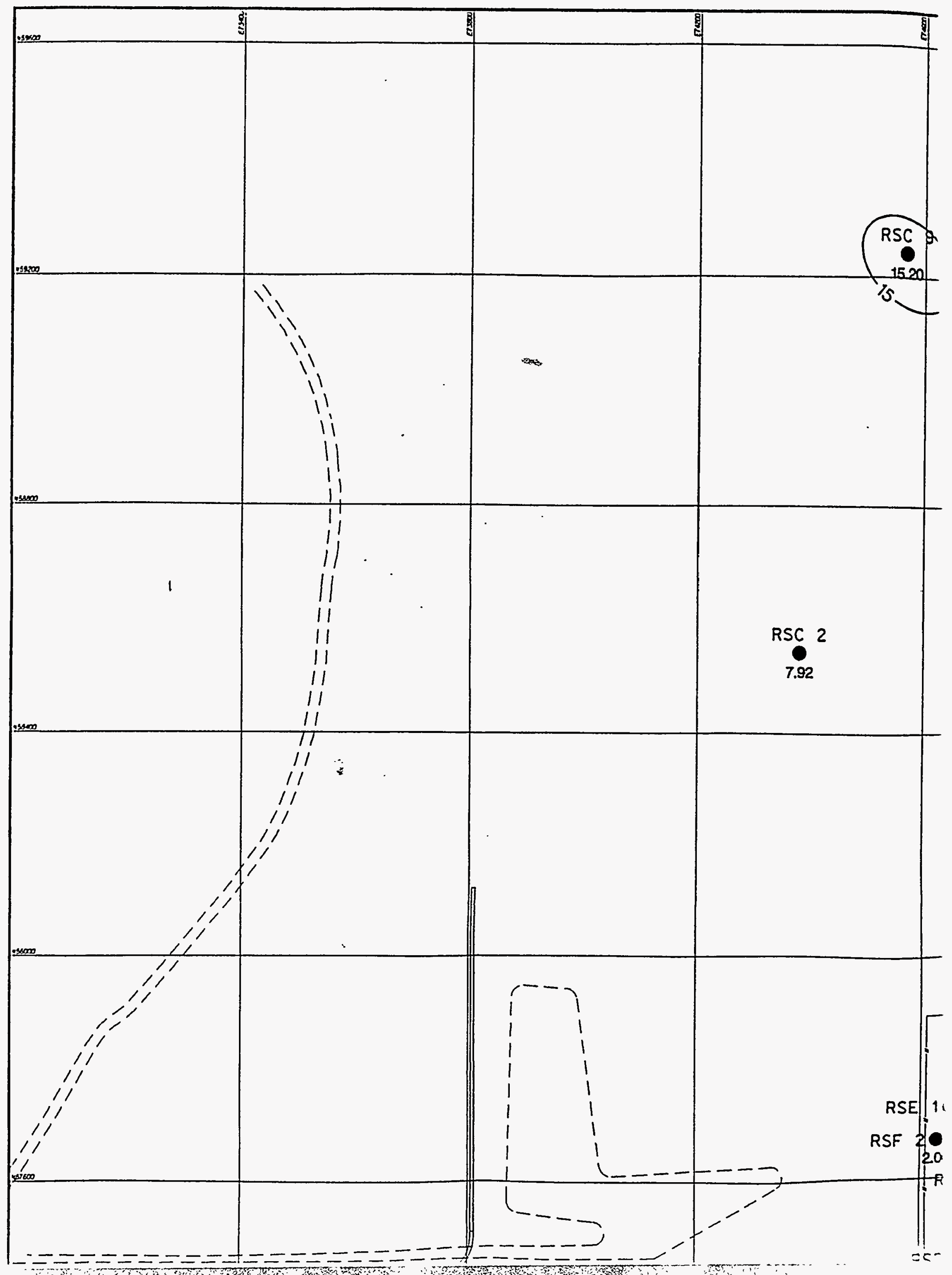




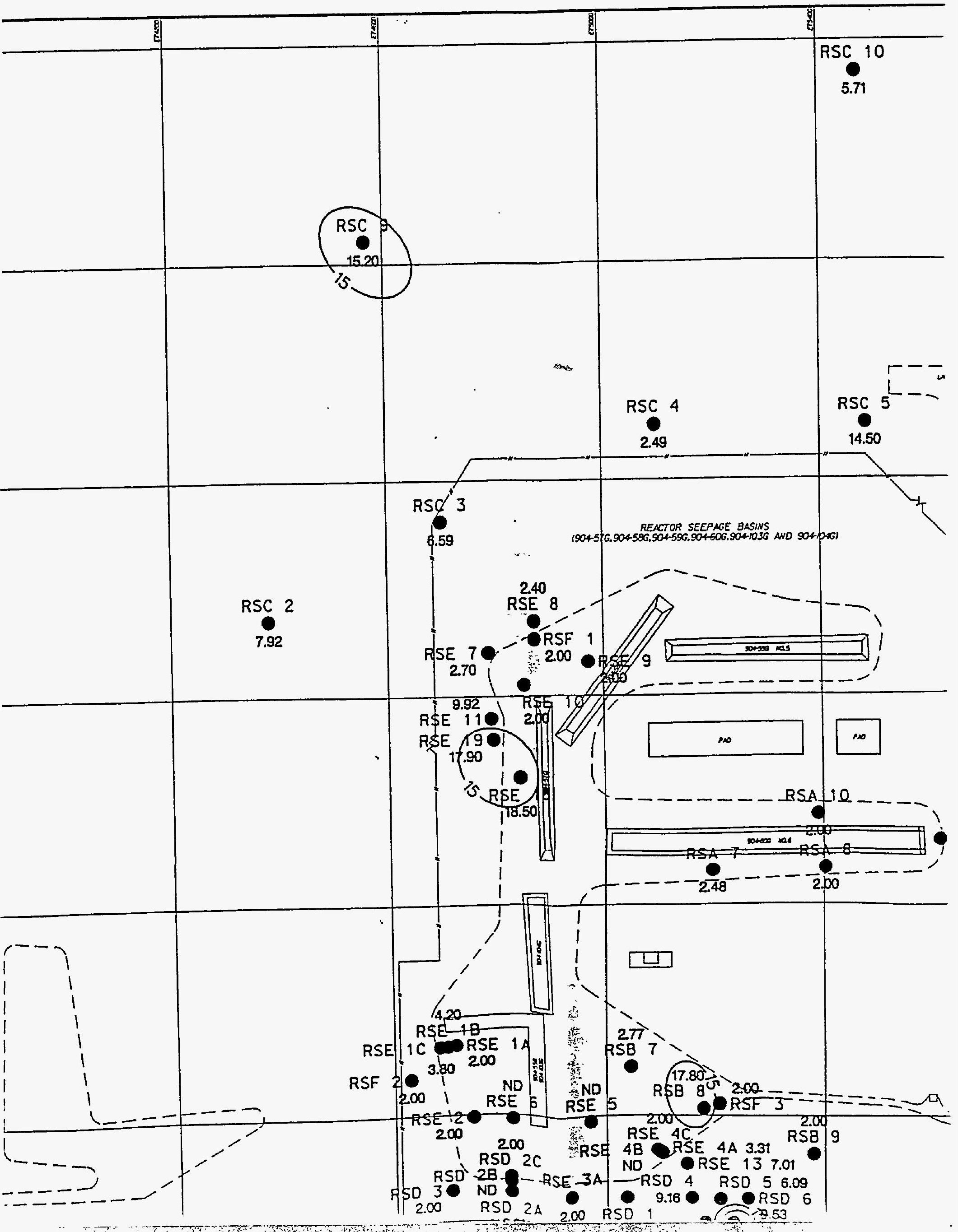




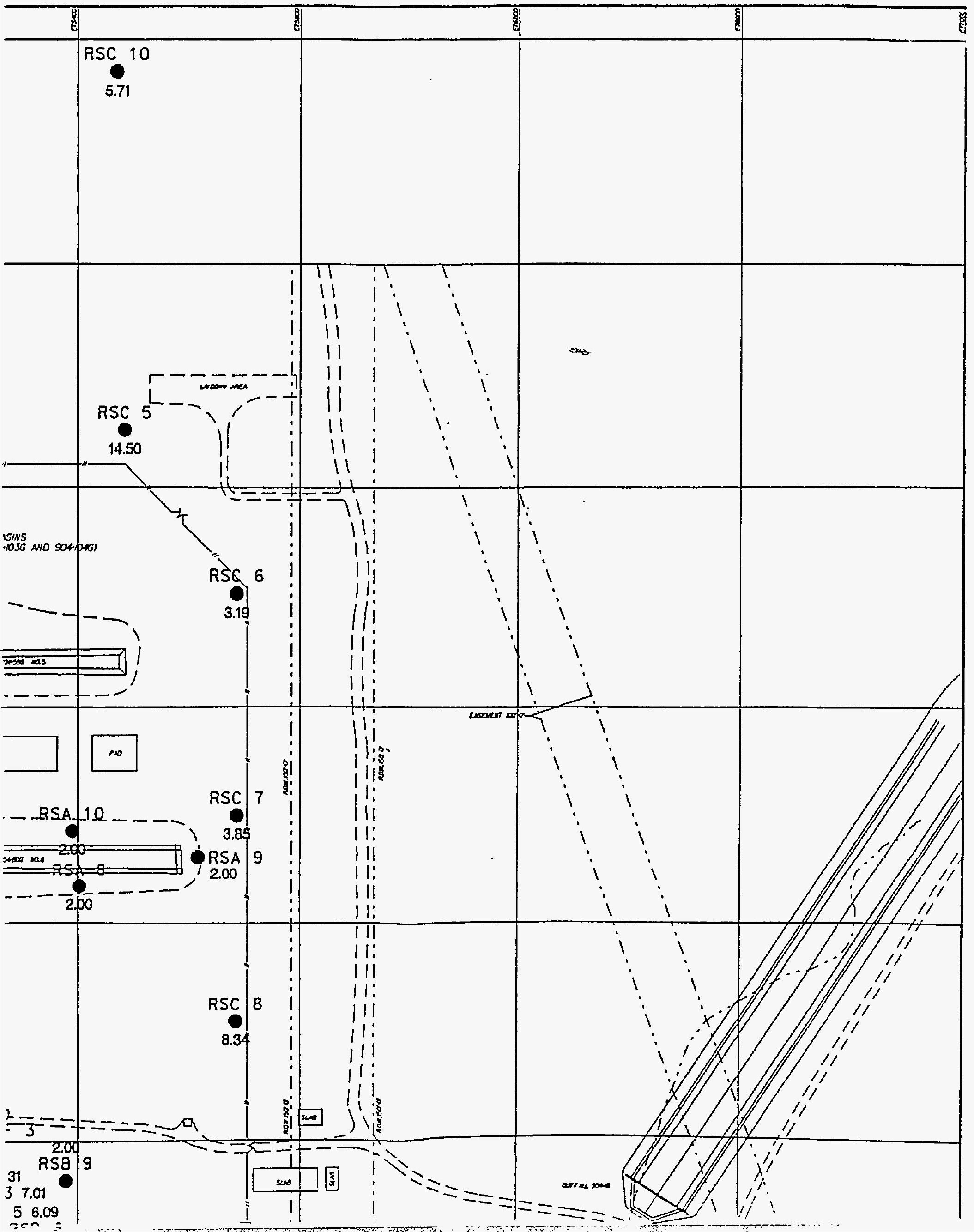




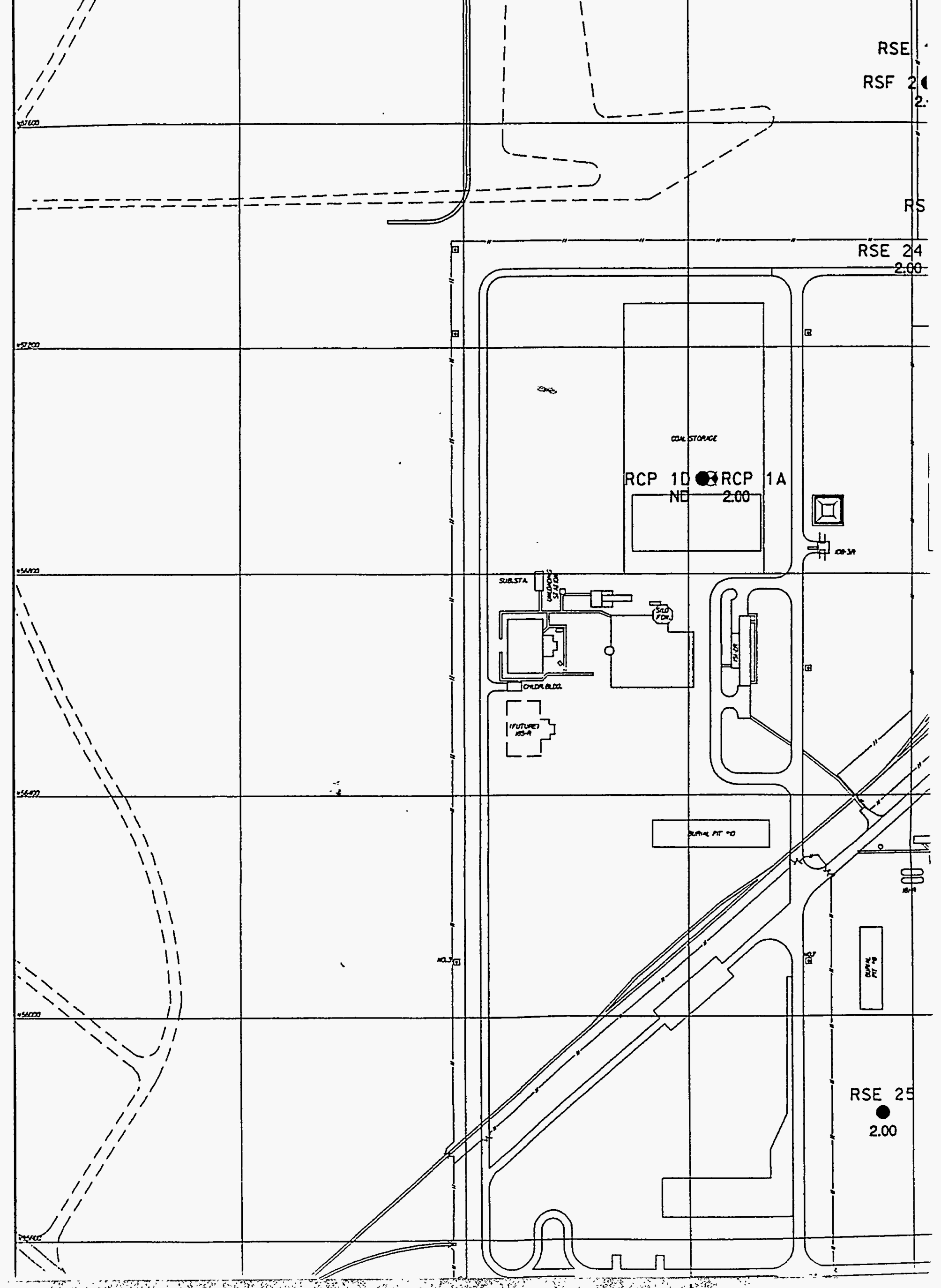




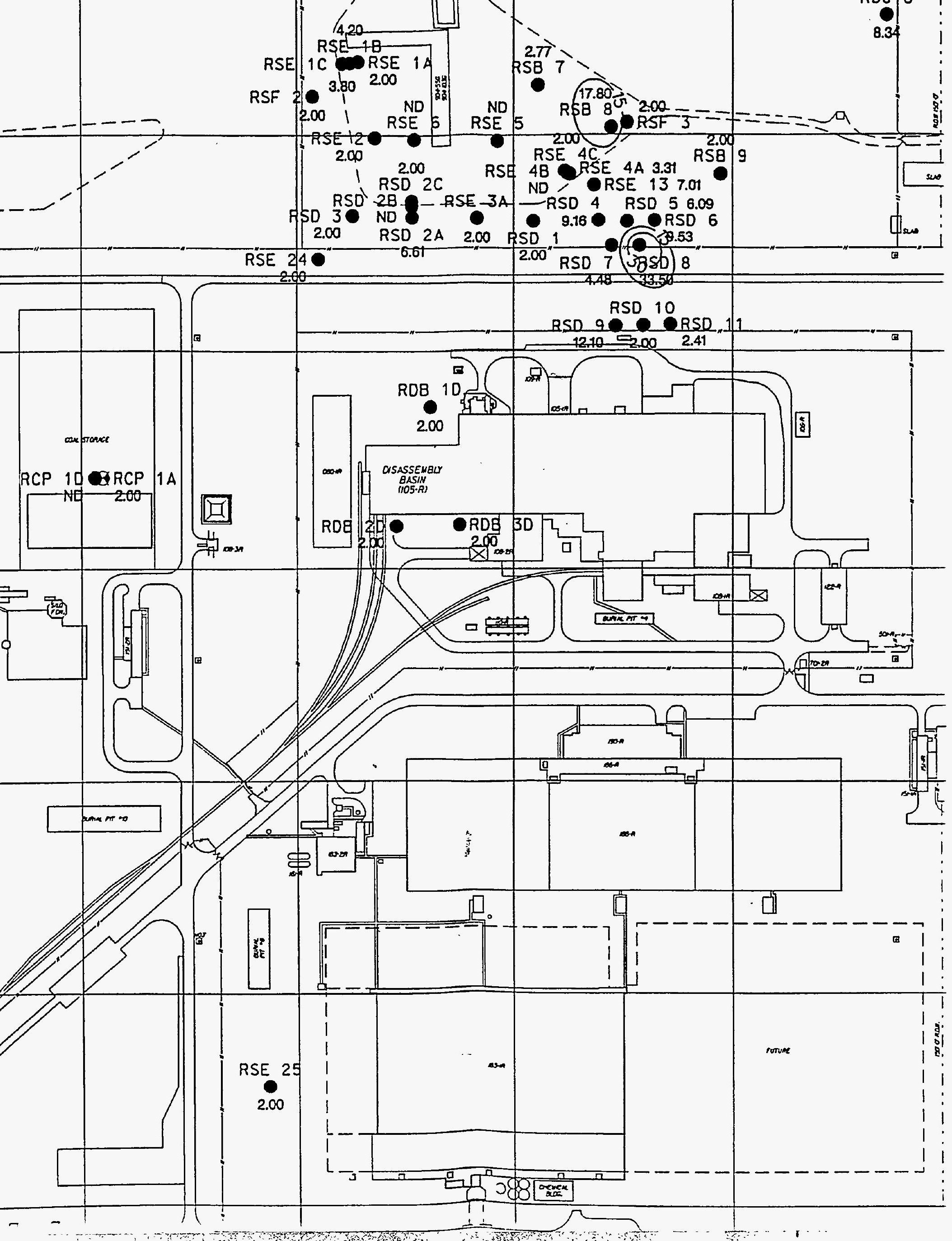




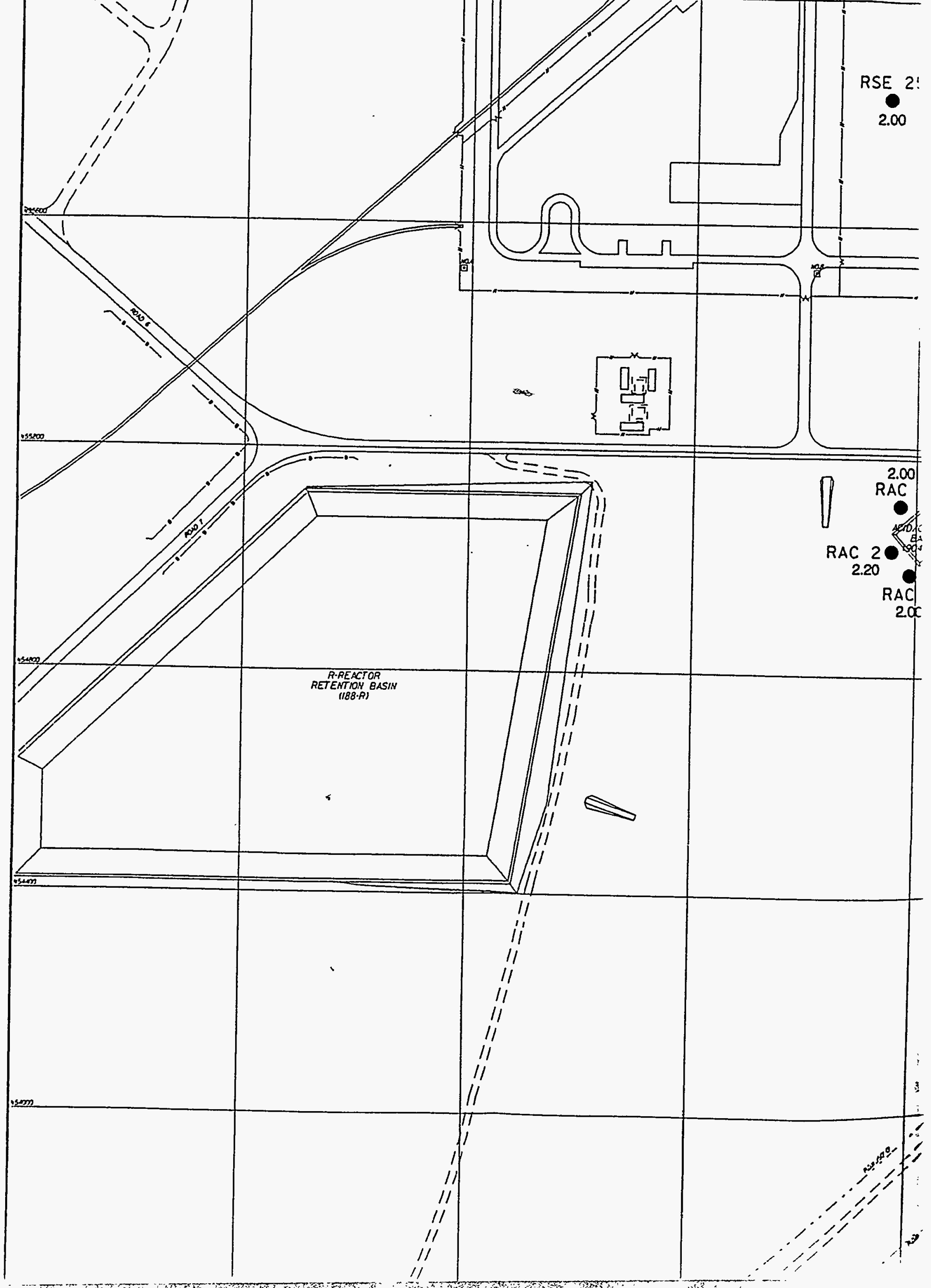




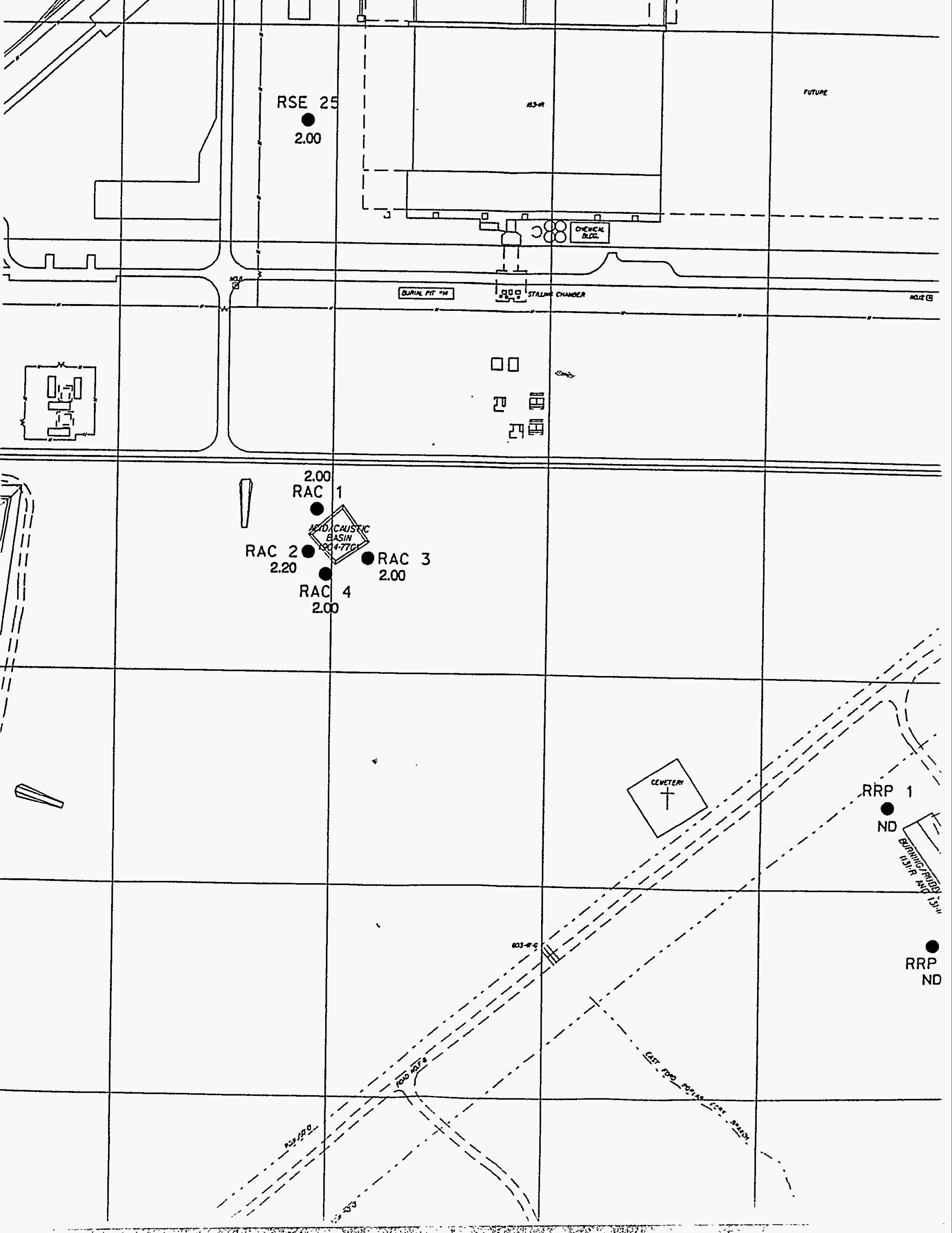




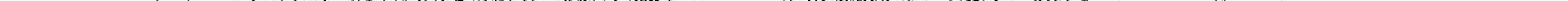




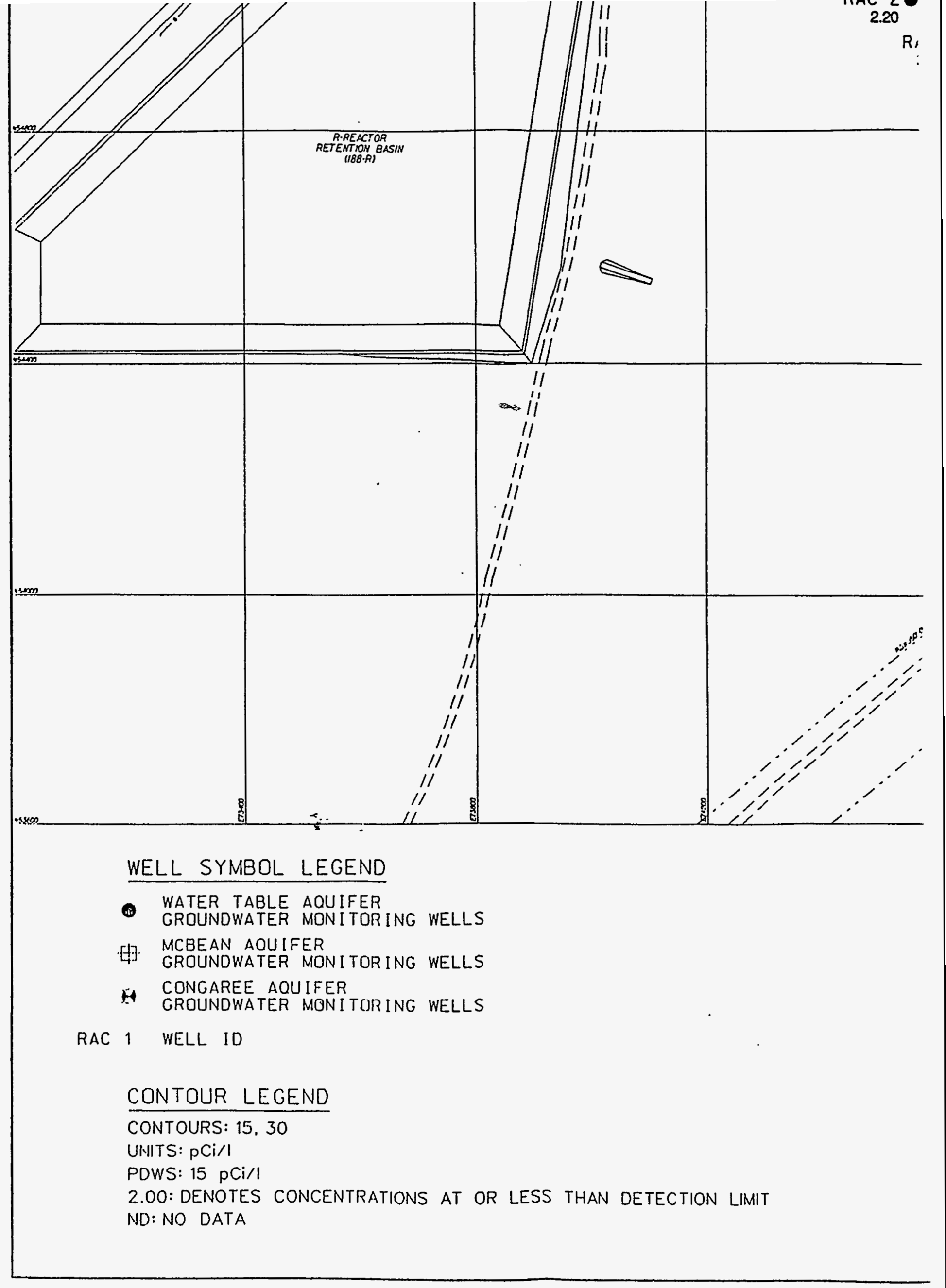





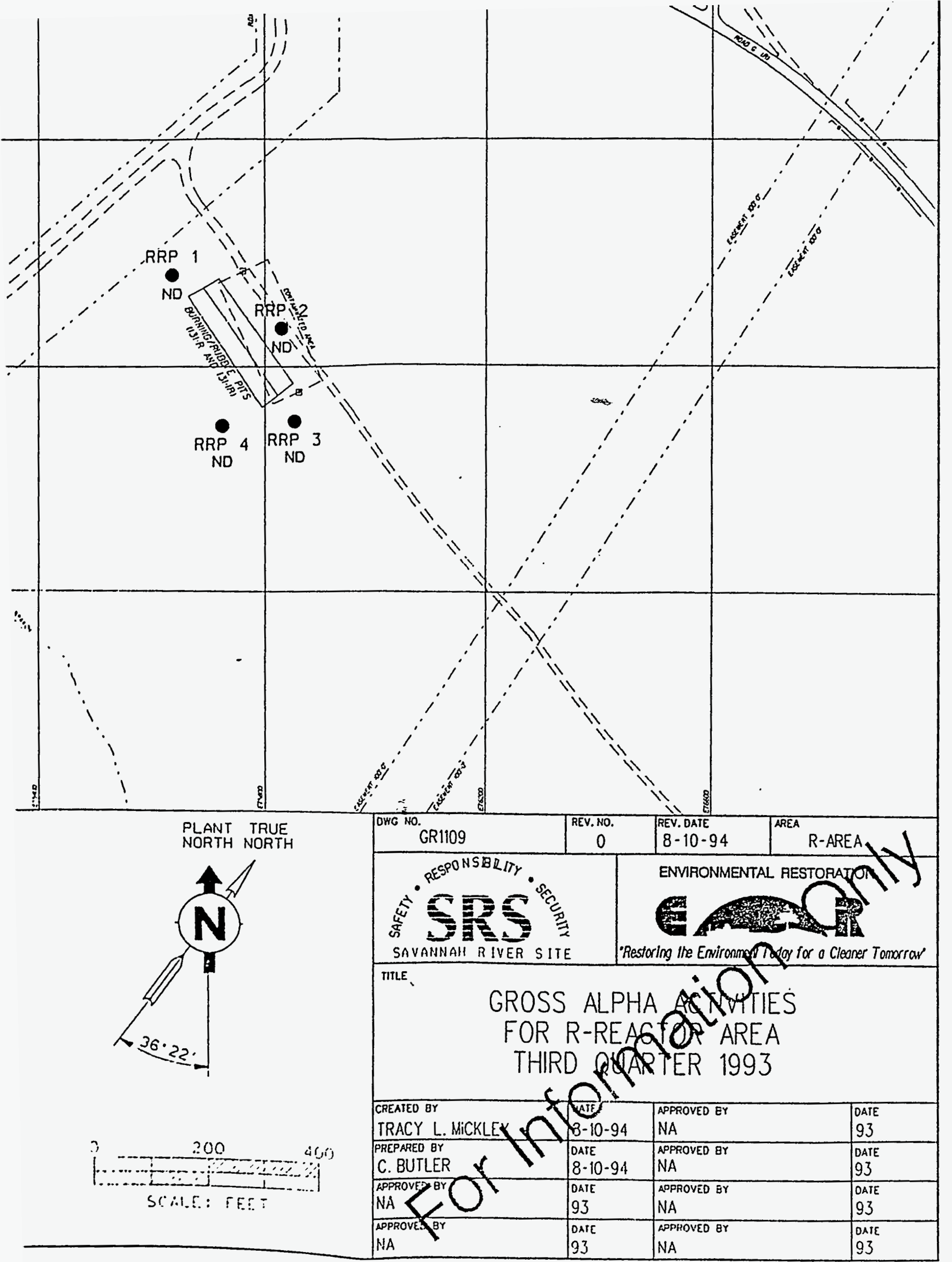




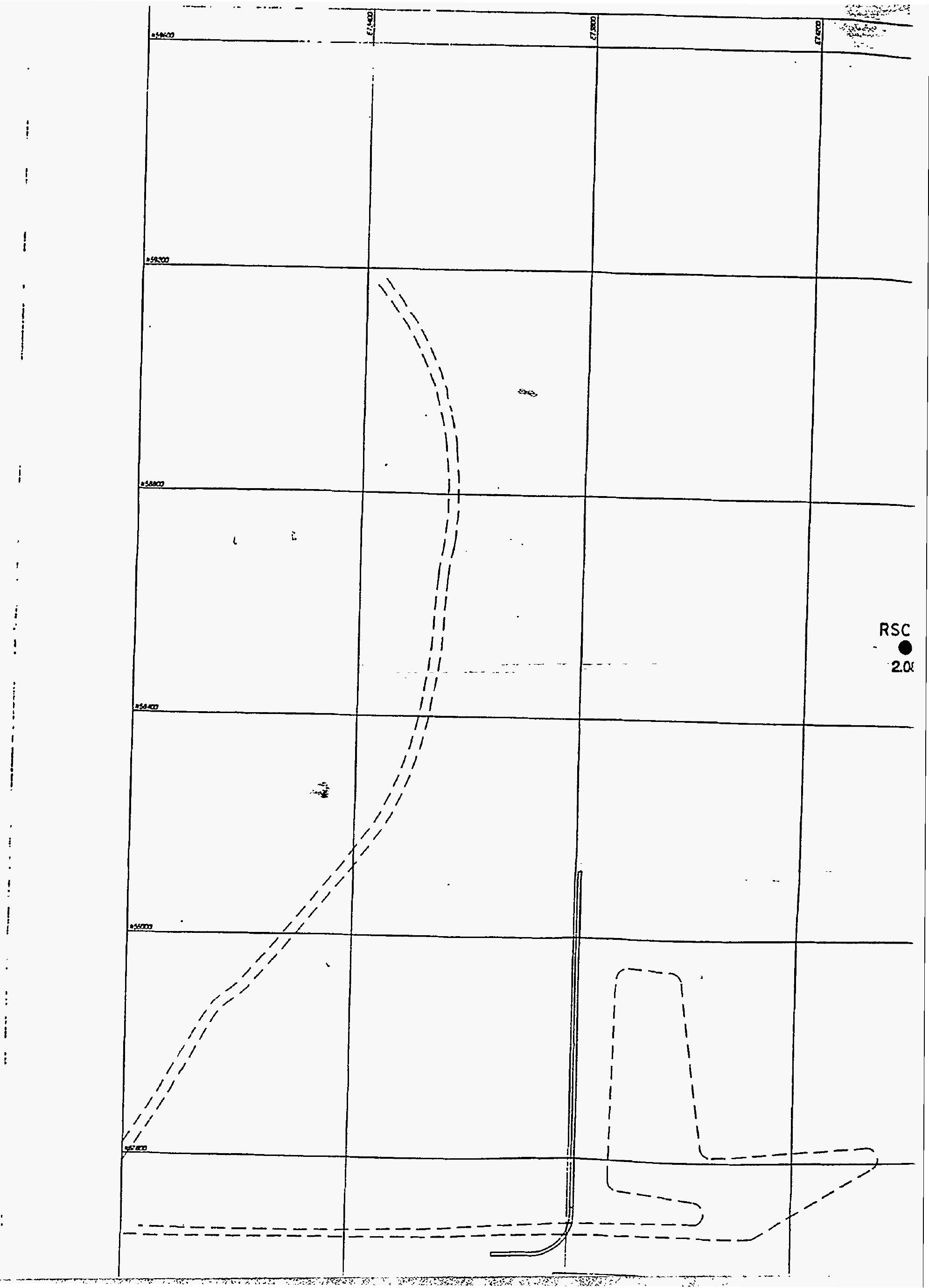




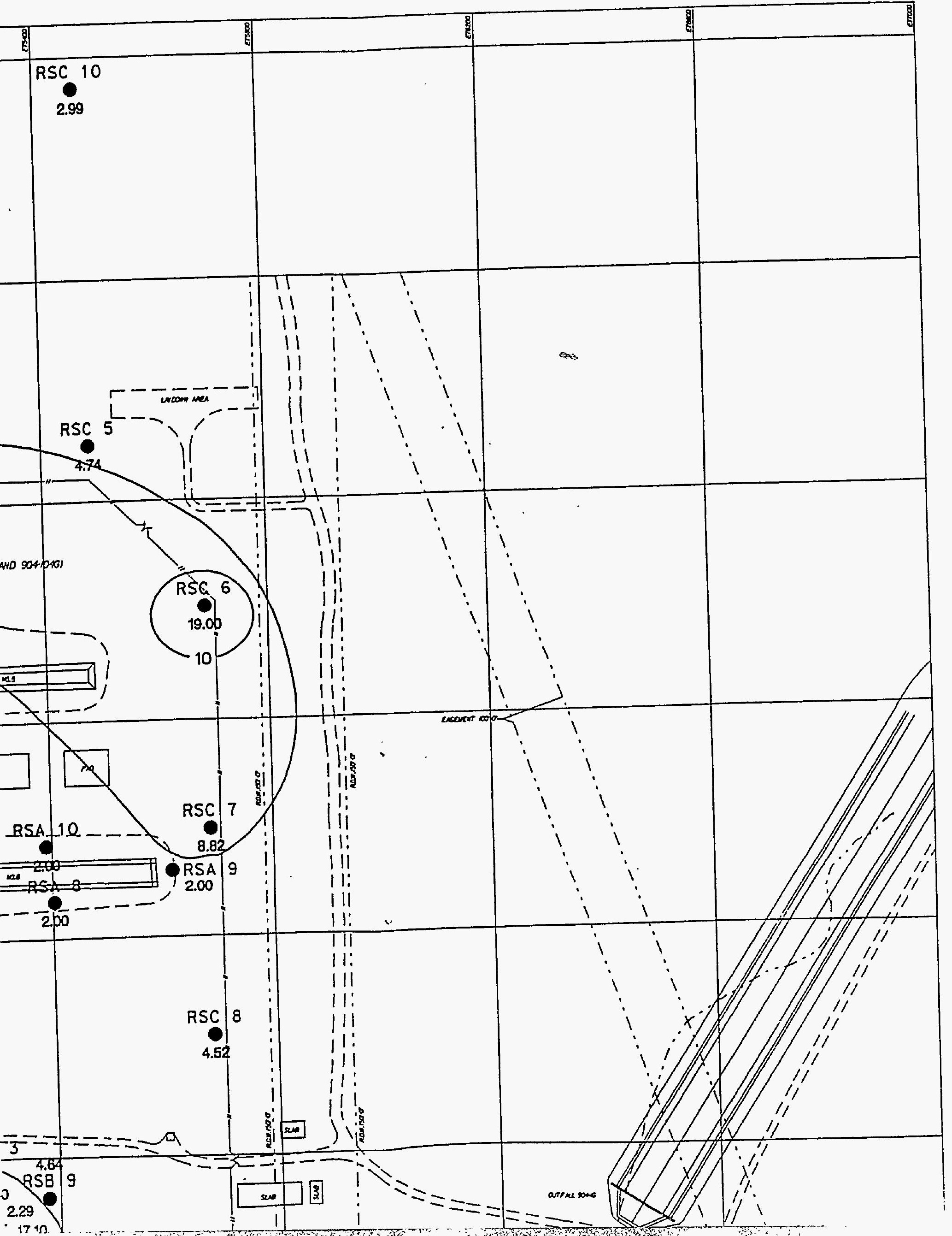




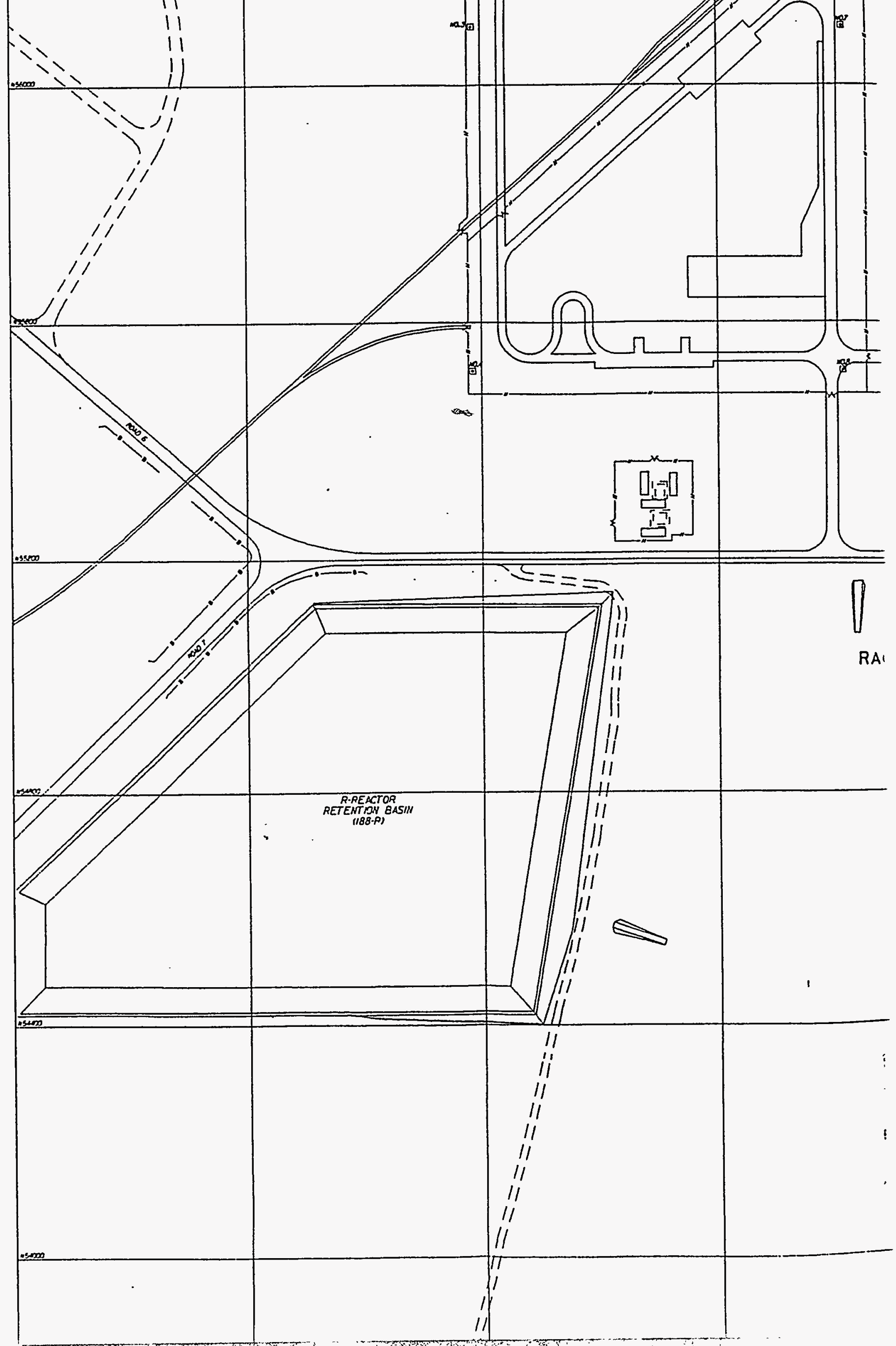




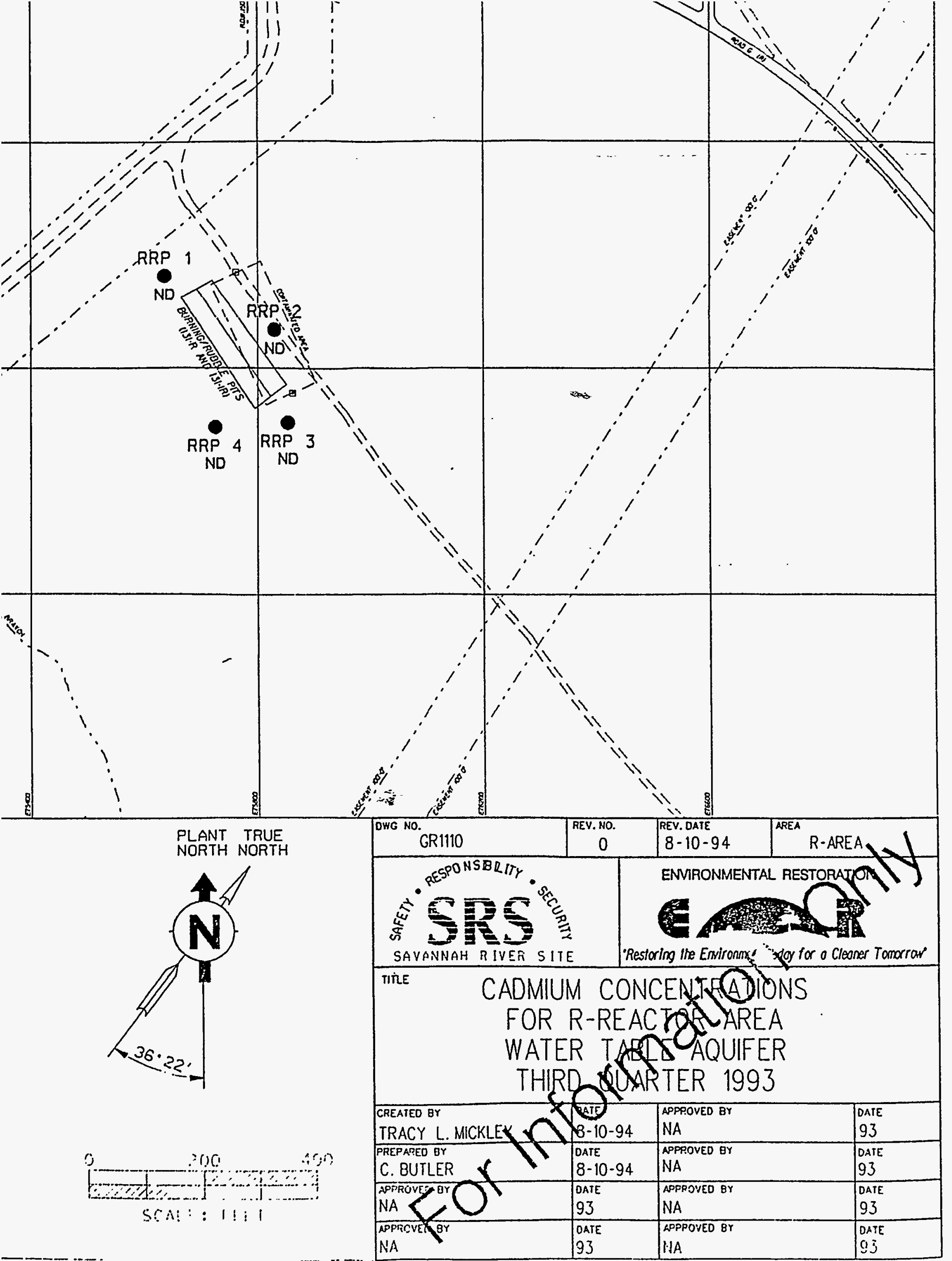




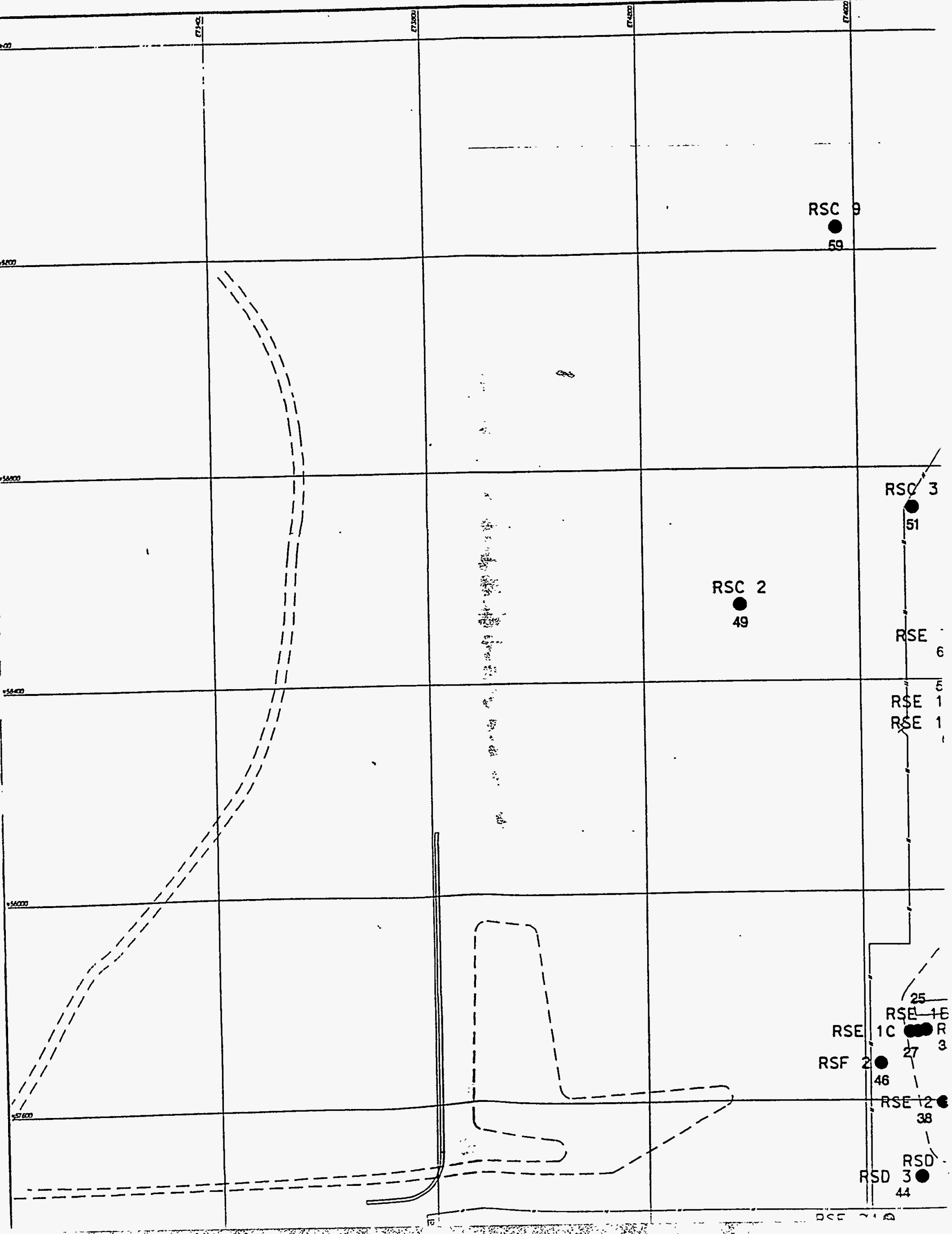




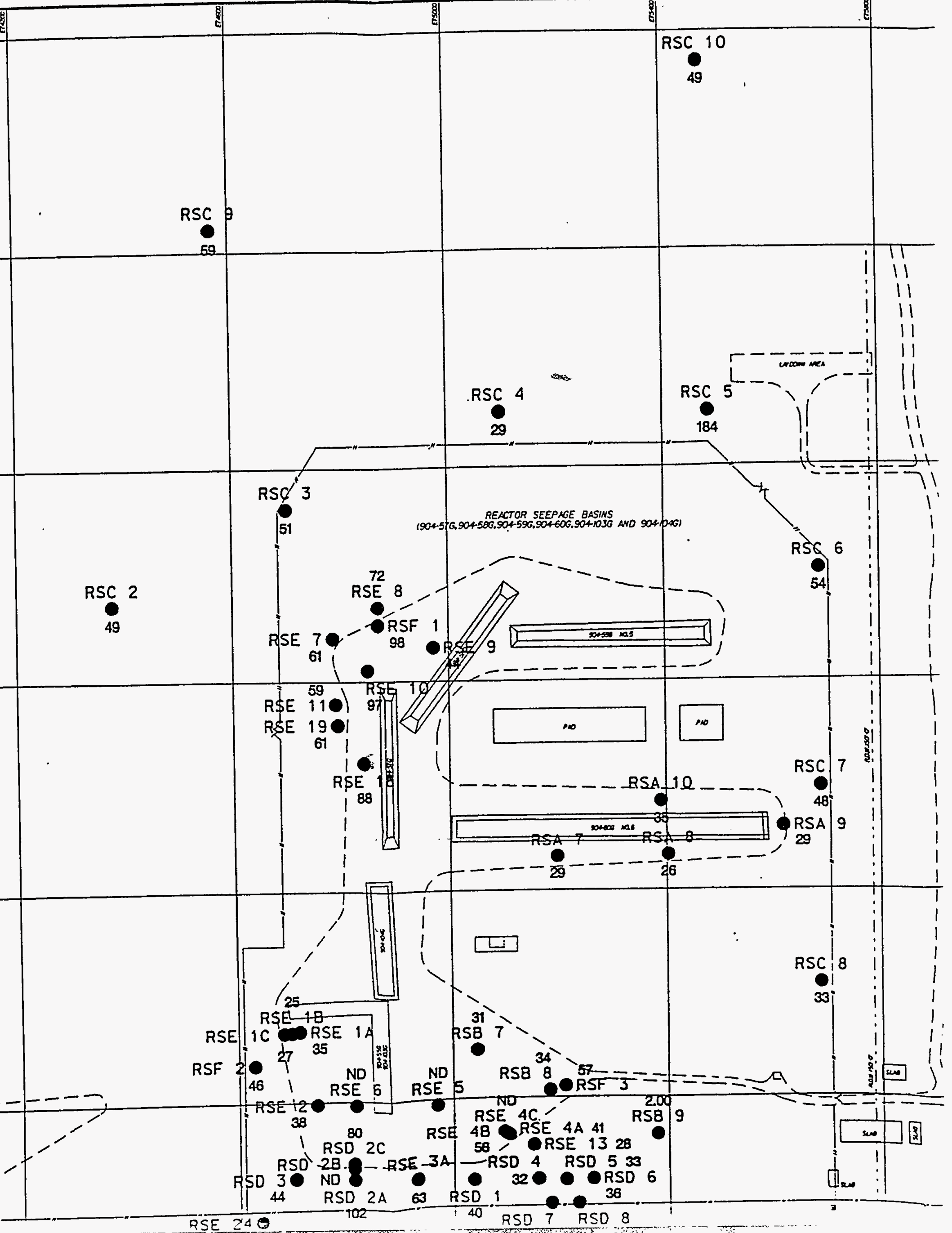




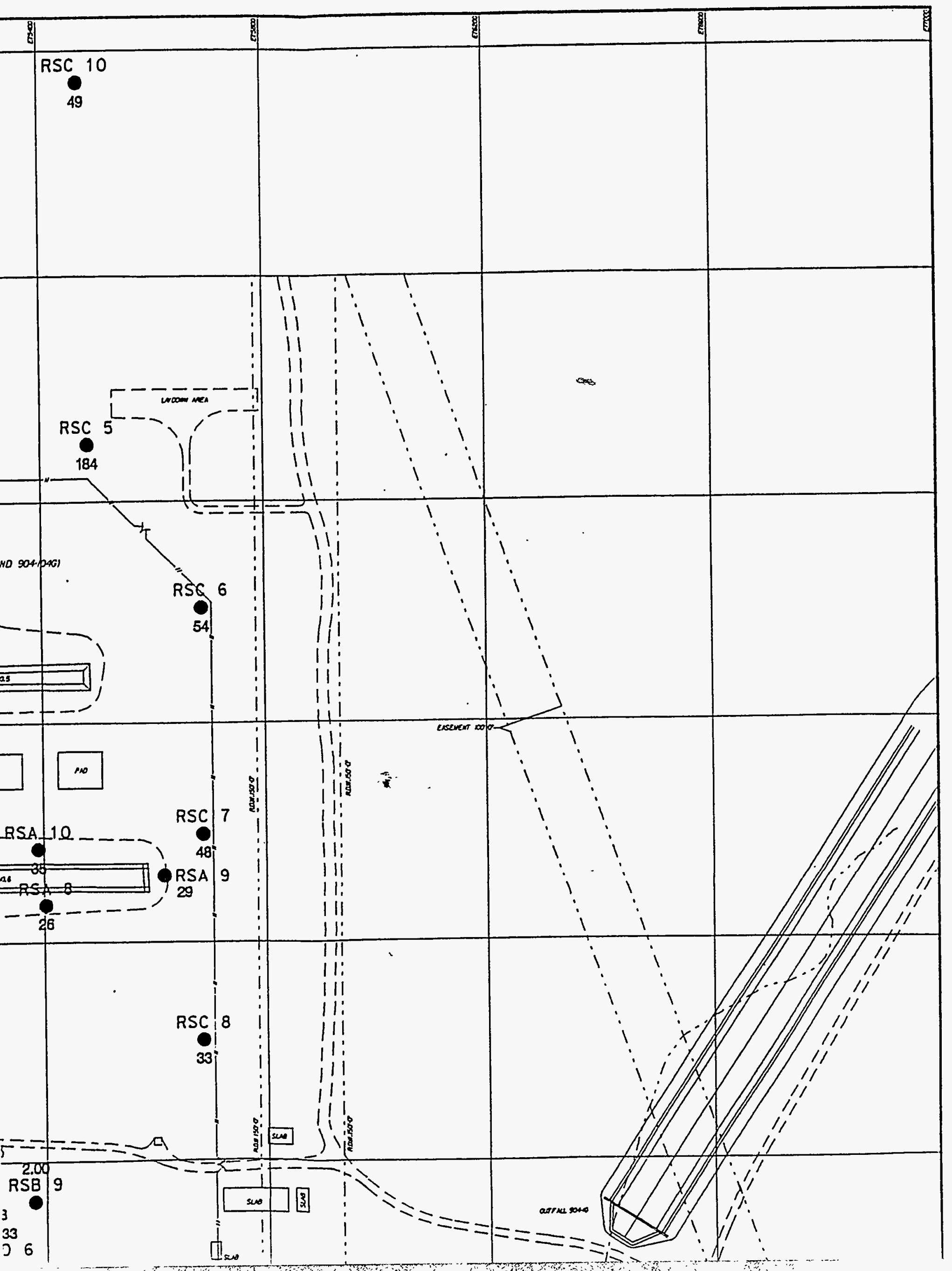




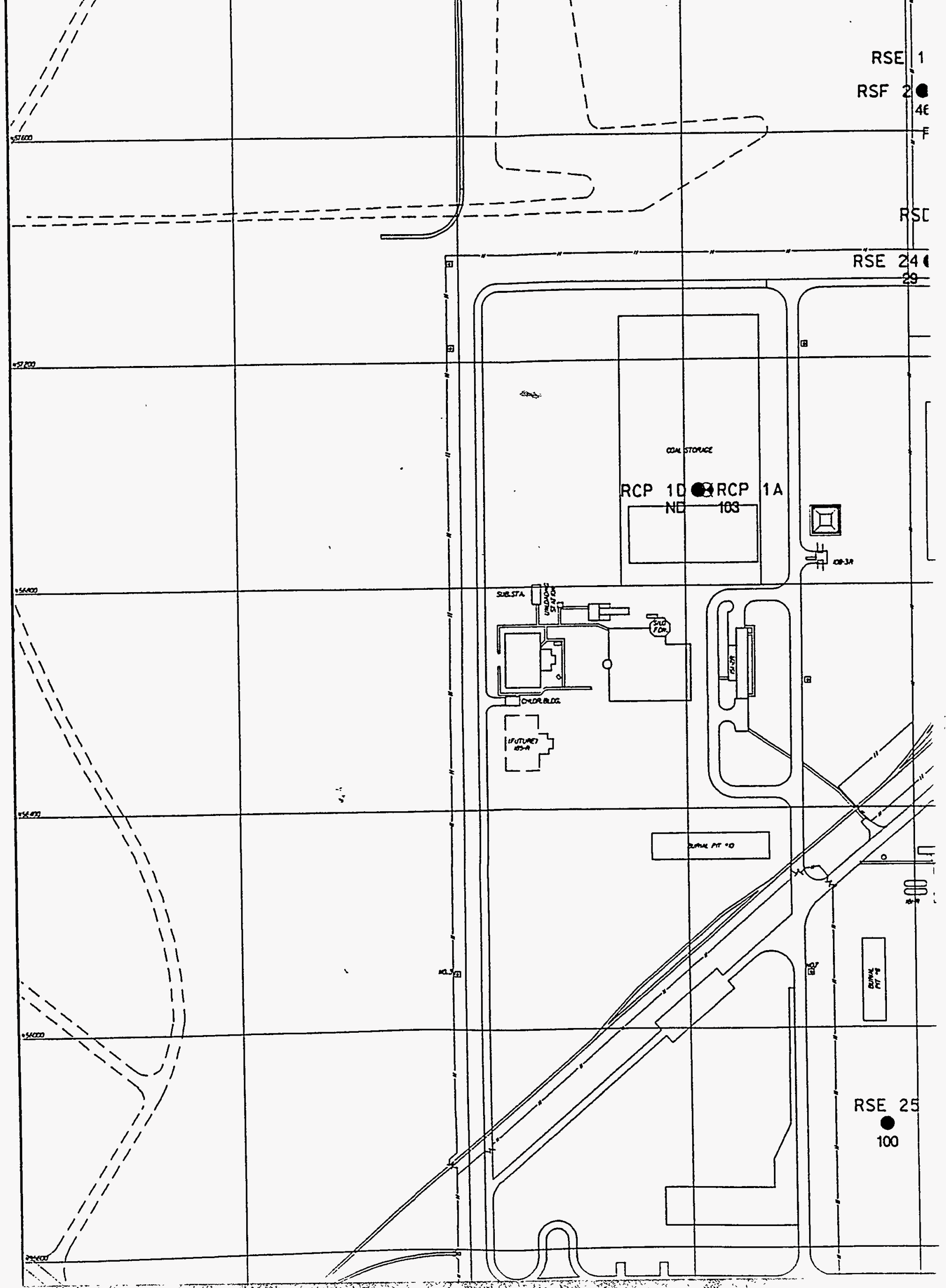




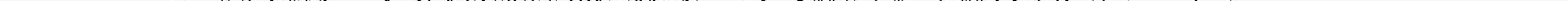




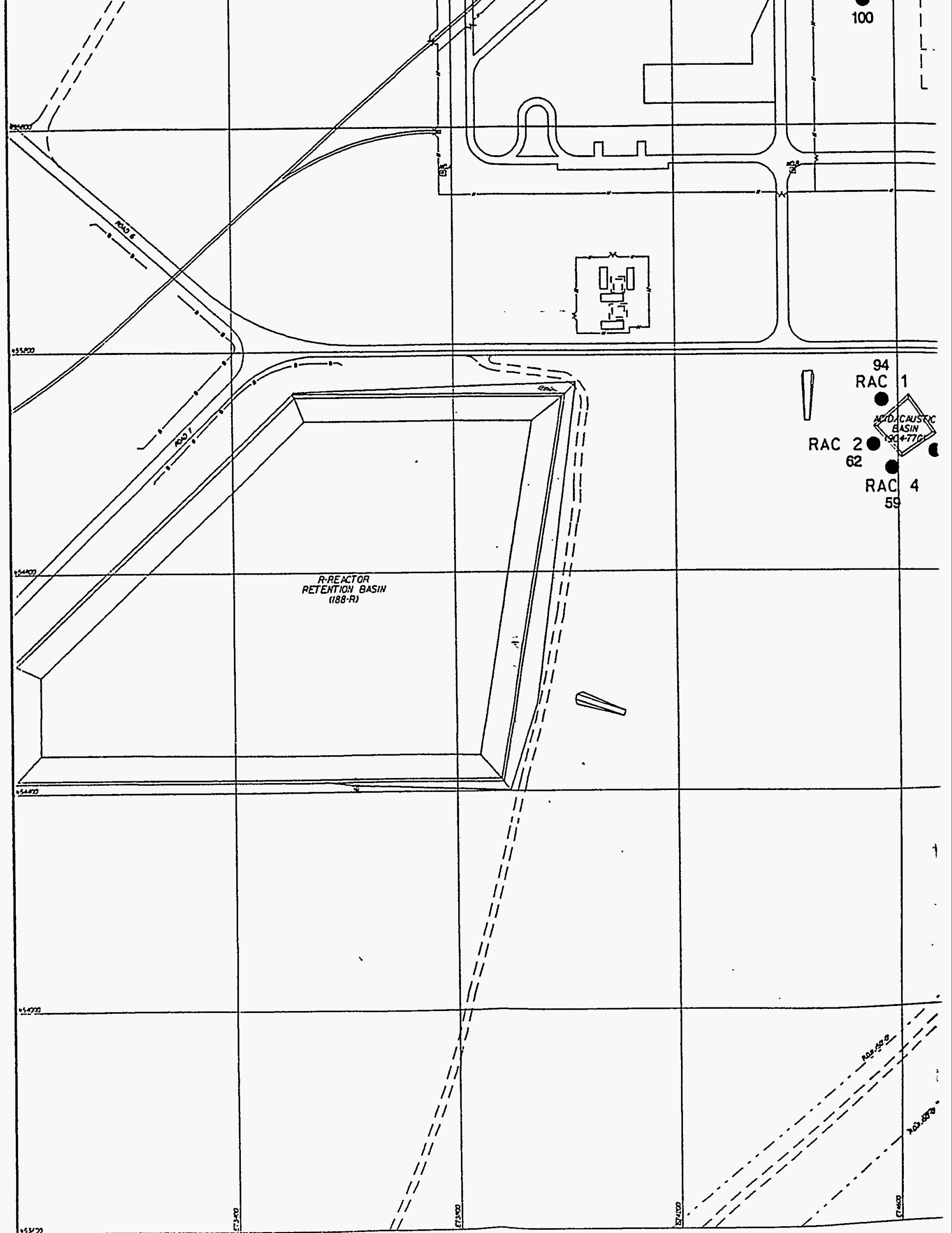




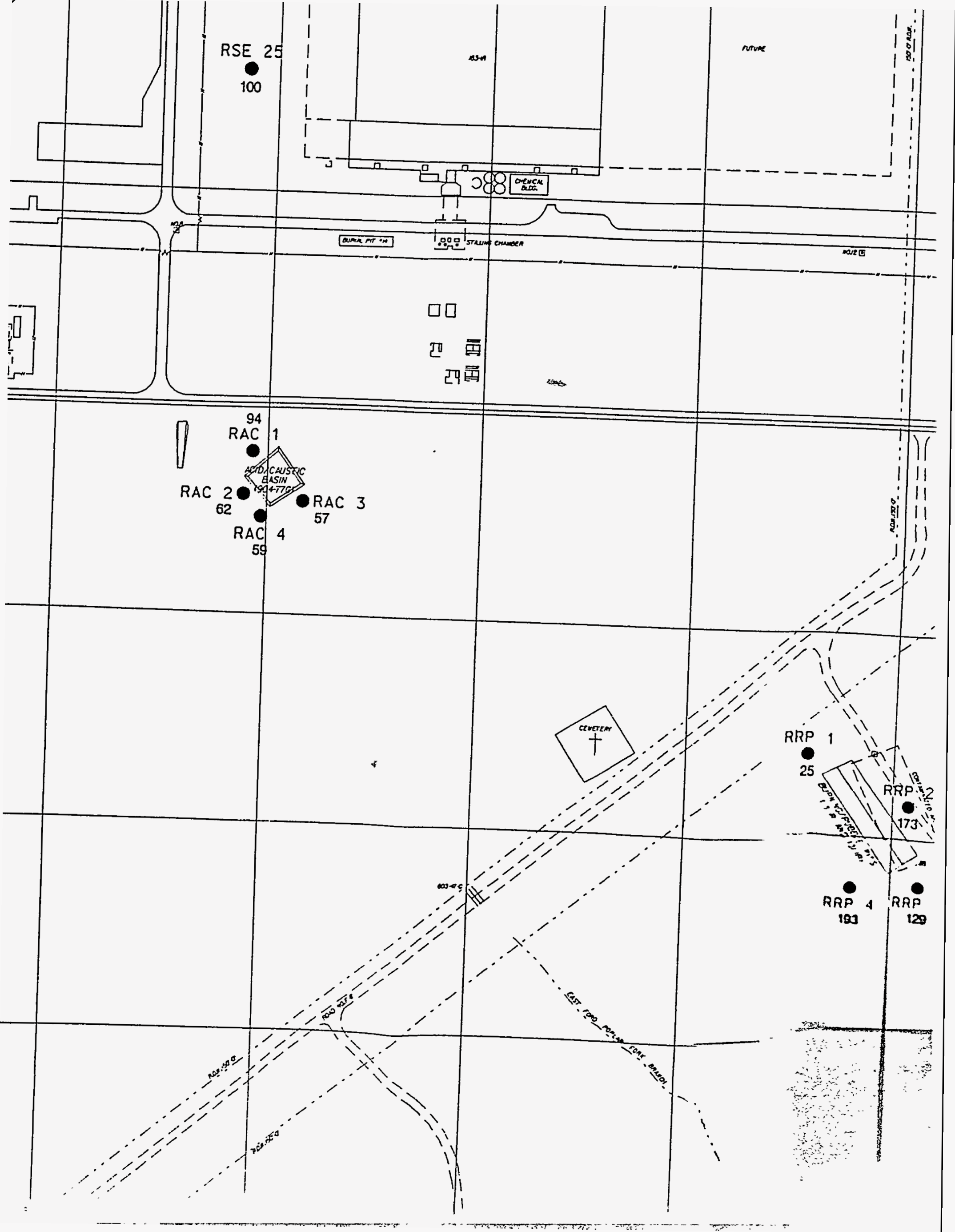




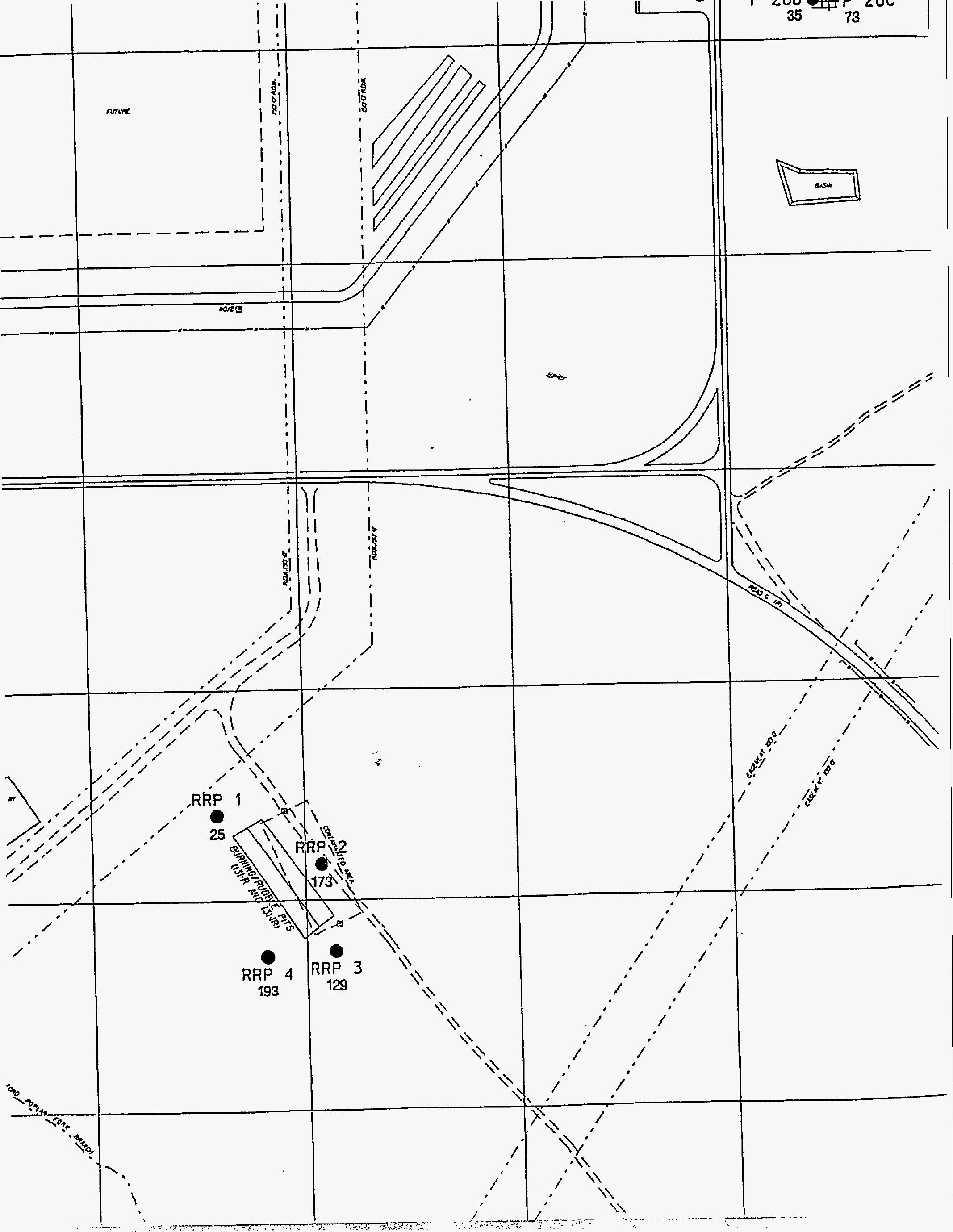




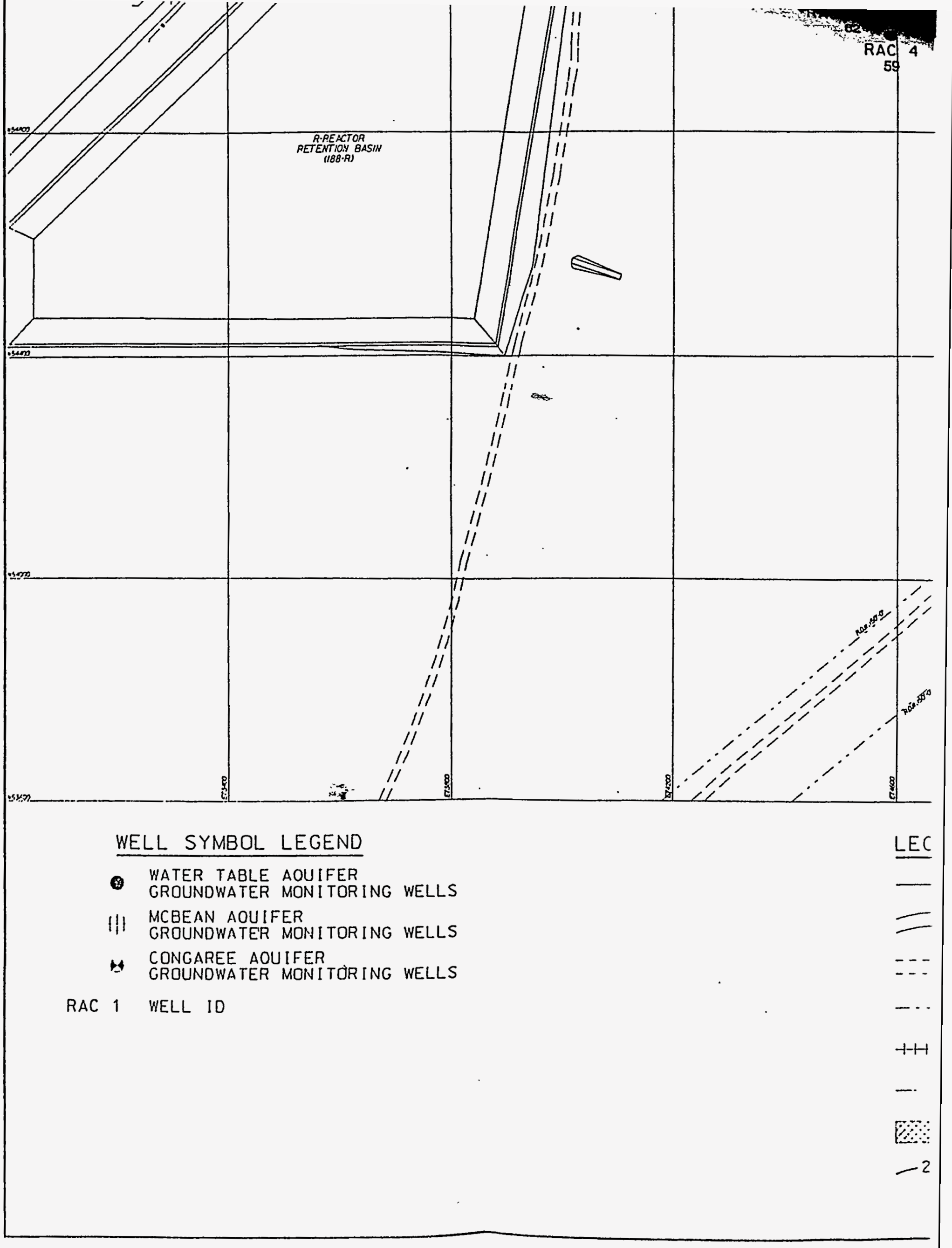




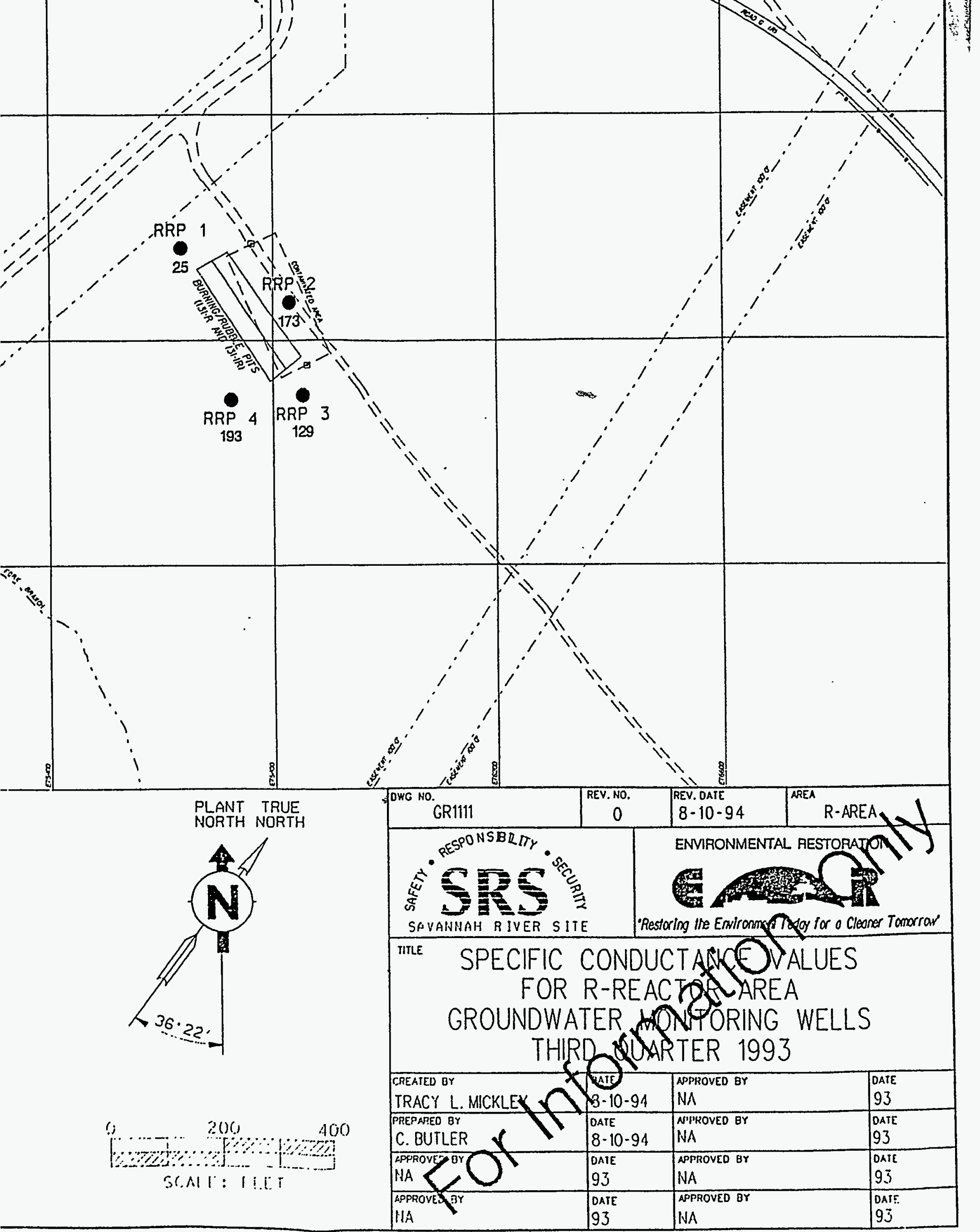




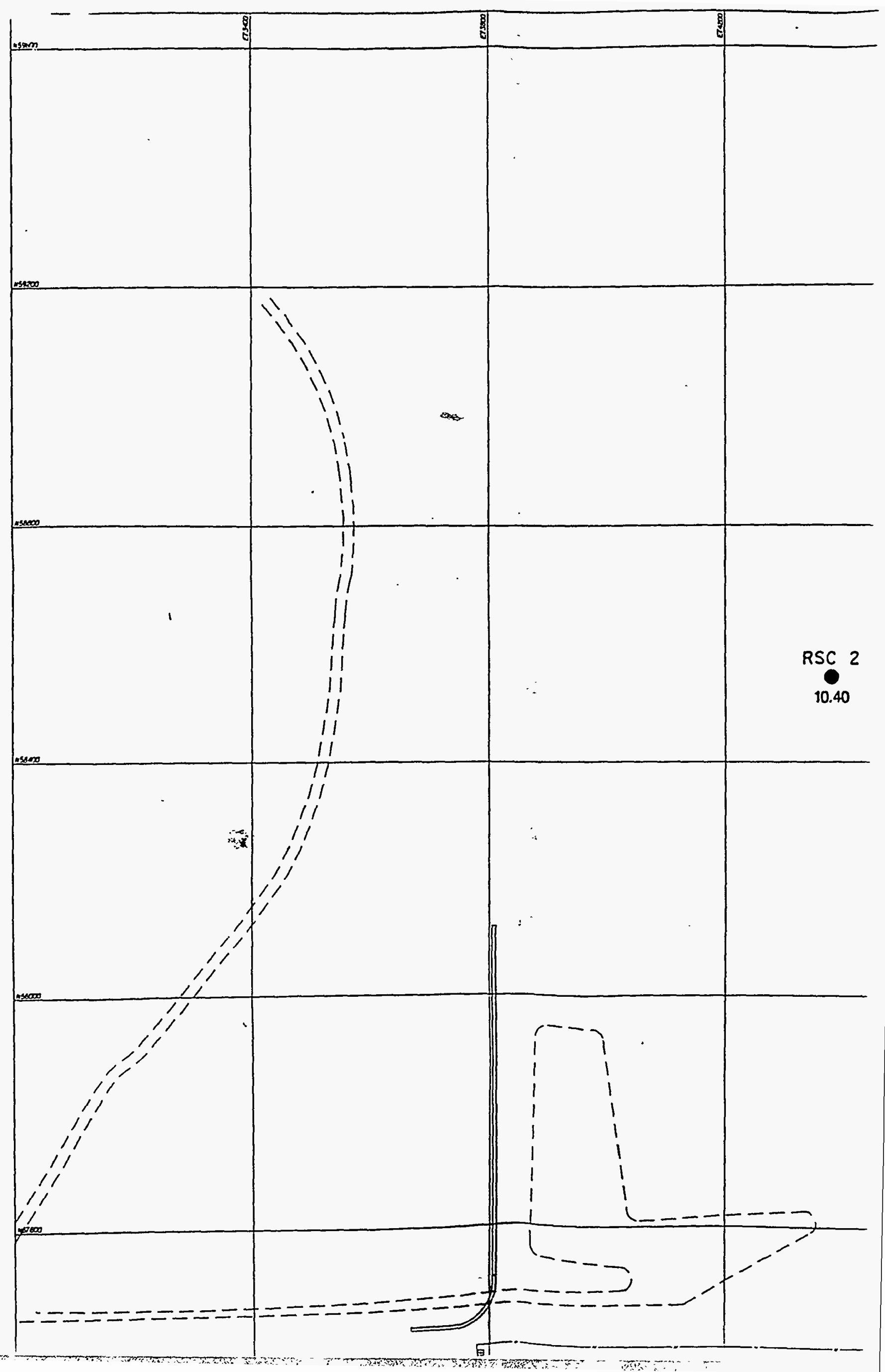




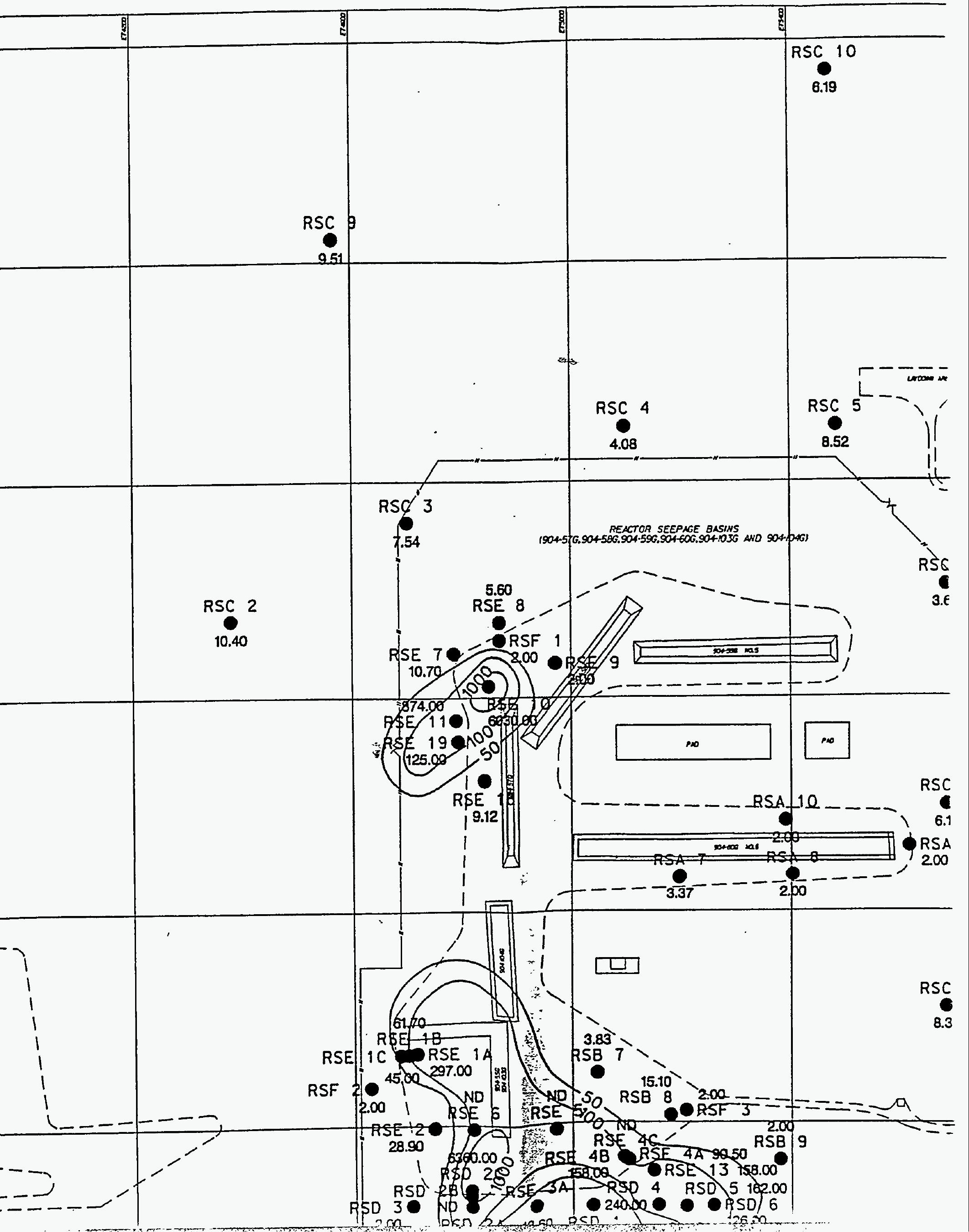




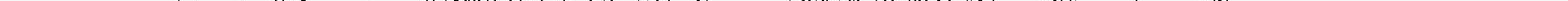




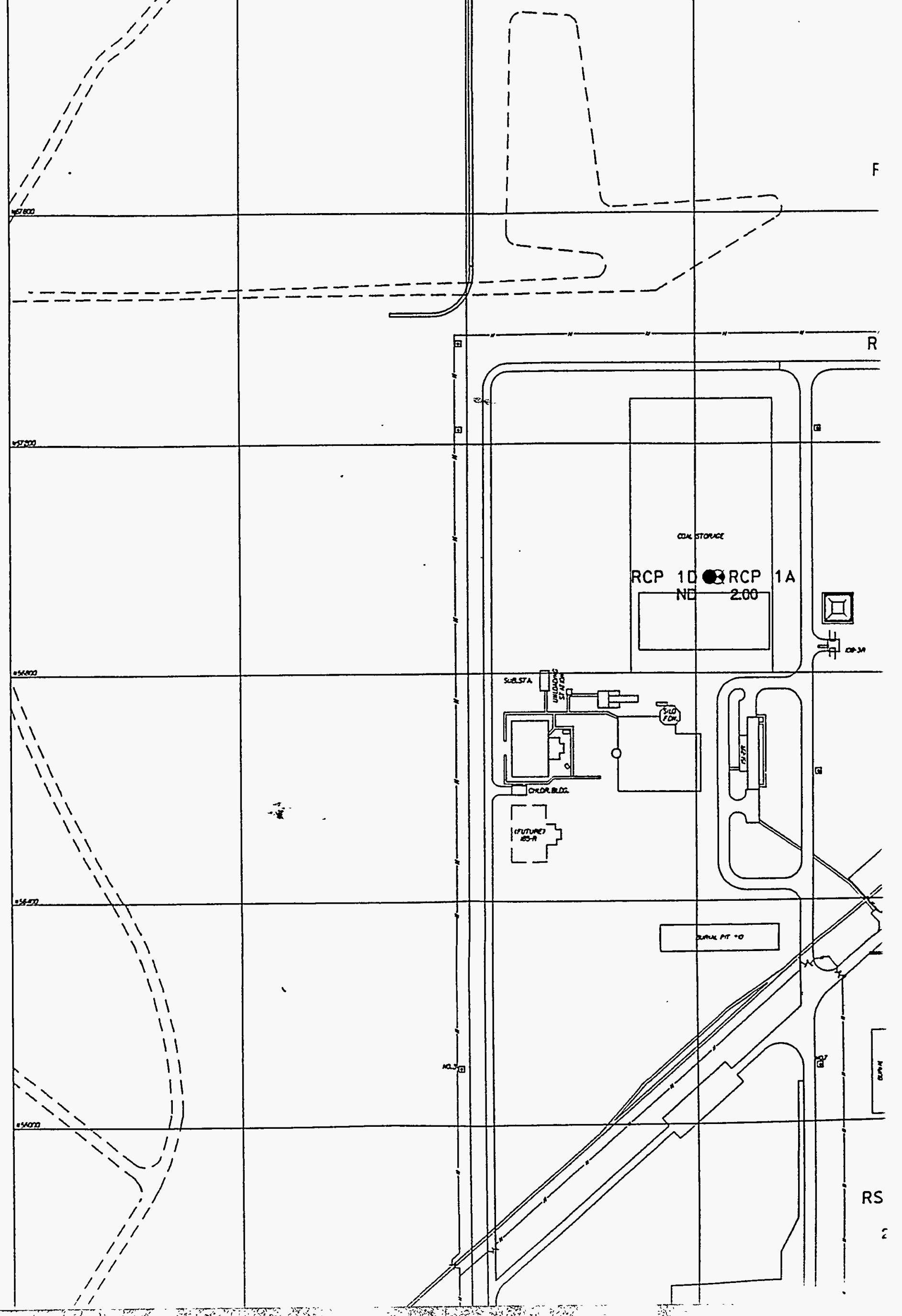




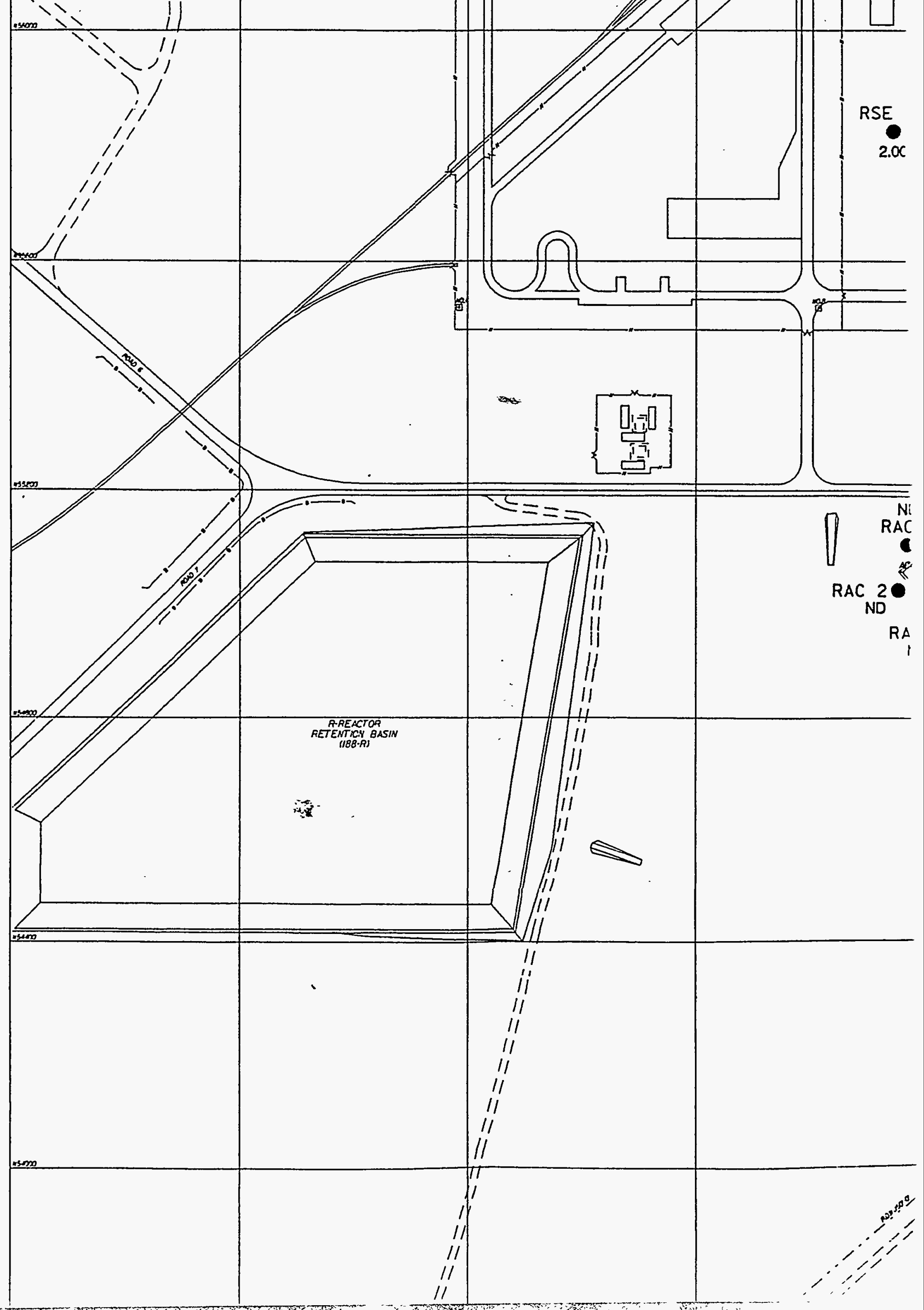




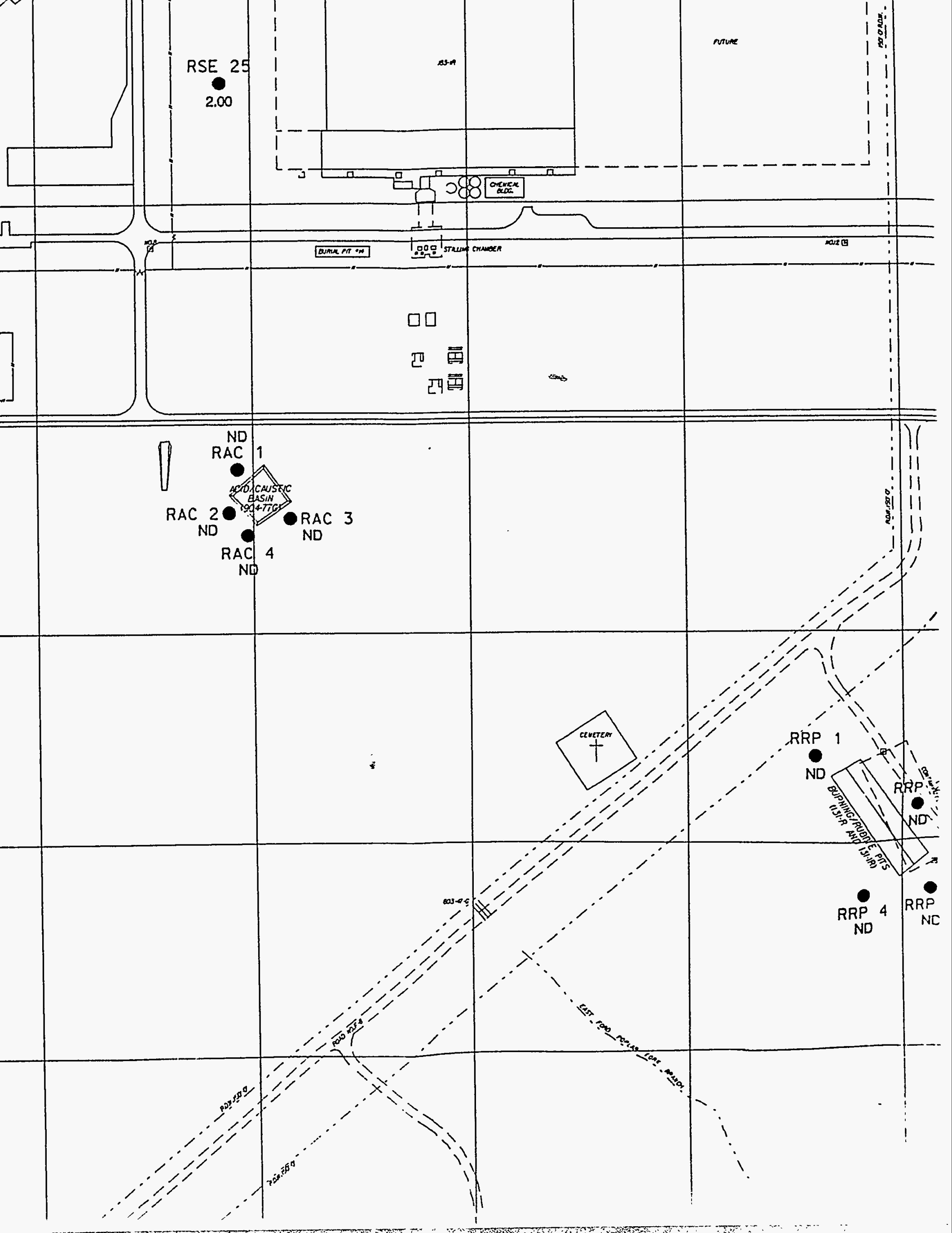




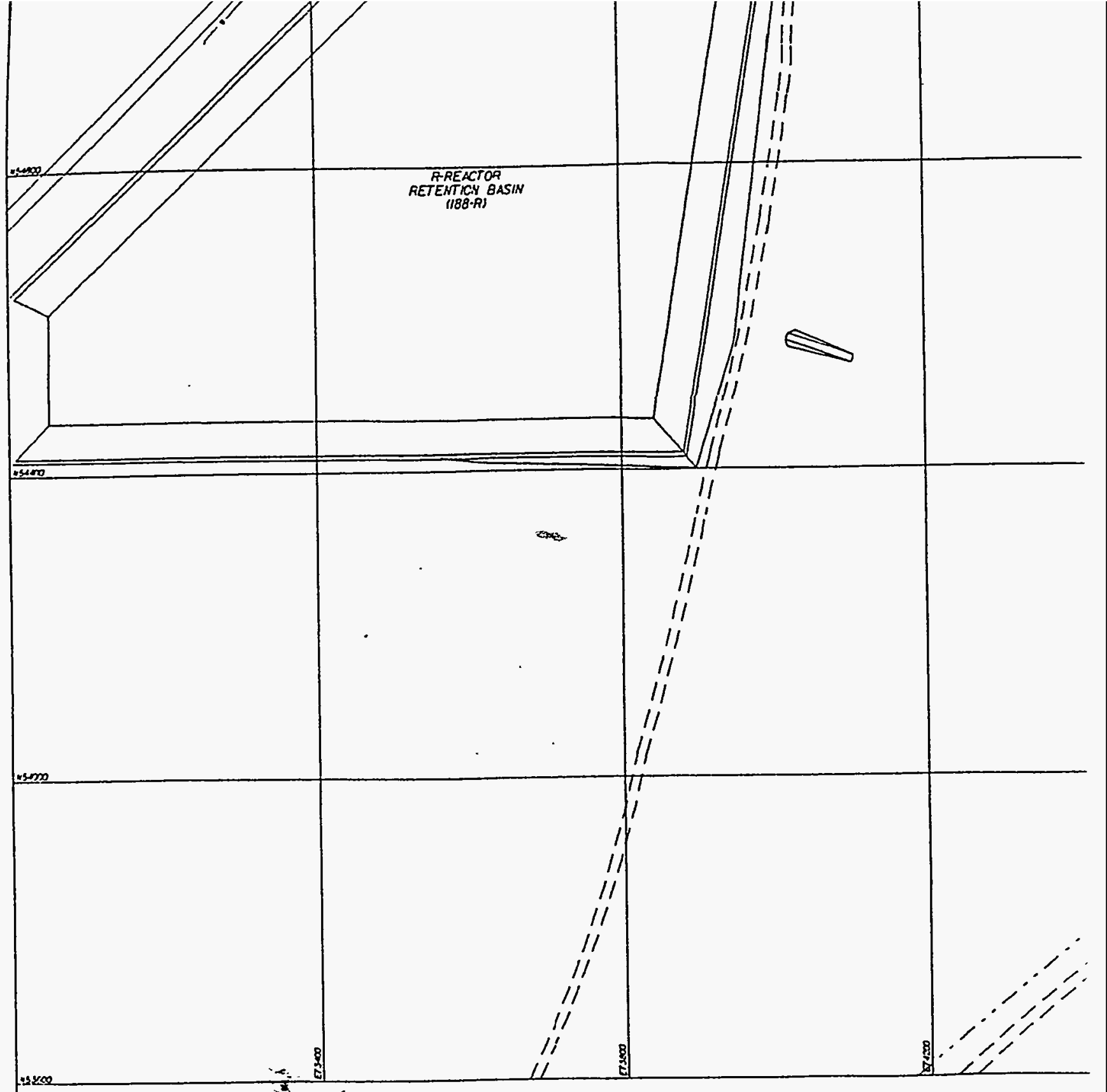

\section{WELL SYMBOL LEGEND}

- water table aQuifer

- GROUNOWATER MONITORING WELLS

MCBEAN AQUIFER

tF. GROUNDWATER MONITORING WELLS

CONGAREE AQUIFER

ts GROUNDWATER MONITORING WELLS

RAC 1 WELL ID

CONTOUR LEGEND

CONTOURS: $50,100,1000$

UNITS: $\mathrm{pCi} / 1$

PDWS: $50 \mathrm{pCi} / \mathrm{I}$

2.00: DENOTES CONCENTRATIONS AT OR LESS THAN DETECTION LIMIT ND: NO DATA 


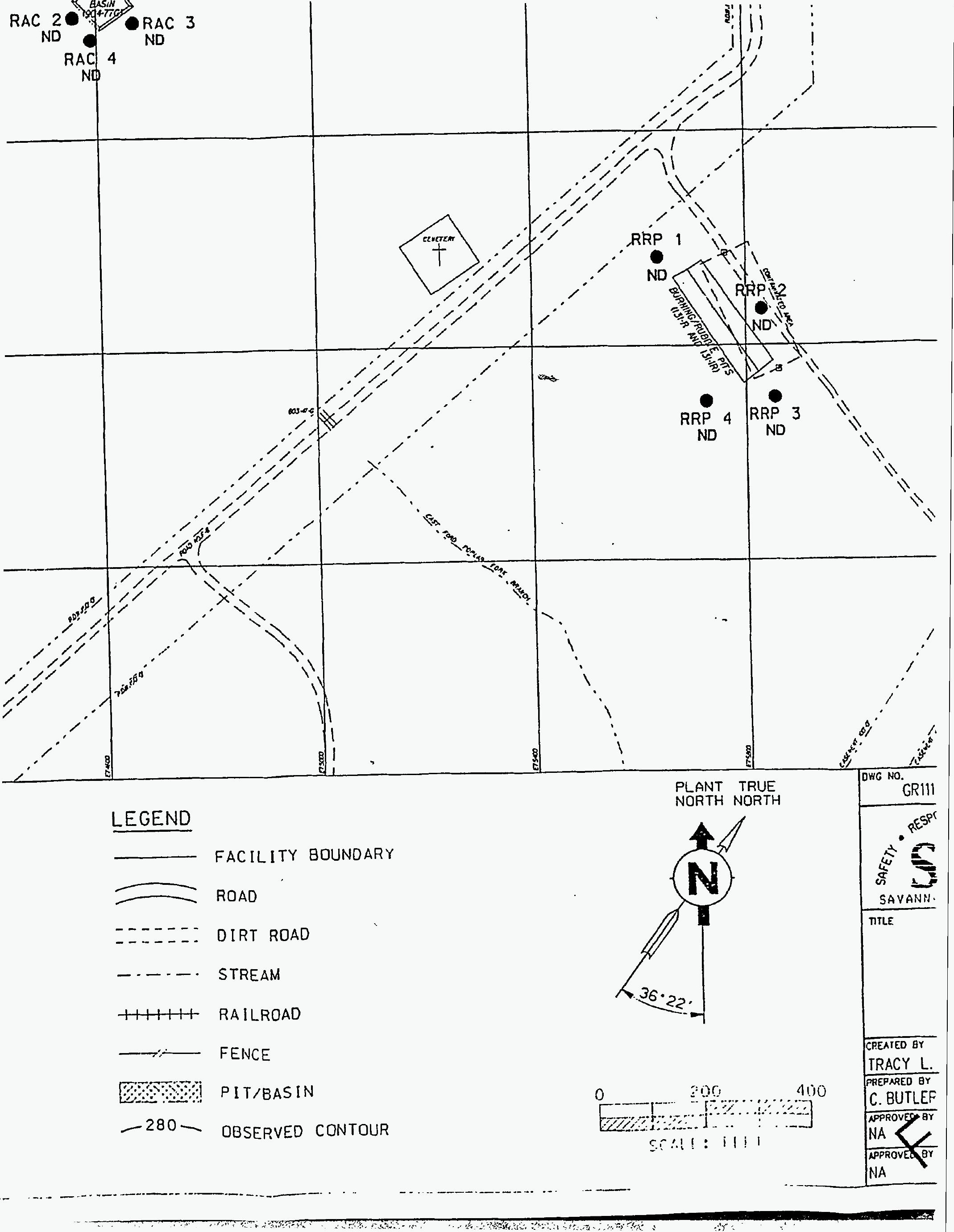




$$
\text { 泣 }
$$




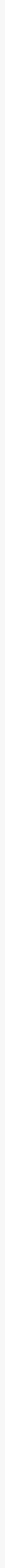




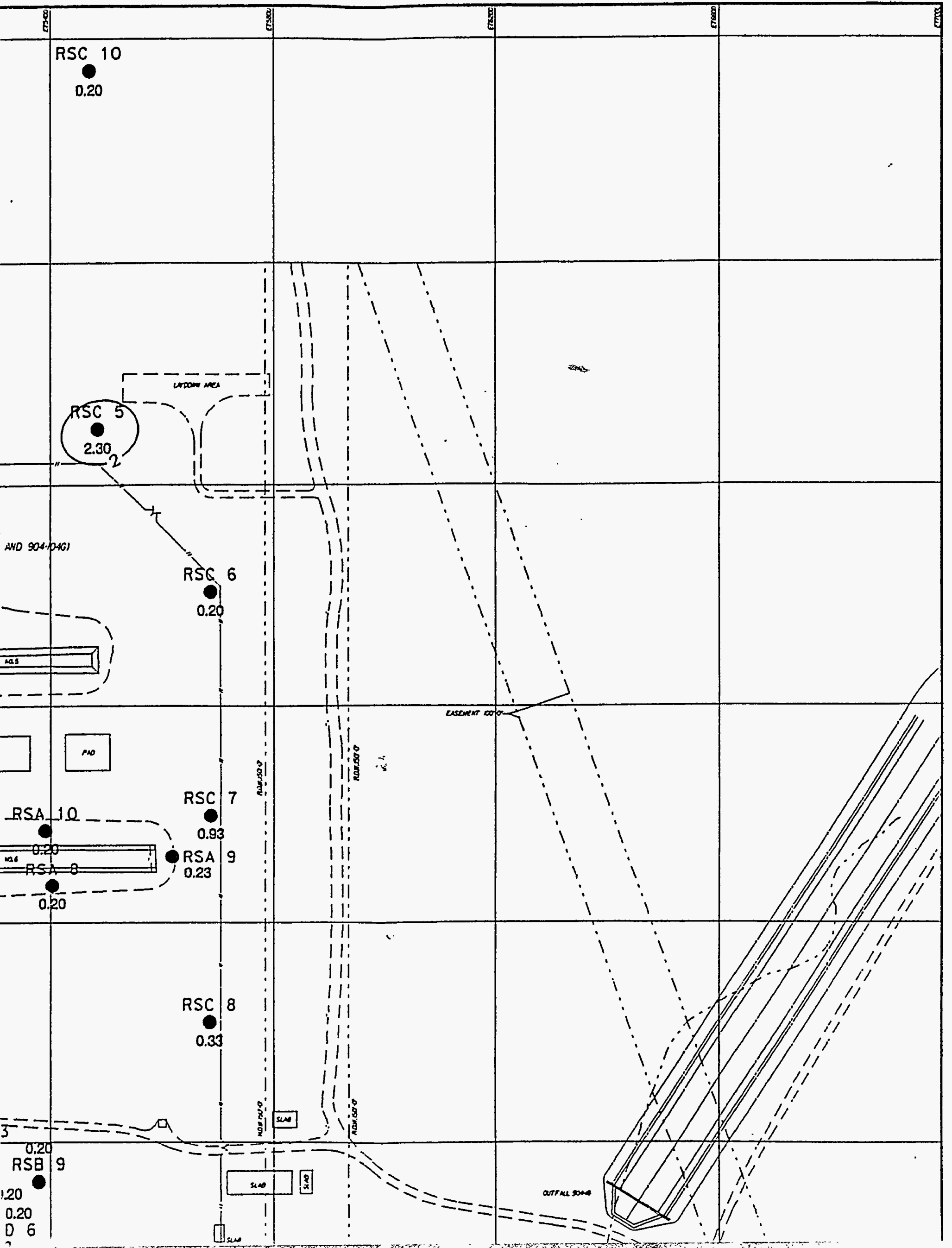




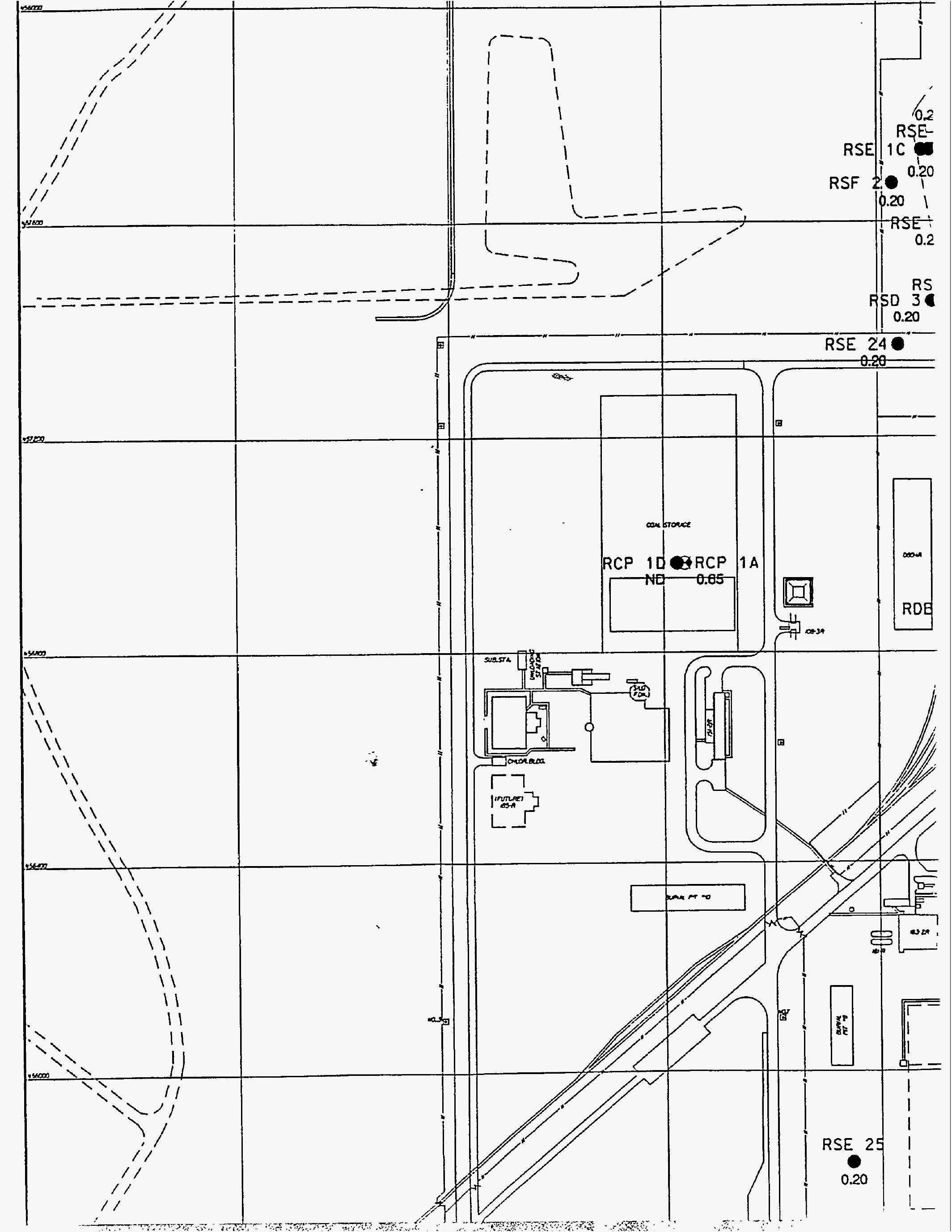




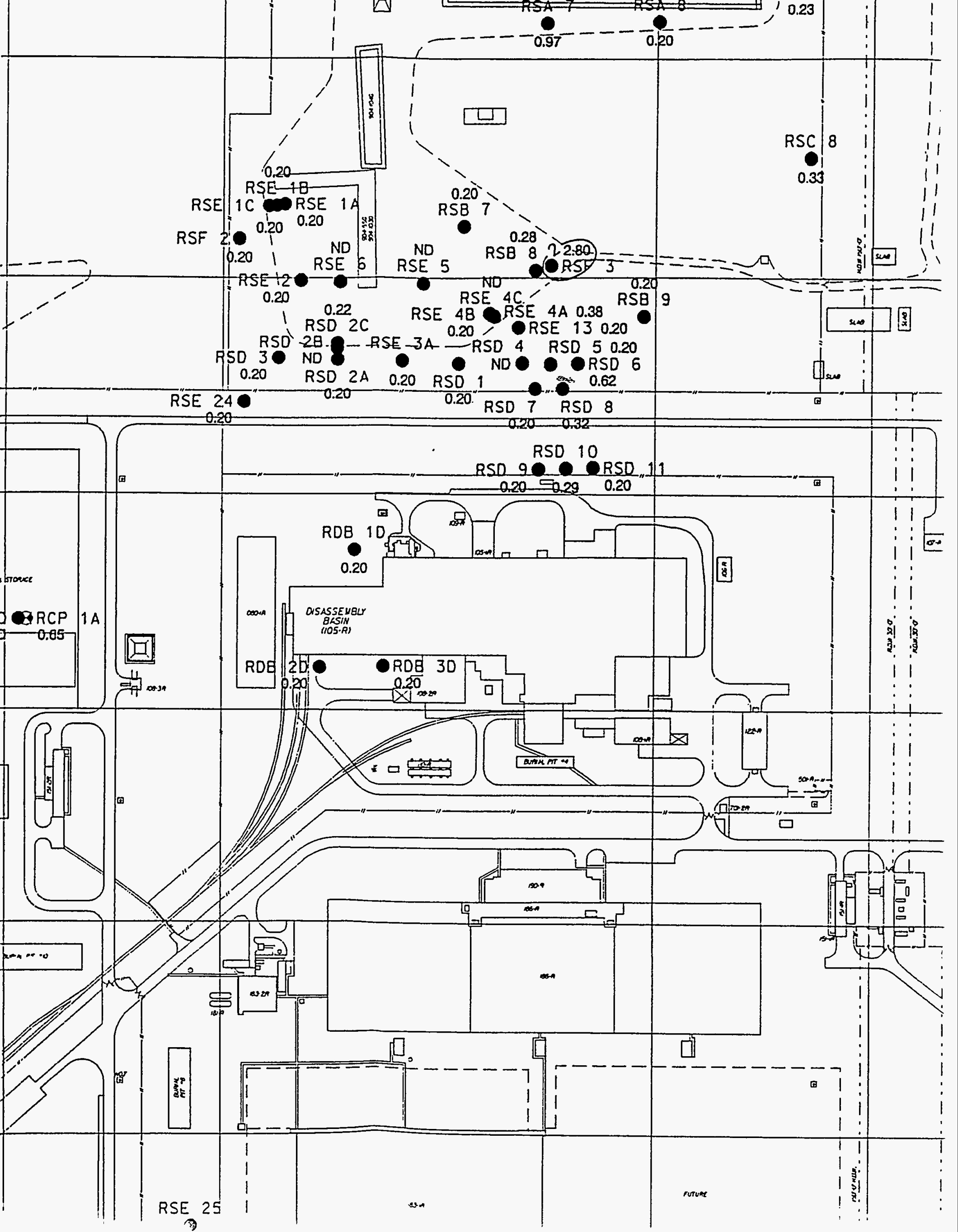




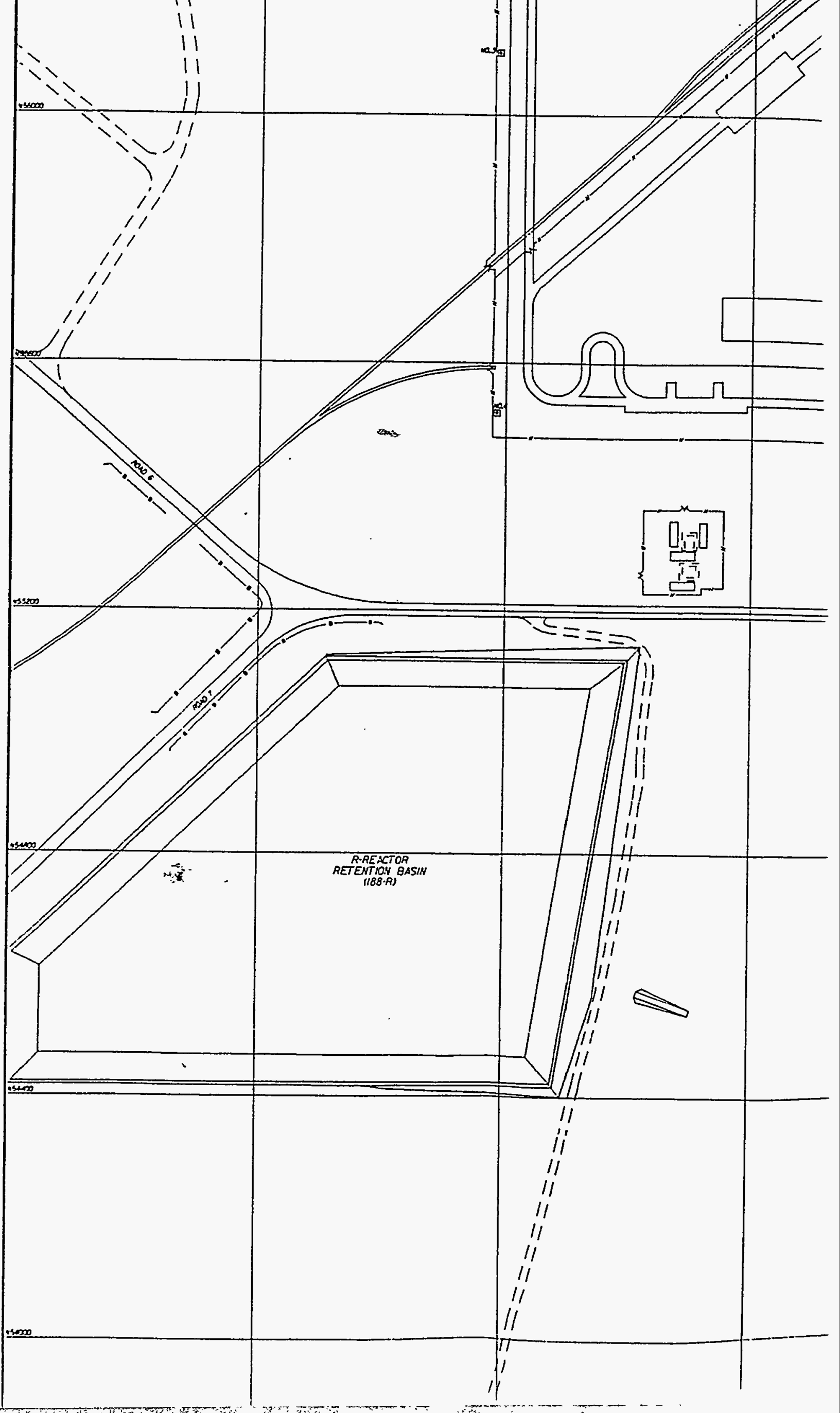




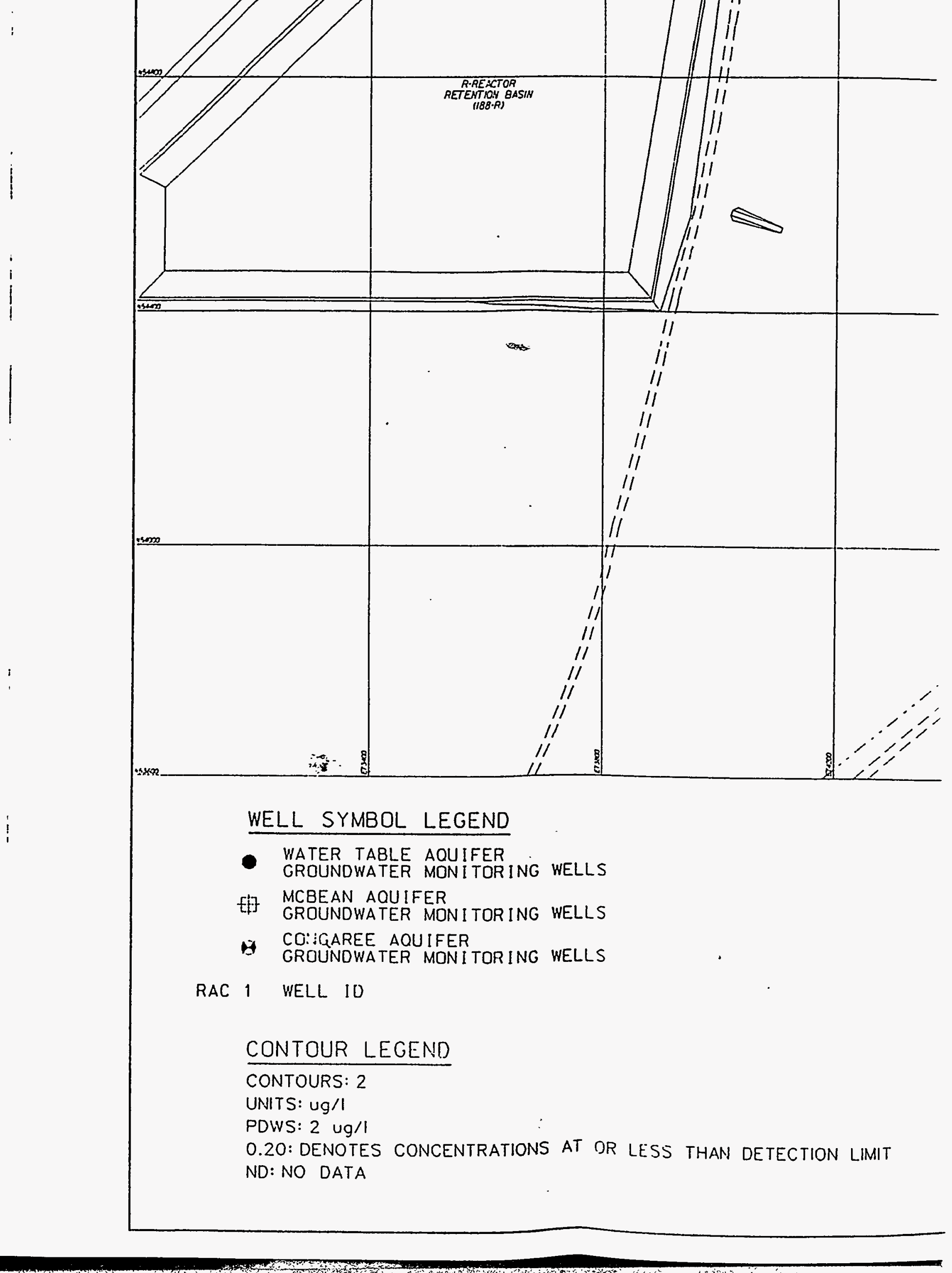





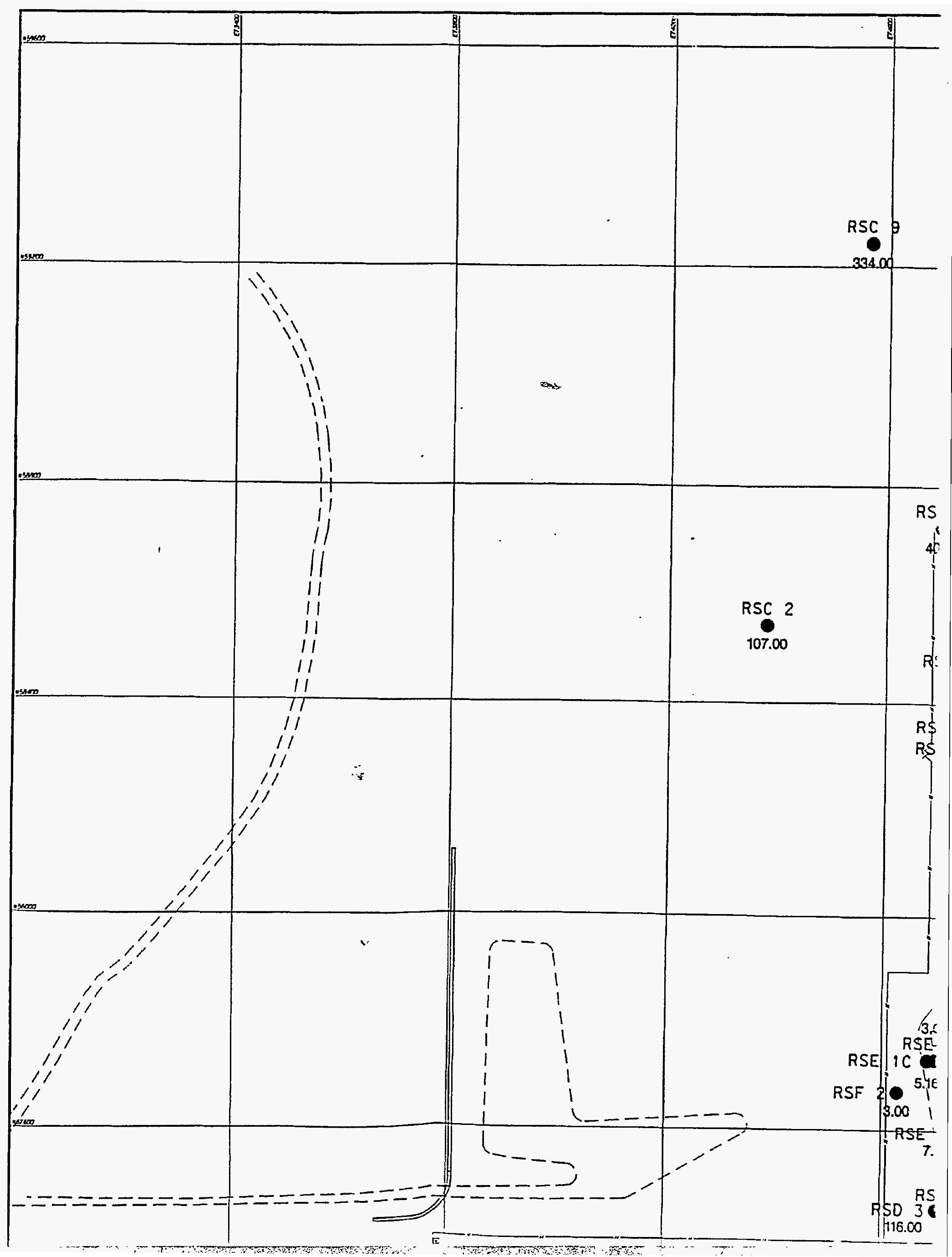




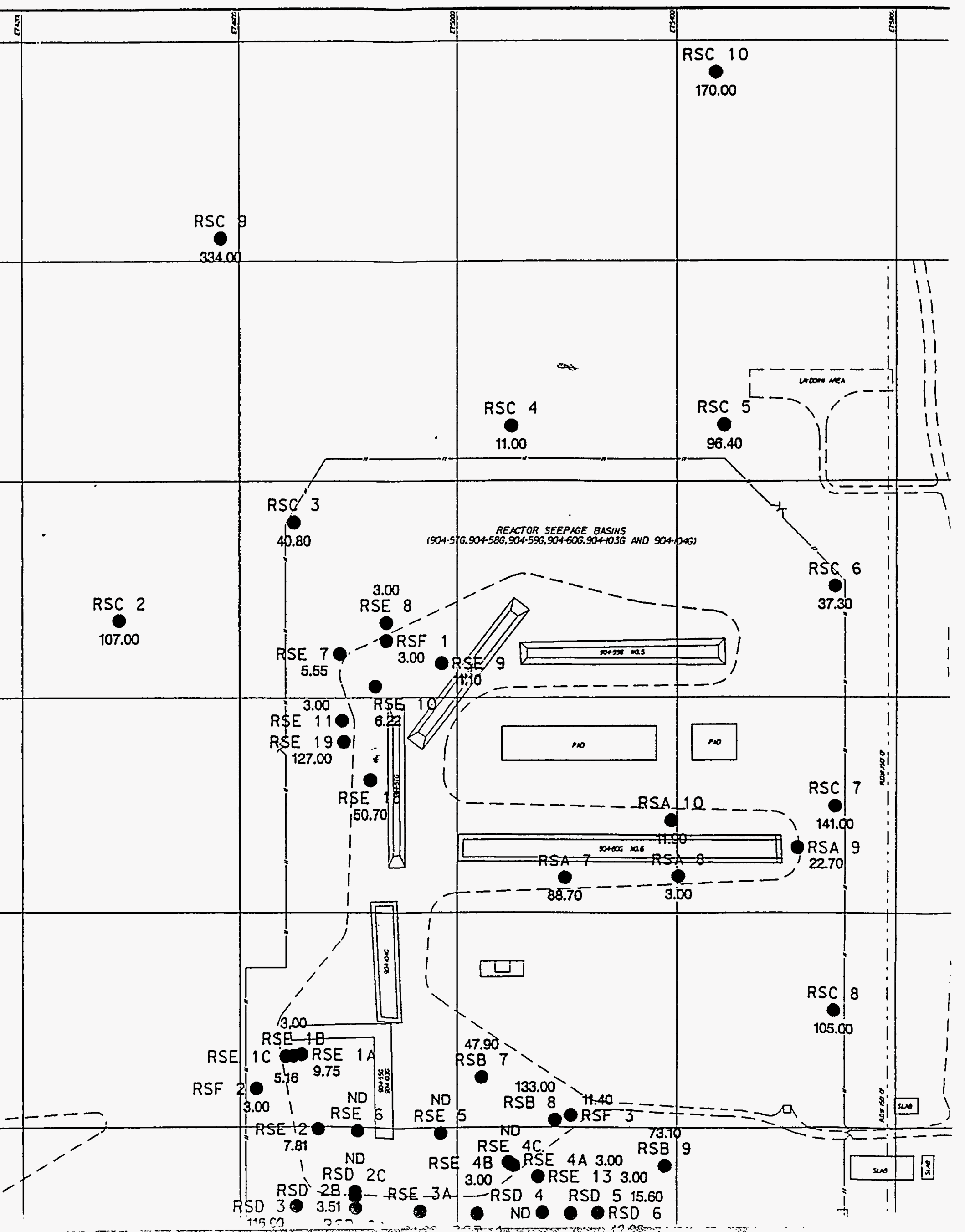




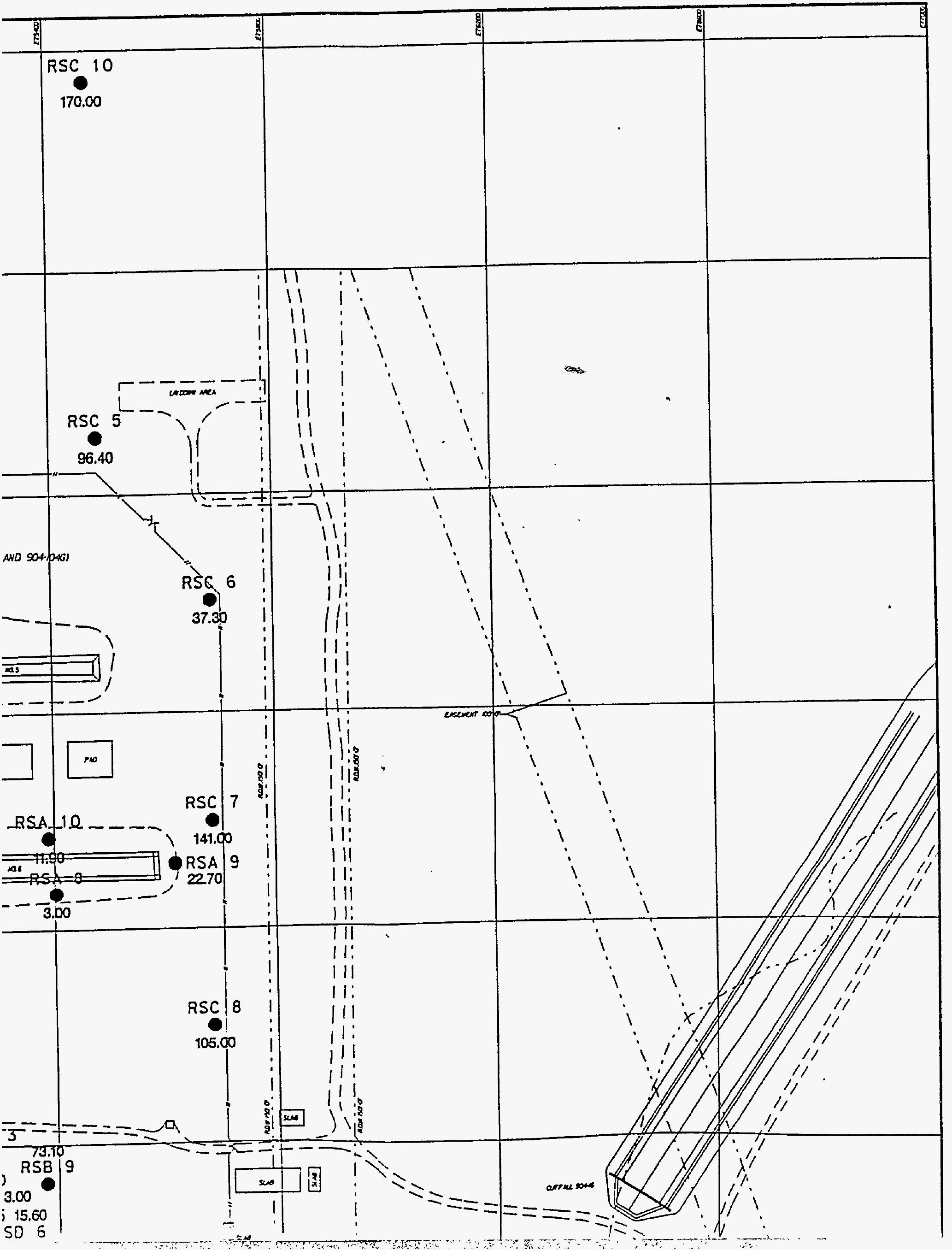




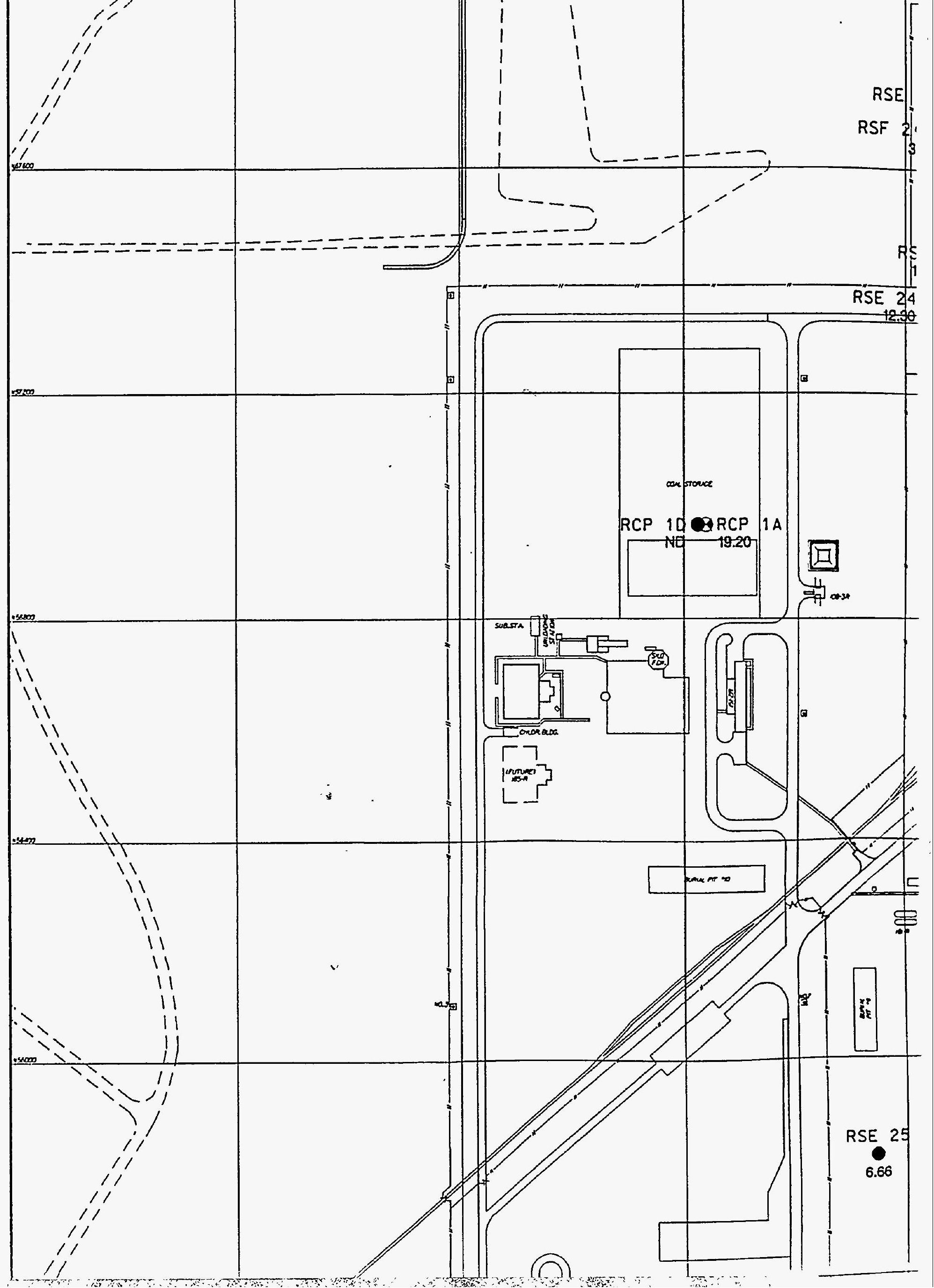





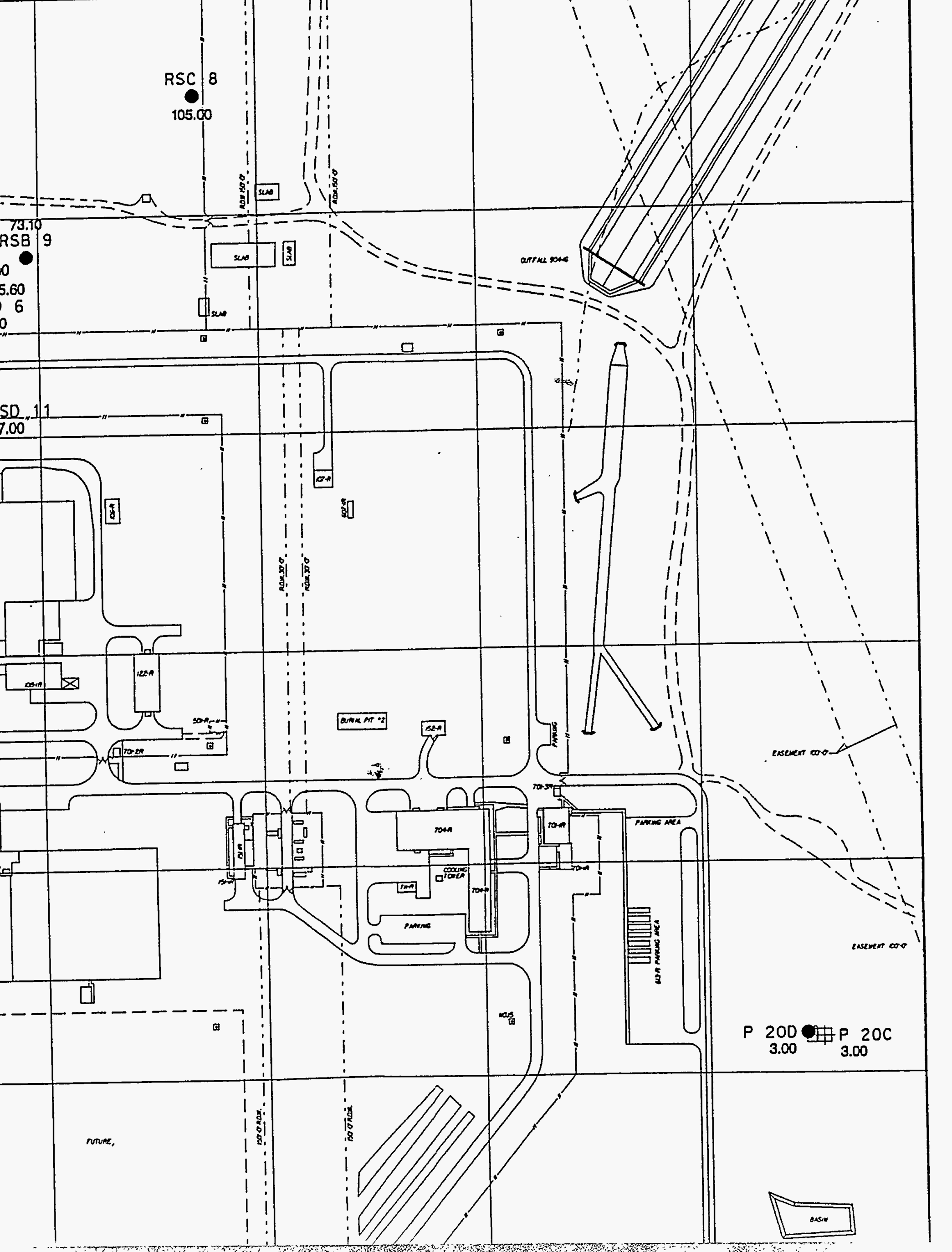




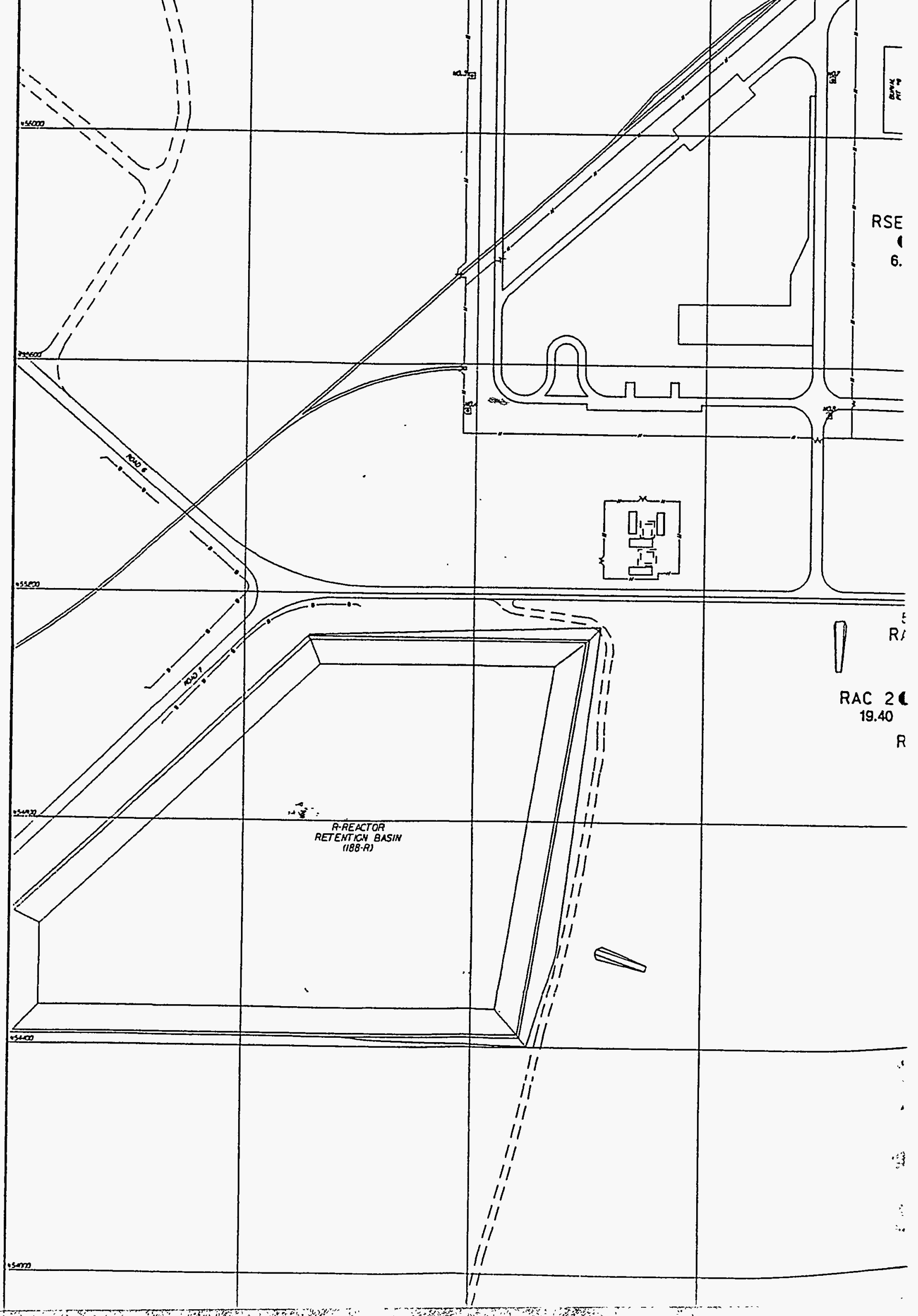




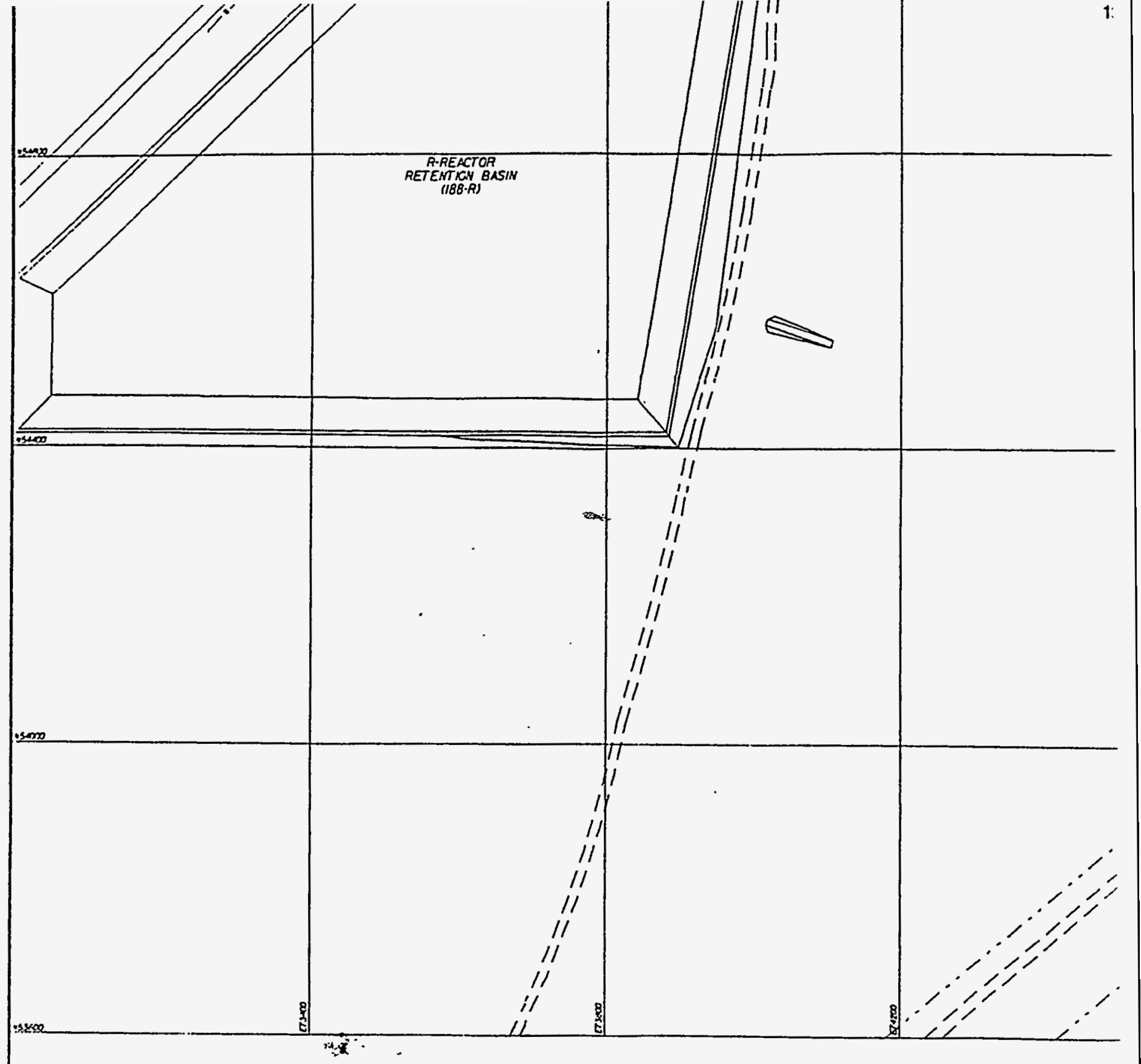

WELL SYMBOL LEGEND

- water table aQuifer

GROUNOWATER MONITORING WELLS

\#f MCBEAN AOUIFER

If GROUNDWATER MONITORING WELLS

a CONGAREE AQUIFER

9 GROUNOWATER MONITORING WELLS

RAC 1 WELL ID

CONTOUR LEGEND

UNITS: ug/I

PDWS: 50 ug/I

3.00: DENOTES CONCENTRATIONS AT OR LESS THAN DETECTION LIMIT ND: NO DATA 


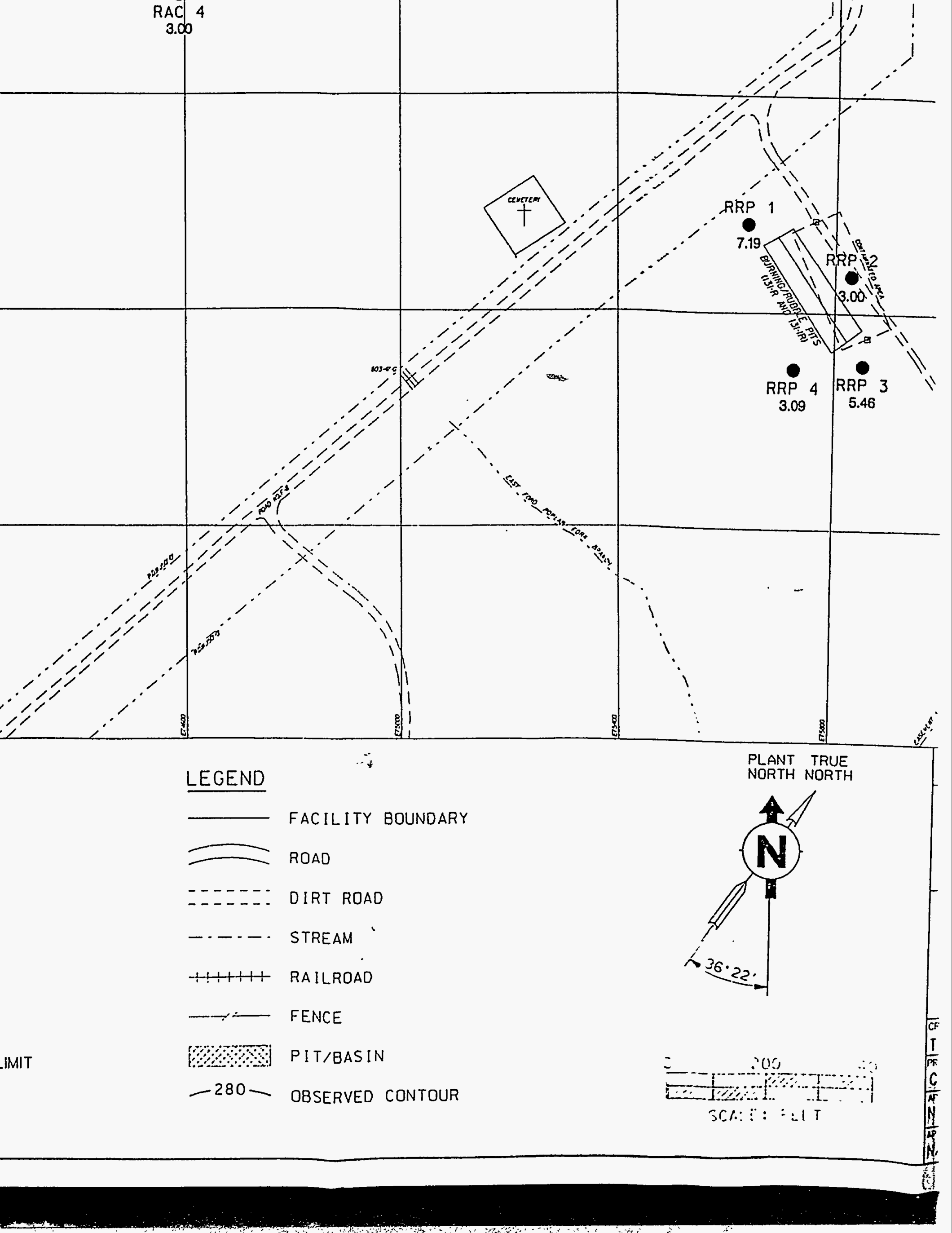




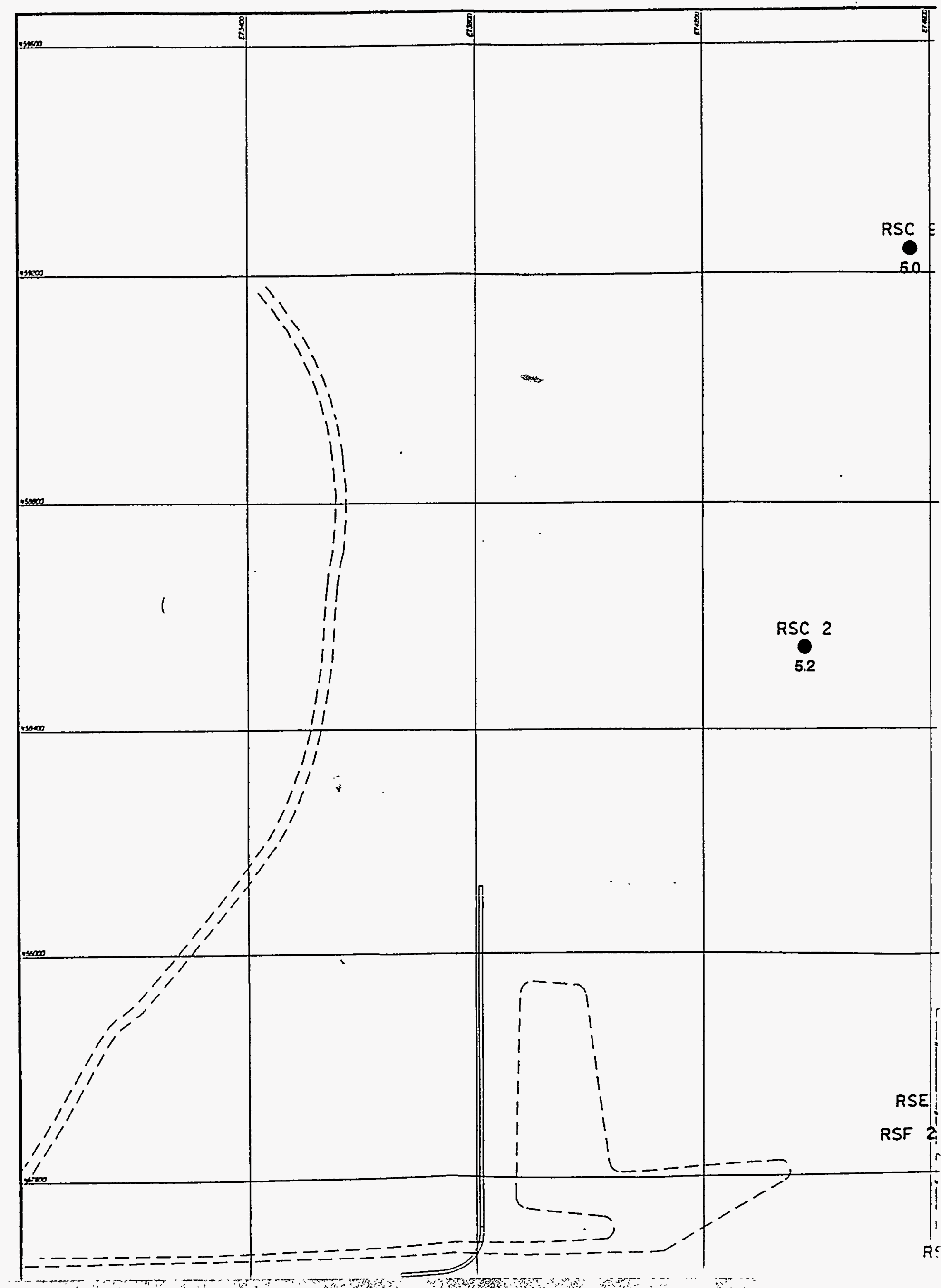




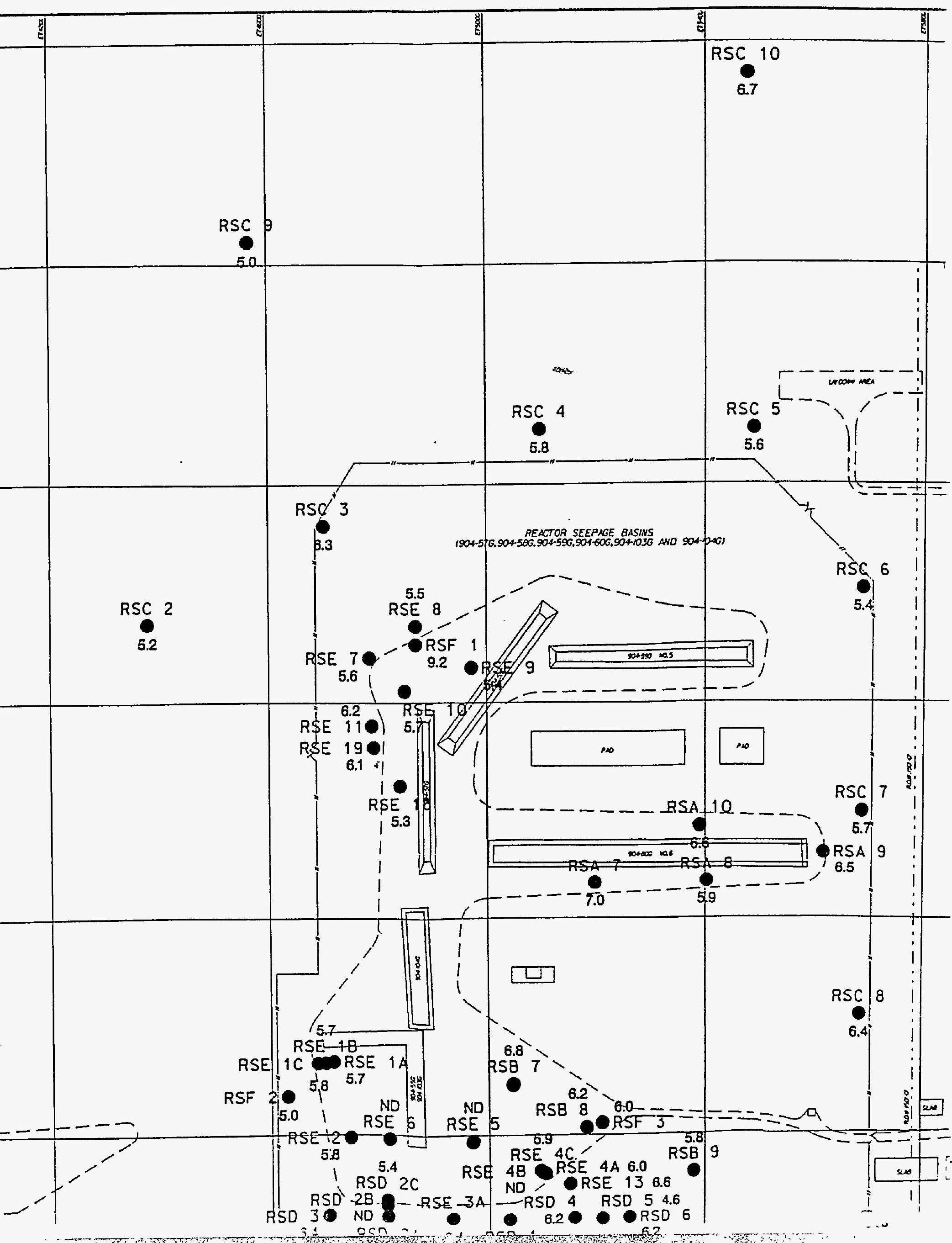




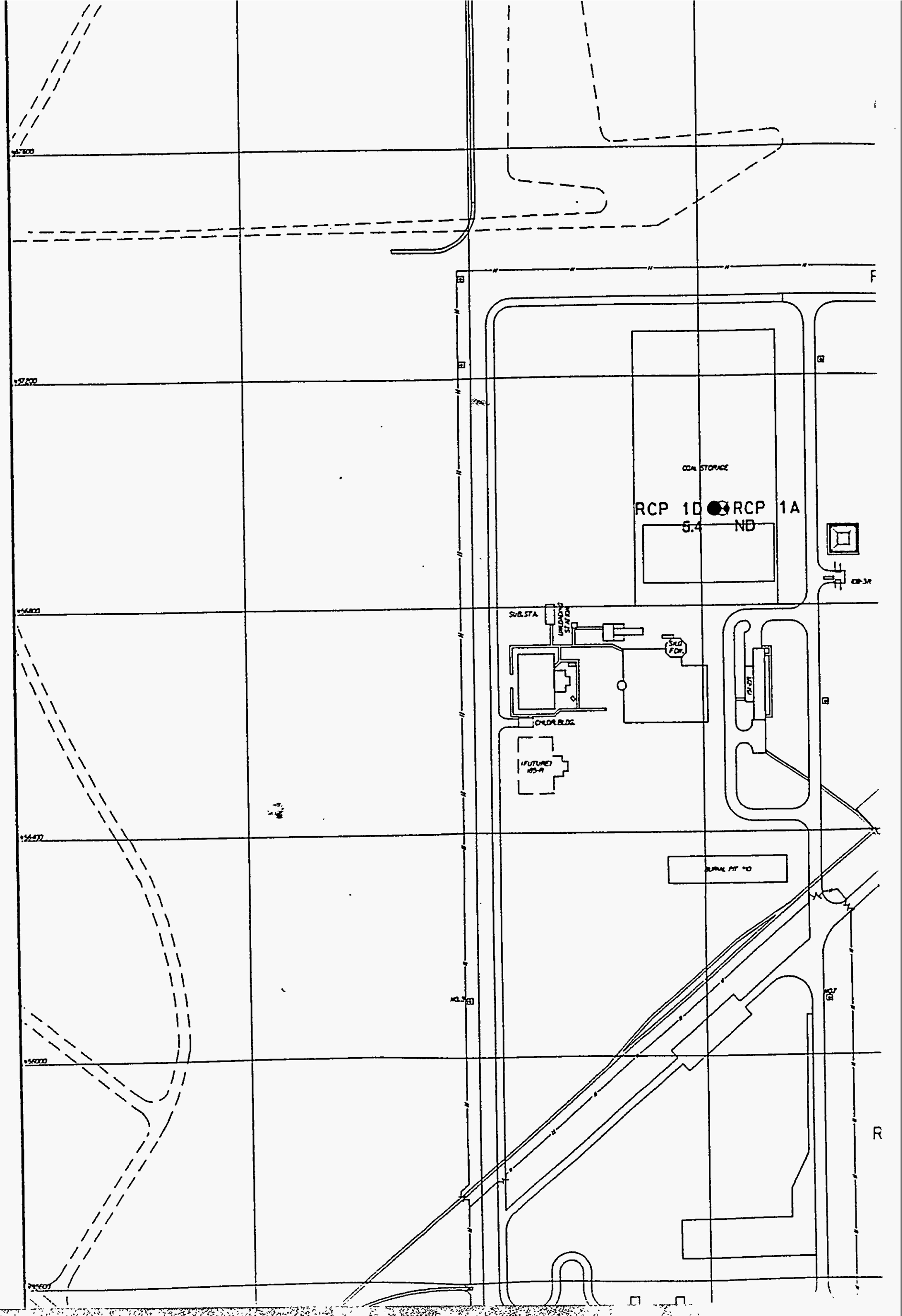




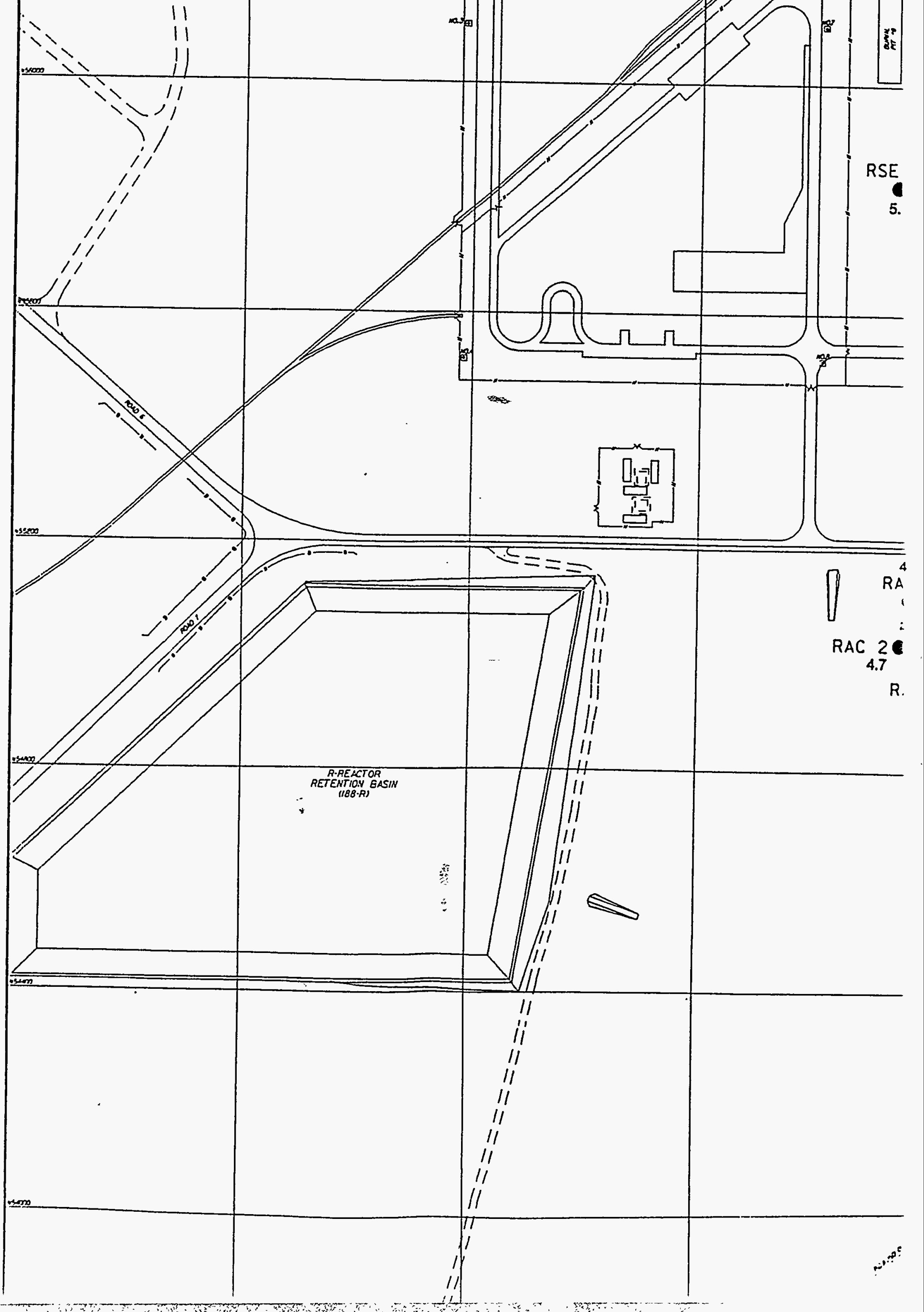




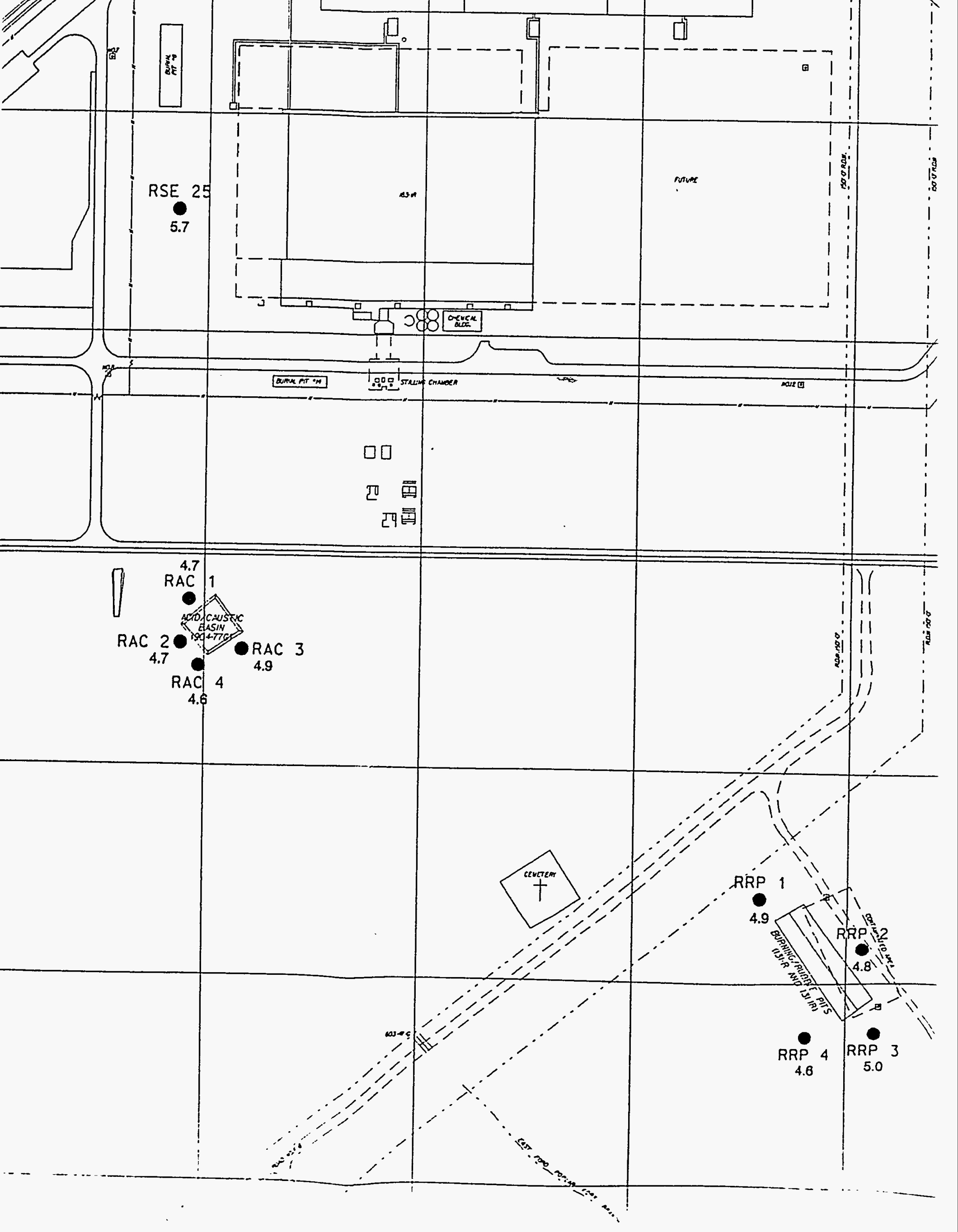




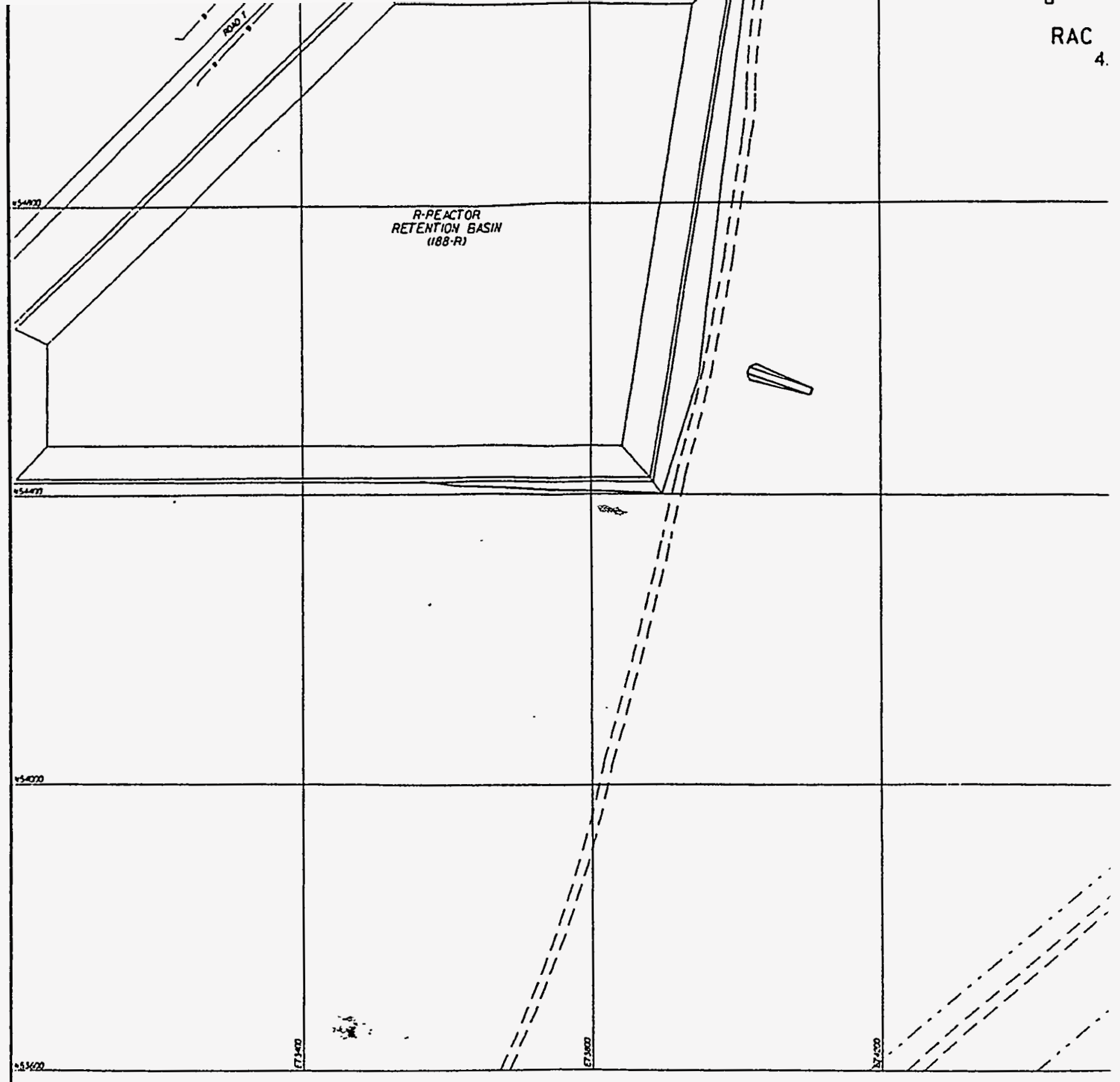

\section{WELL SYMBOL LEGEND}

- water table auvifer

- gROUNDWATER MONITORING WELLS

H MCBEAN AQUIFER

H GROUNDWATER MONITORING WELLS

- congaree aOUIFER

* GROUNDWATER MONITORING WELLS

RAC 1 WELL ID 


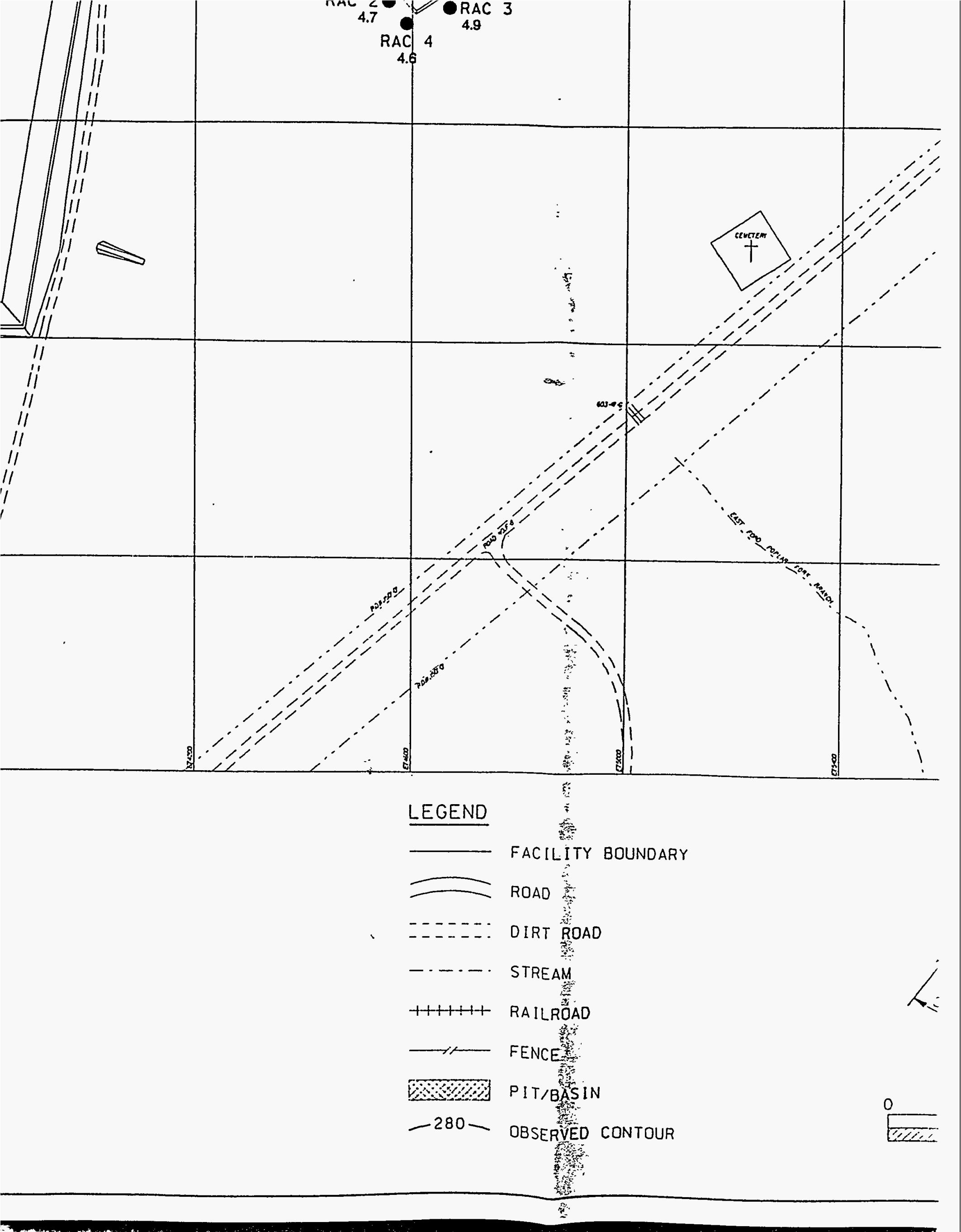




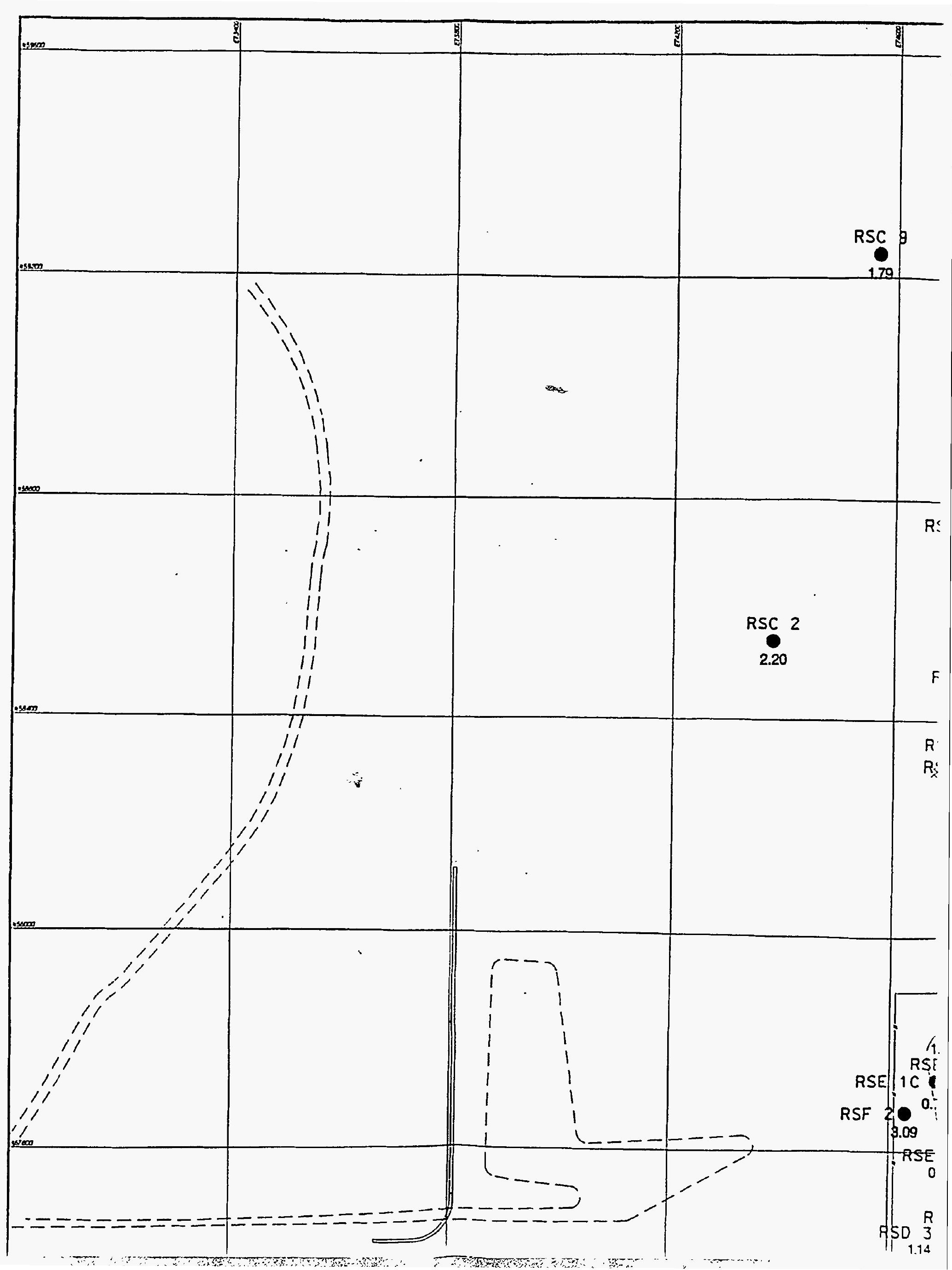




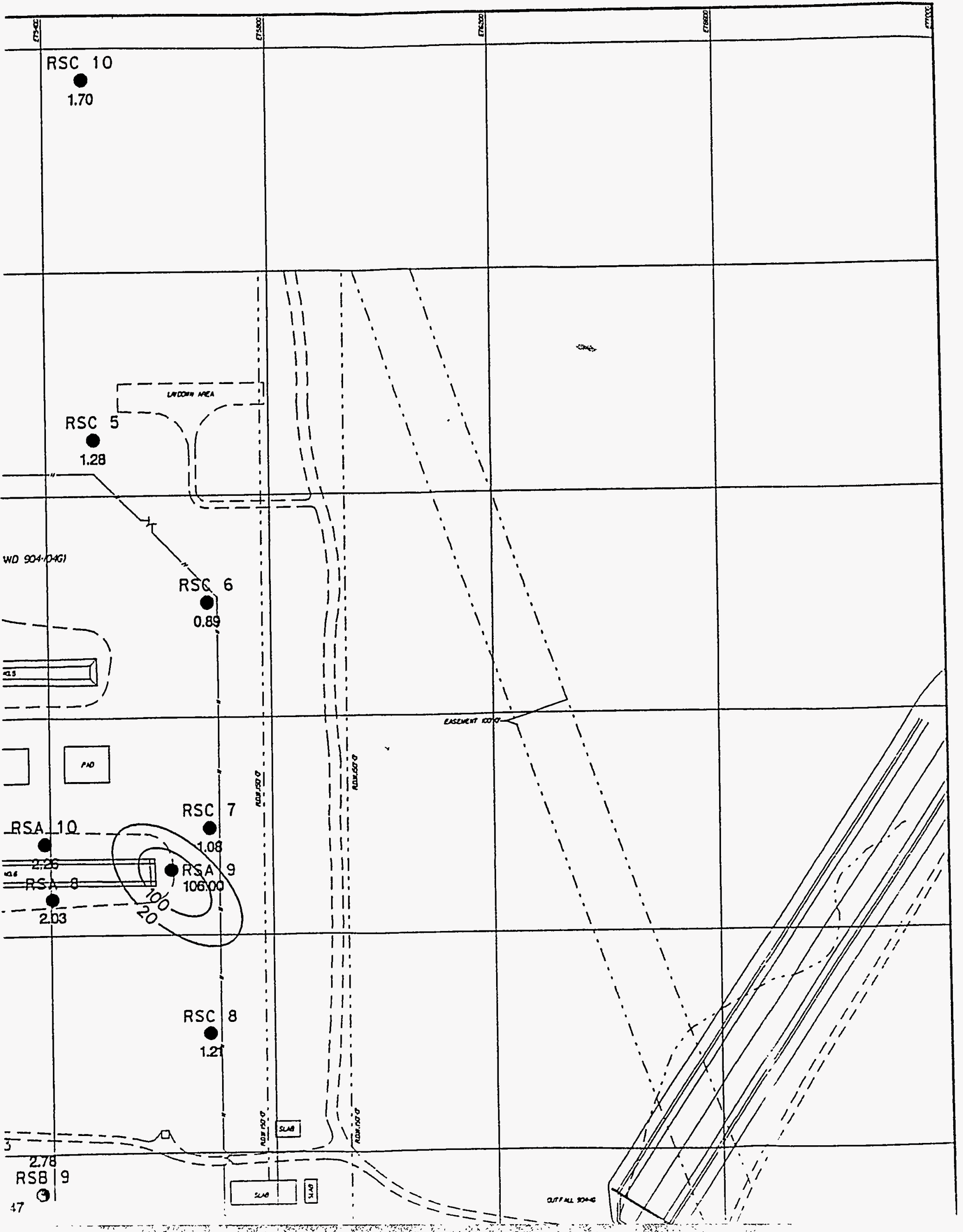




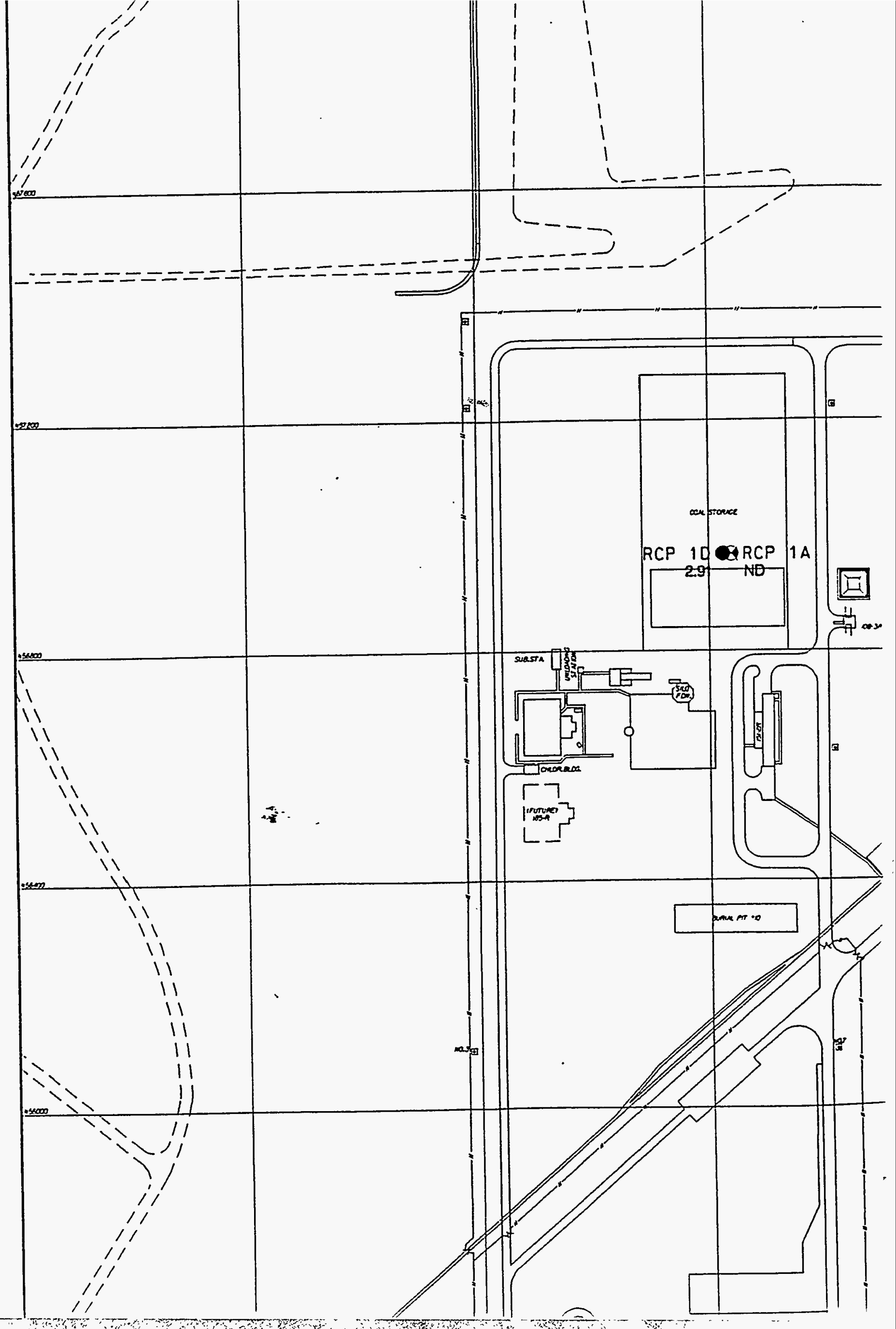




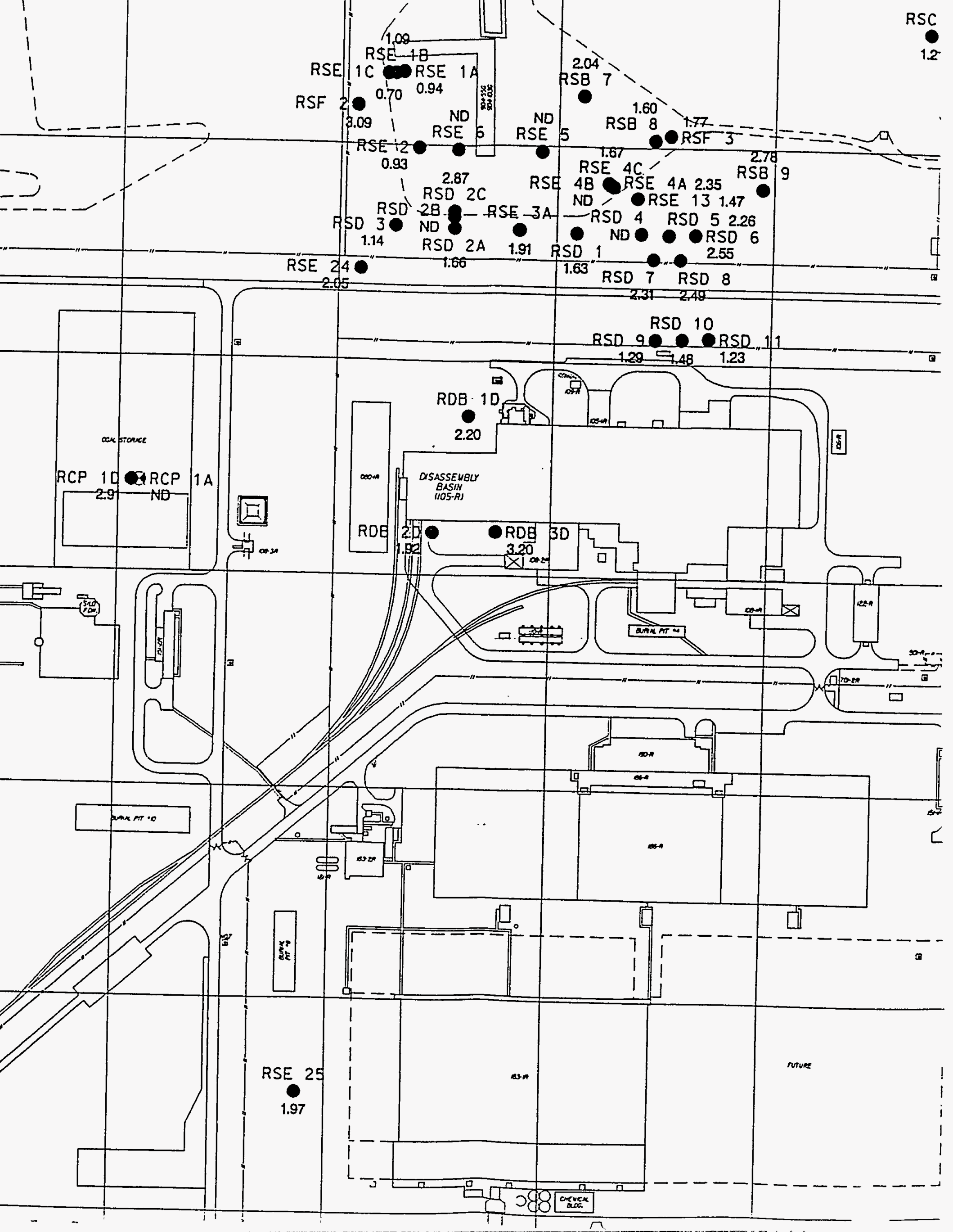




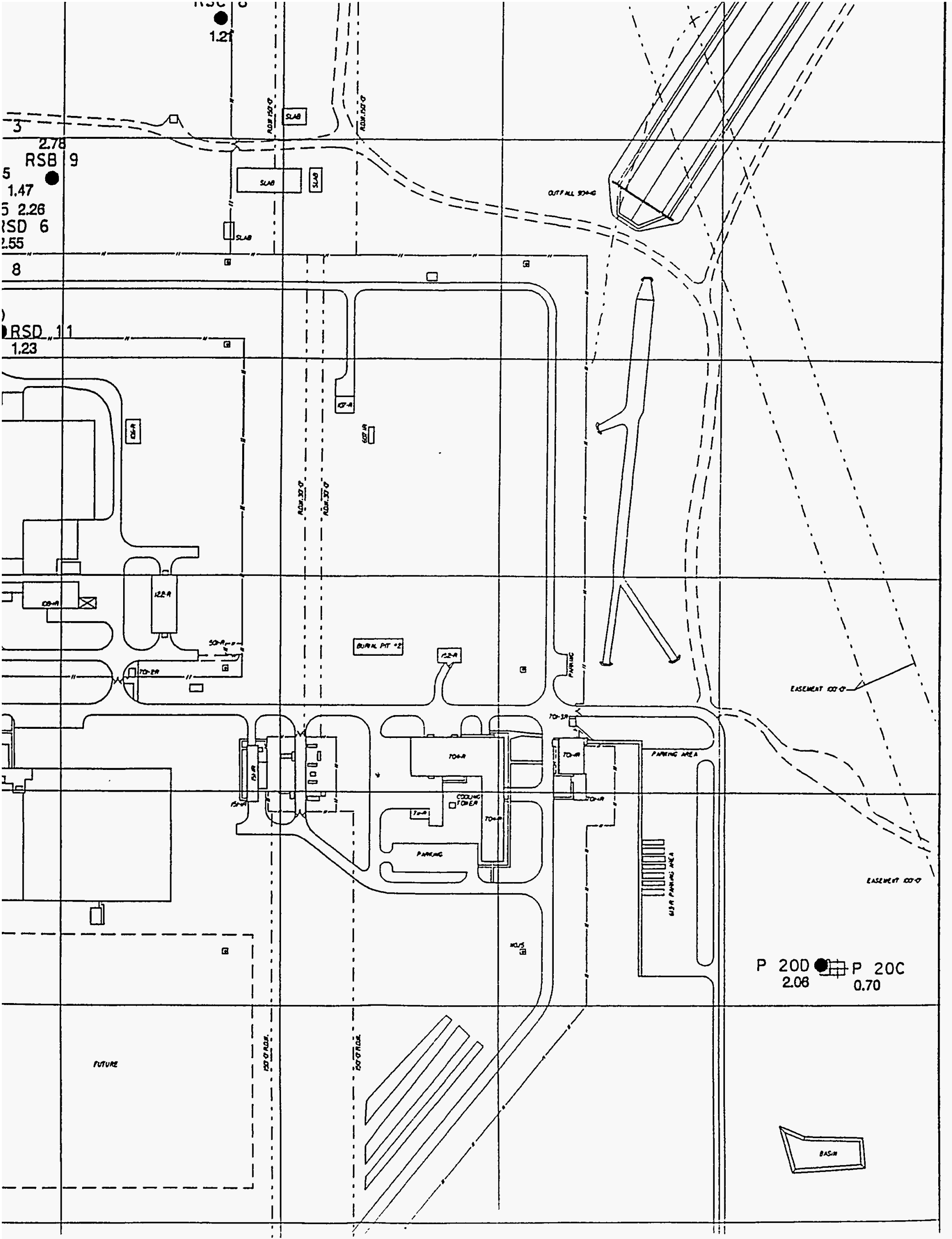




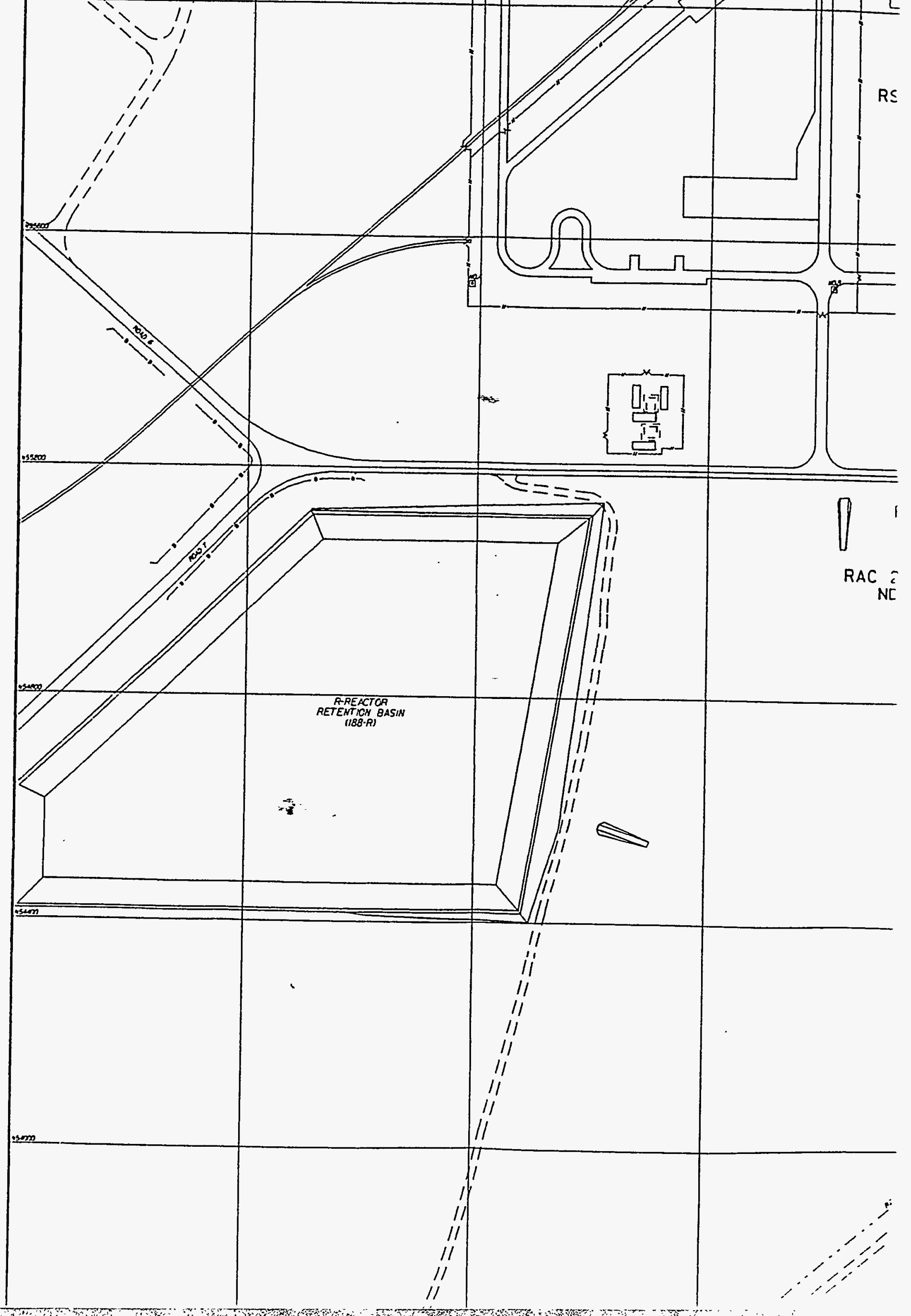


